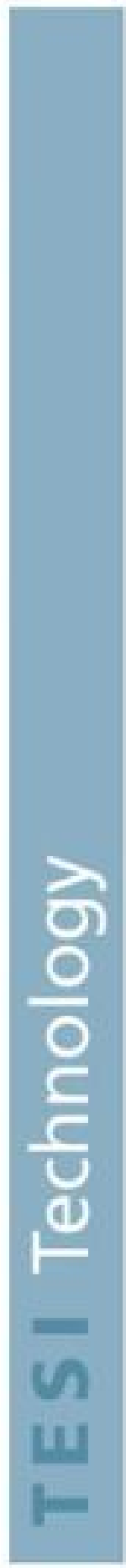

\title{
La frammentazione del paesaggio periurbano
}

\author{
Criteri progettuali per la riqualificazione \\ della piana di Firenze
}

Gabriele Paolinelli 


\section{UNIVERSITÀ DEGLI STUDI DI FIRENZE}

Tesi: Technology

1

\section{LA FRAMMENTAZIONE DEL PAESAGGIO PERIURBANO \\ Criteri progettuali per la riqualificazione della piana di Firenze}

Gabriele Paolinelli 


\begin{tabular}{l}
\hline La frammentazione del paesaggio periurbano / Gabriele Paolinelli. - Firenze : \\
Firenze University Press, 2003. \\
(Tesi. Technology / Università degli Studi di Firenze, 1) \\
http://digital.casalini.it/fulltext/is.asp?isbn=8884530636 \\
Stampa a richiesta disponibile su http://epress.unifi.it \\
\\
ISBN 88-8453-063-6 (online) \\
ISBN 88-8453-070-9 (print) \\
712.094551 (ed. 20 ) \\
Architettura del paesaggio - Firenze <provincia >
\end{tabular}

Print on demand is available

(C) 2003 Firenze University Press

Università degli Studi di Firenze

Firenze University Press

Borgo Albizi, 28, 50122 Firenze, Italy

http://epress.unifi.it/

Printed in Italy 
Niente è immutabile

e poco è irrezersibile

(Richard T.T. Forman) 
Dipartimento di Urbanistica e Pianificazione del territorio

Dottorato di ricerca in Progettazione paesistica

http://www.unifi.it/drprogettazionepaesistica

Il dottorato di ricerca in Progettazione paesistica è stato attivato con il XII ciclo nel 1997, su iniziativa della professoressa Mariella Zoppi, che lo ha coordinato fino all'aprile 2000, quando il coordinamento è pasato al professor Giulio G. Rizzo. È in corso il XVIII ciclo.

Il dottorato ha attivato tre percorsi formativi da intendersi come linee guida per orientare le singole ricerche dei dottorandi: aree naturali, piano e progetto; verde urbano, piano e progetto; le risorse naturali nel paesaggio urbano: l'acqua. Gli esiti che si prefigurano alla fine del triennio di ricerca sono due: formazione di personale altamente qualificato da inserire in organismi di ricerca pubblici (università, CNR) o privati (fondazioni operanti nel settore) e formazione di una figura di paesaggista, sensibile ai valori dell'ambiente, delle aree protette e del paesaggio, in grado di coprire ruoli d'elevata responsabilità presso ministeri, agenzie per l'ambiente, regioni, province e comuni, parchi e riserve naturali. Il dottorato, oltre le normali lezioni previste dall'ordinamento, organizza periodicamente seminari, conferenze e convegni aperti agli studenti ed agli specializzandi.

Questo volume riporta gli esiti degli studi svolti dal 1999 nell'ambito del Dottorato di ricercain Progettzzione paesistica dell'Università di Firenze. L'elaborazione della ricerca è terminata nel novembre 2001. Latesi è stata discussa nel marzo 2002, presso il Dipartimento di Urbanistica e Pianificazione del territorio dellaFacoltàdi Architettura di Firenze (presidente della commissione Andrea Tosi, del Politecnico di Milano, commissar Biagio Cillo, dell'Università della Calabria, e Pompeo Fabbri, del Politecnico di Torino). La revisione perla pubblicazione è stata conclusa nel novembre 2002.

Il lavoro ha tratto importanti benefici dal supporto delle molte persone con cui ho avuto modo di confrontarmi in più occasioni di studio e di discussione teorica, come anche di applicazione in atre esperienze di ricerca.

Dei preziosi indirizzi scientifici per l'impostazione e lo sviluppo della ricerca, ringrazio i coordinatori del dottorato Giulio G. Rizzo e Mariella Zoppi, il tutor Augusto Boggiano, a cui devo inoltre importanti suggerimenti riguardanti le specificità del territorio fiorentino, e gli altri componentiil Collegio dei docenti, fra i quali in particolare Antonello Boatti, Gabriele Corsani, Guido Ferrara, Massimo Olivieri e AndreaTosi,peri molti contributi di discussione, risultati determinanti nella stesura dello studio.

Tutta la mia gratitudine va inoltre a Giuliana Campioni, Mario Di Fidio, Matteo Guccione, Bernardino Romano che, in ambiti scientifici ed occasioni di incontro diverse, hanno sostenuto e stimolato varie fasi della ricerca ed al caro amico Biagio Guccione, che ho avuto vicino dalle prime elaborazioni, fino a quelle conclusive.

Un ringraziamento speciale ad Antonella Valentini, per i costanti e sostanziali contributi scientifici alla revisione critica del lavoro, ed a Simona Cappellini e Chiara Tesi, per l'attenta predisposizione di alcunebai cartografiche, utilizzate nella parte applicativa della ricerca.

L'autore. 
Ad Antonella 



\section{Indice}

$\begin{array}{lr}\text { Prefazione di Giulio G. Rizzo } & 9\end{array}$

PREMESSA: IL TEMA E LA METODOLOGIA DI STUDIO 11

1 IL PAESAGGIO PERIURBANO CONTEMPORANEO ATTRAVERSO I FENOMENI DI FRAMMENT AZIONE STRUTTURALE

1.1 Processi di modificazione del paesaggio di pianura delle aree ad elevata pressione insediativa 17

1.2 La frammentazione paesistica come fenomeno di modificazione e criterio di descrizione $\begin{array}{ll}\text { del paesaggio periurbano } & 32\end{array}$

2 LE CONOSCENZE ANALITICHE FINALIZZATE

$2.1 \quad$ Individuazione dei temi di analisi finalizzata 41

2.2 I fattori territoriali di frammentazione paesistica 43

2.3 Gli indicatori primari di frammentazione paesistica 56

3 LA DIAGNOSI STRUTTURALE DI FRAMMENTAZIONE PAESISTICA

3.1 Elementi di impostazione metodologica della diagnosi

3.2 Criteri interpretativi afferenti alla diagnosi di incidenza dei fattori territoriali 80

3.3 La diagnosi delle condizioni di stato del paesaggio $\quad 89$

3.4 La diagnosi delle alterazioni potenziali del paesaggio 99

4 VERSO UN MODELLO DI SCENARIO STRATEGICO PER LA RIQUALIFICAZIONE DIFFUSA DEL PAESAGGIO PERIURBANO

$4.1 \quad$ Il contributo simulativo degli scenari teorici di riqualificazione diffusa del paesaggio $\quad 103$

4.2 Criteri progettuali per la riqualificazione diffusa delle aree periurbane: i sistemi lineari $\begin{array}{ll}\text { di continuità paesistica } & 108\end{array}$

5 LA FRAMMENTAZIONE DEL PAESAGGIO PERIURBANO: ESITI ED INDIRIZZI DI SVILUPPO DELLA RICERCA 137

\section{BIBLIOGRAFIA}

Riferimenti di interesse generale per lo studio del paesaggio e degli insediamenti 141

Riferimenti di interesse specifico per lo studio della frammentazione paesistica $\quad 144$

$\begin{array}{ll}\text { - paesaggi culturali e naturalità diffusa } & 145\end{array}$

- frammentazione paesistico-ambientale e reti ecologiche $\quad 145$

$\begin{array}{ll}\text { - greenways } & 147\end{array}$

$\begin{array}{ll}\text { - corridoi paesistici e corridoi ambientali } & 148\end{array}$

$\begin{array}{lr}\text { Riferimenti relativi al territorio di indagine } & 149\end{array}$

APPENDICE

A Schede di identificazione morfologica dei fattori territoriali di frammentazione paesistica

B I corridoi paesistici secondo alcuni casi studio di piani e progetti per la conservazione della natura e del paesaggio in Italia 



\section{Prefazione}

di Giulio G. Rizzo

Gabriele Paolinelli ha concluso brillantemente il dottorato di ricerca in Progettazione paesistica e la sua tesi di dottorato ha meritato apprezzamenti che ci sono pervenuti da più parti. La nostra convinzione sulla validità del lavoro scientifico, unitamente agli apprezzamenti, ci ha spinto a chiedere a Gabriele Paolinelli di pubblicare la sua ricerca, allo scopo di rendere partecipe sia la comunità scientifica, sia il pubblico degli studenti, di un lavoro ben costruito.

Gabriele Paolinelli è noto agli studiosi delle tematiche paesaggistiche perché ha già pubblicato numerosi contributi interessanti tra i quali si ricordano i volumi, dei quali è stato curatore insiemecon altri, V erso nuovi modelli di pianificazione integrata, Disegnare il verde, Piani del V erde \& Piani del Paesagoio. Dunque si tratta di presentare, e lo facciamo con piacere, il lavoro di un ricercatore ormai noto.

Il libro affronta il tema della frammentazione paesistica estendendolo dalla mera accezione ambientale, alle alterazioni strutturali del paesaggio ${ }^{1}$.

Il concetto di frammentazione è esteso dalla sola accezione ambientale, alle alterazioni strutturali del paesaggio, indagando la frammentazione paesistica come modificazione complessa, responsabile di effetti negativi, oltre che sugli habitat e le popolazioni animali e vegetali, su molte funzioni ambientali importanti per l'ecologia umana, per le permanenze storiche e per l'articolazione morfologica del paesaggio. La ricerca affronta il problema delle caratteristiche delle entità paesistiche lineari (cornidoi), e ne studia l'idoneità ad interagire con la parcellizzazione e la congestione degli spazi periurbani. Paolinelli, pur ricorrendo al lessico dell'ecologia applicata, usa la denominazione di sistema di continuitù in luogo di quella di corridoio, ritenuta più adatta, dal punto di vista definitorio, a coprire la complessità delle configurazioni degli spazi aperti che a diversi livelli possono svolgere funzioni di continuità e riequilibrio strutturale. I principi teorici di diversificazione delle modalità d'uso e gestione degli spazi aperti per l'equilibrio paesistico-ambientale costituiscono la base dell'articolazione dei ruoli individuati per tali sistemi, con riferimento ad ipotesi di scenari d'intervento e di governo calibrati sulla congestione e sui deficit qualitativi dei paesaggi periurbani di pianura. Proprio in questi ultimi luoghi i sistemi lineari di spazi aperti assumono un potenziale ruolo strategico nelle politiche territoriali per il riequilibrio paesistico ed ambientale ${ }^{2}$.

Utilizzando le specificità disciplinari della progettazione paesistica, talvolta ancora marginali nell'ambito degli studi riguardanti la frammentazione, lo studio sviluppa un percorso di analisi, diagnosi e proposta progettuale, incentrato sulla ricerca di relazioni scientificamente significative tra le categorie della frammentazione paesistica e quelle dei sistemi lineari degli spazi aperti. L'autore

${ }^{1}$ La ricerca, di tipo applicato, è basata su osservazioni condotte sul paesaggio periurbano occidentale di Firenze, con il supporto scientifico di appropriati riferimenti bibliografici e delle indicazioni derivanti dall'esame di alcuni significativi çasi.

${ }^{2}$ Un'accezione strategica, riferita a sistemi complessi di spazi aperti differenziati, per destinazioni d'uso e ruoli paesisticoambientali, è distinta da un'accezione morfologica, riferita a sistemi semplici di spazi aperti, relativamente omogenei, normalmente parti costitutive anche dei precedenti. 
sottolinea la necessità di effettuare un'esplicita distinzione fra i concetti di continuità paesisticoambientale e di reticolarità ecologica. Infatti, trattare la frammentazione paesistica come fenomenologia e problematica territoriale, e i corridoi, o sistemi di continuità, come soggetti di studio e di analisi/progettazione, non coincide con il definire e progettare le reti ecologiche. Paolinelli ritiene che le politiche di riqualificazione diffusa, impostate secondo tale approccio, possano comprendere le azioni per la conservazione e lo sviluppo della reticolarità ecologica e, conseguentemente, della biodiversità, consentendo condizioni di qualità paesistica delle aree ad elevata pressione insediativa in grado di supportare la conservazione dinamica degli stessi equilibri ecosistemici del paesaggio ${ }^{3}$.

Lo studio di Gabriele Paolinelli è il convincente epilogo di un complesso percorso conoscitivo in una realtà assai diffusa in Italia, quella della frammentazione del paesaggio nelle aree di pianura e di frangia urbana, che si conclude con la proposizione di un modello di scenario strategico per la riqualificazione diffusa del paesaggio periurbano. Al di là della metodologia di studio, che pur delinea validi elementi di novità nell'analisi conoscitiva e nella diagnosi strutturale della frammentazione paesistica, è interessante il tentativo di simulazione di scenari teorici di modificazione del paesaggio.

Paolinelli giunge alla proposizione di criteri progettuali per la riqualificazione delle aree periurbane e alla definizione ed esemplificazione del concetto, relativamente nuovo, di sistema lineare di continuità paesistica. $\mathrm{E}^{\prime}$ chiara la derivazione del concetto di sistema lineare di continuità paesistica dal concetto già noto di corridoio ecologico, ma la ricerca evidenzia con altrettanta nettezza gli elementi di autonomia disciplinare propri del Dottorato di Progettazione paesistica, brillantemente frequentato da Gabriele Paolinelli, che rendono tali sistemi lineari delle tessere fondamentali nella ricostruzione dell'unità del paesaggio periurbano.

I risultati definitori e metodologici contenuti nel testo aprono varie possibilità di applicazione. Si segnalano, in particolare, i territori che le amministrazioni pubbliche di livello diverso hanno il compito di governare: dalle attività di formazione dei piani regolatori generali comunali di nuova generazione, a quelle relative ai piani territoriali provinciali di coordinamento, come ad un livello superiore, ai piani regionali d'indirizzo territoriale ed ai piani di parchi ed aree protette. Infine, importanti spazi d'applicazione, dal punto di vista tecnico-scientifico, si possono avere nella progettazione delle infrastrutture viarie e tecnologiche, nelle sistemazioni di bonifica idraulica e di messa in sicurezza idrogeologica, di riordino agricolo. Un utilizzo dei modelli di conoscenza, sperimentati in questa ricerca, riguarda le possibili applicazioni nell'ambito delle valutazioni strategiche interne ai processi di formazione dei piani, come in quelle ex post. Soprattutto nelle valutazioni ex ante, è possibile sviluppare applicazioni funzionali ad obiettivi di pianificazione di politiche d'uso differenziato degli spazi per l'equilibrio paesistico-ambientale.

In conclusione Paolinelli ha svolto, come appare evidente, un lavoro non comune, punto di arrivo di un percorso assai articolato, che conduce ad una diversa ed innovativa modalità di uso delle conoscenze per il controllo dell'uso dello spazio fisico.

Firenze, dicembre 2002.

3 E sono a interessanti e per nulla secondari gi approfondimenti proposti nel testo sui temi del rapporto tra frammentazione del paesaggio nelle aree di frangia urbana e pianificazione comunale e territoriale e quelli delle relazioni intercorrenti tra paesaggio e fenomeni strutturali, quali le dinamiche economiche e sociali. 


\section{Premessa: il tema e la metodologia di studio}

"La società contemporanea è, nel suo complesso, impreparata o non disposta ad intendere il paesaggio nella sua vera essenza, ad osservarne la particolare fisionomia insita nell'aspetto orografico, geologico e vegetale, a valutarne i contenuti storici e le qualità artistiche. Da questa incapacità del nostro tempo ad intendere il paesaggio deriva la difficoltà ad assicurarne la tutela e la protezione: l'erroneo credo nella illimitatezza delle risorse naturali ha determinato un processo irreversibile di dilapidazione del paesaggio naturale, di alterazione di quello storico" ${ }^{\prime 4}$.

In Italia, occorre considerare anche il peso non indifferente della concezione del paesaggio come bellezza naturale e culturale che, a partire da radici estetiche, letterarie, storiche e filosofiche, ha contribuito a diffonderne la considerazione come immagine della realtà, in luogo della realtà stessa ${ }^{5}$. Nell'opera di Rosario Assunto, alla concezione ottocentesca di tipo vedutistico, del paesaggio-quadro, è succeduta la concezione filosofica, di assai più ampia portata culturale, del "paesaggio come luogo della memoria e del tempo" ${ }^{\prime \prime}$. Ma, mentre in E uropa e negli Stati Uniti la concezione degli aspetti percettividel paesaggio si è sviluppata insieme agli studi sulle sue articolazioni strutturali essa, nel nostro paese, ha indubitabilmente condizionato in maniera decisiva gli orientamenti dei sistemi legislativi ed amministrativi di governo del territorio, come gli indirizzi e gli sviluppi della ricerca scientifica e della formazione tecnica.

In realtà "ciò che noi chiamiamo paesaggio, non in senso estetico, ma geografico, è sovente un ecosistema o una costellazione di ecosistemi. L'armonia estetica è sovente il riflesso di una armonia molto più sostanziale di fenomeni, che solo una sottile e attenta indagine può rilevare e valutare in termini quantitativi"'7.

La complessità degli aspetti definitori che sono stati individuati dai molteplici studi sviluppati intomo al paesaggio, in particolare nella seconda metà del XX secolo, e la conseguente articolazione del panorama delle discipline e delle relative competenze, comportano regolarmente l'esigenza della collocazione culturale e scientifica dei lavori, come elemento minimo di chiarezza, utile ad evitare parte degli equivoci che ad oggi persistono su questi temi. Questa ricerca fa espressamente riferimento al paradigma disciplinare dell'architettura del paesagoio, nell'ambito del quale gli studi di pianificazione paesistica si avvalgono anche degli sviluppi prodotti dalla pianificazione ecologica e dalle applicazioni di ecologia del paesagoio.

Il paesaggio è ritenuto l'espressione del prodotto delle interazioni fra le risorse naturali e culturali e le diverse popolazioni viventi in un dato territorio. Esso è caratterizzato da una articolazione strutturale e da processi funzionali ed evolutivi di tipo dinamico. L'analisi della struttura profonda del paesaggio, derivante dalla sedimentazione storica - naturale e culturale - produce la base conoscitiva su cui si

\footnotetext{
4 Maniglio Calcagno A., A rchitettura del paesaggio. Ezoluzione storica, Calderini, Bologna, 1983.

${ }^{5}$ Romani V., Il paesagio: teoria e pianificazione, Franco Angeli, Milano, 1994.

${ }^{6}$ Romani V., 1994, cit.

${ }^{7}$ Giacomini V., I parchi nazionali, in Per la salzezza dei beni culturali in Italia, Atti e documenti della Commissione di indagine per la tutela e la valorizzazione del patrimonio storico, archeologico, artistico e del paesaggio, vol. II, Colombo, Roma, 1967.
} 
fondano le interpretazioni diagnostiche delle caratteristiche di sensibilità e vulnerabilità e le conseguenti valutazioni dei potenziali di uso e gestione delle risorse del territorio.

All'esplicitazione di tali presupposti teorici allo studio del paesaggio contribuiscono decisamente anche gli orientamenti culturali e tecnico-scientifici di due recenti documenti di livello internazionale e nazionale. La Convenzione europea, ove si constata che "il paesaggio svolge importanti funzioni di interesse generale, sul piano culturale, ecologico, ambientale e sociale e costituisce una risorsa favorevole all'attività economica" ${ }^{\prime 8}$. La proposta di carta del paesaggio denominata Carta di Napoli ${ }^{9}$, che fa riferimento esplicito all'accezione di paesaggio come sistema vivente in evoluzione, caratterizzato da una struttura, dal relativo funzionamento e da processi di cambiamento.

Trattando questioni definitorie, queste premesse non sono volte tanto ad una contrapposizione tra concezioni diverse del paesaggio ${ }^{10}$, quanto ad indicare i punti di partenza dello studio; esula infatti dai compiti di questo lavoro, e pare anche sempre più aleatorio, allungare la ragguardevole quantità di scritti che da anni continuano a vedere impegnate energie nella discussione delle diverse interpretazioni dei termini paesagoio, ambiente, territorio.

La perdita di identità del paesaggio, rimasto fino all'ultima guerra mondiale strettamente legato alle condizioni naturali e culturali da cui si è sviluppato, ha assunto nella seconda metà del secolo una intensità di progressione e diffusione assolutamente incomparata nella storia. La capacità di modificazione acquisita dall'uomo con lo sviluppo tecnologico lo ha portato, in un periodo relativamente molto breve, al di fuori dei ritmi coevolutivi che per secoli hanno governato le trasformazioni paesistiche; ritmi nei quali gli uomini, secondo culture empiriche ed intuitive, prima che scientifiche e tecniche, sono stati attori essenziali degli equilibri dinamici, insieme alle altre popolazioni viventi.

La predisposizione umana a questa perdita di contatto, rivelatasi in realtà tutt'altro che un affrancamento dalla dipendenza dalla natura, in una qualche forma, era probabilmente caratteristica anche delle società precedenti alla nostra ed è forse intrinseca alla stessa umanità. Occorre, senza idealizzare il passato, riconoscere che se nel Rinascimento fossero state disponibili le tecnologie contemporanee, con tutta probabilità si sarebbe andati già allora ben oltre quei mirabili ed artefatti equilibri tra natura e cultura che sono i giardini all'italiana e le sistemazioni idraulico-agrarie delle

${ }^{8}$ Consiglio d'E uropa, Conzenzione E uropea del Paesaogio, Firenze, 20 ottobre 2000.

9 AIAPP-FEDAP, Carta di Napoli. Il parere degli specialisti sulla riforma degli ordinamenti di tutela del paesaggio in Italia, raccomandazioni per la redazione di una carta del paesaggio avanzate al Convegno Nazionale FEDAP-AIAPP, La trasformazione sostenibile del paesaggio, con il patrocinio del Ministero per i Beni e le Attività Culturali e del Ministero dell'Ambiente, Napoli, 8 ottobre 1999. Il documento è stato approvato nel 1999 a Napoli dalle associazioni professionali e dalle società scientifiche italiane che si occupano di paesaggio ed ambiente, sotto l'egida della rispettiva federazione nazionale (FEDAP), e sottoposto all'attenzione dei lavori della prima Conferenza Nazionale sul Paesaggio, tenutasi a Roma nel 1999.

10 Sono numerose le formulazioni del concetto di paesaggio adottate o elaborate da autori di grande importanza che si distinguono nettamente da quella a cui si fa riferimento in questo studio, fortemente legata ai concetti di realtà e di struttura. Basti per tutti citare i nomi di Rosario Assunto, oppure di Joachim Ritter, del quale riteniamo utile riportare a titolo esemplificativo un breve passo riguardante l'essenza dell'entità paesaggio, tratto da "Paesaggio - Uomo e natura nell'età moderna" (Guerini, 1994). "Paesaggio è natura che si rivela esteticamente a chi la osserva e la contempla con sentimento: né i campi dinanzi alla città né il torrente come confine, strada mercantile e ostacolo per costruire ponti, né i monti e le steppe dei pastori e delle carovane (o dei cercatori di petrolio) sono, in quanto tali, "paesaggio". Lo diventano solo quando l'uomo si rivolge a essi senza uno scopo pratico, intuendoli e godendoli liberamente per essere nella natura in quanto uomo. Il suo "uscire" nella natura ne trasforma il volto. Ciò che è normalmente utile o inutile, in quanto terra incolta, ciò che per secoli era rimasto trascurato o considerato elemento estraneo, ostile e terrificante, diviene grande, sublime e bello: diviene esteticamente paesaggio". Anche Eugenio Turri fa riferimento al paesaggio come prodotto della percezione, delle emozioni, della rappresentazione, ma distinguendolo dal territorio, risultante da indagini, ricerche, informazioni. Ne "Il paesaggio come teatro" (Marsilio, 1998), Turri rileva che "una concezione come questa del paesaggio va poco d'accordo con quella proposta da R. Assunto là dove scrive: la realtà che dobbiamo studiare e su cui, se necessario, dobbiamo intervenire, è sempre il paesaggio e non l'ambiente, e meno che mai il territorio, in quanto, a suo dire, include in sé il territorio e l'ambiente. È invece più giusto e più positivo partire dal dato percettivo (cioè il paesaggio che esiste solo dal momento in cui c'è l'uomo che guarda e metabolizza in sé, nel proprio patrimonio culturale, quello sguardo), per arrivare poi alla concretezza del territorio e dell'ambiente con cui occorre veramente fare i conti se si vuole sopravvivere. Il paesaggio quindi come elemento mediatore all'interno del sistema natura-cultura e non come contenitore di ecologia e storia (Assunto, 1976)". 
pianure e delle pendici collinari. Del resto, anche in quell'epoca ed in quelle precedenti e successive, per le quali ben poco si addice, rispetto ad oggi, parlare di pressione antropica, non mancavano gli effetti sul paesaggio della fiducia incontrastata nel genio umano e della sua applicazione sconsiderata ad opere di disboscamento, dissodamento e di modificazione idraulica ${ }^{11}$.

La progressione del fenomeno dell'inurbamento, che nel XX secolo ha assunto proporzioni tali da avviare il pianeta al bilanciamento tra popolazioni abitanti nelle città e popolazioni abitanti nelle campagne, ha risvolti paesistici decisivi, non solo nei confronti degli equilibri ecologici, ma anche della articolazione morfologica del paesaggio e dei gradi di permanenza storica, che lo configurano come testo non riproducibile, quanto fragile, della evoluzione culturale umana. In tal modo il paesaggio periurbano contemporaneo, in particolare nei territori di pianura ad elevata pressione insediativa, costituisce la risultante dell'avvicendarsi di modalità di utilizzo delle risorse e dello stratificarsi di alterazioni strutturali profonde e sempre meno reversibili, anche in ragione dei relativi effetti cumulativi.

Da ormai oltre vent'anni, importanti filoni di ricerca afferenti all'ecologia applicata ed alla biologia conserzazionale si sono concentrati sul fenomeno della frammentazione ambientale derivante dai processidi trasformazione spaziale. Oggetto delle indagini e delle definizioni teoriche sono le popolazioni animali e vegetali ed i loro habitat, nell'ambito della problematica generale della conservazione della biodiversità.

In questo studio si sostiene la tesi che oltre che di frammentazione ambientale occorra parlare di frammentazione paesistica, anche per quanto essa stessa costituisca una fondamentale concausa della prima. Secondo tali presupposti, nella misura in cui non si tratti esclusivamente degli effetti sulle popolazioni animali e vegetali, assumono rilievo gli approcci di ecologia umana e di ecologia urbana, come quelli di saperi scientifici inerenti la conoscenza degli aspetti culturali della morfologia e della storia del paesaggio.

Gli sviluppi statunitensi della pianificazione ecologica, assunta come "processo di elaborazione di informazioni biofisiche e socioculturali volte a mettere in evidenza e considerare le opportunità e le limitazioni d'uso del paesaggio"12, mostrano tutta la loro attualità generale, quanto la marginalità delle loro applicazioni nel nostro paese, per la definizione di politiche e misure di controllo attivo delle alterazioni paesistiche riconducibili alla categoria complessa della frammentazione.

Oggi inoltre, le applicazioni delle teorie dei sistemi suggeriscono alcuni elementi di riflessione critica sulle teorie ed i metodi di conoscenza del paesaggio, che invitano a distinguere l'approssimazione della sua struttura, secondo le reali condizioni di complessità sistemica e le relative caratteristiche di risposta, da quanto produce la meccanica sovrapposizione di informazioni che, con forti limitazioni conoscitive, risulta difficilmente sensibile all'intreccio di relazioni ed al più probabile peso che caratterizzano i diversi fattori ${ }^{13}$. Nonostante l'interesse che questi strumenti di ordinamento e trattamento delle informazioni possono suscitare relativamente alla domanda di conoscenza attendibile del paesaggio, il continuo riferimento ai riscontri empirici, derivabili dall'osservazione scientifica della realtà, si configura comunque come l'ingrediente assolutamente insostituibile in ogni tipo di indagine paesistica, per quanto, come normalmente accade, è dall'osservazione della realtà, che le teorie ed i modelli possono trarre conferme o smentite.

L'elevata articolazione di molti paesaggi europei e di quelli italiani in genere, rapportata alle dimensioni sensibilmente diverse di questi territori rispetto a quelli americani, richiede inoltre una attenzione più spinta verso le configurazioni spaziali che le distinte componenti paesistiche concorrono a realizzare, sempre in maniera distinta ed unica, ma secondo ordini e relazioni

11 Sereni E., La degradazione del paesaggio collinare e montano nel Rinascimento, in Storia del paesagoio agrario italiano, Laterza, Ban, 1961-1999.

12 Steiner F., Costruire il paesagoio, McGraw Hill Italia, Milano, 1994.

13 Bertuglia C.S., Staricco L., Complessità, autoorganizzazione, città, Franco Angeli, Milano, 2000; Fabbri P., Natura e cultura del paesaggio agrario, CittàStudi, Milano, 1997; Ingegnoli V., Fondamenti di ecologia del paesaggio, CittàStudi, Milano, 1993. 
riconoscibili. In tal senso, la lettura della articolazione morfologica del paesaggio, secondo criteri che superino l'approccio esclusivamente percettivo, consente l'elaborazione di conoscenze strutturali dettagliate della realtà paesistica.

Allo stesso tempo, l'importanza del pensiero progettuale come valore culturale essenziale per la concezione di politiche di conservazione attiva, recupero e modificazione del paesaggio, non risulta mai abbastanza affermata ed illustrata. Non si tratta di distinguere tra città e campagna, quanto di riuscire a considerare il paesaggio nella sua complessa articolazione e poliedricità, per cui, dall'urbano al rurale, esso muta i propri caratteri nelle varie forme intermedie, con diversi gradienti di rilievo dei fattori naturali e di quelli culturali. In questo senso, lo sforzo di riconoscimento dell'identità dei paesaggi contemporanei delle pianure ad elevata pressione insediativa è inevitabilmente vacuo, nell'ipotesi in cui parta da questioni di nomenclatura, anziché di comprensione e gestione della realtà. A poco vale in questi territori pensare alla progettazione urbana, piuttosto che extraurbana, degli spazi verdi pubblici, piuttosto che di quelli privati e delle colture agricole. La diffusa assenza di specifica e circoscritta identità è l'unica loro attuale e riconoscibile identità.

"Nasce (...), di fronte alla constatazione della nuova perdita di identità del territorio rurale perfettamente omologa alla perdita di identità delle nostre città e coerentemente con la perdita di identità culturale delle popolazioni rurali - l'esigenza di una maggiore riflessione sullo sviluppo del nuovo paesaggio, sui propri riferimenti culturali, tradizionali e storici; nasce la necessità di riempire il vuoto lasciato dalla ruralità che ha ceduto il passo all'industrializzazione, prima che sia completamente occupato dalla ruralità urbana di ritorno. I metodi sono quelli della pianificazione e progettazione paesaggistica che affondi le radici su solide basi culturali. Non bastano, infatti, le cognizioni tecniche, architettoniche o agronomiche che siano, ma occorrono idee valide in termini di immagine dello spazio che si intende proporre e realizzare ${ }^{\prime 14}$.

Ben si prestano ad essere estese alla progettazione paesistica le considerazioni di Kevin Lynch su quella urbana, che "è praticata assai di rado, o meglio è praticata erroneamente come architettura o come ingegneria di grandi dimensioni (...). La vera progettazione urbana non inizia mai con una situazione vergine e non prevede mai un lavoro finito. Più propriamente essa ragiona in termini di processo, di prototipi, di guida, di incentivo, di controllo ed è in grado di concepire sequenze ampie, fluide, insieme a dettagli concreti e semplici. Non può essere ancora definita una disciplina progredita ma, piuttosto, un nuovo tipo di progettazione o un nuovo modo di guardare all'argomento di cui si occupa. Una riserva ben sviluppata di modelli che siano in grado di integrare processo e forma sarebbe di grandissima utilità a questo fine. Questi modelli e queste costruzioni teoriche dovrebbero esser tuttavia sufficientemente indipendenti e semplici, in modo da permettere quella continua rifondazione di obiettivi, analisi e possibilità che è tipica della progettazione urbana"15.

Contributi del tutto complementari a quelli sviluppati nel campo della pianificazione spaziale da studiosi come Lewis, McHarg e Steiner, ed ispirati agli stessi caratteri di indipendenza e semplicità dei modelli, richiamati da Lynch, derivano da quella che Forman individua coma la terza generazione di ricerche nel campo dell'ecologia del paesagoio, sviluppatasi a partire dagli ultimi anni Ottanta, ed in particolare dalla concezione strutturale del paesaggio come mosaico ${ }^{16}$. Il modello di riferimento di questo approccio conoscitivo, fondato sul riconoscimento della realtà paesistica come mosaico articolato in matrici, macchie e corridoi, non assume necessariamente funzioni di strumento di settore, bensì può costituire una efficace base interpretativa della struttura e dei processi di modificazione del paesaggio, nel complesso dei diversi caratteri che lo identificano.

14 Pirani A. (a cura), Progetti della natura e dell'uomo, Franco Angeli, Milano, 1998.

15 Lynch K., Progettare la città: la qualità della forma urbana, ETAS libri (The MIT Press), Milano (Cambridge, Massachusetts), 1990 (1981).

16 Forman R.T.T., Land mosaics, the ecology of landscapes and regions, Cambridge, 1995. La definizione di paesaggio (landscape)

è: a mosaic where a cluster of local ecosystems is repeated in similar form ozer a kilometers-zide area. La definizione di mosaico (mosaic)

è: a pattern of patches, corridors, and matrix, each composed of small, similar aggregated objects. 
Gli elementi di biologia conserzazionale specificamente inerenti il tema della frammentazione e lo studio dei sistemi spaziali lineari, vengono colti in questo lavoro come espressione orientativa degli esiti di ricerche proprie di altre competenze scientifiche, complementari alla pianificazione e progettazione paesistica. Nell'assoluto rispetto dell'identità di ogni competenza, questo livello basilare di consapevolezza è ritenuto utile al dialogo con altri campi che, pur da punti di vista diversi da quelli dei paesaggisti, studiano inevitabilmente lo stesso paesaggio, in riferimento al quale i processi di pianificazione richiedono sempre più efficaci ed articolati confronti.

Secondo quanto fin qui premesso, la ricerca riguarda specificamente le condizioni di frammentazione del paesaggio periurbano di pianura. Esse incidono su una gamma articolata di aspetti che, oltre le dinamiche ecologiche delle popolazioni animali e vegetali, coinvolgono decisamente le condizioni, ambientali, funzionali e morfologiche, degli insediamenti umani, con manifestazioni complesse e profonde, in particolare laddove gli stessi insediamenti investono la quasi totalità del territorio.

Argomento topico dello studio sono i caratteri di idoneità dei sistemi lineari di continuità paesistica (corrida paesistici) per la riqualificazione diffusa del paesaggio attraverso effetti di contenimento e riduzione delle tendenze e degli stati di frammentazione. I sistemi lineari di spazi aperti vengono considerati, inchiave propositiva, come soggetti e/o strategie di intervento, incentrati sulla considerazione prioritaria delle valenze ambientali e culturali del paesaggio, laddove esso regolarmente non è all'attenzione, ma, piuttosto, viene bistrattato ed ignorato dalla generalità delle forme di governo territoriale, che si occupano di altro, sommando elementi ed argomenti, senza la consapevolezza della struttura che l'insieme di questi va assumendo, ovvero senza un'idea contemporanea di paesaggio, riconosciuta e coltivata. Il tema della frammentazione paesistica, in assenza di un inquadramento teorico organico, viene affrontato, a partire dalla introduzione delle sue forme di manifestazione e degli specifici aspetti definitori, attraverso l'indagine dei fattori territoriali causali, degli indicatori per l'analisi, dellemodalità di diagnosi. La ricerca procede attraverso il costante riferimento strumentale alla realtà paesistica del territorio periurbano occidentale di Firenze, prescelta come soggetto di studio applicato, e si conclude con la definizione sperimentale di un modello per la riqualificazione diffusa del paesaggio, incentrato sulle specifiche problematiche ed opportunità che caratterizzano il tema affrontato.

Alle diversità di ruolo degli strumenti di pianificazione territoriale si riferisce l'individuazione di alcuni casi studio significativi italiani, proposti come riferimento teorico-pratico attraverso la schedatura mirata riportata in appendice. La scelta di limitare geograficamente i casi studio al nostro paese discende dalla considerazione dell'importanza che assumono in questo ambito di ricerca $\mathrm{i}$ caratteri paesistici peculiari, in relazione agli elementi concettuali e metodologici che possono essere derivati. L'ampia bibliografia specialistica, prevalentemente nordeuropea e statunitense, costituisce comunque un supporto costantemente ricorrente, ordinato secondo un'impostazione strettamente finalizzata.

Tre scale risultano complementari e importanti per i diversi ruoli che sono in grado di svolgere: la scala locale della pianificazione comunale, la scala sovralocale intermedia della pianificazione di coordinamento provinciale, la scala sovralocale di area vasta della pianificazione di indirizzo regionale e statale, i cui caratteri sono estendibili ai sistemi transnazionali.

Un campo di studio basilare riguarda il riconoscimento delle relazioni strutturali intercorrenti tra i modelli territoriali ed i processi di modificazione del paesaggio delle pianure, con riferimento alla crescita della pressione insediativa, relativamente alle principali soglie storiche. È in tal senso di particolare interesse, la lettura dei contributi della ricerca Itaten ${ }^{17}$ sulla identificazione delle forme insediative del territorio italiano.

17 La ricerca nazionale Itaten è coordinata da A. Clementi, P.C. Palermo, G. Dematteis, delle Università di Chieti, Milano e Torino, ed organizzata in 16 unità regionali che fanno riferimento alle Università locali; l'unità che fa capo all'Università degli Studi di Firenze è coordinata da R. Innocenti. Si veda Urbanistica 106/1996, e A. Clementi, P.C. Palermo, G. Dematteis, (a cura), Le forme del territorio italiano, 2 voll., Laterza, Bari, 1996. 
Nell'ambito generale della descrizione dei processi di modificazione del paesaggio collegati alle condizioni morfologiche e socioeconomiche dei territori considerati, vengono in secondo luogo isolati gli elementi fondamentali di identificazione dei fenomeni di frammentazione del paesaggio. Non si richiama qui un elenco esaustivo dei contributi utili alla discussione di questi aspetti, ma occorre citare l'esperienza della ricerca Planeco ${ }^{18}$, che ha prodotto studi per l'identificazione delle condizioni di frammentazione ambientale e dei possibili ruoli della pianificazione territoriale per il mantenimento delle connessioni ambientali.

18 La ricerca nazionale Planeco, finanziata dal Ministero dell'Università e della Ricerca Scientifica e Tecnologica per il biennio 1998-2000, coinvolge alcune strutture universitarie dell'Italia centrale, L'Aquila, Camerino, Chieti, ed ha attivato collaborazioni con l'Agenzia Nazionale per la Protezione dell'Ambiente (ANPA) e con l'Istituto Nazionale di Urbanistica (INU) che hanno prodotto ulteriori azioni di ricerca ed hanno fornito contributi nell'ambito dell'innovazione legislativa di livello regionale. È responsabile, per l'Università di L'Aquila, Bernardino Romano. Si veda il sito web all'indirizzo http://dau.ing.univaq.it/planeco. 


\section{1 - Il paesaggio periurbano contemporaneo attraverso $i$ fenomeni di frammentazione strutturale}

\section{1 - Processi di modificazione del paesaggio di pianura delle aree ad elezata pressione insediatiza}

Nella seconda metà del secolo scorso l'urbanizzazione e l'infrastrutturazione del territorio hanno determinato, con il concorso delle pratiche di meccanizzazione agraria e di regimazione dei corsi d'acqua, una decisa tendenza alla semplificazione ed omologazione del paesaggio, che nelle aree metropolitane ha assunto il carattere di fenomeno pervasivo, tuttora in progressione, nonostante si siano verificati nel tempo alcuni mutamenti dei fattori causali concorrenti. Nella generalità dei casi, ne conseguono effetti di alterazione e destrutturazione del paesaggio, verso un mosaico con gradi di frammentazione crescenti in relazione alla pressione antropica.

"Le aree inedificate del territorio vengono trasformate da una molteplice gamma di interventi di dimensioni sempre maggiori che ne annullano i valori paesistici e ne distruggono gli equilibri ecologici: grandi stabilimenti industriali, centrali elettriche, autostrade, ferrovie, viadotti sono divenuti i protagonisti del paesaggio di oggi alterandone il senso di scala. (...) Queste opere (...) che devono essere accettate come parte integrante del paesaggio moderno (...), possono ancora conciliarsi armonicamente con la natura se l'intervento attivo e utilitaristico dell'uomo nella processualità della natura viene accompagnato da una coscienza e da una volontà estetica; se l'intervento dell'uomo sul territorio è preceduto da uno studio del paesaggio, volto ad individuarne ed analizzarne caratteri e valori"$^{\prime 19}$.

La sempre minore riconoscibilità della città come paesaggio culturale identificato ed equilibrato, è fortemente connotata da una gamma crescente di deficienze funzionali ed ambientali. Nelle pianure, più facilmente e frequentemente soggette a dispersione urbana, le configurazioni del rapporto cittàcampagna, ovunque mutate sul piano sociale ed economico, perdono anche i segni di permanenza storica che le identificavano come testi culturali di grande significato, fino ad avviare la loro stessa considerazione verso argomentazioni di archeologia del paesaggio, in ragione del radicale mutamento del modello insediativo. Pur con livelli di intensità diversi, il paesaggio rurale subisce pertanto pesanti alterazioni strutturali, in un processo di inesorabile erosione della articolazione semiologica stratificata nei secoli.

Mentre ad una lettura strutturale istantanea basata sulla dimensione spaziale corrispondono forti difficoltà di identificazione delle linee di demarcazione dei connotati del paesaggio, ad una lettura di ordine storico, che registri gli elementi identificativi rispetto alla dimensione temporale, corrisponde quella che, neppure troppo estremizzando, risulta una contrapposizione di due paesaggi decisamente estranei.

“Due paesaggi dunque: uno il paesaggio dell'Italia rurale, l'Italia del passato, l'Italia bella, delle dolcezze sempre più rare, dall'altra il paesaggio dell'urbanesimo dilatato, diffuso, dell'industrializzazione leggera, mobile, invadente, della modernità già post-industriale,

${ }^{19}$ Manigio Calcagno A., A rchitettura del paesagoio. E zoluzione storica, Calderini, Bologna, 1983. 
dell'urbanesimo globale. Essi convivono a breve distanza. Il primo è, per gli uomini di una certa generazione, il paesaggio della nostalgia, del desiderio, della distensione appagante, il secondo il paesaggio della realtà d'oggi, nervosa, rumorosa, senza tregua, ma anche il paesaggio della necessità, quella che fa vivere il primo paesaggio, il quale ormai è solo un frammento, una permanenza residuale del passato, immagliata dentro la rete delle strade e delle autostrade, della città continua $(\ldots)^{\prime \prime 20}$.

Le più forti inversioni di tendenza devono essere collegate, quasi esclusivamente, all'abbandono delle pratiche agricole, dovuto alle condizioni di marginalità economica e di interstizialità spaziale, ed incoraggiato dalla pressione della rendita fondiaria urbana. Nella generalità dei casi, questi processi, al di fuori di ogni minimo controllo progettuale riferito ad un'idea contemporanea del paesaggio periurbano di pianura, determinano in tal modo una definitiva perdita di permanenza storica, dovuta alla forte invasività delle trasformazioni nei confronti dei valori culturali testimoniali, che, una volta persi, non sono affatto recuperabili, alla stessa stregua dell'estinzione di specie naturali endemiche o rare.

"Imbalsamare il paesaggio come un documento d'archivio, conservarlo intatto, come si conserva un'opera d'arte, un quadro d'autore, non è possibile, poiché il paesaggio, già per sua natura mutevole, deve trasformarsi per adattarsi alle diverse irrinunciabili esigenze dell'umanità; ma poiché la nostra civiltà del $\mathrm{XX}$ secolo sta contribuendo in modo più rapido e massiccio, rispetto ai secoli passati, alla modificazione del paesaggio, bisogna fare in modo che nell'operare in una così varia e complessa stratificazione storica non vengano alterati irrimediabilmente i valori e turbati gli equilibri esistenti nell'ambiente ${ }^{\prime \prime 21}$.

Eppure, occorre considerare realisticamente come, ancora oggi, sia difficile riscontrare un concreto riconoscimento dei ruoli degli spazi aperti secondo la complessa articolazione che li caratterizza in termini ambientali, storici e culturali, sociali ed economici. Non solo non emerge l'importanza delle loro funzioni biologiche ed ecologiche; di fatto non viene riconosciuta la specificità delle componenti naturali nella realizzazione e nel funzionamento della città, che tende ad esser pensata ancora come meccanismo, formato essenzialmente da componenti artificiali ${ }^{22}$. Negli sviluppi insediativi contemporanei caratterizzati dall'urbanizzazione diffusa e dalle configurazioni fortemente instabili ed ibride dei paesaggi periurbani, queste difficoltà e carenze descritte per la città consolidata si manifestano, sotto forme ed intensità diversificate, in un quadro complessivo di squilibri e consumo di risorse di difficile governo.

A Firenze, come nelle città delle più diverse dimensioni, non soltanto gli spazi verdi sono pochi, ma la loro stessa esistenza risulta costantemente minacciata. "Il verde urbano, come quello extraurbano, soffre per l'inquinamento; su di esso poi grava tutto il carico delle pressioni antropiche tipiche delle aree urbane (...). Se il verde, non solo quello di città, ha valore storico e culturale, ha però anche una funzione ambientale che, negli ultimi tempi, sta acquisendo un ruolo sempre maggiore. Il verde urbano interviene nel regolare quei parametri ambientali che, tipicamente, soffrono di una sindrome "da città". (...) Il verde cittadino riduce le turbolenze dell'aria, permette la vita di numerosi animali (...), filtra ed abbatte il carico degli inquinanti gassosi e particolati, accumula (allontanandole dall'ambiente circostante) sostanze di elevata tossicità come i metalli pesanti, regola l'equilibrio del rapporto $\mathrm{O} / \mathrm{CO}^{2}$, riduce la carica batterica, abbatte il rumore, riduce le sostanze radioattive in circolazione, regola gli estremi termici controllando l'estensione dell'isola di calore ${ }^{\prime 23}$.

20 Turri E., La megalopoli padana, Marsilio, Venezia, 2000.

${ }^{21}$ Maniglio Calcagno A., 1983, cit.

${ }^{22}$ Gambino R., I parchi naturali, La Nuova I talia Scientifica, Roma, 1991.

${ }^{23}$ Gellini R., Grossoni P., Valori culturali e ambientali del werde urbano e periurbano, in Ulivieri L. (a cura), PTCP conoscere per progettare: zoci, frammenti, esperienze nel "cantiere" del Piano, ad Arnum, Quaderni dell'Assessorato all'Urbanistica e Assetto del Territorio della Provincia di Firenze, 2, 1994. 

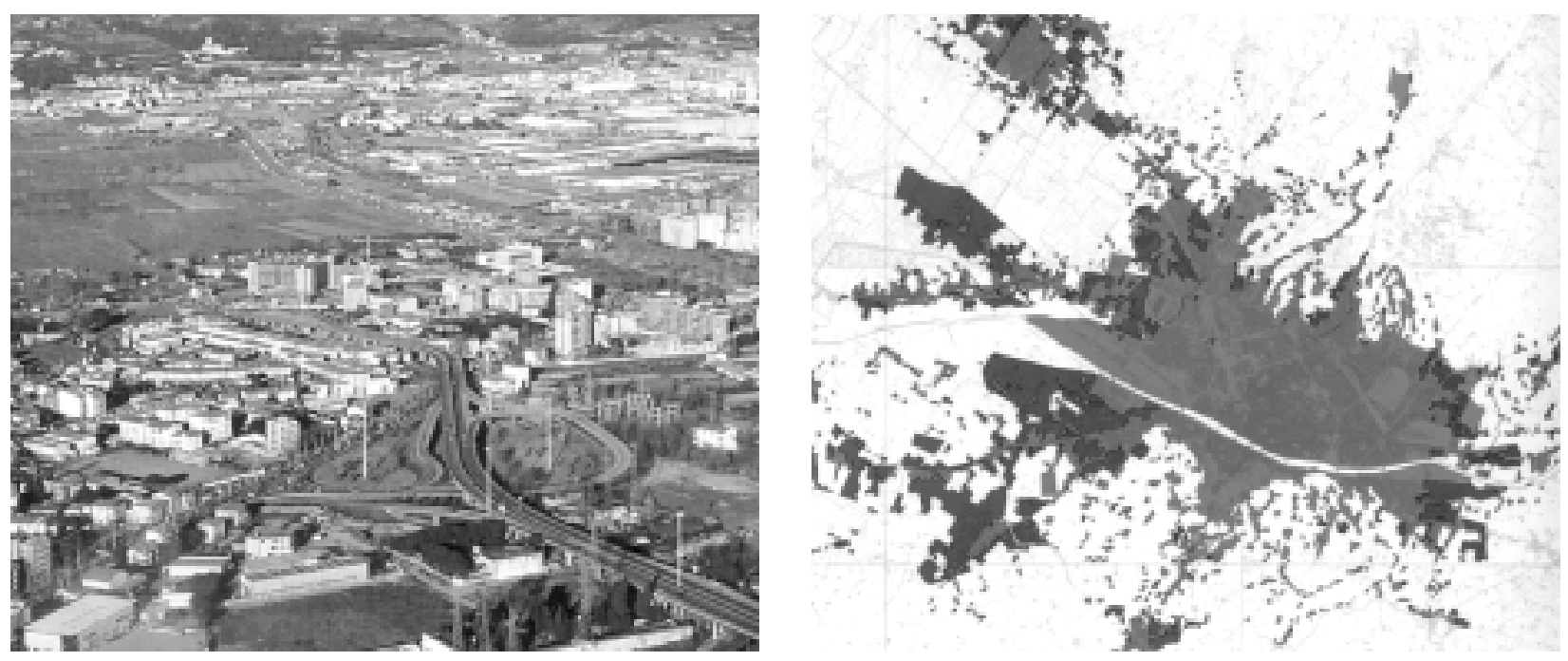

Figura 1 (a sinistra) - La piana di Castello al margine occidentale di Firenze alla fine degli anni Ottanta (immagine tratta da: G. Di Pietro, Un progetto per Firenze - La nuoza città nella piana di Castello, Ponte alle Grazie, Firenze, 1990).

Figura 2 (a destra) - L'articolazione territoriale degii spazi urbanizzati nell'area fiorentina al 1981. La progressione degli sviluppi insediativi, priva di una guida strategica definita su base paesistico-ambientale, ha portato negli ultimi cinquanta anni ai ben noti fenomeni di consumo di suolo e di dispersione urbana. Già agli inizi degii anni '80 del secolo scorso, nell'area ad ovest del nucleo consolidato, hanno assunto un'estensione territoriale non trascurabile le espansioni di Scandicci, e, a nord, di Torri a Cintoia e Osmannoro (aree evidenziate in grigio scuro). In queste ed altre aree i venti anni seguenti vedranno spesso la saturazione indiscriminata e la frammentazione dello spazio rurale in una struttura polverizzata interclusa dall'urbanizzato (immagine tratta da Boggiano A., Zoppi M., Toscana, in: Astengo A., Nucci C. (a cura), IT URB 80 - Rapporto sullo stato dell'urbanizzazione in Italia, Quaderni di Urbanistica Informazioni, 8, 1990).

Se è stato detto come si debba riconoscere che processi di degradazione del paesaggio abbiano caratterizzato anche varie altre epoche storiche, è allo stesso tempo importante notare come ad essi si siano regolarmente alternati la rinascita e lo sviluppo di forme paesistiche nuove e caratterizzanti, legate alle specifiche tecnologie ed economie. Ne è un esempio significativo lo sviluppo delle piantagioni colturali che ha segnato il Settecento e l'Ottocento in Toscana, Umbria e Marche, dando luogo ad un rinascimento paesistico che ha prodotto forme di equilibrio di altissimo valore culturale $^{24}$. Diversamente, oggi questa alternanza è un'ipotesi sempre più difficile, in ragione del progredire dell'artificializzazione del paesaggio, talvolta fino alla sua mineralizzazione ${ }^{25}$. Nuclei urbani, margini urbani e campagne urbanizzate sono, nella congestione delle aree metropolitane, sempre più deficitari di spazi. Sono deficit qualitativi, spesso pesantemente influenzati dalla loro consistenza quantitativa. La frammentazione degli spazi ne determina la loro crescente indisponibilità in dimensioni unitarie significative dal punto di vista paesistico. I processi di modificazione tendono a determinare configurazioni con caratteristiche di sempre minore reversibilità, anche in considerazione della fragilità delle risorse non rinnovabili.

"Non si tratta di una scelta fra città o campagna: entrambe sono essenziali; ma oggi è la natura, assediata in campagna, troppo scarsa nella città, ad essere diventata preziosa"26.

${ }^{24}$ Sereni E., Storia del paesaggio agrario italiano, Laterza, Bari, 1961-1999.

25 Con il termine mineralizzazione si intende, nell'ambito di questo studio, il processo di alterazione paesistica che per effetto dell'edificazione, dell'urbanizzazione e/o dell'infrastrutturazione, porta alla perdita delle componenti e funzioni biotiche del paesaggio ed ai conseguenti disturbi alle componenti e funzioni fisiche (ad esempio quelle relative ai suoli ed alle acque). Non rientrano nell'utilizzo qui fatto del termine gii effetti di mineralizzazione dei suoli fertili a seguito dell'impiego nelle colture agrarie di agenti chimici.

${ }^{26}$ McHarg I.L., Progettare con la natura, Muzzio (Doubleday \& Company, Inc. Garden City, New York), Padova, 1989 (1969). Considerando che McHarg condusse queste osservazioni su processi già allora in atto, più che contenuti premonitori alle indicazioni di questo autore recentemente scomparso, occorrerebbe riconoscere concretamente il permanere, ad oltre trent'anni di distanza, della inadeguatezza delle politiche di governo territoriale. 
Lo sviluppo socioeconomico dovuto alla progressiva industrializzazione dei processi produttivi ha notoriamente innescato un complesso di fattori con diverse ricadute di tipo territoriale e conseguenti fenomeni di modificazione del paesaggio. Del concetto di paesaggio rurale viene meno il significato precipuo di espressione di una realtà sociale, economica ed ambientale, ormai sostanzialmente soppiantata da altre forme legate alla società industriale.

Due fenomeni sono individuabili come fondamentali in questo processo diffuso ed intenso di modificazione paesistica: “(...) tra la popolazione che abita le campagne, quella che trae sostegno dall'agricoltura non è più predominante; la facilità dei mezzi di trasporto e telecomunicazione ha profondamente inciso sulla composizione socioeconomica. A fianco di chi trascorre l'intera giornata nell'ambiente rurale esiste chi è occupato da un'attività diversa da quella agricola e lavora in un luogo diverso da quello di residenza. Con costoro bisogna considerare chi torna al mondo rurale il fine settimana o per trascorrervi le vacanze. Questi fenomeni di vario part-time hanno provocato profonde trasformazioni fisiche, oltre che sociali, nel tradizionale mondo contadino che perde vieppiù una sua precisa connotazione, con implicazioni importanti anche dal punto di vista paesaggistico $^{\prime 27}$.

La piana di Firenze non ha costituito alcuna eccezione rispetto a tutte queste dinamiche di trasformazione. “(...) Nel 1917 si redige il piano industriale per il quartiere di Rifredi (...); dal1924 la società elettrica Valdarno realizza diverse cabine e linee elettriche da $30.000 \mathrm{KW}$ nella zona delle "Due Case" (...); dal 1928 al 1929 comincia la trasformazione da corrente continua a corrente alternata, che porta un incremento alla produzione industriale dell'area (la Fiat, la Pignone, la Galileo); negli anni Trenta il progetto di "Bonifica integrale" della piana di Sesto redatto dall'ing. De Horatis separa le acque alte da quelle basse, elimina quasi interamente i ristagni d'acqua, e rende la piana utilizzabile per essere infrastrutturata con autostrade, ferrovie ed industrie (...). L'industrializzazione induce una forte affluenza di popolazione da tutta la provincia (soprattutto dal Mugello) e dal resto della regione con la conseguente creazione di insediamenti periferici intorno a Firenze (prevalentemente prima a nord-ovest e poi a sud-ovest) e ai piccoli centri fino ad allora a vocazione agricola (...). I microequilibri interni, che giudiziosamente avevano costruito il paesaggio fiorentino, saltano: la fabbrica risucchia energie umane dal contado, l'economia mezzadrile diventa residuale, con il conseguente abbandono delle opere di drenaggio artificiale della collina (...) che consentivano alle acque di infiltrarsi nel sottosuolo. (...) La condizione di degrado della piana non è evidente ad un approccio sommario, perché il crollo del sistema non è lineare, inscrivibile all'interno della categoria causa-effetto, ma deriva dalla crisi a rete dell'intero sistema. Tutte le variabili ambientali, sociali, culturali - iniziano a degenerare e lo stress di ognuna crea ripercussioni a catena sulle altre ${ }^{\prime 28}$.

Si tratta di trasformazioni che generano perdita di capacità figurativa del paesaggio, per la sua profonda alterazione e semplificazione spaziale, ma anche perdita di valori culturali storici e pesante caduta di efficienza ambientale per la protezione ambientale delle colture agrarie e delle risorse idriche e per la protezione e regolazione ambientale degli stessi insediamenti.

I limitati condizionamenti ambientali all'insediamento umano caratteristici dei territori di pianura hanno fatto sì che l'incremento progressivo della domanda di risorse da parte degli sviluppi insediativi e infrastrutturali trovasse ampi raggi di azione in questi ambiti. Il progredire dello sviluppo tecnologico ha determinato una deleterea illusione di efficacia illimitata dei mezzi a disposizione per affrontare gli ostacoli realmente decisivi tipici di questi territori: i delicati equilibri idraulici, legati, oltre che alle stesse aree di pianura ed ai relativi sistemi di bonifica, anche alle aree collinari e montane dei sottobacini idrografici. Si è innescato un comportamento di occupazione indiscriminata di spazi di pertinenza funzionale del sistema idrologico: la presunzione dell'assenza di rischi e della

${ }^{27}$ Fabbri P., Natura e cultura del paesagoio agrario, CittàStudi, Milano, 1997.

28 Poli D., La piana fiorentina. Una biografia territoriale narrata dalle colline di Castello, Alinea, Firenze, 1999. 
possibilità tecnica di correggere e controllare le alterazioni indotte negli equilibri idrualici è stata diffusa ed applicata oltre ogni limite di ragionevolezza, anche economica.

Dalla fine dell'Ottocento ad oggi e soprattutto negli ultimi cinquanta anni, si assiste in tal modo ad un aumento generalizzato della pressione antropica sugli equilibri naturali e culturali dei paesaggi di pianura, distinguibile essenzialmente in quattro matrici fondamentali:

- la diffusione di forme di conduzione agraria legate alle logiche ed alle tecnologie della produzione industriale;

- l'incremento quantitativo e le trasformazioni tipologiche degli insediamenti urbani, a fronte del regresso dell'economia rurale;

- l'incremento dell'infrastrutturazione viaria e tecnologica del territorio;

- l'incremento del prelievo di risorse naturali per le attività agrarie, industriali e urbane e per la realizzazione degli stessi interventi da queste richiesti.

La domanda di risorse, assolutamente senza precedenti, riguarda innanzitutto e sempre più pesantemente lo spazio, ma anche altre risorse ambientali, quali le falde acquifere, i suoli fertili, $\mathrm{i}$ sottosuoli estrattivi. Alla pressione insediativa si legano indissolubilmente i rischi di modificazione della struttura profonda del paesaggio, per quanto riguarda le permanenze storiche, come per le configurazioni geomorfologiche, caratterizzate da maggior metastabilità, ma anch'esse rispondenti ad equilibri dinamici non immuni da perturbazioni antropiche o sconvolgimenti naturali.

Questo avviene in maniera diretta ed intensa con le attività di escavazione di inerti, influenzando, in particolare nei territori di pianura, gli equilibri biologici e fisici delle falde acquifere, basti pensare nel caso in questione all'area dei Renai di Signa, in destra d'Arno, a valle di Firenze, oggi gestita a parco per l'interesse naturalistico e sociale, ma di fatto caratterizzata da un'elevata sensibilità ambientaleper l'affioramento della falda, dovuto all'escavazione.

Anche i prelievi idrici innescano processi di alterazione ambientale con effetti paesistici diffusi e talvolta di severa intensità. Malgrado allo stato degli equilibri idrici dei suoli siano legate le effettive possibilità di sviluppo della vegetazione e pertanto le funzioni ambientali che essa può svolgere, il progressivo inquinamento delle acque superficiali e l'incremento del bisogno idrico urbano stanno progressivamente alterando gli equilibri idrogeologici, con emungimenti sempre più elevati di acque di falda, e conseguente loro abbassamento. Allo stesso tempo, si verificano in altre aree a forte antropizzazione crisi di segno opposto, come nel caso della risalita delle falde di Milano. La crisi idrogeologica di un territorio relativamente limitato, come quello nazionale, può provocare, anche in uno stesso periodo, pesanti siccità ed alluvioni. L'estate 2002 ha lasciato solo le ultime manifestazioni della lunga serie di testimonianze dell'evidente inadeguatezza, non solo italiana, dei modi contemporanei di governo del territorio e di utilizzo delle risorse ambientali. Quanto è avvenuto nel nord Europa conferma che anche la maggiore attenzione riservata tradizionalmente a questi aspetti da paesi come l'Austria e la Germania, non garantisce efficaci forme di controllo e riduzione dei rischi di dissesto idrogeologico, dovuti alla generalizzazione di forme produttive ed insediative ad elevato impatto ambientale, in parallelo alla progressiva riduzione delle funzioni di presidio svolte dalle attività agricole e forestali. In Italia peraltro, solo con le riforme legislative regionali degli ultimi anni '90, in alcune regioni fra cui la Toscana, la Liguria e l'Emilia Romagna, è stato sancito il passaggio dalla regolamentazione urbanistica all'adozione di principi e strumenti di governo del territorio e di attenzione alle risorse ambientali, compresi lo spazio ed il suolo fra quelle non rinnovabili, alle quali è stata riconosciuta un'ovvia centralità.

In realtà, siccità ed alluvioni, due estremi critici che spesso vengono descritti con informazioni approssimative come opposte manifestazioni di una situazione paradossale, possono avere forti legami causa-effetto relativi ai tipi ed all'intensità della pressione antropica.

Nonostante il fenomeno della subsidenza sia noto ed ampiamente studiato da tempo, si assiste al progredire dei suoi effetti. Nella pianura lucchese, il prelievo idrico per usi industriali provoca 
sprofondamenti del terreno anche a chilometri di distanza dagli insediamenti produttivi dell'industria cartaria, generando crateri profondi anche alcuni metri, a seguito del cedimento di cavità sotterranee, e cunicoli variamente lunghi ed articolati, con rischi anche per la sicurezza statica di edifici ed infrastrutture viarie e tecnologiche. Nella pianura bolognese la pressione insediativa genera un elevato prelievo di acqua potabile, a cui si aggiunge il carico rilevante di quella per usi produttivi, ad esempio per le colture di barbabietola da zucchero. "L'ordine di grandezza dell'abbassamento del terreno è, purtroppo, assai elevato (...) (fino ad oltre due metri complessivi, con punte di 8 centimetri all'anno negli ultimi venticinque anni). Tutto ciò ha comportato un aumento diffuso del rischio idraulico dovuto alla perdita di funzionalità della capacità di scolo dell'attuale sistema di canali di bonifica (soprattutto per gli abbassamenti delle arginature e la perdita delle pendenze degli alvei). Il sistema scolante, infatti, concepito per portare le acque da sud verso nord, in caso di piena non permette più un loro transito efficiente verso gli impianti di pompaggio, situati nella bassa pianura, con la conseguenza che già a partire da pochi chilometri a monte della conurbazione bolognese si registrano con sempre maggior frequenza le esondazioni. A complicare la questione interviene anche la elevata artificializzazione di tutto il sistema, che si traduce in corsi d'acqua rigidamente inalveati, in alvei pensili, in un sistema scolante sottodimensionato rispetto alla diffusa impermeabilizzazione del suolo ed alla diminuizione dei tempi di corrivazione; senza dimenticare l'aumento dei drenaggi sotterranei" ${ }^{\prime 2}$. "Nell'ultimo decennio, in particolare, si è assistito ad un raddoppio della quantità di acqua attinta ai corpi d'acqua superficiali, mentre l'acqua emunta mediante pozzi è passata da circa $89.000 \mathrm{l} / \mathrm{sec}$. a circa $115.000 \mathrm{l} / \mathrm{sec}$. (...) Oltre alla quantità emunta va rilevato che la concentrazione degli emungimenti, a parità di acqua emunta, può indurre focolai concentrati di desertificazione $\mathrm{e}^{\prime 30}$.

"L'espansione degli aggregati urbani risulta quasi sempre indifferente alla qualità delle risorse rinnovabili e non rinnovabili, che consuma. Nei processi di pianificazione urbanistico-territoriale il suolo è spesso considerato come supporto inerte per molteplici attività umane; ne vengono ignorate le funzioni ecologiche (produzione di biomassa, trasformazione di materia organica, filtro per le acque) o i maggiori fattori di rischio di degradazione e sottovalutati gli aspetti connessi al loro potenziale produttivo" ${ }^{\prime 31}$.

Tra Firenze e Pistoia “(...) la crescita edilizia è avvenuta (...) per ispessimento di insediamenti filiformi localizzati lungo le direttrici viarie storiche, ma anche per effetto delle previsioni compiacenti di molti piani urbanistici, che hanno invaso la piana erodendo via via le aree interne. Mentre nel 1951 la piana si presentava come una sequenza di centri distribuiti ai bordi della valle (margine collina-pianura) connessi da borghi sorti sulla viabilità maggiore (Pistoiese, Pisana, Pratese, Senese), al 1984 le aree inedificate rappresentano degli ambiti interclusi nel quadro di una sequenza edilizia pressoché continua, che interessa non più soltanto i bordi della piana, ma vaste aree centrali (ad esempio la direttrice Scandicci-Campi). La tendenza alla saturazione delle zone centrali è ancor più evidente se raffrontata allo stato delle previsioni urbanistiche vigenti (alla metà degli ultimi anni Ottanta, n.d.r.). Una gran parte dei piani regolatori, infatti, ipotizza previsioni che appaiono sovradimensionate soprattutto nelle destinazioni industriali che supportano un allontanamento delle

\footnotetext{
${ }^{29}$ Provincia di Bologna, Assessorato Ambiente, Servizio Pianificazione Paesistica, Piano programmatico per la conserzazione e $\mathbb{1}$ miglioramento degli spazi naturali nella prozincia di Bologna, Bologna, 2000.

30 Ministero dell'Ambiente, Comunicazione Nazionale per la Lotta alla Siccità ed alla Desertificazione, Comitato Nazionale per la Lotta alla Desertificazione, Roma, 1999.

${ }^{31}$ Ufficio di Piano per la redazione dello Schema Direttore - SDTM, Primo rapporto sulle condizioni di sostenibilità delle prezisoni urbanistiche dell'area ansta bolognese, Bologna, 1997.
} 
unità produttive dal comune di Firenze verso la cintura ${ }^{32}$ e nel disinvolto uso di zone commerciali, direzionali e di cosiddetto terziario avanzato" ${ }^{\prime 33}$.

"Fra il '61 e il '71 crescono in modo eccezionale tre comuni della prima fascia di Firenze: Scandicci, di 9.233 abitanti, Sesto di 19.223 (quasi raddoppio), Campi (che è di prima fascia anche per Prato) di 8.963 (...) Per capire qual era la situazione edilizia in quegli anni basta citare solo i dati ufficiali: al 1967 nei comuni da noi considerati (l'intera area metropolitana Firenze Prato Pistoia, n.d.r.) erano state approvate 423 lottizzazioni che prevedevano 34.361.654 metri cubi di edificato totale, per una estensione di 1.892 ettari (il 4,4\% dell'intera estensione della piana, che è di 43.400 ettari circa). Alcune situazioni locali erano particolarmente inquietanti: (...) a Scandicci (32.186 residenti) 39 per 4.206.010 $\mathrm{mc}(\ldots)^{\prime \prime 34}$ erano le lottizzazioni approvate. "Quando i piani regolatori comunali vengono redatti non possono fare a meno di "fotografare" questa situazione, adattandosi - nel migliore dei casi - alle situazioni in atto o pregresse. Il riflesso di questa scelta adattativa si tramuta in una pesante ipoteca economica per l'eccessiva offerta di aree edificabili per la residenza e, soprattutto, per l'industria e le attività terziarie. Ipoteca che è stata alla base del nesso esistente tra rendita fondiaria e meccanismi dello sviluppo areali nell'intera piana ${ }^{35}$. È stato anche questo condizionamento che fissando lo stato di diritto dei suoli favorirà i processi di rilocalizzazione industriale nella seconda metà degli anni settanta ${ }^{36}$. Così alla già cospicua urbanizzazione della piana, calcolata in 11.481 ettari al 1986, i vari piani urbanistici comunali prevedevano di aggiungerne altri 5.434 ettari. Il che significa che il 38,8\% dell'intero bacino può ormai considerarsi suolo totalmente urbanizzato. (...) Quello che finisce per prevalere, nonostante i primi piani regolatori, è una tendenza spontanea che finirà per saldare parti consistenti del territorio. Firenze si espande verso ponente, cioè verso Prato $(\ldots)$ che porterà ad uno stravolgimento della delicata gerarchia storica degli insediamenti, dei percorsi lineari antichi, dei collegamenti ragionevoli da un nodo all'altro del tessuto territoriale, così facendo le stesse tracce della centuriazione romana sostengono un processo di neocolonizzazione industriale e residenziale, multidirezionale, pieni più vuoti, discontinua, smagliata, differenziata da punto a punto, e nella lunga distanza, da Firenze verso il mare, straordinariamente monotona e omogenea nella riproposizione continua delle modulazioni territoriali accentrate e decentrate $\mathrm{e}^{\prime \prime 37}$.

“Per quanto riguarda i mutamenti del periodo più recente, i risultati dei censimenti del 1991 pongono in evidenza un andamento differente dell'evoluzione socioeconomica delle regioni dell'Italia nordorientale e centrale. (...) Nelle trasformazioni degli insediamenti non vi è stata un'estensionegeografica del processo di urbanizzazione in nuove aree della regione (Toscana, n.d.r.), ma si è avuto piuttosto un consolidamento dei sistemi insediativi esistenti. (...) Tuttavia la crescita del territorio urbanizzato è continuata nell'ultimo intervallo infracensuario (1981-1991, n.d.r.) - come effetto ritardato di pianie progetti di sviluppo edilizio impostati negli anni Settanta - in modo più marcato nelle corone suburbane di alcuni capoluoghi di provincia e in alcune aree periferiche della regione (...). Il rallentamento

\footnotetext{
32 "Fenomeno sancito dal Prg del '62 in termini urbanisticamente corretti, in quanto riferito ad un quadro di piano intercomunale che ha avuto una accelerazione incontrollata dopo il 1976 a seguito del trasferimento delle Officine Galileo Galilei del gruppo Montedison a Campi Bisenzio, inteso come processo di autofinanziamento e reso possibile aldilà di ogni possibile controllo urbanistico". Cit. in Boggiano A., Zoppi M., Toscana, in Astengo A., Nucci C., IT. URB. 80 Rapporto sullo stato dell'urbanizzazione in Italia, volume primo, Quaderni di Urbanistica Informazioni, n. 8, 1990.

33 Boggiano A., Zoppi M., 1990, cit.

${ }^{34}$ Bortolotti L., De Luca G., Come nasce un'area metropolitana Firenze Prato Pistoia: 1848-2000, Alinea, Firenze, 2000.

35 "Su questo rimandiamo alle interessanti riflessioni di R. Caselli, A. Cavalieri, Rendita e accumulazione in Toscana. Un'analisi del mercato immobiliare, relazione presentata alla XIV Conferenza italiana di Scienze regionali, Bologna 6-8 ottobre 1993". Cit. in Bortolotti L., De Luca G., 2000, cit.

36 "Le nuove direttrici di sviluppo divorano allora l'intero territorio, riattivando produttivamente sistemi urbani minori, incorporando mercati di lavoro locali, lasciandosi guidare dalla tessitura stessa degii organismi territoriali: ogni insediamento umano guadagnato diventa base di conquista di nuovi spazi. Viene utilizzato in questo processo - nella piana di Firenze ciò è particolarmente evidente - lo strumento più forte di conquista: la casa. La residenza diventa cellula elementare del nuovo ordine produttivo, residenza-officina, casa-laboratorio, centro di riorganizzazione di un sistema multiplo di lavori della famiglia (...)", Paba G., Urbanistica e cultura a Firenze: una show-toun per la shoe-toun, Atti dell'Istituto di ricerca territoriale e urbana 1982, Tip. Capponi, Firenze, 1982. Cit. in Bortolotti L., De Luca G., 2000, cit.

37 Bortolotti L., De Luca G., 2000, cit.
} 
o l'inversione di tendenza della dinamica demografica e dell'urbanizzazione nel capoluogo regionale e nei capoluoghi di provincia, la crescita dei centri minori delle corone sub-urbane e della campagna urbanizzata, l'ulteriore diffusione territoriale delle attività dei settori tipici dell'industria leggera producono un'attenuazione delle differenze tra aree urbane, campagna urbanizzata e aree turisticoindustriali. La dilatazione del reticolo della campagna urbanizzata ha interessato nel periodo più recente (l'ultimo decennio del Novecento, n.d.r.) interstizi interni o appendici periferiche delle aree già formatesi nelle precedenti fasi di sviluppo. Inoltre la campagna urbanizzata - anche in seguito allo sviluppo di attività terziarie nei centri urbani medi e piccoli - sembra evolvere verso specifiche forme di insediamento urbano ("città diffusa")

Il recente discutere di desertificazione, anche relativamente ai paesi settentrionali del Mediterraneo, esprime fondate preoccupazioni per l'avanzamento di profonde alterazioni ambientali: il paesaggio ne costituisce l'indicatore più immediato e completo, per le spiccate e diversificate sensibilità caratteristiche.

"Il processo di urbanizzazione incide sul fenomeno di desertificazione in termini di sottrazione di suoli fertili all'impiego agricolo, determinando, in ultima analisi, la riduzione delle capacità produttive. (...) Ancora oggi si calcola che ogni anno in Italia, per cause diverse, vengono sottratti alle attività agricole non meno di 30.000 ettari di terreni ad alta produttività. Casi eclatanti si possono registrare nella valle dell'Arno, nelle pianure della Campania, nell'hinterland di Cagliari, nei dintorni di Palermo ed in generale nelle aree costiere, dove all'espansione urbana ed industriale si è aggiunto lo sviluppo turistico. (...) Un processo analogo a quello dell'urbanizzazione, in termini di sottrazione di risorsa, avviene per effetto della crescente diffusione sul territorio, soprattutto in certe realtà, di discariche e di attività estrattive spesso incontrollate. (...) In Italia, caratterizzata da un territorio fortemente antropizzato, l'estendersi del processo di desertificazione è in diretto rapporto con la crisi dei centri urbani storici che a un assetto tradizionale del paesaggio costituito da sistemi abitativi a forte compenetrazione naturale e a basso consumo di risorse, sostituisce un modello basato sulla cementificazione massiccia, il dispendio energetico e l'inquinamento ambientale ${ }^{\prime \prime 39}$.

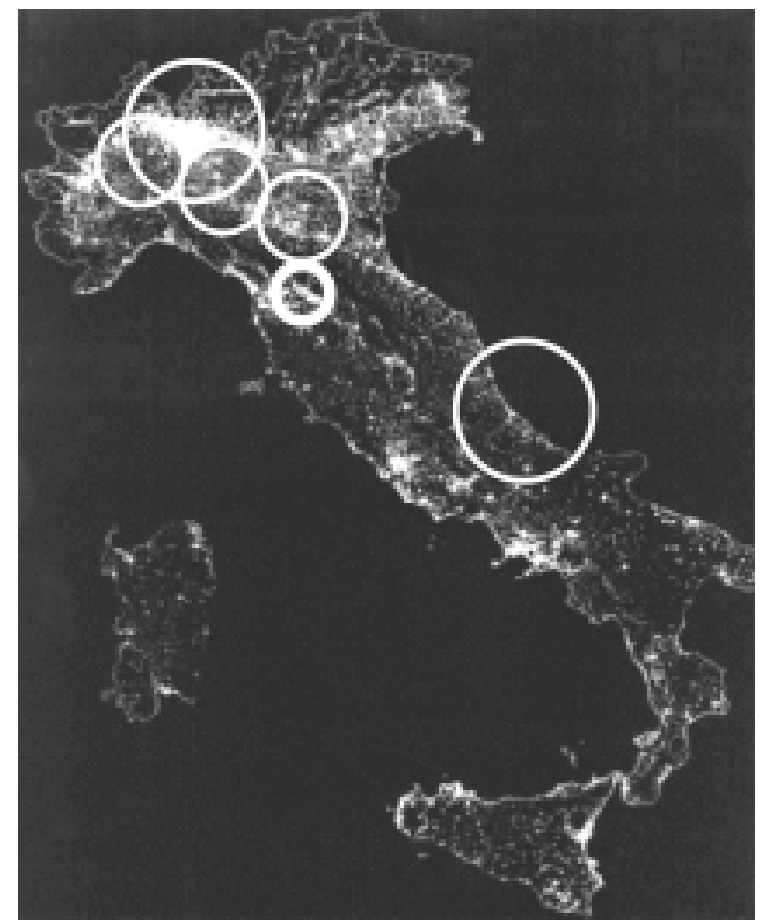

Figura 3 - La distribuzione geografica dell'urbaniz-zazione del territorio italiano secondo i dati elaborati nell'ambito della ricerca Itaten (immagine tratta da Clementi A., La ricerca Itaten: forme del territorio italiano, Urbanistica, 106, 1996).

Il dato risultante da questa rappresentazione difetta, dal punto di vista dei fenomeni di frammentazione paesisticoambientale, dell'incidenza effettiva delle manifestazioni di urbanizzazione diffusa e della rete infrastrutturale minore, evidentemente non risultanti a questa scala di lettura. Sono ben evidenti i bacini metropolitani, fra cui quello di Firenze, nel settore metropolitano della Toscana settentrionale, caso studio della presente ricerca (cerchiato con maggiore spessore), a cui si riferiscono le schede di descrizione tipologica riportate nell'appendice A. Con i cerchi più fini sono indicati i territori dei cinque casi studio selezionati e descritti in modo mirato nelle specifiche schede riportate nell'appendice $\mathrm{B}$. Si noti che tutti i casi studio, selezionati con riferimento alle diverse scale, sono relativi a territori di pianura soggetti ad elevata pressione insediativa, ad eccezione dell'Abruzzo, unica esperienza disponibile alla scala regionale.

\footnotetext{
38 Innocenti R. (a cura), Toscana, in A. Clementi, P.C. Palermo, G. Dematteis, (a cura), Le forme del territorio italiano, II volume, Laterza, Bari, 1996.

${ }^{39}$ Ministero dell'Ambiente, 1999, cit.
} 
Se la pressione antropica di matrice agraria può indurre effetti di perdita di habitat (umano, animale, vegetale), alla pressione di matrice insediativa, oltre a tali fenomeni generalizzati di alterazione, sono connessi effetti diversificati in funzione dei modelli territoriali a cui ha dato luogo, in particolare a seguito degli sviluppi verificatisi dopo la seconda guerra mondiale:

- espansione urbana di margine;

- insediamento lineare misto residenziale e produttivo lungo infrastrutture viarie di rilievo locale;

- insediamento lineare produttivo lungo infrastrutture viarie di rilievo sovralocale;

- diffusione urbana nella campagna;

- incremento delle reti viarie;

- incremento delle reti tecnologiche.

Un esito generale di grande peso, dovuto alla rapidità ed alla intensità di questi processi di trasformazione, coincide con la crisi di significato del paesaggio come autorappresentazione ed espressione dinamica di una cultura. Il paesaggio, come espressione teatrale, ovvero rappresentativa, della società “( ...) risulta irto di complessità, la quale attiene alle difficoltà di operare e direi quasi di vivere in una società come la nostra, che non è una società olistica, in cui gli individui sono integrati, formano una sorta di corpo unico che si muove secondo spinte, credenze, ideali collettivi comuni. Siamo in una società nella quale le spinte individualistiche sono fortissime, divergenti gli interessi, le visioni del mondo, le passioni territoriali, i rapporti quotidiani con lo spazio eccetera. Basta vedere del resto la stessa varietà di oggetti che formano i nostri paesaggi, nei quali poi sembra venir meno ogni adesione della cultura alla natura e nei quali si sentono infiniti scollamenti rispetto ai paesaggidel passato $^{\prime \prime 40}$.

Nei territori soggetti ad elevata pressione insediativa, il paesaggio periurbano è andato così evolvendo verso forme a sempre minor tasso di naturalità e di reversibilità degli assetti antropici. Questi processi evolutivi, nonostante il calo della pressione demografica avvenuto negli ultimi decenni, non sono assolutamente arrestati, né tanto meno invertiti, né infine bilanciati dagli effetti di politiche di compensazione paesistico-ambientale, nel nostro paese del tutto assenti, sia in termini di strumenti di legge, che di prassi pianificatoria e progettuale.

“Dopo più di cinquant'anni di intensa trasformazione degli spazi rurali, di sviluppo turistico delle coste e delle montagne, di diffusione incontrollata degli insediamenti e delle grandi reti infrastrutturali non sono più (...) in gioco soltanto la forma fisica e la funzionalità ecologica del mondo che ci circonda. E il suo stesso significato che fa problema, di fronte al collasso dei delicati equilibri che fino al recente passato hanno legato le società locali alla produzione del loro paesaggio di vita $(\ldots)^{\prime \prime 41}$. Per avere una dimensione generale dell'entità di questi fenomeni basti considerare, fra gli altri dati che sono stati qui riportati per cenni, il quadro quantitativo dell'urbanizzazione del territorio nazionale, di cui peraltro la ricerca Itaten definisce i connotati qualitativi attraverso la lettura morfologica.

I numeri degli "(...) ultimi studi analitici risalgono alla ricerca sull'urbanizzazione in Italia fino agli anni Ottanta, coordinata da Giovanni $\operatorname{Asteng}^{42}(\ldots)$ : fra gli anni Cinquanta e gli anni Ottanta, a fronte di crescite di popolazione mai superiori al 70 per cento, l'incremento di consumo di suolo ha raggiunto punte anche di oltre il 700 per cento. Un aggiornamento assai complesso operato agli inizi degli anni Novanta, utilizzando la Carta tematica sulla occupazione del suolo, prodotta dall'Istat parallelamente al censimento generale dell'agricoltura del 1990, porta ad evidenziare casi di crescita

\footnotetext{
40 Turri E., Il paesaggio come teatro, Marsilio, Venezia, 1998.

41 Clementi A., La rigenerazione dei paesagoi italiani, in TOURING Club ITALIANO, Il paesaggio italiano. Idee Contributi Immagini, Touring Club Italiano, Milano 2000.

42 Si tratta della ricerca nazionale IT. URB. 80 , più volte citata.
} 
del consumo di suolo del quarantennio 1950-1990 con punte del 1300 per cento nell'area di Taranto e dell'800 per cento nella pianura modenese ${ }^{\prime \prime 43}$.

Alcune esemplificazioni significative possono fornire un'ulteriore cognizione dell'intensità delle trasformazioni paesistiche dell'ultimo secolo ed in particolare degli ultimi cinquanta anni. "Nell'ultimo mezzo secolo la popolazione residente nelle zone costiere è aumentata di 4,6 milioni di unità. Oggi quasi un terzo degli italiani risiede nei comuni costieri. Lungo 1.260 chilometri di costa adriatica fino alla fine del secolo scorso c'erano altrettanti chilometri di dune a uno o a più cordoni. Oggi ne sopravvivono appena 123 chilometri, nemmeno il 10 per cento (...). Mezzo secolo fa eravamo 47.500.000 abitanti. Oggi siamo poco più di 57.000.000. Ma i vani di abitazione sono balzati nello stesso periodo da 37.300 .000 ad oltre 120.000 .000 (comprese seconde e terze case). (...) I chilometri di strade sono passati da poco più di 100.000 (1950) ai 300.000 di oggi. Ricomprendendo nei due conteggi le strade comunali extraurbane, i chilometri di autostrade sono passati in un cinquantennio da 480 a 6.500 chilometri circa" ${ }^{\prime 4}$.

Riferendosi agli assetti insediativi globali della Val Padana, Turri osserva che "la megalopoli è una divoratrice di spazio e di storia formidabile, semina distruzioni, cancella memorie: talora avvicinandola dalle direttrici padane che attraversano le residue campagne, la si vede avanzare con le sue muraglie di cemento, eruttando file di macchine come sua propria deiezione, inquinante, continua, senza requie, tra paesaggi che risentono sgradevolmente del suo respiro ${ }^{\prime 45}$.
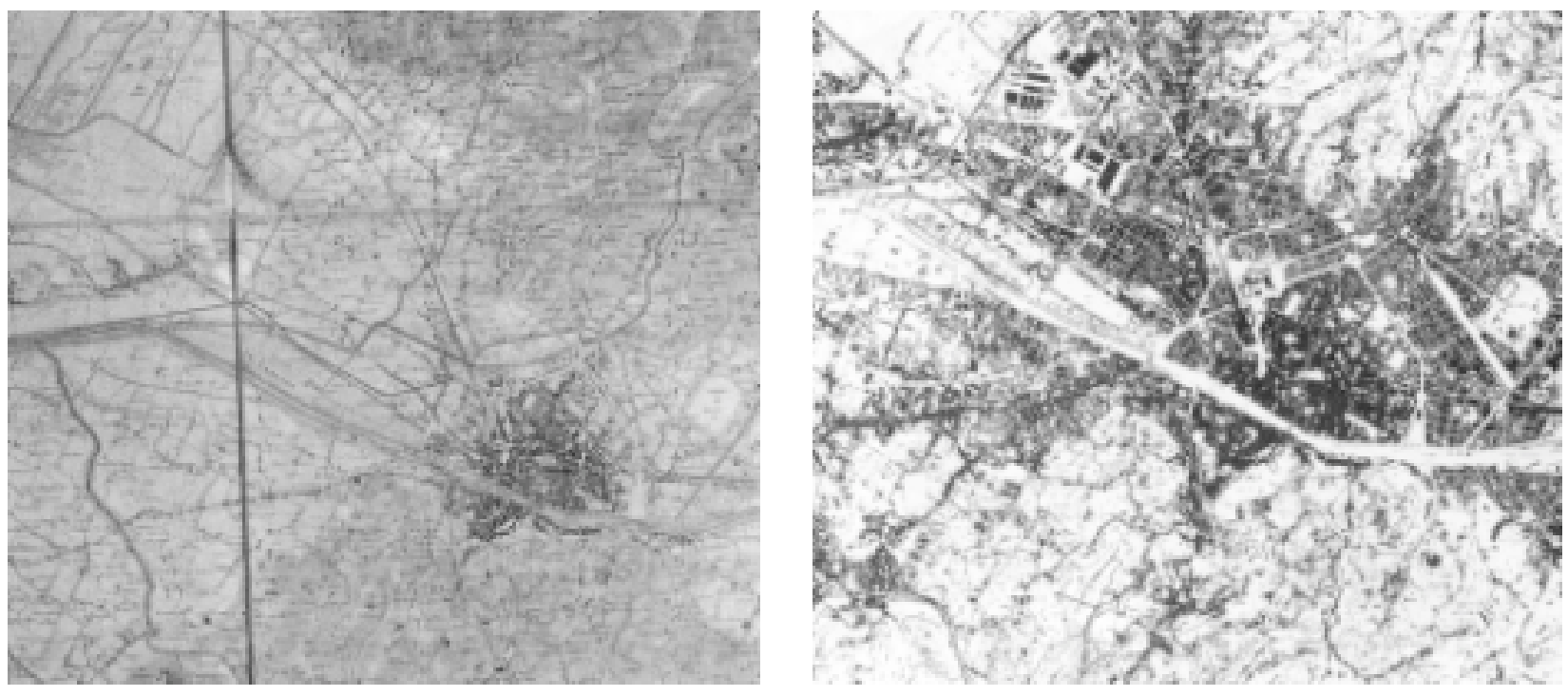

Figura 4 - L'evoluzione dell'estensione e della morfologia insediativa di Firenze nell'arco di un secolo. L'immagine a sinistra è una riproduzione parziale e ridotta di una carta basata su rilievi del 1870-74, aggiornata fino al 1880, eseguita dall'allora Istituto Topografico Militare. L'immagine a destra è una riproduzione parziale e ridotta di una carta della Regione Toscana aggiomata al 1985. È evidente il passaggio dalla città ottocentesca che manteneva ancora la configurazione sviluppata nel Medioevo e nel Rinascimento, modificata essenzialmente solo dai viali di circonvallazione e da alcuni sviluppi lungo la via Pistoiese, e la città contemporanea nella quale ad ovest tre forti direttrici di sviluppo lungo altrettante vie di comunicazione e la saldatura con i centri minori lungo queste configurano una struttura insediativa radicalmente diversa, caratterizzata da una decisa alterazione della campagna e dell'intero sistema insediativo (immagini tratte da: G. Di Pietro, Un progetto per Firenze - La nuoza città nella piana di Castello, Ponte alle Grazie, Firenze, 1990).

43 Comitato per la Bellezza e Centro Studi TCI (a cura), Un Paese spaesato. Rapporto sullo stato del paesaggio italiano, I Libri Bianchi del Touring Club Italiano, n. 12, Milano, 2001.

${ }^{44}$ Comitato per la Bellezza e Centro Studi TCI (a cura), Un Paese spaesato. Rapporto sullo stato del paesagoio italiano, 2001, cit.

45 Turri E., 2000, cit. 
Una prima articolazione spaziale, riportata nella fotografia zenitale della figura 5, permette l'identificazione orientativa di queste manifestazioni nel caso del paesaggio periurbano di pianura di Firenze.

Indipendentemente dal grado di dettaglio con cui è possibile condurre le indagini, la riconoscibilità delle configurazioni paesistiche storiche, che con il governo dei Lorena raggiunsero nel Settecento i livelli più evoluti di articolazione, è oggi praticamente venuta meno, per la progressiva eliminazione ed obliterazione anche dei loro sistemi primari.

In quel periodo "(...) continuarono (...) le migliorie alle opere di bonifica, che introdurranno definitivamente il classico paesaggio dell'alberata anche in quelle aree di pianura che erano state fino ad allora caratterizzate da unità poderali più etese coltivate a seminativi nudi, intervallati da prati e sodi. Ora invece i vasti appezzamenti tradizionali vennero frazionati dall'introduzione di filari di vite maritata all'acero e da alberi da frutta consociati al gelso. (...) La maggior parte del territorio fuori le mura era "uno spazio aperto", destinato all'agricoltura produttiva, che rappresentava uno dei maggiori introiti dell'economia del tempo. A causa delle opere di regimazione idraulica, da poco ultimate, che ancora non impedivano i frequenti straripamenti dei canali (Macinante, Dogaja, Fosso Reale, Osmannoro), soltanto la bassa pianura che si estendeva (...) verso l'Osmannoro, Brozzi e Peretola, risultava ancora poco sviluppata per quanto concerne la coltura della vite e soprattutto dell'olivo" ${ }^{\prime \prime 4}$.

I mirabili equilibri di architettura del paesaggio raggiunti attraverso l'attenta politica agraria avevano identificato, come mai prima, la pianura: alla minuta articolazione degli spazi ed alla loro elevata diversificazione, corrispondeva la forza di una struttura unitaria riconoscibile. La coltura promiscua, presente per circa un terzo dell'estensione superficiale del territorio, con l'alberata, connotava decisamente il paesaggio rispetto all'incertezza morfologica delle precedenti configurazioni stratificatesi nei secoli. La superficie del territorio coltivato si aggirava intorno al sessanta per cento, di cui quasi il sessanta per cento, era arborato ${ }^{47}$.

“È possibile affermare che nei dintorni di Firenze l'ultimo atto territorializzante sia quello lorenese, che si protrasse fino agli anni Cinquanta del Novecento, periodo in cui l'insorgere di un nuovo modello socio-economico portò con sé l'abbandono delle regole storiche di costruzione del territorio. Lo studio della Carta Austriaca (1851), mostra un'immagine del territorio che possiamo assumere come una fotografia della regione fiorentina nel pieno della sua maturità (...) la stessa immagine è ancora rintracciabile in una cartografia dell'IGM degli anni Cinquanta del Novecento. I centri della piana si situavano ai margini, sul bordo esterno dell'antico lago e gli insediamenti avevano un aspetto puntiforme, tanto da consentire le connessioni biotiche fra i vari ecosistemi. Soltanto un'esile viabilità attraversava la delicata parte interna della piana, mentre le città e i paesi si collocavano allo sbocco delle valli sulle conoidi di deiezione dei fiumi ${ }^{\prime 48}$.

Oggi, concordemente con molti tratti tipici degli sviluppi insediativi di pianura, la crescita lungo direttrici viarie e le dimensioni da essa spesso assunte rispetto a quelle fisiografiche del contesto di riferimento, fanno sì che non si determini complessivamente una situazione nella quale risulta rintracciabile un margine della città ed, allo stesso tempo, siano sempre più labili e frammentate le strutture che per secoli hanno identificato la campagna, anche secondo alterne dinamiche evolutive, fino alla sostituzione dell'economia rurale a vantaggio della produzione agricola industrializzata, propria dell'economia di mercato contemporanea. A nord ed a sud, gli sviluppi insediativi verso ovest risultano ormai saldati ai tessuti periurbani del capoluogo compresi tra Scandicci, Peretola e Castello.

\footnotetext{
46 Poli D., 1999, cit.

${ }^{47}$ Rombai L., Insediamenti e paesagoio agrario dall'età comunale al XIX sec., in Barbieri G., Canigiani F., Fonnesu J., RombaiL., I zalori geografico-storici del paesaggio fiorentino - proposte di uso e tutela, Atti dell'Istituto di Geografia dell'Università di Firenze, quaderno n. 11, 1982.

48 Poli D., 1999, cit.
} 
Tre importanti ambiti di spazi aperti non urbanizzati o debolmente urbanizzati risultano evidentemente isolati tra aree ad intenso sviluppo insediativo, fortemente saldate alla città. L'Arno, regimato e canalizzato, costituisce il corso d'acqua principale a cui afferisce l'intero sistema idrografico dell'area, caratterizzato in pianura da corsi d'acqua naturali regimati e canali artificiali di bonifica. Le colline sono caratterizzate dalle matrici paesistiche dei boschi e delle colture agricole. Nel caso dei boschi di latifoglie prevale la forma di governo a ceduo, e la composizione vegetazionale originaria caratterizzata, nei versanti esposti a settentrione, dalla dominanza del cerro sui substrati prevalentemente argillosi e del carpino nero su quelli a maggior permeabilità e, nei versanti più bassi ed in quelli soleggiati, dalla compresenza del bosco deciduo e lucivago di roverella e orniello e del bosco denso di specie mediterranee, nei versanti più ripidi ed assolati, con sempreverdi come il leccio. Fra le colture agricole collinari prevalgono l'oliveto e subordinatamente il vigneto specializzati in alternanza ai seminativi; sono presenti tracce localizzate della coltura promiscua storica dell'olivo con la vite in filare; la struttura insediativa è connotata da una fitta trama viaria strettamente legata ai caratteri geomorfologici e da borghi di origine medioevale, edifici sparsi di origine mezzadrile e dimore signorili, molte delle quali di origine rinascimentale.

Figura 5 - Articolazione metastrutturale del paesaggio dell'area di studio (rappresentazione su fotografia aerea zenitale - Regione Toscana, originale in scala 1:30000):

(1) paesaggio forestale collinare con alternanza di boschi di latifoglie e boschi di conifere di impianto;

(2) paesaggio rurale collinare con prevalenza di colture arboree ad olivo, caratterizzato da un complesso sistema insediativo storico, di strade, borghi, case coloniche e dimore nobiliari;

(3) paesaggio rurale di pianura con prevalenza di colture erbacee ed orticole;

(4) paesaggio agrario di pianura con colture erbacee intensive e zone umide artificiali; sistemi infrastrutturale e residenziale rurali in abbandono;

(5) paesaggio di pianura in aree soggette a vincolo aereoportuale;

(6) paesaggio urbano fluviale del parco storico de Le Cascine;

(7) paesaggio periurbano fluviale in aree degradate per abbandono ed usi impropri;

(8) paesaggio di pianura urbano e periurbano delle aree di frangia del sistema insediativo metropolitano;

(9) paesaggio urbano dei fondovalle dei rilievi collinari.
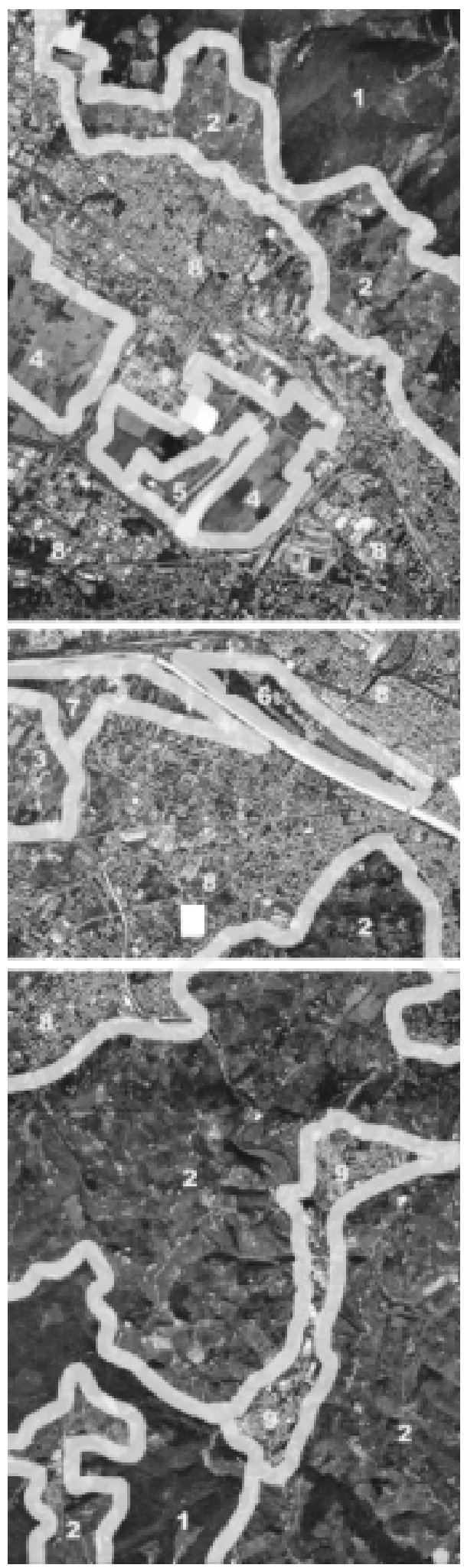

In pianura prevalgono i seminativi semplici con una presenza rilevante di incolti produttivi; sono presenti tracce di ordinamento agrario tipico del modello storico dell'alberata tosco-umbromarchigiana; non sono presenti formazioni relitte della vegetazione forestale planiziale originaria; alcune aree, in sinistra dei fiumi Arno e Greve, hanno costituito uno dei maggiori bacini di produzioni orticole a servizio della città. 
Dove l'accesso e l'utilizzo degli spazi sono interdetti per motivi di sicurezza infrastrutturale, come nel caso dell'aereoporto, il paesaggio è caratterizzato da una configurazione in tutto analoga a quella delle colture erbacee intensive, salva l'inaccessibilità e l'estesa presenza di recinzioni. I parchi urbani dell'area occidentale sono essenzialmente due, essendo quello della piana di Castello e quello delle Grandi Cascine, nell'area di Argingrosso, ancora solo previsioni di piano. Il Parco delle Cascine, complesso di rilevante interesse storico e caricato di importanti funzioni di servizio come maggiore parco urbano di Firenze, è stato anche fino alla metà del secolo scorso sede dell'unica formazione forestale di pianura che, per la rilevante presenza di farnie ed olmi, ricordasse la struttura vegetazionale dei boschi planiziari originari. Il Parco dell'Area Fiat di Novoli, di prossima realizzazione in concomitanza con gli altri lavori di ristrutturazione urbanistica che investono l'area, sarà chiamato ad assumere un rilievo strategico nel sistema urbano degli spazi aperti, dovendo far fronte ad una crescente densità di servizi che si aggiunge alla già elevata densità residenziale dell'area. Non sono estranee a questa parte della città le aree di pianura in cui gli spazi aperti sono utilizzati per attività diverse da quelle agricole: si tratta di spazi per servizi pubblici per attività produttive di estrazione, lavorazione o deposito e commercializzazione di beni. È rilevante anche la presenza di aree degradate e di incolti produttivi, in alternanza agli spazi utilizzati. Nei rilievi collinari gli insediamenti hanno configurazioni diverse in ragione dei caratteri geomorfologici del paesaggio, lineari o centrali, di fondovalle o di crinale, sono formazioni di origine storica, in taluni siti antecedente all'epoca medioevale, con sviluppi recenti ai margini dei nuclei e lungo le strade. Fra gli insediamenti urbani di pianura prevalgono invece i tessuti di sviluppo recente, caratterizzati generalmente da una decisa specializzazione funzionale. Risultano ormai interclusi e trasformati i nuclei storici appartenenti alla struttura rurale del contado fiorentino, come Torre Galli, Peretola, Castello.

Esiste pertanto una netta distinzione tra "il tessuto delle permanenze, assai variegato, ed il tessuto formato da quello che abbiamo definito l'alluvionamento, legato agli sviluppi più recenti, cioè della seconda metà del Novecento. In confronto alle manifestazioni più uniformi e senza radicamento nelle diverse situazioni territoriali di questo strato antropico che, pur con la diversità dei suoi manufatti sembra assimilabile ad un'unica poltiglia urbana, quelle degli strati anteriori si rivelano profonde, umorali, legate ai processi di lunga durata, cioè con un solido radicamento degli uomini e delle loro cose nell'ambiente naturale e nella cultura che quelle cose ha prodotto. (...) Ma questa è la città diffusa, che ha dilatato lo spazio urbano, ha riempito la pianura di edificazioni, con sprechi enormi di spazio, di verde, di silenzi. E che comporta il moltiplicarsi del traffico con la reticolarità degli insediamenti, la loro diffusione particolare che distanzia l'abitare dal lavorare, lo spazio pubblico dallo spazio privato" ${ }^{49}$.

Rispetto a questo stato di omologazione, a ben vedere senza nessun paradosso coincidente con la forte eterogeneità di componenti del paesaggio contemporaneo, occorre fare riferimento al concetto di dizersità paesistica, per distinguerlo nettamente da quello di frammentazione, legato agli stati criticidi cui fin qui si è fatto cenno.

L'accezione con cui è utilizzato in questo lavoro il termine frammentazione paesistica, alla cui definizione è espressamente dedicato il paragrafo successivo, è di tipo negativo. Pur considerando il fatto che un paesaggio con caratteri di elevata frammentarietà possa avere qualità positive in determinate condizioni specifiche interne e di contesto, tale accezione viene assunta con significato univocamente negativo, sia per esigenze di chiarezza, sia per analogia all'utilizzo che ne viene fatto nelle discipline ambientali, ed infine per attinenza alle realtà territoriali a cui lo studio si riferisce, nelle quali il paesaggio periurbano di pianura è soggetto ad intense e diffuse manifestazioni patologiche, a causa della elevata pressione insediativa.

Il concetto di dizersità paesistica, in analogia con quello di diversità biologica, coincide invece generalmente con condizioni di stato e con dinamiche positive. Esso risulta decisamente di difficile

49 Turri E., 2000, cit. 
definizione e applicazione, nella misura in cui non venga circoscritto ad un approccio di ecologia del paesaggio, trovando in questo caso forti elementi di coincidenza con quello di dizersità biologica.

Dal punto di vista ecologico, la diversità paesistica ha un peso determinante nel bilancio qualitativo del paesaggio ed è in diretto rapporto con la diversità biologica. In questo ambito gli ecologi hanno condotto studi approfonditi e risultano spesso i primi e più decisi fautori della conservazione del paesaggio culturale. Anche le forme più costruite, quali ad esempio i terrazzamenti colturali delle aree collinari con muri a secco, costituiscono infatti importanti serbatoi di biodiversità, risultando habitat caratterizzati dalla compresenza di specie floristiche e/o faunistiche in quantità alle volte maggiori rispetto a realtà ben più evolute sul piano ecosistemico ${ }^{50}$.

Nei territori di pianura la diversità del paesaggio è storicamente connessa in buona misura alle oscillazioni della presenza dell'equipaggiamento vegetale agroforestale. In una configurazione caratterizzata da elevata densità e continuità spaziale di siepi e macchie di campo, a seguito di un aumento iniziale di diversità dovuto alla loro riduzione, si raggiunge progressivamente una soglia critica dopo la quale inizia una rapida caduta, corrispondente a stadi negativi di crescente semplificazione spaziale e biologica.

$\mathrm{La}$ diversità data dalla struttura spaziale del paesaggio e dalla composizione delle diverse configurazioni biotiche che ne costituiscono una parte sostanziale, ha assunto sempre più rilievo anche a seguito del diverso orientamento maturato dall'ecologia applicata, rispetto alle teorie iniziali. "Gli ecologi sono stati convinti per lungo tempo che gli ecosistemi ricchi di specie, cioè quelli caratterizzati da una grande varietà di forme di vita e da una consistente diversità delle specie presenti, siano molto più dotati di stabilità e insieme di tollerabilità rispetto a quelli più poveri di specie. (...) Questa ipotesi della "stabilità attraverso la diversità", che è stata a lungo discussa, ha avuto origine dal fatto che tra la diversità delle specie e la stabilità di un ecosistema esiste una correlazione di tipo positivo. Nel 1979 Haber ha analizzato criticamente questa ipotesi, osservandola nell'ottica della pianificazione ambientale orientata secondo principi ecologici ed è giunto alla conclusione che accanto alla "diversità", o diversità delle specie, esiste una "diversità", che può essere indicata anche come varietà strutturale o diversità biotopica. (...) Haber comunque ritiene che per la pianificazione spaziale più importante sia la diversità spaziale o il mosaico (pattern) di alcune unità spaziali, cioè cellule di un paesaggio, differenti ma sostanzialmente affini ${ }^{\prime \prime 51}$.

"La diversità di un sistema combina il numero di elementi differenti presenti (ricchezza, varietà) e i relativi rapporti quantitativi. Tale caratteristica è studiata in molteplici realtà ambientali. (...) In un ambiente reale sono in genere presenti numerose specie animali e vegetali (...) Il livello di diversificazione delle specie presenti viene definito in ecologia dizersità biotica, ma tale concetto è estendibile anche ad altri campi: si può analizzare la diversità degli usi del suolo su un territorio, o la diversificazione delle fonti di alimentazione di una popolazione umana, o la varietà degli elementi che costituiscono un paesaggio. In ecologia la diversità biotica è tradizionalmente definita attraverso il numero di specie presenti nell'ambiente considerato (definito anche ricchezza specifica) e l'abbondanza relativa della specie (definita anche equitabilità o equiripartizione). Tale modello è del tutto generale: la teoria dell'informazione l'ha utilizzato per descrivere l'eterogeneità di qualsiasi insieme di elementi (dai fraseggi musicali agli elenchi telefonici). La diversità biotica è quindi più elevata in una realtà con un numero maggiore di specie (una maggiore ricchezza specifica) e, a parità di numero di specie, in una realtà con una distribuzione più omogenea delle presenze (una maggiore equiripartizione). (...) Come si è detto, l'applicazione di questo concetto in campo ambientale non deve essere limitata al

\footnotetext{
50 Carlo Blasi, nel suo intervento al convegno promosso da Italia Nostra sul tema Biodizersità nel paesagoio agrario storico (Firenze, 19 novembre 1999), ha documentato ampiamente l'importanza di queste sistemazioni anche dal punto di vista naturalistico, fornendo una ulteriore motivazione alle istanze più tradizionali di conservazione del paesaggio culturale, riferite ai valori storico testimoniali, oltre che ambientali e fisionomico-percettivi.

${ }^{51}$ Finke L., Introduzione all'E cologia del paesagoio, (Landschaftokologie), Franco Angeli, Milano, 1993 (Verlags-GmbH Holler und Zwick, Braunschweig, 1986). Il testo riporta anche la trattazione dei concetti di diversità e stabilità ed un'ampia bibliografia.
} 
settore strettamente ecologico. Ad esempio nelle analisi territoriali, uno spazio in cui gli usi del suolo siano molteplici, o ben equiripartiti, ha una diversità più alta rispetto ad altri spazi più omogenei; zone agricole ove un unico tipo di coltura sia assolutamente prevalente rispetto alle altre, da considerare quindi a bassa diversità, devono essere distinte da altre ove gli usi agricoli siano differenziati. Una caratteristica concettualmente vicina alla diversità è la complessità, che esprimeanche aspetti di tipo relazionale: complesso è un sistema che combina una elevata diversificazione dei suoi elementi costitutivi con una rete di molteplici relazioni tra di essi. (...) La diversità varia con le dimensioni dell'ambiente considerato, a secondo del contesto geografico, in relazione allo stato dinamico del sistema complessivo. La diversità non è una caratteristica assoluta, ma entro certi limiti è funzione delle modalità di analisi. Ad esempio il numero di specie trovate in una stazione aumenta con le dimensioni dei campioni analizzati o con le dimensioni dell'area considerata. (...) Tale condizione impone evidentemente attenzioni specifiche nelle analisi della diversità ambientale. (...) Per evitare errori, le analisi di diversità devono evidentemente essere fatte tra situazioni del medesimo livello geografico. (...) La diversità ambientale può essere stimata in modo relativamente oggettivo, attraverso indici sintetici convenzionali" ${ }^{\prime 52}$.

Non risultano ad oggi indici e tecniche di misurazione per l'analisi della dizersità paesistica, ad eccezione dell'indice di eterogeneità, sviluppato in ecologia del paesaggio con riferimento all'indice di dizersità biologica. Non si dispone di una letteratura scientifica sistematica, che abbia specificamente ed approfonditamente affrontato l'argomento, sul piano del suo ordinamento teorico, come della sperimentazione delle tecniche di conoscenza, al punto che pare lecito considerare il dubbio che esso possa avere una consistenza teorica e pratica, anche in funzione della sua stessa possibilità di discussione con gli strumenti che abbiamo accumulato, pur rimanendo certamente auspicabile l'approfondimento scientifico di questi aspetti. La natura complessa del paesaggio, governata da un elevato numero di variabili e difficilmente determinabile in tutta la sua articolazione, fa sì che vi siano evidenti difficoltà per una estensione del concetto di diversità e di sue applicazioni conoscitive e progettuali che lo coinvolgano proficuamente e concretamente. È però comunque possibile pensare alla misurazione di singole caratteristiche responsabili in parte della diversità paesistica complessiva, ad esempio gli usi del suolo, oppure le componenti ed i sistemi di interesse storico-culturale o di rilievo percettivo, e così altri ancora. Anche l'incidenza dei contesti culturali, sanciti a livello istituzionale come generatori fondamentali del paesaggio con la ratifica della Convenzione europea, fa sì che sia di difficile sistematizzazione il problema della conoscenza dei suoi gradi di diversità e dei relativi caratteri di metastabilità o criticità.

Sarà probabilmente utile ed interessante seguire ed approfondire gli sviluppi delle iniziative in corso sulle relazioni tra diversità biologica e paesistica. Sebbene sia opportuno che questi due concetti restino rigorosamente distinti, si ritiene importante il ruolo assunto dal paesaggio nei recenti documenti prodotti dal Consiglio d'Europa nell'ambito delle azioni per la conservazione della biodiversità. Con il supporto scientifico e tecnico del Centro Europeo per la Conservazione della Natura (ECNC) è infatti stata definita ed è in corso di sviluppo, con il secondo piano d'azione 20012005, la Strategia Pan-E uropea per la Dizersità Biologica e Paesistica ${ }^{53}$.

"Il fatto che la diversità sia componente fondamentale della qualità ambientale da proteggere è stato da tempo acquisito in principi fondamentali raccomandati in sedi internazionali; ad esempio già nel programma MAB (Man and Biosphere Program, 1974) dell'UNESCO una raccomandazione per l'istituzione di riserve della biosfera era la seguente: è desiderabile che riserze rappresentatize della biosfera contengano la massima zarietà possibile di ecosistemi, comunità ed organismi caratteristici del bioma. Una ragionedi base per cui la diversità biotica deve esser considerata una componente importante della qualità

${ }^{52}$ Malcevschi S., Qualità ed impatto ambientale, E taslibri, Milano, 1991.

${ }^{53}$ ECNC - E uropean Center for Nature Conservation, sito WE B: www.ecnc.nl/ . 
ambientale è che essa implica anche una elevata diversità genetica: un elevato numero di specie possiede per definizione un patrimonio genetico complessivo maggiore rispetto ad un insieme di poche specie. (...) Mantenere la diversità significa mantenere il massimo possibile di diversità genetica, la cui conservazione è un'assicurazione ed un investimento nei confronti delle generazioni future. Un'altra probabile ragione, che la psicologia ambientale potrà aiutare a verificare, è che il disporre di un ambiente variato corrisponde ad un'esigenza profonda della psiche umana. (...) Una motivazione che spesso è stata adottata in passato per giustificare i rapporti tra diversità e qualità è una presunta corrispondenza tra elevati livelli di diversità ed alti livelli di stabilità nei sistemi ambientali $(. .$.$) : tale corrispondenza non è stata confermata dai fatti { }^{\prime \prime 54}$.

Restano pertanto, anche sul piano dell'ordinamento teorico, ragionevoli dubbi sul fatto che sia possibile contare su relazioni biunivoche tra la diversità biologica e quella paesistica e tra i relativi segni qualitativi e risulta comunque del tutto assente un quadro organico in base al quale sia possibile apprezzare tali relazioni, nelle loro qualità positive e negative.

\section{2 - L a frammentazione paesistica come fenomeno di modificazione e criterio di descrizione del paesaggioperiurbam}

Il rilievo che nelle aree periurbane di pianura assumono le manifestazioni di frammentazione paesistica, indotte sostanzialmente dalla elevata pressione insediativa e dai modelli di governo territoriale, fa sì che questa fenomenologia possa rappresentare una componente determinante nello studio dei processi di modificazione del paesaggio e costituire pertanto un indicatore complesso della qualità paesistico-ambientale, secondo opportuni sviluppi metodologici di analisi e diagnosi.

Il concetto di frammentazione nella pubblicistica afferente alle discipline territoriali è talvolta legato all'attributo ambientale $e^{55}$, talvolta all'attributo paesistica ${ }^{56}$, che occorre leggere anche secondo le differenze linguistiche e culturali fra gli autori, provenienti da paesi appartenenti a diverse aree geografiche. In ogni caso il termine sta generalmente ad indicare uno stato di alterazione strutturale dovuto alla parcellizzazione di habitat animali e vegetali, di unità ecosistemiche, o di unità spaziali omogenee per uso del suolo, a cui consegue la perdita di diversità biologica e paesistica, in uno scenario complessivo di congestione e disarticolazione spaziale ${ }^{57}$.

"The overall structural and functional integrity of a landscape can be understood and evaluated in terms of both pattern and scale. (...) A common landscape pattern is fragmentation, which is often associated with the loss and isolation of habitat. Alternatively, fragmentation is considered as one of several land trasformation processes, which together may produce a diminution and isolation of habitat. Fragmentation also results from natural disturbances, such as fires and herbivore invasions, but has become an international land policy issue because of the widesperad alteration of land mosaics by human activities.

The spatial scale at which fragmentation occurs is important when identifying strategies to cope with continued habitat loss and isolation. For example, fragmented habitat at a fine scale may be perceived as intact habitat at a broad scale.

\footnotetext{
54 Malcevschi S., 1991, cit.

55 Bisogni L.G., Gariboldi A., Malcevschi S., Reti ecologiche ed interzenti di miglioramento ambientale, Il Verde E ditoriale, Milano, 1996 e Romano B., Continuità ambientale. Pianificare per il riassetto ecologico del territorio, Andromeda E ditrice, Teramo, 2000. ${ }^{56}$ Forman R.T.T., Land mosaics, the ecology of landscapes and regions, Cambridge, 1995.

${ }^{57}$ La definizione di fragmentation riportata da R.T.T. Forman nel più completo dei suoi lavori recenti è: "the breaking up of a habitat, ecosystem, or land-use type in small parcels" (si veda Forman R.T.T., 1995, cit.).
} 
Only by recognizing and addressing landscape changes across different scales (perhaps at least three) can planners and designers maximize protection of biodiversity and natural processes ${ }^{\prime \prime 58}$. Considerando che la perdita delle funzioni ambientali di habitat e di connessione di habitat delle popolazioni animali e vegetali è in diretto rapporto con alterazioni di qualità e quantità biotiche ed abiotiche del paesaggio, si ritiene corretto adottare il termine frammentazione paesistica per denominare una fenomenologia più complessa di quella ambientale e concausa di quest'ultima relativamente alle condizioni delle popolazioni animali e vegetali nei paesaggi culturali. In sostanza si ritiene prioritario il principio che le modificazioni del paesaggio indotte dai processi di uso e gestione delle risorse del territorio contribuiscono sostanzialmente a determinare le condizioni di frammentazione ambientale ${ }^{59}$. In ragione della matrice decisamente culturale del paesaggio dei territori di pianura soggettiad elevata pressione insediativa, è inoltre importante comprendere nel concetto di frammentazione paesisticala ridurione delle qualità e quantità di habitat umano, per quanto le modificazioni paesistiche che inducono isuddetti effettidi frammentazione ambientale, generano inevitabilmente perdita di identità e struttura funzionale, spaziale, storica ed ecologica, anche relativamente alle esigenze di vita delle popolazioni insediate. Ai fini specialistici di conoscenza delle dinamiche ecologiche animali e vegetali, in ecologia del paesaggio, sono stati distinti i processi di trasformazione spaziale identificati come cofattori della perdita di habitat secondo cinque diversi tipi e gradi di alterazione, ordinati per criticità progressiva (si veda lo schema della figura 6): perforazione (perforation), suddivisione (dissection), frammentazione (fragmentation), riduzione (shrinkage), progressiva eliminazione (attrition) ${ }^{60}$.

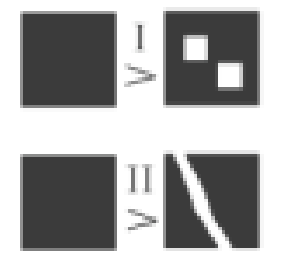

I Perforation: i processi spaziali producono trasformazioni del mosaico paesistico puntuali e pertanto discontinue, perforandone la matrice rurale; possono essere causate da edifici e gruppi di edifici isolati, come dalla dispersione urbana nelle aree rurali con buone condizioni di permanenza strutturale.

II Dissection: i processi spaziali producono trasformazioni del mosaico paesistico a prevalente sviluppo lineare, continue e generalmente costituenti reti molto estese, dizidendone la matrice rurale e, nei casi di reti sovralocali, anche quella urbana; possono essere causate da strade, ferrovie, canali di bonifica ed infrastrutture in genere.

III Fragmentation: i processi spaziali producono trasformazioni profonde e continue del mosaico paesistico, frammentandone la matrice rurale e producendo talvolta ulteriori alterazioni anche di quella urbana; dovute alla diffusione dell'insieme delle trasformazioni citate (I e II), danno luogo a effetti generalizzati di alterazione del paesaggio, con incremento dell'artificialità, perdita di equipaggiamento vegetale e funzioni ecologiche, perdita di permanenza storica e identità spaziale.

IV Shrinkage: l'incremento della diffusione ed intensità delle condizioni di frammentazione produce una riduzione di alcuni tipi di componenti e sistemi del mosaico paesistico, con conseguenti possibilicali di diversità biologica, morfologica e storica, e complessivamente con profonde e diffuse alterazioni del paesaggio.

V Attrition: la progressione dei processi di riduzione della presenza di alcuni tipi di componenti e sistemi del mosaico paesistico ne produce l'eliminazione, con conseguenti possibili perdite di diversità biologica, morfologica, storica, come talvolta di caratteri di rilevante interesse intrinseco, alterando completamente il paesaggio.

Figura 6 - Ideogramma dei processi di alterazione spaziale utili allo studio della frammentazione paesistica (adattato daForman R.T.T., L and mosaics, the ecology of landscapes and regions, Cambridge, 1995).

${ }^{58}$ Dramstad W.E., Olson J.D., Forman R.T.T., Landscape E cology Principles in Landscape A rchitecture and Land-Use Planning Harvard University Graduate School of Design - Island Press - American Society of Landscape Architects, Washington, 1996. Nostra traduzione del testo, riportato dall'edizione originale: "La totale integrità strutturale e funzionale di un paesaggio può essere capita e valutata nei termini di modello e scala (...). Un modello usuale è la frammentazione, che spesso è associata alla perdita ed all'isolamento degi habitat. In alternativa, la frammentazione è considerata come uno dei numerosi processi di trasformazione territoriale, che può produrre sia la diminuzione che l'isolamento degi habitat. La frammentazione risulta anche da disturbi naturali, come gii incendi e le invasioni degli erbivori, ma è diventata una urgenza politica internazionale a causa della alterazione diffusa del mosaico territoriale determinato dalle attività umane. La scala spaziale alla quale la frammentazione interviene è importante quando vengono identificate le strategie per fronteggiare la perdita e l'isolamento degli habitat continui. Per esempio, un habitat frammentato a scala di dettaglio, può essere percepito come un habitat intatto alla scala vasta. Solo riconoscendo ed indirizzando i cambiamenti del paesaggio attraverso le differenti scale (forse al minimo tre) i pianificatori ed i progettisti possono ottimizzare la protezione della biodiversità e dei processi naturali".

59 Dramstad W.E., Olson J.D., Forman R.T.T., 1996, cit.: “Natural processes as well as human activities change landscapes". Nelle aree periurbane di pianura, sono le attività umane e le loro strutture insediative a produrre i maggiori effetti di alterazione del paesaggio e pertanto anche di frammentazione della sua struttura.

60 Forman R.T.T., 1995, cit. 
Tale articolazione costituisce di fatto semplicemente un modello di analisi dei processi di trasformazione spaziale basato sul riconoscimento fisionomico delle diverse tipologie di componenti che concorrono a formare il mosaico del paesaggio e sull'osservazione dinamica delle modalità di trasformazione del mosaico stesso.

Poiché l'impostazione fisionomica può essere correttamente sganciata dagli specifici obiettivi di analisi, il modello risulta in questa forma applicabile alla interpretazione dei processi di trasformazione spaziale, oltre che per l'ecologia animale e vegetale, anche per gli aspetti di ecologia umana e per quelli culturali, relativi ai caratteri storici e morfologici del paesaggio.

La rappresentazione del processo di alterazione spaziale ${ }^{61}$ che il modello restituisce adduce ulteriori elementi di chiarezza sulla consistenza, la diffusione e l'intensità delle alterazioni caratteristiche della fenomenologia della frammentazione paesistica. È sufficiente infatti un semplice esperimento di lettura di una fotografia aerea zenitale secondo queste categorie, per avere idea della diffusione e della continuità spaziale caratteristiche delle criticità che esse rappresentano. Oggi, è frequente che nelle aree di pianura si parta da stadi di avanzata alterazione, che spesso coincidono con manifestazioni di frammentazione intensa e diffusa. Questo è di fatto il motivo principale per cui si ritiene significativo assumere come centrale il concetto di frammentazione rispetto agli altri considerati dal modello, che possono essere visti come stadi antecedenti o successivi, in ragione dei quali sono minori o maggiori le difficoltà di attuare efficacemente politiche capaci di invertire le tendenze critiche. In tal senso lo stesso Forman, pur rilevando l'importanza ecologica della distinzione di queste specifiche geometrie di modificazione spaziale per gli effetti sulle popolazioni naturali, segnala l'opportunità dell'utilizzo del concetto esteso di frammentazione paesistica nei processi di analisi spaziale ${ }^{62}$.

La consistenza scientifica del concetto di frammentazione paesistica risulta direttamente implicata anche dalla definizione delle teorie e dei metodi per l'interpretazione della frammentazione ambientale. Sia l'analisi della biopermeabilità proposta nella ricerca Planeco in Italia, che il modellodi interpretazione ecosistemica delle trasformazioni spaziali di cui si sta parlando, legato ad esperienze di ecologia del paesaggio statunitensi ed europee, fanno riferimento a categorie di modificazione spaziale, o se vogliamo riferisi ad una denominazione esplicitamente negativa, di alterazione spaziale. Ciò comporta il diretto coinvolgimento del paesaggio in tutte le sue articolazioni strutturali profonde: un'evidenza scientifica, alla quale fa eccezione solo il caso in cui tale osservazione sia riferita alla concezione del paesaggio come entità estetica, o comunque esclusivamente dipendente dalle variabili culturali della percezione sociale ed individuale dei luoghi, estranea all'impostazione di questa ricerca. È un'evidenza dovuta essenzialmente alla costituzione stessa del paesaggio, complesso sistemico, la cui struttura può essere letta in chiave molecolare, secondo la più spinta disaggregazione delle sue componenti, ma anche rispetto a livelli di organizzazione maggiore che, pur non coincidendo in tutto con la sua reale complessità, offrono significativi elementi di conoscenza. Considerando il livello di organizzazione del paesaggio che ha come entità di riferimento gli spazi aperti, nella loro articolazione minuta del mosaico paesistico in tessere omogenee, si accede ad una tipologia di elementi già in sé complessi, sezioni verticali complete del paesaggio, che restano subordinate rispetto al livello organizzativo massimo che afferisce a quest'ultimo, per il fatto che non ne contengono autonomamente tutta la struttura relazionale. È tale struttura che determina il paesaggio come massimo livello di organizzazione considerato in questo ragionamento, conferendogli caratteristiche diverse dagli spazi che lo compongono, secondo il principio olistico per cui il tutto è più della somma delle parti. Pertanto, tornando alla riflessione sulla consistenza scientifica del concetto e della problematica della frammentazione paesistica, se si riconosce l'articolazione spaziale come livello organizzativo strutturale del paesaggio, ne viene appunto l'evidenza che i processi di

${ }^{61}$ La categoria dell'alterazione, riferita all'analisi del paesaggio, è stata proposta da G.G. Rizzo in Tuscia Romana. Paesagoio e territorio: metodi e modelli di zalutazione (a cura di G.G. Rizzo; Gangemi, Roma, 1990).

62 Forman R.T.T., 1995, cit. 
trasformazione spaziale, oltre ad avere i documentati effetti di frammentazione ambientale degli habitat animali e vegetali, possono avere effetti di frammentazione del paesaggio.

Acquisito il principio che i fenomeni di frammentazione paesistica dipendono dai processi di trasformazione spaziale, risulta l'importanza dell'intepretazione di questi ultimi, anche per la definizione metodologica stessa.

Osservando che nelle aree di pianura i processi di trasformazione spaziale sono in atto nel nostro paese da due millenni e che tali processi negli ultimi cinquanta anni hanno assunto connotati generalizzati di alterazione del paesaggio, più che di modificazione intesa come contributo positivo alla stratificazione storica della sua struttura, si può dedurre come la frammentazione paesistica sia una patologia particolarmente diffusa, che nelle aree ad elevata pressione insediativa e/o produttiva raggiunge livelli rilevanti di copertura del territorio.

La Pianura Padana è certamente il bacino territoriale italiano più significativo da questo punto di vista e, l'area milanese in particolare, si colloca al terzo posto in Europa, dopo Londra e Parigi, per strutturazione megapolitana. "La superficie edificata è ormai così estesa che degli originari ecosistemi padani non si è conservato più nulla o ben poco. Ora si deve parlare di nuovi ecosistemi nei quali l'elemento antropico appare come il fattore dominante, che ne fa dei sistemi continuamente aperti ed oscillanti per effetto dei progressivi interventi, sia aggiuntivi che correttivi. Vi sono aree della megalopoli di cui si può parlare come di sistemi degenerati, dove cioè i normali processi naturali, basati su relazioni fra suolo, aria, vita vegetale e vita animale, non hanno più luogo, con effetti dannosi per l'uomo, per la sua salute fisica, per la sua psicologia, il suo senso del vivere, del produrre e dello stesso riprodursi come membro di una società. (...) Il comune di Rho, ad esempio, ha una superficie che per il $62 \%$ del totale è destinata ad uso urbano (...). In primo luogo sono scomparse molte delle formazioni vegetali che pur un tempo l'uomo aveva mantenuto ed utilizzato (...); sono scomparsi i fontanili e i corsi d'acqua sono diventati cloache (...). Gli apporti idrici non riescono poi a rimpinguare sufficientemente le falde idriche, dato il forte prelievo, sia da parte dell'industria che degli usi pubblici. La zona serba ancora le tracce inquinanti della raffineria IP costruita negli anni Sessanta alle porte di Milano (...): nei suoli usati per le coltivazioni si trovano ancora benzene, pirene, naftaline, idrocarburi e oli minerali; ciò unitamente ai sedimenti delle piogge acide rende mal praticabile l'agricoltura (circa il 38\% della superficie territoriale, n.d.r.), peraltro ormai presente in pochi spazi interstiziali, e rende i suoli incapaci di autodepurarsi ${ }^{\prime \prime 63}$.

La conoscenza delle alterazioni paesistiche di origine antropica, che nel secolo passato hanno avuto una progressione assolutamente unica nella storia, rende decisamente evidente il grado di intensità che le manifestazioni di frammentazione strutturale possono raggiungere, fino alla eliminazione completa delle configurazioni a cui si riferisce lo stesso processo di frammentazione. Sempre più difficilmente, con il progredire della edificazione e della infrastrutturazione del territorio, di pari passo alla marginalizzazione delle aree agricole, nella pianura fiorentina è ad esempio oggi possibile riconoscere la frammentazione della struttura insediativa storica rurale, le cui componenti, edifici, strade, alberate, sistemi di drenaggio, sono sempre più profondamente ridotte, avvicinandosi evidentemente allo stato processuale critico della eliminazione. Altrettanto, se non più gravemente, accade per la struttura spaziale della quale sono difficilmente rintracciabili, perfino a livello di frammenti, configurazioni equilibrate e con precise identità, tanto è intenso e diffuso il processo di riduzione delle formazioni paesistiche ad esse riconducibili.

Nel proseguire il parallelo con le indicazioni delle applicazioni ecologiche, si può rilevare come secondo queste "la framentazione del paesaggio produce una serie di aree naturali relitte circondate da una matrice territoriale di natura più o meno profondamente diversa. (...) Ai fini del governo del territorio è importante sapere se le zone relitte hanno un valore effettivo per la conservazione della

63 Turri E., 2000, cit. 
biodiversità e, se il valore esiste, come devono essere gestite affinchè esso venga mantenuto. Una valutazione delle attuali condizioni di frammentazione dell'ambiente naturale deve considerare:

- le esigenze delle specie potenzialmente interessate;

- gli impatti intrinseci alla frammentazione in quanto tale;

- i tipi e le geometrie della frammentazione;

- il comportamento della matrice di riferimento;

- il ruolo dei corridoi ecologici.

La criticità della frammentazione dipende dalle caratteristiche delle specie a cui si fa riferimento. Un primo evidente impatto è la riduzione della superficie complessiva di ambiente naturale, e quindi della quantità di habitat a disposizione delle specie di interesse presenti negli ecosistemi. Vi sono poi una serie di conseguenze specifiche della frammentazione, che si traducono in svantaggi aggiuntivi rispetto alla semplice perdita di habitat. In una revisione di molteplici studi effettuati al riguardo su mammiferi ed uccelli, Andrein (1994) rileva che, mentre al di sopra del 30\% di habitat idoneo sul territorio la perdita di animali è proporzionale alla perdita di habitat, al di sotto di questa soglia, il decremento aumenta in modo relativamente maggiore ${ }^{\prime \prime 64}$.

Alcuni studi di ricerca applicata sviluppati nell'ambito disciplinare della pianificazione ambientale, hanno considerato il concetto di continuità ambientale in contrapposizione a quello di frammentazione, introducendo quello complementare, già citato, di biopermeabilità del territorio.

"La metodologia della continuità ambientale è stata elaborata attraverso diverse fasi di sperimentazione, ritenendo questo aspetto territoriale, dipendente prevalentemente dalla geografia delle componenti urbanistico-insediative e dalle modalità di uso del suolo, un riferimento basale per le considerazioni attinenti invece la sfera della reticolarità ecologica, dipendente a sua volta essenzialmente dalle componenti di carattere biologico-naturalistico"165.
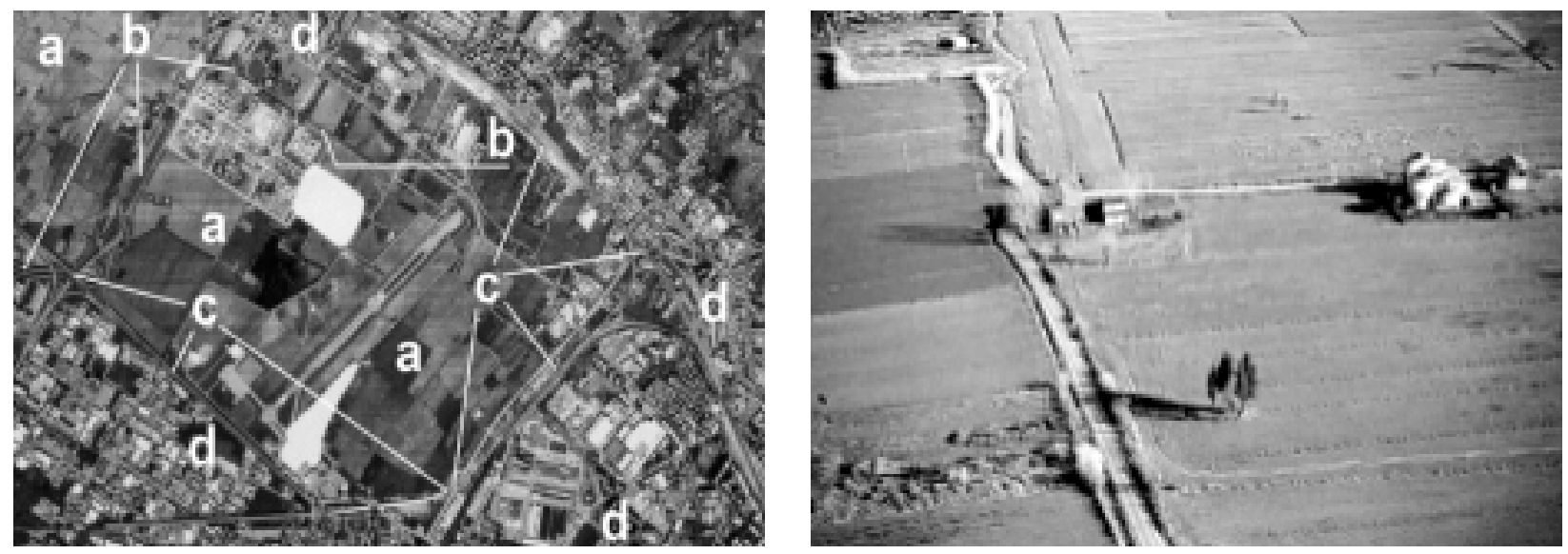

Figura 7 (a sinistra) - Il fenomeno della frammentazione paesistica nell'area periurbana occidentale di Firenze tra le colline di Castello e Scandicci, secondo quattro categorie generali: a) omologazione e semplificazione spaziale e biologica del paesaggio rurale - b) assenza di diversificazione spaziale e biologica e rigidità di conformazione dei canali artificiali di bonifica - c) infrastrutturazione congestionata e priva di sistemi di compensazione ed inserimento paesistico-ambientale d) espansione urbana priva di sistemi di compensazione ed equipaggiamento paesistico-ambientale (rappresentazione ridotta, dall'originale in scala 1:30000 - fotografia aerea zenitale Regione Toscana).

Figura 8 (a destra) - La "desertificazione" spaziale delle colture agricole della piana di Castello alla fine degi anni ottanta del '900. L'intensità del fenomeno fa sì che queste configurazioni risultino degradate ma anche degradanti e, nello specifico della frammentazione paesistica, sia aree ad elevata frammentazione, che fattori di frammentazione. Le condizioni odierne di questi paesaggi sono caratterizzate rispetto alle immagini qui riportate, da evidenti forme di recupero spontaneo di naturalità, dovute essenzialmente all'abbandono dei campi ed in progressione, senza il controllo di alcuna forma di governo ed in assenza di qualunque idea contemporanea del paesaggio di pianura (immagini tratte da: $G$. Di Pietro, Un progetto per Firenze - La nuoza città nella piana di Castello, Ponte alle Grazie, Firenze, 1990).

${ }^{64}$ Bisogni L.G., Gariboldi A., Malcevschi S., 1996, cit.

65 Romano B., 2000, cit. Per gi sviluppi delle ricerche sulla continuità ambientale e le relazioni con la reticolarità ecologica, si rimanda, oltre che al testo citato, al sito WE B PLANE CO Project: http://dau.ing.univaq.it/planeco. 
Gli effetti diversificati della frammentazione paesistica richiedono la definizione di congrue risposte nell'ambito delle azioni per la riqualificazione diffusa del paesaggio. In Europa, ed ancor più in Italia, la denominazione di paesaggio naturale è correttamente applicabile a rare situazioni relitte ed, in quanto tali, inevitabilmente minoritarie ed isolate. Il tema della conservazione della natura assume allora come soggetto di conoscenza e progetto il paesaggio culturale, che caratterizza i nostri parchi naturali ed è diffuso in tutto il territorio a regime ordinario, notoriamente connotato da condizioni di seminaturalità diffusa. La semplificazione e l'alterazione degli equilibri dinamici dei paesaggi culturali ha prodotto una riduzione importante di habitat animali e vegetali, con pesanti effetti di isolamento delle aree strategiche dal punto di vista naturalistico, fino a metterne a rischio la funzionalità ecosistemica. Ripercussioni di non poco peso gravano anche sull'ecologia umana: la carenza di vegetazione è fra i fattori responsabili dei problemi di regolazione microclimatica e di protezione dall'inquinamento che colpiscono gli insediamenti urbani contemporanei, come della ridotta capacità di autoprotezione e conservazione della produttività delle moderne monocolture agrarie intensive. A questi grandi gruppi di effetti, in realtà molto articolati, si aggiunge la riduzione del potenziale ricreativo del paesaggio, di evidente importanza strategica, sia sul piano sociale, che su quello economico, e capace di condizionare anche la stessa efficacia delle politiche delle aree protette, soprattutto a causa delle qualità delle aree ad esse contigue.

Un'impostazione delle problematiche di frammentazione strutturale basata su principi comprensivi, può attivare processi di conservazione e recupero del paesaggio attraverso misure efficaci nei confronti di molteplici obiettivi che, nei paesaggi culturali, possono presentare spesso relazioni di complementarietà:

- conservazione e miglioramento della biodiversità e delle condizioni di vita e riproduzione delle popolazioni animali e vegetali, attraverso la salvaguardia e la neocostituzione degli habitat e dei sistemi paesistici di connessione;

- rafforzamento dell'efficacia delle politiche per le aree protette, attraverso il superamento del modello insulare e la costituzione di sistemi territoriali dotati di livelli di protezione effettivamente graduati rispetto alle fonti di impatto;

- conservazione e miglioramento delle condizioni di produttività e di protezione ambientale degli ecosistemi agrari che presentano un elevato grado di consumo delle risorse non rinnovabili, attraverso l'equipaggiamento e la diversificazione del paesaggio;

- difesa e riequilibrio idrogeologici, attraverso la progressiva rinaturazione dei corsi d'acqua e la messa in sicurezza delle aree sensibili, con strategie di rispetto e di riserva di spazi per le dinamiche idrauliche;

- salvaguardia di risorse per le popolazioni future, attraverso il concreto riconoscimento di tali finalità come base strategica fondamentale per qualunque tipo di politica per lo sviluppo sostenibile;

- miglioramento ambientale urbano dal punto di vista microclimatico, della mitigazione dei fenomeni di inquinamento, dell'offerta qualitativa e quantitativa di spazi per la ricreazione, attraverso la conservazione e l'equipaggiamento paesistico di spazi aperti organizzati in termini sistemici;

- recupero morfologico dell'identità del paesaggio, attraverso il riconoscimento e l'interpretazione progettuale dei caratteri storici e lo sviluppo di caratteri contemporanei identificanti;

- riappropriazione culturale dei valori del paesaggio e sviluppo delle identità territoriali progressivamente omologate dall'evoluzione sociale contemporanea, attraverso la fruizione del paesaggio secondo condizioni formative, informative e logistiche idonee alla sua comprensione ed al conseguente riconoscimento di valore;

- miglioramento qualitativo dell'offerta turistica, particolarmente importante dal punto di vista socioeconomico, attraverso la diversificazione e la realizzazione di circuiti di qualità diffusa fra i siti 
tradizionali di maggior rilievo storico, culturale e naturalistico e l'attuazione di politiche di marketing territoriale sostenibili sui piani paesistico ed ambientale ${ }^{66}$.

Vi sono alcune coincidenze con quanto Luigi Boitani ha chiamato la "protezione delle funzioni". Nell'ambito delle politiche per la natura ed il paesaggio, "non c'è dubbio che questa tattica della conservazione sia di gran lunga la più moderna ed efficace. Spostando l'enfasi dalle singole specie e dai singoli habitat, l'attenzione dell'azione di conservazione viene posta sul mantenimento di alcune funzionalità interne dei sistemi ecologici, coinvolgendo in una unica lettura sia le specie che gli ambienti che le ospitano. La difficoltà di realizzazione di questo approccio risiede nella identificazione delle funzionalità e poi degli ambiti spaziali in cui esse possono essere delimitate. Le funzioni ecosistemiche sono quindi lette soprattutto nei termini riduttivi di servizi forniti dai sistemi ecologici all'uomo, come ad esempio il mantenimento della qualità delle riserve idriche o di risorse vegetali o faunistiche. È certo una lettura molto antropocentrica ma ha due grandi vantaggi, legare l'azione di conservazione ad un immediato e concreto ritorno per le popolazioni locali, e la possibilità di quantificare in termini economici i costi e i benefici dell'azione di conservazione ${ }^{\prime \prime 67}$.

L'applicazione monocorde delle teorie delle reti ecologiche risulta decisamente parziale in confronto alle potenzialità molteplici della promozione di politiche di piano basate su logiche di differenziazione degli utilizzi degli spazi: potenzialità di compensazione e miglioramento ambientale che si rivelerebbero fortemente incisive anche in termini di benefici alle popolazioni umane, senza negare gli spazi di efficacia per la salvaguardia della vita e della diversità animale e vegetale.

"Il punto di partenza può considerarsi la teoria dell'uso differenziato del territorio per garantire l'equilibrio ecologico. Essa trasferisce alla pianificazione territoriale ed urbanistica principi di organizzazione ecologica dedotti dal funzionamento degli ecosistemi, la cui stabilità dipende dalla diversità. (...) L'aumento dell'entropia e quindi del disordine degli ecosistemi è caratterizzato da uniformità spaziale e instabilità temporale, mentre al contrario l'aumento della negentropia, ossia dell'ordine, è caratterizzato da diversità spaziale e stabilità temporale. La modernità tende notoriamente a livellare il territorio, cancellando la diversità ed aumentando l'entropia. Tipica per esempio è la distribuzione pressoché ubiquitaria di insediamenti residenziali e produttivi. Al contrario, l'alternanza tra zone con forti carichi ambientali e zone con forti capacità di assorbimento dei carichi stessi, oppure di sviluppo di ecosistemi naturali, pur assicurando spazi adeguati alle esigenze della modernità, appare ecologicamente più favorevole ${ }^{\prime \prime 68}$.

Il modello insediativo delle aree di pianura ad elevata pressione insediativa più rispondente a questi criteri e con il potenziale di frammentazione paesistica minore, o forse è meglio dire più governabile,

\footnotetext{
66 Testo adattato dalla nota curata dall'autore per l'introduzione del seminario di studio del Dottorato di Ricerca in Progettazione Paesistica tenuto dal prof. Bernardino Romano sul tema Frammentazione ambientale e politiche di gozerno territoriale - Significati e potenzialità delle reti ecologiche nella pianificazione dei paesagoi culturali (Università degli Studi di Firenze, Dipartimento di Urbanistica e Pianificazione del territorio, Firenze, 5 aprile 2000) e da Connettizità ecologica e qualità del paesaggio nei lineamenti territoriali per il riordino degli spazi aperti urbani e periurbani della Valdiniezole (PT), in Guccione B., Paolinelli G. (a cura), Piani del zerde E Piani del paesagoio, Alinea, Firenze, 2001.

${ }^{67}$ Boitani L., La tutela e la anlorizzazione della biodizersità terrestre in Italia: appunti per la Rete E cologica Nazionale, intervento al convegno nazionale Conserzazione della natura e sziluppo locale: il sistema delle aree protette e la Rete Ecologica Nazionale, ECOLAVORO99, Legambiente, Ministero dell'Ambiente, Federazione Italiana Parchi e Riserve Naturali, Firenze 14 dicembre 1999.

${ }^{68}$ Di Fidio M., Teoria e prassi delle reti ecologiche. Dall'isolamento all'integrazione della difesa della natura, intervento al seminario di studio del Dottorato di ricerca in Progettazione Paesistica Le reti ecologiche. Strategie di equipaggiamento paesaggistico e mighioramento ambientale, Università degli Studi di Firenze, Dipartimento di Urbanistica e Pianificazione del territorio, Firenze 26 maggio 1999.
} 
sembra essere quello policentrico a rete ${ }^{69}$. Darebbero utili approfondimenti di questi aspetti in considerazione del fatto che la configurazione policentrica è stata confermata dalla ricerca Itaten come "caratteristica specifica del modo di manifestarsi del fenomeno metropolitano nelle aree di pianura della Toscana settentrionale ${ }^{\prime \prime 70}$, e della conseguente opportunità di considerarne i possibili sviluppi contemporanei verso paesaggi di pianura maggiormente equilibrati, nei quali idonee politiche territoriali e misure di intervento risultino efficaci nel contenimento e nella riduzione della frammentazione del paesaggio e nella sua qualificazione ecologica, morfologica e storica.

Anche nell'ambito delle riflessioni sviluppate dall'urbanistica, si è rintracciato il concetto di frammentazione: esso risulta riferito alle città dei paesi in via di sviluppo, in contrapposizione alla città occidentale alla quale, viene attribuita una struttura spaziale gerarchizzata, ma unitaria, se pur fortemente articolata al proprio interno. "La frammentazione della città dei paesi in via di sviluppo è la diretta conseguenza della fase storica coloniale. (...) L'urbanizzazione del dopo indipendenza ha avuto luogo invece (e ancora oggi ha luogo, salvo poche eccezioni assolutamente peculiari) nel quadro di una assoluta incapacità di pianificare e governare la crescita e le trasformazioni della città. (...) Le città multimilionarie che in misura crescente costellano i paesi in via di sviluppo, insieme a tassi di crescita demografica tuttora molto elevati, non permettono di "governare" l'espansione urbana, soprattutto tenendo conto delle limitate risorse (non solo finanziarie) a disposizione. Sta di fatto che i risultati a livello dell'organizzazione spaziale sono stati, nel dopo-indipendenza, non dissimili da quelli del periodo della dominazione coloniale. La frammentazione spaziale non è dunqueun fenomeno temporaneo, anzi, essa sembra configurarsi come un fatto strutturale, con il quale ci si dovrà confrontare per molto tempo a venire. Occorre assumerla come condizione sostanziale e non come una "anomalia" rispetto ad una situazione di "normalità", che il modo di organizzarsi delle nostre città rappresenterebbe ${ }^{\prime \prime 71}$.

A Firenze, nell'area periurbana occidentale della piana compresa tra le colline di Castello e Scandicci, sono riscontrabili manifestazioni intense e diffuse di frammentazione paesistica, afferenti a quattro categorie generali, tipiche delle aree di pianura soggette ad elevata pressione insediativa (figura 7): omologazione e semplificazione spaziale e biologica del paesaggio rurale, artificializzazione dei corsi d'acqua naturali regimati ed assenza di diversità biologica e spaziale dei canali artificiali di bonifica, infrastrutturazione congestionata e priva di sistemi di inserimento e compensazione paesisticoambientale ed infine, espansione urbana priva di sistemi di inserimento e compensazione paesisticoambientale.

\footnotetext{
69 "Applicato alla pianificazione di un'area metropolitana a scala vasta il modello a rete può condurre a unità due modelli di orgnanizzazione territoriale estremi, che da soli non possono generare un disegno ottimale. Il primo modello è quello tradizionale di organizzazione territoriale su una rete radiocentrica, fondato su grandi infrastrutture di trasporto collettivo e privato, che tuttavia, a causa della crescente polarizzazione che genera fra posti di lavoro (al centro) e residenze (alla periferia), non è più in grado da solo di garantire un ordinato sviluppo territoriale. Il secondo modello, in parte opposto, è quello della ville éclatée, della città diffusa o "sparpagliata", in cui tendenzialmente non vi è più un centro ma una serie infinita di piccole polarità integrate in reti assai connesse di trasporto e comunicazione a livello della città-regione. È il modello di Los Angeles, per intendersi, un modello che utilizza appieno tutti i gradi di libertà concessi da due tecnologie moderne, il telefono (tendenzialmente connesso a una rete di computer) e l'auto privata; un modello ad alto consumo di suolo che effettivamente vediamo realizzarsi nelle megalopoli americane ed europee, sull'onda di una crescente deregulation urbanistica. Anche questo modello incontra presto due limiti vistosi: esso ha bisogno di innervature maggiori di trasporto su gomma per evitare gii ingorghi crescenti di traffico e, allorché presenta, come nel caso di tutta la fascia centrale lombarda, una commistione fra residenze e piccole unità di produzione industriale, genera guasti ambientali irreparabili. Il modello policentrico a rete risponde a questi problemi mantenendo da una parte il senso della città compatta tradizionale, attraverso il riferimento al centro e all'effetto città; ma d'altra parte moltiplica i centri (che non sono solo "nodi" di una rete, ma veri "luoghi") diffondendoli su un territorio vasto e integrandoli in una rete efficiente, ma non più radiocentrica, di trasporto (come la rocade del progetto Orbitale parigino)". Testo tratto da Camagni R., Processi di utilizzazione e difesa dei suoli nelle fasce periurbane: dal conflitto alla cooperazione, in Boscacci F., Camagni R. (a cura), Tra città e campagna, Milano, 1994, cit. anche in Turri E ., La megalopoli padana, Marsilio, Venezia, 2000.

${ }^{70}$ Innocenti R. (a cura), Toscana, in A. Clementi, P.C. Palermo, G. Dematteis, (a cura), Le forme del territorio italiano, II volume, Laterza, Bari, 1996.

${ }^{71}$ Balbo M., La città di frammenti, Urbanistica, 100, 1990.
} 
Nelle aree di pianura, "il sistema rurale essendo esso stesso fattore di degrado ambientale non è più in grado di costituire antidoto al degrado urbano. Ciò tenendo conto della trasformazione nell'uso dei fondi agricoli che hanno subito le pesanti riduzioni dei terreni in regime sodivo e la drastica riduzione delle piante legnose di campo e di ripa. La valutazione ecologica delle modificazioni antropiche assume del resto una particolare importanza nelle aree periurbane, dove i conflitti per l'uso delle risorse originarie (in primo luogo il suolo), e gli effetti distruttivi che vengono esercitati sulle stesse risorse, rendono del tutto evidente il concetto di rischio causato dalle trasformazioni urbanistiche ed agricole attualmente in corso e postulano come necessaria l'adozione di misure di controllo globale dell'ambiente. Infatti è proprio in questi ambienti periurbani che più urgente si presenta la necessità di ridare naturalità al sistema territoriale. Si tratta in particolare di considerare il sistema della vegetazione come il tessuto unificante in grado di contrastare l'output dell'ecosistema urbano, e di contrapporsi alle gravi conseguenze dello sprazd delle aree periurbane, anche per avviare un processo di conciliazione-integrazione tra le metodologie della pianificazione dell'ecosistema urbano e quelle della pianificazione dell'ecosistema agricolo'"72.

A questo tipo di considerazione, per quanto già detto, occorre affiancare la presa in carico degli effetti sui caratteri storici e spaziali del paesaggio. L'immagine della piana di Firenze riportata nella figura 8 è estremamente significativa nel descrivere questa evidenza. Alla frammentazione della struttura e delle relazioni biotiche, dovuta in questo caso essenzialmente alla eliminazione dei soprassuoli vegetali arborei ed arbustivi, si lega evidentemente la profonda alterazione degli equilibri morfologici di questi spazi, che nelle alberate avevano i più importanti caratteri di diversificazione, ed identificazione dimensionale e semiologica. Le stesse alberate, che hanno costituito un soprassuolo con completa dignità ed efficacia di architettura del paesaggio, fanno parte con le strade e l'edilizia rurale, i complessi civili e religiosi, di una struttura paesistica storica di rilevante valore culturale, che oggi risulta solo faticosamente rintracciabile a causa della pervasività e della congestione degli sviluppi insediativi e infrastrutturali metropolitani e della connessa marginalizzazione delle attività agricole, nonostante l'elevata resistenza che il paesaggio oppone alle modificazioni attraverso la propria struttura semiologica profonda.

In questa direzione, della ricerca di tecniche di analisi e diagnosi comprensive delle principali componenti fenomenologiche della frammentazione paesistica, è stata orientata l'individuazione dei fattori causali e degli indicatori primari.

\footnotetext{
72 Tosi A. (a cura), Degrado ambientale periurbano e restauro naturalistico, Quaderni del Dipartimento di Scienze del territorio del Politecnico di Milano, Angeli, Milano, 1999.
} 


\section{2 - Le conoscenze analitiche finalizzate}

\section{1 - Individuazione dei temi di analisi finalizzata}

I riferimenti scientifici per le analisi territoriali della frammentazione ambientale ${ }^{73}$ afferiscono generalmente alle discipline applicate della biologia conservazionale, dell'ecologia del paesaggio e della pianificazione ambientale, interessando specificamente l'ecologia delle popolazioni animali e vegetali e complessivamente il livello di biodiversità dei diversi ambienti.

Nell'ambito di questo lavoro, come si è sottolineato fino dalla premessa, si ritiene importante prefigurare la possibilità di estendere lo studio di questa fenomenologia alla considerazione di altri caratteri strutturali del paesaggio, relativi alla stratificazione storica, alla morfologia degli spazi, alle funzioni ambientali incidenti sull'habitat umano.

Negli studi paesistici, che siano mirati alla definizione progettuale di piani per l'uso e la gestione delle risorse o di interventi per la realizzazione di opere, come anche in quelli destinati alle sole finalità conoscitive, si è soliti fare riferimento a due fasi principali di elaborazione delle informazioni: l'analisi e la diagnosi. Alla prima competono il rilievo e l'organizzazione sistematica dei dati, alla seconda l'interpretazione delle conoscenze analitiche, secondo specifici obiettivi ed attraverso idonei metodie strumenti.

In entrambe queste tipologie di elaborazione conoscitiva, ricorre in genere una ulteriore distinzione, che si ritiene utile tenere presente per ragioni di chiarezza e comunicabilità del processo di trattamento delle informazioni: si tratta della distinzione tra conoscenze tematiche e conoscenze di sintesi.

Nel caso in questione, date per note le principali conoscenze tematiche di base, in quanto assolutamente strumentali ed ininfluenti nella costruzione del ragionamento proposto, si pone un interrogativo essenziale in merito alla natura delle analisi finalizzate alla diagnosi di frammentazione paesistica, con specifico riferimento alle peculiarità del paesaggio periurbano di pianura, come tipologia critica caratteristica dei territori soggetti ad elevata pressione insediativa.

Il soggetto di studio, nell'area metropolitana di Firenze, è stato assunto come campione sperimentale per la forte rappresentatività che lo caratterizza nel panorama italiano, in cui prevalgono, anche nelle realtà insediative più importanti, centri di dimensioni medie e medio-piccole.

73 Oltre al fondamentale lavoro di Forman R.T.T., Land mosaics, the ecology of landscapes and regions, Cambridge, 1995, si vedano, fra gi altri: Bisogni L.G., Gariboldi A., Malcevschi S., Reti ecologiche ed interzenti di miglioramento ambientale, Il Verde E ditoriale, Milano, 1996; Dramstad W.E., Olson J.D., Forman R.T.T., Landscape E cology Principles in Landscape A rchitecture and Land-Use Planning, Harvard University Graduate School of Design - Island Press - American Society of Landscape Architects, Washington, 1996; Fabbri P., Natura e cultura del paesaogio agrario, CittàStudi, Milano, 1997; Ingegnoli V., Fondamenti di ecologia del paesaggio, CittàStudi, Milano, 1993; Romano B., Continuità ambientale. Pianificare per il riassetto ecologio del territorio, Andromeda E ditrice, Teramo, 2000. 
Come primo genere di conoscenza finalizzata, risulta utile che l'analisi dei fattori territoriali di frammentazione paesistica sia riferita alle cause reali o potenziali di discontinuità ed impoverimento della struttura del paesaggio, sia che ciò avvenga da un solo punto di vista, sia nel caso in cui vi siano ricadute su più aspetti: oltre che su quelli ecosistemici, anche su quelli storici o morfologici.

Ad un secondo genere di analisi afferisce la lettura delle condizioni del paesaggio tramite indicatori idonei a cogliere la consistenza delle manifestazioni di frammentazione dovute ai fattori causali rilevati.

Attraverso tali analisi, necessariamente condotte come distinte elaborazioni su base cartografica, la sintesi diagnostica potrà individuare le condizioni di stato e le dinamiche, riportando le conoscenze disaggregate ad un grado di unitarietà rispondente agli obiettivi della descrizione strutturale del paesaggio e della sua interpretazione progettuale.

In ragione delle principali relazioni intercorrenti tra la struttura del paesaggio ed i processi di crescita insediativa, si riscontrano, anche nel caso del territorio fiorentino, quattro forme principali di utilizzazione delle risorse, tipiche delle aree metropolitane per diffusione ed intensità delle manifestazioni: gli insediamenti, le infrastrutture viarie, le infrastrutture e le strutture tecnologiche, le attività produttive primarie e secondarie.

Tali tipi di utilizzazione degli spazi costituiscono, singolarmente e per aggregazione, cause di frammentazione paesistica ed ambientale. Ai fini della relativa diagnosi essi vengono pertanto classificati come fattori territoriali di frammentazione (paragrafo 2.2; appendice A: schede di identificazione morfologica dei fattori territoriali), distinti secondo la loro articolazione spaziale (fattori lineari, areali, puntuali) ed in base al grado di omogeneità della loro configurazione (fattorisemplici, complessi).

In merito agli indicatori di frammentazione paesistica vengono proposte cinque analisi tematiche, relative ad altrettanti caratteri strutturali del paesaggio sensibili alle alterazioni: mineralizzazione del paesaogio, equipaggiamento zegetale del paesagoio, permanenza storica del paesaggio, articolazione spaziale del paesagio, articolazione funzionale del paesagoio (paragrafo 2.3). Esse, ove sia possibile considerare la scala temporale oltre quella spaziale, possono coprire la descrizione della fenomenolgia, sia dal punto di vista della struttura del paesaggio, che dai punti di vista del suo funzionamento e del suo cambiamento.

Risulta evidente anche dalla composizione di questo gruppo di indicatori il peso che si attribuisce, secondo gli orientamenti già espressi, ad alcune conoscenze distinte e complementari rispetto a quelle proprie dell'ecologia del paesaggio.

Mentre però per i fattori territoriali la motivazione delle distinzioni proposte discende direttamente dal riconoscimento di quanto nella realtà paesistica si ritiene causa di frammentazione strutturale, per gli indicatori le scelte sono, come avviene in genere nei processi conoscitivi che utilizzano entità concettuali di questo tipo, meno immediatamente e direttamente motivabili, soprattutto nei termini elementari del perché un determinato indicatore viene prescelto al posto di un altro. Questo non vale per la mineralizzazione del paesaggio, che costituisce il principale indicatore macroscopico della fenomenologia della frammentazione, certamente parziale e grossolano nella descrizione, ma altrettanto ineludibile per l'importanza e l'intensità del tipo di alterazione che rileva.

Il criterio discriminante con cui sono stati individuati gli altri ha coinciso sostanzialmente con la riscontrabilità di una rilevante incidenza dei fenomeni di frammentazione paesistica sulle formazioni, sulle relazioni o sulle valenze che l'indicatore rileva, ovvero di caratteristiche di elevata sensibilità alle alterazioni paesistiche connesse ai fattori di frammentazione. Non v'è dubbio che ne possano esistere altri, come che ognuno di essi possa prevedere una articolazione interna in indicatori secondari, atti ad identificare la complessità del paesaggio.

Pertanto, quelli proposti vengono assunti come indicatori primari di frammentazione paesistica, funzionali alla prefigurazione di uno scenario metodologico per l'estensione dello studio a tutti i caratteri 
strutturali che identificano il paesaggio. Si tratta evidentemente di uno scenario non esaustivo e, in quanto sperimentale, bisognoso di progressive precisazioni e revisioni, la cui funzione essenziale è di base ed ipotesi di ricerca, piuttosto che di punto di arrivo sul quale fissare conclusioni e teorizzazioni.

\section{2 - I fattori territoriali di frammentazione paesistica}

"Le modificazioni nei processi d'uso del territorio sono la risultante di una molteplicità di fattori non sempre facili da individuare: concorrono infatti elementi di tipo tecnologico, demografico sociale ed economico. Aumenta il livello di antropizzazione del territorio, ma soprattutto si modificano gli schemi e le regole del gioco, in un processo di continua rincorsa di un equilibrio ideale che è sempre più trasformazione e riqualificazione del vecchio più che creazione del nuovo" ${ }^{\prime 74}$.

È essenziale che l'analisi della frammentazione paesistica proceda al rilevamento della distribuzione territoriale (caratteri topologici) dei diversi fattori (caratteri tipologici), attraverso il censimento sistematico su base cartografica delle forme di utilizzazione dello spazio. Occorre però non prescindere complessivamente dalla lettura dei processi e delle dinamiche culturali di ordine socioeconomico, che hanno parte sostanziale nella evoluzione del paesaggio ed anche nella fenomenologia della frammentazione della sua struttura.

Le condizioni di frammentazione possono avere origini molteplici e complementari: la promozione di politiche territoriali, l'attuazione di previsioni di piani territoriali, la realizzazione di opere progettate, la diffusione di tecnologie, l'avvento di mutamenti socioeconomici. Nel caso specifico l'analisi dei processi e delle dinamiche socioeconomiche riguarda in particolare gli sviluppi insediativi, l'evoluzione delle pratiche agrarie, i cambiamenti di assetto delle proprietà e le pressioni dovute alla rendita fondiaria. Questi aspetti sono intrecciati da strette relazioni di condizionamento, di cui il paesaggio costituisce una sorta di punto di partenza e di arrivo, nella configurazione ciclica delle diverse forme di uso e gestione delle risorse territoriali.

La classificazione dei fattori territoriali di frammentazione paesistica costituisce un campo di approfondimento scientifico che potrebbe sviluppare strumenti di conoscenza e controllo delle alterazioni indotte sulle risorse, utili alla lettura delle relazioni tra il paesaggio ed il contesto socioeconomico.

Si è detto come sia fondamentale considerare che le modificazioni del paesaggio possono dipendere, oltre che dai suoi caratteri strutturali e dai processi socio-economici endogeni, anche dalla promozione ed attuazione di scelte politiche.

Per la piana fiorentina, la marginalità dell'agricoltura deve in tal senso essere legata, oltre che alle condizioni strutturali non particolarmente favorevoli al suo sviluppo, anche agli indirizzi che la città ha assunto in modo strutturato a partire dal periodo fascista. Il settore primario risultava allora fortemente subordinato rispetto agli sviluppi prefigurati per quello secondario, in particolare con l'artigianato, e per quello che diventerà il terziario, con il turismo ed il commercio ${ }^{75}$. In realtà, non siè di fronte ad una vera e propria discontinuità storica, quanto all'interpretazione, da parte di quella società e sotto l'influsso ideologico di quell'epoca, delle valenze delle risorse territoriali, nel particolare rapporto storico con la campagna che, dai periodi di maggior splendore del Rinascimento e dell'Illuminismo, era improntato ad una mirabile urbanità di quest'ultima. Un'interpretazione che

74 De Rita G., Proliferazioni e addensamenti del territorio: 1985-1995, in A. Clementi, P.C. Palermo, G. Dematteis, (a cura), Le forme del territorio italiano, I volume, Laterza, Bari, 1996.

75 "La politica ideata per Firenze da Pavolini, segretario della federazione fiorentina del Partito Fascista, è stata efficacemente illustrata da M. Palla: cultura, turismo ed artigianato. Da questa impostazione nascono nel '31 la Fiera dell'artigianato (...) e, come richiamo per il turismo qualificato, il Maggio Musicale Fiorentino nel 1933 (...). Per i gusti meno raffinati dal 1930 c'è il calcio in costume. Tutto quadra: per il collegamento autostradale con la Toscana costiera viene scelto il percorso turistico, per Montecatini e Lucca, a preferenza di quello commerciale-industriale per Livorno". Testo tratto da Bortolotti L., De Luca G., Come nasce un'area metropolitana Firenze Prato Pistoia: 1848-2000, Alinea, Firenze, 2000 . 
possiamo oggi giudicare discutibile in taluni aspetti, per gli effetti determinati, non tanto relativamente all'epoca in cui è stata diffusa, quanto al progredire degli indirizzi e dei processi allora innescati. Nonostante questo però, risulta difficilmente controvertibile l'esistenza di evidenti relazioni di tale interpretazione con le citate radici storiche del rapporto città-campagna tipico del paesaggio fiorentino.

Le tecniche di analisi territoriale sperimentate in questa ricerca si avvalgono di elaborazioni di genere topologico e, pertanto, sono idonee ad individuare quei fattori di cui sia riscontrabile una incidenza sulla realtà, attraverso le documentazioni cartografiche e fotografiche. Può trattarsi di una incidenza dovuta a modificazioni paesistiche pregresse o in corso, oppure sotto forma di condizioni attendibili in ragione delle politiche di governo territoriale.

L'analisi dei fattori di frammentazione paesistica fa riferimento ad una classificazione impostata su criteri di ordine morfologico. La morfologia del paesaggio è assunta a carattere descrittore transcalare fondamentale, in quanto espressione del rapporto fra risorse del territorio e relative esigenze e modalità di uso e gestione, frutto di una complessa metabolizzazione di natura e cultura, comprensiva, in termini di effetti, dei processi e delle dinamiche socioeconomiche.

È utile considerare la filosofia interpretativa a cui fa riferimento la ricerca Itaten, nella quale si osserva come "il metodo di lavoro più adatto per intercettare le innovazioni che si stanno dispiegando nell'Italia di questi anni nasca proprio dallo studio dei rapporti tra morfologie insediative e morfologie sociali, osservate nel loro autonomo dispiegarsi e analizzate nelle trasformazioni delle loro reciproche interdipendenze territoriali. (...) Questa è una delle ipotesi principali della ricerca, che muove appunto dalla identificazione delle morfologie come passaggio obbligato per risalire alla conoscenza dei processi di utilizzazione del territorio e delle strutture che determinano il loro cambiamento $^{\prime \prime 76}$.

Se il riconoscimento morfologico della struttura del paesaggio permette di costruire un efficace quadro analitico è anche vero però che l'approfondimento di alcuni aspetti di primaria importanza, relativamente a determinati ambiti territoriali segnalati dall'analisi stessa, può fornire utili dettagli intorno alla effettiva natura delle configurazioni considerate. Ci si riferisce in particolare alla analisi delle dinamiche che caratterizzano il regime fondiario, per quanto riguarda il parametro economico del valore di mercato dei suoli, a quelle che caratterizzano i processi di mutamento della articolazione della proprietà fondiaria, peraltro legati anche agli aspetti precedenti, ed infine alle politiche agricole locali, fortemente condizionate dagli elementi citati. La lettura di questi aspetti, attraverso il confronto di soglie storiche significative con le condizioni attuali, permette la definizione dei processi strutturali di ordine socioeconomico che hanno contribuito alla formazione di un determinato paesaggio ed alla determinazione dei relativi stati di frammentazione. Queste indagini, richiamate anche per ragioni di completezza teorica, non rientrano evidentemente nelle potenzialità peculiari degli studi di analisi e progettazione paesistica, che dei loro esiti, come di altre informazioni specialistiche, possono fare un uso proficuo, mantenendo la distinzione delle rispettive competenze disciplinari. Non si ravvisa in ogni caso alcun vizio teorico in una sequenza ipotetica nella quale esse venissero elaborate in misura mirata, dopo un primo livello di analisi dei fattori territoriali, che individuasse specifici ambiti e temi di approfondimento conoscitivo degli aspetti socioeconomici, permettendo in questo modo una ottimizzazione del loro impiego.

Pare essenziale, infine, che l'analisi della frammentazione paesistica non prescinda dal considerare, la specifica natura, sia il carattere reale che il carattere potenziale a cui sono diversamente ascrivibili i fattori, a secondo se vengano riscontrati in essere o se ne venga presunta la possibilità di sviluppo

76 A. Clementi, P.C. Palermo, G. Dematteis, Itaten. Ricerca sulle forme del territorio italiano, in A. Clementi, P.C. Palermo, G. Dematteis, (a cura), Le forme del territorio italiano, I volume, Laterza, Bari, 1996. 
sulla base delle politiche territoriali documentate. I fattori reali si riferiscono alle entità esistenti sul territorio, che costituiscono componenti del paesaggio e partecipano a determinarne le condizioni di stato. I fattori potenziali sono invece determinati dalle previsioni di piano, facenti capo alle distinte scale e tipologie di competenza istituzionale, dalla pianificazione sovraordinata di bacino, fino a quella attuativa comunale. Tale ottica binaria è evidentemente utile per rendere il più possibile fedele l'approssimazione della realtà prodotta attraverso il processo conoscitivo: non solamente perché il paesaggio stesso è governato da equilibri dinamici ma, innanzitutto, per la consapevolezza della progressione dei fenomeni di alterazione paesistica nelle aree periurbane di pianura, malgrado la nota e consolidata assenza di crescita demografica. Si tratta infatti di territori soggetti a processi insediativi contemporanei in gran parte afferenti alla localizzazione di attività terziarie. Si pensi nell'area metropolitana fiorentina ai nuovi centri commerciali e complessi terziari tra Campi Bisenzio, Calenzano, Sesto Fiorentino e Osmannoro, come anche all'espulsione dai centri urbani consolidati di funzioni di livello sovralocale, ad esempio il nuovo polo scientifico universitario di Sesto Fiorentino, ed al potenziamento degli apparati infrastrutturali, l'alta velocità ferroviaria Milano-Napoli, la terza corsia della Autostrada del Sole, la tramvia metropolitana di Firenze, la viabilità tangenziale traCampi Bisenzio e Signa e le previsioni di potenziamento nell'area di Castello. È necessario pertanto prescindere da qualunque generalizzazione che intenda sostenere che il paesaggio di pianura di queste aree non sia ormai più a rischio di consumo e destrutturazione e pertanto esigente esclusivamente misure di recupero.

È possibile fare riferimento a due criteri principali di identificazione morfologica dei fattori territoriali di frammentazione paesistica. In base all'articolazione spaziale, i fattori possono essere distinti in lineari, areali e puntuali. In base all'omogeneità di composizione, i fattori possono essere distinti in semplici e complessi.

La classificazione ${ }^{77}$, sviluppata sulla scorta dei diversi casi censiti nell'ambito di studio, risulta articolata secondo cinque generi:

1 fattori areali complessi: caratterizzati dalla compresenza di più componenti in configurazioni areali sviluppate su significative superfici;

2 fattori areali semplici: caratterizzati dalla dominanza di una componente con configurazione areale sviluppata su significative superfici;

3 fattori lineari complessi: caratterizzati dalla compresenza di più componenti in configurazioni lineari sviluppate su significative lunghezze;

4 fattori lineari semplici: caratterizzati dalla dominanza di una componente con configurazione lineare sviluppata su significative lunghezze;

5 fattori puntuali: caratterizzati dalla dominanza di una sola componente con configurazione a sviluppo decisamente circoscritto.

Fra gli studi pubblicati in Italia nello specifico ambito di ricerca riguardante la frammentazione dell'ambiente e del paesaggio, l'unico caso in cui si è riscontrato un censimento sistematico su base tipologica delle cause territoriali del fenomeno è stato prodotto, relativamente al territorio abruzzese e, per certi temi e scale, a livello nazionale, nell'ambito della citata ricerca Planeco. Sebbene la ricerca sia specificamente riferita alla continuità ambientale ed utilizzi come criterio principale di indagine la biopermeabilità del territorio, anche in quel caso gli autori hanno predisposto una classificazione in cui le "aree considerate non biopermeabili alla scala territoriale" sono responsabili di frammentazione ambientale, mentre quelle biopermeabili, "superfici naturali e seminaturali, superfici agricole frammiste a spazi naturali", sono ritenute favorevoli alla continuità ambientale.

\footnotetext{
77 Per la trattazione completa dell'argomento si vedano, in appendice, le schede di identificazione morfologica dei fattori territoriali di frammentazione paesistica e le relative annotazioni metodologiche introduttive.
} 
Fra le aree non biopermeabili, ovvero i fattori causali della frammentazione ambientale, lo studio riscontra le seguenti categorie ${ }^{78}$ : tessuto urbano continuo, tessuto urbano discontinuo, aree industriali o commerciali, reti stradali e ferroviarie e spazi accessori, aree estrattive, aree sportive e ricreative, seminativi in aree non irrigue, risaie, frutteti, oliveti, prati stabili, colture annuali associate a colture permanenti, sistemi colturali e particellari complessi.

"Il concetto di biopermeabilità presenta un limite importante rispetto al suo significato trans-scalare. Può essere ritenuto di buona utilità quando è chiamato a rappresentare la fisionomia della naturalità complessiva del territorio a dimensioni medio-grandi, mentre perde indubbiamente di significato interpretativo quando se ne tenta una lettura a dimensione locale. (...) La lettura della biopermeabilità si rivela perciò utile prevalentemente nei casi in cui spazi antropizzati e non antropizzati realizzino condizioni di commistione, sovrapposizione, interferenza reciproca continua e complessa. Evidenziare la biopermeabilità di una regione territoriale significa sostanzialmente valutare il punto a cui il processo di modificazione ingenerato dalla attività umana è giunto in termini di disgregazione della matrice naturale ${ }^{\prime \prime 79}$.

Rispetto a questo approccio focalizzato sugli aspetti territoriali incidenti sulla frammentazione degli habitat animali, l'analisi della fenomenologia della frammentazione paesistica necessita di strumenti conoscitivi di tipo diverso, pur costruiti ed utilizzati con logiche simili.

Relativamente al paesaggio periurbano di pianura, sul piano teorico è possibile ipotizzare la lista di controllo per l'identificazione morfologica dei fattori di frammentazione paesistica riportata nell'appendice A, considerando che essa è necessariamente aperta e non esaustiva, in quanto soggetta a variazioni in ragione dello specifico soggetto di studio. Ad ogni voce della lista è associata una scheda descrittiva dei caratteri tipologici del relativo fattore di frammentazione paesistica.

L'analisi dei fattori reali di frammentazione paesistica non richiede dati conoscitivi di base sofisticati, né in numero elevato. È comunque necessario disporre di cartografia aereofotogrammetrica, di fotogrammi aerei zenitali e di cartografia tematica dell'uso del suolo. Il grado di definizione di tali informazioni può incidere ovviamente in via teorica sulla qualità dell'analisi, ma i requisiti di precisione raggiunti normalmente oggi rendono questo aspetto relativamente poco consistente. Ha importanza preminente invece il lavoro di osservazione che compete chi effettua l'analisi. Assodato che maggiore è l'esperienza specifica dell'operatore, più l'analisi produce una rappresentazione fedele della realtà, è possibile pensare all'utilità di una codifica dei caratteri di base delle singole tipologie di fattori territoriali, da operare nella fase di preparazione dello studio (si vedano le schede di identificazione tipologica riportate in appendice). Tale utilità assume valenze scientifiche nella fase di identificazione metodologica, ma potrebbe avere anche valenze tecniche per le applicazioni pratiche ai casi reali, caratterizzati da grandi estensioni territoriali e relativa necessità di attivare più analisti. In questo caso, per l'analisi del paesaggio periurbano di Firenze sono state utilizzate fotografie aeree zenitali (formati digitali in scala 1:30.000, Regione Toscana) e la cartografia dell'uso del suolo ${ }^{80}$, elaborata in scala 1:10000 per integrazione e comparazione di fonti (Carta dell'uso del suolo 1:25000, edizione 1985, dati 1978, Regione Toscana; CTR 1:10000, con indicazioni topografiche e di uso del suolo, Regione Toscana; Fotografie aeree zenitali 1:30000, volo 1998, Regione Toscana; Rilievi diretti, 2000). La costruzione della carta tematica di base dell'uso del suolo ha fatto riferimento alla

\footnotetext{
78 Per la descrizione completa delle singole categorie si veda Romano B., Continuità ambientale. Pianificare per il riassetto ecologico del territorio, Andromeda Editrice, Teramo, 2000. Per gii sviluppi delle ricerche sulla continuità ambientale e le relazioni con la reticolarità ecologica, si rimanda, oltre che al testo citato, al sito WEB PLANECO Project: http://dau.ing.univaq.it/planeco.

79 Romano B., Continuità ambientale. Pianificare per il riassetto ecologico del territorio, Andromeda E ditrice, Teramo, 2000.

${ }^{80}$ L'elaborazione della cartografia tematica di base dell'Uso del suolo, a cura degi architetti Simona Cappellini e Chiara Tesi, ha fatto riferimento alla specifica esperienza della loro tesi di laurea, discussa nel 2000 presso il Dipartimento di Urbanistica e Pianificazione del territorio della Facoltà di Architettura di Firenze, sul tema della frammentazione paesistica, nella quale è stato considerata una parte di territorio dell'area metropolitana Firenze-Prato-Pistoia comprensiva anche delle aree periurbane indagate in questo studio. Chi scrive è stato correlatore della tesi (relatore è stato il Prof. Biagio Guccione).
} 
classificazione sperimentata dalla Regione Toscana sull'area di Lucca nell'ambito di uno studio pilota cofinanziato dall'Autorità di Bacino del Fiume Serchio ${ }^{81}$.

L'analisi rileva una elevata diffusione di configurazioni responsabili della fenomenologia indagata. Alla rilevante presenza dei fattori areali complessi, costituiti dai tessuti urbani, si aggiunge una consistenza non trascurabile di fattori areali semplici, costituiti sia da spazi aperti a colture erbacee intensive ed incolti produttivi, che da spazi aperti soggetti a vincolo aereoportuale. Il margine urbano compreso tra il corso dell' Arno a sud e le pendici collinari di Castello a nord è caratterizzato da più fattori lineari complessi, costituiti da fasci di infrastrutture viarie e tecnologiche, che per oltre due chilometri si trovano a loro volta affiancati.

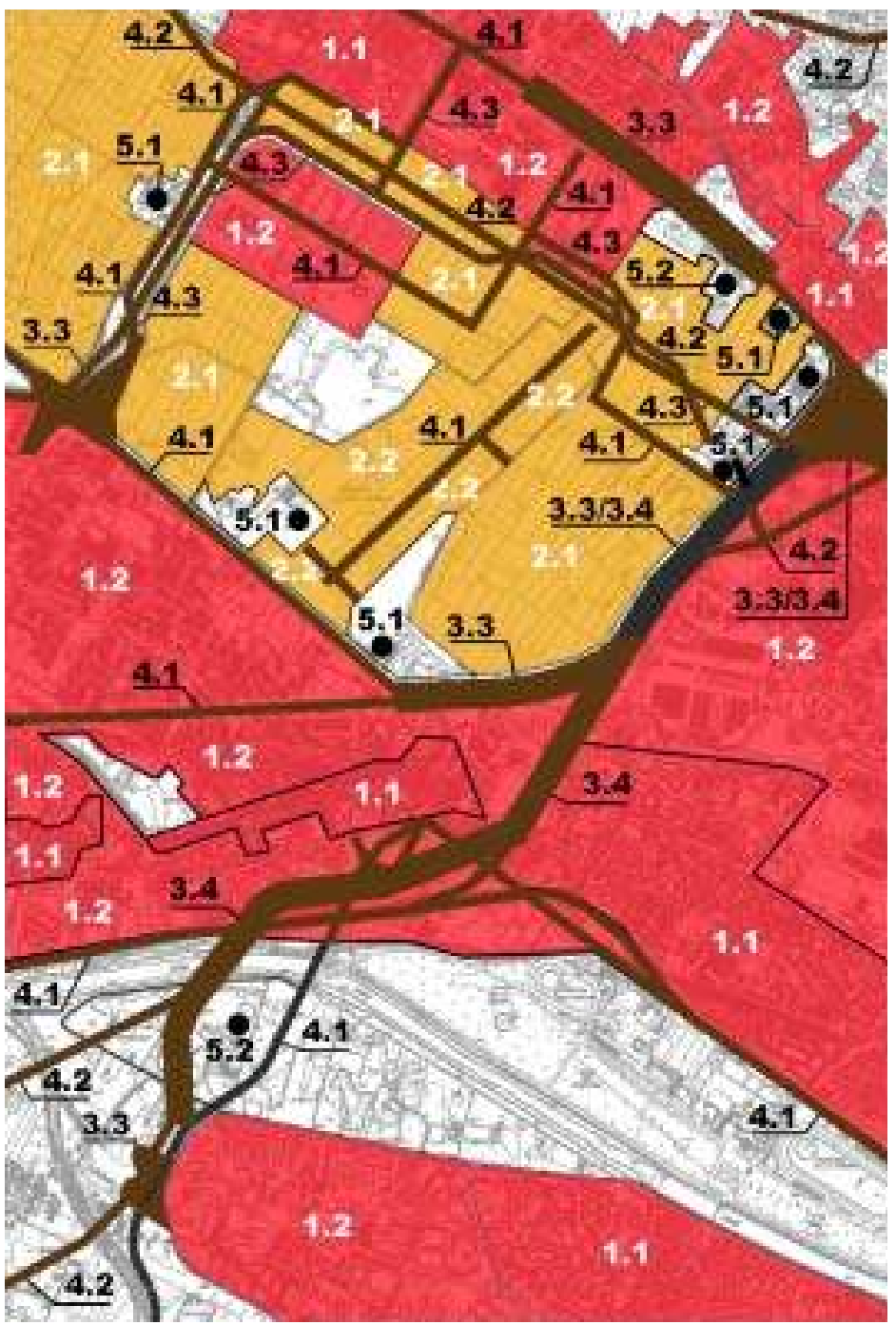

Figura 9 - Carta di analisi dei fattori reali di frammentazione paesistica e collocazione del campione territoriale nell'immediato contesto di riferimento. Gli indici numerici sono riferiti alle tipologie considerate nella lista di controllo per l'identificazione morfologica ed alle relative schede riportate in appendice (Cartografia ridotta da originale 1:10000 - CTR Regione Toscana - fotografia aerea zenitale da originale 1:30000 - Regione Toscana).
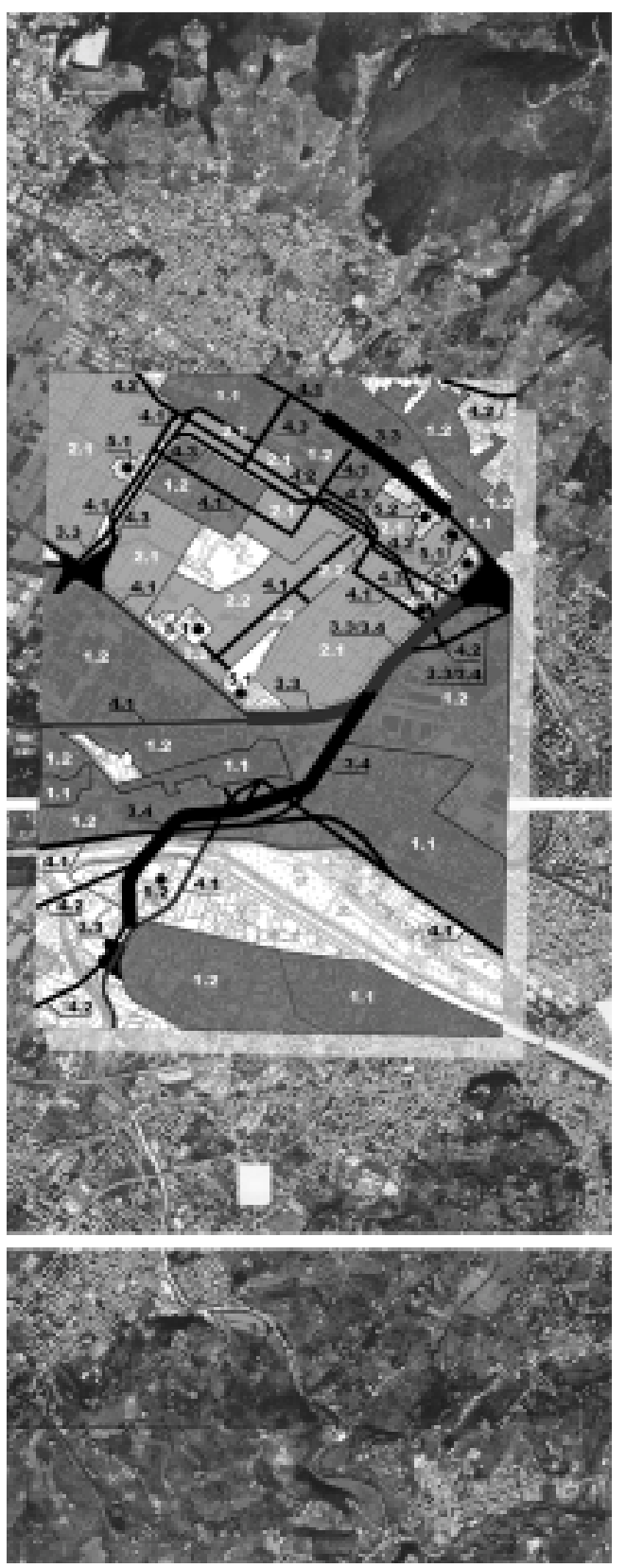

81 Si veda Greggio A., Hofmann A., Rossi R., Trevisani M., Vincia A., Una nuoza base dati di uso del suolo: la sperimentazione fatta nel bacino pilota del fiume Serchio, Documenti del territorio, 39, 1998. 
A nord i primi lembi di paesaggio privi di elementi di rilievo dal punto di vista dell'analisi di frammentazione coincidono con le pendici pedecollinari di Castello.

Nella pianura a sud di Sesto Fiorentino si trova una zona umida artificiale di interesse naturalistico, isolata in un contesto praticamente ormai privato di ogni consistente formazione di equipaggiamento vegetale e sempre più interessato invece dal progredire di urbanizzazioni ed edificazioni.

A sud, in destra e sinistra d'Arno, spazi diversi costituiscono un sistema paesistico di interesse strategico per l'intero territorio fiorentino: dal Parco storico delle Cascine, alle aree di Argingrosso, Mantignano e Ugnano.

Indipendentemente dalla spiccata transcalarità che caratterizza la fenomenologia della frammentazione paesistica e di conseguenza i relativi processi conoscitivi, l'analisi dei fattori territoriali deve necessariamente essere condotta su ambiti di estensione idonea a cogliere sia nella stessa fase di rilevamento analitico, che nella successiva di interpretazione diagnostica, l'effettiva articolazione delle manifestazioni, coprendo nella maniera più esaustiva possibile il complesso delle relazioni che identificano il soggetto di studio.

In tal senso, anche nel caso di rilievi di dettaglio o relativi ad aree di limitata estensione, occorre comunque collocare il processo conoscitivo in un quadro di riferimento di idonea estensione. La casistica aperta dei fattori territoriali, che nasce inevitabilmente dalle specificità strutturali del paesaggio e dei modelli di insediamento e produzione caratteristici di un dato ambito territoriale, richiede di considerare l'estensione minima degli studi di frammentazione paesistica non tanto in riferimento ad una dimensione superficiale, quanto ad un congruo grado di copertura tipologica dei fattori e di copertura topologica delle loro relazioni spaziali.

L'analisi dei fattori potenziali di frammentazione paesistica fa riferimento al quadro complessivo delle politiche territoriali georiferite, ovvero a tutte quelle che vengano tradotte in piani supportati da cartografie. Escludendo i piani di settore, per i quali è stata considerata avvenuta o in corso l'effettiva implementazione nei piani generali per quanto riguarda gli aspetti di governo del territorio, si tratta dei seguenti strumenti: Piano di bacino (Piano stralcio riduzione rischio idraulico); Piano di indirizzo territoriale regionale (Schema strutturale dell'area metropolitana Firenze-Prato-Pistoia); Piano territoriale di coordinamento provinciale; Piani regolatori generali comunali; Piani particolareggiati attuativi d'area o di sistema.

In ragione delle differenze di impostazione dovute alle finalità e di conseguenza ai ruoli ed alle scale di questi strumenti di governo territoriale non risulta significativa una classificazione unitaria delle politiche in termini di potenziali di frammentazione del paesaggio. L'analisi procede invece alla lettura separata dei diversi piani ed alla ricomposizione unitaria dei fattori di frammentazione paesistica nella relativa cartografia (figura 14). È a questo quadro che si riferiscono le possibilità di lettura complessiva delle relazioni tra le politiche territoriali di diversa scala.

Come accade per l'analisi dei fattori reali, in cui la morfologia del paesaggio è assunta come espressione del rapporto fra risorse e modalità di uso e gestione, comprendendo la metabolizzazione dei processi e delle dinamiche socioeconomiche, le configurazioni spaziali e normative delle politiche di piano ai vari livelli esprimono le stesse relazioni per quanto appartiene alla sfera delle previsioni di uso delle risorse $\mathrm{e} / \mathrm{o}$ delle loro modalità di governo.

La ricerca delle tendenze di frammentazione attraverso la lettura dei piani, può essere considerata esaustiva anche dei diversi aspetti socioeconomici che condizionano questa fenomenologia, relativamente alla prima costituzione di un quadro della articolazione spaziale e della tipologia dei fattori. Anche in questo caso, occorre osservare però che l'approfondimento degli specifici aspetti socioeconomici non direttamente leggibili nei piani territoriali, può permettere una migliore indentificazione delle dinamiche in corso e pertanto un miglior grado di attendibilità delle successive misure di intervento. 
Lo scollamento ad oggi ancora esistente fra il quadro reale delle dinamiche di trasformazione del paesaggio ed il quadro propositivo delle politiche di governo delle risorse costituito dai piani, fa sì che l'analisi non rilevi elementi di particolare peso nel territorio di studio per quanto concerne le strategie e le misure di controllo attivo delle problematiche considerate in questo lavoro. Tutto ciò, nonostante che di molte questioni si abbia una consapevolezza disciplinare consolidata: già alla metà degli ultimi anni Sessanta, il Piano Intercomunale del Comprensorio Fiorentino riconosceva ad una ricerca specifica sul paesaggio, quanto meno sul piano teorico-metodologico, il ruolo di interpretazione strutturale guida ${ }^{82}$. Il paesaggio veniva considerato come manifestazione strutturale complessa sensibile alle dinamiche territoriali, anticipandone la concezione come indicatore ambientale e sociale, che oggi va diffondendosi.

Complessivamente l'analisi dei fattori potenziali di frammentazione paesistica (figura 14) rileva una situazione relativamente semplice, connotata da due grandi ambiti di rischio: la cosiddetta piana di Castello, immediatamente ad ovest di Firenze e, più ad occidente, la pianura a sud di Sesto Fiorentino. Le previsioni di sviluppo insediativo relative a queste aree, per localizzazione e per tipologia di tessuto, tendono a confermare il modello della espansione urbana a macchia e della conurbazione. Non si riscontrano fenomeni significativi di dispersione insediativa né tracce di innesco di fenomeni di diffusione urbana nelle aree agricole intercluse. Le grandi opere infrastrutturali riguardano sostanzialmente completamenti ed integrazioni di assi esistenti, mentre i due interventi di rilievo che interessano il bacino dell'area metropolitana, la ferrovia ad alta velocitàe la terza corsia autostradale, pur limitrofi, risultano esterni all'ambito di studio.

Il primo livello di pianificazione sovraordinata - Piano di bacino, Piano stralcio per la riduzione del rischio idraulico - può essere considerato, in via teorica, privo di potenziale di frammentazione paesistica relativo alle forme di governo ed alle misure di intervento che gli competono. È d'obbligo fare riferimento ad una visione teorica, per quanto gli effetti paesistici degli interventi strutturali previsti dal piano dipendono in buona misura dalle modalità di realizzazione, in esso non specificate e correttamente demandate agli approfondimenti progettuali necessari, oltre che per specificare $\mathrm{i}$ contenuti delle opere in termini tecnici, anche per riconoscere le differenze e le specifiche identità dei diversi luoghi di intervento. Casse di espansione, casse di laminazione (estranee per tipologia all'area di studio, completamente pianeggiante), adeguamenti spondali ed in genere il complesso delle opere previste per la messa in sicurezza idraulica del territorio ed il governo delle acque superficiali, non costituiscono infatti a priori cause di alterazioni paesistiche, né specificamente di frammentazione, bensì si configurano piuttosto come opportunità contemporanee di costruzione di paesaggi, nei quali l'attenzione ai requisiti funzionali non esclude la ricerca di connotati di qualità spaziale e di equilibrio con la stratificazione storica del paesaggio e la sua articolazione ecologica. Distinguendo il piano teorico da quello pratico, nelle attuali condizioni di regime idrogeologico e di evoluzione delle pratiche manutentive dei corsi d'acqua, è però necessario considerare come le tipologie di intervento argomento della pianificazione di bacino ed in particolare di quella per la riduzione del rischio idrogeologico non siano in realtà estranee a rischi di frammentazione del paesaggio.

82 “È nota l'importanza, per Firenze e per tutto il comprensorio considerato, del rapporto città-paesaggio circostante. Molto meno diffusa è la coscienza che non si tratta, più che mai in questo caso, soltanto di un rapporto paesistico, di un rapporto tra città e campagna. La realtà del paesaggio, quale si è venuta, attraverso gli interventi dell'uomo, a realizzare, si caratterizza, si qualifica, in questo caso, come una vera e propria struttura urbanistica altrettanto complessa e integrata, in un tessuto organico articolatissimo, quanto quello della città; è evidente da ciò l'importanza di una coscienza esauriente e completa di tale realtà. Praticamente inesistenti sono però ancora oggi $(1965$, n.d.r.) studi approfonditi e sistematici che restituiscano una oggettiva analisi della struttura di questo paesagoio e costituiscano la indispensabile base per la pianificazione di qualsiasi intervento interessi il paesaggio stesso. Questo è appunto il fine che si è proposta questa ricerca nella quale dunque il termine paesaggio viene assunto nel suo significato globale derivante dalla considerazione di tutti $\mathrm{i}$ fattori che contribuiscono a definire, sia a livello strutturale che percettivo, la forma del territorio" (Di Pietro G., Greppi C., Pettini P., Sica P., Dall'E rba G., Piano Intercomunale del Comprensorio Fiorentino, Ufficio Tecnico del Piano Intercomunale Fiorentino, Studi Ricerche Documenti, Firenze, 1965). 
In merito alle questioni connesse alle modalità di gestione dei corsi d'acqua, i maggiori problemi vi sono relativamente agli effetti di impoverimento paesistico, sia ecologico, che morfologico, conseguenti agli interventi di manutenzione imposti dalle necessità di raggiungimento di determinati requisiti di portata idraulica, dettati a loro volta dalle sempre crescenti necessità di drenaggio delle pianure alluvionali bonificate. A questo circolo vizioso corrispondono l'impoverimento idrico delle pianure, paradossalmente legato al loro elevato rischio idraulico, e la gestione dei corsi d'acqua di origine naturale come canali. In tal senso sono strategiche le misure di salvaguardia delle risorse di cui si è detto a propostito delle aree di pertinenza fluviale: è necessario infatti invertire il progressivo bisogno di drenaggio delle pianure, per rivitalizzare il loro paesaggio ed allo stesso tempo permettere concrete condizioni di applicabilità di interventi di riqualificazione paesistico-ambientale dei corsi d'acqua naturali regimati e di miglioramento ecologico e morfologico dei canali di bonifica.

Altre problematiche responsabili potenziali di fenomeni di ulteriore frammentazione paesistica sono legate alla realizzazione o all'adeguamento di arginature di golene, di casse di espansione e di casse di laminazione a garanzia della efficienza degli impianti idrovori di sollevamento, come dei potenziamenti delle canalizzazioni esistenti. Anche in questo caso occorre progressivamente introdurre, a fianco dei criteri di ingegneria idraulica, criteri di realizzazione e manutenzione rispondenti alle esigenze di miglioramento paesistico-ambientale, contribuendo anche al loro recepimento all'interno delle voci dei costi di realizzazione e manutenzione, esattamente come è necessario fare per le infrastrutture viarie. Argini trapezoidali con pendenze dettate sostanzialmente solo da criteri di stabilità delle terre e di tenuta dei carichi idraulici, superfici di casse di espansione ed alvei di canali e torrenti totalmente privi e privati di vegetazione, nonché tecnologie improprie, possono essere sostituite da modalità diverse, ormai mature sul piano tecnico, compatibili con più obiettivi di qualità. $\mathrm{Ma}$, anche in questo caso, non si presenta solo la questione culturale del recepimento di questi concetti, bensì il problema di dover costruire concrete condizioni strutturali di progressivo riequilibrio idrogeologico per cui tali operazioni siano veramente praticabili, uscendo dalla mera gestione idraulica del paesaggio.

Nell'area di studio, il Piano stralcio segnala, ad est del complesso sistema di casse di espansione del fiume Bisenzio, un ampio ambito a prevalenza di colture erbacee ed incolti produttivi, prioritario per la salvaguardia ed il miglioramento idrogeologico (aree di pertinenza fluviale), ponendo quanto meno forme di limitazione o di ostacolo all'urbanizzazione indiscriminata della campagna. In realtà, ove fosse ricercata una opportuna e concreta interpretazione di queste indicazioni nell'ambito dei piani provinciali e soprattutto di quelli comunali, esse costituirebbero una sostanziale spinta alla riqualificazione diffusa del paesaggio, da realizzare attraverso progetti di equipaggiamento vegetale delle campagne e delle reti idrografiche e di sviluppo di zone umide per il riequilibrio idraulico del territorio, ma anche per il recupero di specifici equilibri idrogeologici ed ecologici della pianura.

Il caso studio trattato, trovandosi in Toscana, fa riferimento ad una delle più avanzate legislazioni italiane in materia di governo del territorio, che ha posto la salvaguardia delle risorse al centro delle politiche per il perseguimento dell'obiettivo generale dello sviluppo sostenibile, considerando lo spazio come una risorsa primaria. Questo fa sì che il territorio toscano si trovi oggi governato attraverso un Piano di indirizzo territoriale estremamente esigente nei confronti della pianificazione di coordinamento ed operativa, ai diversi livelli di competenza provinciale e comunale. Da questo punto di vista, anche per la pianificazione di indirizzo troviamo una serie di modelli di comportamento di indubbio interesse e spessore, riguardanti ad esempio le aree di frangia urbana, come le aree rurali marginali, e molte altre configurazioni a spiccata sensibilità paesistico-ambientale. In realtà, però, tali modelli divengono efficaci nel momento in cui riescono a promuovere la formazione e l'attuazione di politiche locali ad essi conseguenti. È anche vero inoltre che questo livello di governo del territorio è coinvolto, sebbene non per competenze esclusive, nell'intera questione della infrastrutturazione, sia nelle forme sovraordinate (competenze statali e transnazionali) che in quelle subordinate (competenze provinciali e comunali). Si è in questi casi nell'ambito in cui i rischi di frammentazione 
dovrebbero essere preventivati e valutati, scegliendo le opportune misure di localizzazione, realizzazione e compensazione. Di fatto, se il Piano non si fa carico di un'attenta, esplicita e concreta politica di promozione della compensazione paesistico-ambientale per le infrastrutture, tanto meno questo avviene per altri importanti fattori potenziali di frammentazione, come le trasformazioni insediative, residenziali e produttive, e quelle agrarie, anche se per queste ultime occorre sospendereil giudizio, in vista degli esisti di applicazione del recente Piano regionale di sviluppo rurale, considerando le possibili efficacie dell'articolata strumentazione ed argomentazione che lo caratterizza. È difficile credere che la sostenibilità di tutti gli interventi sia garantita dalle valutazioni degli effetti ambientali previste dalla legge, come che le trasformazioni possano uscire da tali procedure con congrue prescrizioni e definizioni delle compensazioni necessarie ed, infine, se di valutazioni ambientali si parla, non si riesce a ravvisare in tali procedure quali efficaci misure di garanzia possano essere attivate contro la diffusione dei fenomeni di alterazione del paesaggio.
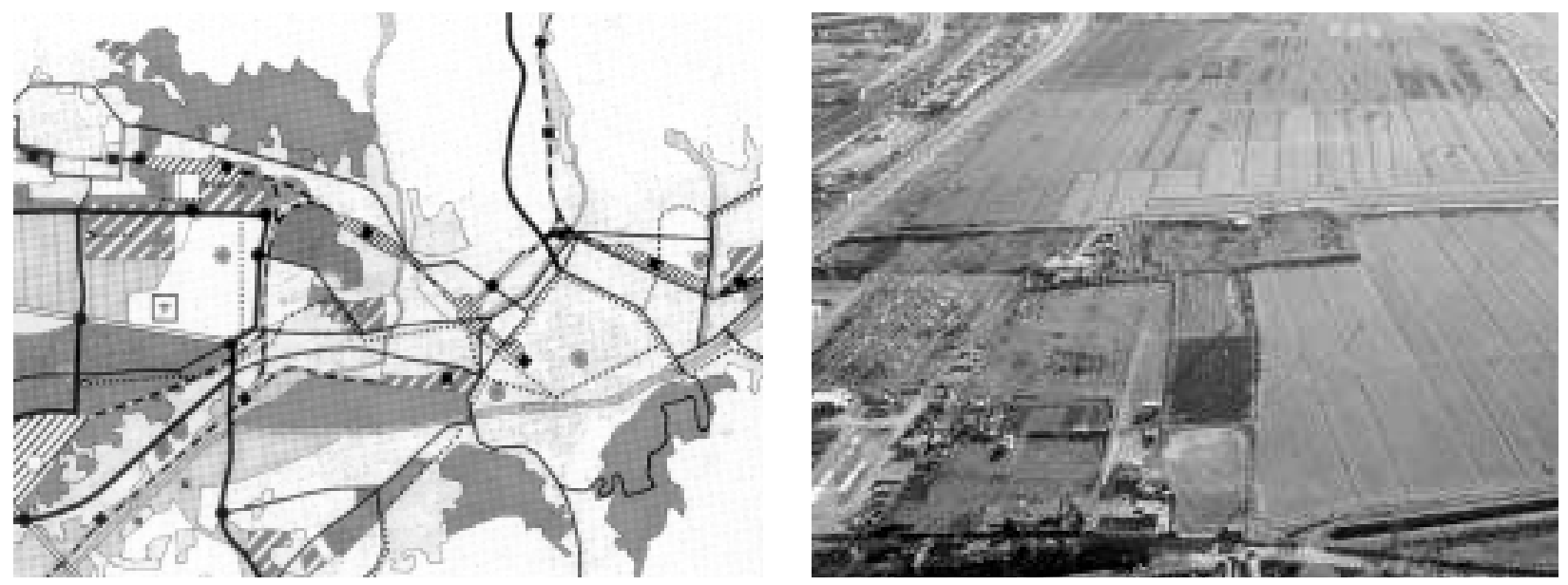

Figura 10 (a sinistra) - Piano di indirizzo territoriale regionale - Schema strutturale dell'area metropolitana Firenze Prato Pistoia. Stralcio dell'area fiorentina (immagine tratta da: G. De Luca (a cura) La pianificazione regionale in Toscana: 1984-1990, Quaderni di Urbanistica Informazioni, 10, supplemento a Urbanistica Informazioni n. 116, marzo-aprile 1991).

Figura 11 (a destra) - Le aree periurbane della piana di Castello da nord (da: G. Di Pietro, Un progetto per Firenze - La nuora città nella piana di Castello, Ponte alle Grazie, Firenze, 1990).

Per quanto concerne l'area metropolitana Firenze Prato Pistoia, il Piano fa riferimento al relativo Schema strutturale, uno strumento che dobbiamo, nella sua prima ed innovativa concezione, a quanto proposto da Astengo negli ultimi anni Ottanta. Una lettura di questo quadro territoriale dal punto di vista dell'analisi dei potenziali di frammentazione paesistica non può non registrare come, nella sua progressiva formazione esso abbia sostanzialmente ridotto la propria carica propositiva come politica sovraordinata, luogo deputato alla definizione ed all'indirizzo di importanti e concrete politiche paesistico-ambientali di bacino metropolitano, per divenire sostanzialmente un quadro di recepimento e sintesi della pianificazione comunale. Se questo da una parte non permette di attribuire allo Schema strutturale significativi potenziali aggiuntivi di frammentazione della struttura del paesaggio, dall'altra fa sì che non si riscontrino neppure misure positive di governo e riduzione del fenomeno, rispetto al quale perfino le aree verdi, su cui tanto si è speso in termini di dibattiti e progetti, non hanno ad oggi nessuna connotazione sistemica e risultano evidentemente squilibrate e deficitarie rispetto al bacino territoriale ed al carico urbano ed infrastrutturale che lo caratterizza.

Nell'ambito del Piano di indirizzo è in corso di definizione il problema della frammentazione degli habitat animali dal punto di vista della determinazione tipologica e normativa delle cosiddette aree di collegamento ecologico-funzionale, secondo i riferimenti teorici ed empirici sviluppati dalle teorie sulla reticolarità ecologica e dagli studi di biologia conservazionale. Dovranno essere risolti i nodi 
riguardanti gli effetti complessi di frammentazione del paesaggio, da cui dipende sostanzialmente la frammentazione degli stessi habitat naturali.

Anche il Piano territoriale di coordinamento provinciale di Firenze ha affrontato, nell'ambito dello Statutodel territorio la problematica della frammentazione degli habitat. Va riconosciuto il non trascurabile anticipo con cui questo è avvenuto rispetto agli altri piani, come si deve rilevare in questo caso una esplicita attenzione alle alterazioni del paesaggio. Si può osservare però come, nella filosofia del Piano, la considerazione del paesaggio sia di tipo prevalentemente conservativo e molto più ampiamente sviluppata in riferimento alle aree di maggior pregio, dei rilievi collinari e montani. Pur richiamando la necessità di operare per salvaguardare e ripristinare congrui gradi di continuità ambientale, il Piano non contiene politiche strategiche georiferite, ovvero non compie scelte che possano tradursi, in forza del coordinamento sovracomunale, in misure di intervento e questo accade, oltre che per il territorio cosiddetto a regime ordinario, anche per quanto riguarda il sistema delle aree protette di interesse locale (unica forma di area protetta prevista nel territorio indagato), chenon sono ancora state inserite in un quadro strategico unitario dove, nel rispetto delle singole specificità, potrebbero costituire un insieme di scala vasta con potenziali ben superiori alle singole unità e con possibile ruolo di impalcatura di una rete territoriale, in grado di supportare e promuovere politiche di conservazione attiva e miglioramento paesistico-ambientale a scala metropolitana. Non si riscontrano in ogni caso indicazioni del Piano che possano configurarsi come fattori potenziali di frammentazione paesistica in aggiunta al quadro della pianificazione comunale.

Mentre per l'analisi dei primi tre livelli di pianificazione sovracomunale si tratta di utilizzare basi informative immediatamente interpretabili, per quanto riguarda i Piani regolatori generali comunali risulta necessario considerare il mosaico relativo al territorio di indagine, con le note esigenze di unificazione delle prescrizioni ${ }^{83}$.

Premesso che nessuno dei piani considerati in quanto competenti su territori interessati dallo studio (Firenze, Sesto Fiorentino, Scandicci) ha alcuna concreta forma di controllo valutativo e progettuale, non solo degli effetti di frammentazione paesistica potenziali, ma neppure delle alterazioni paesistiche in genere, è indubbio che gli effetti maggiori siano riferibili agli sviluppi insediativi previsti nella piana di Castello.

I fattori di frammentazione potenziale riscontrati afferiscono essenzialmente agli sviluppi insediativi (fattori 1.1, 1.2) ed infrastrutturali (fattori 4.1). Questi ultimi riguardano completamenti e raccordiai margini degli insediamenti interni alla piana: a sud di Sesto Fiorentino, a nord di Osmannoro, ad est di Firenze (figura 14), senza comprendere la rete viaria di distribuzione della nuova area direzionaledi Castello, considerata all'interno del fattore complesso di tipo insediativo. A sud di Sesto Fiorentino, sono presenti importanti previsioni di espansione urbana per attrezzature e residenza, che costituiscono una fascia con sviluppo est-ovest, disposta lungo il margine dell'insediamento; un'altra area di sviluppo insediativo si trova più a sud, ad est del nuovo polo scientifico universitario.

\footnotetext{
83 L'elaborazione della cartografia tematica di base dell'Mosaico dei piani regolatori generali comunali, a cura degii architetti Simona Cappellini e Chiara Tesi, ha fatto riferimento alla specifica esperienza della loro tesi di laurea, discussa nel 2000 presso il Dipartimento di Urbanistica e pianificazione del territorio della Facoltà di Architettura di Firenze, sul tema della frammentazione paesistica, nella quale è stata considerata una parte di territorio dell'area metropolitana Firenze-PratoPistoia comprensiva anche delle aree periurbane indagate in questo studio. Chi scrive è stato correlatore della tesi, relatore è stato il Prof. Biagio Guccione.

I dati riportati fanno riferimento al quadro conoscitivo del PTC Provinciale di Firenze (1998). Durante l'elaborazione della ricerca (1999-2001), i comuni interessati dal campione di studio avevano in corso il processo di revisione dei piani generali secondo i disposti della riforma legislativa toscana del 1995; i dati relativi ai nuovi piani non sono stati considerati per le difficoltà connesse allo stato di avanzamento delle elaborazioni, senza alcuna limitazione operativa per la ricerca che, per le proprie specifiche finalità di studio teorico-metodologico, ne avrebbe fatto comunque un uso esclusivamente strumentale, pari all'impiego fatto dei piani vigenti.
} 

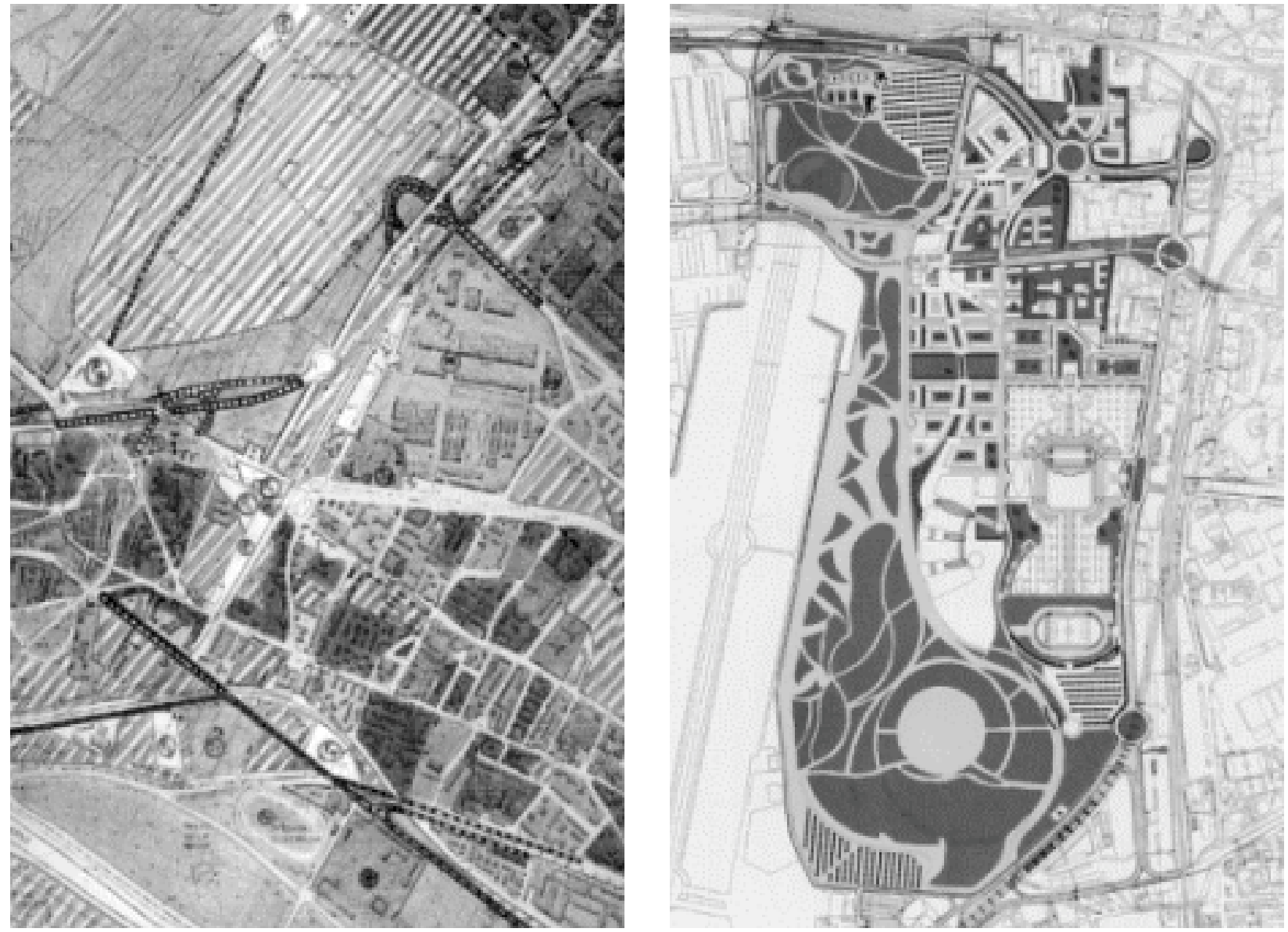

Figura 12 (a sinistra) - Mosaico dei Piani regolatori generali comunali (stralcio di cartografia ridotta dall'originale 1:10000). Figura 13 (a destra) - Piano Urbanistico Esecutivo di Castello - Planimetria del sistema del verde (immagine tratta da: Comune di Firenze, Sette idee per il parco urbano di Castello, Ufficio Pianificazione Grandi Progetti, Firenze, 2001).

Come accennato, le previsioni di sviluppo insediativo dei piani particolaregoiati di attuazione, risultano in questo caso indubbiamente quelle più importanti in termini di effetti potenziali di frammentazione paesistica: per le dimensioni complessive, per la tipologia, per la localizzazione. Gli interventi previsti dal Piano urbanistico esecutioo (PUE) di Castello investono un'area di poco inferiore ai 250 ettari, collocata tra il margine occidentale di Firenze e l'aereoporto e delimitata a sud dal tratto terminale dell'autostrada e a nord dalla ferrovia. Le superfici si articolano in circa 150 ettari di spazi edificati, circa 18 ettari di viabilità, percorsi e relative pertinenze e circa 80 ettari di parco urbano.

L'estensione del parco urbano fa sì che vi sia una sua funzione attendibile di compensazione, condizionabile certamente in positivo o negativo dalle modalità di progettazione, realizzazione, gestione, ma potenzialmente rilevante. La sua collocazione, fra l'aeroporto e le zone di sviluppo insediativo direzionale a margine di Firenze, lo investe di un'ovvia funzione di separazione di queste ultime dall'area aereoportuale, sia dal punto di vista visivo che, in parte, dal punto di vista acustico.

Di fatto la configurazione morfologica e funzionale del parco (figura 13) recepita all'interno del piano esecutivo $^{84}$ è decisamente estranea ai caratteri semiologici e culturali dei luoghi. Esula comunque dai compiti di questo studio una lettura critica di tali aspetti, richiamati esclusivamente per ragioni di inquadramento. Il parco nasce come grande sistema verde con andamento nord-sud, un corridoio

${ }^{84}$ Si tratta dello schema progettuale prodotto sotto forma di Piano guida da un gruppo di studio coordinato da Richard Rogers. Rispetto a tale scenario, nell'ambito di un successivo concorso ad inviti, sette gruppi di studio composti da vari esperti hanno elaborato altrettante proposte progettuali, producendo una ampia base di discussione che investe anche la stessa impostazione tipologica del parco. Si veda Comune di Firenze, Sette idee per il parco urbano di Castello, Servizio pianificazione grandi progetti, Firenze, 2001. 
tendenzialmente omogeneo secondo l'impostazione progettuale che è stata sommariamente descritta, che verrebbe a strutturare il nuovo margine occidentale della città, a seguito dell'espansione dovuta alla realizzazione del centro direzionale. La struttura di equipaggiamento paesistico prevista per questo insediamento, nonostante sia da realizzare interamente ex novo, risulta in realtà alquanto carente. Non si rintraccia infatti la carica innovativa delle linee guida suggerite dal piano iniziale, per quanto riguarda la depurazione delle acque, la regolazione microclimatica, l'equipaggiamento arboreo ed arbustivo delle strade, il ripristino di corsi d'acqua in superficie e la previsione di aree allagabili. Gli esiti progettuali complessivi mostrano un sistema di spazi verdi estremamente debole in termini di quantità di alberature, quanto in termini di identità semiologica della loro composizione. ${ }^{85} \mathrm{Il}$ paesaggio urbano pare fatto essenzialmente dal tessuto di spazi aperti disegnato dagli edifici all'interno del quale gli alberi compaiono come arredi vegetali in filari incerti o aggruppamenti incapaci di affermare una dimensione urbana, caratterizzata da spazi ben identificati ed equipaggiati, ponendo ancora una volta il rischio di produrre una nuova periferia. Nonostante la presenza di una spina sinuosa con andamento nord-sud e la configurazione estraniante del complesso per la nuova sede dei Carabinieri, la morfologia "normale" del resto del tessuto insediativo risulta molto più relazionata e relazionabile di quella del parco alla trama dei segni e delle tracce storiche che caratterizza il contesto in cui l'intervento si inserisce.

Complessivamente, la lettura degli strumenti di pianificazione territoriale sotto il punto di vista della individuazione dei fattori potenziali di frammentazione paesistica, ne rileva la concentrazione nella piana compresa fra il margine urbano occidentale di Firenze e gli insediamenti di Sesto Fiorentino, a nord, e di Osmannoro, a sud. Ciò è dovuto essenzialmente al fatto che in questo territorio insistono le aree meno consolidate dal punto di vista urbanistico. Sono invece essenzialmente prive di previsioni potenzialmente causa di frammentazione le aree collinari di Castello, protette da vincoli e proprietà pubbliche, nonché dallo stesso regime fondiario e sociale delle aree private, che governa, prima ancora degli strumenti di pianificazione, il paesaggio delle colline fiorentine. Non vengono riscontrati fattori potenziali di frammentazione afferenti ai tessuti urbani di pianura esistenti. Nell'area di Argingrosso le previsioni ed i vincoli del Piano di Bacino rafforzano quelle di spazi verdi della pianificazione comunale e provinciale.

Nell'intento di risalire alle strutture ed alle dinamiche dei processi di alterazione paesistica può essere utile considerare una tipologia di fattore potenziale, o meglio di fattore di rischio, che fa riferimento alle possibili ricadute socioeconomiche di determinati caratteri strutturali del paesaggio, in base alle quali tutto viene ad essere ricondotto ancora alle categorie delle alterazioni paesistiche ad esse conseguenti. Si consideri a titolo di esempio che, nella piana ad ovest di Firenze, la lettura dei pianiha confermato la tendenza all'urbanizzazione del paesaggio e pertanto alla marginalizzazione delle aree agricole di pianura in destra d'Arno. Ma è lecito pensare che parte delle motivazioni di questo destino territoriale risieda appunto in condizioni strutturali per cui l'agricoltura si trova ad essere particolarmente debole sul piano economico e pertanto ancora più facilmente marginalizzabile che altrove. Non si può omettere il fatto, tanto ovvio quanto sostanziale, che si sta parlando per lo più di spazi aperti periurbani, con tutto quanto questo comporta in termini di peso della rendita fondiaria. Ma se si osservano le caratteristiche di idoneità dei suoli agli usi agricoli si trova proprio l'ulteriore motivazione di ordine strutturale delle attuali condizioni delle aree periurbane occidentali comprese fra l'autostrada, la ferrovia e l'aereoporto. L'area è divisa praticamente in due parti di uguale estensione (figura 15) dalla distinzione dei terreni più alti della piana di Castello, il settore settentrionale, rispetto a quelli bassi, ad elevato rischio idraulico, del settore meridionale fino a Peretola. Nella classificazione di idoneità agli usi agricoli, i due settori risultano afferire alle tipologie

${ }^{85}$ La planimetria del sistema del verde del Piano Urbanistico Esecutivo di Castello è stata pubblicata in Comune di Firenze, 2001, cit. 
di minor qualità ${ }^{86}$. Sembra pertanto significativo considerare come anche le caratteristiche strutturali del paesaggio, nel caso in questione la scarsa idoneità all'uso agricolo, per i condizionamenti che comportano per gli utilizzi umani, possano costituire precondizioni per determinati tipi di trasformazioni e pertanto fattori di rischio dell'innesco di processi di alterazione, seppure non si configurino come diretti fattori causali.

Questo tipo di chiave interpretativa è evidentemente di maggior interesse, come lettura dei rischi di alterazione, nei casi in cui il paesaggio non sia ancora imbrigliato all'interno di indirizzi territoriali definiti e consolidati. Ma, abbandonando l'ottica della ricerca delle alterazioni potenziali, lo stesso tipo di analisi può essere finalizzata anche alla interpretazione delle condizioni di stato del paesaggio, risalendo processualmente alle loro motivazioni storiche, con un particolare interesse conoscitivo proprio nelle aree più strutturate, caratterizzate generalmente da una complessa stratificazione ed interazione di componenti e relazioni.
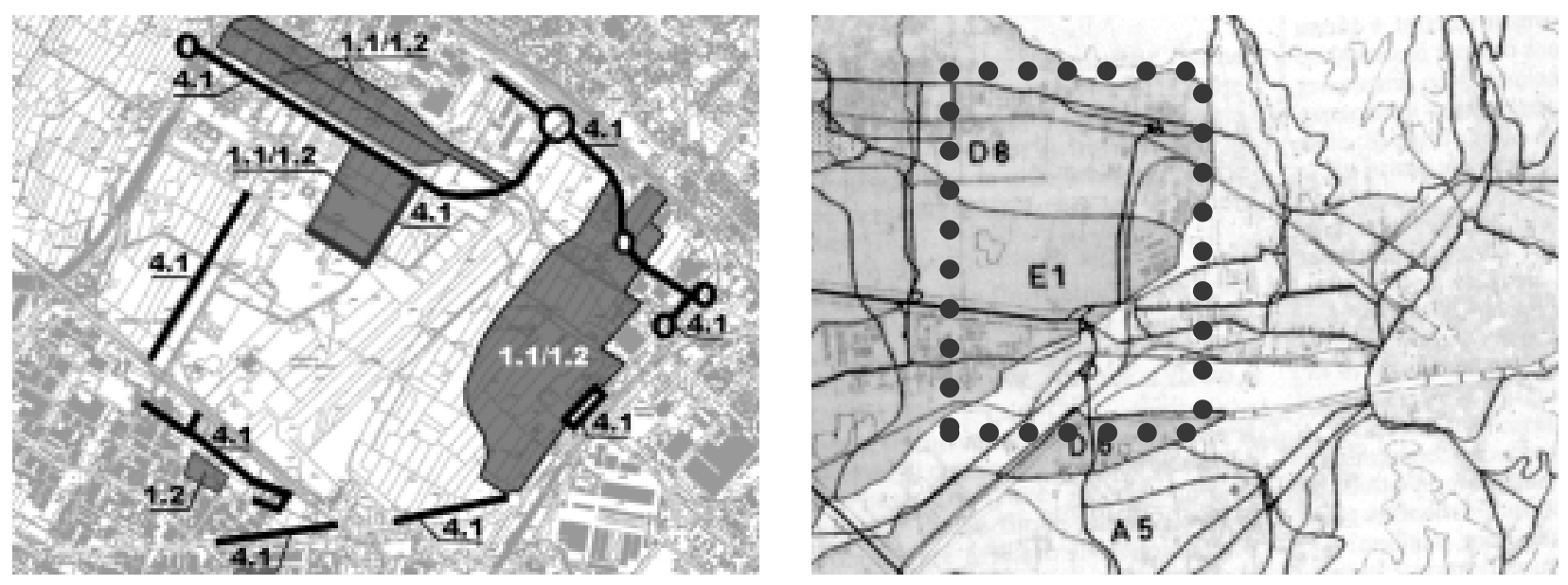

Figura 14 (a sinistra) - Carta di analisi dei fattori potenziali di frammentazione paesistica: gli indici numerici sono riferiti alle tipologie riportate nella lista di controllo per l'identificazione morfologica (cartografia ridotta da originale 1:10000 CTR Regione Toscana).

Figura 15 (a destra) - Carta delle caratteristiche di idoneità alle coltivazioni agricole: l'ambito di studio, contraddistinto dal riquadro puntinato, risulta avere tutti gii spazi non urbanizzati nelle classi di idoneità più bassa, la $\mathrm{D}$ e la $\mathrm{E}$ (immagine tratta da: Bianchi F.R., Favi E., Le caratteristiche agricole della piana, Quaderni di Urbanistica Informazioni, n. 7, 1990).

$86 \mathrm{Si}$ fa riferimento allo studio di Bianchi F.R., Favi E., Le caratteristiche agricole della piana, Quaderni di Urbanistica Informazioni, n. 7, 1990, elaborato su tutta l'area metropolitana Firenze Prato Pistoia, nell'ambito delle analisi per la formazione del relativo Schema Strutturale. I risultati delle valutazioni di idoneità (figura 15) hanno classificato la metà settentrionale dell'ambito di studio di questa ricerca nella IV classe (D8) e la metà meridionale nella V classe (E1). La classe generale D comprende le "aree senza o con poche limitazioni fisiche in cui l'agricoltura è pesantemente influenzata dagli usi extragricoli e non ha saputo sviluppare idonee soluzioni organizzative". Nello specifico l'unità D8 a cui afferisce parte dell'ambito di studio "occupa la fascia pedecollinare tra Firenze e Sesto Fiorentino, fino alla ferrovia per Bologna e la parte di pianura a nord dell'area aeroportuale. I suoli in genere presentano buone caratteristiche, essendo dotati di efficiente drenaggio, di tessiture franche, privi di ciottoli e con una adeguata quantità di carbonato di calcio. (...) A sud della ferrovia, nel Piano di Castello, si trovano ancora alcuni poderi di piccole e piccolissime dimensioni, con strutture assolutamente tradizionali, coltivati a part-time e/o manodopera anziana. (...) Le buone risorse fisiche originarie sono state completamente consumate da una massiccia e disorganica espansione urbana e dalla rilevante presenza di tratte infrastrutturali $(\ldots)^{\prime \prime}$. È pertanto piuttosto per il settore meridionale dell'area di studio che vale la considerazione proposta in merito al peso dei caratteri strutturali di ordine naturale (idrogeologici, geomorfologici e pedologici) sulle dinamiche evolutive di ordine culturale relative agli usi degli spazi. Questo ambito è risultato nella classe $E$, che comprende le "aree con notevoli limitazioni fisiche e con agricoltura degradata". Nello specifico l'unità E1 di cui fa parte è quella "più interessata da opere idrauliche fisse, quali il Fosso Reale e il Canale Macinante, cui è affidato il compito di raccogliere le acque nella zona. (...) I suoli sono caratterizzati da una tessitura piuttosto argillosa, con qualche difficoltà di drenaggio, resa meno evidente dalla presenza di estese superfici a prato. L'uso del suolo è costituito esclusivamente da seminativi semplici e da seminativi arborati a vite, con alcune superfici a ortaggi. La parte centrale dell'area, con terreni piuttosto argillosi e con drenaggio moderato, è interessata da un'agricoltura cerealicola-zootecnica. L'inserimento di famiglie coltivatrici immigrate in tempi più o meno recenti, ha consentito la diffusione dell'allevamento ovino mediante lo sfruttamento dei terreni non solo dell'azienda propria, ma anche delle risorse foraggere comunque reperibili in area". 


\section{3 - Gli indicatori primari di frammentazione paesistica}

"Partiamo dal postulato che la natura è un processo, che interagisce, che risponde a leggi che rappresentano valori e possibilità per l'uso umano, con certe limitazioni e perfino proibizioni per alcuni usi" ${ }^{\prime \prime 7}$. Secondo un'accezione strutturale del paesaggio, per cui esso trascende la sfera culturale della percezione, assumendo valenze più ampie e connotati identificabili in maniera più oggettiva, è possibile parafrasare questo celebre enunciato, affermando l'occorrenza di considerare innanzitutto che il paesaggio è un processo. Ciò risulta congruente con la formulazione secondo cui il paesaggio ha una struttura, è caratterizzato da più relazioni di funzionamento, è soggetto a determinate forme di cambiamento $^{88}$. Sebbene l'esplicitazione sistematica di questi concetti discenda dalle teorie sviluppate negli studi di ecologia del paesaggio, essi sono parte integrante degli approcci di tipo fisiografico che, a partire da McHargh e dagli autori a cui egli si riferì, talvolta ne hanno precorso i risultati. Struttura, funzionamento e cambiamento sono pertanto categorie determinanti per la descrizione del paesaggio, a cui possono essere riferiti altrettanti generi di indicatori, indipendentemente da specifici obiettivi conoscitivi e progettuali.

L'indicatore ambientale è una "variabile statistica quantitativa o qualitativa, rappresentativa di unaspetto, di un fattore (ambientale o umano) e di interesse di un contesto specifico (...). Un indicatore è dunque una variabile soggettiva scelta oggettivamente. Un indicatore può essere definito in una o più dimensioni, generalmente è espresso in funzione del tempo e/o dello spazio. Con riferimento al loro aspetto descrittivo, gli indicatori (quantitativi o qualitativi) sono anche chiamati descrittori. Con riferimento a misurazioni e/o stime, gli indicatori quantitativi sono anche chiamati parametri" ${ }^{\prime 89}$.

L'utilizzo degli indicatori discende dal fatto che "la maggior parte delle caratteristiche della qualità ambientale riflette apprezzamenti di natura astratta, non direttamente analizzabili attraverso unità di misura fisiche, dirette e oggettive. Tali caratteristiche possono essere stimate sulla base di parametri ambientali a cui sia stata preventivamente attribuita una corrispondenza con la qualità analizzata. Tali parametri danno indicazioni sulla qualità dell'ambiente, e vengono per questo chiamati indicatori ambientali. Indicatore ambientale è pertanto un elemento dell'ambiente o una sua caratteristica in grado di rappresentare, singolarmente $\mathrm{o}$ in combinazione con altri parametri, caratteristiche ambientali non direttamente misurabili attraverso unità di misura fisiche. In altre parole un indicatore ambientale può essere definito come un parametro a cui sia stata riconosciuta una corrispondenza con un'entità astratta (un dato evento, un altro elemento dell'ambiente, un sistema complesso, una scala di giudizio) di cui costituisce testimonianza. Esso è soprattutto uno strumento concettuale, che costituisce a sua volta strumento per l'utilizzo di altri strumenti e metodologie ${ }^{\prime \prime 90}$.

"Occorre ricordare che l'indicatore deve aiutare ad interpretare lo stato dell'ambiente, le pressioni delle attività umane, con riferimento ad una soglia, deve avere una valenza scientifica in termini di prevedibilità e di metodo da seguire, deve essere misurabile. Nella definizione degli indicatori debbono essere previsti meccanismi di feed-back per cui gli indicatori saranno legati a sistemi di monitoraggio e di aggiornamento dei dati ${ }^{\prime \prime 1}$.

Queste definizioni proprie delle teorie e pratiche dell'analisi e della valutazione ambientale si attagliano pienamente ai processi di indagine paesistica, che accentuano però le citate difficoltà

${ }^{87}$ McHarg I.L., Progettare con la natura, Muzzio (Doubleday \& Company, Inc.), Padova (Garden City, New York), 1989 (1969).

${ }^{88}$ Forman R.T.T., Land mosaics - The ecology of landscapes and regions, Cambridge, 1995.

89 Colombo A.G., Malcevschi S., (a cura), Manuale A A A degli Indicatori per la V alutazione di Impatto A mbientale - Indicatoride paesaggio, coordinatore Delsante S., Vol. 5, Centro VIA Italia, Associazione Analisti Ambientali, Federazione delle Associazioni Scientifiche e Tecniche, Milano, 1999.

${ }_{90}^{90}$ Malcevschi S., Qualità ed impatto ambientale, E taslibri, Milano, 1990.

91 Bettini V., Screening dello studio di zalutazione delle alternatize di tracciato in zariante alla strada statale n. 63, dattiloscritto, Università di Venezia - IUAV, Corso di laurea in Pianificazione Territoriale Urbanistica Ambientale, Corso di diploma in Sistemi Informativi Territoriali, Venezia, 1999 (?). 
valutative e di oggettivazione. Nell'ambito delle ricerche condotte in materia di pianificazione ambientale, sono stati definiti alcuni indici per la valutazione delle condizioni di frammentazione ambientale dovuta alla pressione insediativa ${ }^{92}$.

Nella analisi della frammentazione dovuta alla urbanizzazione "in via preliminare si può prevedere l'utilizzo dei seguenti indicatori:

- incidenza dell'urbanizzazione rispetto alle diverse forme di uso del suolo in ambiti geografici significativi;

- dispersione dell'urbanizzazione sul territorio (indice indirettamente collegato alla densità viaria);

- caratteri spaziali di organizzazione dell'insediamento;

- coefficienti di forma dell'area urbanizzata' ${ }^{\prime \prime 93}$.

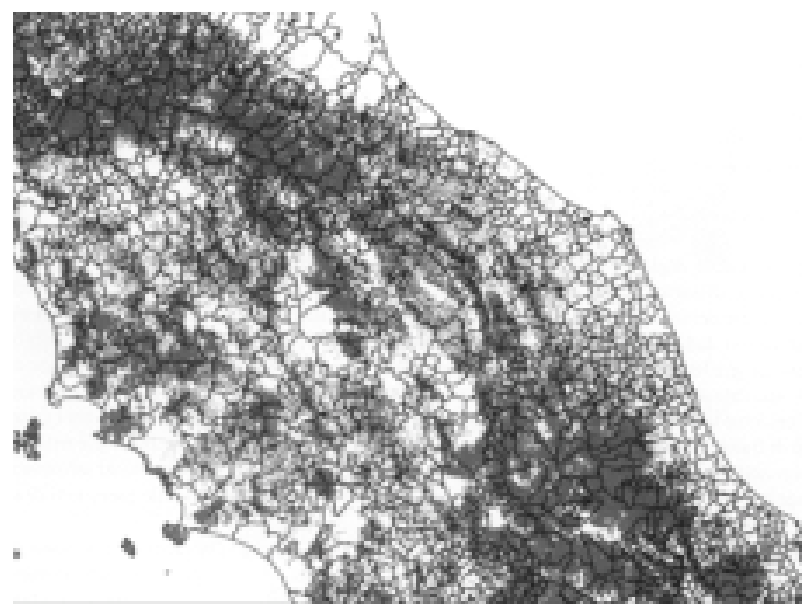

Figura 16 - L'articolazione e la dimensione territoriale degi ambiti comunali dell'I talia centrale (perimetri a tratto) a confronto con la geografia della biopermeabilità (le aree in grigio indicano gii ambiti di importanza primaria alle quali si aggiungono su gran parte del territorio nazionale ambiti di paesaggio rurale con naturalità diffusa) (immagine tratta da: Romano B., Continuità ambientale. Pianificare per il riassetto ecologico del territorio, Andromeda E ditrice, Teramo, 2000).

Indicatori ed indici numerici sono stati definiti per la frammentazione da urbanizzazioni e da infrastrutture. L'utilizzo di tali indici non è tanto finalizzato alla valutazione finale di dettaglio della intensità e localizzazione delle manifestazioni di frammentazione, quanto ad utilizzare i sistemi informativi territoriali per la predisposizione di valutazioni su grande scala, che permettano di approssimare la realtà del fenomeno per gradi successivi di approfondimento, ma tenendo il controllo delle relazioni geografiche e paesistiche. Ciò è mirato ad una prima costruzione del quadro della biopermeabilità ${ }^{94}$ del paesaggio, con ruolo di dato orientativo fondamentale per l'ottimizzazione degli approfondimenti conoscitivi e l'indirizzo di base dei piani territoriali di area vasta.

Già questo primo dato di ricerca ${ }^{95}$, indubbiamente da approfondire per una sua applicabilità ad obiettivi progettuali operativi, mette in risalto in termini geografici un problema oggettivo e sostanzialmente noto, che l'evoluzione della strumentazione legislativa e tecnica della pianificazione per il governo del territorio dovrà affrontare efficacemente. L'articolazione territoriale della continuità biotica del paesaggio risulta di difficile governo per la elevata parcellizzazione amministrativa del territorio italiano, soggetto alle politiche di oltre ottomila amministrazioni comunali (figura 16).

92 In Italia ha trattato questo tema la ricerca PLANECO, soprattutto per quanto riguarda l'individuazione di indicatori utilizzabili sulla base di informazioni di tipo territoriale di reperimento relativamente semplice rispetto ai dati ecologici specifici. Una sintesi dei risultati si trova in Romano B., Continuità ambientale. Pianificare per il riassetto ecologico del territorio, Andromeda Editrice, Teramo, 2000 e vari contributi sul tema si trovano anche sulla News Letter Planeco, n. 1-6, disponibile sul sito web:http://dau.ing.univaq.it.

${ }_{93}$ Romano B. (f), Continuità ambientale. Pianificare per il riassetto ecologico del territorio, Andromeda E ditrice, Teramo, 2000.

${ }^{94}$ Termine utilizzato per denominare condizioni di continuità della struttura biotica del paesaggio indipendentemente dai diversi caratteri intrinseci di importanza naturalistica, ecologica, storico-culturale, fisionomico-percettiva; si veda Romano B. (f), 2000, cit.

95 Le cartografie elaborate su scala nazionale nell'ambito della ricerca Planeco, compresa anche quella della biopermeabilità possono essere consultate sul sito WE B http://dau.ing.univaq.it. 
Relativamente allo specifico tema della frammentazione paesistica, occorre individuare quali indicatori siano idonei alla descrizione delle principali alterazioni paesistiche che caratterizzano questa fenomenologia. Il loro impiego analitico, a supporto della diagnosi degli stati e delle dinamiche del paesaggio, può essere funzionale alla definizione di politiche e progetti di riqualificazione diffusa.

Per definizione, l'utilizzo degli indicatori prevede l'effettuazione di misurazioni parametriche, finalizzate alla descrizione delle quantità e qualità che essi rappresentano. Questo non coincide però necessariamente e direttamente con l'adozione di tecniche numeriche di ponderazione dei dati, al fine di ottenere giudizi diagnostici complessi (si veda il capitolo 3).

Tutti gli indicatori fanno riferimento ad una rappresentazione cartografica, essenziale, sia come base dimensionale per i rilevamenti di tipo numerico, che non possono ovviamente in questi casi essere completamente condotti in campagna, ma devono avvalersi di idonei modelli in scala, sia per la considerazione analogica delle configurazioni rilevate, per ovvie ragioni fondamentale in sede di analisi, quanto in sede di diagnosi.

La complessità costitutiva e relazionale che caratterizza la struttura del paesaggio ed i relativi processi di funzionamento e cambiamento comportano una eterogeneità e gerarchia degli indicatori di frammentazione difficilmente eliminabile, più probabilmente assumibile, invece, come carattere identificativo utile a guidare le successive fasi diagnostiche di interpretazione dei dati analitici prodotti attraverso gli indicatori stessi.

La funzione analitica degli indicatori di frammentazione paesistica non è tanto alternativa quanto complementare a quella dei fattori territoriali, costituendo un approfondimento conoscitivo in termini qualitativi e quantitavi delle situazioni rilevate attraverso il censimento dei fattori medesimi. Relativamente alle caratteristiche del paesaggio individuabili come indicatori, è possibile definire un quadro sperimentale mirato alla copertura tematica degli aspetti che risultano prioritari nella fenomenologia della frammentazione paesistica (figura 17).

Queste analisi, come accade per tutte quelle riguardanti le trasformazioni del paesaggio, assumono particolare efficacia conoscitiva ove possano essere elaborate su base diacronica. Al fine di vedere meglio le loro potenzialità conoscitive è utile considerare la stretta complementarietà con le descrizioni bibliografiche attraverso un esempio di osservazione delle modificazioni del paesaggio di pianura intervenute nell'ultimo secolo.

A Firenze, alla metà dell'Ottocento, ma fino alla prima guerra mondiale “(...) in tutta la piana dominava la coltura promiscua, per lo più seminativi vitati, che producevano oltre il grano un vino molto mediocre, o arborati e vitati (...). La prosperità agricola delle varie parti della pianura può essere rapportata alla densità degli insediamenti e in particolare delle ville padronali. Sotto questo aspetto appaiono favoriti i terreni a sinistra dell'Arno, a valle di Firenze, che oggi formano l'estremità di ponente del territorio comunale (...). Qui le ville, quasi sempre nobiliari in origine, hanno una densità all'incirca di tre a chilometro quadro. (...) Molto più bassa è la densità delle ville nell'area centrale a destra dell'Arno, specie verso il margine sud, dove il livello del suolo è più basso $(\ldots)^{\prime \prime 96}$.

La Carta d'Italia IGM del 1934 mostra configurazioni degli spazi rurali apparentemente invariate, caratterizzate dalla permanenza e prevalenza del seminativo vitato e/o arborato, dei connotati spaziali dell'alberata, tipici della coltura promiscua. Ma in realtà si era avviato un processo socioeconomico che avrebbe avuto decisive ricadute sul paesaggio della piana. Ne sono una precondizione lo sviluppo dei tracciati ferroviari e la realizzazione del rettifilo della autostrada di collegamento con la costa tirrenica, che, già allora, risultano leggibili come segni evidentemente sovraordinati e diversi rispetto a quelli che componevano la matrice rurale del paesaggio. Era iniziata l'infrastrutturazione moderna del territorio: ad essa si legava il processo di sviluppo sociale ed economico industriale del paese.

96 Bortolotti L., De Luca G., Come nasce un'area metropolitana Firenze Prato Pistoia: 1848-2000, Alinea, Firenze, 2000. 


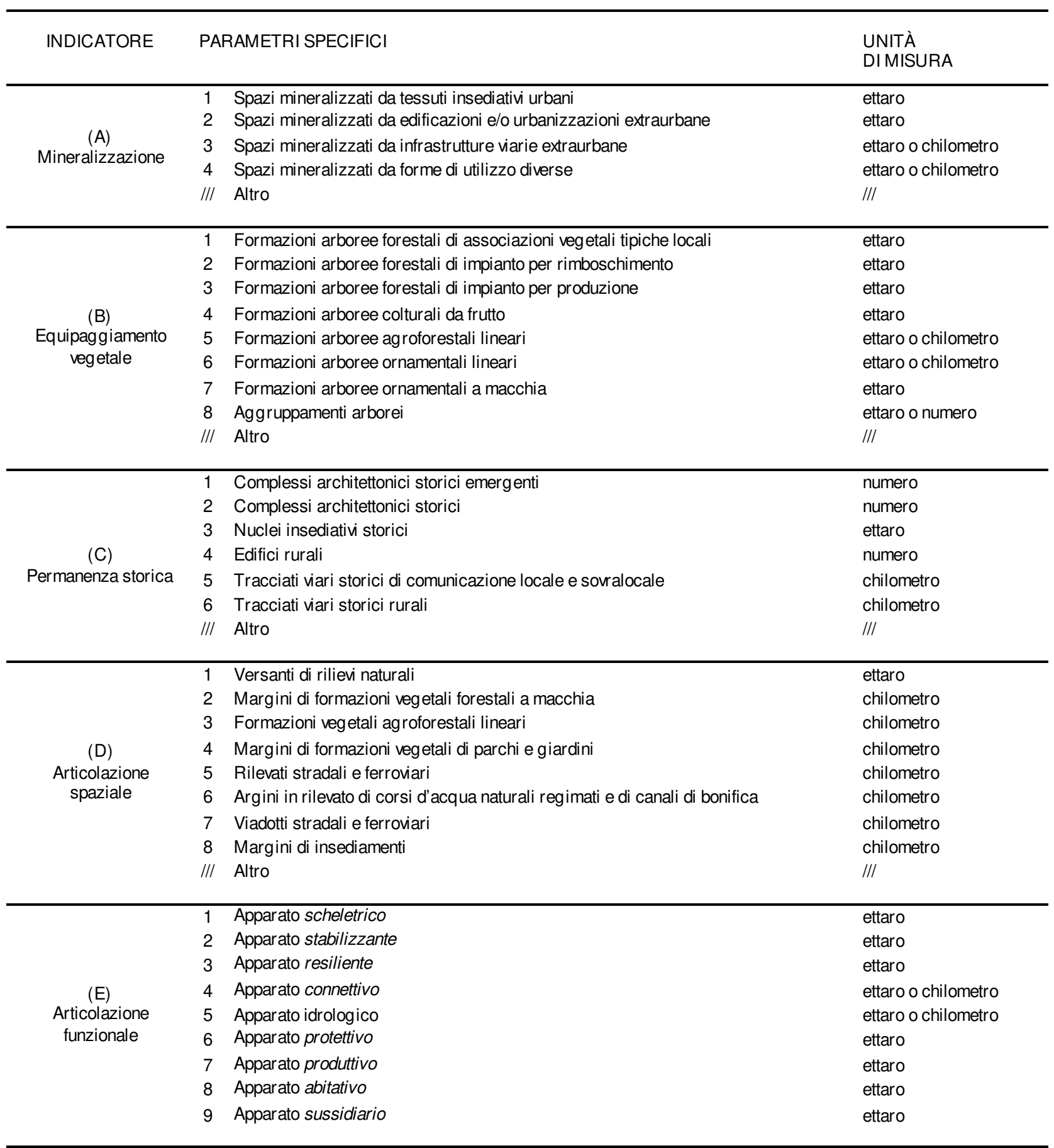

Figura 17 - Quadro degii indicatori primari di frammentazione paesistica (le denominazioni riportate in corsivo fanno riferimento a definizioni convenzionali utilizzate in ecologia del paesaggio ${ }^{97}$ ).

97 Vittorio Ingegnoli (Ingegnoli V., 1993, cit.) propone le seguenti definizioni degli apparati paesistici, riferite alle tipologie di spazi che vi afferiscono: scheletrico: “(...) elementi del paesaggio le cui funzioni paesistiche e biotiche sono dominate dai processi geomorfologici"; connettizo: elementi del paesaggio responsabili dei livelli di connettività che esso è in grado di esprimere (n.d.r.); stabilizzante: “(...) elementi ad alta metastabilità, a cui spettano generalmente le funzioni regolatrici $\mathrm{e}$ protettive dominanti rispetto agli altri ecosistemi"; resiliente: “(...) elementi con grande capacità di ripresa (...) spesso complementare al precedente"; escretore: "(...) reticolo di corridoi fluviali del quale viene utilizzata la capacità di trasporto e depurazione"; protettizo: "(...) elementi capaci di influire sulla regolazione microclimatica, l'isolamento acustico e la strutturazione degi spazi negli insediamenti, sulla regolazione e la protezione dei coltivi agricoli, sulla ricreazione della popolazione"; produttiz: "(...) elementi del paesaggio con funzione agricola"; abitatiz: "(...) funzioni insediative residenziali e di servizio"; sussidiario: "(...) elementi con funzione industriale, (...) infrastrutture territoriali". 
Ancora prima della fine degli anni Trenta “(...) la proprietà rurale (specie quella media) si fraziona negli intorni dei centri maggiori, ponendo una delle premesse all'esplosione edilizia del secondo dopoguerra ${ }^{98}$. Dopo il conflitto gli esponenti della proprietà terriera appaiono meno restii ad impegnarsi in prima persona nelle imprese industriali ${ }^{99}(\ldots)$. A Firenze si sviluppa una discreta zona industriale, grazie anche all'annessione di Brozzi, Peretola e parte di Scandicci; ma il distacco dalla città più industriale, Milano, rimane enorme: 52 società industriali contro circa $800^{100}$. Nei trasporti pubblici, la cui rete andava sempre più infittendosi ${ }^{101}$, raggiungono posizioni di predominio la Sita e la Lazzi (...). Occorre accennare ai consorzi di bonifica, per le trasformazioni attuate nella pianura. I consorzi vennero raggruppati in due soli, quello dell'Ombrone, di $120 \mathrm{kmq}$ circa, costituito nel '37 ed esteso in pianura fino agli argini del Bisenzio, fiume dal corso pensile, e quello di Sesto (Fiorentino, n.d.r.), di 72,2 kmq. A valle di Sesto venne costruita una doppia canalizzazione, per separare le acque alte (quelle che possono scolare naturalmente) dalle acque basse $(\ldots)^{\prime \prime 102}$.

La trasposizione e l'interpretazione cartografica di questo tipo di informazioni permette l'identificazione spaziale dei processi di trasformazione paesistica. Per l'esame dello sviluppo delle condizioni di elevata pressione insediativa, le soglie storiche significative sono essenzialmente tre: la soglia contemporanea (fine $\mathrm{XX}$ sec.), relativa alla identificazione dei sistemi strutturali attuali destinatari della diagnosi degli stati di frammentazione del paesaggio e le soglie passate (seconda metà e fine XIX sec. e metà XX sec.), precedenti l'avvio dei processi di espansione insediativa e pertanto funzionali alla identificazione dei sistemi strutturali storici di cui analizzare gli stati di permanenza.

La soglia contemporanea può fare riferimento al paesaggio attuale ed alla sua descrizione attraverso $\mathrm{i}$ dati fotografici e cartografici più aggiornati. Il quadro relativo alle soglie storiche può fare riferimento al supporto di conoscenze di periodi precedenti, al fine di comprendere la strutturazione antropica profonda del paesaggio: la cartografia della prima levata dell'Istitituto Geografico Militare Italiano (fine '800); la cartografia del Catasto Lorenese (fine '800); la cartografia dello Stato Pontificio e del Granducato di Toscana (metà '800). La soglia storica intermedia, coincidente con gli anni '70 e '80 del secolo scorso, è relativa ad una fase di mutazione di orientamento dei processi insediativi, che vede il passaggio dalla priorità della ricostruzione postbellica e della espansione edilizia residenziale, proprie dei due decenni precedenti, alla priorità della infrastrutturazione e strutturazione di servizio, con una forte espansione insediativa delle attività terziarie. Tale soglia storica, ampiamente documentata anche per il territorio di Firenze dalla citata ricerca IT URB 80, non risulta fondamentale da un punto di vista cartografico, pur mantenendo valore documentario. Infatti, per le problematiche poste dall'obiettivo della definizione di un modello progettuale di scenari di riduzione della frammentazione e di riqualificazione diffusa del paesaggio, diviene essenziale, oltre la conoscenza delle condizioni attuali, quella del paesaggio ancora non profondamente e diffusamente alterato dalla crescita insediativa metropolitana.

L'intensa "perdita di paesaggio" che caratterizza la nostra epoca, dovuta al progressivo ed improprio utilizzo degli spazi aperti per realizzare insediamenti ed infrastrutture, talvolta paradossalmente coincidente con le problematiche di rinnovare il destino, oltre che le destinazioni, delle molte aree urbane e periurbane dismesse, costituisce indubbiamente la manifestazione più macroscopica e generale del comportamento di consumo delle risorse non rinnovabili proprio della società contemporanea. A tale consumo, in ragione dei caratteri qualitativi e quantitativi che lo identificano in termini di alterazioni paesistiche, sono dovute decise manifestazioni di frammentazione paesistica,

98 Lunganelli M., cit. in Bortolotti L., De Luca G., 2000, cit.

99 Palla M., cit. in Bortolotti L., De Luca G., 2000, cit.

100 Annuario Italiano del Capitalista, cit. in Bortolotti L., De Luca G., 2000, cit.

101 Dainelli G. e Innocenti P., cit. in Bortolotti L., De Luca G., 2000, cit.

102 Bortolotti L., De Luca G., 2000, cit. 
che interessano la struttura ed i relativi processi funzionali, afferenti alle componenti biotiche, comea quelle abiotiche, ma coinvolgono profondamente anche l'articolazione morfologica del paesaggio, come le sue condizioni di permanenza storica, nella misura in cui si tratta nella generalità dei casi di fenomeni di sostituzione e sovrapposizione, anziché di stratificazione paesistica.

Sulla consistenza di queste manifestazioni incidono decisamente le quantità di spazi in cui il paesaggio viene ad essere mineralizzato a seguito della realizzazione di edifici, infrastrutture e relativi spazi pertinenziali. Queste condizioni sono misurabili in termini assoluti (superficie degli spazi mineralizzati) e relativi (superficie degli spazi mineralizzati per ettaro o chilometro quadro di territorio, oppure percentuale di spazi mineralizzati relativa ad un ambito). La considerazione della mineralizzazione del paesaggio è pertanto esattamente rispondente ai requisiti di misurabilità e monitorabilità, citati in precedenza a proposito delle definizioni di indicatore. Facendo riferimento ai parametri indicati nel quadro della figura 17, risulta la situazione riportata nella tabella della figura 18.

\begin{tabular}{lcccccc} 
& \multicolumn{7}{c}{ FORME DI MINERALIZZAZIONE } \\
& \multicolumn{7}{c}{ DEGLI SPAZI } & TOT. \\
\cline { 2 - 6 } & 1 & 2 & 3 & 4 & 238 \\
\hline SUPERFICIE DEGLI SPAZI (Ha) & 164 & 46 & 27 & 1 & 49 \\
\hline PERCENTUALE DEGLI SPAZI (\%)* & 33,8 & 9,5 & 5,6 & 0,2 & 49 \\
\hline
\end{tabular}

${ }^{*}$ Riferita alla superficie totale del campione di indagine pari a $485 \mathrm{Ha}$

Figura 18 - Rilevamento dell'indicatore mineralizzazione del paesagoio - Parametri riscontrati e quantificati: (1) spazi mineralizzati da tessuti insediativi urbani, (2) spazi mineralizzati da edificazioni e/o urbanizzazioni extraurbane, (3) spazi mineralizzati da infrastrutture viarie extraurbane, (4) spazi mineralizzati da forme di utilizzo diverse (specifamente: canale collettore in calcestruzzo).

Il territorio analizzato presenta condizioni relative (al campione di indagine) di elevata mineralizzazione del paesaggio: il fenomeno interessa complessivamente circa la metà della superficie (49\%). La maggior incidenza è dovuta ai tessuti urbani $(33,8 \%)$, che risultano molto estesi e caratterizzati da discontinuità degli spazi mineralizzati sporadiche e quantitativamente poco influenti (figura 19).

Si noti che il complesso degli spazi di tipo 1 e 2, afferenti ai sistemi insediativi urbani, è responsabile di un tasso relativo di mineralizzazione del paesaggio pari ad oltre il $43 \%$. Un'altra indicazione significativa viene dalla considerazione del complesso degli spazi di tipo 2, 3 e 4, afferenti al paesaggio periurbano non interessato da tessuti insediativi e da insediamenti per servizi, responsabile di un tasso relativo di mineralizzazione pari ad oltre il 15\%. Tale dato fornisce una descrizione eloquente dell'intensità del processo di consumo degli spazi nel paesaggio periurbano; la distribuzione degli spazi mineralizzati, rilevata dall'indagine cartografica (figura 19), completa il quadro analitico evidenziando la "geografia" locale del fenomeno della frammentazione rilevata da questo indicatore.

Nell'area considerata la mineralizzazione del paesaggio dovuta a canali artificiali ha un peso che occorre vedere relativamente alla presenza generalmente limitata di queste situazioni nei tratti extraurbani dei corsi d'acqua: il fenomeno ha in questo caso una estensione lineare di oltre $950 \mathrm{ml}$ per una superficie di spazi aperti mineralizzati che si avvicina ad 1 ettaro.

L'articolata gamma di effetti e relazioni che questo tipo basilare di alterazione paesistica produce fa sì che le condizioni di mineralizzazione assumano una collocazione gerarchica particolare fra gli indicatori di frammentazione paesistica, non tanto collocandosi al vertice di un modello piramidale 
per il quale le altre manifestazioni deriverebbero in tutto da questa, ma identificandosi come manifestazione complessa, sia in termini di diversificazione delle forme in cui si concretizza, sia in termini di diversificazione delle ricadute sul paesaggio. La dimostrazione della incongruenza di un ipotetico modello piramidale delle relazioni fra gli indicatori di frammentazione paesistica, in cui la mineralizzazione del paesaggio dovesse trovarsi al vertice, può fare riferimento anche alla sola considerazione che non sono esclusivamente gli sviluppi insediativi edilizi ed infrastrutturali ad essere responsabili della rimozione dell'equipaggiamento vegetale del paesaggio, notoriamente e sostanzialmente avvenuta anche a seguito delle evoluzioni delle pratiche agrarie, rispetto alle quali l'indicatore mineralizzazione non è sensibile. Secondo questa osservazione, la logica a cascata non risulta applicabile agli indicatori tematici utilizzati per descrivere la fenomenologia della frammentazione paesistica per la parzialità della copertura descrittiva che li caratterizza singolarmente.
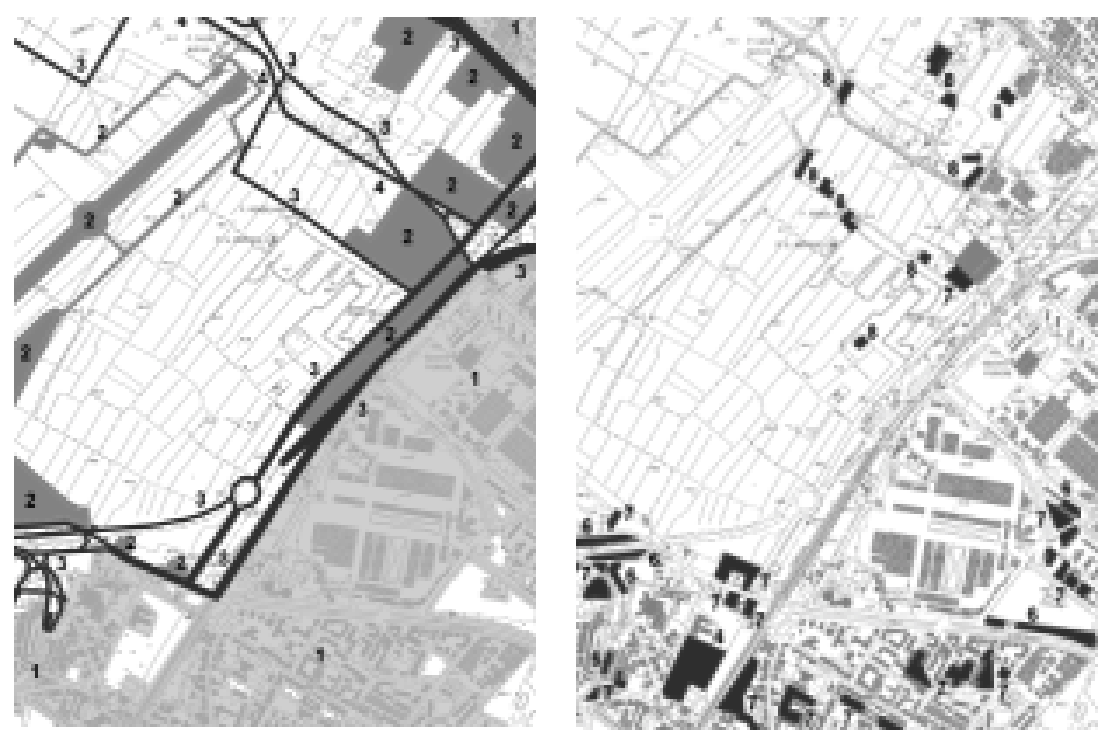

Figura 19 - Analisi degli indicatori di frammentazione paesistica: mineralizzazione del paesaggio (a sinistra), equipaggiamento vegetale del paesaggio (a destra). Gli indici numerici fanno riferimento alle tipologie riportate nel quadro sinottico della figura 17 (cartografie ridotte da originale 1:10000 - CTR Regione Toscana).

Ai fini dell'utilizzo dell'indicatore della mineralizzazione del paesaggio in sede di diagnosi, occorre rilevare che la descrizione numerica da esso fornita circa le quantità di spazi mineralizzati non copre la questione dell'articolazione topologica che ne identifica la distribuzione. Essendo tale dato di fondamentale importanza in relazione agli effetti di frammentazione del paesaggio, sia per quanto riguarda la loro intensità, sia per la loro identificazione qualitativa, diviene necessario individuare parametri di misurazione che permettano di approssimare tali caratteri. A tal fine è utile affiancare alla misurazione assoluta della quantità di spazi mineralizzati in un dato ambito l'incidenza percentuale rispetto alla realtà complessiva riscontrata nell'ambito di riferimento, restando la referenziazione cartografica dei dati rilevati di peculiare importanza.

A tale genere di informazioni di alterazione strutturale del paesaggio, l'analisi degli stati e delle dinamiche di frammentazione paesistica richiede di affiancare dati specificamente riferiti ad alcuni caratteri paesistici che risentono profondamente degli effetti di questa fenomenologia. Si tratta essenzialmente dei caratteri di equipaggiamento vegetale, di permanenza storica, di articolazione spaziale, e di articolazione funzionale, proposti, con la mineralizzazione, come indicatori primari della frammentazione del paesaggio (figura 17). È evidente che, sia gli indicatori di struttura, che quelli di funzionamento, permettono di descrivere il cambiamento del paesaggio nella misura in cui sia possibile praticarne una lettura diacronica riferita a soglie storiche significative.

L'equipaggiamento zegetale, arboreo e arbustivo, costituisce un carattere assolutamente trasversale in termini di connotazione del paesaggio. Dalla presenza di vegetazione sono influenzate le condizioni ambientali delle popolazioni umane ed animali. Ma la quantità e la tipologia di equipaggiamento 
vegetale costituiscono elementi determinanti la connotazione morfologica del paesaggio ed allo stesso tempo sono, in taluni casi di formazioni ornamentali o agroforestali relitte, testimonianze delle sue configurazioni storiche.

In analogia con la mineralizzazione, occorre che anche l'equipaggiamento vegetale sia rilevato sì in termini assoluti (ad esempio, nelle scale proprie dell'analisi di frammentazione paesistica, in terminidi superfici, per le formazioni a sviluppo planimetrico areale, e di lunghezze, per le formazioni lineari), ma anche nei termini relativi di incidenza percentuale complessiva delle superfici e di incidenza percentuale unitaria relativa ad ambiti di riferimento con caratteristiche omogenee (percentuale di superficie con soprassuoli vegetali arborei e/o arbustivi rispetto al totale dell'area di studio e rispetto all'ambito spaziale omogeneo di appartenenza). Nel caso specifico delle formazioni vegetali lineari, filari e siepi di campo, alle misure di estensione superficiale occorre spesso sostituire quelle di lunghezza, in ragione della irrilevanza della loro dimensione trasversale rispetto alla scala di analisi.

Per tutti gli indicatori, è anzitutto necessario il riconoscimento fisionomico delle diverse tipologie alle quali vengono associate le misurazioni parametriche. Attraverso i sistemi informativi geografici (GIS), è possibile infatti procedere agevolmente al calcolo delle misure complessive per semplice somma, mentre sarebbe impossibile disaggregare per tipologie un dato rilevato unitariamente, per l'assenza delle informazioni necessarie. Rispetto agli otto parametri individuati in via teorica nel quadro riportato nella figura 17 , nell'area di studio sono state riscontrate quattro tipologie, la cui misurazione produce i dati riportati nella tabella della figura 20.

\begin{tabular}{ccccccc} 
& \multicolumn{5}{c}{ TIPOLOGIA } \\
\cline { 2 - 5 } & \multicolumn{3}{c}{ DI EQUIPAGGIAMENTO } & TOT. \\
\hline SUPERFICIE DEGLI SPAZI (Ha) & 4 & 6 & 7 & 8 & 16,5 \\
PERCENTUALE DEGLI SPAZI (\%)* & 2,5 & 3 & 8 & 3 & 3,4 \\
\hline
\end{tabular}

${ }^{*}$ Riferita alla superficie totale del campione di indagine pari a $485 \mathrm{Ha}$

Figura 20 - Rilevamento dell'indicatore equipaggiamento zegetale del paesaggio - Parametri riscontrati e quantificati: (4) formazioni arboree colturali da frutto, (6) formazioni arboree ornamentali lineari, (7) formazioni arboree ornamentali a macchia, (8) aggruppamenti arborei.

Il territorio analizzato presenta condizioni relative (al campione di indagine) di elevata semplificazione biologica del paesaggio (si consideri anche la conseguente incidenza sull'articolazione spaziale), dovute ad un grado di equipaggiamento vegetale assai basso: risultano presenti formazioni vegetali per una superficie complessiva di poco superiore a 16 ettari $(3,4 \%)$.

Nonostante la carenza di sistemazioni vegetali caratteristica delle aree urbane, anche in questo caso si rilevano le condizioni tipiche ormai di molti paesaggi periurbani di pianura, dove ai tessuti urbani corrispondono le maggiori quantità di equipaggiamento vegetale, mentre le aree agricole risultano sempre più desertificate (figura 19).

Ai 170 ettari di aree extraurbane non mineralizzate corrispondono 3 ettari di spazi con equipaggiamento vegetale, peraltro valutati per eccesso, essendo stati compresi anche quelli presumibilmente appartenenti a formazioni colturali o loro relitti. Se ne ricava un grado di equipaggiamento del paesaggio agrario di pianura (o meglio di quello che mantiene tale potenziale di utilizzo degli spazi, nel quale debbono essere compresi i molti ettari di suoli attualmente non coltivati) che non supera comunque l'incidenza del 1,8\% anche considerando tutte le formazioni arboree e miste arboreo/arbustive rintracciabili dall'esame delle fotografie aeree. A questo si deve 
inoltre aggiungere la scarsa qualità generalizzata dell'equipaggiamento presente che, per gli stati di conduzione dei fondi, risulta soggetto a sviluppo e composizione vegetale spontanea, con diffusione di specie infestanti, e privo di ogni misura di governo e di incremento (ad eccezione di quelle di manutenzione praticate per i corsi d'acqua, che tendono piuttosto a ridurre le presenze).

La permanenza storica è un indicatore essenziale per la conoscenza delle condizioni di frammentazione della struttura culturale profonda del paesaggio e costituisce, anche nella forma istantanea relativa all'attualità, una identificazione precisa dei processi di cambiamento che lo caratterizzano.

Il riconoscimento delle diversità tipologiche che identificano le diverse componenti della storia culturale del paesaggio, permette di procedere ad una iniziale misurazione separata della presenza delle stesse, da cui ricavare i conseguenti dati aggregati.

Per quanto riguarda le emergenze relative ai beni di interesse storico-architettonico e monumentale il rilievo viene effettuato in termini di numero di presenze. Per quanto concerne invece le configurazioni sistemiche degli ordinamenti storici, insediativi e paesistici in genere, occorrerà procedere al loro rilevamento attraverso unità di misura congrue ai diversi soggetti (ad esempio lunghezze per le strade e la rete idrografica relativa ai sistemi di bonifica agraria, oppure superfici per quanto riguarda gli insediamenti aggregati a geometria areale o lineare ed infine ancora numero di presenze per quanto riguarda gli edifici isolati afferenti agli ordinamenti rurali).
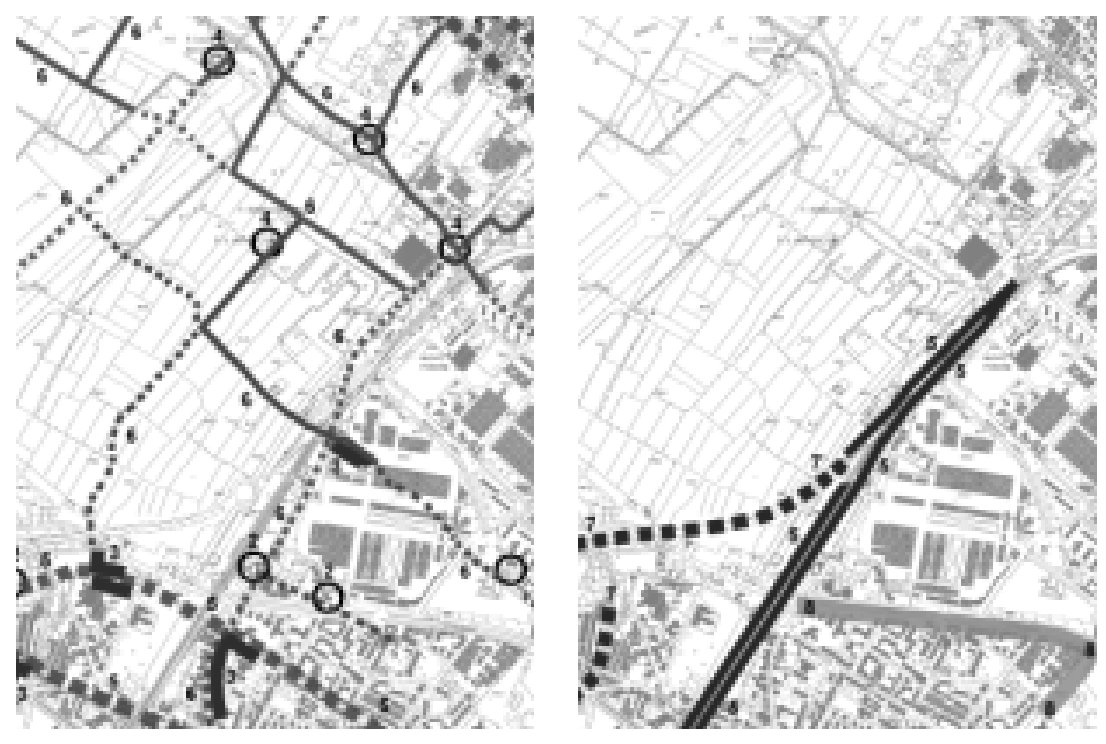

Figura 21 - Analisi degli indicatori di frammentazione paesistica: permanenza storica del paesaggio (a sinistra; i tratteggi sono relativi a tracciati stradali perduti o completamente obliterati dalle trasformazioni incorse), articolazione spaziale del paesaggio (a destra; i tratteggi sono relativi a tracciati stradali su viadotto, distinti da altre componenti per caratteri semiologici). Gli indici numerici fanno riferimento alle tipologie riportate nel quadro sinottico della figura 17 (cartografie ridotte da originale 1:10000 - CTR Regione Toscana).

Nonostante sia possibile utilizzare indici numerici sensibili alla distribuzione spaziale ed alla concentrazione delle risorse, l'identificazione della frammentazione dei sistemi paesistici storici deve avvalersi necessariamente della rappresentazione cartografica (figura 21) dei loro segni e delle tracce ancora riconoscibili.

Rispetto ai parametri individuati in via teorica nel quadro riportato nella figura 17, nell'area di studio sono state riscontrate cinque tipologie su sei: (2) complessi architettonici storici, (3) nuclei insediativi storici, (4) edifici rurali, (5) tracciati viari storici di comunicazione locale e sovralocale, (6) principali tracciati viari storici rurali.

L'articolazione spaziale del paesaggio, fra gli indicatori di struttura considerati, risulta il più eterogeneoe transcalare. Occorre comunque evitare ogni equivoco rispetto ad altri tipi di indicatore finalizzati al rilevamento delle condizioni di visibilità del paesaggio, considerando come l'articolazione spaziale sia un carattere risultante dalla classificazione di quanto presente nell'ambito di studio e non anche di quanto da questo è possibile percepire. 
L'evidente transcalarità dell'indicatore è legata alla forte diversità che vi può essere tra le componenti che in un determinato ambito concorrono a definire gli spazi. Si pensi ai diversi pesi paesistici che possono avere nella struttura una siepe di campo, rispetto ad un bosco, ed ancora entrambi rispetto al versante di un rilievo collinare: eppure tutti concorrono a determinare gli spazi. Considerazioni analoghe valgono per le componenti minerali o comunque artificiali, fra cui nel paesaggio di pianura assumono rilievo spaziale i rilevati infrastrutturali, i margini degli insediamenti, le grandi strutture edilizie, i rilevati di discariche di rifiuti urbani e di stoccaggio finale di inerti, le infrastrutture tecnologiche fuori terra.

Per l'articolazione che caratterizza questo indicatore, occorre più che per gli altri procedere alla individuazione delle specifiche componenti da rilevare in relazione all'ambito di studio e, in funzione della loro natura, utilizzare unità di misura idonee, calibrando anche il dettaglio del rilievo in funzione della domanda di conoscenza data dalle finalità ultime dell'indagine, che, nel campo della frammentazione del paesaggio, oscilla dall'area vasta delle macrorelazioni al singolo spazio finale, dove determinate azioni e non altre dovranno essere progettate in termini esecutivi ed attuate.

Rispetto ai parametri individuati nel quadro riportato nella figura 17 , nell'area di studio sono state riscontrate tre tipologie su otto: (5) rilevati stradali e ferroviari, (7) viadotti stradali e ferroviari, (8) margini di insediamenti. Anche in questo caso valgono le considerazoni fatte in merito agli indici numerici per il precedente indicatore.
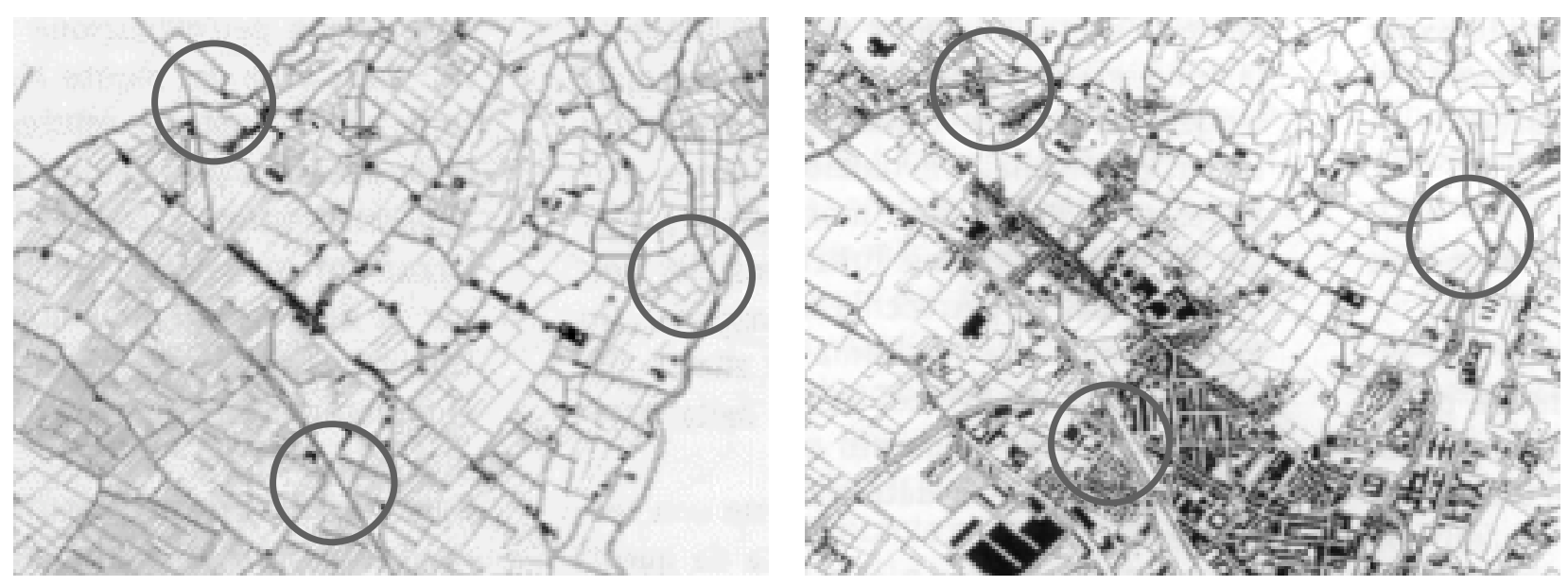

Figura 22 - Confronto storico di una parte dell'area di studio, la piana e le colline di Castello, relativamente ad un periodo di circa cento anni, tra la fine dell'Ottocento e la fine del Novecento. È ben evidente la consistenza del fenomeno dell'evoluzione del paesaggio dalla matrice rurale dominante, alla contaminazione di questa con quella urbana in alcune aree ed alla sostituzione di quella urbana come dominante in altre. I cerchi indicano alcuni punti di riferimento per facilitare il raffronto delle cartografie relative alle due soglie storiche: 1873 - Catasto Lorenese (a sinistra) e 1985 - Catasto contemporaneo (a destra), immagini tratte da: Poli D., La piana fiorentina, Alinea, Firenze, 1999.

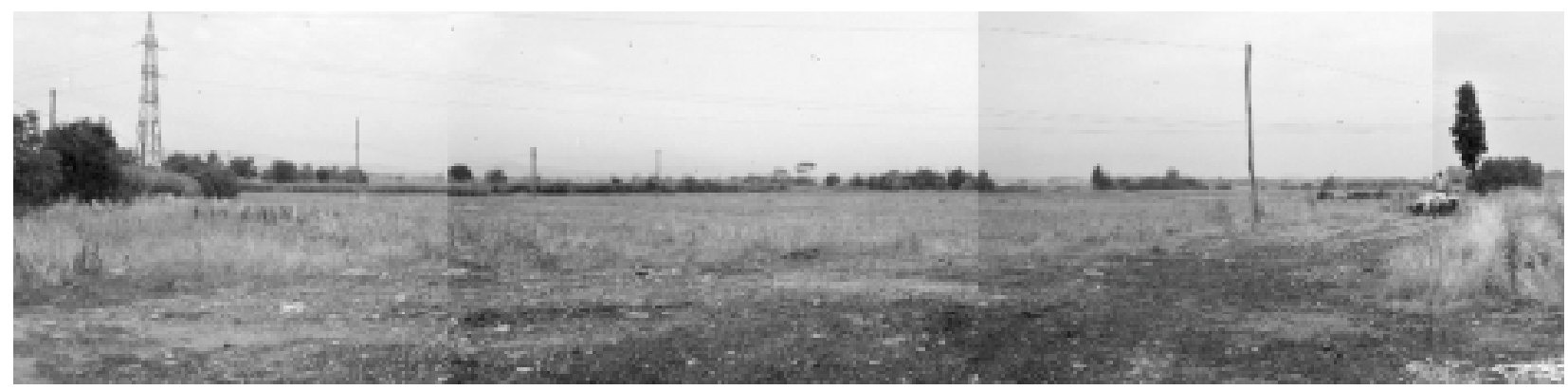

Figura 23 - La desertificazione spaziale del paesaggio della piana di Castello. Alle perdite di identità e riconoscibilità dei caratteri dimensionali e morfologici degi spazi, dovute alla progressiva rimozione delle forme colturali storiche, non ha corrisposto finora una nuova strutturazione del paesaggio, basata su una concezione contemporanea del suo destino (fotografie di Gabriele Paolinelli, 2001). 


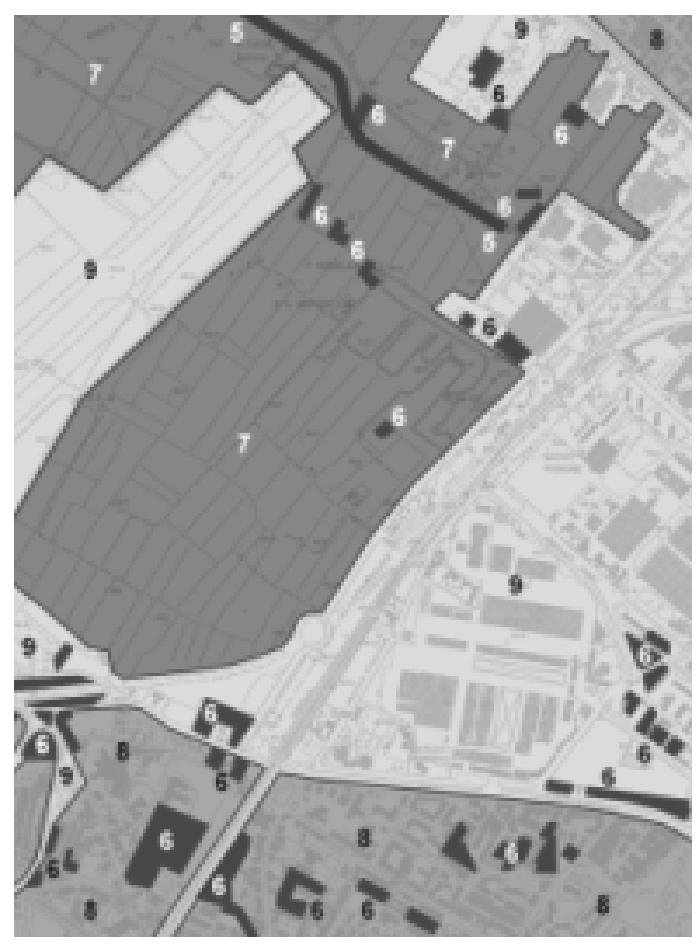

Figura 24 - Analisi degli indicatori di frammentazione paesistica: articolazione funzionale del paesaggio (le campiture in toni di grigio indicano l'estensione dei diversi apparati).

Questo elaborato fa riferimento al criterio di prevalenza della funzione a cui gli spazi vengono ascritti: esso comporta una approssimazione della complessità della struttura reale del paesaggio, strumentale alla sua descrizione cartografica.

Da un punto di vista di bilancio ecologico occorre integrare l'elaborazione cartografica, definendo con il miglior grado di approssimazione possibile la ripartizione percentuale dell'importanza di più apparati su di uno stesso spazio ed eventualmente elaborando singoli strati cartografici tematici per ogni apparato.

Questi due ordini di approssimazione delle funzioni ecosistemiche del paesaggio possono avere applicazioni diverse: ai fini della analisi e diagnosi di frammentazione paesistica si ritiene essenziale il quadro cartografico di sintesi che fa riferimento al criterio di prevalenza, per la priorità, almeno in una prima fase di indagine, della considerazione delle relazioni geografiche fra i diversi apparati.

Gli indici numerici della cartografia fanno riferimento alle tipologie riportate nel quadro sinottico della figura 17 (cartografia ridotta da originale 1:10000 - CTR Regione Toscana).

Avendo coperto con questo gruppo di indicatori primari le configurazioni strutturali del paesaggio più sensibili ai fenomeni di frammentazione, resta da considerarne l'articolazione funzionale, a cui anche queste concorrono in ragione di strutture relazionali, che gli studi di ecologia del paesaggio sono soliti codificare sotto la denominazione di apparati paesistici, essendone sottointeso l'attributo funzionali.

Anche l'indagine dell'articolazione funzionale del paesaggio mostra una decisa transcalarità ed, in ragione di questo motivo, si vedrà nel successivo paragrafo sulla diagnosi di frammentazione paesistica come, per quanto riguarda i paesaggi periurbani, laddove questa assume connotati di forte congestione e conflittualità degli spazi, occorra affiancare l'approfondimento interpretativo delle specifiche funzioni reali o latenti degli spazi aperti all'analisi delle funzioni paesistico-ambientali condotta per tipologie di paesaggio.

Assunto che la classificazione del paesaggio condotta nell'ambito della costruzione interpretativa della carta degli apparati paesistici deriva dalle applicazioni di ecologia del paesaggio e non di ecologia, ovvero tiene conto per definizione degli aspetti naturali e culturali, si ritiene importante precisare lo specifico utilizzo di questa forma di interpretazione nell'ambito del processo conoscitivo della fenomenologia della frammentazione paesistica. Gli apparati paesistici vengono definiti come "insiemi funzionali che legano diversi elementi"103. Più estesamente è possibile dire che gli apparati paesistici sono quelle configurazioni territoriali date dalle parti di paesaggio alle quali sono state riconosciute le stesse funzioni prevalenti. È evidente che, come accade in tutte le forme di lettura del paesaggio, il modello che ne deriva costituisce una rappresentazione approssimata della realtà e che questa è ben più complessa, in quanto difficilmente ogni parte di paesaggio è ascrivibile ad una sola categoria funzionale, costringendo per questo l'analisi ad adottare il criterio della prevalenza. Ma anche il solo fatto, più volte dimostrato, che i modelli di funzionamento del paesaggio che derivano da questo tipo di lettura si trovano regolarmente in conflitto con i modelli di uso e gestione delle risorse ed in particolare di insediamento, costituisce una precisa indicazione di quanto il controllo dei processi territoriali sia ancora ben lontano anche dalla sola capacità di essere conseguente

103 Ingegnoli V., 1993, cit. 
all'articolazione funzionale macrostrutturale del paesaggio, riscontrabile con questo grado di conoscenza ${ }^{104}$.

Nel caso specifico dell'utilizzo nell'ambito del processo conoscitivo finalizzato alla diagnosi di frammentazione paesistica, non è stata ritenuta rilevante la distinzione tra gli apparati paesistici afferenti all'habitat umano e quelli afferenti all'habitat naturale. Divengono invece particolarmente significative la ricostruzione e l'interpretazione topologica delle configurazioni spaziali a cui risultano associate le funzioni paesistiche soggette ai processi di frammentazione.

\begin{tabular}{lccccccc} 
& \multicolumn{7}{c}{ TIPOLOGIA } \\
\cline { 2 - 7 } & 5 & 6 & 7 & 8 & 9 & TOT. \\
\hline SUPERFICIE DEGLI SPAZI (Ha) & 9,5 & 16,5 & 166 & 79 & 214 & 485 \\
PERCENTUALE DEGLI SPAZI (\%)* & 2 & 3,4 & 34,2 & 16,3 & 44,1 & 100 \\
\hline
\end{tabular}

${ }^{*}$ Riferita alla superficie totale del campione di indagine pari a $485 \mathrm{Ha}$

Figura 25 - Rilevamento dell'indicatore articolazione funzionale del paesagoio - Parametri riscontrati e quantificati: (5) apparato idrologico, (6) apparato protettivo, (7) apparato produttivo, (8) apparato abitativo, (9) apparato sussidiario.

Rispetto alla classificazione convenzionale degli apparati paesistici (figura 17 e nota 94) che prevede la distinzione in apparato scheletrico, connettion, stabilizzante, resiliente, escretore, protettizo, produttizo, abitatio, sussidiario, occorre osservare peraltro che gli spazi afferenti ad alcuni di questi rientrano fra i fattori territoriali di frammentazione paesistica, mentre altri coincidono con funzioni che ne compensano gli effetti o che comunque hanno segno opposti rispetto ai primi.

Una considerazione a sé è utile per l'apparato cosiddetto escretore, essendo i corsi d'acqua classificatifra i fattori di frammentazione paesistica ed allo stesso tempo fondamentali sistemi di continuità. Anche nella schedatura identificativa dei fattori di frammentazione paesistica in appendice, è evidenziatoche i corsi d'acqua, pur generando alcuni effetti di frammentazione ${ }^{105}$, hanno la funzione strutturale di veicolazione di una risorsa essenziale per tutte le forme di vita (umana, animale, vegetale) che nel paesaggio esprimono diversamente la propria presenza. Tanto è importante tale funzione che non si ritiene fine a se stesso segnalare l'opportunità di una revisione della denominazione di questo apparato, che nella forma di escretore fa pensare più propriamente ad un sistema finalizzato ad espellere il fluido, piuttosto che alla complessa gamma di interazioni ecologiche ma anche sociali, economiche e percettive, che caratterizzano o potrebbero caratterizzare normalmente i corsi d'acqua. A tal fine in questo studio, senza ritenere questa denominazione l'unica adottabile, si preferisce

104 Di questo tipo di riscontro gli studi applicativi che utilizzano l'ecologia del paesaggio ed in particolare l'analisi degli apparati forniscono ampie dimostrazioni. Chi scrive può riferirsi specificamente allo studio condotto nel 1995 per conto della Provincia di Pistoia sul paesaggio periurbano della Valdinievole in Toscana. Anche in quel caso la classificazione del paesaggio secondo criteri di tipo funzionale ha prodotto una articolazione descrittiva di un complesso di alterazioni profonde responsabili dei forti squilibri paesistico-ambientali che caratterizzano il territorio compreso fra i rilievi collinari tra Pescia e Monsummano Terme a Nord e le zone umide del Padule di Fucecchio a Sud. Si veda Campioni G., Ferrara G., Tutela della naturalità diffusa, pianificazione degli spazi aperti e crescita metropolitana, Il Verde E ditoriale, Milano, 1997.

105 Relativamente al reticolo idrografico di superficie, occorre sottolineare come effetti di frammentazione paesistica non derivino solo dalla presenza di canali artificiali con argini in rilevato. Anche i corsi d'acqua naturali e seminaturali possono costituire barriere per le popolazioni di talune specie animali e conseguentemente per la diffusione delle popolazioni delle specie vegetali ad esse legate per la propagazione riproduttiva. Sull'argomento si vedano ad esempio le conclusioni dello studio dell'influenza del corso della Dora Baltea in Val D'Aosta sulle popolazioni di ungulati, prodotto nell'ambito del Programma di monitoraggio delle reti ecologiche promosso dall'Agenzia nazionale per la protezione dell'ambiente (ANPA www.ecoreti.it). 
parlare di apparato idrologico, in quanto rappresentativo del funzionamento dei sistemi delle acque di superficie.

Rispetto ai parametri individuati in via teorica nel quadro riportato nella figura 17, nell'area di studio sono state riscontrate cinque tipologie su nove, la cui misurazione produce i dati riportati nella tabella della figura 25 .

Il territorio analizzato presenta condizioni tipiche dei paesaggi periurbani di pianura. Oltre l'assenza degli apparati da cui dipendono direttamente le condizioni di equilibrio ecologico del paesaggio, risulta evidente il deficit di spazi con funzioni di protezione ambientale (apparato protettivo) e l'incidenza minoritaria dell'apparato produttivo $(16,3 \%)$, a fronte degli apparati normalmente più diffusi nelle aree periurbane, abitativo e sussidiario, che complessivamente incidono per il 78,3\%. 


\section{3 - La diagnosi strutturale di frammentazione paesistica}

\section{1 - Elementi di impostazione metodologica della diagnosi}

La spiccata transcalarità che caratterizza notoriamente la struttura del paesaggio, comporta l'utilizzo di tecniche composite nell'ambito delle applicazioni conoscitive e progettuali, in cui l'impiego di diversi strumenti è finalizzato ad ottenere la maggiore sensibilità possibile: questo vale anche per le problematiche di frammentazione paesistica e ambientale ${ }^{106}$.

Con specifico riferimento ai paesaggi periurbani, se risulta assolutamente necessaria l'ottica di scala vasta ai fini delle determinazione dell'articolazione paesistica del contesto, all'opposto, la complessità di componenti e di relazioni che caratterizza il mosaico degli spazi aperti ne richiede il dettagliato censimento delle funzioni paesistico-ambientali, non legate alle destinazioni d'uso da relazioni univoche. Quest'ultimo approccio è adatto a supportare i processi di conoscenza e progettazione del paesaggio urbano e periurbano, ma richiede una scala di indagine e rappresentazione grafica idonea alla individuazione dei singoli spazi come componenti "elementari": non minore di 1:10000, considerando che le scale più idonee sono 1:5000, nelle realtà meno articolate, e 1:2000 in quelle in cui il grado di complessità raggiunge livelli in ragione dei quali la scala precedente non permette interpretazioni e rappresentazioni chiare ${ }^{107}$.

106 Si può osservare, ad esempio, che "un (...) ordine di complessità è collegato al problema del mantenimento, e dell'eventuale ripristino, delle condizioni di continuità ambientale che costituiscono l'aspetto pregiudiziale sul quale incardinare poi ogni politica di deframmentazione degii habitats. È intuitivo che per conseguire tale risultato è necessario operare con lo strumento del piano, ma ad ogni livello di espressione di esso. Sarebbe infatti del tutto inutile recepire i connotati della continuità ambientale nei piani di coordinamento se poi gli strumenti urbanistici generali ed esecutivi non affrontano il problema. Questa istanza ripropone nuovi scorci sul tema del rapporto tra i livelli di pianificazione. Infatti gli interventi di deframmentazione degli habitats naturali possono essere operativamente e decisivamente gestiti unicamente al livello del piano comunale e sub-comunale (contiguità tra gli spazi verdi territoriali, soluzioni alternative alla realizzazione di opere di delimitazione della proprietà troppo estese, rinaturazione di porzioni di suolo, by-pass infrastrutturali, orientamento delle politiche agricole locali). Ma a questo livello, che le controlla, le configurazioni strategiche della ecocontinuità non sono visibili e rilevabili, mentre lo sono al livello di indirizzi nazionali e di coordinamento regionale che però possiedono una irrilevante capacità di incidenza sulla gestione minuta degii interventi. Indubbiamente una delle risposte che le ricerche in atto dovranno fornire è anche quella connessa proprio con questi aspetti di ordine relazionale normativo". Romano B., Seminario DUPT - UNIFI, Firenze, 5 aprile 2000.

107 Guido Ferrara, Gilberto Oneto, Guido Ronzani, sono fra gli autori che nel nostro paese hanno elaborato i primi contributi di sviluppo teorico ed applicativo di questo tipo di indagine. Si riportano alcuni criteri metodologici sviluppati dalla ricerca MURST "Piani del verde", conclusa nel 2000 presso il DUPT di Firenze, tratti da Guccione B., Paolinelli G. (a cura), Piani del zerde E Piani del paesaggio - Elementi di eroluzione metodologica nell'ambito del dibattito sui nuozi piani comunali, Alinea, Firenze, 2001. "Il riconoscimento delle condizioni di stato degli spazi aperti, oltre le categorie canoniche delle destinazioni d'uso, è un passaggio essenziale di lettura paesistica, particolarmente importante relativamente alle aree soggette ad intensa e diffusa urbanizzazione ed infrastrutturazione, caratterizzate dai conseguenti stati di congestione spaziale e frammentazione del paesaggio. La V alutazione diagnostica dei ruoli paesistici ed ambientali, riferita alla articolazione elementare degli spazi aperti, costituisce infatti una interpretazione del paesagoio che, per grado di dettaglio e tipo di contenuti, può supportare efficacemente sia la verifica delle strategie paesistiche di area vasta, che la definizione, in sede di piano locale, delle specifiche progettuali di indirizzo per la progettazione dei singoli interventi di modificazione e gestione del paesaggio. Tre presupposti complementari motivano lo sviluppo di questo tipo di metodologia e ne determinano l'interesse applicativo nell'ambito della pianificazione territoriale di livello comunale:

- il paesaggio è articolato in spazi, dalle cui caratteristiche, relazioni e condizioni di utilizzo, dipende l'insieme della sua struttura; 
Una specifica questione di indirizzo degli studi di ricerca teorica ed applicata della frammentazione del paesaggio riguarda i tipi e livelli di conoscenza disponibili o raggiungibili ed i relativi tempi di acquisizione, relativamente alle diverse finalità di diagnosi. Sotto questo aspetto è necessario innanzitutto tenere distinti i concetti della connessione biotica, esitente/ottenibile in un dato paesaggio, e della connettività floro/faunistica, degli ecosistemi esistenti e dei neoecosistemi previsti ${ }^{108}$.

Mentre sul primo concetto, analisi e progetto procedono attraverso strumenti ed informazioni che dispongono già di importanti fondamenti e sviluppi, avvalendosi dell'osservazione morfologica del paesaggio e del rilevamento di specifici caratteri della sua struttura, di ordine biotico ed abiotico, naturale e culturale, per quello che riguarda il secondo concetto della connettività ecologica, il campo delle variabili conoscitive aumenta in maniera rilevante, in ragione dei diversi tipi di ecosistema e delle specificità delle singole specie naturali o, quanto meno, dei loro raggruppamenti.

Le indagini richiedono inoltre rilevamenti statistici, per la deduzione empirica dei comportamenti, e monitoraggi, per la verifica dei risultati in relazione alla scala temporale, nonché per la gestione delle dinamiche a cui il paesaggio nel complesso è soggetto, alle quali le comunità viventi, animali per prime, possono reagire con decise modificazioni di comportamento e assetto demografico.

Da questo punto di vista, relativamente alle conoscenze sviluppate e sviluppabili intorno ai corridoi come sistemi lineari di spazi aperti, l'obiettivo della riqualificazione del paesaggio periurbano di pianura si articola pertanto innanzitutto per diversi livelli di difficoltà delle indagini tematiche. Mentre alla pianificazione e progettazione di misure idonee al soddisfacimento delle esigenze umane di riordino e miglioramento ambientale e di connotazione spaziale e valorizzazione dei potenziali ricreativi, corrispondono requisiti conoscitivi ampiamente raggiungibili con le disponibilità reali attuali, alla definizione delle politiche e delle misure di intervento per la salvaguardia della biodiversità, la conservazione e lo sviluppo di habitat animali e delle loro connessioni, corrispondono requisti conoscitivi il cui soddisfacimento per il territorio nazionale è ben lontano dall'essere sufficiente, per copertura degli argomenti e delle specie.

Questo comporta la necessità di procedere nella predisposizione di dati scientifici di ecologia animale e vegetale e nella loro sistematizzazione per il migliore uso applicato alla pianificazione, ma anche la parallela necessità di procedere alla attenta analisi dei caratteri paesistici da punti di vista ecologici non specie-specifici, come da quelli dell'ecologia umana e della protezione ambientale in genere, dei

- il grado di definizione delle conoscenze e delle scelte progettuali di pianificazione deve arrivare alla scala degi spazi per rispondere alla reale articolazione strutturale del paesaggio;

- l'attuabilità delle strategie di piano e delle misure di intervento richiede procedure di analisi e diagnosi dotate di precisa referenziazione spaziale; le elaborazioni analogiche su base cartografica risultano le più idonee; le elaborazioni numeriche possono risultare preziose se mirate alla descrizione approfondita delle prime ed elaborate con le tecnologie appropriate dei sistemi informativi geografici (GIS).

“E necessario far riferimento alle grandi classi di gerarchizzazione di ruolo (ecologico, percettivo, funzionale) in cui tutti gii spazi aperti possono trovare corretta collocazione, per capire quali sono le finalità che ciascuno di essi gioca attualmente, rispetto a quelle che potrebbe giocare in un processo di riequilibrio. Si badi che quando si parla di spazi aperti non ci si riferisce solo al "verde", ma (...) ci si riferisce complessivamente al sistema dei "vuoti", cioè al negativo della città costruita e "mineralizzata", ovvero agli spazi entro cui può ancora aver luogo la riproduzione della vita animale e vegetale e in cui in una parola è presente la biosfera. Se ci si riferisce agli spazi aperti, si noterà subito che il loro ruolo non sempre è obbligatoriamente una funzione urbana in senso stretto, ovvero un modo d'uso direttamente collegato alle attività e all'impiego degi spazi, ma esprimono sempre preoccupazioni ed esigenze d'ordine ecologico riguardanti la riproduzione o meno di uno stato di equilibrio. (...) La ricomposizione unitaria delle conoscenze è quanto occorre per operare le scelte, e per consentire un confronto non perdente con le innovazioni in corso nel tessuto edificato della città. (...) Occorre allora ritrovare una logica interna, un quadro di riferimento globale per le aree inedificate della città, per sapere in anticipo gi obiettivi da perseguire ed escludere le azioni contraddittorie ed incompatibili. Si tratta infine di identificare in modo non occasionale compiti, ruoli e contenuti delle diverse categorie di spazi aperti che configurano il paesaggio". (Ferrara G., La considerazione degli spazi aperti nella pianificazione urbanistica comunale, in: Fiorani F., GiovannelliG, Paolinelli G., (a cura), Verso nuozi modelli di pianificazione integrata, Alinea, Firenze, 1994)".

108 Romano B., Continuità e reticolarità ambientali, nuozi protagonisti del piano territoriale, intervento al Convegno Internazionale Reti ecologiche, azioni locali di gestione territoriale per la conserzazione dell'ambiente, Centro Studi Valerio Giacomi, Gargnano, 12 e 13 ottobre 2000. 
caratteri storico-culturali e fisionomico-percettivi e dei conseguenti potenziali sociali ed economici che il paesaggio può sviluppare secondo adeguate politiche di uso e gestione delle risorse.

L'applicazione del principio di precauzione ambientale è un tema di ricerca scientifica e di pratica gestionale ben preciso, da cogliere relativamente ad un necessario periodo di formazione e implementazione di conoscenze ecologiche idonee alla pianificazione, che non è ragionevole pensare possa occupare solo qualche anno, ma da considerare allo stesso tempo come normale filosofia di governo territoriale.

In tal senso risulta non casuale l'atteggiamento del Consiglio d'E uropa, che ha ricondotto le politiche di conservazione della natura e del paesaggio riguardanti il tema della diversità sotto una strategia unitaria già citata in questo lavoro ${ }^{109}$.

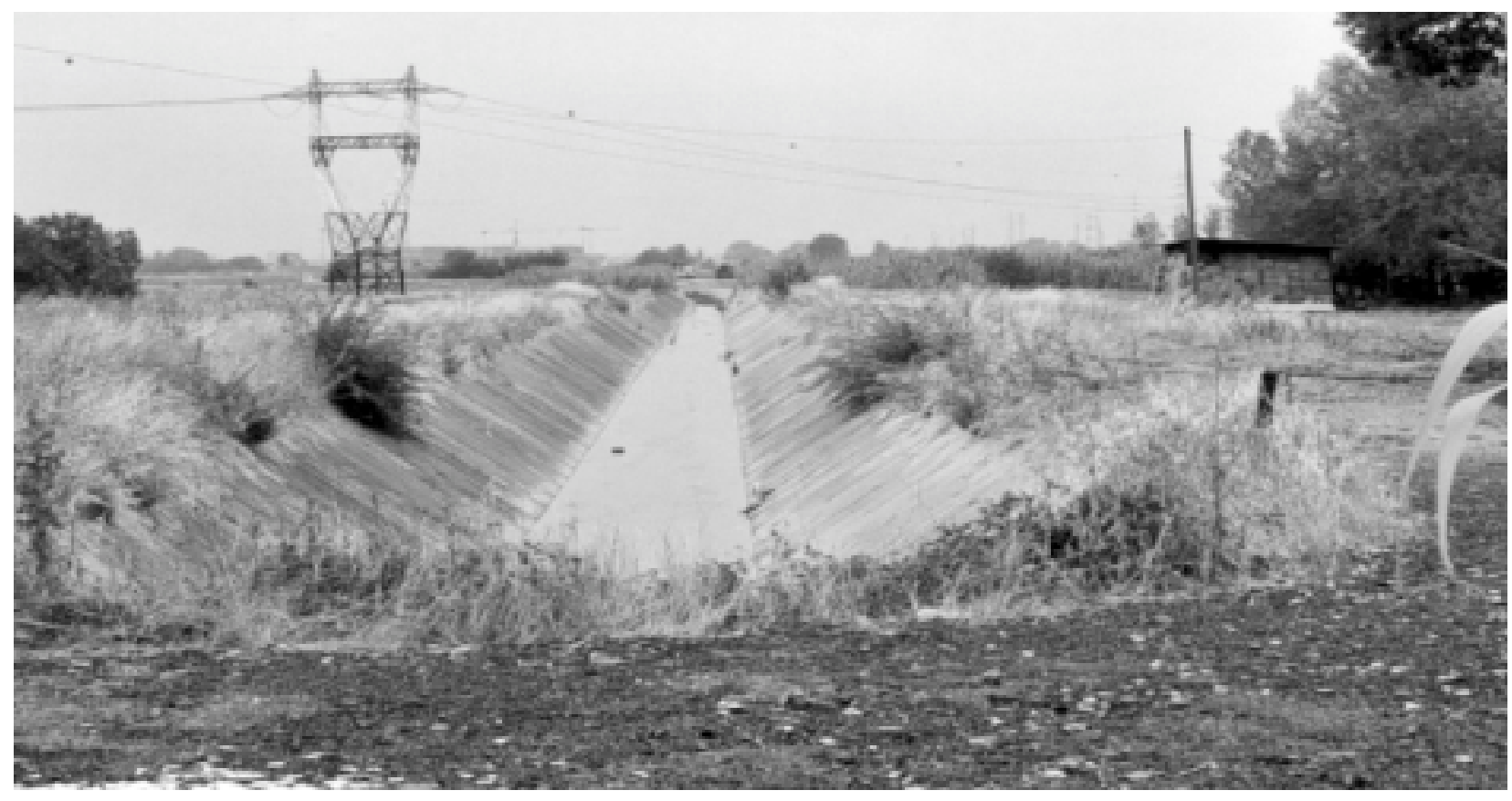

Figura 26 - Un canale collettore della piana di Castello con l'alveo murato in calcestruzzo (fotografia di Gabriele Paolinelli, 2001).

Le relazioni sviluppabili tra conservazione della diversità biologica, relativa alle comunità animali e vegetali e patrimonio sostanziale per l'esistenza umana, e conservazione della diversità del mosaico dei soprassuoli, configurazione strutturale del paesaggio, espressione delle dinamiche di equilibrio tra natura e cultura, sono evidentemente un importante terreno su cui è necessario fondare politiche territoriali non settoriali, finalizzate anche a sopperire a contingenti carenze di conoscenza ad elevata specializzazione. "In questo panorama dotazionale delle informazioni, si tenta pertanto di condurre avanti il discorso metodologico che mira a raggiungere migliori assetti di pianificazione, attraverso una serie di "buchi" cognitivi che divengono sempre più condizionanti il risultato man mano che si procede nel dettaglio operativo" ${ }^{\prime 110}$.

L'impostazione della diagnosi di frammentazione paesistica proposta di seguito fa riferimento ad un utilizzo combinato di tecniche analogiche e tecniche numeriche; a queste ultime è demandato il compito importante delle misurazioni di qualità paesistica attraverso gli indicatori primari di

${ }^{109}$ La Strategia pan-europea per la dizersità biologica e paesistica è stata promulgata nel 1996. Il quarto meeting in cui è stato varato il secondo piano quinquennale di azione 2001-2005, si è tenuto a Strasburgo e Ginevra il 7 gennaio 2000 (STRA$\mathrm{CO}(00) 4)$.

110 Romano B. (f), 2000, cit. 
frammentazione (paragrafo 2.3). L'esigenza dell'utilizzo delle tecniche analogiche congiuntamente alle tecniche numeriche fa capo essenzialmente a motivi di parzialità degli indici numerici per la trattazione di taluni tipi di informazioni finalizzate alla conoscenza e/o all'indirizzo progettuale. Si consideri ad esempio, nello specifico caso in questione, la perdita di permanenza storica: a prescindere dal tipo e intensità di storicizzazione del paesaggio documentabile con le analisi, quanto interessa finalisticamente la diagnosi è l'identificazione delle relazioni fra gli elementi di permanenza ed i fattori di frammentazione, al fine di poter orientare il progetto secondo misure di rimozione o controllo delle cause. In tal senso, non è sufficiente conoscere i chilometri di reticolo viario storico relitto o il numero di case coloniche interposte al tessuto urbano periferico, ma occorre considerare anche le relazioni spaziali che investono i diversi casi per identificarne gli effetti, operando quindi anche in termini di osservazione della realtà attraverso la sua rappresentazione con modelli iconografici idonei.

La frammentazione paesistica può costituire un efficace indicatore di sintesi della qualità del paesaggio. Indicatore paesistico di sintesi in quanto non settorialmente afferente ad uno specifico tipo di carattere (caratteri ecologici e ambientali, caratteri storici e culturali, caratteri semiologici e visuali), bensì costruito secondo finalità di bilancio. Occorre però riflettere sul concetto di bilancio e considerarne le applicazioni sviluppate su problematiche analoghe. Infatti, senza opportuni criteri di controllo e correzione, la valenza di indicatore di sintesi resta limitata a fenomeni dello stesso "segno", non essendo idoneo ad apprezzare la diversa natura dei fenomeni soggetti ad effetti di compensazione, dovuti a valori di segno opposto e non correttamente confrontabili con quelli derivanti da fenomeni di segno omogeneo. Possono in ogni caso generarsi effetti di compensazione dei valori di sintesi: ad esempio due ambiti possono risultare con grado di frammentazione paesistica molto simile, pur essendo profondamente diversa l'incidenza specifica dei singoli fattori che vi concorrono. È pertanto opportuno mantenere disaggregate le informazioni diagnostiche relative ai cinque indicatori di frammentazione paesistica individuati, al fine di poter comunque collaudare ed identificare le aggregazioni sintetiche (figura 30).

Le tecniche di valutazione numerica strutturate secondo modelli matematici appositamente progettati o adattati per rispondere ai problemi conoscitivi posti, utilizzano generalmente grandezze di calibrazione dell'importanza dei diversi indicatori considerati, spesso denominati pesi, proprio a rappresentare il loro significato nei modelli di calcolo ${ }^{111}$.

L'utilizzo di indici numerici permette peraltro, oltre l'elaborazione di dati diagnostici funzionali al progetto, l'elaborazione di dati di monitoraggio per il controllo degli effetti paesistici e la gestione processuale delle politiche di conservazione e sviluppo del paesaggio. Se si ritiene fondamentale la misurazione delle specifiche qualità paesistiche relative ai diversi indicatori di frammentazione attraverso parametri numerici, al fine di trarre le relative conclusioni di diagnosi tematica in base a dati quantitativi in grado di completare l'identificazione analogica delle qualità indagate, allo stesso modo non si ritiene essenziale in riferimento alle specifiche finalità di questo tipo di diagnosi l'utilizzo di tecniche numeriche di ponderazione e sintesi, finalizzate a far confluire i risultati delle misurazioni tematiche in valori relativi ad indicatori di maggior complessità, come nella fattispecie potrebbe accadere per un ipotetico indice di frammentazione paesistica.

111 Un'ampia ricognizione dei modelli di valutazione territoriale su base numerica si trova in Girard L.F., Nijkamp P., Le zalutazioni per lo sziluppo sostenibile della città e del territorio, Franco Angeli, Milano, 1997. Una trattazione mirata delle possibilità di applicazione dei GIS si trova in Pozzana G., Campari I., Franchini D., Valutazione di impatto ambientale $e$ Geographic Information Systems, Franco Angeli, Milano, 1993 ed in Poletti A., Metodi e strumenti per un nuozo gozerno della aittà e del territorio, Maggioli, Rimini, 2001. Per l'ampiezza della trattazione di questioni attinenti a questo studio, risulta significativo il modello definito in Rizzo G.G. (a cura), Tuscia Romana - paesagoio e territorio - metodi e modelli di zalutazione, Gangemi, Roma, 1990. Il quadro metodologico sviluppato in quest'ultimo lavoro, per affinità di soggetti e criteri di studio, risulta infatti pienamente congruente con le esigenze di elaborazione della diagnosi di frammentazione paesistica, che può esser riferita alla categoria generale denominata alterazione, utilizzata nella ricerca e qui riproposta. 


\begin{tabular}{|c|c|c|c|c|c|}
\hline I & $\begin{array}{l}\text { Articolazione metastrutturale } \\
\text { del paesaggio } \\
\text { in ambiti spaziali complessi } \\
\text { (unità di paesag gio) } \\
1 \text { cartografia generale }\end{array}$ & $\begin{array}{l}\text { Riconoscimento dei caratteri e delle } \\
\text { relazioni paesistiche fondamentali } \\
\text { a livello di scala vasta, finalizzato } \\
\text { a considerare le specificità } \\
\text { del soggetto di studio nel contesto } \\
\text { di cui fa parte. }\end{array}$ & $\begin{array}{c}\mathrm{Da} \\
1: 100000 \\
\text { a 1:25000 }\end{array}$ & \multirow{2}{*}{ 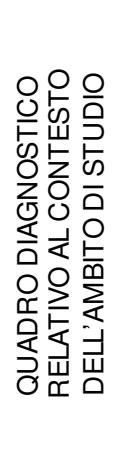 } & \\
\hline II & $\begin{array}{l}\text { Articolazione strutturale } \\
\text { del paesaggio } \\
\text { in componenti fisionomiche di base } \\
\text { (mosaico paesistico) } \\
1 \text { cartografia generale }\end{array}$ & $\begin{array}{l}\text { Approfondimento del quadro } \\
\text { contestuale delle relazioni } \\
\text { paesistiche e primo livello } \\
\text { di approssimazione } \\
\text { delle specifiche condizioni } \\
\text { di frammentazione paesistica } \\
\text { del soggetto di studio. }\end{array}$ & 1:10000 & & \\
\hline $\begin{array}{c}\text { III } \\
\mathrm{a}\end{array}$ & $\begin{array}{l}\text { Grado di frammentazione paesistica } \\
1 \text { cartografia generale }\end{array}$ & $\begin{array}{l}\text { Sintesi delle condizioni } \\
\text { di frammentazione paesistica } \\
\text { derivanti dalle analisi tematiche } \\
\text { degli indicatori. }\end{array}$ & 1:10000 & & 焉 \\
\hline $\begin{array}{l}\text { III } \\
b\end{array}$ & $\begin{array}{l}\text { Incidenza dei fattori territoriali } \\
\text { sug li stati } \\
\text { di frammentazione paesistica } \\
5 \text { cartog rafie tematiche } \\
1 \text { cartog rafia generale }\end{array}$ & $\begin{array}{l}\text { Applicazione del modello } \\
\text { di incidenza dei fattori territoriali } \\
\text { sugli stati di frammentazione } \\
\text { paesistica alla articolazione spaziale } \\
\text { rilevata dalle analisi tematiche } \\
\text { degli indicatori. }\end{array}$ & 1:10000 & & 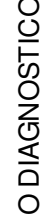 \\
\hline IV & $\begin{array}{l}\text { Censimento delle funzioni } \\
\text { paesistico-ambientali } \\
\text { degli spazi aperti } \\
1 \text { cartografia generale }\end{array}$ & $\begin{array}{l}\text { Interpretazione riferita ad unità } \\
\text { paesistiche elementari (spazi aperti), } \\
\text { funzionale all'approfondimento } \\
\text { dei precedenti stadi conoscitivi } \\
\text { e preliminare alla definizione } \\
\text { degli indirizzi diag nostici } \\
\text { per il prog etto di riqualificazione } \\
\text { del paesaggio periurbano. }\end{array}$ & $\begin{array}{l}\text { Da } 1: 5000 \\
\text { a } 1: 2000\end{array}$ & & $\frac{\mathrm{Q}}{2}$ \\
\hline
\end{tabular}

Figura 27 - Schema generale del processo di diagnosi della frammentazione paesistica.

Nelle applicazioni paesistiche, l'utilizzo di modelli matematici complessi per valutazioni multicriteri può incontrare difficoltà non trascurabili nell'oggettivazione delle attribuzioni dei criteri e coefficienti di correzione del peso dei fattori.

La sostanziale eterogeneità degli indicatori ritenuti essenziali (indicatori primari), pone in questo caso ulteriori ostacoli alle procedure di calcolo di eventuali valori derivati dagli indicatori stessi, per il trattamento dei quali occorrono strumenti sensibili alle differenze intrinseche oppure è necessario eliminare le differenze stesse, ottenendo si una omogeneità delle informazioni, ma incorrendo anche nel rischio di una decisa semplificazione e riduzione dello specifico potenziale informativo di ogni tema. Ad esempio, le differenze esistenti tra gli elementi conoscitivi che identificano l'articolazione spaziale del paesaggio e quelli relativi alla mineralizzazione, denotano gradi di complessità assolutamente diversi per questi due indicatori.

Mentre la descrizione numerica della mineralizzazione, in valore assoluto e relativo, fornisce una identificazione nitida di un determinato paesaggio, ancora più efficace quando sia praticabile il confronto con altre realtà note, la misurazione dell'articolazione spaziale, ancorchè possibile, comporta una disaggregazione sostanziale dell'indicatore in elementi conoscitivi di natura diversa che, in quanto non semplicemente sommabili, richiederebbero tecniche di ponderazione numerica, già nell'ambito della valutazione interna relativa ad un solo indicatore. 
L'applicazione di sistemi di calcolo alla valutazione diagnostica degli stati rilevati dai singoli indicatori e dal loro complesso dovrebbe pertanto procedere alla definizione, per ognuno di questi, di uno o più indici adatti alla reciproca interazione trasversale fra più indicatori, oltre che al soddisfacimento delle domande di conoscenza relative agli specifici caratteri indagati.

In linea teorica, qualunque carattere paesistico può essere ricondotto ad una serie di parametri numerici. Non si tratta pertanto di discutere la possibilità tecnica di condurre tale operazione, quanto di finalizzarne sempre prelminarmente l'impiego.

Per le diagnosi tematiche di frammentazione paesistica, si ritiene importante la definizione di basi analitiche attraverso l'utilizzo di indici numerici e delle relative misurazioni (rilievo e interpretazione dei singoli indicatori). È possibile invece condurre la diagnosi di sintesi degli stati e delle tendenze di frammentazione paesistica sia condotta per via analogica, con tecniche di sovrapposizione cartografica (interpretazione unitaria degli indicatori).

L'utilizzo di coefficienti numerici, nel caso della diagnosi di sintesi, non coincide pertanto con misurazioni di qualità del paesaggio (espressioni numeriche reali, assolute e relative, di quantità corrispondenti a qualità), ma con la sua classificazione secondo il complesso delle risultanze riscontrate attraverso gli indicatori (espressioni numeriche astratte di appartenenza a classi di qualità). Data la dipendenza della diagnosi di frammentazione paesistica da un'ottica transcalare, occorre osservare quali siano i ruoli peculiari delle intepretazioni diagnostiche di scala vasta, riferite ad articolazioni del paesaggio in entità complesse, come di quelle di dettaglio, riferite ad articolazioni in entità elementari.

La figura 27 riporta lo schema generale di diagnosi proposto, nell'ambito del quale i diversi livelli di interpretazione fanno riferimento a specifici dati conoscitivi ed in particolare il terzo livello, nucleo fondamentale della diagnosi, si avvale dei criteri proposti nel modello di incidenza dei fattori territorialisugi stati di alterazione identificativi della frammentazione paesistica, descritto nel quadro di sintesi riportatonella figura 29 ed esplicitato nelle pagine ad essa successive e nel paragrafo 3.2.

Il primo livello di diagnosi della frammentazione paesistica fa riferimento alla articolazione in ambiti spaziali proposta nel paragrafo 1.1, come prima descrizione analitica del paesaggio (figura 5). A questo livello possono essere identificate le condizioni macroscopiche di frammentazione e le relazioni territoriali tra le configurazioni paesistiche responsabili e quelle sensibili (paragrafo $3.3-1^{\circ}$ livello di diagnosi).

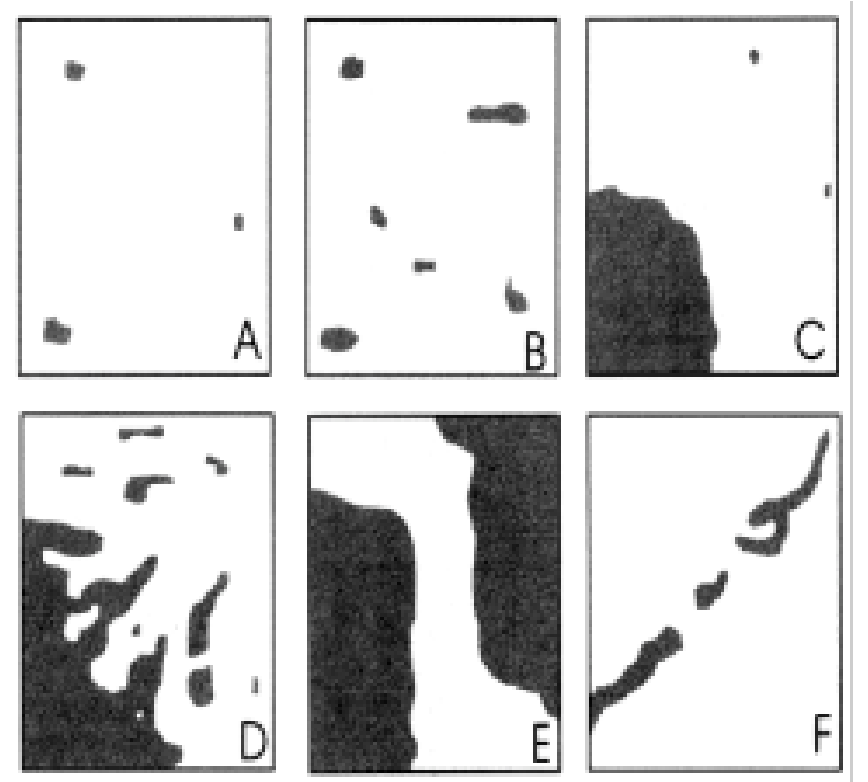

Figura 28 - Descrizione tipologica della frammentazione con riferimento alla articolazione macroscopica del mosaico paesistico dei soprassuoli (adattato da Bisogni L.G., Gariboldi A., Malcevschi S., Reti ecologiche ed interzenti di migioramento ambientak, Il Verde Editoriale, Milano, 1996): (A) piccole unità ecosistemiche isolate $\mathrm{e}$ distanti in una matrice paesistica a forte antropizzazione; (B) complesso di piccole unità isolate ma relativamente vicine, in una matrice paesistica a forte antropizzazione; (C) piccole unità isolate in una matrice paesistica a forte antropizzazione, vicine ad un ambito vasto a maggior naturalità; (D) piccole unità isolate in una matrice paesistica a forte antropizzazione, vicine ad unità connesse ad un ambito vasto a maggior naturalità; $(E)$ ambiti ad alto grado di naturalità separati da fasce poco permeabili agli scambi ecologici; (F) unità di dimensioni variabili a sviluppo lineare dislocate a loro volta secondo una sequenza lineare, in una matrice paesistica a forte antropizzazione. 
Un secondo livello di interpretazione strutturale degli stati di frammentazione deriva dal riferimento alla morfologia del mosaico paesistico ed alle caratterizzazioni che di questo forniscono gii indicatori di mineralizzazione e di equipaggiamento vegetale del paesaggio (paragrafo $3.3-2^{\circ}$ livello di diagnosi). Per questo tipo di elaborazione sono supporti essenziali le cartografie topografiche di base e le fotografie aeree zenitali.

Prima di passare alla descrizione del terzo livello di diagnosi, che riveste evidente centralità in questo lavoro, si ritengono utili alcune osservazioni inerenti il quarto livello, relativo al censimento delle funzioni paesistico-ambientali degli spazi aperti.

Riferendosi a quanto premesso all'inizio del paragrafo, occorre utilizzare una lista di controllo ${ }^{112}$ per la loro classificazione, considerando alcuni aspetti di interesse metodologico:

- “(...) la lista vale sia per le elaborazioni conoscitive di diagnosi, che per quelle progettuali di pianificazione, con le variazioni derivanti dalle diversità fra le condizioni di stato riscontrate e le previsioni di progetto; in tal modo, essa costituisce un elemento di stretta continuità tra il soggetto di studio e le misure di intervento ad esso indirizzate;

- la casistica riportata non è esaustiva e la lista è pertanto aperta alle integrazioni che i singoli casi di studio possono richiedere, sia come variazione della composizione delle classi, che come loro incremento o riduzione;

- le classi di censimento e gerarchizzazione degli spazi aperti si distinguono innanzi tutto in classi di destinazione d'uso e classi di ruolo paesistico-ambientale;

- le classi di ruolo paesistico-ambientale sono ordinate, per quanto possibile, relativamente alla crescente intensità degli interventi per la conservazione e/o il conferimento di ruolo agli spazi aperti: dalla tutela, alle forme di conservazione attiva, a quelle di recupero, agli interventi di integrazione e trasformazione del paesaggio;

- ad una sottoclasse di destinazione d'uso possono corrispondere normalmente, in un determinato spazio, una o più sottoclassi di ruolo paesistico-ambientale;

- la conoscenza dell'equipaggiamento vegetale del paesaggio, sia in termini di indici quantitativi, che di tipi, costituisce un fondamentale supporto nella attribuzione delle classi di ruolo ed in particolare nel riconoscimento del reale grado di efficienza degli spazi aperti rispetto alle funzioni latenti, individuabili in termini di potenziali;

- la valutazione del rapporto fra uso e ruolo/i paesistico-ambientali degli spazi aperti costituisce il cardine interpretativo e decisionale su cui si incentra la metodologia;

- carenza e/o frammentazione degli spazi afferenti alle diverse classi di ruolo paesistico-ambientale costituiscono indicazioni di squilibri e patologie, da interpretare attraverso un attento e documentato riferimento alle condizioni reali del contesto, per le quali occorre che le misure di intervento individuino le opportune integrazioni e compensazioni, sulla base dei risultati diagnostici;

- gli spazi aperti in condizioni di degrado paesistico-ambientale costituiscono una tipologia particolare per la quale l'analisi dei caratteri specifici e delle relazioni contestuali è strettamente mirata alla loro ridestinazione d'uso e di ruolo" ${ }^{113}$.

L'applicazione diagnostica elaborata (paragrafo 3.3) omette il quarto livello per la disponibilità di esemplificazioni in altri studi già citati: è opportuno in generale che la sua presenza venga strettamente considerata in funzione della scala e delle finalità a cui la diagnosi si riferisce. Si tratta di

$112 \mathrm{La}$ metodologia in questione risulta strumentale rispetto agi obiettivi specifici di questa ricerca. Si rimanda ad un esempio di lista di controllo per la classificazione degli spazi aperti, omettendone l'applicazione esemplificativa alla realtà del campione di studio, elaborata per gii altri livelli diagnostici. Si veda a tal fine Guccione B., Kroegmeier L., Martelli S., Paolinelli G., Valentini A., Venturi P., L'interpretazione diagnostica e progettuale del mosaico degli spazi aperti nel piano generale comunale, in Guccione B., Paolinelli G. (a cura), Piani del Verde E Piani del Paesagoio - Elementi di ewoluzione metodologica nell'ambito del dibattito sui nuori piani comunali, Alinea, Firenze, 2001.

${ }^{113}$ Guccione B., Kroegmeier L., Martelli S., Paolinelli G., Valentini A., Venturi P., 2001, cit. 
approfondimenti conoscitivi riguardanti le connessioni diagnostiche e progettuali tra le indicazioni di uso e gestione delle risorse (strategie di governo di competenza della pianificazione) e quelle successive di modificazione degli spazi (misure di intervento di competenza della progettazione). In particolare essi non sono significativi in tutti i casi in cui il processo conoscitivo non sia finalizzato alla individuazione dei caratteri paesistici tipicamente necessari alla pianificazione locale, generale ed attuativa, ed alla progettazione degli interventi per la sistemazione degli spazi aperti.

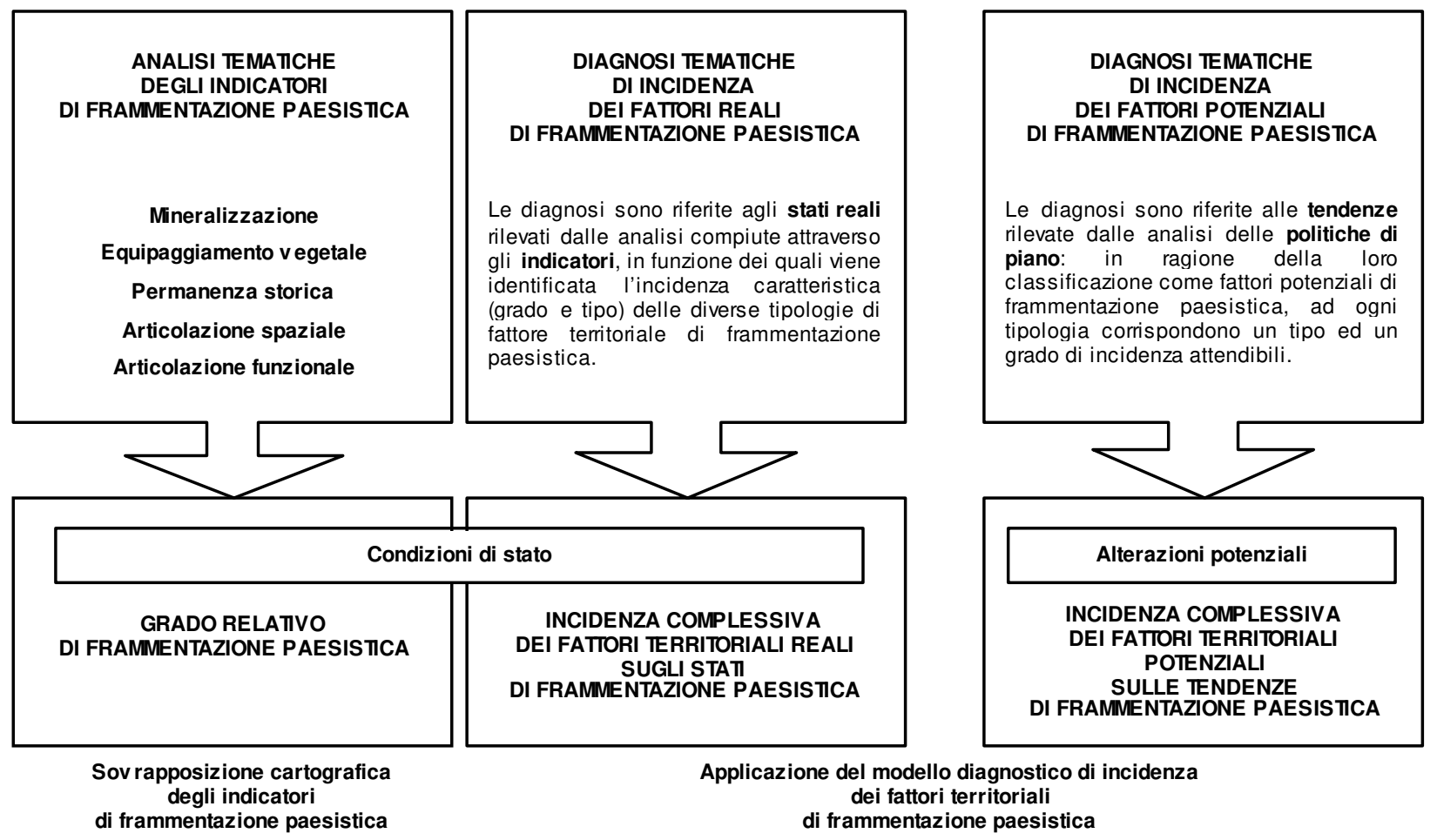

Figura 29 - Schema specifico del $3^{\circ}$ livello di diagnosi - Gli indicatori vengono utilizzati esclusivamente nelle due diagnosi relative alle condizioni di stato: grado di frammentazione paesistica (schema a sinistra) e incidenza complessiza dei fattori territoriali reali sugli stati di frammentazione paesistica (schema al centro). La diagnosi delle alterazioni potenziali fa riferimento alla classificazione dei fattori ed ai relativi criteri interpretativi, non essendo possibile applicare gi indicatori alla misurazione di stati potenziali a causa del livello di indeterminatezza delle informazioni (schema a destra).

Il terzo livello di diagnosi delle condizioni di stato del paesaggio, è articolato essenzialmente in due elaborazioni riguardanti la sintesi delle situazioni riscontrate attraverso gii indicatori primari e la sintesi delle relazioni di incidenza dei fattori su quelle stesse situazioni. Non si tratta tanto di due quadri interpretativi conseguenti, né in rapporto di subordinazione, bensì di due identificazioni complementari della fenomenologia.

Nel caso della sintesi diagnostica del complesso di informazioni riportate dai cinque indicatori, attraverso un processo di sovrapposizione cartografica, è possibile ottenere una identificazione dell'articolazione territoriale della frammentazione paesistica per gradi relativi di intensità, connessi alla presenza di situazioni critiche: dalla classe " 0 ", relativa alla assenza di ogni indicatore, alla classe " 5 ", in cui tutti gli indicatori segnalano il fenomeno. Si tratta pertanto di una classificazione in ambiti spaziali omogenei per grado di complessità e conseguente gravità delle condizioni del paesaggio relativamente agli stati di frammentazione strutturale (paragrafo $3.3-3^{\circ}$ livello di diagnosi).

Questo quadro diagnostico, impostato secondo una classificazione graduata, è integrato dal secondo genere di elaborazione, che riguarda l'identificazione cartografica del tipo e grado di incidenza dei fattori sugli stati di frammentazione.

L'applicazione del modello di incidenza dei fattori territoriali (proposto di seguito) alle cartografie di analisi dei cinque indicatori primari, produce altrettante diagnosi tematiche. 
La sovrapposizione cartografica non è necessaria per l'elaborazione di sintesi del grado di incidenza complessiva, in quanto è possibile controllare questo dato nell'ambito della definizione del modello diagnostico, attribuendolo in base agli specifici livelli di alterazione di cui i fattori territoriali sono risultati responsabili relativamente ai cinque indicatori primari proposti (figura 30).

A tal fine è possibile adottare l'attribuzione di un coefficiente numerico relativo $(\mathrm{CR})$ per individuare le tre classi di incidenza: $C R=0$ per incidenza unitaria irrilevante; $C R=1$ per incidenza unitaria minima; $C R=3$ per incidenza unitaria media; $C R=9$ per incidenza unitaria massima.

Come si può osservare nel quadro diagnostico della figura 30, non si verificano casi di incidenza complessiva irrilevante, per i quali tutti i cinque indicatori primari si dovrebbero trovare in condizioni di incidenza unitaria irrilevante. Si ottiene pertanto una articolazione in tre classi di incidenza complessiva: CR compreso fra 1 e 15 per incidenza complessiva minima; CR compreso fra 16 e 30 per incidenza complessiva media; CR compreso fra 31 e 45 per incidenza complessiva massima.

Mentre il grado di frammentazione fornisce un quadro sintetico dell'entità della problematica in termini di consistenza complessiva riscontrata sul territorio, il grado di incidenza dei fattori fornisce un quadro sintetico del peso che essi vengono ad assumere in una determinata area come cause. Pertanto, il primo identifica la gravità degli effetti del fenomeno, il secondo si riferisce al peso delle cause. Insieme costituiscono il quadro complessivo delle priorità e limitazioni/opportunità di correzione della frammentazione paesistica.

Ancora relativamente a questo livello, occorre approfondire la descrizione del modello di diagnosi di diagnosi dell'incidenza dei fattori territoriali sugli stati di alterazione identificativi della frammentazione paesistiae di attribuzione dei lizelli di correzione possibili, per il ruolo essenziale di chiave di lettura da esso rivestito. Considerato che le classi di analisi dei fattori territoriali e degli indicatori paesistici sono state individuate fra quelle strettamente attinenti la tematica specifica e che pertanto risultano tutte caratterizzate da molteplici relazioni di incidenza, è utile infatti identificare specificamente tali relazioni, per comprendere i tipi di sensibilità del paesaggio rispetto al complesso dei fattori territoriali e le possibilità di intervento per il miglioramento delle condizioni di stato ed il controllo delle tendenze. Il concetto di incidenza dei fattori territoriali è proiettato in ottica progettuale, comprendendo la considerazione dell'intensità dell'alterazione paesistica (figura 30 - quadro in alto) relativamente alle possibilità effettive di correzione e miglioramento delle condizioni di stato (figura 30 - quadro in basso).

Dalla diagnosi di frammentazione paesistica è stato escluso il concetto di rezersibilità delle alterazioni in ragione delle limitazioni ad esso connaturate quando sia riferito al paesaggio. Da una parte vi sono ragioni di ordine pragmatico, relative al quadro culturale, sociale ed economico reale, per cui sarebbe del tutto aleatorio considerare reversibili alterazioni come quelle dovute alla costruzione di una autostrada o di un quartiere residenziale. Ma vi sono anche ragioni di ordine filosofico-scientifico, in base alle quali la reversibilità di una condizione del paesaggio costituisce di fatto una contraddizione in termini, nella misura in cui ogni modificazione porta ad una nuova configurazione, unica e diversa dalla precedente.

Il livello di correzione possibile, segnalato nella identificazione delle singole relazioni di incidenza, è relativo a quanto può essere fatto per ridurre gli stati di frammentazione paesistica riscontrati, fino alla situazione positiva estrema della rigenerazione, ovvero della produzione di nuove condizioni di equilibrio. Questi aspetti si traducono in livello di attenzione prezentiza quando si tratti di alterazioni potenziali.

Un dato che non può sorprendere, dal momento che il modello è riferito a paesaggi culturali, è che la più alta sensibilità alle modificazioni è relativa all'indicatore " $\mathrm{C}$ " permanenza storica, sul quale 13 tipologie di fattori territoriali sul totale considerato di 17 risultano avere incidenza massima.

I fattori che risultano responsabili dei maggiori livelli di incidenza complessiva sono, come è noto dalla descrizione fatta della realtà, quelli costituiti dalle diverse tipologie insediative, dalle infrastrutture viarie e dalle monocolture agrarie industrializzate. 


\section{MODELLO DIAGNOSTICO quadro di sintesi}

Figura 30 - Schema del modello di incidenza ( $3^{\circ}$ livello di diagnosi).

Il quadro in alto indica i livelli di incidenza dei fattori territoriali nelle alterazioni di frammentazione paesistica (condizioni di stato o alterazioni potenziali)

Il quadro sottostante indica i relativi livelli di correzione possibili (da interpetare in termini di misure di intervento per la riduzione, nel caso delle condizioni di stato, e di politiche di governo per il contenimento, nel caso delle alterazioni potenziali).

\section{INCIDENZA DEI FATTORI TERRITORIALI \\ SU GLI STATI DI ALTERAZIONE IDENTIFICATIVI DELLA FRAMMENTAZIONE PAESISTICA}

$\max$

Incidenza massima (Coefficiente Relativo CR = 9)

I fattori sono responsabili dei massimi livelli di alterazione del paesaggio

med Incidenza media (Coefficiente Relativo $\mathrm{CR}=3$ ) I fattori sono responsabili di livelli medi di alterazione del paesaggio

min Incidenza minima (Coefficiente Relativo $C R=1$ ) I fattori sono responsabili dei minimi livelli di alterazione del paesaggio

irr Incidenza irrilevante (Coefficiente Relativo $\mathrm{CR}=0$ ) I fattori non sono responsabili di alterazioni del paesaggio di rilievo relativamente all'indicatore considerato

\begin{tabular}{lll}
\hline & A & Mineralizzazione del paesaggio \\
\cline { 2 - 2 } $\begin{array}{ll}\text { INDICATORI DI } \\
\text { FRAMMENTAZIONE } \\
\text { PAESISTICA }\end{array}$ & B & Equipaggiamento vegetale del paesaggio \\
\cline { 2 - 2 } & C & Permanenza storica del paesaggio \\
\cline { 2 - 2 } & D & Articolazione spaziale del paesaggio \\
\hline E & Articolazione funzionale del paesaggio \\
\hline
\end{tabular}

VALORI TOTALI DEI COEFFICIENTI RELATIVI (CR)

INCIDENZA COMPLESSIVA (min: CR 1-15; med: CR 16-30; max: CR 31-45)

LIVELLI DI CORREZIONE POSSIBILI DEGLI STATI DI ALTERAZIONE IDENTIFICATIVI DELLA FRAMMENTAZIONE PAESISTICA

$*$ Possibilità di correzione remote Le alterazioni sono caratterizzate da spazi di operatività severamente ridotti

** Possibilità di correzione condizionate Le alterazioni sono caratterizzate da apprezzabili spazi di operatività soggetti a sostanziali condizionamenti tecnici

$* * *$ Possibilità di correzione elevate Le alterazioni sono caratterizzate da rilevanti spazi di operatività fino a possibili effetti di rigenerazione del paesaggio

Nota: II concetto di possibilità di correzione degli stati di frammentazione paesistica fa riferimento a criteri tecnici e prescinde dai condizionamenti sociali ed economici connessi alle diverse realtà territoriali.

\begin{tabular}{lll}
\hline & A & Mineralizzazione del paesaggio \\
\cline { 2 - 3 } $\begin{array}{l}\text { INDICATORI DI } \\
\text { FRAMMENTAZIONE } \\
\text { PAESISTICA }\end{array}$ & B & Equipaggiamento veg etale del paesaggio \\
\cline { 2 - 3 } & C & Permanenza storica del paesaggio \\
\hline D & Articolazione spaziale del paesaggio \\
\hline E & Articolazione funzionale del paesaggio
\end{tabular}


FATTORI TERRITORIALI DI FRAMMENTAZIONE PAESISTICA

\begin{tabular}{|c|c|c|c|c|}
\hline \multicolumn{2}{|c|}{ Areali } & \multicolumn{2}{|c|}{ Lineari } & \multirow{2}{*}{ Puntuali } \\
\hline complessi & semplici & complessi & semplici & \\
\hline
\end{tabular}

\begin{tabular}{|c|c|c|c|c|c|c|c|c|c|c|c|c|c|c|c|c|}
\hline 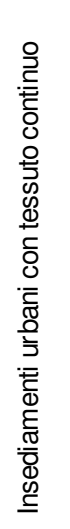 & 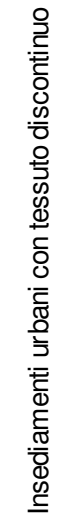 & 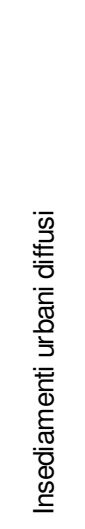 & 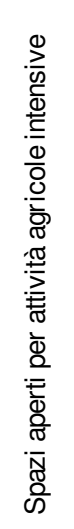 & 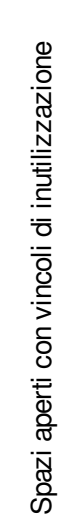 & 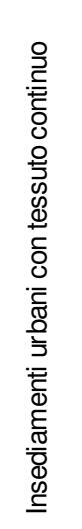 & 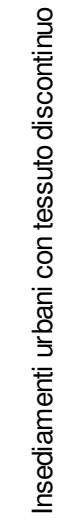 & 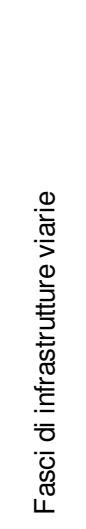 & 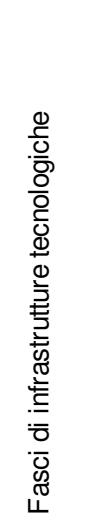 & 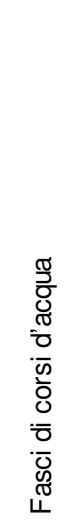 & 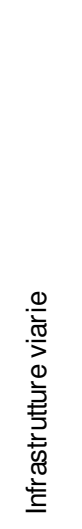 & 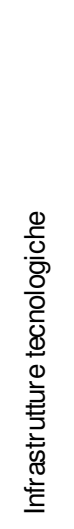 & 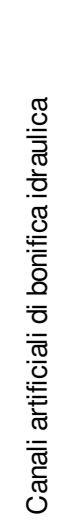 & 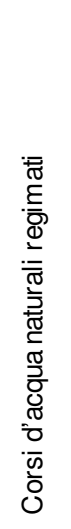 & 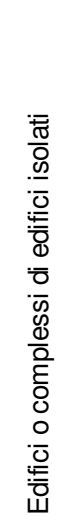 & 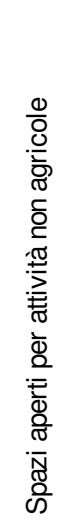 & 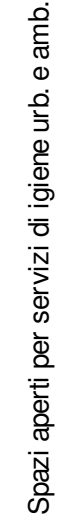 \\
\hline 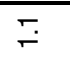 & $\stackrel{\text { Tִ }}{\longrightarrow}$ & $\stackrel{m}{\circ}$ & $\overline{\mathrm{N}}$ & กิ & $\bar{m}$ & ָָ & $\underset{m}{m}$ & ঙे & $\stackrel{\llcorner}{\text { p }}$ & 广 & テ̛ & $\stackrel{m}{+}$ & $\stackrel{ナ}{\forall}$ & is & ஸึ่ & ڤై \\
\hline $\max$ & $\max$ & $\max$ & irr & irr & $\max$ & $\max$ & $\max$ & $\min$ & med & $\max$ & $\min$ & med & $\min$ & max & med & $\mathrm{med}$ \\
\hline max & med & med & max & $\max$ & $\max$ & med & $\max$ & med & $\max$ & $\max$ & med & $\max$ & $\max$ & med & $\max$ & med \\
\hline $\max$ & $\max$ & $\max$ & $\max$ & $\max$ & med & med & $\max$ & $\max$ & med & $\max$ & $\max$ & $\max$ & $\min$ & max & $\max$ & nax \\
\hline med & $\max$ & max & $\max$ & $\max$ & med & max & $\max$ & $\max$ & $\max$ & max & $\max$ & med & $\min$ & max & $\max$ & $\max$ \\
\hline $\max$ & $\max$ & max & $\min$ & med & $\max$ & $\max$ & $\max$ & $\min$ & med & $\max$ & $\min$ & med & $\min$ & $\max$ & med & med \\
\hline 39 & 39 & 39 & 28 & 30 & 33 & 33 & 45 & 23 & 27 & 45 & 23 & 27 & 13 & 39 & 33 & 27 \\
\hline & & & & & & & & & & & & & & & & \\
\hline
\end{tabular}

\begin{tabular}{|c|c|c|c|c|c|c|c|c|c|c|c|c|c|c|c|c|}
\hline 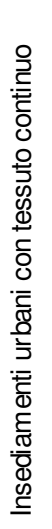 & 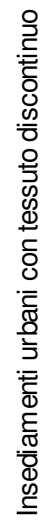 & 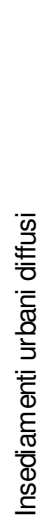 & 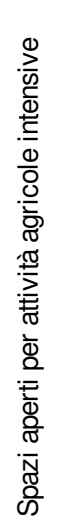 & 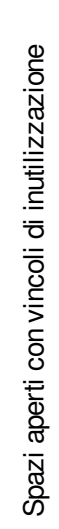 & 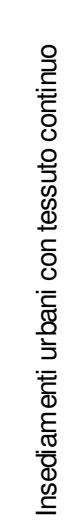 & 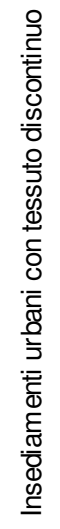 & 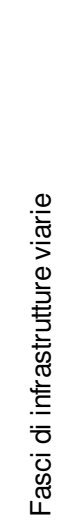 & 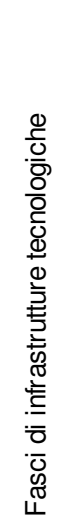 & $\begin{array}{l}\frac{\pi}{0} \\
\overline{0} \\
\bar{\pi} \\
\overline{0} \\
\overline{0} \\
\overline{0} \\
\overline{\overline{0}} \\
\overline{0} \\
\bar{c} \\
\bar{L}\end{array}$ & 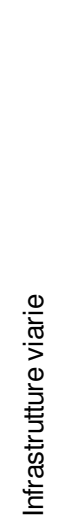 & 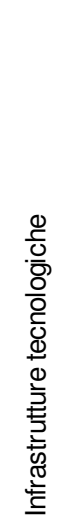 & 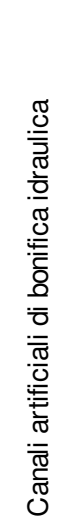 & 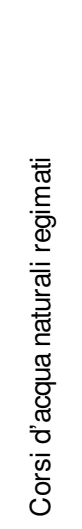 & 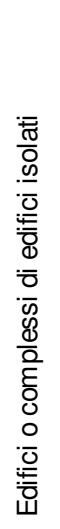 & 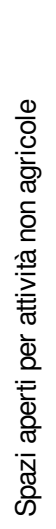 & 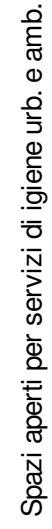 \\
\hline 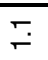 & $\stackrel{N}{\stackrel{N}{\prime}}$ & $\stackrel{m}{\longrightarrow}$ & $\overline{\mathrm{N}}$ & ָ̃ & $\bar{m}$ & $\stackrel{\sim}{\sim}$ & $\stackrel{m}{m}$ & ঙ্লে & $\stackrel{10}{\infty}$ & $\bar{f}$ & $\stackrel{\sim}{\sim}$ & $\stackrel{m}{+}$ & $\underset{+}{+}$ & $\overline{10}$ & กָ & మై \\
\hline$*$ & $*$ & $* *$ & - & - & $*$ & $*$ & $* *$ & $* *$ & $* * *$ & $* *$ & $* *$ & $* * *$ & $* * *$ & $*$ & $* *$ & ** \\
\hline$*$ & $* *$ & $* *$ & $* * *$ & $* *$ & $*$ & $* *$ & $* * *$ & $* *$ & $* * *$ & $* * *$ & $* *$ & $* * *$ & $* * *$ & $* *$ & $*$ & $* *$ \\
\hline * & $*$ & $* *$ & $*$ & $* *$ & $*$ & $*$ & $*$ & * & $*$ & $* *$ & $*$ & $* *$ & $* * *$ & $* *$ & $* *$ & $* *$ \\
\hline & $*$ & $* *$ & $* * *$ & $* *$ & $* *$ & $* *$ & $* *$ & $*$ & $* *$ & $* * *$ & $*$ & $* *$ & $* * *$ & $* *$ & $*$ & * \\
\hline
\end{tabular}




\section{2 - Criteri interpretativi afferenti alla diagnosi di incidenza dei fattori territoriali ${ }^{114}$}

I criteri interpretativi riportati in questo paragrafo esplicitano i contenuti diagnostici dei quadri di sintesi del modello proposto (figura 30); un'applicazione esemplificativa di tali criteri si trova nei successivi paragrafi 3.3 e 3.4 .

La definizione dei giudizi diagnostici fa sostanzialmente capo alle relazioni causa/effetto tra i fattori territoriali e gli indicatori di frammentazione paesistica ed ha pertanto contenuti di ordine tipologico. La sua utilità è legata alla sistematizzazione delle diagnosi di frammentazione paesistica su vasta scala, sia per quanto riguarda le esigenze di omogeneità di giudizio di condizioni ascrivibili ad una stessa classe di fattori, sia per le possibilità di informatizzazione delle procedure di archiviazione ed elaborazione dei dati.

I criteri interpretativi non risultano però sensibili alle diversità paesistiche che identificano specificamente un dato ambito territoriale, essendo riferiti univocamente ai caratteri tipologici dei fattori e privi di ogni elemento di informazione geografica specifica. Come accade generalmente nel caso dell'utilizzo di criteri tipologici di lettura del paesaggio relativi a configurazioni elementari o comunque semplici, risulta quindi necessario che gli esiti diagnostici vengano vagliati rispetto alle unità di paesaggio di appartenenza ( $1^{\circ}$ livello diagnostico) ed ai relativi approfondimenti derivanti dal $2^{\circ}$ livello diagnostico, per assumere nei giudizi finali gli effettivi pesi dei fattori, in funzione delle condizioni caratteristiche del contesto in cui essi agiscono. Se tale aspetto non assume rilievo nel caso studio in questione, per la relativa omogeneità del paesaggio considerato dalla diagnosi, appartenente in tutto alla pianura periurbana metropolitana, è sufficiente immaginare di estendere l'applicazione delle analisi e diagnosi alle vicine aree collinari a nord ed a sud, per percepire l'importanza dell'interpretazione topologica dei pesi diagnostici derivanti dalla lettura tipologica dei fattori.

\section{Mineralizzazione del paesagoio}

I massimi livelli di incidenza dei fattori territoriali relativamente agli stati reali e potenziali di mineralizzazione del paesaggio sono dovuti al complesso dei fattori di tipo insediatizo (tipologie: 1.1, 1.2, $1.3,3.1,3.2,5.1$ ) e dei fattori di tipo infrastrutturale viario (tipologie: $3.3,4.1$ ).

In larga misura ne è responsabile la sostituzione della matrice rurale preesistente con quella a prevalenza di spazi mineralizzati propria dei tessuti urbani a sviluppo areale e lineare o con quella propria della dispersione insediativa, caratterizzata da edifici e complessi di edifici isolati. La realizzazione di infrastrutture viarie nell'ambito di matrici rurali o rurali con dispersione insediativa, assume in ogni caso una consistenza di evidente rilievo.

Anche nei casi di tessuti urbani discontinui, in ragione della profondità dell'alterazione, la maggior disponibilità di spazi aperti rispetto ai tessuti urbani continui non determina un incremento rilevante delle possibilità di correzione che restano remote. Per le specifiche caratteristiche delle forme di urbanizzazione diffusa, il livello relativo di correzione possibile può mutare sensibilmente verso possibilità di correzione condizionate, in funzione delle modalità di uso e gestione degli spazi aperti non mineralizzati, interclusi in quantità rilevanti e talvolta con livelli non trascurabili di continuità, determinando requisiti idonei ad un progressivo recupero paesistico verso nuove forme di equilibrio.

\footnotetext{
114 Per la classificazione tipologica dei fattori territoriali di frammentazione paesistica si vedano il paragrafo 2.2 e la lista di controllo e le schede riportate nell'appendice A. Per comodità di lettura si riportano i riferimenti numerici delle diverse tipologie di fattore: 1 fattori areali complessi; 1.1 insediamenti urbani con tessuto continuo; 1.2 insediamenti urbani con tessuto discontinuo; 1.3 insediamenti urbani diffusi; 2 fattori areali semplici; 2.1 spazi aperti per attività produttive agricole intensive; 2.2 spazi aperti soggetti a vincoli di inutilizzazione; 3 fattori lineari complessi; 3.1 insediamenti urbani con tessuto continuo; 3.2 insediamenti urbani con tessuto discontinuo; 3.3 fasci di infrastrutture viarie; 3.4 fasci di infrastrutture tecnologiche; 3.5 fasci di corsi d'acqua; 4 fattori lineari semplici; 4.1 infrastrutture viarie; 4.2 infrastrutture tecnologiche; 4.3 canali artificiali di bonifica idraulica; 4.4 corsi d'acqua naturali regimati; 5 fattori puntuali; 5.1 edifici e complessi di edifici isolati; 5.2 spazi aperti per attività produttive non agricole; 5.3 spazi aperti per servizi di igiene urbana e ambientale.
} 
Il livello di correzione possibile è in generale decisamente basso (4 tipologie di fattori su 5 sono caratterizzate da possibilità di correzione remote) per la carenza e la discontinuità degli spazi aperti non mineralizzati residui.

Il margine di recupero di funzionalità biologica del paesaggio è connesso all'efficacia di interventi di equipaggiamento vegetale con specie arboree d'alto fusto ed arbustive idonee all'impiego in condizioni difficili, per inquinamento e condizionamenti spaziali e di soluzioni progettuali e tecnologiche che massimizzino le capacità di drenaggio delle pavimentazioni, considerando la relativa fattibilità tecnica, anche in relazione ai rischi di inquinamento degli acquiferi sotterranei. Risultano inoltre strettamente complementari le misure di protezione e miglioramento ambientale specificamente mirate alle risorse primarie (depurazione delle acque utilizzate e di scolo e protezione delle acque superficiali e di falda, fissazione delle polveri e compensazione delle emissioni inquinanti). Rispetto alle alterazioni potenziali, occorre che al livello di attenzione preventivo ad esse connesso corrisponda la pianificazione e la regolamentazione di una serie di congrue misure di contenimento delle condizioni critiche, che considerino concretamente la salvaguardia di spazi aperti strategici ai fini della separazione di ambiti insediativi e la costituzione di sistemi di spazi aperti per la compensazione e l'inserimento paesistico-ambientale degli sviluppi previsti. Nell'ambito dello stesso ordine di misure rientra la progettazione di tessuti urbani di completamento od espansione secondo requisiti di congrua dotazione qualitativa e quantitativa di spazi aperti.

Per quanto riguarda le infrastrutture viarie, la frequente compresenza di più entità affiancate determina configurazioni caratterizzate da rilevanti impegni di spazi e prevalente continuità di spazi mineralizzati anche nella direzione trasversale agli assi longitudinali (fasci di infrastrutture).

Le possibilità di correzione sono riferite in particolare agli spazi aperti interclusi tra le infrastrutture e a margine delle stesse e condizionate da aspetti tecnici e normativi.

Anche nel caso delle configurazioni semplici riguardanti singole infrastrutture, alla minore estensione superficiale unitaria delle alterazioni non corrispondono sostanziali differenze di incidenza assoluta del fattore, né di possibilità di riduzione degli effetti.

Il margine di recupero di funzionalità biologica del paesaggio fa riferimento agli stessi criteri citati per i tessuti urbani, sia per quanto riguarda le modalità di intervento che le misure di protezione e miglioramento ambientale delle risorse primarie.

Rispetto alle alterazioni potenziali, occorre che al livello di attenzione preventivo ad esse connesso corrisponda, in sede di pianificazione e progettazione ed, ancora prima, ai livelli decisionali inerenti le infrastrutture primarie e le relative procedure di Studio e Valutazione di Impatto Ambientale, una serie di congrue misure di prevenzione e contenimento degli effetti critici, che consideri concretamente la salvaguardia di spazi aperti strategici ai fini della continuità biotica e morfologica del paesaggio e la costituzione di sistemi di spazi aperti per la compensazione e l'inserimento paesistico-ambientale delle opere previste.

Manifestazioni di minor rilievo con medio livello di incidenza dipendono dai corsi d'acqua artificiali (tipologie: 3.5 e 4.3) e dagli spazi aperti per attività produttize non agricole e per servizi di igiene ambientale (tipologie: 5.2 e 5.3).

Nel caso delle opere di canalizzazione e regimazione funzionali all'adeguamento dei corsi d'acqua alle portate idrauliche pianificate e delle conseguenti configurazioni complessive da essi assunte, il livello di correzione possibile è elevato e può giungere alla parziale rinaturazione dei tratti con alveo mineralizzato, attraverso interventi con tecnologie e specie vegetali idonee per condizioni ambientali e rispondenza ai condizionamenti normativi. Nei casi di configurazioni a fascio di più corsi d'acqua, importanti opportunità di compensazione e miglioramento paesistico sono date dalla possibilità di funzionalizzazione ed equipaggiamento paesistico degli spazi interclusi, generalmente degradati ed inutilizzati.

Le possibilità di correzione connesse agli effetti delle attività non agricole nell'ambito di matrici rurali o rurali con dispersione insediativa e delle aree per servizi di igiene ambientale sono condizionate dalle stesse forme di utilizzo degli spazi. 
Il margine di recupero di funzionalità biologica del paesaggio fa riferimento agli stessi criteri citati per i tessuti urbani, sia per quanto riguarda le modalità di intervento che le misure di protezione e miglioramento ambientale delle risorse primarie. Sono inoltre da considerare fra le possibilità di correzione le misure di sistemazione paesistico-ambientale praticabili a seguito della dismissione dei siti, per esaurimento o per altre motivazioni tecniche e/o economiche.

Rispetto alle alterazioni potenziali, occorre che al livello di attenzione preventivo ad esse connesso corrisponda, in sede di pianificazione e di regolamentazione, una serie di congrue misure di prevenzione e contenimento delle condizioni critiche, che consideri concretamente la salvaguardia di spazi aperti strategici ai fini della continuità biotica e morfologica del paesaggio e di spazi aperti idonei per collocazione e dimensione a svolgere funzioni di separazione ed in generale di compensazione paesistico-ambientale.

L'incidenza minima è propria delle infrastrutture tecnologiche (tipologie: 3.4 e 4.2) e dei corsi d'acqua naturali regimati (tipologia: 4.4). I livelli di correzione possibili risultano elevati per i corsi d'acqua, peri quali sono plausibili attese di sostanziale riduzione e/o rimozione delle condizioni critiche. Per quanto riguarda le infrastrutture tecnologiche occorre considerare che, sebbene esse comportino la presenza di sistemi artificiali interrati, questi hanno però generalmente una sezione trasversale complessiva che può essere considerata poco influente dal punto di vista biotico generale, non determinando gravi disturbi. Assolutamente irrilevanti risultano da questo punto di vista le infrastrutture aeree, alle quali è imputabile la sola presenza delle opere di fondazione delle strutture portanti puntiformi.

Le misure di correzione consistono sostanzialmente nella riduzione delle opere artificiali e nella limitazione del loro utilizzo nei nuovi interventi che, in particolare nel caso dei corsi d'acqua, possono avvalersi delle tecnologie dell'ingegneria naturalistica e delle conseguenti possibilità di ripristino di condizioni migliori di equipaggiamento vegetale.

\section{Equipagiamento zegetale del paesaggio}

I massimi livelli di incidenza dei fattori territoriali relativamente agli stati reali e potenziali di equipaggiamento vegetale del paesaggio sono dovuti ai fattori insediativi e specificamente ai tessuti urbani continui (tipologia: 1.1,3.1), alle colture agrarie intensize (tipologia: 2.1) ed ai zincoli di inutilizzazione (tipologia: 2.2), ai fattori infrastrutturali viari $(3.3,4.1)$, ai corsi d'acqua artificiali e naturali (tipologie: 3.5, $4.3,4.4$ ) ed infine alle attività produttize non agricole (tipologia: 5.2 ).

Le maggiori estensioni ed intensità di alterazione sono imputabili agli insediamenti con tessuto continuo ed alle monocolture agrarie. Questi fattori sono però responsabili di condizioni che presentano livelli di correzione possibili diversi.

I tessuti insediativi sono caratterizzati da una strutturazione e da una carenza di spazi aperti per le quali le possibilità di correzione risultano remote ed il margine di recupero di equipaggiamento vegetale del paesaggio è connesso all'efficacia delle misure di conservazione degli spazi aperti che ne risultano dotati e di sistemazione di quelli che, pur essendo idonei, ne risultano privi.

Nel caso delle colture agrarie, invece, non vi sono condizionamenti tecnici, né spaziali, alla ricostituzione di congrue configurazioni di equipaggiamento vegetale del paesaggio, per le quali occorre privilegiare l'introduzione di configurazioni sostenibili in questi contesti sociali ed economici, a retoriche politiche di ripristino di forme ormai sostanzialmente estranee agli assetti territoriali contemporanei, pensando alla progressiva costituzione di nuovi paesaggi in equilibrio.

Il livello di attenzione preventivo rispetto alle alterazioni potenziali suggerisce in entrambi i casi citati l'adozione di misure di conservazione degli spazi aperti dei tessuti urbani continui, come componenti strategiche delle quali, oltre che ostacolare la riduzione, occorre incentivare l'incremento attraverso un idoneo indirizzo delle politiche per le aree dismesse e, per quanto riguarda le aree agricole, l'adozione di misure di conservazione, in ragione del valore di riserve di risorse che esse comunque 
svolgono, essenziale anche per la stessa fattibilità di interventi di progressiva ricostituzione dell'equipaggiamento vegetale del paesaggio.

Per quanto riguarda le aree soggette a vincoli di inutilizzazione, i livelli di correzione possibili risultano condizionati dalla natura stessa dei vincoli (ad esempio motivazioni di sicurezza). Gli interventi possibili non sono però privi a priori di margini di configurazione di paesaggi apprezzabili per qualità intrinseche e per capacità relazionali rispetto al contesto.

Un peso non indifferente rivestono anche i fattori di tipo lineare ed in una forma particolare i corsi d'acqua naturali regimati. Mentre infatti per tutte le entità artificiali, sia strade, che canali di bonifica, si tratta della eliminazione di formazioni vegetali preesistenti e della assenza di equipaggiamento vegetale per mancata predisposizione di queste formazioni paesistiche, nel caso dei corsi d'acqua naturali regimati si è in presenza della nota progressiva riduzione della vegetazione di ripa in forza delle esigenze di ordine idraulico di canalizzazione e manutenzione. Tale peso è relativo in particolare al fatto che queste tipologie costituiscono una rete di spazi pubblici con elevate caratteristiche di continuità e diffusione territoriale, in particolare ove siano viste in un quadro unitario. Le possibilità di correzione risultano generalmente elevate (strade e corsi d'acqua naturali regimati) salvo nel caso dei canali di bonifica per i quali occorre considerare il maggiore peso che assumono i condizionamenti tecnici sovraordinati di ordine idraulico.

Nello specifico il recupero di equipaggiamento vegetale del paesaggio può vedere nella rete infrastrutturale viaria e idrografica naturale i sistemi paesistici di intervento prioritario per i quali non sussistono condizionamenti assoluti: ad essi può essere affidato il ruolo di struttura primaria di equipaggiamento e di innesco di processi complementari di recupero di qualità paesistica. L'equipaggiamento vegetale dei corsi d'acqua arginati (naturali e artificiali) è però strettamente legato in termini di fattibilità al riequilibrio idraulico del territorio ed alla diminuzione della domanda di drenaggio delle aree di pianura soggette a rischio idraulico. Da questi aspetti dipendono infatti le possibilità di modificazione dei corsi d'acqua con attribuzione di caratteri strutturali di seminaturalità e le possibilità di adozione di pratiche diverse di manutenzione.

Il livello di attenzione preventiva rispetto alle alterazioni potenziali, dato dalla sensibilità delle risonse, nei casi dei corsi d'acqua, e gli ampi margini di riqualificazione paesistica in entrambi i casi dei corsi d'acqua e delle infrastrutture viarie, richiedono idonee misure di pianificazione e regolamentazione che salvaguardino in maniera efficace gli spazi aperti ai loro margini e incentivino la loro sistemazione con equipaggiamento vegetale, anche in funzione dei potenziali sviluppabili, oltre che in termini di rigenerazione e protezione ambientale, per gli usi ricreativi e di mobilità alternativa.

Possibilità di correzione remote corrispondono agli effetti dei fattori puntuali relativi alle attività produttive non agricole, per i quali reali opportunità di intervento sul paesaggio si prospettano per lo più solo a seguito della dismissione delle attività stesse, suggerendo l'importanza di riservare idonei spazi di separazione da altri utilizzi e di sottoporli ai relativi interventi di sistemazione paesistica.

Mentre non si riscontrano fattori ascrivibili alla classe di incidenza minima sulle condizioni di equipaggiamento vegetale del paesaggio, tutti i fattori rimanenti risultano afferire alla classe di incidenza media. A tale situazione corrisponde anche un livello omogeneo delle possibilità di correzione riscontrate, che risultano condizionate.

Si tratta specificamente dei fattori insediativi relativi ai tessuti urbani discontinui, diffusi o con caratteridi dispersione (tipologie: 1.2, 1.3, 3.2, 5.1), alle infrastrutture tecnologiche (tipologie: 3.4 e 4.2) ed infine agli spazi per servizi di igiene ambientale (tipologia: 5.3). Sebbene in tutti questi casi si sia in presenza di una sostituzione della preesistente matrice rurale, l'incidenza in termini di perdita o riduzione della dotazione paesistica di equipaggiamento vegetale risulta minore rispetto alle situazioni descritte in precedenza, in forza della quota di equipaggiamento presente (quanto meno a livello quantitativo), per quanto riguarda gli insediamenti, e in forza di una tendenza generalizzata a non intervenire su quanto non necessario a fini di sicurezza o di produzione, per quanto riguarda rispettivamente le aree vincolate e le infrastrutture tecnologiche e le aree per attività non agricole, che spesso ai margini 
mantengono presenze arboree ed arbustive preesistenti o sviluppatesi in forma spontanea durante le fasi di conduzione produttiva.

È intuibile la diversificazione che caratterizza il livello di correzione omogeneo attribuito a questi casi. Mentre per gli insediamenti le possibilità di intervento per la costituzione di sistemi di equipaggiamento paesistico sono condizionate essenzialmente da aspetti di congestione e carenza di spazi, per quanto riguarda le infrastrutture viarie e le infrastrutture e strutture tecnologiche $\mathrm{i}$ condizionamenti sono per lo più circoscritti ad aspetti funzionali (inerenti requisiti di sicurezza) e progettuali (inerenti i requisiti quantitativi e qualitativi degli spazi con funzioni di compensazione ed inserimento paesistico-ambientale). Per gli insediamenti diviene strategica l'attenzione a riservare gli spazi residui a destinazioni compatibili con una rilevante dotazione di equipaggiamento vegetale, possibilmente spazi verdi destinati alle diverse funzioni che essi possono accogliere, ed a programmare interventi di realizzazione di sistemazioni di equipaggiamento con specie vegetali di alto fusto e arbustive per gli spazi esistenti che ne sono privi, nonché di miglioramento di quanto esistente.

Il livello di attenzione preventiva rispetto alle alterazioni potenziali deve tenere conto delle note tendenze a non considerare nell'ambito di queste forme di utilizzo la presenza di formazioni vegetali come elemento strutturale del paesaggio. Diviene pertanto strategico prevedere in ambito di pianificazione e regolamentazione idonei spazi fisici e di fattibilità normativa ed economica delle sistemazioni di equipaggiamento vegetale a stretto corredo di tali forme di uso e/o dirette ad effetti di inserimento e compensazione paesistico-ambientale delle stesse.

\section{Permanenza storica del paesagoio}

Dei massimi livelli di incidenza dei fattori territoriali relativamente agli stati reali e potenziali di permanenza storica del paesaggio è responsabile la maggior parte delle tipologie: gli insediamenti urbani nelle manifestazioni comuni sviluppatesi nella seconda metà del secolo scorso (tipologie: 1.1, 1.2, 1.3, 5.1), gli spazi per attività agricole intensize (tipologia: 2.1) e quelli sottoposti a vincoli di inutilizzazione (tipologia: 2.2), le infrastrutture viarie e tecnologiche (tipologie: 3.3, 3.4, 4.1, 4.2), i canali di bonifica (tipologia: 4.3), gli spazi aperti per attività produttize non agricole (tipologia: 5.2) e gli spazi per servizi di igiene ambientale (tipologia: 5.3).

Il livello di incidenza è dovuto essenzialmente alla grave carenza di forme equilibrate di stratificazione storica e di rapporto con il contesto paesistico preesistente. Ciò ha comportato e comporta infatti la frammentazione delle matrici paesistiche storiche, sia di quelle continue, che hanno i massimi livelli di sensibilità alle trasformazioni, come quella colturale, sia di quelle discontinue, strutturate su modelli spaziali puntiformi o a rete che, pur avendo livelli di sensibilità minori rispetto alle precedenti, risentono pesantemente dei processi di modificazione paesistica e, in conseguenza della loro stratificazione insensibile alle configurazioni storiche, mostrano in genere condizioni di severa criticità, basti pensare alla frammentazione della rete viaria rurale $\mathrm{o}$ alla perdita della matrice puntiforme dell'edilizia rurale.

Le possibilità di correzione risultano in larga misura remote in ragione del carattere intrinseco del paesaggio di risorsa irriproducibile, particolarmente legato al suo valore culturale di testo storico comprensivo delle diverse espressioni umane.

Le possibilità di correzione condizionate attribuite alle aree con urbanizzazione diffusa o dispersione insediativa, alle aree soggette a vincolo di inutilizzo, sono legate alla maggior presenza di spazi aperti ed alle loro maggiori dimensioni relative e si riferiscono essenzialmente alle possibilità di salvaguardia preventiva e di conservazione dei valori di permanenza storica residui, limitatamente ad una loro apprezzabilità in relazione alla tipologia a cui essi afferiscono. Le possibilità di correzione condizionate attribuite alle infrastrutture viarie ed ai canali di bonifica fanno riferimento all'appartenenza tipologica di questi fattori a due dei principali sistemi di strutturazione antropica del paesaggio che insieme a profonde radici storiche mostrano talvolta rispetto agli insediamenti una 
maggior stabilità territoriale, mantenendo nel tempo alcune coincidenze di tracciato ed, in parte, di tipologia. Le possibilità di intervento si riferiscono pertanto alla conservazione di tali forme di permanenza ed alla riduzione degli effetti di alterazione che le riguardano. Infine per quanto concerne i servizi di igiene ambientale, sono state attribuite possibilità di correzione condizionate in ragione dei margini di ottimizzazione che dovrebbero afferire agli interventi pubblici o comunque finalizzati alla fornitura di servizi di interesse pubblico, margini ritenuti pertanto praticabili quanto meno come obiettivi di innovazione delle modalità di definizione di tali interventi.

Livelli di incidenza media risultano caratterizzare per motivazioni diverse gli insediamenti urbani lineari con tessuto continuo e con tessuto discontinuo (tipologie 3.1 e 3.2) ed i fasci di corsi d'acqua (tipologia: 3.5) nei casi in cui siano costituiti dall'affiancamento di uno o più canali artificiali ad uno o più (confluenza) corsi naturali.

Nel caso degli insediamenti, la permanenza della configurazione lineare, ove vi siano origini storiche, costituisce un indice di apprezzabile permanenza del tessuto che, in mancanza di sviluppi infrastrutturali integrativi, non ha subito rilevanti alterazioni. Le possibilità di correzione remote attribuite a queste tipologie di fattori sono invece riferite al caso in cui l'insediamento lineare derivida una alterazione profonda della struttura storica del paesaggio, come per le configurazioni lineari sviluppatesi lungo una infrastruttura fra nuclei storici a seguito dell'espansione terziaria e secondaria $\mathrm{o}$ anche residenziale.

Il livello di attenzione preventiva rispetto alle alterazioni potenziali si articola in priorità di conservazione, relativamente agli elevati valori di permanenza storica, ed in priorità di salvaguardia e prevenzione di ulteriori aggravamenti delle condizioni critiche, nel caso in cui l'insediamento risulti caratterizzato da bassi gradi di permanenza storica.

Gli unici fattori ascrivibili a livelli di incidenza minima risultano essere i corsi d'acqua naturali regimati (tipologia: 4.4), in quanto loro stessi fanno parte della storia del paesaggio e pertanto non sono responsabili di sovrapposizioni od obliterazioni rilevanti del patrimonio storico culturale. I livelli di correzione possibili sono elevati, e trovano nel valore strategico dei corsi d'acqua un importante incentivo alla necessaria rifunzionalizzazione del patrimonio storico. Basti pensare agli opifici di diverso tipo ed alle relative sistemazioni idrauliche che, in un ottica di sistema museale e di utilizzo per servizi culturali e ricreativi compatibili, divengono componenti strettamente complementari al sistema dei percorsi nell'ambito di politiche complesse di riqualificazione e conservazione del paesaggio.

Rispetto alle alterazioni potenziali l'intensità e la progressione della perdita di permanenza storica del paesaggio impone un livello di attenzione preventivo nei confronti di tutte le tipologie di fattore territoriale, alle quali occorre riferire idonee politiche di salvaguardia e di conservazione delle risorse.

\section{Articolazione spaziale del paesaggio}

Anche per gli stati reali e potenziali di articolazione spaziale del paesaggio sono ritenuti responsabili dei massimi livelli di incidenza gran parte dei fattori territoriali, nello specifico, i tessuti urbani discontinui e quelli caratterizzati da urbanizzazione diffusa o dispersione insediatiza (tipologie: 1.2, 1.3, 3.2, 5.1), le attività agricole intensize, le aree soggette a vincoli di inutilizzazione (tipologie: 2.1 e 2.2), le infrastrutture (tipologie: 3.3, 3.4, 4.1, 4.2), i fasci di corsi d'acqua (tipologia: 3.5) ed infine i fattori puntuali, le attriatì produttize non agricole ed i servizi di igiene ambientale (tipologie: 5.2 e 5.3).

Il livello di incidenza è dovuto alla generalizzata carenza ove non assenza di identità spaziale caratteristica dei tessuti urbani prodotti dagli sviluppi insediativi governati con i criteri della zonizzazione monofunzionale e degli standards urbanistici. Tale caratteristica, in funzione della rilevante estensione dimensionale e diffusione di queste configurazioni insediative, coincide non solo con la loro evidente incapacità di identificazione semiologica positiva del paesaggio, bensì anche con l'effetto, assolutamente negativo e strettamente conseguente, della disarticolazione della sua struttura spaziale, che viene ad essere allo stesso tempo decisamente semplificata, sui piani tipologico e 
dimensionale, e congestionata, dalla reiterazione e sovrapposizione di componenti e sistemi spesso conflittuali, per caratteri intrinseci e/o per localizzazione reciproca.

Le possibilità di correzione risultano remote per i fattori insediativi della tipologia 1.2, in ragione del rapporto di forma, per il quale l'interazione con tipologie paesistiche diverse è proporzionalmente ridotta al minimo, e della loro elevata strutturazione, per la quale gli interventi possibili, salvo i casidi ristrutturazione urbana, sono limitati in genere alla realizzazione di sistemazioni vegetali con funzioni di separazione, di marginatura, di schermatura, finalizzate nel loro complesso ad un recupero di qualità morfologica del paesaggio. Le possibilità di correzione risultano invece condizionate per $\mathrm{i}$ fattori insediativi della tipologia 1.3, per la densità spaziale che le caratterizza che, se da una parte è responsabile di forme di alterazione devastanti, dall'altra è quella che permette i più ampi margini di intervento in termini di disponibilità potenziale di spazi. Alle infrastrutture tecnologiche corrispondono possibilità di correzione remote ogniqualvolta si tratti di tipologie aeree, per le quali spesso, indipendentemente dalla qualità intrinseca dei manufatti, gli stati di congestione e conflitto che vengono a determinarsi non sono risolvibili. Possibilità di correzione remote si hanno anche per le attività produttive non agricole in ragione delle difficoltà di concreto miglioramento delle condizioni degli spazi aperti nelle fasi di esercizio della produzione; per queste tipologie devono però essere considerate le possibilità di intervento per il recupero paesistico successivo alla dismissione delle aree.

Il livello di attenzione preventivo rispetto ai rischi di sviluppo di tali tipologie deve essere commisurato al binomio che le caratterizza in termini di rilevante potenziale di alterazione del paesaggio e remote possibilità di correzione ed orientamento degli effetti.

Si hanno invece possibilità di correzione condizionate per i restanti fattori responsabili dei massimi livelli di incidenza, ad eccezione delle colture agrarie intensive e delle infrastrutture viarie singole, per le quali le possibilità di correzione divengono elevate, con potenziali di rigenerazione della articolazione spaziale del paesaggio.

Nello specifico, gli insediamenti urbani lineari con tessuto discontinuo ed, ancorché in misura minore, gli edifici ed i complessi di edifici isolati, per il rapporto di forma favorevole ad una forte interazione con le tipologie paesistiche limitrofe, mostrano infatti margini di intervento più ampi delle configurazioni areali afferenti allo stesso tipo di tessuto urbano. Oltre che con interventi strutturali di completamento e riordino insediativo, atti a definire i margini e gli spazi di relazione, è possibile in questi casi intervenire con interventi di inserimento paesistico nei quali l'utilizzo di formazioni vegetali arboree ed arbustive può assumere, pur soggetto a condizionamenti, significato di modifica strutturale del paesaggio con effetti di riequilibrio ed integrazione delle diverse tipologie (in particolare urbano-rurale ed urbano-infrastrutturale). Gli spazi aperti soggetti a vincoli di inutilizzo vedono le possibilità di correzione delle criticità di articolazione spaziale condizionate dalle specifiche esigenze di esercizio della funzione alla base della istituzione del vincolo. Ciò non toglie comunque un apprezzabile margine di intervento che il progetto può sviluppare in diverse forme possibili nel rispetto dei requisiti suddetti. Se, a titolo esemplificativo si considerano le aree di pertinenza delle piste aereoportuali, non esistono motivi univoci ed incontrovertibili per i quali questi paesaggi così importanti dal punto di vista della qualità dei sistemi infrastrutturali di accesso alle aree urbane e metropolitane debbano essere necessariamente ed esclusivamente caratterizzati da prati falciati, né avere sistemi di mediazione spaziale rispetto all'immediato intorno nei confronti del quale innescano generalmente squilibri di scala. Fasci di corsi d'acqua ed aree con spazi aperti per servizi di igiene ambientale possono ancora essere assoggettati ad interventi di miglioramento ed inserimento paesistico-ambientale, calibrati su alcuni condizionamenti sostanziali legati alle sezioni ed alle dinamiche idrauliche dei corsi d'acqua ed alle esigenze di esercizio e caratteristiche morfologiche e dimensionali degli spazi per l'igiene ambientale.

Il livello di attenzione preventivo rispetto alle alterazioni potenziali deve orientare, in ragione dei livelli di correzione possibili, le scelte tipologiche e localizzative definendo scenari di contenimento degli effetti. 
Al livello di incidenza media afferiscono gi insediamenti con tessuto continuo (tipologie: 1.1 e 3.1) ed i canali artificiali per la bonifica idraulica nella configurazione dei fattori semplici (tipologia: 4.3). Gli insediamenti che rientrano in questa tipologia comprendono talvolta tessuti di rilevante qualità spaziale ed interesse storico. In tali casi, le caratteristiche di identità escludono completamente la stessa ascrivibilità fra $\mathrm{i}$ fattori territoriali di frammentazione paesistica per quanto concerne l'articolazione spaziale, determinando una diagnosi di irrilevanza. Restano i casi in cui a questi tipi di tessuto insediativo non risultano corrispondere tali caratteri qualitativi: ad essi è attribuibile una incidenza minore delle altre tipologie 1.2, 1.3 e 3.2, ma responsabile comunque di effetti di squilibrio spaziale non trascurabili. Per i canali artificiali, tale attribuzione è dovuta ad una situazione intermedia di effetti di alterazione spaziale tra quella caratteristica dei fasci di corsi d'acqua e quella dei corsi d'acqua naturali regimati (rispettivamente attribuiti alle classi di incidenza massima e minima). Rispetto a tali situazioni possono essere pertanto considerati intermedi sia il livello di correzione possibile (possibilità di correzione condizionate) che il livello di attenzione preventiva, ancorchè, peri canali artificiali, il primo sia, nelle attuali condizioni di governo territoriale, più vicino a quello dei fasci di corsi d'acqua, che non a quello dei corsi d'acqua naturali regimati.

Incidenza minima è attribuita esclusivamente ai corsi d'acqua naturali (tipologia: 4.4) che, rispetto a questo ordine di alterazione paesistica, non sono nella generalità dei casi significativamente apprezzabili come fattori di alterazione, ma risultano piuttosto fra le poche configurazioni dotate di una propria identità spaziale e di rapporti di equilibrio rispetto alla scala paesistica, sia per quanto concerne l'articolazione spaziale minuta, che come rilievo semiologico complessivo. Si tratta in questi casi delle tipiche condizioni in cui il livello relativo di elevate possibilità di correzione coincide con la situazione limite rispetto alla quale viene meno la stessa idoneità del termine correzione e gli interventi possono concretamente superare la categoria del miglioramento paesistico, producendo effetti di rigenerazione del paesaggio, attraverso misure di consolidamento e rafforzamento dei caratteri positivi che identificano queste tipologie.

\section{A rticolazione funzionale del paesagoio}

Rispetto alle alterazioni della articolazione funzionale ecosistemica del paesaggio, viene attribuita incidenza massima ai tessuti urbani (tipologie: 1.1, 1.2, 1.3, 3.1, 3.2, 5.1), alle infrastrutture viarie (tipologie: $3.3,4.1$ ), in ovvia coincidenza con quanto avviene per la mineralizzazione del paesaggio, che da sola costituisce un pesante effetto di alterazione funzionale ecosistemica.

Tutti questi fattori sono responsabili di un impoverimento biologico del paesaggio, di tipo qualitativo, ma anche quantitativo, che incide sulla riduzione delle configurazioni con funzioni ecosistemiche primarie di stabilizzazione e resilienza, determina un carico ambientale al quale non corrisponde in genere una idonea compensazione, ma piuttosto una assoluta carenza di sistemi paesistici di protezione, ed infine riduce pesantemente, ove non la comprometta, l'efficienza di apparati paesistici che svolgono funzioni strategiche di connessione ambientale (basti pensare alla tombatura dei corsi d'acqua e alla loro completa alterazione con l'abbassamento della portata al di sotto del deflusso minimo vitale, a seguito degli eccessivi prelievi, abusivi e concessi).

Si trovano in possibilità remote di correzione gli insediamenti con tessuto continuo e quelli con tessuto discontinuo a sviluppo areale. Carenza di spazi, estensione superficiale e forte strutturazione, sono gli elementi che contribuiscono sostanzialmente a ridurre le possibilità di intervento. Attraverso misure di equipaggiamento vegetale è possibile migliorare le condizioni di protezione e di connessione ambientale; queste nel caso dei corsi d'acqua, se affiancate ad interventi più profondi di depurazione delle acque, modificazione del regime idraulico e recupero di naturalità, possono contribuire ad un miglioramento delle condizioni idrologiche complessive.

Il livello di attenzione preventivo rispetto alle alterazioni potenziali deve essere commisurato al binomio che le caratterizza in termini di rilevante potenziale di alterazione del paesaggio e remote possibilità di correzione ed orientamento degli effetti. 
Vi sono invece possibilità di correzione condizionate per le alterazioni dovute alle altre tipologie insediative ed alle infrastrutture viarie. In tutti questi casi vi sono margini di intervento più ampi, sia in termini di disponibilità di spazi, da riservare e destinare a funzioni di compensazione e protezione ambientale, che in termini di modificazione strutturale di alcune configurazioni responsabili di particolari tipi di criticità (si pensi ad esempio agli effetti critici sul piano dei rischi idraulici dovuti, nelle aree di pianura, ai terrapieni stradali, ampiamente mitigabili attraverso l'utilizzo di carreggiate sovraelevate nei punti di maggior sensibilità, per il mantenimento di congrui livelli di continuità della rete idrografica minore ove nonostante tutte le precauzioni localizzative si riscontri o si confermi la necessità dell'infrastruttura e la sua incompatibilità).

Anche rispetto ai rischi di sviluppo di tali tipologie il livello di attenzione preventiva deve fare riferimento al rilevante potenziale di alterazione del paesaggio; occorre pertanto orientarne attentamente i processi localizzativi e progettuali al fine di contenere gli effetti di alterazione paesistica e provvedere alla loro migliore compensazione, anche attraverso interventi esterni ai sedimi di edifici ed infrastrutture e dei relativi spazi di immediata pertinenza.

Livelli di incidenza media sono attribuiti, fra i fattori areali, agli spazi aperti con vincoli di inutilizzazione (tipologia: 2.2), in considerazione del minor peso che in questi casi ha l'estensione superficiale rispetto a quelli descritti sopra, ai fattori lineari coincidenti con i fasci di corsi d'acqua e con i canali di bonifica idraulica (tipologie: 3.5 e 4.3), per le relazioni che hanno con gli equilibri di questa risorsa primaria, pur con l'elevato grado di artificialità che li caratterizza, ed ai i fattori puntuali coincidenti con gli spazi per attività produttize non agricole e per servizi di igiene ambientale (tipologie: 5.2, 5.3), in ragione del rapporto di forma e della relativamente limitata estensione superficiale che li accomuna.

Le possibilità di correzione si trovano a livelli diversi in ragione delle specificità delle diverse tipologie. Esse risultano remote per gli spazi aperti per attività produttive, salvo la considerazione delle opportunità di intervento a seguito della loro dismissione. Il livello di attenzione preventivo rispetto a queste tipologie deve pertanto fare riferimento agli effetti di posticipazione della gran parte dei possibili interventi di recupero paesistico-ambientale. Risultano invece caratterizzate da possibilità di correzione condizionate le alterazioni dovute ai vincoli di inutilizzazione, per la presumibile quota di salvaguardia ambientale che essi esprimono al di fuori degli obiettivi per i quali sono stati istituiti. Ancora possibilità di correzione condizionate vengono attribuite alle aree per servizi di igiene ambientale, per le possibilità di intervento presumibili in ragione della prevalente proprietà fondiaria pubblica ed in relazione all'evoluzione delle politiche ambientali verso una progressiva maggiore attenzione ed efficacia. Possibilità di correzione condizionate si hanno ancora per i fasci di corsi d'acqua e per i canali artificiali singoli, in ragione delle attuali forme di governo idraulico del territonio e dei relativi limiti alla introduzione di modalità di gestione ambientale e paesistica rispondenti a scenari complessi, caratterizzati da molteplici fattori di qualità.

Il livello di attenzione preventivo rispetto alle alterazioni potenziali deve essere commisurato al significato strategico delle funzioni investite ed ai condizionamenti che caratterizzano le possibilità di correzione, al fine di produrre scelte localizzative e tipologiche che contengano gli effetti critici.

Incidenza minima è attribuita alle monocolture agricole intensize (tipologia: 2.1), alle infrastrutture tecnlogidhe (tipologie: $3.4,4.2$ ) ed ai corsi d'acqua naturali regimati (tipologia: 4.4), per i limitati effetti di alterazione ecosistemica caratteristici, a cui corrispondono ampi margini di intervento.

Possibilità di correzione elevate afferiscono alle monocolture agricole, relativamente alle opportunità di conversione attraverso pratiche agrarie diverse caratterizzate da sostenibilità ambientale e socioeconomica, ed ai corsi d'acqua naturali regimati. Le due tipologie restanti di fattori sono caratterizzate da possibilità di correzione condizionate. 


\section{3 - La diagnosi delle condizioni di stato del paesagoio}

Come si è accennato, occorre che la fenomenologia della frammentazione paesistica venga indagata, sia relativamente alle condizioni di stato, che alle alterazioni potenziali del paesaggio. Questa esigenza fa sì che la distinzione analitica dei fattori territoriali in reali e potenziali debba essere mantenuta anche nelle osservazioni diagnostiche.

Con riferimento allo schema generale di diagnosi proposto (figura 27, paragrafo 3.1), la diagnosi delle condizioni di stato del paesaggio si esplica su tre o quattro livelli, in ragione delle specifiche finalità conoscitive. La considerazione congiunta dei risultati dei livelli diagnostici permette la formulazione del giudizio completo relativo alle condizioni dell'ambito territoriale soggetto di studio, in ragione anche delle relazioni contestuali significative.

$1^{\circ}$ lizello di diagnosi: articolazione metastrutturale del paesagoioin ambiti spaziali complessi (unità di paesagoio)

L'analisi del paesaggio per ambiti spaziali omogenei consente il riconoscimento macroscopico delle configurazioni fondamentali ed, in ragione dei loro caratteri intrinseci, come della collocazione e della distribuzione geografica, permette una prima identificazione delle forme di frammentazione paesistica. Questo livello di indagine, essenziale nel processo di conoscenza del paesaggio, è relativo ad estensioni territoriali di ordine regionale e subregionale e trova nei piani territoriali di coordinamento provinciali gli strumenti che, per scala e per competenze di legge, risultano migliori per la gestione del difficile rapporto tra le politiche territoriali operative di livello comunale e quelle di indirizzo di livello sovracomunale.

Nell'ambito di studio, è possibile distinguere essenzialmente tre tipi di paesaggio in ragione degli stadi di alterazione dominanti. Un primo tipo di paesaggio caratterizzato da elevati livelli di qualità ecosistemica, di permanenza storica e di identità morfologica (indice " $\mathrm{A}$ " nella figura 31).

Si tratta, nella generalità dei casi, coincidente con i paesaggi pedecollinari e collinari (A1), di situazioni fortemente condizionate dalle dinamiche socioeconomiche e culturali, a cui sono legate le diverse forme di uso e gestione delle risorse. Vi fa eccezione

Figura 31 - Diagnosi di frammentazione paesistica - condizioni di stato - $1^{\circ}$ livello diagnostico: unità di paesaggio (rappresentazione su fotografia aerea zenitale - Regione Toscana, scala originale 1:30000).
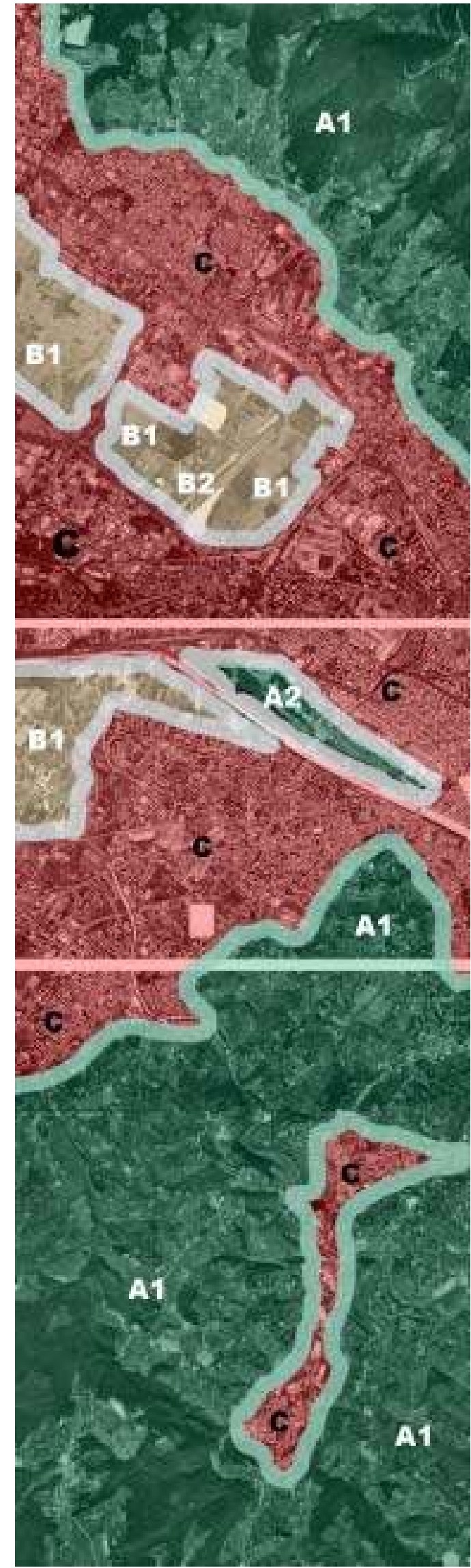
solamente il caso del Parco delle Cascine, unica area di rilievo nel paesaggio urbano di pianura per qualità paesistica, sia sul piano dell'interesse ecologico che su quelli storico e visuale (A2). Le condizioni strutturali del paesaggio denotano una resilienza complessiva non trascurabile, pur essendo questo caratterizzato dalla elevata fragilità di alcune componenti (ad esempio delle sistemazioni idraulico-agrarie di collina). Non si riscontrano presenze di rilievo di fattori di frammentazione, né per estensione, né per diffusione ad eccezione del caso dell'area planiziale del Parco delle Cascine, il cui unico sistema di connessione con il paesaggio extraurbano risulta ormai il fiume, con i suoi spazi di pertinenza sempre più ridotti o utilizzati in maniera impropria.

Un secondo tipo di paesaggio risulta avere livelli di qualità ecosistemica tendenti a stati critici e rilevanti perdite di permanenza storica ed identità morfologica (indice " $B$ " nella figura 31). Anche con le profonde alterazioni che ne connotano la configurazione contemporanea, questi paesaggi mantengono elevati potenziali di rigenerazione. Si riscontrano manifestazioni di frammentazione paesistica di rilevante intensità e diffusione, sostanzialmente incrementata dalla infrastrutturazione e dai fenomeni di dispersione insediativa. A differenza delle aree con colture agricole (B1), gli spazi recintati di pertinenza dell'aereoporto (B2) costituiscono anche fattori di frammentazione funzionale del paesaggio, relativamente alle possibilità di fruizione umana, in ragione della specifica disposizione sul margine urbano occidentale, che risulta completamente interessato da questi, a partire dalla conurbazione meridionale, fino a quella settentrionale.

Vi sono infine paesaggi in condizioni di severa criticità, per deficit biotico, per perdita di permanenza storica e di identità morfologica (indice " $\mathrm{C}$ " nella figura 31). Si tratta di aree urbanizzate, caratterizzate da diffuse e diversificate manifestazioni di frammentazione paesistica.

Oltre le condizioni "interne" specificamente caratteristiche dei diversi ambiti, del territorio indagato, interamente compreso nella provincia di Firenze, risultano evidenti la discontinuità fra i paesaggi di elevata qualità (ambiti "A") e le condizioni di frammentazione del paesaggio caratteristiche degli ambiti " $\mathrm{B}$ " relativamente all'elevato grado di isolamento dei primi, dovuto alla continuità territoriale degli sviluppi insediativi che hanno progressivamente occupato la piana (figura 31).

$2^{\circ}$ lizello di diagnosi: articolazione strutturale del paesagoio in componenti fisionomiche di base (mosaico paesistico)

Secondo i criteri metodologici illustrati nel precedente paragrafo 3.1, questo livello di diagnosi riveste un ruolo di connessione tra le necessità conoscitive di scala vasta e quelle di scala locale. L'approfondimento diagnostico individua in tal senso categorie e relazioni che costituiscono una descrizione delle situazioni delineate dal primo livello interpretativo ed un impianto di riferimento per il terzo livello, che costituisce l'elaborazione propriamente operativa della diagnosi, dai cui risultati dovranno ripartire le considerazioni per la pianificazione sovralocale e locale, come quelleper la pianificazione d'area e la conseguente programmazione e progettazione degli interventi.

In termini di approfondimento del precedente primo livello diagnostico, l'interpretazione del quadro territoriale consentita dalla lettura del mosaico paesistico (figura 32) mette in evidenza alcuni caratteri strutturali del paesaggio urbano e periurbano, che si ritiene utile richiamare.

A nord, il cordone insediativo che da Firenze, attraverso Castello e Sesto Fiorentino, prosegue verso Calenzano e Prato, mostra, in corrispondenza dell'insediamento di Sesto Fiorentino, una presenza rilevante di macchie all'interno della matrice paesistica urbana, coincidenti con spazi verdi, talvolta limitrofi; in queste stesse aree una presenza rilevante di corridoi di corsi d'acqua naturali regimati afferenti a piccoli sottobacini collinari è andata riducendo la propria effettiva importanza paesisticoambientale, con la progressiva erosione di spazi dovuta agli sviluppi insediativi, che hanno in taluni casi completamente obliterato queste risorse. 
A nord-est, fino a tutta l'area di Castello, la matrice urbana risulta sostanzialmente continua, priva di corridoi e macchie con rilevanza paesistica; in relazione a questi tessuti, ed in particolare ai margini urbani settentrionali, è importante considerare la ricchezza di macchie arboree che caratterizza il paesaggio pedecollinare, sia per le formazioni agro-forestali, che per quelle dei molti parchi storici, di cui alcuni di grande rilievo.

Anche ad est la matrice urbana risulta decisamente caratterizzata dalla continuità del mosaico degli spazi mineralizzati, priva di ogni strutturazione di spazi verdi di rilievo paesistico, mentre a sud ad una presenza non trascurabile di spazi verdi corrisponde una loro polverizzazione.

L'unica entità di rilievo paesistico, che interrompe la continuità degli spazi mineralizzati della matrice urbana, è costituita dal complesso delle aree delle Cascine e dell'Argingrosso lungo l'Arno e dei relativi corridoi del fiume Arno e del torrente Mugnone e marginalmente del fiume Greve.

Ad ovest la matrice rurale del paesaggio di pianura, caratterizzata da forti livelli di semplificazione e destrutturazione generatori di frammentazione è a sua volta evidentemente frammentata da due macchie di tipo urbano di cui una a prevalenza di spazi mineralizzati (il polo scientifico universitario), l'altra a prevalenza di spazi aperti non mineralizzati, ma soggetti a vincoli di inutilizzazione (l'aereoporto). In questo stesso ambito si trova una zona umida di origine artificiale che fa parte diun più ampio complesso di aree distribuito nella piana, nate per utilizzi venatori ed oggi protette per l'interesse che rivestono per l'ecologia animale. Ad esse non corrisponde alcuna formazione di equipaggiamento vegetale con sviluppo e composizione tali da poter svolgere funzioni di protezione ambientale, nè di articolazione spaziale e caratterizzazione dello scenario visuale.

L'articolazione strutturale del paesaggio riscontrata attraverso la classificazione fisionomica delle configurazioni del mosaico (figura 32) è caratterizzata da quattro tipi di matrici, sei tipi di macchie, tre tipi di corridoi:

\section{Matrici:}

1 paesaggio forestale con formazioni seminaturali a prevalenza di latifoglie e con formazioni di impianto a prevalenza di conifere;

2 paesaggio rurale con prevalenza di colture arboree da frutto (oliveti specializzati);

3 paesaggio di origine rurale con prevalenza di colture erbacee (seminativi semplici e campi a foraggio) e incolti produttivi;

4 paesaggio urbano con prevalenza di spazi mineralizzati.

\section{Macchie:}

5 boschi con prevalenza di vegetazione di impianto e boschi di parchi;

6 colture agricole con prevalenza di forme arboree specializzate (oliveti);

7 neoecosistemi acquatici (zone umide di pianura);

8 spazi aperti urbani prevalentemente verdi soggetti a forme di uso e/o gestione;

9 spazi aperti urbani prevalentemente degradati per attività antropiche e/o abbandono;

10 spazi urbani prevalentemente mineralizzati.

\section{Corridoi:}

11 corsi d'acqua naturali regimati (fiumi, torrenti e rii) con presenza limitata e discontinua di equipaggiamento vegetale arboreo ed arbustivo e con canalizzazioni nelle aree di pianura;

12 corsi d'acqua artificiali (canali di bonifica) prevalentemente privi di equipaggiamento vegetale arboreo ed arbustivo e con sezioni in rilevato di terra inerbito;

13 strade prevalentemente prive di equipaggiamento vegetale arboreo ed arbustivo.

I corridoi stradali sono considerati e rappresentati ove attraversino matrici extraurbane, ovvero dove costituiscano caratteri di diversità strutturale del paesaggio; non è rilevante invece a questo livello di 
lettura indicarne la presenza nelle aree urbane per la omogeneità di mineralizzazione che ad essi corrisponde rispetto agli spazi con cui entrano in relazione.

Il quadro prodotto corrisponde ad un primo grado di approssimazione strutturale delle condizioni di frammentazione della piana e delle conseguenti discontinuità paesistiche con i sistemi dei rilievi collinari e submontani.

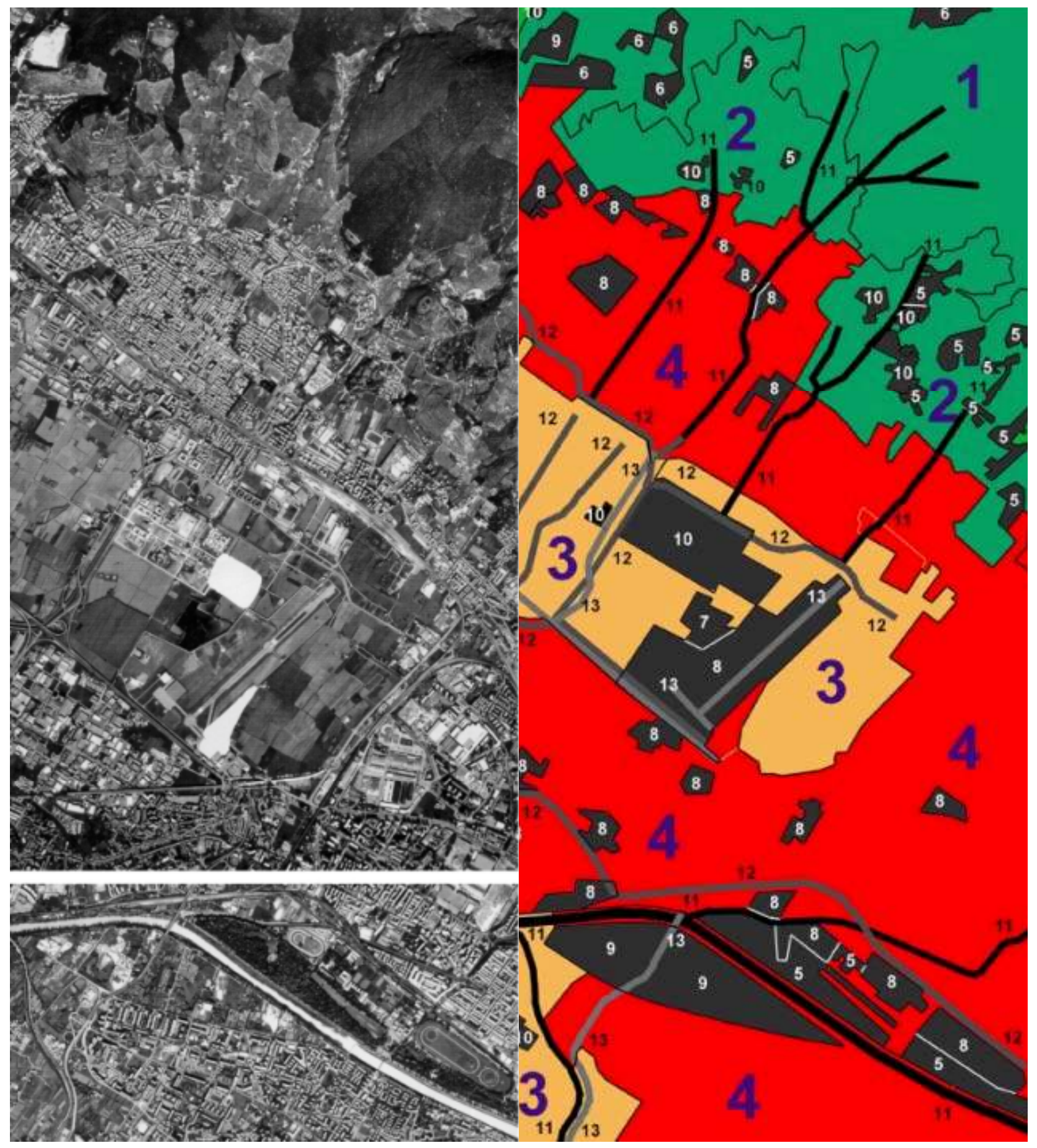

Figura 32 - Diagnosi di frammentazione paesistica - condizioni di stato - $2^{\circ}$ livello diagnostico: mosaico paesistico. L'articolazione strutturale del paesaggio riscontrata attraverso la classificazione fisionomica delle configurazioni del mosaico è caratterizzata da quattro tipi di matrici (1-4), sei tipi di macchie (5-10), tre tipi di corridoi (11-13). I corridoi stradali sono considerati e rappresentati ove attraversino matrici extraurbane, ovvero dove costituiscano caratteri di diversità strutturale del paesaggio (fotografia aerea zenitale da originale 1:30000 - Regione Toscana). 


\section{$3^{\circ}$ lizello di diagnosi (sottolizello " $a$ ") $)^{115}$ : grado di frammentazione paesistica}

L'aggregazione dei dati delle singole analisi tematiche degli indicatori di frammentazione paesistica permette di elaborare i relativi schemi cartografici di sintesi, mantenendo lo stesso livello di dettaglio geometrico delle prime. Le cinque analisi tematiche condotte tramite gli indicatori primari di frammentazione paesistica hanno consentito di elaborare altrettante rappresentazioni delle condizioni critiche del paesaggio (figura 33), utili alla sintesi diagnostica del grado relativo di frammentazione: presenza di spazi mineralizzati, assenza di formazioni di equipaggiamento vegetale, assenza di complessi o sistemi di permanenza storica, assenza di complessi o sistemi di articolazione spaziale di interesse morfologico, assenza di apparati di articolazione funzionale di interesse ecosistemico. L'evidente diffusione delle condizioni critiche relative ai singoli indicatori denota una situazione di severa semplificazione e destrutturazione biologica e morfologica del paesaggio, per la quale risulta più significativo soffermarsi direttamente sui risultati della elaborazione diagnostica di sintesi, salva l'opportunità di una breve osservazione relativa allo schema riguardante la mineralizzazione del paesaggio, sia per l'importanza dell'indicatore in questione, che per il fatto che è l'unico che rileva condizioni di forte diversità nel soggetto di studio (paragrafo 2.3). Il 51\% del territorio indagato non risulta in condizioni di paesaggio mineralizzato, per una superficie complessivamente corrispondente a 247 ettari, di cui 170 di paesaggio periurbano ed extraurbano. Ne derivano condizioni relative (al campione di indagine) di potenziale rigenerazione del paesaggio che, osservate da un punto di vista strettamente strutturale ed astratto dai condizionamenti di ordine socioeconomico, risultano di significativo rilievo.
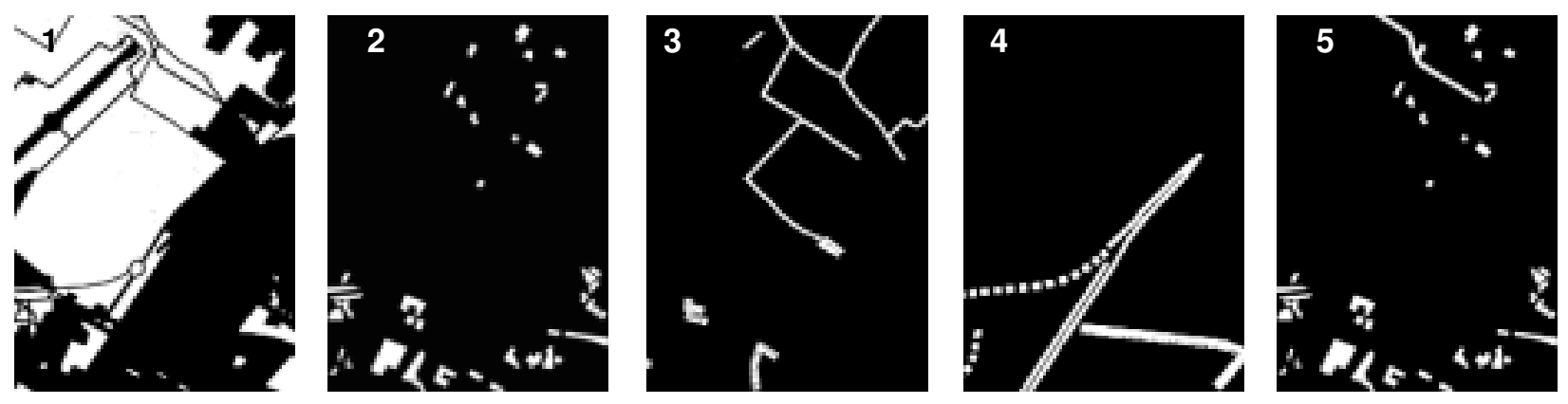

Figura 33 - Diagnosi di frammentazione paesistica - condizioni di stato - $3^{\circ}$ livello diagnostico: schemi cartografici di sintesi della frammentazione paesistica secondo le condizioni critiche (in nero) rilevate dagii indicatori primari: (1) presenza di spazi mineralizzati; (2) assenza di equipaggiamento vegetale; (3) assenza di complessi o sistemi di permanenza storica; (4) assenza di complessi o sistemi di articolazione spaziale di interesse morfologico; (5) assenza di apparati di articolazione funzionale di interesse ecosistemico (schemi ridotti da elaborazioni originali in scala 1:10000).

Questa caratteristica resta in evidenza in ogni caso anche nella cartografia di diagnosi del grado di frammentazione paesistica (figura 34), in cui la compresenza di condizioni critiche singolarmente rilevate dagli indicatori determina un grado relativo di approssimazione della gravità delle manifestazioni di frammentazione del paesaggio, osservate dal complesso dei punti di vista considerati e non più sotto singoli aspetti. Si deve considerare che il quadro derivante da questa cartografia è relativo ad un campione di indagine dimensionalmente circoscritto, per motivazioni esclusivamente strumentali alla sperimentazione applicata, e per questo comprensivo di configurazioni paesistiche tutte più o meno intensamente coinvolte nelle manifestazioni di frammentazione strutturale. L'applicazione del modello diagnostico, al di fuori dei limiti operativi di questa esperienza teorico-metodologica, ad un territorio di estensione maggiore, metterebbe in evidenza anche articolazioni diverse del mosaico spaziale, come, probabilmente, ambiti paesistici privi di manifestazioni significative di

115 Si veda l'articolazione del terzo lizello di diagnosi, in due sottolivelli con diversi contenuti, riportata nel paragrafo 3.1 e sintetizzata nello schema di figura 27. 
frammentazione. In merito a ciò è utile riferirsi in parallelo anche alle considerazioni transcalari proposte nel precedente paragrafo 2.2 per l'analisi dei fattori territoriali (si veda anche la figura 9).

Nel territorio di indagine, la diffusione delle condizioni critiche di frammentazione del paesaggio e la relativa sovrapposizione spesso multipla in uno stesso spazio aperto, ha fatto sì che non si riscontri la classe " 0 ", relativa all'assenza di manifestazioni di frammentazione. Questo è un dato caratteristico generalmente delle aree ad elevata pressione insediativa ed in particolare dei paesaggi urbani e periurbani. Risultano decisamente prevalenti le condizioni relative alle classi " 4 " e " 5 " in cui la compresenza di quattro e cinque indicatori con segnalazione di condizioni critiche comporta gradi relativi di frammentazione "mediamente alta" ed "alta". Occorre notare la continuità, oltre che la diffusione, delle aree afferenti alle classi con grado maggiore di frammentazione a discapito di quelle in condizioni meno critiche, che risultano fortemente polverizzate. Le condizioni di frammentazione strutturale che la diagnosi evidenzia risultano in sintesi caratterizzate dalla presenza dominante dei gradi più alti di manifestazione del fenomeno. A tali condizioni afferiscono ambiti paesistici fortemente continui, rispetto ai quali gli spazi in condizioni più favorevoli risultano evidentemente esigui e polverizzati. L'indagine rileva pertanto condizioni relative di severa frammentazione strutturale del paesaggio, che richiedono sia misure di intervento atte alla modificazione delle condizioni intrinseche di questi ambiti, che misure di salvaguardia e sviluppo di condizioni favorevoli nelle circostanti aree strategiche per la continuità paesistica.

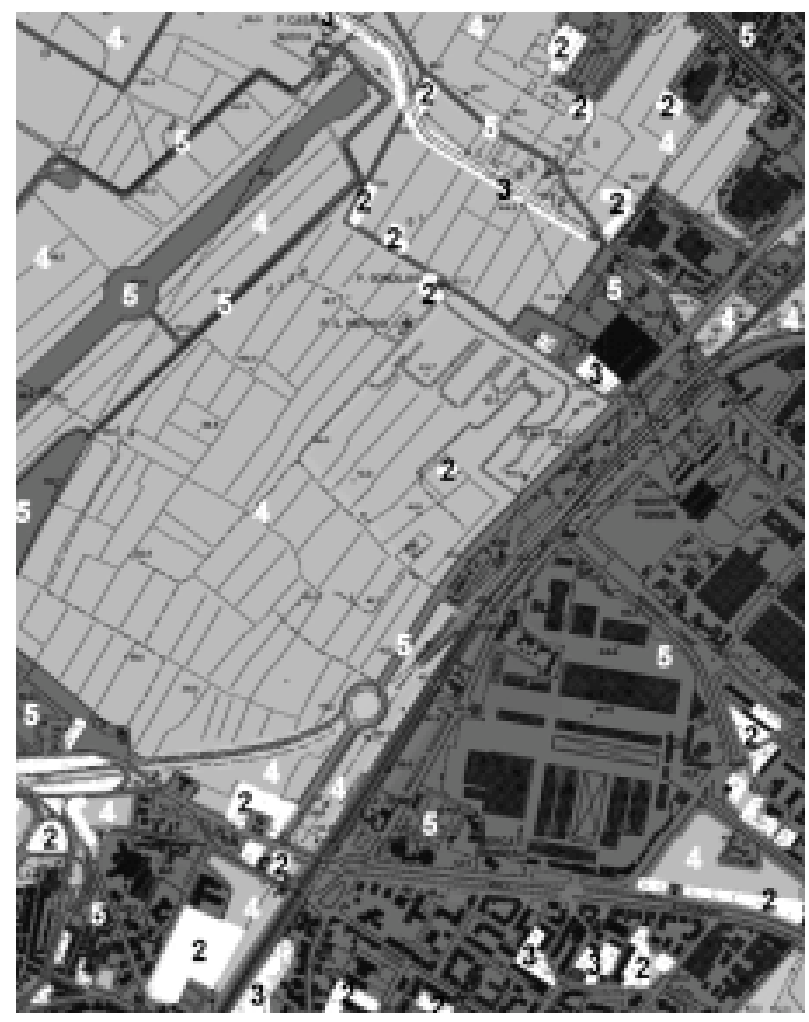

Figura 34 - Diagnosi di frammentazione paesistica condizioni di stato - $3^{\circ}$ livello diagnostico: grado di frammentazione paesistica.

Identificazione dell'articolazione territoriale della frammentazione paesistica per gradi relativi di intensità, connessi alla presenza di situazioni critiche: dalla classe "0", relativa alla assenza di ogni indicatore, alla classe " 5 ", in cui tutti gii indicatori segnalano il fenomeno. Si tratta di una classificazione in ambiti spaziali omogenei per grado di complessità e conseguente gravità delle condizioni del paesaggio relativamente agi stati di frammentazione strutturale (riduzione da originale elaborato su base 1:10000 - CTR Regione Toscana).

$3^{\circ}$ lizello di diagnosi (sottolizello " $b$ ") ${ }^{116}$ : tipo e grado di incidenza dei fattori territoriali sugli stati di frammentazione paesistica

L'applicazione del modello di incidenza dei fattori territoriali sugli stati di frammentazione paesistica permette di interpretare le condizioni del paesaggio rilevate dagli indicatori. $\mathrm{E}$ stata ritenuta importante la definizione sperimentale di una griglia di giudizi diagnostici (paragrafo $3.1-3^{\circ}$ livellodi

116 Si veda l'articolazione del terzo lizello di diagnosi, in due sottolivelli con diversi contenuti, riportata nel paragrafo 3.1 e sintetizzata nello schema di figura 27. 
diagnosi) al fine di ottenere una congrua omogeneità di interpretazione, in considerazione del fatto che le indagini relative a queste tematiche sono legate a ragguardevoli livelli di progressivo affinamento di dettaglio, quanto necessariamente riferite a territori molto vasti. Appare evidenteintal senso l'utilità di disporre di un modello con giudizi di base guidati, in modo da permettere sia l'applicazione da parte di più operatori, che l'utilizzo di tecnologie informatiche di automazione. Una ulteriore fase di sperimentazione, oltre la messa a punto del modello, dovrebbe quindi procedere ad un suo congruo collaudo applicativo, relativamente al rapporto con gli operatori, come alla informatizzazione attraverso l'utilizzo di idonee tecnologie informative territoriali di archiviazione, misurazione ed elaborazione cartografica dei dati.

Si può osservare come il determinismo relativo ${ }^{117}$ di tale procedura sia limitato alla fase iniziale della diagnosi, ovvero alla interpretazione di base dei dati forniti dagli indicatori. Resta invece esclusivamente alla sensibilità interpretativa della diagnosi topologica ed eventualmente di quella statistica, complementare alla prima, considerare il peso ed il significato della articolazione, della estensione e delle relazioni territoriali, che le applicazioni del modello mostrano ed, in ragione di questo complesso di informazioni, definire la diagnosi delle condizioni di frammentazione del paesaggio. Ciò è vero per il terzo livello diagnostico, quanto per quelli precedenti, con compiti di inquadramento contestuale, e per quello successivo, con compiti di approfondimento rispondente all'articolazione elementare degli spazi aperti. In tal senso, l'utilizzo delle tecnologie informatiche permette oggi di attivare processi di conoscenza e monitoraggio effettivamente rispondenti al principio di transcalarità, più volte richiamato come fondamentale negli studi riguardanti il paesaggio.

Secondo la prima applicazione del modello diagnostico descritto nel precedente paragrafo 3.1, relativa alla incidenza dei fattori territoriali sugli stati di mineralizzazione del paesaggio (a sinistra nella figura 35), risultano prevalenti le situazioni di incidenza massima, connesse al grado di diffusione ed intensità degli stati di mineralizzazione del paesaggio caratteristico dei tessuti insediativi (1.1) e (1.2), delle infrastrutture viarie singole (4.1) e dei fasci di infrastrutture viarie (3.3). Le possibilità di correzione sono remote, per gli insediamenti, e condizionate, per le infrastrutture viarie.

Relativamente agli stati di equipagoiamento zegetale del paesaggio (al centro nella figura 35), risulta confermata l'incidenza massima dei fattori insediativi (1.1), relativi ai tessuti a maggior rapporto di copertura edilizia. Ancora massimi risultano i livelli di incidenza delle infrastrutture viarie (3.3 - 4.1), degli ambiti con prevalenza di colture agricole intensive e/o incolti produttivi (2.1), delle aree soggette a vincolo aereoportuale (2.2).

Incidenza media afferisce ai fattori insediativi con tessuto discontinuo (1.2), come a quelli relativi alle aree con edifici e complessi di edifici isolati (5.1) ed alle aree con spazi aperti per attività produttive non agricole (5.2).

Le condizioni di incidenza minima dei fattori infrastrutturali tecnologici complessi (3.4) non hanno rilievo, essendo relative a fasci caratterizzati da incidenza più grave, dovuta alle infrastrutture viarie. L'incidenza dei fattori territoriali su gli stati di permanenza storica del paesaggio è caratterizzata dall'assoluta prevalenza nell'area di studio dei livelli massimi, afferenti ai fattori insediativi ed infrastrutturali (1.1 - 1.2 - 3.3 - 4.1 - 5.1), a quelli produttivi $(2.1-5.2)$ ed a quelli propri delle areedi pertinenza aereoportuale (2.2). Le possibilità di correzione sono prevalentemente remote, per le oggettive difficoltà di recupero di molti dei caratteri storici del paesaggio di pianura nelle aree periurbane.

Anche nel caso degli stati di articolazione spaziale del paesagoio, la diagnosi tematica registra una netta prevalenza dei livelli di massima incidenza. L'unica eccezione di rilievo è costituita dai livelli di

117 L'attributo relatizo è utilizzato per sottolineare il fatto che la componente deterministica della procedura illustrata non riguarda la definizione dei singoli giudizi diagnostici associati ai diversi binomi causa/sensibilità intercorrenti fra i fattori e gii indicatori, quanto l'estensione di uno stesso giudizio di base alle situazioni paesistiche che le analisi riferiscono ad una stessa tipologia. 
incidenza media attribuiti ai fattori insediativi della tipologia 1.1, ai quali corrispondono però anche possibilità di correzione condizionate, in ragione della forte strutturazione raggiunta.

Relativamente ai fattori responsabili dei massimi livelli di incidenza, possibilità elevate di correzione risultano proprie delle colture agrarie (2.1) e delle infrastrutture viarie singole (4.1), mentre entrano in gioco condizionamenti per le aree di pertinenza aereoportuale (2.2) e per i fasci di infrastrutture vianie (3.3). Possibilità di correzione remote corrispondono ai tessuti urbani discontinui consolidati (1.2).

La diagnosi di incidenza dei fattori territoriali sugli stati di articolazione funzionale del paesaggio (figura35), condotta con riferimento al quadro delle funzioni ecosistemiche prevalenti, rileva condizioni di incidenza massima con possibilità di correzione remote rispetto ai fattori insediativi $(1.1-1.2)$ e con possibilità di correzione condizionate, rispetto ai fattori infrastrutturali viari complessi (3.3). Media incidenza è attribuita ai fattori afferenti le aree con vincolo aereoportuale (2.2). In condizioni di incidenza minima si trovano le aree con prevalenza di colture agricole intensive e/o incolti produttivi (2.1), le infrastrutture tecnologiche (4.2 - elettrodotti aerei). Come per l'equipaggiamento vegetale, le condizioni di incidenza minima dei fattori infrastrutturali tecnologici complessi (3.4) non hanno rilievo essendo relative a fasci infrastrutturali caratterizzati da incidenza più grave. Sono classificati con incidenza irrilevante gli spazi aperti con equipaggiamento vegetale, per le funzioni paesisticoambientali che svolgono, afferendo ad apparati che rispetto agli stati di frammentazione hanno effetti positivi (funzioni protettive prevalenti).
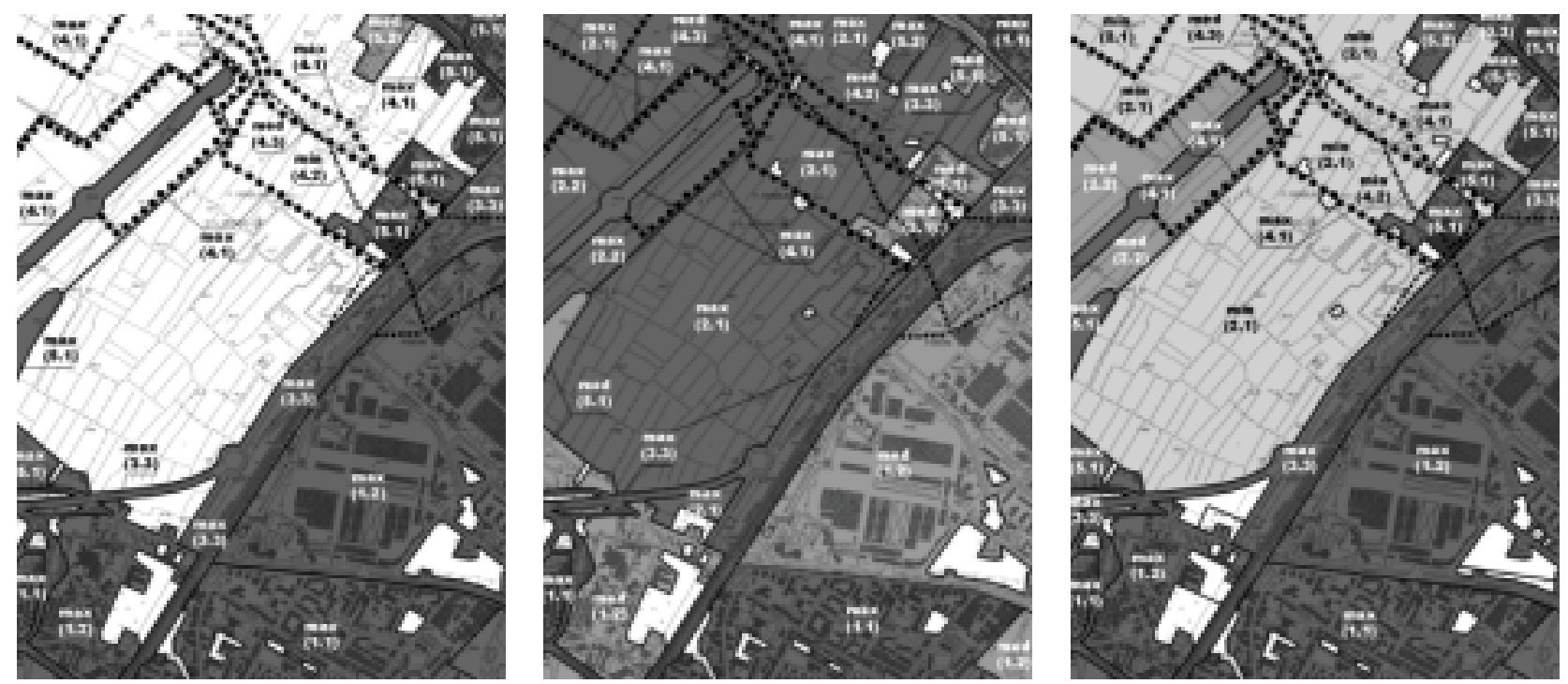

Figura 35 - Diagnosi di frammentazione paesistica - condizioni di stato - $3^{\circ}$ livello diagnostico: incidenza dei fattori territoriali su gi stati di mineralizzazione del paesaggio (a sinistra), su gi stati di equipaggiamento vegetale del paesaggio (al centro), su gi stati di articolazione funzionale del paesaggio (a destra) - esempi di diagnosi tematiche relative a tre dei cinque indicatori primari (cartografie ridotte dagli originali 1:10000 - CTR Regione Toscana). I livelli di incidenza sono indicati dagii indici max, med, min. Per le aree, l'estensione dei livelli di incidenza è messa in rilievo dalle retinature: grigio scuro = $\max$; grigio medio = med; $\min$ (assente); il bianco corrisponde a livelli di incidenza irrilevante; nel caso dell'equipaggiamento vegetale, essi coincidono con gii spazi ove ne sia stata riscontrata la presenza. I fattori lineari semplici sono indicati dalle puntinature. I tipi di incidenza sono identificati univocamente attraverso gi indici numerici doppi (0.0), corrispondenti alle diverse tipologie di fattore.

La sintesi diagnostica del tipo e grado di incidenza dei fattori territoriali su gli stati di frammentazione paesistica permette di considerare la fenomenologia in un quadro sufficientemente unitario per essere confrontato sia allo stesso livello, con la diagnosi del grado di frammentazione del paesaggio (figura $34)$, che ad altri livelli, in chiave transcalare, con le relative interpretazioni diagnostiche elaborate.

Il quadro di incidenza complessiva, di cui la figura 36 riporta l'esemplificazione cartografica, conserva la stessa articolazione in classi utilizzata dalle diagnosi tematiche, facendo ancora parte dello stesso modello di parametrizzazione ( $\mathrm{si}$ veda lo schema in alto nella figura 30). Ciò permette di 
salvaguardare due livelli di lettura necessari per una indicazione sufficientemente completa delle specificità diagnostiche. Alle quattro classi di incidenza (massima, media, minima, irrilevante) corrispondono ambiti omogenei per livello di attenzione, costituiti da spazi in condizioni diverse relativamente ai singoli indicatori primari. Per cui occorre mantenere la perimetrazione delle singole unità spaziali derivanti dall'insieme delle diagnosi tematiche ed associare alla diagnosi di sintesi $l^{\prime}$ indice del fattore territoriale a cui corrispondono gli specifici tipi di incidenza ${ }^{118}$.

$\mathrm{Al}$ fine di chiarire la complementarietà esistente tra i due esiti diagnostici inerenti il grado ed il tipo di incidenza, può essere utile una esemplificazione riferita al soggetto di studio, ricordando la necessità di considerare sempre queste operazioni come dedicate ad applicazioni di tipo dinamico e riferite a vaste estensioni territoriali. Si consideri l'ambito contrassegnato con l'asterisco nella cartografia riportata nella figura 36, relativo ad una porzione di paesaggio periurbano caratterizzato da tessuto insediativo discontinuo (fattore di tipologia 1.2), con prevalenza di destinazioni produttive (attività secondarie e terziarie, miste a servizi pubblici). L'applicazione verticale (relativa a tutti gli indicatori) del modello diagnostico allo specifico fattore censito, produce un giudizio diagnostico connotato da forti limitazioni delle possibilità di correzione ${ }^{119}$ degli stati di frammentazione paesistica, in ragione dell'elevato grado di strutturazione del tessuto urbano, secondo le specificazioni delle opportunità e priorità di intervento di seguito espresse rispetto alle condizioni di alterazione riscontrate attraverso gli indicatori primari.

L'ambito è caratterizzato da possibilità remote di correzione degli stati di mineralizzazione del paesaggio, per la carenza e la discontinuità degli spazi aperti non mineralizzati residui. Il margine di recupero di funzionalità biologica del paesaggio è connesso all'efficacia di interventi di equipaggiamento vegetale con specie arboree d'alto fusto ed arbustive idonee all'impiego in condizioni difficili per inquinamento e condizionamenti spaziali e di soluzioni progettuali e tecnologiche che massimizzino le capacità di drenaggio delle pavimentazioni, considerando la relativa fattibilità tecnica, anche in relazione ai rischi di inquinamento degli acquiferi sotterranei. Risultano inoltre strettamente complementari le misure di protezione e miglioramento ambientale specificamente mirate alle risorse primarie (depurazione delle acque utilizzate e di scolo e protezione delle acque superficiali e di falda, fissazione delle polveri e compensazione delle emissioni inquinanti). Vi sono possibilità condizionate di correzione delle carenze di equipaggiamento vegetale del paesaggio, per ragioni di congestione e carenza di spazi. Divengono strategiche la riserva degli spazi residuali per destinazioni compatibili con una rilevante dotazione di equipaggiamento vegetale, possibilmente spazi verdi destinati alle diverse funzioni che essi possono accogliere, e l'attuazione di interventi di equipaggiamento con specie vegetali di alto fusto ed arbustive per gli spazi esistenti che ne sono privi, nonché di miglioramento delle sistemazioni esistenti in condizioni deficitarie.

Le possibilità di correzione della perdita di permanenza storica del paesaggio sono remote, in ragione del carattere intrinseco di risorsa irriproducibile del paesaggio, particolarmente legato al suo valore culturale di testo storico comprensivo delle diverse espressioni umane, e del grado di strutturazione dei tessuti insediativi, caratterizzato da grave carenza di forme equilibrate di stratificazione storicaedi

118 Relativamente all'esemplificazione riportata nella figura 36, si può osservare come due aree risultate con grado di incidenza complessiva massima possano avere tipi di incidenza diversi in ragione dei fattori responsabili (max(1.1), $\max (1.2), \max (3.3)$ eccetera). Riferendosi allo schema riportato in alto nella figura 30, si consideri come l'incidenza complessiva di un fattore sugii stati di frammentazione paesistica risulti specificamente connotata dalle cinque diagnosi tematiche relative agli indicatori. Ad esempio, secondo una lettura verticale dello schema, l'incidenza dei fattori infrastrutturali viari semplici sarà data dalla considerazione complessiva delle condizioni di stato relative ai binomi causa/sensibilità: 4.1/A, 4.1/B, 4.1/C, 4.1/D, 4.1/E. Il modello non risponde invece ad una lettura orizzontale di sintesi, che darebbe la sensibilità complessiva caratteristica di ogni indicatore. Ciò è dovuto a ragioni connesse con la stessa identificazione degli indicatori (paragrafo 2.3): essendo stati individuati tutti gli indicatori come primari e complementari, sarebbe inutile e discutibile una loro graduatoria per sensibilità.

19 E utile ricordare come tali margini di intervento siano espressi dal punto di vista della fattibilità tecnica, escludendo gli aspetti economici e culturali di ogni ordine e genere che, pur costituendo condizionamenti primari alla praticabilità delle misure, sono variabili che esulano dalle competenze e dalle finalità specifiche di questo studio ed in particolare del modello diagnostico qui considerato (si veda lo schema riportato nella figura 30 in basso). 
rapporto con il contesto paesistico preesistente. I margini di intervento si riferiscono essenzialmente alla salvaguardia preventiva ed alla conservazione attiva dei valori di permanenza storica residuali, limitatamente ad una loro apprezzabilità in relazione alla specifica tipologia di componente paesistica a cui afferiscono.

Risultano remote anche le possibilità di correzione delle carenze e dei conflitti di articolazione spaziale del paesaggio, in ragione del rapporto di forma tipico del fattore, per il quale l'interazione con tipologie paesistiche diverse è ridotta al minimo, e della elevata strutturazione del tessuto urbano, per la quale gli interventi possibili, salvo i casi della ristrutturazione di interi comparti, sono limitati in genere alla realizzazione di sistemazioni vegetali con funzioni di separazione, di marginatura, di schermatura, finalizzate nel loro complesso ad un recupero di qualità morfologica del paesaggio.

Sono remote in questo caso, infine, le possibilità di correzione delle carenze e dei conflitti di articolazione funzionale del paesaggio, per la ridotta disponibilità di spazi aperti idonei e per l'estensione superficiale e la forte strutturazione caratteristica del tessuto urbano. Attraverso misure di equipaggiamento vegetale è possibile migliorare le condizioni di protezione e di connessione ambientale.

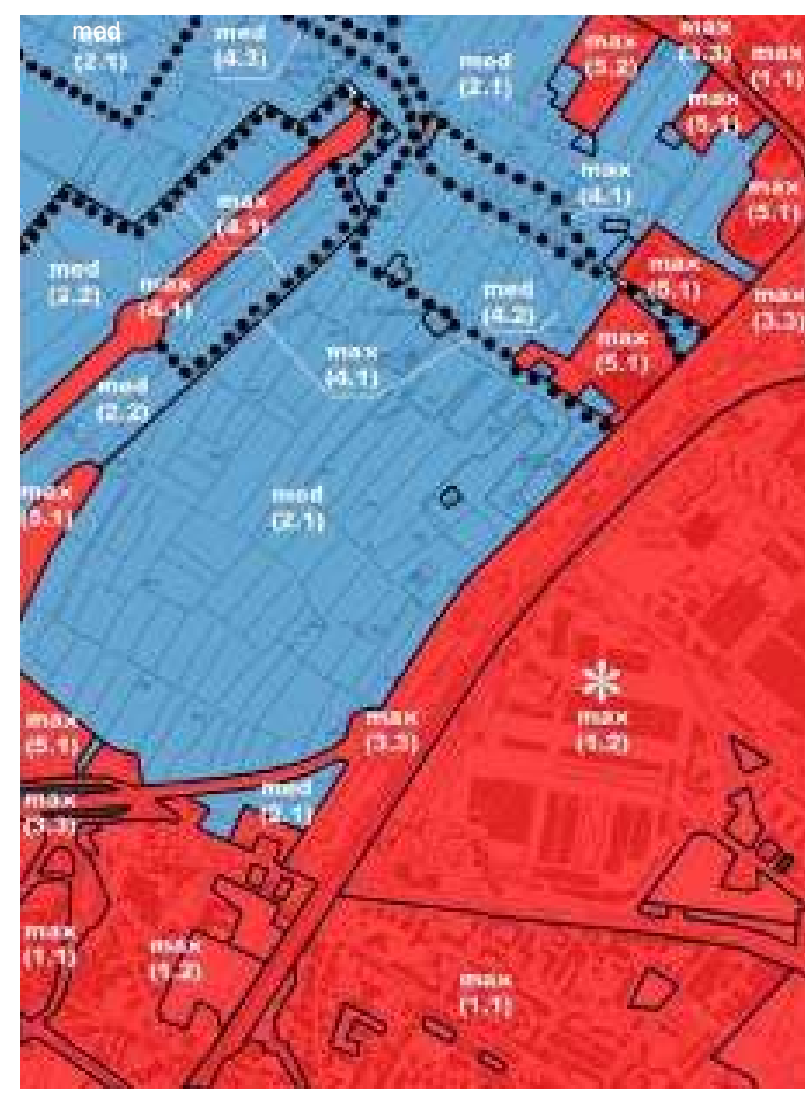

Figura 36 - Diagnosi di frammentazione paesistica condizioni di stato - $3^{\circ}$ livello diagnostico: incidenza complessiva dei fattori territoriali su gli stati di frammentazione paesistica - diagnosi di sintesi (cartografia ridotta dall'originale 1:10000 - CTR Regione Toscana). I livelli di incidenza sono indicati dagii indici max, med, min (assente). Per le aree L'estensione dei livelli di incidenza è messa in rilievo dalle retinature: rosso nella pubblicazione WEB (grigio scuro nella stampa) = max; azzurro nella pubblicazione WEB (grigio chiaro nella stampa) $=$ med. I tipi di incidenza restano specificamente indicati dagii indici numerici doppi (0.0) corrispondenti alle diverse tipologie di fattore.

In conclusione è possibile riferire la diagnosi di sintesi dell'incidenza complessiva (figura 36) a quattro classi teoriche, non sempre riscontrabili in un dato ambito di studio.

La classe di incidenza irrilezante, coincide con l'assenza di fattori territoriali di frammentazione paesistica. La diagnosi rileva condizioni di conservazione delle risorse e di sensibilità alla alterazione degli equilibri paesistici per le quali è di importanza prioritaria adottare un livello di attenzione alto (misure di prevenzione della frammentazione paesistica potenziale), in riferimento ad eventuali processi di frammentazione nelle aree considerate ed in quelle limitrofe. Si tratta di ambiti prioritari per la salvaguardia e/o la conservazione delle risorse (la classe non è rappresentata in questo caso, per la forte pressione insediativa che caratterizza l'ambito considerato). 
La classe di incidenza minima risulta riferita esclusivamente ai corsi d'acqua naturali regimati (si vedalo schema riportato in alto nella figura 30). La diagnosi rileva condizioni di conservazione delle risorse e di alterazione degli equilibri paesistici per le quali occorre adottare un livello di attenzione alto (misure di prevenzione e contenimento della frammentazione paesistica) in particolare in riferimento allo sviluppo di fattori territoriali con elevato potenziale di frammentazione, ad esempio infrastrutture ed urbanizzazioni (la classe non è rappresentata in questo caso, per la completa assenza di corsi d'acqua naturali regimati nell'ambito considerato).

In classe di incidenza media la diagnosi rileva condizioni di rapporto tra stati critici e stati di conservazione delle risorse per le quali occorre adottare idonee forme di intervento (misure attive di contenimento e riduzione della frammentazione paesistica), facendo fronte ai condizionamenti di ordine spaziale, funzionale, normativo.

Infine, in classe di incidenza massima la diagnosi rileva condizioni di rapporto tra stati critici e stati di conservazione delle risorse per le quali è di importanza prioritaria adottare idonee forme di intervento (misure attive di contenimento e riduzione della frammentazione paesistica), oltre che facendo fronte ai condizionamenti di ordine spaziale, funzionale, normativo, considerando il significato strategico della gestione dello spazio in relazione alle gravi carenze quantitative e qualitative.

Come è accaduto per l'analisi dei fattori territoriali, anche per la diagnosi di frammentazione paesistica, è necessario considerare i risultati delle indagini nel contesto di cui il soggetto di studio fa parte parte, relativizzandoli alle condizioni complessive di questo. La collocazione del campione ridotto, che ha permesso di sviluppare le considerazioni di dettaglio sul mosaico degli spazi aperti, nell'ambito del campione più ampio del territorio della piana ad ovest ed a nord-est di Firenze, mostra come ovviamente la situazione muti in modo rilevante nelle vicine aree pedecollinari di paesaggio rurale e urbano, nelle aree collinari forestali o nelle aree urbane dove siano presenti complessi di spazi verdi di rilevante interesse. Sebbene questo concetto di relativizzazione sia qui strumentale alla sola comprensione dei dati sperimentali, in quanto, in caso di applicazione pratica, tali riscontri si avrebbero ben più evidenti dagli esiti delle analisi e diagnosi, è pur vero che in qualunque applicazione si ha sempre a che fare con un soggetto che ha un contesto territoriale di riferimento e che quest'ultimo è facilmente più vasto dell'ambito di studio. Da ciò risulta che può essere comunque utile adottare questa forma di controllo e completamento del processo conoscitivo, in particolare in sede di diagnosi.

In tal senso si consideri infine come i caratteri esemplificati per gli ambiti del tipo di quello contrassegnato con l'asterisco nella cartografia, al quale corrispondono diagnosi di gravi condizioni di frammentazione e possibilità di correzione di questi stati alquanto limitate, non debbano ovviamente deviare la valutazione generale circa le possibilità di intervento nelle aree periubane, che di fatto sono molte e diversificate, appena si allarghi l'orizzonte a tutta la casistica della manifestazione della problematica studiata e della articolazione tipologica degli spazi aperti.

\section{4 - La diagnosi delle alterazioni potenziali del paesagoio}

Il tema della diagnosi delle alterazioni potenziali di frammentazione del paesaggio viene affrontato con esclusivo riferimento alle cause di ordine antropico, in ragione dell'importanza delle componenti culturali in termini di condizionamenti degli equilibri paesistico-ambientali nelle aree periurbane di pianura; non vengono considerate le crisi strutturali e le involuzioni di ordine naturale, per l'oggettiva irrilevanza che esse mostrano.

Le analisi e le diagnosi proposte per i fattori potenziali permettono di considerare gli aspetti connessi alle politiche territoriali esplicitate dagli strumenti di pianificazione. Restano fuori dall'influenza di tale intepretazione tutti quei processi di natura socioeconomica che sfuggono al governo, talvolta anche alla sola identificazione, da parte dei piani territoriali. Occorre però osservare come le tendenze 
evolutive di taluni fenomeni di natura socioeconomica siano già leggibili nella diagnosi delle condizioni di stato, soprattutto quando si disponga di informazioni diacroniche. In questi casi, l'interpretazione delle topologie (dimensione spazio) e delle cronologie (dimensione tempo) degli effetti paesistici riferibili a cause di ordine socioeconomico, permette di identificare le tendenze e quindi i potenziali di alterazione caratteristici di tali fattori. Il paesaggio delle campagne è portatore delle più palesi manifestazioni della intensità e della diffusione che le alterazioni dovute a questo ordine di fattori sono in grado di indurre. Si pensi alle alterazioni dovute ai cambiamenti del mosaico delle proprietà delle terre ed ai relativi cambiamenti di tipologia socioeconomica delle proprietà stesse, ma anche alle involuzioni del paesaggio rurale, dovute all'inurbamento, prima, ed alla urbanizzazione delle campagne, poi, sempre più legata alla terziarizzazione dei processi produttivi. Da fattori di questo genere non sono certo indenni i paesaggi periurbani: l'annoso, controverso ed irrisolto problema del regime dei suoli è senza dubbio fra i più importanti e, ad oggi, assolutamente al di fuori di forme di governo da cui sia lecito attendersi garanzie di salvaguardia delle risorse paesistico-ambientali.

Con riferimento allo schema proposto (si vedano il paragrafo 3.1 e specificamente le figure 27 e 29 ), la diagnosi delle alterazioni potenziali del paesaggio si esplica essenzialmente al terzo livello, pur permettendo il primo livello alcune valutazioni di interesse per quanto riguarda le politiche territoriali di scala vasta. Gli altri livelli non possono essere correttamente e concretamente considerati, non essendo desumibili con attendibilità dai piani, né gli effetti sull'articolazione del mosaico paesistico (secondo livello), né le specifiche modificazioni dell'articolazione degli spazi aperti (quarto livello).

L'analisi dei fattori potenziali di frammentazione paesistica (paragrafo 2.2) non ha rilevato nell'ambito di studio elementi significativi per quanto concerne le politiche territoriali sovracomunali, pertantola diagnosi relativa al primo livello non trova argomenti di criticità, determinando così un giudizio positivo. Per l'entità delle trasformazioni che considera, risulta invece necessario riferire la diagnosidi terzo livello al quadro territoriale in cui ha le più importanti incidenze paesistiche (figura 37) al fine già discusso di avere una corretta visione transcalare, che contribuisca alla miglior identificazione del fenomeno. In ragione dell'analisi dei fattori potenziali di frammentazione paesistica, che ha condotto una lettura dei piani regolatori comunali secondo le corripondenze tipologiche alla classificazione dei fattori territoriali, la diagnosi di incidenza segnala gli elementi di rischio in termini di frammentazione potenziale del paesaggio, permettendo di definire le diverse istanze di contenimento e riduzione.

Nell'eventualità che vengano riscontrate alterazioni potenziali in relazione a previsioni di piano che comprendano anche funzioni di compensazione paesistico-ambientale, occorre ponderare i livelli diagnostici previsti dal modello rispetto a tali potenziali. Infatti, la presenza di spazi e sistemi di spazi con questi ruoli comporta la necessità di una loro valutazione in termini di bilancio paesisticoambientale complessivo, attraverso un approfondimento conoscitivo e progettuale delle stesse indicazioni di piano. È il caso ad esempio dell'ambito contrassegnato con l'asterisco nella figura 37, per il quale si rimanda alla descrizione dello scenario paesistico sviluppato per l'area periurbana di Castello, come specifica simulazione sperimentale di tali concetti (paragrafo 4.2).

Si osservi infine che la cartografia di diagnosi della frammentazione paesistica potenziale evidenzia eclusivamente le previsioni di piano non attuate, mentre le forme di governo delle aree con previsioni attuate rientrano nella valutazione delle condizioni di stato.

Analogamente a quanto è stato fatto per le condizioni di stato, anche per le alterazioni potenziali, è possibile riferire la diagnosi (figura 37) a quattro classi teoriche, non sempre riscontrabili in un dato ambito di studio.

La classe di incidenza irrilezante coincide con l'assenza di fattori territoriali di frammentazione paesistica potenziale. La diagnosi non rileva forme di governo territoriale responsabili di possibile insorgenza o incremento degli stati di frammentazione. 
Nei casi in cui la diagnosi relativa ai fattori potenziali riscontrabili dal quadro della pianificazione territoriale non rilevi rischi, ma si riscontri comunque la corrispondenza di stati di frammentazione a forme di uso e gestione delle risorse, pur non direttamente imputabili di tali effetti, è opportuno ricorrere a specifici approfondimenti socioeconomici. Si pensi, ad esempio, al caso comune delle aree agricole regolamentate come zone omogenee, più o meno diversificate, nelle quali gli effetti dell'evoluzione agraria, tecnologica e politico-economica, si manifestano come sostanziali modificazioni paesistiche.

Fra le misure di prevenzione dei processi di frammentazione risulta prioritaria (eccetto che nel caso dei tessuti urbani consolidati) l'adozione di politiche di conservazione attiva e/o di miglioramento paesistico-ambientale attraverso, la definizione di specifici programmi e progetti di paesaggio, in grado di destinare e governare le aree, evitando i rischi della loro marginalizzazione, isolamento e progessiva riduzione.

La classe di incidenza minima, assente nell'area di studio, è riferita esclusivamente ai corsi d'acqua naturali regimati e pertanto significativa in presenza di fiumi e torrenti con previsioni di piano relative al loro assetto idraulico e più in generale paesistico-ambientale.

La diagnosi rileva in tali casi forme di governo territoriale rispetto alle quali occorre definire idonee politiche di prevenzione dei processi di frammentazione, con attenzione specifica alla localizzazione ed alle modalità di realizzazione delle sistemazioni idrualiche.

La classe di incidenza media, assente nell'area di studio, è riferita a previsioni di spazi per colture agrarie intensive, per attività con aree soggette a vincoli di inutilizzazione, per strutture ed infrastrutture tecnologiche e di canali artificiali. In tali casi, la diagnosi rileva forme di governo territoriale rispetto alle quali occorre definire idonee politiche di prevenzione dei processi di frammentazione, con attenzione specifica alla localizzazione ed alla tipologia di destinazione ed ai relativi conflitti potenziali, prevedendone l'eliminazione o almeno la compensazione.

La classe di incidenza massima è attribuita agli sviluppi insediativi ed infrastrutturali. La diagnosi rileva forme di governo territoriale rispetto alle quali risulta prioritario definire idonee politiche di prevenzione dei processi di frammentazione, con attenzione specifica alla riserva di congrui spazi di compensazione, oltre che alla localizzazione ed alla tipologia delle opere ed al controllo dei relativi conflitti potenziali.

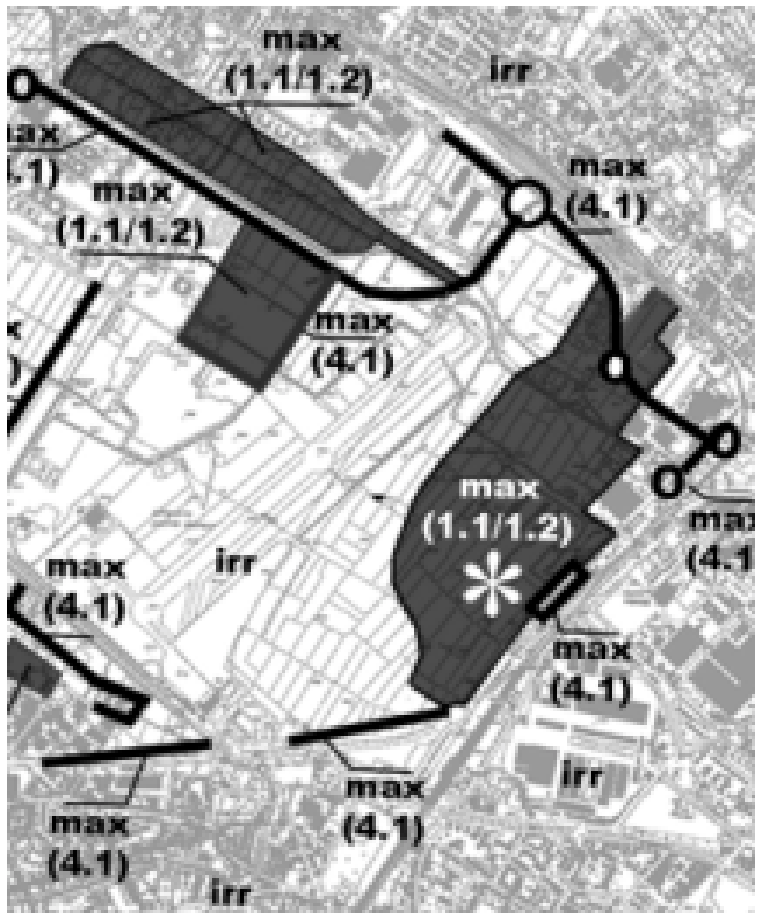

Figura 37 - Diagnosi di frammentazione paesistica alterazioni potenziali - $3^{\circ}$ livello diagnostico - incidenza dei fattori territoriali sugi stati di frammentazione (cartografia ridotta dall'originale 1:10000 - CTR Regione Toscana). I livelli di incidenza sono indicati dagli indici max, med, $\min$ (assente). Il bianco corrisponde a situazioni di presunta incidenza irrilevante. 


\section{4 - Verso un modello di scenario strategico per la riqualificazione diffusa del paesaggio periurbano}

\section{1 - Il contributo simulatizo degi scenari teorici di riqualificazione diffusa del paesagoio}

Per definizione, il progetto paesistico ha come obiettivo prioritario la promozione e la produzione di forme equilibrate di suo e gestione delle risorse, sensibili rispetto ai caratteri strutturali profondi del paesaggio, dovuti alla geolitologia, alla geomorfologia ed alla idrogeologia, come alle configurazioni culturali derivanti dai processi di trasformazione e regimazione idraulica e di strutturazione rurale ed urbana. Secondo questo presupposto, è possibile ipotizzare alcuni scenari teorici di recupero degli stati di frammentazione del paesaggio periurbano di pianura. Essi si basano sulle specificità del contesto di studio, con riferimento alla marginalità di alcune forme socioeconomiche, fra cui per prima l'agricoltura, alle tendenze di sviluppo, che altre forme confermano o manifestano, fra cui la spinta dell'infrastrutturazione e dello sviluppo insediativo, legato alla progressiva articolazione del settore produttivo terziario.

In base alle caratteristiche del paesaggio ed ai relativi livelli di congestione e di deficit qualitativi e quantitativi del tessuto degli spazi aperti, sono stati ritenuti significativi sei scenari rispondenti all'obiettivo primario del contenimento e della riduzione della frammentazione paesistica: (1) equipaggiamento paesistico ${ }^{120}$ delle grandi infrastrutture viarie e delle relative aree di pertinenza e/o interstiziali; (2) equipaggiamento paesistico della rete viaria locale; (3) equipaggiamento paesistico della rete viaria rurale; (4) equipaggiamento paesistico dei corsi e corpi d'acqua e delle aree di intervento per la difesa idrogeologica; (5) equipaggiamento e recupero paesistico di aree degradate, interstiziali o con utilizzi marginali; (6) equipaggiamento paesistico di aree soggette a vincoli infrastrutturali di inutilizzazione ed inaccessibilità.

Questa operazione, evidentemente retorica e strumentale a finalità esclusisvamente dimostrative, è stata condotta riferendosi a tipologie di intervento prive di reali limitazioni tecniche, ottenendo pertanto un quadro la cui reale fattibilità è condizionata solo da fattori di ordine normativo, amministrativo e finanziario, che non hanno rilievo ai fini della discussione teorico-metodologica inerente la ricerca.

Sono stati tralasciati altri possibili obiettivi primari, come l'equipaggiamento delle colture agricolecon siepi di campo, in ragione della loro subordinazione rispetto a sistemi di scala più ampia e della citata marginalità economica che caratterizza l'agricoltura in queste aree. Sono stati tralasciati, inoltre, anche obiettivi praticabili con esiti di riqualificazione paesistica diffusa solo se inseriti in più ampie e sistemiche politiche strutturali: vi rientrano le coltivazioni agricole ad alta redditività e/o biologiche, come anche le coltivazioni a fini sociali, e specificamente, ricreativi, educativi e rieducativi.

120 Per equipagoiamento paesistico, secondo l'accezione anglosassone di equipment, si intende qui un complesso organico di interventi con finalità ecologiche ed ambientali, morfologiche e percettive, sociali e culturali, incentrati sull'utilizzo prevalente di materiali viventi (terre e piante, erbacee, arbustive ed arboree). 
L'attributo teorico, legato alla finalità modellistica di queste simulazioni, costruite con l'intento di rappresentare il tipo di diffusione che le politiche di riqualificazione paesistica potrebbero raggiungere, è riferito essenzialmente a due ordini di caratteri. Innanzi tutto dà conto dell'attribuzione diretta delle misure di riqualificazione a tutte le componenti presenti facenti parte di una determinata tipologia, al fine di non incorrere nella soggettività connaturata alla progettazione, non congruente con le specifiche finalità di queste elaborazioni. In secondo luogo, si riferisce all'astrazione delle misure di intervento dai citati condizionamenti dati dalle realtà socioeconomica e normativa, per la loro spiccata suscettibilità al cambiamento, che conferirebbe caratteri di contingenza alle simulazioni, subordinandole inoltre ai limiti ed all'inadeguatezza di talune leggi, norme e comportamenti.

La simulazione degli scenari teorici relativi agli obiettivi primari individuati è riferita al paesaggio periurbano dello stesso campione territoriale di indagine utilizzato per le analisi e le diagnosi di frammentazione.
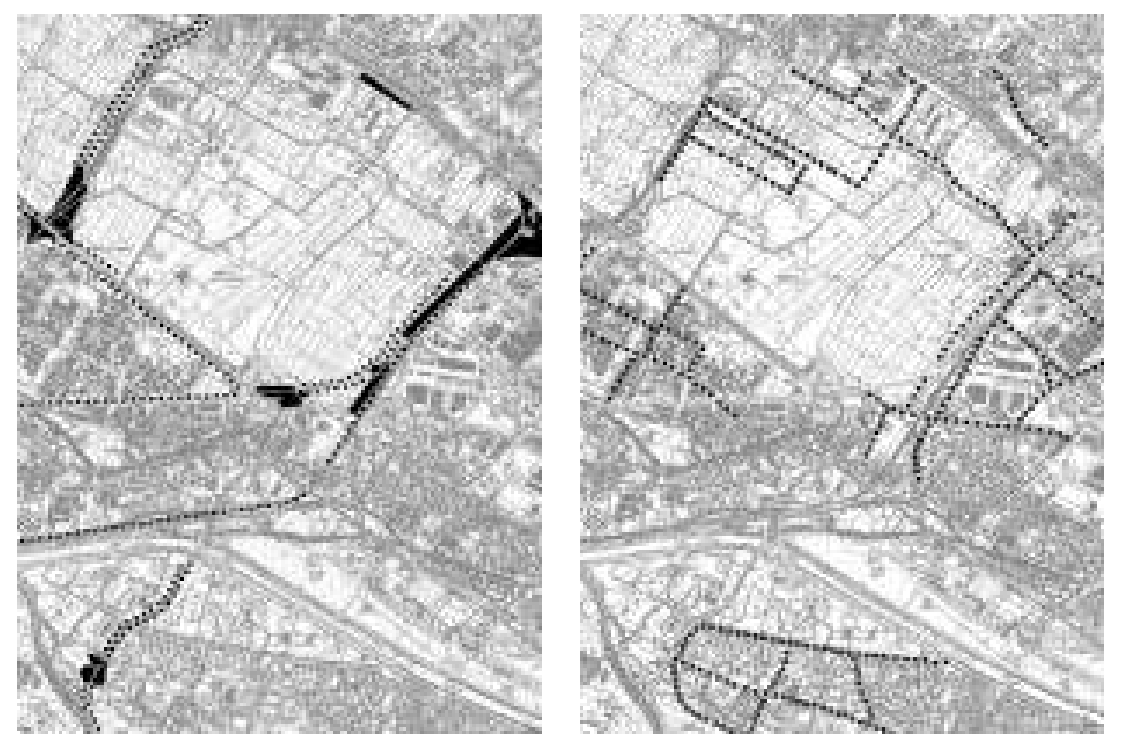
Figura 38 - Simulazioni degli scenari teorici (1) "Equipagoiamento paesistico delle grandi infrastrutture viarie e delle relatize aree di pertinenza e/ o interstiziali", a sinistra e (2) "Equipagoiamento paesistico della rete viaria locale", a destra (cartografia ridotta da originale 1:10000 - CTR Regione Toscana).

Gli scenari 1 - equipagoiamento paesistico delle grandi infrastrutture viarie e delle relatize aree di pertinenza e/ o interstiziali e 2 - equipagoiamento paesistico della rete viaria locale sono legati alla rete infrastrutturale edalle opportunità di riqualificazione del paesaggio, oltre che di qualificazione degli spazi stradali, che potrebbero concretizzarsi nella misura in cui fossero stabiliti, sul piano normativo e finanziario, idonei standard e tipologie di equipaggiamento vegetale arboreo ed arbustivo degli spazi ad esse limitrofi. Entrambi gli scenari sono fortemente trasversali alle diverse configurazioni paesistiche che connotano l'area di studio, dall'urbano denso e consolidato, fino alle estensioni di monocolture agricole con presenza rilevante di spazi abbandonati o sottoutilizzati.

Le opportunità di intervento, che nelle aree urbanizzate si riducono progressivamente al crescere della congestione spaziale, restano in genere comunque rilevanti, soprattutto per quanto riguarda la continuità delle formazioni vegetali ottenibile ed i relativi contributi all'incremento di qualità ecologica e semiologica del paesaggio ed alla sua deframmentazione. Le strade sono infatti, assieme ai corsi d'acqua, reti strategiche, per la continuità della proprietà pubblica dei suoli e le conseguenti possibilità di intervento. Questi caratteri di trasversalità territoriale rispetto alle diversificazioni del paesaggio costituiscono requisiti importanti per la riqualificazione diffusa delle aree periurbane, permettendo la loro strutturazione secondo configurazioni sistemiche a rete, che possono assumere rilievo semiologico e risultare dotate di un apprezzabile potenziale ecologico di rigenerazione e protezione, nonchè idonee a migliorare l'articolazione degli spazi, secondo forme nuove di equilibrio tra i tessuti urbani consolidati, le periferie e le campagne urbanizzate. Nelle simulazioni riportate nella figura 38 è evidente la complementarietà di questi due scenari, che hanno già per proprio conto un rilevante potenziale di diffusione territoriale di misure di recupero di qualità del paesaggio. 
Gli scenari 3 - equipagiamento paesistico della rete viaria rurale e 4 - equipaggiamento paesistico dei corsi e corpi d'acqua e delle aree di interzento per la difesa idrogeologica sono legati a due importanti reti territoriali: quella della viabilità rurale e quella dei corsi d'acqua che, pur coincidendo in pianura prevalentemente con canali di bonifica idraulica e con corsi d'acqua naturali canalizzati, costituiscono un sistema importante della struttura del paesaggio. Possono pertanto rientrare in questo binomio di scenari le misure di riequipaggiamento del paesaggio agrario di pianura indirizzate prioritariamente verso questi sistemi di cui, pur riconoscendo l'importanza del reticolo di equipaggiamento minore delle siepi e macchie di campo, occorre considerare il valore strutturale e strategico, non solo in termini ambientali, ma anche ed in misura non minore, in termini spaziali e storici, per quanto può concernere la conservazione delle tracce e dei segni relittuali di permanenza.

Simili ai precedenti per i caratteri di trasversalità territoriale, questi due scenari permettono strategie di miglioramento paesistico di importanza prioritaria dal punto di vista della fruizione degli spazi aperti da parte delle popolazioni urbane supportata da sistemi di mobilità alternativa, che, attraverso la viabilità rurale, possono risultare connessi a quelli urbani principali ed ai corsi d'acqua, costituendo vie di penetrazione privilegiate, per l'elevato grado di protezione che spesso permettono rispetto alla viabilità tradizionale ad alto carico di traffico.

Per le simulazioni riportate nella figura 39 vale la stessa osservazione fatta per i precedenti scenari $1 \mathrm{e}$ 2, considerando ovviamente, anche in questi casi, la complementarietà che li caratterizza e che, se essa non è estendibile ai precedenti scenari sul piano funzionale della fruizione umana, ciò avviene pienamente per gli aspetti di recupero di qualità morfologica ed ecologica del paesaggio, rispetto ai quali gli scenari 1, 2, 3, 4 contribuiscono a costituire un quadro unitario relativo ad un sistema a rete di spazi aperti differenziati, dotati di congrue quantità e tipologie di equipaggiamento vegetale.
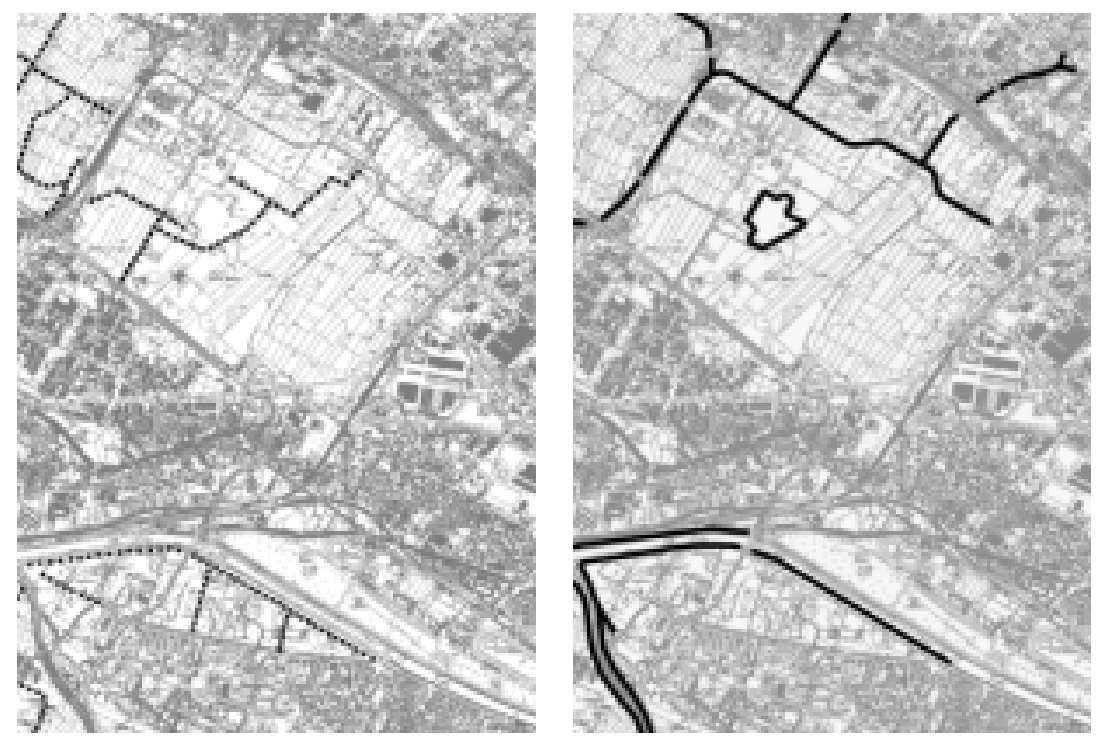

Figura 39 - Simulazioni degi scenari teorici (3) "Equipagoiamento paesistico della rete viaria rurale", a sinistra e (4) "Equipagoiamento paesistico dei corsi $e$ corpi d'acqua e delle aree di interzento perla difesa idrogeologica", a destra (cartografia ridotta da originale 1:10000 - CTR Regione Toscana).

Gli ultimi due scenari considerati, 5 - equipaggiamento e recupero paesistico di aree degradate, interstizialiocon utilizzi marginali e 6 - equipaggiamento paesistico di aree soggette a vincoli infrastrutturali di inutilizzazione ed inaccessibilità, nettamente distinti per tipologia degli spazi di intervento, sono accomunati dal fattoche non sono finalizzabili alla individuazione di sistemi lineari di spazi aperti, ma riguardano piuttosto situazioni relative ad aree, talvolta estese, talvolta anche alquanto limitate, da poter essere considerate di tipo relativamente puntuale. Lo scenario 5 è relativo alla tematica, estremamente delicata e difficile, del recupero delle aree abbandonate o degradate e della conversione di aree con utilizzi marginali, che, nel tessuto congestionato del paesaggio urbano contemporaneo, possono assumere valore strategico, per le potenzialità di miglioramento, come, ove non vi sia una gestione consapevole ed attiva del fenomeno, possono costituire pesanti ipoteche sulla perseguibilità stessa della qualità urbana. Le ipotesi di recupero ambientale e progettazione del paesaggio di queste aree rivestono 
un'importanza rilevante sia nei confronti degli insediamenti urbani nel loro complesso, dal punto di vista della regolazione e protezione ambientale, che dal punto di vista della efficacia di riordino del paesaggio periurbano e di limitazione dei conflitti per intervisibilità e/o promiscuità spaziale. La simulazione riportata nella figura 40 è costruita per difetto, non comprendendo in questa categoria la presenza rilevante di incolti produttivi nella piana di Castello, per l'eplicito destino urbano di queste aree, in forza del quale ad esse non è attribuibile correttamente il carattere di marginalità per abbandono.
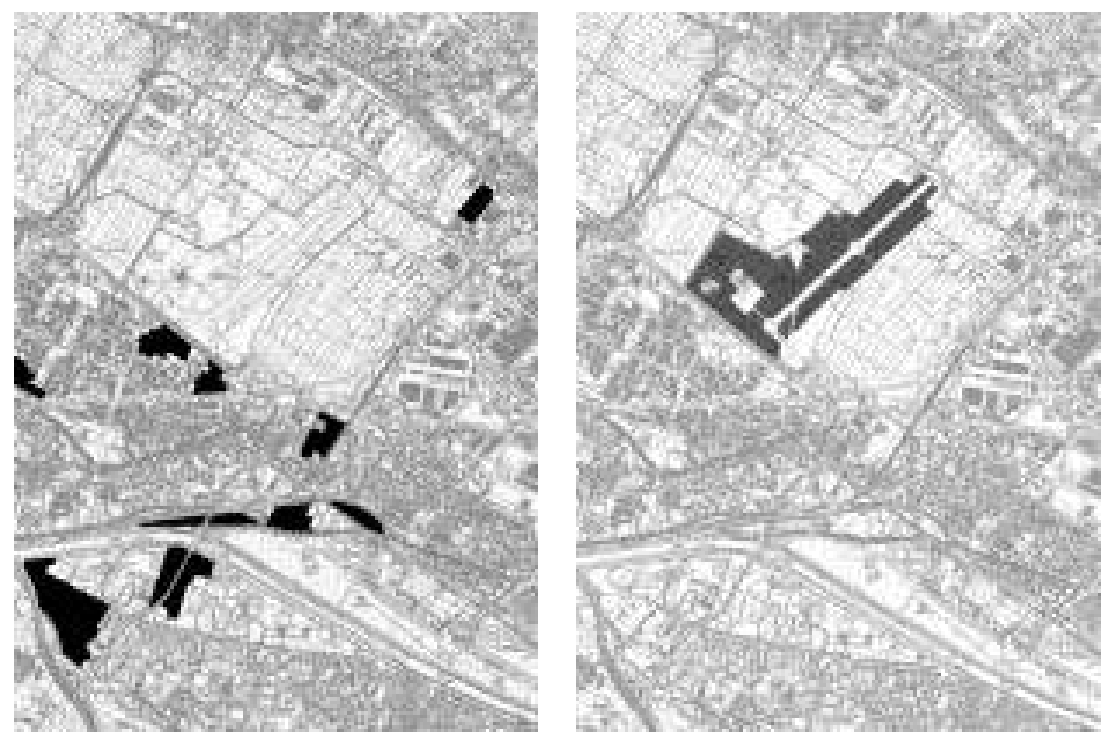

Figura 40 - Simulazioni degii scenari teorici (5) "Equipagoiamento e recupero paesistico di aree degradate, interstiziali o con utilizzi marginali", a sinistra e (6) "E quipagoiamento paesistico di aree soggette a incoli infrastrutturali di inutilizzazione ed inaccessibilità" a destra (cartografia ridotta da originale 1:10000 - CTR Regione Toscana).

Risulta diversa invece la situazione dell'aereoporto, relativa alla simulazione del ruolo potenziale di interventi di sistemazione paesistica di spazi soggetti a vincoli di inutilizzazione ed inaccessibilità. $\mathrm{Nel}$ rispetto di ogni requisito di sicurezza dell'esercizio delle attività, gli spazi di pertinenza non mineralizzati e non interessati dalle manovre di autoveicoli ed aerei, costituscono un complesso assi esteso di aree per le quali è possibile pensare a soluzioni progettuali, tecnologiche e gestionali, rispondenti ad obiettivi di incremento della qualità morfologica ed ecologica del paesaggio, non ultimo considerando le migliori forme di inserimento nel contesto paesistico, in questo caso in ragione della percezione terrestre, ma anche aerea. I problemi tecnici che impediscono l'impiego di specie arboree ed arbustive non costringono, sul piano della fattibilità, ad avere le condizioni attuali prive di qualunque connotazione di architettura del paesaggio. È possibile ipotizzare lo studio di idonee forme di impiego di specie erbacee diverse, di specie tappezzanti, di specie igrofile in aree pilotate in condizioni di allagabilità attraverso la modificazione mirata del sistema di drenaggio dei suoli (con la possibilità di valutare l'utilizzo per fitodepurazione), mantenendo le necessarie attenzioni alle esigenze di sicurezza, legate ad esempio alla preclusione della presenza di determinate specie animali, in particolare di uccelli. In relazione alle limitazioni di fattibilità degli interventi, ove non sia possibile modificare efficacemente i caratteri spaziali, è prioritaria la messa a punto e l'attuazione di misure di miglioramento paesistico che, sotto controllo progettuale, prevedano l'estensione di tali caratteri ad aree limitrofe, favorendo l'integrazione morfologica nel mosaico degli spazi aperti. A Firenze ad esempio, ad est, le aree destinate alla realizzazione del parco di Castello, ad ovest, le aree agricole, sarebbero idonee all'eventuale adozione di misure di questo tipo nei confronti delle aree aereoportuali. La reale importanza della considerazione di interventi relativi a questi aspetti, risulta evidente anche solo pensando che l'ambito di studio costituisce, attraverso l'aereoporto, l'autostrada e la ferrovia ad alta velocità, e malgrado gli evidenti livelli di degrado del paesaggio, la "porta principale" dei transiti internazionali e nazionali relativi ad una delle città universalmente ritenute più importanti. 
Rispetto all'interesse specifico di questo lavoro per le configurazioni di spazi aperti con sviluppo lineare dominante, nel prossimo paragrafo 4.2, viene proposto un loro ordinamento, che fa capo innanzi tutto alla distinzione nelle due categorie principali dei sistemi semplici di spazi aperti e dei sistemi complessi di spazi aperti (si vedano le relative definizioni).

Gli scenari teorici illustrati, individuano prevalentemente configurazioni lineari afferenti alla categoria dei sistemi semplici di spazi aperti, relativi a strade, corsi d'acqua, campi agricoli, eccetera. Ma ampie possibilità di applicazione sono connesse sul piano teorico alle configurazioni afferenti a sistemi complessi di spazi aperti: lo scenario teorico complessivo (figura 41) ne indica più casi, relativi a fasci infrastrutturali, corsi d'acqua ed aree intercluse.

Occorre osservare in tal senso come un complesso di spazi aperti marginali, in parte abbandonati o con utilizzi impropri, difficilmente accessibili a causa dei condizionamenti delle infrastrutture viarie, insieme a spazi aperti di pertinenza di attrezzature pubbliche o di interesse pubblico di diverso tipo, possa essere considerato secondo un disegno unitario, in cui le sistemazioni dei singoli spazi vengano realizzate rispondendo ad una articolazione di funzioni paesistico-ambientali pensata in relazione al contesto insediativo, rispettando requisiti di relazione sistemica, ottenibili solo attraverso il coordinamento dei diversi interventi, finalizzato espressamente ad obiettivi di scala superiore rispetto a quelli ottenibili attraverso la riqualificazione dei singoli spazi.

Rientrano in questo ordine di finalità di livello superiore, gli aspetti semiologici inerenti il conferimento di identità spaziale ai paesaggi periurbani caratterizzati da stridente congestione e parcellizzazione dei segni, ma anche gli aspetti funzionali legati alla costituzione di sistemi di mobilità privata alternativa a quella meccanizzata, come gli aspetti di protezione e regolazione ambientale, per i quali risulta essenziale l'incremento delle quantità e qualità della vegetazione arborea ed arbustiva.

Tipologie di intervento considerate dai sei scenari tematici (misure prevalentemente pubbliche e prioritarie)

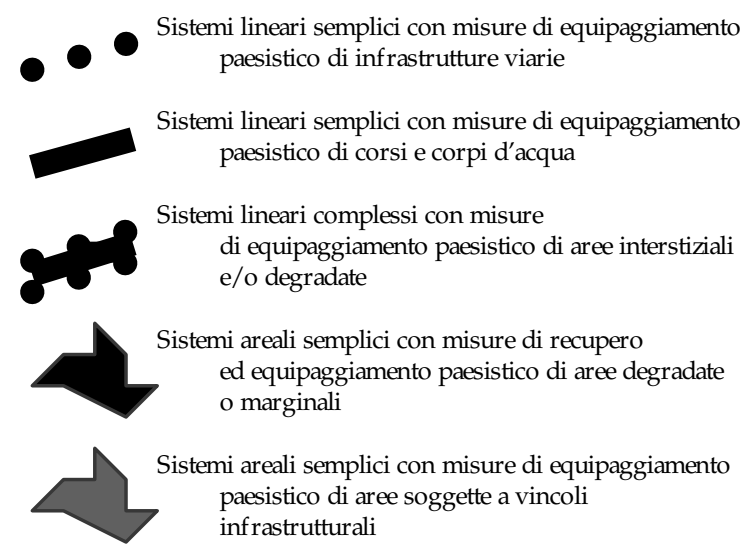

Tipologie di intervento integrative (misure prevalentemente private da incentivare e regolare attraverso convenzioni)

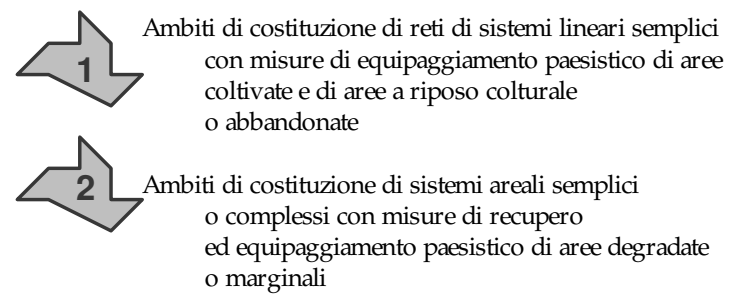

Figura 41 - Simulazione dello scenario teorico complessivo di riqualificazione diffusa del paesaggio periurbano di pianura (cartografia ridotta da originale 1:10000 - CTR Regione Toscana).

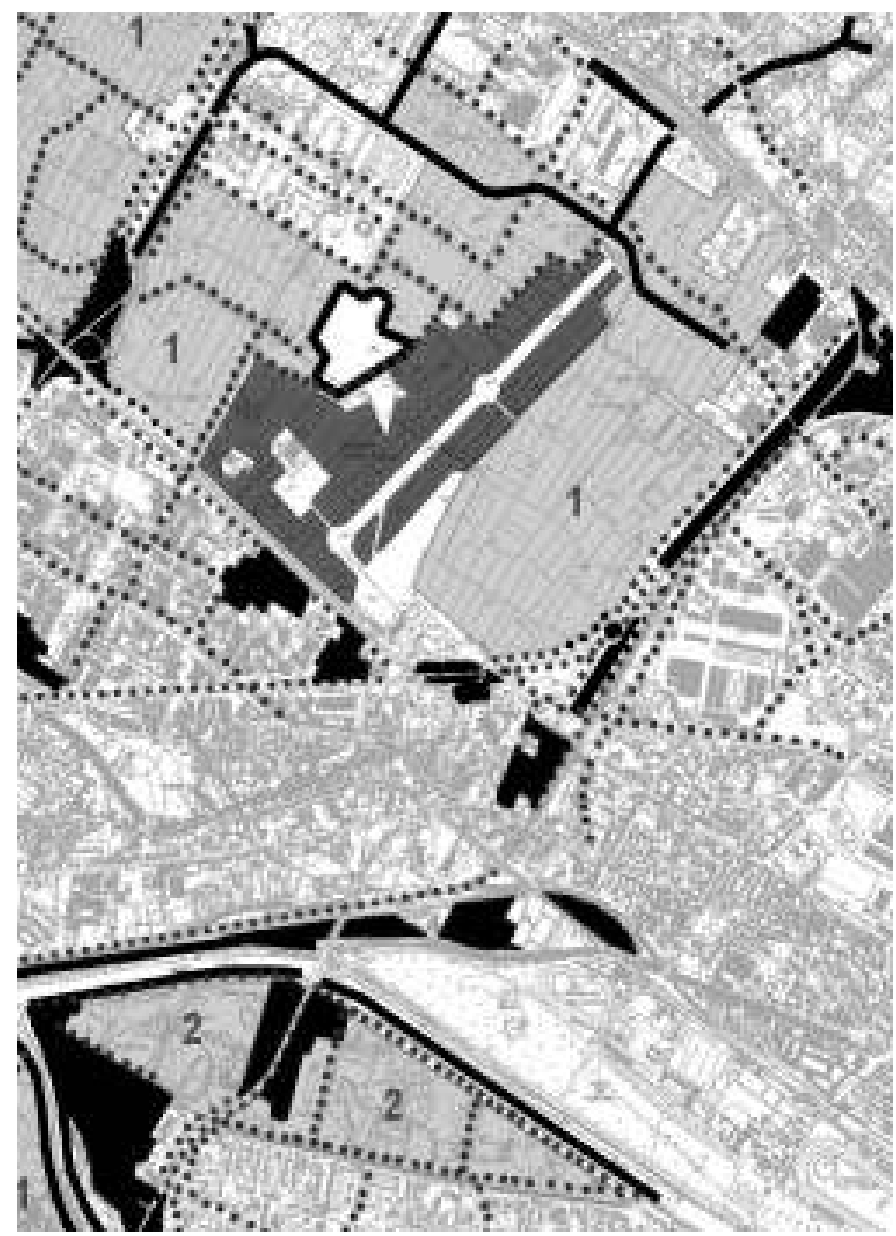


Altri sistemi lineari di tipo complesso potrebbero riguardare strategie territoriali di scala vasta finalizzate ad innescare e garantire processi di progressivo recupero di condizioni di equilibrio paesistico-ambientale delle aree periurbane, anche a favore della praticabilità stessa di eventuali espansioni insediative in condizioni di salvaguardia delle risorse, secondo i principi dello sviluppo sostenibile, o, quanto meno, di compensazione delle alterazioni. Il bilancio complessivo dei sei scenari teorici e dei relativi effetti potenziali di riqualificazione diffusa del paesaggio, mostra l'importanza potenziale delle configurazioni paesistiche lineari, a cui si riferiscono quattro scenari su sei. Un'importanza che indubbiamente ha un valore assoluto, nel paesaggio agrario padano come in quello dell'area metropolitana fiorentina, ma, diviene ancora più significativa nei confronti delle problematiche di congestione e disarticolazione degli spazi dei paesaggi periurbani di pianura, rispetto alle quali le simulazioni mostrate producono un'evidenza, supportata dalle considerazioni tipologiche sviluppate. Dalla articolazione cartografica complessiva degli scenari terorici (figura 41), risulta evidente il potenziale di diffusione e di diversificazione di impiego che caratterizza i sistemi paesistici lineari e pertanto anche il nesso logico tra le procedure di analisi e diagnosi della frammentazione paesistica, sviluppate nei capitoli precedenti, ed i criteri progettuali riguardanti i sistemi lineari dispazi aperti, sviluppati dal paragrafo successivo. Immaginando di applicare le tecniche di conoscenza ad una realtà paesistica diffusamente rivista, come quella prefigurata nello scenario della figura 41, si registrerebbe infatti una netta diminuzione della diffusione e della continuità spaziale dei fattori territoriali di frammentazione e dei relativi effetti.

\section{2 - Criteri progettuali per la riqualificazione diffusa delle aree periurbane: $i$ sistemi lineari di continuità paesistia}

La considerazione delle funzioni paesistico-ambientali degi spazi aperti, per la protezione, la regolazione ed il migioramento degii insediamenti, mette in evidenza l'importanza di individuare tipi di organizzazione sistemica di queste risorse, idonei ad inserirsi realisticamente ed efficacemente nel tessuto congestionato dei paesaggi periurbani delle aree di pianura, dove l'elevata pressione insediativa ha prodotto innanzi tutto il loro consumo, la loro parcellizzazione e marginalizzazione. $\mathrm{Da}$ tempo, alcune ricenche condotte nell'ambito delle discipline dell'architettura del paesaggio e della pianificazione ambientale, come altre, afferenti all'ecologia del paesagjio ed alla biologia conservazionale, hanno trattato la tipologia del corridoio. A secondo degi autori, dei momenti, delle scuole e discipline, ad esso sono stati rifeniti diversi attributi (ambientale, zerde, bidogion, ecalogion, presistico), evidenziandone il ruolo primario rispetto ad una finalità, piuttosto che ad un'altra (conservazione della natura, conservazione del paesaggio, valonizzazione ricreativa e turistica, strutturazione degi spazi verdi urbani in ordinamenti sistemici). Denominazioni a parte, la configurazione topografica di questo tipo di formazione paesistica, carattenizzata, rispetto a quelle areali della machia e della matrice, dalla dominanza dello sviluppo lineare, costituisce l'aspetto sostanziale su cui questi studi hanno incentrato le proprie applicazioni, distinguendone, nei diversi casi ed alle diverse scale, i ruoli strutturali, riconosciuti o possibili. In realtà, la prima considerazione esplicita del concetto di corridaio nell'ambito delle discipline temitoriali, di cui si è riscontrata documentazione nella letteratura scientifica, non deriva dalle scienze naturali, né dall'ecologia, quanto dalle citate applicazioni di pianificazione, sviluppate negi Stati Uniti alla fine degii anni '60 del secolo scorso, in particolare a seguito della evoluzione legislativa in materia di politiche ambientali ${ }^{121}$. Già alcuni anni prima, Philip $\mathrm{H}$. Lewis, a seguito dei risultati di una articolata azione di ricerca universitaria condotta nell'ambito degi insegnamenti di architettura del paesaggio, presso l'Università del Wisconsin, le cui analisi fisiografiche avevano evidenziato una rete di configurazioni linean, aveva sollevato limportanza di attivare una politica preventiva di preservazione di un sistema a rete di comidoi ambientali fra gi stati del Wisconsin e del Iowa ed aveva promosso in tale direzione una vastainiziativa di sensibilizzazione culturalee tecnica, presso gi organi istituzionali locali enazionali ${ }^{122}$.

121 U.S.A., National Environmental Policy Act (NEPA), 1969.

122 L'espressione utilizzata è: a ststem of corridors that would crisscross the states of Wisconsin and Ioum. Si veda: Lew is PH, Quality corridors in Wisconsin, Landscape Architecture, 54, 1964 e Lew is P.H., L'ewluzione della progettazione regionale, Urbanistica, 108, 1997. Si veda anche Flink C.A., Searns R.M., Schwarz L.LaB., (a cura), Greenumys. A guide to Planning Design, and Dezelopment, The Conservation Fund, Island Press, Washington, 1993. 
Ma, già alla fine dell'Ottocento, a partire dall'E merald Necklace di Boston (1876), di Frederick L. Olmsted, le esperienze statunitensi si occupavano, a tutt'altra scala, dello sviluppo dei potenziali degli spazi vendi nelle aree urbane, in termini di concezione di sistemi, costituiti dalla connessione dei parchi tradizionali attraverso spazi lineari, in un tessuto continuo e differenziato. Ci si riferiva espressamenteallegrandi città, manifestando una precisa coscienza ed una sviluppata consapevolezza tecnica, delle necessità degli insediamenti in corso di sviluppo e delle relative potenzialità degli spazi verdi.

A distanza di molti decenni, oggi le aree metropolitane mostrano, nella generalità dei casi, severe condizioni di deficit e squilibrio paesistico ed ambientale. L'inadeguatezza quantitativa e qualitativa degli spazi verdi è uno dei grandi gruppi di fattori di queste condizioni di crisi, peraltro responsabile di gravi ricadute anche in termini di degrado e conflittualità sociali. Non fanno eccezione le aree metropolitane italiane, né fra queste quella fiorentina, di cui si considera una parte nevralgica, a margine del nucleo urbano di Firenze.

Dai punti di vista della connotazione morfologica e funzionale degli spazi aperti, relativamente alla fruizione ed alla percezione umana, i corridoi zerdi sono stati indagati come concetto di pianificazione. In Gran Bretagna, Groome ha avanzato sei argomenti a favore della realizzazione di questi sistemi di spazi aperti, in base ad una ricognizione bibliografica di contributi scientifici significativi. In sintesi sono stati posti in evidenza i seguenti aspetti: libertà di movimento delle persone in spazi protetti dal rumore, dall'inquinamento e da rischi per la loro sicurezza, valenza strutturale nelle strategie di progettazione urbana, differenziazione sistemica dell'offerta ricreativa, valenza strategica nelle politiche di miglioramento urbano e di conservazione dei paesaggi rurali, interesse sociale e culturale dei requisiti funzionali nei confronti delle esigenze di gruppi sociali svantaggiati e della ricreazione all'aperto e peculiare interesse per la valorizzazione dei rapporti di fruizione città-campagna, spiccata capacità di sviluppare il cosiddetto effetto del limite e le relative qualità spaziali di interesse per la fruizione umana ${ }^{123}$.

123 Groome D., Green corridors: a discussion of a planning concept, Landscape and Urban Planning, 19, 1990: “Arguments advanced for the creation of "green corridors". First, linear routeways comprise environmental open space in which people can be free to move without aggravation from noise, pollution, danger or other harmful side-effects. In other words corridor routes can offer an escape from the harshness of the urban environment. Turner records that "even if an individual uses only a small part of the network he gains access to a system and knows that he can use all of its parts" (Turner, 1987). Second, green corridors have been identified, also, for their function in helping to shape particular forms of urban design (Heckscher, 1977). In the planning and development of urban growth in cities in western and central E urope, green corridors have been proposed as a means of reducing or dispersing air pollution (Hough, 1984). Third, it has been argued that green corridors can help serve a wide range of recreational facilities, offering road access at convenient points with car parks and toilet facilities from which walkways lead to other objectives such as picnic sites, public open spaces and woods (Appleton, 1970). Appleton notes that designers can incorporate public transport provisions and allow walkers to walk along a route without having to retrace their steps. Fourth, a further function of corridor routes identified in studies has been their value in providing a framework within which to plan programmes for industrial and environmental improvement in urban areas (Greater Manchester Council, 1983). In rural areas, linear routeways have again been seen as foci for achieving conservation objectives and guiding planning activities (Carlson et al., 1989). Fifth, studies of disused railway lines and canals have also placed considerable value on the ways in which these linear routes afford easy access between town and countryside (Dower, 1963). Such facilities might be seen as widening access opportunities for "non expert" users of the countryside and mobility disadvantaged groups to reach recreational sites (Groome, 1985). Linear routes can be of value, in particular for users for whom movement itself is an important part of leisure experience, notably walking, cycling, riding and jogging (Turner, 1987). It is of interest that these are activities which are experiencing increases in participation at the present time (Office of Population Censuses and Surveyes, 1989). Finally, corridor routes can also be considered in relation to the notion of "edge", a measure used by recreational designers to alter the perimeter of a site and thus increase or decrease the capacity of recreational sites (Beazley, 1969; Heytze, 1975). Linear open spaces providing long edges and offer a close relationship between built-up areas and open spaces (Whyte, 1968)". Riferimenti bibliografici citati: Appleton J.H, Disused railuays in the countryside of England and Wales, Countryside Commission, Cheltenham, 1970; Beazley E., Desioned for recreation, Faber and Faber, London, 1969; Carlson C., Canty D., Steiner S., Mack N., A path for the Palouse: an example of conserzation and recreational planning Landscape and urban planning, 17, 1989; Dower M., Greenumy: a positize future for Britain's cast off railuays, Architectural Review, September 1963; Greater Manchester Council, Medlock V alley Local Plan, The Council, Manchester, 1983; Groome D. M., Recreational corridors: a study of the use of a Middlewood Way and Macclesfield Canal, Occasional Paper $\mathrm{n}$ 20, Department of Town and Country Planning, Manchester University, 1985; Heckscher A., Robinson P., Open spaces. The life of A merican cities, Harper and Row, New York, 1977; Heytze J.C., The field experiment in open air recreational research, Sate Forest Service, Netherlands, 1975; Hough M., City form and natural processes, Croom Helm, Beckenham, 1984; Office of Population Censuses and Surveyes (OPCS), General Household Surzey 1986, HMSO, London, 1989; Turner T., Landkape planning Hutchinson, London, 1987; Whyte W.H., The last landscape, Doubleday, Garden City, New York, 1968. 
Analogamente a quanto deriva dagli sviluppi più recenti delle applicazioni territoriali di biologia conservazionale, anche nella pianificazione paesistica può essere utile introdurre la denominazione di sistema di continuità, più adatta dal punto di vista definitorio a coprire la complessità delle configurazioni di spazi aperti che, a vari livelli, possono svolgere diverse funzioni di continuità della struttura paesistica, in ambiti territoriali caratterizzati da evidenti processi di riduzione degli spazi in cui essa riesce a mantenere apprezzabili gradi di equilibrio ecosistemico, morfologico e storico.

Si attribuiscono alla morfologia lineare dei corridoi paesistici, intesi come sistemi di spazi aperti, alcuni potenziali di riqualificazione diffusa dei paesaggi di pianura: preservazione delle risorse paesistiche ed ambientali, protezione e miglioramento ambientale degli insediamenti, miglioramento morfologico degli spazi, recupero e costituzione di connessioni funzionali, alla scala delle relazioni locali tra gli spazi ed alla scala delle relazioni fra paesaggi diversi.

È possibile adottare una distinzione strumentale di due accezioni di corridoio paesistico, in ragione della utilità teorica ed applicativa derivabile da questa articolazione definitoria. Una accezione morfologica ${ }^{124}$ è relativa a sistemi semplici di spazi aperti, tendenzialmente omogenei. Una accezione strategica $^{125}$ è relativa a sistemi complessi pianificati di spazi aperti, diversificati, per destinazioni d'uso e funzioni paesistico-ambientali, in ragione dell'eterogeneità del mosaico paesistico di riferimento. Fra le discriminanti tra un'accezione e l'altra non rientra la scala o se si vuole il livello territoriale a cui si riferiscono: esse sono piuttosto in relazione di complementarietà da questo punto di vista, nella misura in cui i corridoi di tipo morfologico possono essere componenti dei sistemi complessi di spazi aperti dei corridoi di tipo strategico.

Infatti, alla microscala, una siepe di campo costituisce un corridoio paesistico del primo tipo, con una specifica identità morfologica, esattamente come, a scala geografica, la realtà del corso fluviale principale di un grande bacino idrografico, ben più complessa dal punto di vista strutturale e vasta in termini di dimensioni. Nonostante la profonda diversità di peso dal punto di vista della scala, entrambi i casi sono relativi a formazioni paesistiche, sono realtà unitarie che hanno una struttura ecosistemica, una configurazione spaziale, che sono governate da equilibri e dinamiche proprie e caratterizzate da valori culturali e naturali specifici.

Allo stesso modo, nell'ambito dei progetti di piano, qualunque sia il loro livello di competenza, è possibile avere corridoi del secondo tipo, strategici. Essi sono sistemi differenziati di spazi, a cui possono afferire sia previsioni di piano su ambiti di limitate dimensioni e relative a problematiche locali, sia politiche di scala vasta, ad esempio di relazione tra parchi naturali o aree protette in genere. In questo caso è ancora evidente la diversità netta tra le due situazioni, che potrebbero riguardare una piccola area urbana, la prima, come una regione o un'area ancora più vasta, la seconda. Ma entrambe le situazioni si riferiscono a realtà paesistiche complesse ed alle relative politiche di uso e gestione delle risorse, hanno una delimitazione di riferimento, sono caratterizzate da relazioni interne, interrelazioni con il contesto, complessità di destinazioni d'uso e funzioni paesistico-ambientali degli spazi. Tutte queste caratteristiche possono essere strumentali al raggiungimento di obiettivi pianificati di qualità paesistica ed ambientale, nel caso essi vengano assunti come parti integranti e strutturali di politiche di governo territoriale.

124 Il termine viene proposto secondo il significato letterale, relativo alla identificazione strutturale di una data configurazione reale attraverso l'osservazione dei caratteri percepibili.

125 Il termine viene proposto secondo il significato che ha acquisito nella pianificazione territoriale, relativo ai caratteri delle politiche strutturali per l'uso e la gestione delle risorse. 
Si può osservare come il concetto di corridoio ecologico si riferisca ad una accezione di carattere funzionale, relativa alle popolazioni animali e vegetali. In ragione di determinate condizioni ambientali ${ }^{126}$, si verifica l'idoneità più o meno spiccata di una sequenza lineare di spazi, ad assolvere a talune funzioni rispondenti alle esigenze ecologiche delle popolazioni. I corridoi ecologici di movimento di determinate specie animali o quelli di dispersione di specie vegetali possono esistere ed essere riconosciuti attraverso processi conoscitivi altamente specializzati, senza avere necessariamente una rispondenza nel paesaggio percepibile anche dall'uomo, semplicemente perché i movimenti possono avvenire attraverso spazi trasversali alla articolazione del mosaico paesistico, in ragione delle peculiari preferenze delle specie. L'assegnazione dell'attributo paesistico prevede, diversamente, la coesistenza di caratteri funzionali con una struttura spaziale percepibile, di matrice culturale o naturale, caratterizzata da una propria rispondenza semiologica.

Oggi, il tema dei corridoi di connessione degli habitat animali e vegetali è uno degli aspetti più dibattuti nell'ambito degli studi delle reti ecologiche, da cui dipende parte della stessa loro formulazione teorico-pratica. Luigi Boitani ${ }^{127}$ è intervenuto in questo dibattito, affermando decisamente l'imprecisione e l'insostenibilità scientifica, dal punto di vista biologico, dell'impostazione generale contenuta nel documento programmatico ${ }^{128}$ della Rete Ecologica Nazionale italiana (1998), in cui ai corridoi di connessione è specificamente attribuita la funzione di dispersione delle specie attraverso il collegamento degli habitat. Due elementi di fondo sono ritenuti erronei: l'assumere a priori le attuali aree protette e quelle proposte come siti di maggiore naturalità rispetto al resto del territorio e l'assumere, in maniera generica, i corridoi di connessione come strutture capaci di favorire la dispersione di molte specie. La superficiale traduzione di questi assunti nella pianificazione del territorio può svuotare le previsioni di corridoi biologici di ogni effettiva attendibilità, ad eccezione solo delle previsioni di connessioni specie-specifiche, per le quali siano stati condotti appositi studi sperimentali di osservazione.

"Se è vero che qualche caso di strutture lineari è stato verificato in natura, è certamente vero che l'ecologia ancora non ha trovato una metodologia per progettare un solo corridoio funzionale. Se una prospettiva esiste in questo senso, essa è limitata alla sperimentazione di corridoi specie-specifici, mai multispecifici. (...) La conservazione non può restringere la sua attenzione alle sole aree protette $\mathrm{e}$ alla rete delle interconnessioni, perché la vera sfida di conservazione si gioca nella matrice nella quale le aree sono inserite. (...) Dimentichiamo per un momento l'esistenza delle aree protette e affrontiamo il territorio nella sua globalità. L'Italia è un paese ricco di vaste aree che possiamo definire a naturalità diffusa. (...) La totalità delle aree a naturalità diffusa costituisce una matrice che, probabilmente, copre la maggior parte del territorio italiano: immaginiamo questa matrice dalla quale emergono in positivo alcuni centri di particolare concentrazione di biodiversità e, in negativo, le aree compromesse da un eccessivo degrado. Questo potrebbe essere lo sfondo sul quale operare una analisi del mosaico ambientale per disegnare una serie di reti ecologiche specie-specifiche, destinate alla conservazione ottimale di tutte le specie per le quali possediamo dati adatti a una simulazione delle loro dinamiche ecologiche. (...) Ciò che paradossalmente appare più difficile da introdurre nella pianificazione territoriale è un approccio che permetta di accogliere la dinamica spazio-temporale delle comunità naturali. (...) Ma è anche necessario comprendere che la gestione del paesaggio discende dalla comprensione della sua dinamica evolutiva"129.

126 Condizioni determinate da caratteri ambientali strutturali: biogeografici, morfologici, fitosociologici, geolitologici, pedologici, microclimatici, idrologici, idrogeologici.

127 Docente di ecologia all'Università La Sapienza di Roma, presso la quale è responsabile del Laboratorio di E cologia applicata.

${ }_{128}$ Ministero dell'Ambiente, Servizio Conservazione della Natura, La alorizzazione delle risorse ambientali nelle politiche di sziluppo "La rete ecologica nazionale", note informative, Ministero dell'Ambiente, Roma, 1999.

129 Boitani L., La tutela e la zalorizzazione della biodizersità terrestre in Italia: appunti per la Rete E cologica Nazionale, seminario nazionale Conserazione della natura e sziluppo locale: il sistema delle aree protette e la Rete Ecologica Nazionale, ECOLAVORO99, Legambiente, Ministero dell'Ambiente, Federazione Italiana Parchi e Riserve Naturali, Firenze, 14 dicembre 1999. 
L'autore sostiene che la progettazione delle reti ecologiche secondo questi presupposti viene ad essere libera dalla costrizione di comprendere in modo dominante l'articolazione delle aree protette e risulta invece guidata dalle esigenze delle specie e dalle idoneità dell'ambiente. Si costituisce così un sistema reticolare di aree e corridoi che esclude solo le aree irrimediabilmente compromesse dal punto di vista biologico.
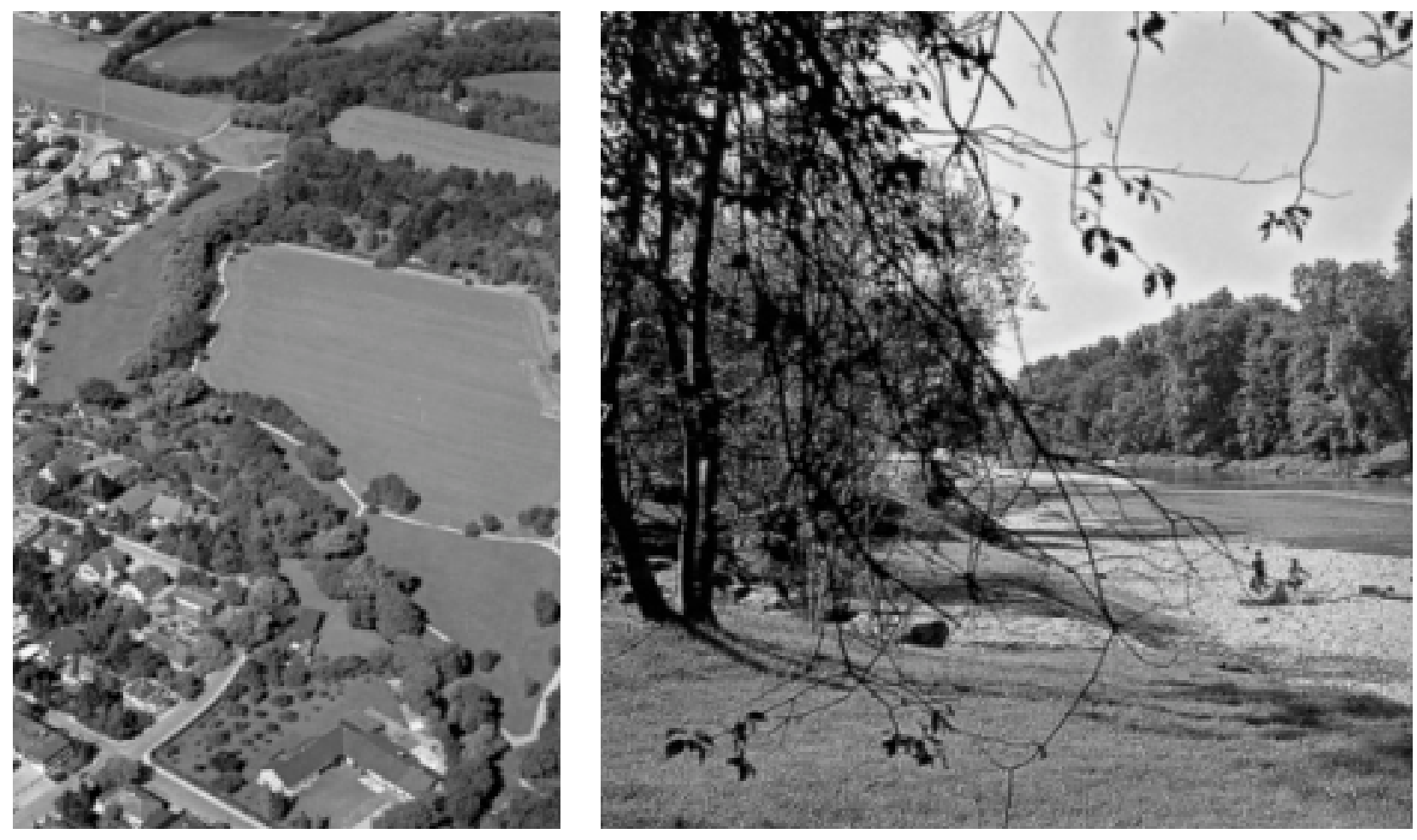

Figura 42 - In Baviera, dove la conservazione del paesaggio ha forti radici culturali, sono facilmente riscontrabili esemplificazioni delle due tipologie di corridoio alla diverse scale. A sinistra, siepi e macchie di campo costituiscono una rete di equipaggiamento agroforestale in cui le formazioni lineari si presentano come corridoi del tipo morfologico con spiccate valenze ecologiche e spaziali. A destra il corso del fiume Isartal, qui ripreso in una delle sezioni urbane di Monaco, costituisce allo stesso modo un corridoio morfologico di rilevanti dimensioni, che contribuisce alla regolazione climatica della metropoli, caratterizzato da elevati potenziali di uso ricreativo (immagini tratte da: Grub H., Lejeune P., Muenchen: Stadt-Land-Gruen, Prestel, Muenchen, New York, 1997).

A conferma della necessità di approfondimento di questi studi vengono segnalazioni da più parti. Nonostante in Germania dalla metà degli anni '80 si conducano ricerche con applicazioni sperimentali riguardanti i sistemi a rete di biotopi, Jurgen Ott ha sostenuto, in un recente contributo, che non si hanno riscontri della efficacia generale di questi corrido ${ }^{130}$. Ott si richiama ad un compendio teorico-pratico di biologia conservazionale, in cui il tema della efficacia biologica dei corridoi è stato affrontato sotto forma di comparazione ragionata degli studi esistenti. Egli sottolinea come non si abbiano dimostrazioni del fatto che sia sufficiente inserire corridoi verdi in un paesaggio di qualunque configurazione per risolvere le problematiche di connessione degli habitat isolati. Viene notato inoltre come nello studio citato non vengano presi in considerazione gli habitat urbani, lasciando presupporre l'inesistenza di studi qualificati riguardanti l'approccio a questa problematica nelle città.

130 Ott J., Collegamenti fra biotopi e reti ecologiche nelle aree urbane della Germania. Che senso hanno i corridoi zerdi nelle città?, in Ghiringhelli R., Dimaggio C., (a cura), Reti ecologiche in aree urbanizzate, Provincia di Milano - Agenzia Nazionale per la Protezione dell'Ambiente, atti del seminario di Milano del 5 febbraio 1999, Franco Angeli, Milano, 1999. 
Nella discussione di un lavoro di ricerca relativo alle associazioni arbustive del paesaggio vegetale costiero della California, Peter A. Bowler ${ }^{131}$ considera il tema dell'efficacia dei corridoi di dispersione delle specie e delle connessioni paesistiche ${ }^{132}$. Egli rileva come sia stato riscontrato quanto meno l'uso di corridoi vegetali per lo spostamento fra habitat, da parte di animali che si cibano di insetti, ma, nella generalità dei casi, per lo più a causa del passaggio di infrastrutture viarie, la maggior parte di questi collegamenti siano frammentati, con decise limitazioni per molte specie di animali.

In sintesi, a differenza del corridoio ecologico, o ambientale, o naturalistico, o biocanale, o biocorridoio, che si voglia dire, al corridoio paesistico, sia nella forma di sistema semplice di spazi aperti (con identità morfologica), come in quella di sistema complesso pianificato (con identità strategica), corrisponde sempre una configurazione distinta dagli spazi limitrofi, in essere oppure attendibile in ragione delle misure di intervento e gestione.
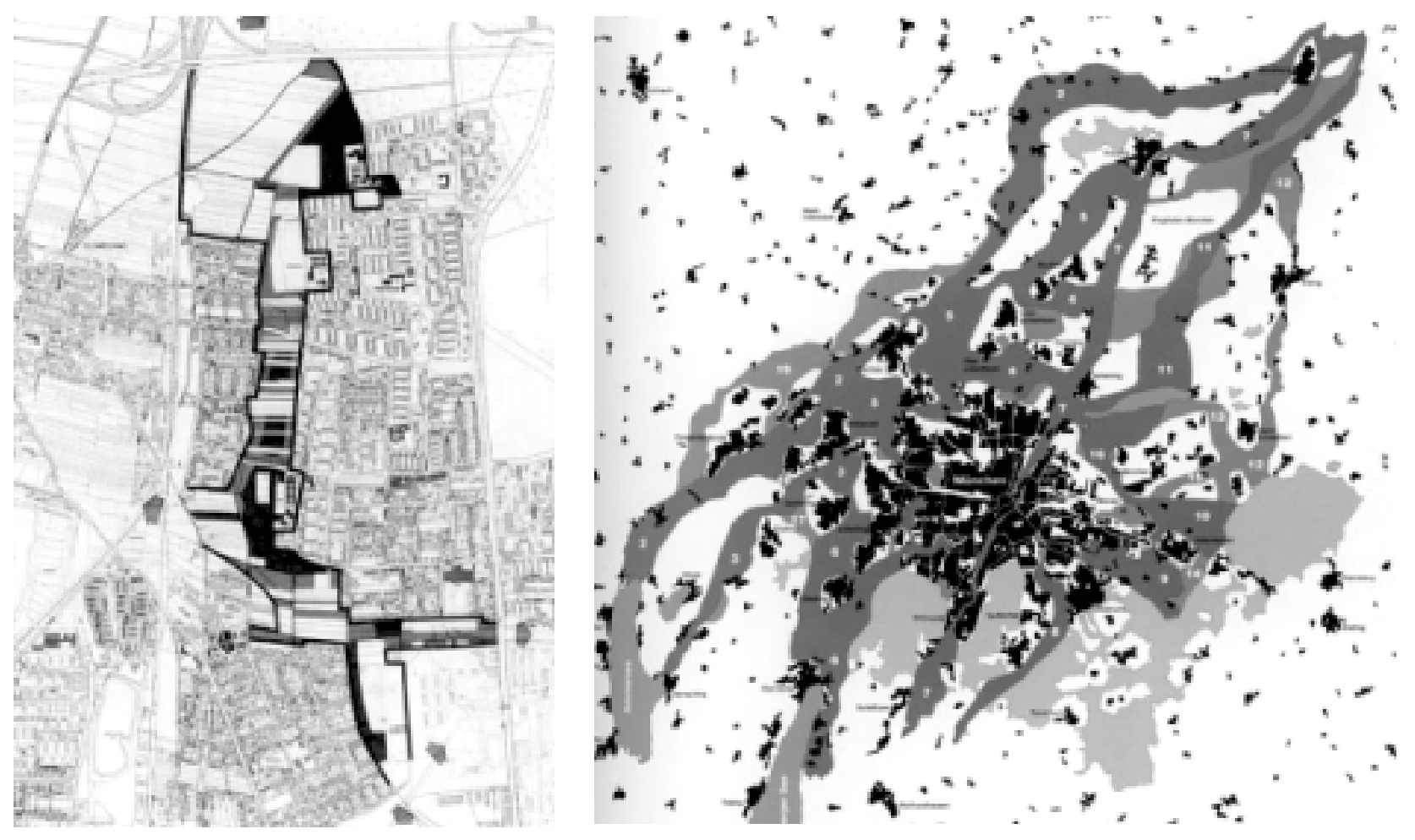

Figura 43 - Nella Repubblica Federale Tedesca alcuni Lander sono particolarmente attenti e coerenti nell'utilizzo degli strumenti di piano per la conservazione attiva della natura e del paesaggio. Nonostante si faccia riferimento ad un modello di pianificazione parallela, come condizione necessaria per la dialettica fra la pianificazione territoriale e la pianificazione paesistica, in taluni casi, come in Baviera, per legislazione regionale, la pianificazione paesistica risulta essere in stretta relazione con i piani di governo del territorio anche al livello comunale, per il quale è prevista l'obbligatoria integrazione dei piani regolatori generali con le indicazioni dei piani del paesaggio. Alle varie scale di pianificazione sono riscontrabili esemplificazioni di corridoio del tipo strategico. A sinistra, un caso a scala urbana, relativo ad un microsistema di spazi aperti nell'ambito di un quartiere residenziale periferico di Monaco; a destra, corridoi dello stesso tipo, alla scala della regione metropolitana, assumono ruoli di relazione tra aree protette in un sistema territoriale a rete (immagini tratte da: Grub H., Lejeune P., Muenchen: Stadt-Land-Gruen, Prestel, Muenchen, New York, 1997).

${ }^{131}$ E sperto di ecologia, opera negli Stati Uniti presso il Dipartimento di E cologia e Biologia evolutiva dell'Università della California.

${ }^{132}$ L'espressione utilizzata è: dispersal corridors and landscape linkages. 
Andando ad analizzare nello specifico le due tipologie fin qui introdotte, pare logico discutere innanzitutto i caratteri del corridoio paesistico come sistema morfologico, sia per essere questa l'accezione più tradizionale e diffusa, sia per una ragione di consequenzialità, essendo questa prima forma, a sua volta, una possibile componente dei sistemi lineari complessi di spazi aperti.

Secondo il modello teorico del mosaico paesistico ${ }^{133}$, ogni configurazione riscontrabile nella realtà è classificabile e cartografabile, con l'ausilio delle informazioni fotografiche aeree e satellitari, come appartenente ad uno dei tre tipi di componenti strutturali: matrice (matrix), macchia (patch) e comidoio (corridor).

\begin{tabular}{|c|c|c|c|c|}
\hline Termine & $\begin{array}{l}\text { Area geog rafica } \\
\text { di utilizzo del termine }\end{array}$ & Funzioni & Scala & $\begin{array}{l}\text { Caratteri spaziali } \\
\text { primari }\end{array}$ \\
\hline Ecological networks & Europa & biotiche & $\begin{array}{l}\text { continentale } \\
\text { nazionale } \\
\text { regionale } \\
\text { locale }\end{array}$ & biologici \\
\hline Habitat networks & Europa - America & biotiche & $\begin{array}{l}\text { nazionale } \\
\text { regionale } \\
\text { locale }\end{array}$ & biologici \\
\hline Ecological infrastructures & Europa & biotiche & $\begin{array}{l}\text { continentale } \\
\text { nazionale } \\
\text { regionale } \\
\text { locale }\end{array}$ & biologici \\
\hline Greenways & America & $\begin{array}{l}\text { biotiche } \\
\text { culturali } \\
\text { multiple }\end{array}$ & $\begin{array}{l}\text { regionale } \\
\text { locale }\end{array}$ & fisici, culturali \\
\hline Wildlife corridors & America & biotiche & $\begin{array}{l}\text { regionale } \\
\text { locale }\end{array}$ & biologici \\
\hline Riparian buffers & Europa - America & $\begin{array}{l}\text { biotiche } \\
\text { multiple }\end{array}$ & $\begin{array}{l}\text { regionale } \\
\text { locale }\end{array}$ & fisici \\
\hline Ecological corridors & America & biotiche & $\begin{array}{l}\text { regionale } \\
\text { locale }\end{array}$ & fisici \\
\hline Environmental corridors & America & multiple & $\begin{array}{l}\text { regionale } \\
\text { locale }\end{array}$ & fisici \\
\hline Greenbelts & Europa - America & culturali & $\begin{array}{l}\text { regionale } \\
\text { locale }\end{array}$ & culturali \\
\hline Landscape linkages & America & biotiche & $\begin{array}{l}\text { regionale } \\
\text { locale }\end{array}$ & biologici \\
\hline
\end{tabular}

Figura 44 - Confronto dei significati attribuiti ai diversi termini fondamentali connessi agli studi sui corridoi nell'ambito delle discipline territoriali in Europa e negi Stati Uniti d'America (schema tradotto e adattato da: J. Ahern, Greenumys as a planning strategy, Landscape and Urban Planning, 33, 1995).

"Questa universalità spaziale consente una comparazione diretta e facilitata di paesaggi differenti per individuarne le configurazioni generali. (...) I patch sono stati analizzati inizialmente dal punto di vista della loro dimensione, forma, numero e foggia del margine. Un'ampia letteratura documenta l'eccezionale valore dei patch di vegetazione naturale per la tutela delle risorse idriche e delle specie dominanti. (...) I corridoi e gli altri elementi lineari attraversano e connettono il paesaggio. Esempi noti di queste forme sono le strade, le linee elettriche, i frangivento, le siepi, i boschi, i torrenti e i corridoi fluviali. I corridoi sono elementi spaziali che svolgono molteplici funzioni e ruoli all'interno del paesaggio. (...) I mosaici hanno esito dalla combinazione di questi elementi spaziali. La configurazione degli spazi prossimi ai patch e ai corridoi è di particolare interesse per le abbondanti

${ }^{133}$ Forman R.T.T., Land mosaics, the ecology of landscapes and regions, Cambridge, 1995. 
interazioni che avvengono tra di essi. (...) I mosaici del paesaggio cambiano continuamente di forma a seconda che i patch e i corridoi compaiano o scompaiano, si espandano o si restringano, si combinino tra loro o si frammentino. I processi naturali, insieme alle attività umane e alla loro organizzazione sul territorio, provocano le trasformazioni territoriali con conseguenti cambiamenti nei flussi e nei movimenti. I modelli spaziali sono utilizzati per identificare la configurazione ottimale, dal punto di vista ecologico, dei mosaici nel caso di interventi di trasformazione territoriale"134.
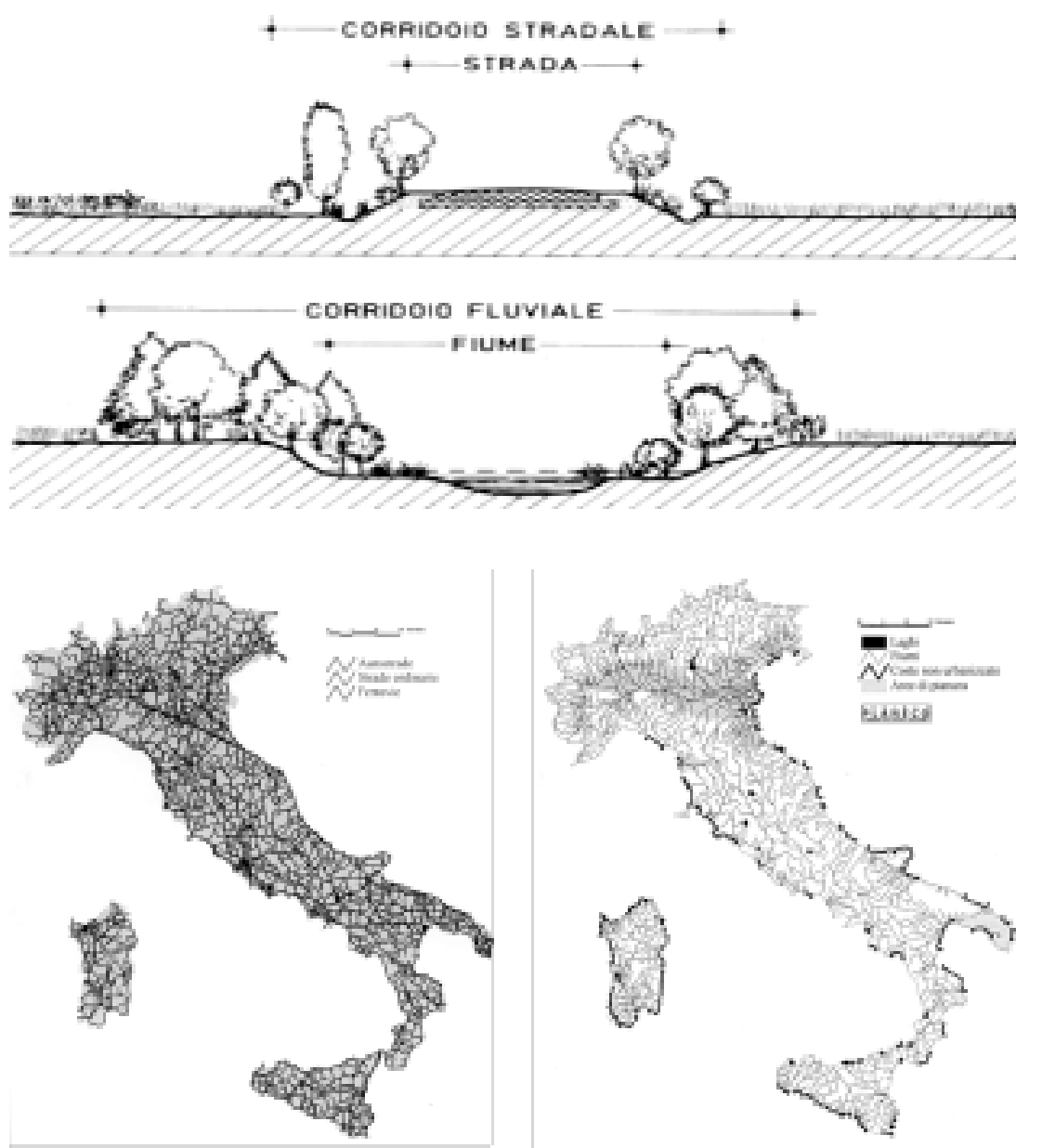

Figura 45 - Confronto tipologico tra i corridoi stradali e fluviali: i primi più frequentemente in rilevato, i secondi in trincea (immagine tratta da: Ingegnoli V., Fondamenti di ecologia del paesagoio, CittàStudi, Milano, 1993).

Figura 46 - Le reti dei principali corridoi infrastrutturali, a sinistra, e fluviali, a destra (in nero sono indicati i tratti di costa non urbanizzati: l'approssimazione deve essere considerata per difetto in quanto, per le scale utilizzate, non rileva il peso reale del complesso delle alterazioni di limitata entità unitaria). Se questi sistemi divenissero soggetti di studio paesistico di piani e progetti di miglioramento ambientale, spaziale e funzionale, attraverso interventi di ingegneria naturalistica e di equipaggiamento vegetale arboreo ed arbustivo, si potrebbe avviare una sistematica attuazione di opere di riqualificazione diffusa (immagini tratte da: Romano B., Continuità ambientale. Pianificare per il riassetto ecologico del territorio, Andromeda E ditrice, Teramo, 2000).

Lo sviluppo di questo modello sintetico di interpretazione fisionomico-strutturale del paesaggio, dovuto alle applicazioni ecologiche ai processi di analisi e diagnosi per la pianificazione, suggerisce l'opportunità di considerare l'estensione di criteri analoghi ad altre finalità conoscitive, per le quali risulti determinante la sintesi strutturale di caratteri morfologici (si veda anche il paragrafo 1.2). Si pensa alle indagini per la conservazione delle permanenze culturali storiche, come dei caratteri naturali geologici e vegetazionali endemici, o semplicemente specifici, dei luoghi e, non ultimo, alle possibili applicazioni nei processi di pianificazione e progettazione delle forme di utilizzo ricreativo, locale e turistico, e di sviluppo insediativo ed infrastrutturale. L'evidente complementarietà o comunque interazione di questi campi di studio e intervento riporta inevitabilmente alla centralitàdel paesaggio come risorsa territoriale complessa, dovuta alle relazioni crociate tra i processi (naturali e culturali) che agiscono sulle specifiche configurazioni di risorse (naturali e culturali) di ogni territorio. Come si è potuto osservare nel precedente paragrafo attraverso gli scenari teorici di intervento, la forte articolazione geomorfologica e la conseguente complessità della rete idrografica del paesaggio italiano, come la densità estremamente elevata della rete viaria, fanno sì che anche solamente la 
definizione e l'attuazione di concrete politiche di recupero e miglioramento paesistico e ambientale di questi corridoi, si configurerebbe come una sostanziale azione di riqualificazione diffusa, particolarmente efficace nelle aree congestionate delle pianure ad elevata pressione antropica, insediativa, come anche produttiva. In realtà si assiste invece al normale maltrattamento dei corsi d'acqua ed alla mancata progettazione delle infrastrutture come opere di paesaggio, con le note conseguenze di desolazione e congestione spaziale, particolarmente acute nei territori di pianura, in ragione dei minori condizionamenti geomorfologici imposti all'intervento umano dalla struttura naturale del paesaggio.

Un altro tema di recupero di valori storico-culturali e ambientali del paesaggio riguarda l'equipaggiamento agroforestale. I sistemi di siepi e macchie di campo, con sviluppi alterni ed evoluzione tipologica, hanno caratterizzato decisamente il paesaggio collinare e planiziale fino ai recenti effetti della industrializzazione agraria, che, in particolare nelle aree di pianura, ha progressivamente determinato l'abbandono e l'abbattimento di queste piantagioni non colturali, storicamente di supporto alla funzionalità della struttura aziendale.
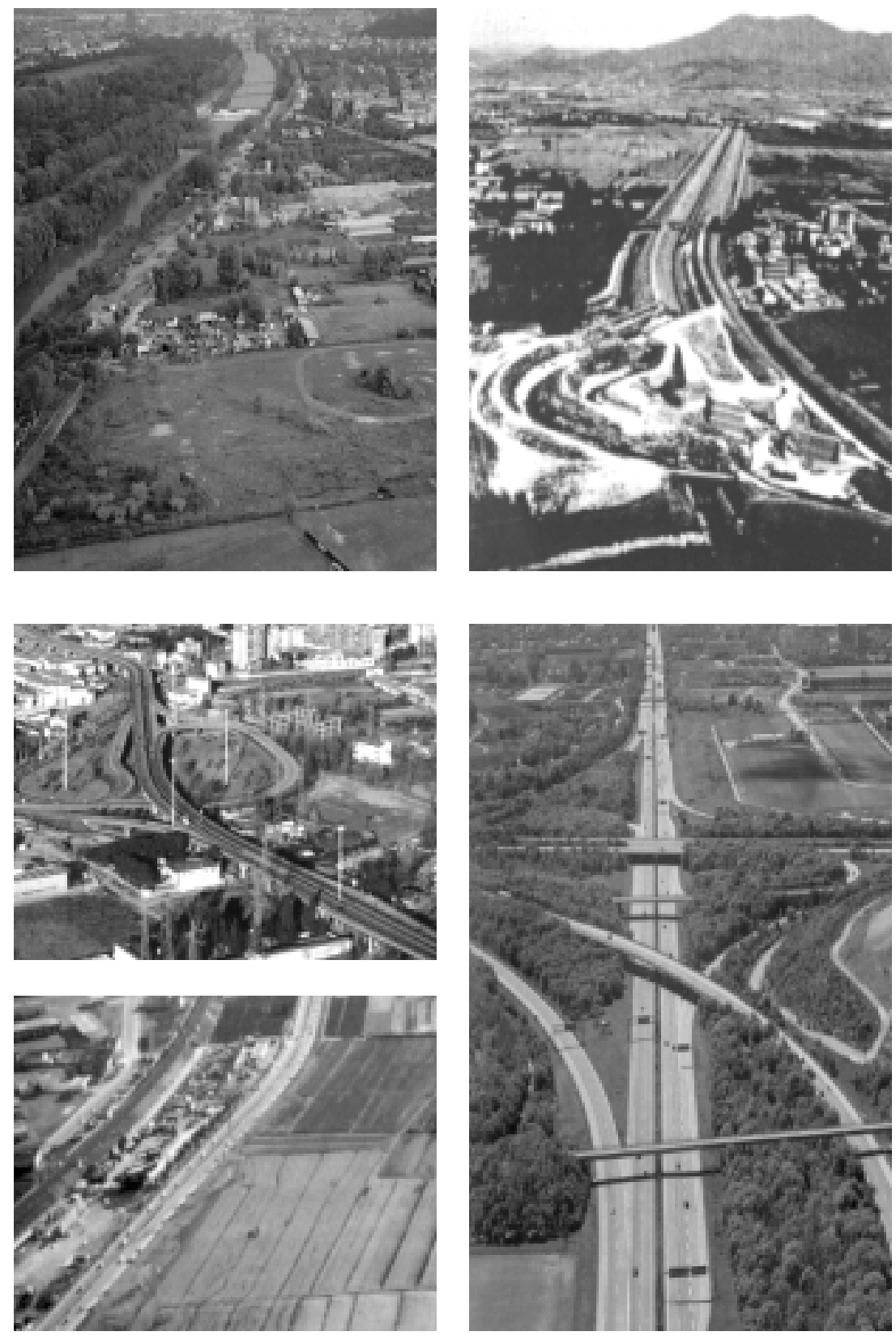

Figura 47 - Il corso canalizzato dell'Arno nell'area urbana fiorentina, in alto a sinistra, in uno scorcio dell'area di pertinenza fluviale di Argingrosso (immagine tratta da: Autorità di bacino del fiume Arno, Piano di bacino del fiume A rno - Rischio idraulico - Sintesi del progetto di piano stralcio, Quaderni, 5, Firenze, 1996). In alto a destra, decisamente non distinguibile da una grande infrastruttura, uno dei canali di bonifica dell'area metropolitana fiorentina, oggetto di recente progettazione e realizzazione di interventi di sistemazione idraulica (immagine tratta da: Consorzio di bonifica Area Fiorentina, Il Consorzio di bonifica dell'area fiorentina - Piani, progetti, intercenti, Firenze, 1998). In basso a sinistra, lo stato delle infrastrutture viarie primarie nell'area metropolitana fiorentina denuncia l'assoluta assenza di consapevolezza paesistica, sia negli strumenti di pianificazione territoriale, che nei progetti di realizzazione delle opere stradali (immagini tratte da: G. Di Pietro, Un progetto per Firenze - La nuoza città nella piana di Castello, Ponte alle Grazie, Firenze, 1990). In basso a destra, nell'area metropolitana di Monaco di Baviera, ad elevati requisiti di funzionalità della rete stradale sono associate politiche di piano atte a riservare i necessari spazi per la realizzazione dei sistemi paesistici di separazione, ed equipaggiamento (immagine tratta da: Grub H., Lejeune P., Muenchen: StadtLand-Gruen, Prestel, Muenchen, New York, 1997). 
A seguito di un panorama di studi ormai denso ed evoluto, oggi molti ripensamenti di tecnica e politica agraria fanno sì che si vada sempre più lavorando intorno al ripristino delle piantagioni agroforestali, con particolare riguardo alle opportunità di recupero ambientale e spaziale dei paesaggi di pianura, desertificati dalla bonifica e dalla industrializzazione colturale agraria.

Alla microscala anche la siepe di campo, per composizione fitosociologica e morfologica, assume valenza di corridoio paesistico. Le funzioni di queste formazioni sono state variamente studiate e presentano una elevata articolazione, pur essendo oggi venute meno alcune funzioni storiche primarie, come quelle di chiusura dei campi e di fornitura di materiali. Esse sono essenzialmente identificabili come funzioni di limite, relativa all'articolazione spaziale ed alla conseguente percezione del paesaggio, di filtro, relativa alla mitigazione dell'aggressività degli agenti climatici e inquinanti, di origine, di flussi di materia ed energia, di assorbimento, di flussi di materia ed energia, di habitat, animale e vegetale, di connessione, di habitat animali e vegetali.

La progettazione delle barriere frangivento costituisce infine un argomento, in parte compreso nel tema precedente dei sistemi agroforestali, che va trovando una sua autonomia di diffusione in forza della specifica funzione di protezione ambientale. Tale funzione deve essere considerata relativamente alla protezione delle colture agrarie soggette a particolari condizioni di aggressione dovuta alle correnti eoliche, ma anche relativamente alla regolazione dei venti per la mitigazione degi effetti di erosione del suolo, in ragione dell'importanza preminente di questa risorsa naturale non rinnovabile. Vi è poi una gamma di funzioni ambientali, tanto rilevanti quanto poco indagate in termini di applicazioni progettuali, che riguarda la protezione e la regolazione microclimatica degli insediamenti.

È importante quindi, che la progettazione delle barriere frangivento sia collocata in modo organico e coordinato nel quadro complessivo delle azioni di riqualificazione paesistica e sia impostata, dai rilievi iniziali alla progettazione spaziale finale, in termini di progettazione paesistica e non esclusivamente ambientale. Il rischio concreto che si corre infatti, malgrado la completa legittimità dell'obiettivo specifico, è quello di generare squilibri e danni paesistici, a causa del mancato controllo conoscitivo e progettuale dell'insieme del contesto in cui le opere vengono ad essere previste. Alla specifica funzione di regolazione dell'azione dei venti sono connessi inoltre anche rischi ambientali, nella misura in cui gli effetti attesi dagli interventi non siano determinati attraverso modelli comprensivi dei principali scenari di qualità, ma riguardino al contrario in modo settoriale solo alcune finalità, assunte erroneamente come primarie in senso assoluto ed esclusivo. Occorre, ad esempio, che la progettazione di sistemi agroforestali per la protezione di colture agrarie controlli gli effetti di alterazione microclimatica, anche relativamente alle esigenze ambientali di ordine insediativo. La finalità generale del riequilibrio ambientale e morfologico del paesaggio si dimostra ancora una volta un obiettivo complesso, che richiede una pianificazione ed una progettazione comprensive, improntate a tecniche diagnostiche multicriteri.

Passando alla accezione denominata strategica, si consideri come, nella definizione delle politiche per la conservazione della natura e del paesaggio, il corridoio biologico venga visto dagli esperti di pianificazione ambientale come misura migliorativa, rispetto all'attuale isolamento delle aree protette, e preventiva, rispetto ai processi di riduzione spaziale degli habitat nei territori a governo ordinario ${ }^{135}$.

135 Si veda in particolare il quadro organico derivante da alcuni contributi di Bernardino Romano: Romano B., Oltre $i$ parchi. La rete zerde regionale. Una ricerca sulle idoneità territoriali per i corridoi ecologici dell'A ppennino Centrale, Università degiStudi dell'Aquila, Dipartimento di Architettura e Urbanistica, Andromeda Editrice, Teramo, 1996; Romano B., La continuità ambientale in Italia, corridoi ecologici per i parchi e le aree protette, Atti XVIII Conferenza Italiana di Scienze Regionali, E uropa e Mediterraneo, Siracusa, ottobre 1997, Volume 3, 1997; Romano B., Planning for environmental continuity in Italy, in PLANECO Project. Planning in ecological network, ricerca nazionale MURST, pubblicazione WE B, Newsletter 1, 1998; Romano B., Continuità ambientale ed interferenza antropica tra le grandi aree protette e le città dell'A ppennino, workshop nazionale Reti e corridoi ecologici per gli interzenti di conserzazione e salanguardia della natura in ambiente urbano e suburbano, Agenzia Nazionale per la Protezione Ambientale ANPA, Catania, 1-3 ottobre 1999; Romano B., La continuità ambientale nella pianificazione, Urbanistica, n. 112, 1999; Romano B., Continuità ambientale. Pianificare per il riassetto ecologico del territorio, Andromeda E ditrice, Teramo, 2000; Romano B., Politiche ambientali nell'A bruzzo dei parchi, Urbanistica Informazioni, n. 169, 2000. 
Ciò assume significati importanti, in questa fase storica di evoluzione degi studi scientifici e delle modalità tecniche ed istituzionali della pianificazione territoriale, sia nelle realtà regionali che a livello nazionale. Si è detto come l'espansione e la diffusione di insediamenti ed infrastrutture, le modalità della corsa alla messa in sicurezza idraulica degli spazi urbanizzati, il progressivo ritiro delle attività agricole e la loro frequente riduzione in condizioni di interstizialità, siano tutti fattori responsabili di effetti di frammentazione e semplificazione dei caratteri strutturali del paesaggio, sia di quelli abiotici, che di quelli biotici, sia nelle loro configurazioni naturali, che in quelle culturali. Carenza di spazio, deficienza delle funzioni di protezione ambientale e storico-culturale, perdita di idoneità all'uso ricreativo, determinano in realtà effetti non trascurabili, oltre che nel territorio in cui si verificano, anche in territori profondamente diversi, che subiscono pesanti condizionamenti paesistici ed ambientali, a seguito di pressioni di vario genere indotte dall'esterno.

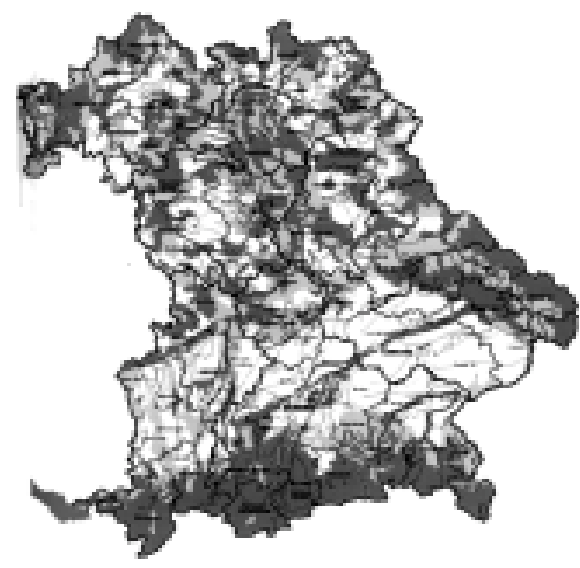

Figura 48 - La rete dei biotopi della Baviera (immagine tratta da: ARGE ALP, Nuoze vie per la tutela della natura e del paesaggio, Comunità di lavoro delle regioni alpine, Bolzano, 2000).

Il dibattito sulla conservazione del paesaggio e della natura, tradizionalmente legato in maniera quasi esclusiva ai parchi naturali ed alle aree protette in genere, va progressivamente spostando i propri modelli territoriali da quelli di tipo insulare a quelli di tipo sistemico e reticolare. Questi orientamenti stanno sviluppando modelli di connessione degli habitat naturali che non si basano tanto sulla individuazione di reti di corridoi concepiti come elementi spaziali omogenei e continui, bensì sulla progettazione di sistemi unitari, in ragione delle prestazioni paesistico-ambientali ottenibili, considerando spazi con destinazioni d'uso diverse compatibili.

Da alcuni anni ormai la pianificazione ambientale considera diffusamente il corridoio come strategia paesistica complessa, legando la denominazione specificamente alle funzioni di conservazione che svolge o potrebbe svolgere. "Dove si verifichi che le popolazioni animali residenti e i processi ecologici non riescono a sostenersi, allora dovrebbero essere suggeriti dei collegamenti tra le aree protette attraverso corridoi. I corridoi varieranno in termini di consistenza geografica e pertanto i gestori dell'area protetta avranno bisogno in molti casi di lavorare con varie organizzazioni governative e non governative in un processo di pianificazione dell'uso del suolo regionale ${ }^{\prime \prime 136}$.

L'applicazione delle teorie dell'uso differenziato degli spazi per l'equilibrio ecologico ${ }^{137}$, può fornirein tal senso contributi conoscitivi e progettuali utili alla definizione di modelli strategici di riqualificazione paesistica. Nei territori di pianura soggetti ad elevata pressione insediativa, in considerazione della congestione e del consumo degli spazi, vi è infatti la necessità di individuare gli

136 IUCN, Parks for life, Workshop III.9, Corridors, transiting zones and buffers: tools for enhancing the effectizeness of proteded areas, IUCN, Gland Switzerland, 1993.

137 Si vedano gli studi sviluppati in Germania, negli anni "70 del secolo scorso, da W. Haber e H.J. Schemel, a partire dai fondamenti terorici pubblicati da E.P. Odum circa dieci anni prima (Odum E.P., The strategy of ecosystem derzlopment, Science 164, 1969). Attente ed articolate illustrazioni e contributi allo sviluppo di tali teorie si trovano in Finke L., Introduzione all'E cologia del paesagio, (Landschaftokologie), Franco Angeli, Milano, 1993 (Verlags-G mbH Holler und Zwick, Braunscweig, 1986) ed in Di Fidio M., A rchitettura del paesaggio, Pirola, Milano, 1993. Fra le esperienze più recenti si trovano i lavori dell'International studygroup on multiple use of Land (ISOMUL) dell'Università di Wageningen, in Olanda. 
ambiti e le politiche attraverso cui perseguire il riconoscimento e l'attuazione di criteri di uso e gestione delle risorse idonei a produrre effetti di protezione e di compensazione paesisticoambientale. In questi territori, in cui il paesaggio è stato ed è soggetto ad intense trasformazioni, non si tratta per lo più di pensare nuovi regimi normativi di tutela e limitazione degli effetti dell'urbanizzazione e dell'infrastrutturazione, quanto di pensare nuovi paesaggi, caratterizzati da forme più mature e stabili di governo delle dinamiche evolutive naturali e culturali, definendo ed attuando scenari progettuali fortemente propositivi ed aperti al maggior numero possibile di soggetti sociali ed economici, oltre che istituzionali.

“(...) La teoria dell'utilizzazione differenziata del territorio per l'equilibrio ecologico, in sede pianificatoria, si è tradotta nella definizione delle cosiddette zone ecologiche prioritarie, con funzioni complementari per la compensazione dei carichi ambientali e per la difesa della natura, tra loro collegate a rete, sia a grande che a piccola scala. (...) Le zone prioritarie per la compensazione dei carichi ambientali sviluppano potenziali naturali (...) in rapporto funzionale diretto con le zone urbano-industriali ed agricole intensive, al cui servizio sono strettamente subordinate, collocandosi sia all'interno che ai margini delle stesse, in una scala dimensionale medio-piccola (intra-aziendale ed intra-comunale), con l'eccezione della funzione ricreativa, che richiede anche una scala maggiore. Invece le zone prioritarie per la difesa della natura comprendono gli ecosistemi di maggior pregio (zone di riserva), che sviluppano potenziali naturali floro-faunistici in rapporto solo indiretto con le aree sovraccariche e devono anzi essere difesi dagli influssi nocivi provenienti dalle stesse, mediante adeguata schermatura (zone cuscinetto), con una scala dimensionale di entrambe le zone da media a grande (escluso il tessuto connettivo). Tra i due sistemi di zone prioritarie ci sono punti di contatto; per esempio gli spazi di compensazione passiva (...) possono sovrapporsi alle zone cuscinetto; inoltre queste ultime possono svolgere anche funzioni di compensazione attiva, costituendo potenziale climatico di rigenerazione e potenziale idrico direttamente disponibili per le aree urbane. Siamo dunque in presenza di una strategia binaria di grande respiro, in grado di ospitare, in una delle sue metà, l'intera teoria delle reti ecologiche nei suoi sviluppi più recenti. A confronto, l'altra metà della strategia appare assai meno sviluppata e carente di dati sperimentali, se si eccettua la componente del potenziale ricreativo"138. In riferimento al contesto scientifico e tecnico fino qui delineato, è utile premettere una esplicita distinzione dei concetti di continuità paesistico-ambientale e di reticolarità ecologica alla definizione di una ipotesi quadro delle idoneità dei sistemi lineari di spazi aperti per il contenimento e la riduzione della frammentazione paesistica. Come si è visto, "il rapporto che intercorre tra la struttura della continuità ambientale e le reti ecologiche vere e proprie (riferite alle specie naturali, ovvero specie-specifiche, n.d.r.) è un argomento tutt'ora in esame in sede di ricerca ${ }^{139}$. Confronti disciplinari ripetuti hanno dimostrato che il sistema della biocontinuità non coincide necessariamente con il sistema delle ecoconnessioni relative a diverse specie ${ }^{\prime \prime 140}$.

138 Di Fidio M., Teoria e prassi delle reti ecologiche. Dall'isolamento all'integrazione della difesa della natura, intervento al seminano Le reti ecologiche: strategie di equipagiamento paesistico e miglioramento ambientale, 26 maggio 1999, DUPT Firenze, Dottorato di Ricerca in Progettazione Paesistica. Questi temi sono studiati da tempo anche in Italia: si vedano in merito anche le citazioni di Guido Ferrara riportate nel precedente paragrafo 3.1 in relazione alla interpretazione diagnostica del censimento degli spazi aperti. Tale metodologia è infatti espressamente finalizzata a riconoscere ed orientare progettualmente le caratteristiche strutturali del mosaico degli spazi aperti verso scenari equilibrati di differenziazione degi usi e di controllo consapevole delle funzioni paesistiche ed ambientali di cui essi si fanno carico o potrebbero farsi carico.

139 Si veda Boitani L., La tutela e la zalorizzazione della biodizersità terrestre in Italia: appunti per la Rete Ecologica Nazionale, seminario nazionale Conserzazione della natura e sziluppo locale: il sistema delle aree protette e la Rete Ecologica Nazionale, ECOLAVORO99, Legambiente, Ministero dell'Ambiente, Federazione Italiana Parchi e Riserve Naturali, Firenze 14 dicembre 1999 ed il quadro degli studi di Ecologia e Biologia conservazionale segnalati nel database bibliografico Lynx accessibile presso il sito WE B del Centro E uropeo per la Conservazione della Natura (ECNC).

140 Brucculeri R., Di Ludovico D., Perrotti A., Properzi P., Romano B., Tamburini G., La continuità ambientale in A bruzzo. Riferimenti e criteri metodologici per la pianificazione della rete ecologica regionale, ANPA, Agenzia Nazionale per la Protezione dell'Ambiente, Piano Stralcio per lo sviluppo del sistema nazionale conoscitivo e dei controlli in campo ambientale, Progetto Monitoraggio delle Retri Ecologiche, Regione Abruzzo, Università dell'Aquila, sintesi del rapporto tecnico finale consegnato il 10 novembre 1999, 2000. 
Da questo deriva che trattare di frammentazione paesistica, come fenomenologia territoriale, e di corridoi, o sistemi di continuità, come soggetti di studio e di analisi e progettazione, non coincide con il definire e progettare le reti ecologiche. Si tratta piuttosto di un approccio alla interpretazione del paesaggio strettamente legato al riconoscimento delle evoluzioni di matrice antropica a cui questo è andato soggetto, in particolare in epoca contemporanea, con gradi di intensità sensibilmente rilevanti nelle aree ad elevata pressione insediativa.

$\grave{E}$ indubbio che queste argomentazioni afferiscano ad un complesso di problematiche che, per quanto interrelate, necessitano di congrue distinzioni, per produrre contributi scientifici validi a prescindere dagli approcci ideologici, che costituiscono un rischio costante per la pianificazione territoriale. Allo stesso tempo, il fatto che gli obiettivi ed i processi di contenimento e riduzione della frammentazione paesistica siano generalmente in linea con le istanze di conservazione della biodiversità e di salvaguardia della reticolarità ecologica, costituisce certamente una componente di sinergia non trascurabile.

Il modello proposto non riguarda tanto un approccio tematico, come quello delle reti ecologiche per le popolazioni animali, ma è incentrato piuttosto sull'obiettivo generale della riqualificazione e conservazione diffusa rispetto alle specifiche criticità della frammentazione del paesaggio.

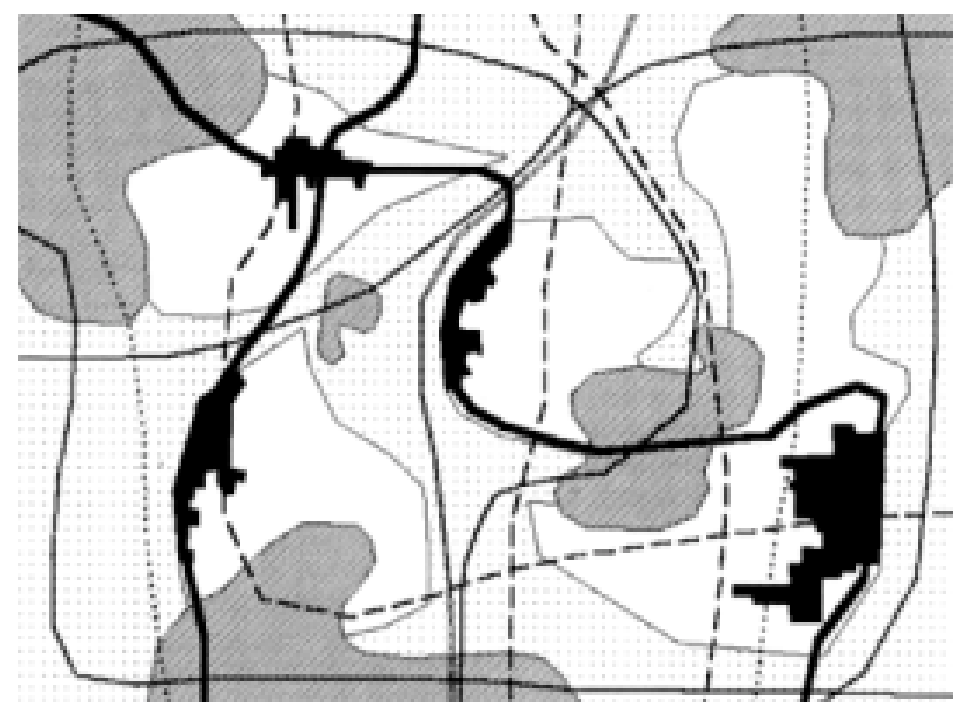

Figura 49 - Schema esemplificativo delle relazioni possibili tra gli spazi di continuità biotica del paesaggio, e di conseguente connessione ambientale degii habitat naturali, e le reti ecologiche di movimento delle diverse specie (animali) nel paesaggio stesso (immagine tratta da: Romano B., Continuità ambientale. Pianificare per il riassetto ecologico del territorio, Andromeda Editrice, Teramo, 2000). È utile considerare che: (1) a diverse specie animali non necessariamente corrisponde, in uno stesso territorio, la stessa rete ecologica; (2) le reti ecologiche specie-specifiche non sono necessariamente comprese nelle aree di continuità biotica del paesaggio, pur essendo queste probabilisticamente le più favorevoli alla presenza ed allo spostamento di animali.

Gli elementi di idoneità dei sistemi lineari di spazi aperti per il contenimento e la riduzione della frammentazione paesistica nei territori periurbani di pianura fanno riferimento alla articolazione delle misure di riqualificazione paesistica secondo 17 obiettivi primari, rispetto ai quali i sistemi lineari di spazi aperti vengono distinti, in base alla configurazione, in sistemi semplici, identificati dal punto di vista morfologico, ed in sistemi complessi, identificati dal punto di vista strategico. L'attributo lineare, riferito ai sistemi paesistici considerati, è evidentemente utilizzato con finalità di identificazione relativamente al carattere topologico distintivo ritenuto più importante in termini di diversificazione rispetto ad altri tipi di configurazioni paesistiche. Esso non si riferisce ad alcun connotato dimensionale quantificabile, bensì alla riconoscibilità della prevalente estensione spaziale in una determinata direzione.

Considerando i 17 obiettivi primari rispetto alle due tipologie di sistemi lineari di spazi aperti, semplici e complessi, si hanno pertanto 34 casi, con diversi livelli di idoneità di queste configurazioni per la riqualificazione diffusa del paesaggio, che costituiscono altrettante categorie interpretative e 
progettuali, di cui si dà, nelle pagine successive, una descrizione specifica ed un quadro di sintesi e confronto (figura 50).

Idoneità dei sistemi lineari di spazi aperti per il contenimento e la riduzione della frammentazionepaesistia Criteri per la zalutazione diagnostica e la definizione progettuale di misure di interzento ${ }^{141}$

A Sistemi semplici di spazi aperti (identità morfologica)

A(1) Costituzione di riserze di risorse - idoneità massima

Ai sistemi semplici afferiscono funzioni paesistico-ambientali di conservazione, essendo composti, oltre che da spazi non mineralizzati, da piantagioni prevalentemente non colturali. La valenza di riserve di risorse, che essi possono assumere attraverso una congrua conservazione e/o diffusione nelle aree periurbane, diviene strategica, per l'efficacia ottenibile in ragione delle loro caratteristiche spaziali, particolarmente idonee ad inserirsi in tessuti frammentati e congestionati.

$\mathrm{A}(2) \quad$ Incremento della biomassa zegetale - idoneità massima

Le formazioni vegetali lineari dei sistemi semplici possono costituire tipologie di piantagione idonee in presenza di frammentazione delle proprietà e degli spazi per ottenere effetti di riqualificazione ambientale diffusa del paesaggio periurbano. Questo elemento di idoneità risulta strettamente connesso al precedente $\mathrm{A}(1)$ per quanto riguarda le forti valenze ambientali e le caratteristiche di idoneità spaziale, ma si distingue per essere specificamente relativo a misure di incremento della biomassa vegetale.

A(3) Costituzione di habitat regetali - idoneità media

Pur non coprendo i requisiti esigenziali di tutte le specie di pianura, le formazioni vegetali lineari dei sistemi semplici permettono il reinserimento e la diffusione di molte specie arboree ed arbustive tipiche del paesaggio planiziale. La loro presenza, per gli effetti di regolazione delle condizioni microclimatiche, può favorire lo sviluppo di habitat vegetali di specie esigenti, che non prediligono la forte esposizione all'azione dei venti e dell'irraggiamento solare, migliorando complessivamente la diversità biologica e morfologica del paesaggio.

A(4) Costituzione di habitat animali - idoneità media

Il recupero di spazi con funzioni di habitat animali non può efficacemente essere affidato solo all'equipaggiamento paesistico ed ai caratteri ecosistemici complessivi dei sistemi semplici per l'esiguità della loro dimensione trasversale più frequente, rispetto ai requisiti esigenziali di colonizzazione che caratterizzano molte specie (fanno eccezione sostanzialmente solo i corridoi fluviali). Nonostante questo non sono pochi gli animali di piccola taglia che trovano anche nei sistemi costituiti da formazioni vegetali lineari minori, in particolare nelle siepi di campo, condizioni di vita idonee alle proprie esigenze. Il livello di idoneità può salire sensibilmente al crescere della dimensione trasversale, fino a raggiungere elevati livelli di interesse strategico, nel caso dei corridoi fluviali.

A(5) Connessione biotica di aree di interesse naturalistico e/ o ecologico - idoneità massima

Le formazioni vegetali lineari dei sistemi semplici possono costituire strutture di continuità biotica tra spazi e sistemi di spazi più complessi e con geometria più compatta, idonei ad assolvere funzioni di habitat (la funzione di connettività però è limitata a situazioni speciespecifiche, di cui occorre avere idonea documentazione statistica).

141 La definizione dei criteri è stata astratta dall'attuale contesto legislativo nazionale riguardante le strade extraurbane ed i corsi d'acqua, in ragione dei molti elementi di conflittualità ed incongruenza, dei quali sono auspicabili nette e sollecite soluzioni, nell'interesse generale della conservazione e dello sviluppo di paesaggi di buona qualità. 
A(6) Costituzione di habitat umano con spazi aperti di servizio - idoneità media

Con esclusione delle tipologie più elementari e compatte (siepi di campo e assimilabili), i sistemi semplici relativi a corsi d'acqua ed infrastrutture viarie possono costituire componenti sostanziali del sistema degli spazi aperti con funzioni di servizio.

Congrui livelli e tipi di equipaggiamento vegetale permettono varie forme di diversificazione degli spazi in essi compresi, rendendoli idonei all'utilizzo per la mobilità e/o la ricreazione all'aperto.

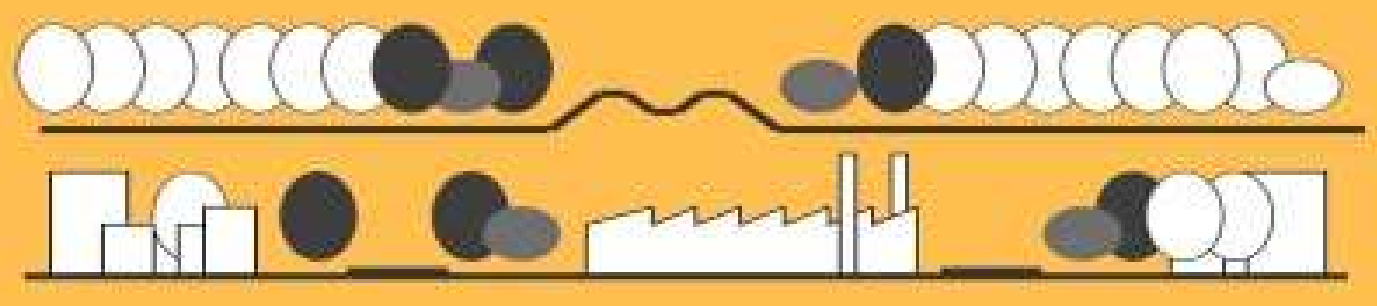

A(7) Collegamento funzionale di parchi e spazi zerdi - idoneità media

Secondo il precedente elemento di idoneità $\mathrm{A}(6)$ e con ulteriore esclusione delle tipologie afferenti alle infrastrutture viarie ad elevato carico di traffico, soprattutto i sistemi semplici relativi ai corsi d'acqua, ma anche quelli della viabilità secondaria, possono divenire collegamenti strategici di spazi verdi, determinando, se opportunamente messi a sistema, una rete di percorrenze alternative funzionali agli spostamenti urbani casa/scuola e casa/lavoro, oltre che a quelli con finalità ricreative.

A(8) Definizione morfologica degii spazi - idoneità massima

Le formazioni vegetali lineari di equipaggiamento paesistico dei sistemi semplici possono costituire tipologie di piantagione idonee a recuperare congrue qualità dimensionali e di articolazione morfologica degli spazi nel paesaggio di pianura, sviluppando la diffusione del cosiddetto effetto del limite.

L'esempio considerato nello schema altimetrico si riferisce a margini di campi ed a fasce a ridosso degli argini di un corso d'acqua in area di bonifica: la situazione priva di chiara struttura spaziale (schema in alto) è messa a confronto con una ipotesi di equipaggiamento vegetale, con margini costituiti da filari o fasce arboree e/o arbustive (schema in basso).

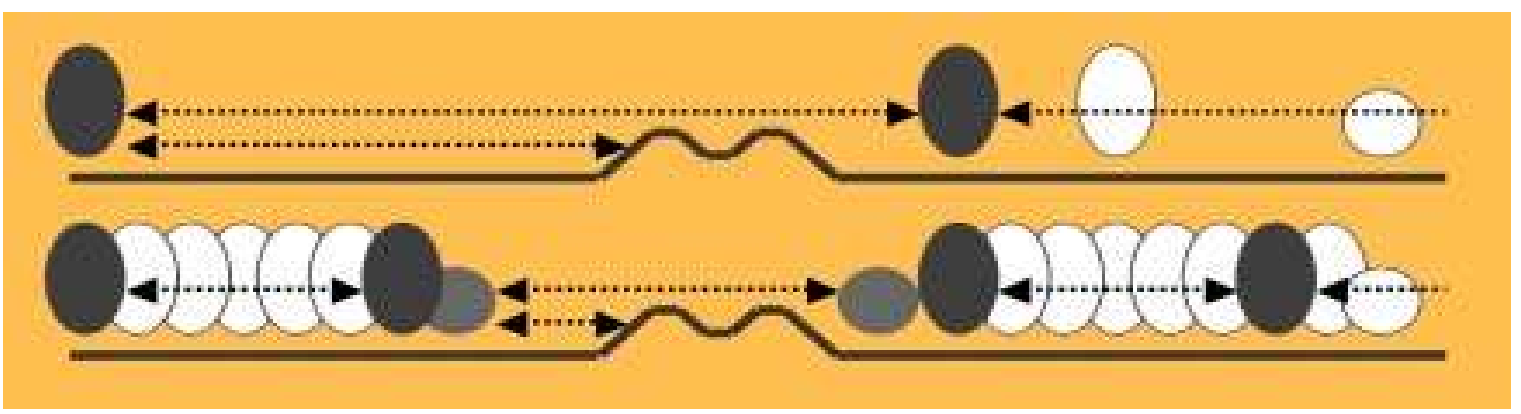

Alla diffusione di sistemi paesistici lineari, in particolare nelle aree periurbane sono legate molte delle possibilità di recupero di qualità morfologica del paesaggio e del conseguente potenziale ricreativo, aspetto non trascurabile in relazione alle caratteristiche di onerosità ed alle carenze degli spazi verdi pubblici di tipo tradizionale. Entrambi questi effetti concatenati sono ottenibili minimizzando le interferenze con la proprietà privata delle aree. 


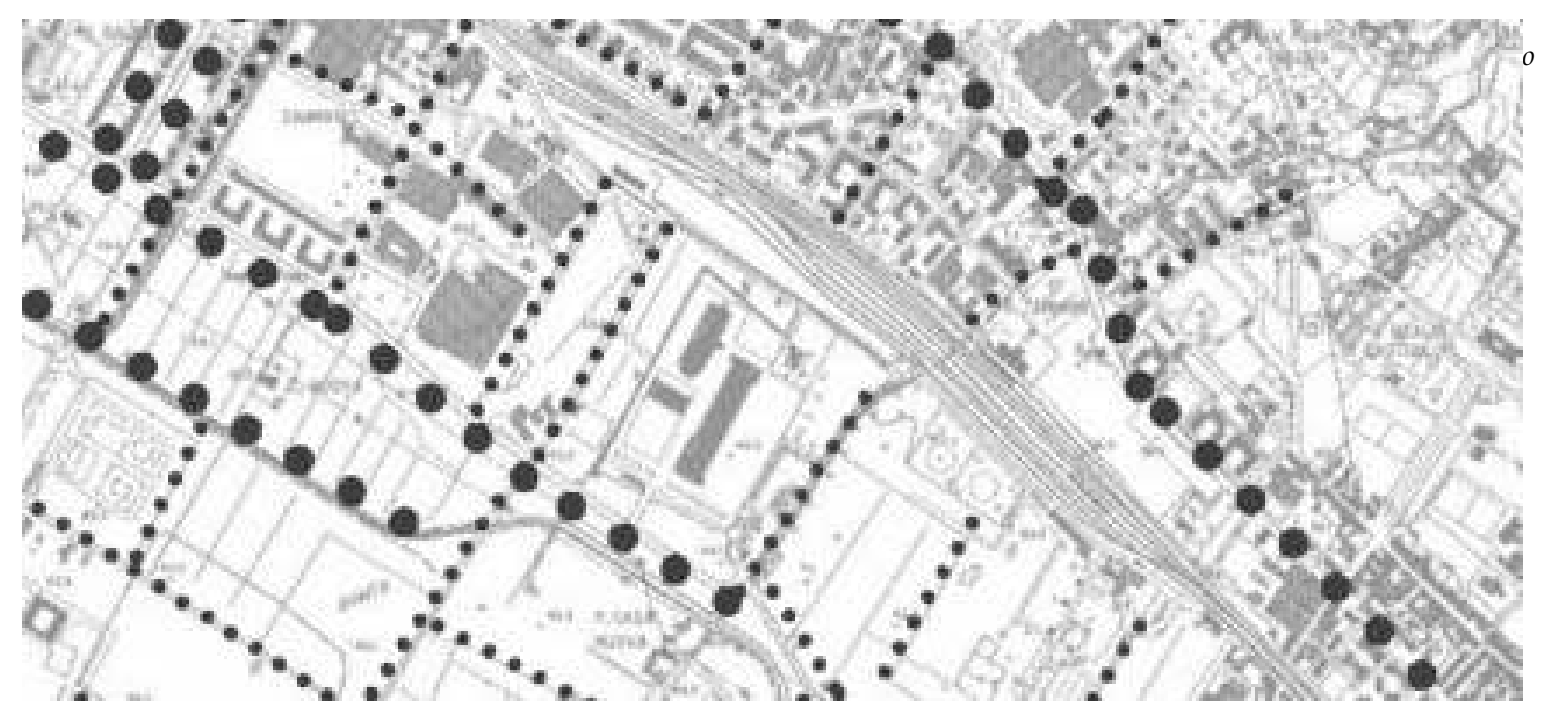

Lo schema planimetrico da conto delle possibilità di impiego di sistemi vegetali lineari per il riordino ed il miglioramento dell'articolazione degli spazi aperti di margine urbano, con benefici sostanziali in termini di recupero di identità spaziale e di limitazione delle conflittualità visive (si veda il successivo elemento di idoenità A9).

A(9) Separazione visuale di usi del suolo conflittuali - idoneità media

Il livello di idoneità, dipendente dalle scelte di composizione vegetale delle piantagioni e dalle loro dimensioni trasversali, può raggiungere requisiti di elevata efficacia.

Nello schema è confrontata la situazione frequente di assenza o carenza di separazione degli usi del suolo conflittuali (schema in alto) con la situazione ottenibile attraverso formazioni vegetali (schema in basso), con condizionamenti progressivamente più pesanti al diminuire degli spazi disponibili.

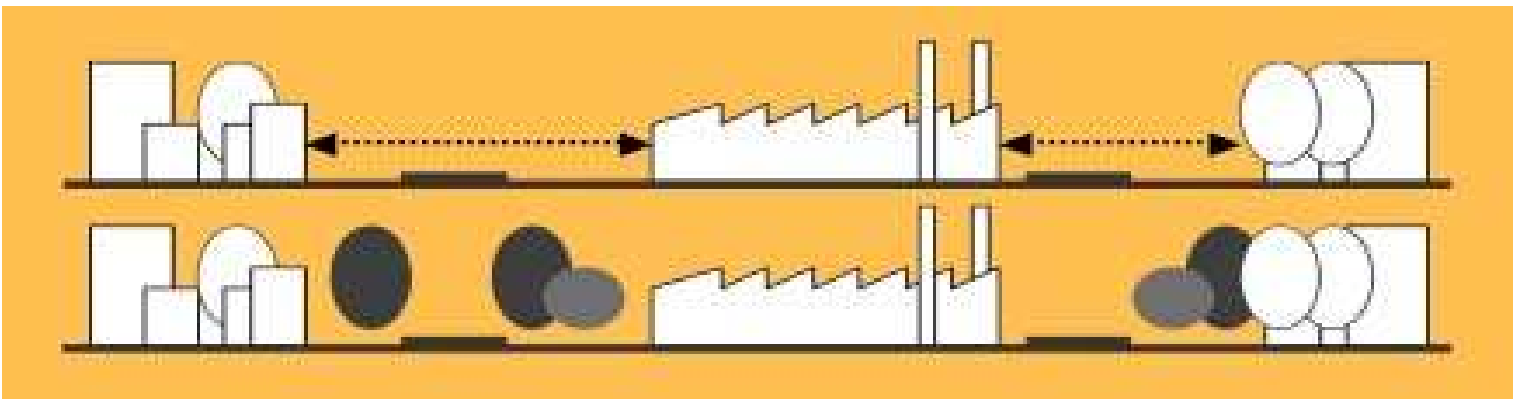

A sinistra è esemplificata la conflittualità di insediamenti industriali/artigianali con insediamenti residenziali, a destra la conflittualità di insediamenti industriali/artigianali con spazi aperti di servizio alla residenza (campi gioco, centri sportivi di base, eccetera).

$\mathrm{A}(10)$ Separazione acustica e/ o olfattiza di usi del suolo conflittuali - idoneità minima

L'efficacia delle piantagioni vegetali dei sistemi semplici è decisamente condizionata dalle loro dimensioni trasversali, nei casi di siepi e filari comunque non idonee a garantire requisiti prestazionali soddisfacenti. L'idoneità complessiva di questi sistemi può divenire apprezzabile, anche con dimensioni trasversali esigue, nel caso siano presenti rilevati in terra, per le spiccate capacità di assorbimento acustico.

$\mathrm{A}(11)$ E quipagoiamento zegetale di infrastrutture viarie - idoneità massima

Le formazioni vegetali dei sistemi semplici possono migliorare il microclima e la percepibilità degli spazi stradali, differenziandoli in ragione dell'intorno e contribuendo alla piacevolezza ed alla sicurezza del percorso, e svolgere funzioni di protezione ambientale, contribuendo alla fissazione delle polveri ed alla depurazione dell'aria dalle emissioni inquinanti, come alla depurazione delle acque di scolo, prima della immissione nel reticolo idrografico superficiale e della penetrazione in falda. Secondo questi requisiti, è opportuno che l'assenza su entrambii 
lati della strada di formazioni arboree e/o arbustive sia circoscritta essenzialmente a due ordini di ragioni:

- funzionali, riguardanti la necessità di segnalare una determinata situazione relativa ad incrocio quant'altro possa condizionare la sicurezza del transito di veicoli e pedoni;

- percettive, riguardanti la salvaguardia e la valorizzazione delle opportunità visive di emergenze paesistiche culturali o naturali.

Nel caso dell'equipaggiamento vegetale di una infrastruttura extraurbana, non occorre che le alberate stradali e le eventuali piantagioni arbustive che completano queste formazioni siano pedissequamente continue. Nei tratti stradali in cui è possibile percepire un paesaggio di pregio, la sezione di equipaggiamento vegetale può essere asimmetrica (schema in alto) per aprire lo spazio della strada verso quelli circostanti, identificabili anche con il concorso di formazioni vegetali lineari ordite secondo direzioni trasversali allo sviluppo della strada.

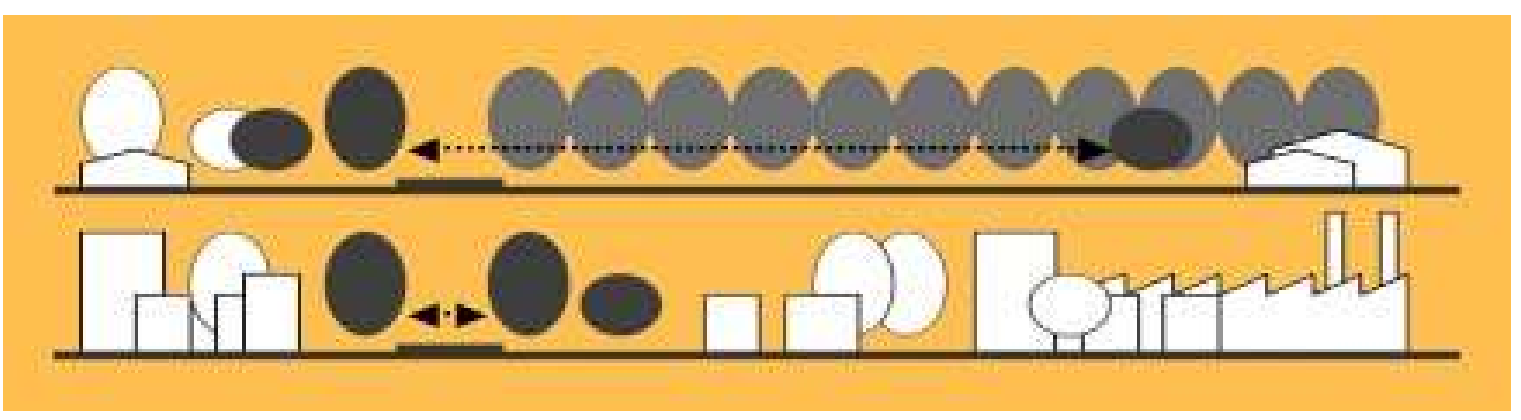

Nel caso dell'equipaggiamento vegetale di una infrastruttura viaria urbana o che comunque attraversa un paesaggio congestionato e profondamente alterato da insediamenti e infrastrutture di scadente qualità architettonica, è opportuno che le alberate stradali e le eventuali piantagioni arbustive che completano queste formazioni siano il più possibile continue, al fine di conferire identità spaziale ai luoghi, interessando prioritariamente i lati della strada che presentano le condizioni maggiormente critiche (schema in basso).

A(12) Inserimento visuale di infrastrutture viarie - idoneità massima

Le formazioni vegetali lineari di sistemi semplici opportunamente individuati nel paesaggio circostante infrastrutture viarie di grande impatto, possono limitarne la percezione a vedute parziali, riducendo la compresenza di elementi conflittuali, dovuti all'infrastruttura stessa, come a detrattori preesistenti, percepibili attraverso il suo utilizzo. Si veda anche l'elementodi idoneità $\mathrm{A}(11)$.

A(13) Equipagoiamento regetale del paesaggio rurale - idoneità massima

Le formazioni vegetali lineari dei sistemi semplici possono migliorare la qualità ambientale e morfologica del paesaggio rurale di pianura, sviluppandone inoltre il potenziale ricreativo rispetto alla domanda sociale delle aree urbane e metropolitane.

Lo schema planimetrico descrive le opportunità di intervento alle quali sono connessi effettidi miglioramento anche per gli obiettivi di qualità considerati dagi elementi di idoneità $\mathrm{A}(1), \mathrm{A}(2), \mathrm{A}(8)$.

$\mathrm{A}(14)$ Regolazione anemometrica e mitigazione degli impatti sul suolo - idoneità massima

Nel paesaggio rurale, l'effetto frangivento delle formazioni vegetali lineari dei sistemi semplici può ridurre i fenomeni di erosione eolica dei suoli e quelli di inaridimento per evapotraspirazione e di esposizione al gelo. Particolare attenzione nella predisposizione di formazioni vegetali con queste funzioni deve essere riservata alla valutazione preventiva degli effetti sugli insediamenti limitrofi, che possono essere di beneficio, con miglioramento della ventilazione e conseguente regolazione dell'isola di calore, ma anche di peggioramento, per distrazione delle correnti esistenti. 


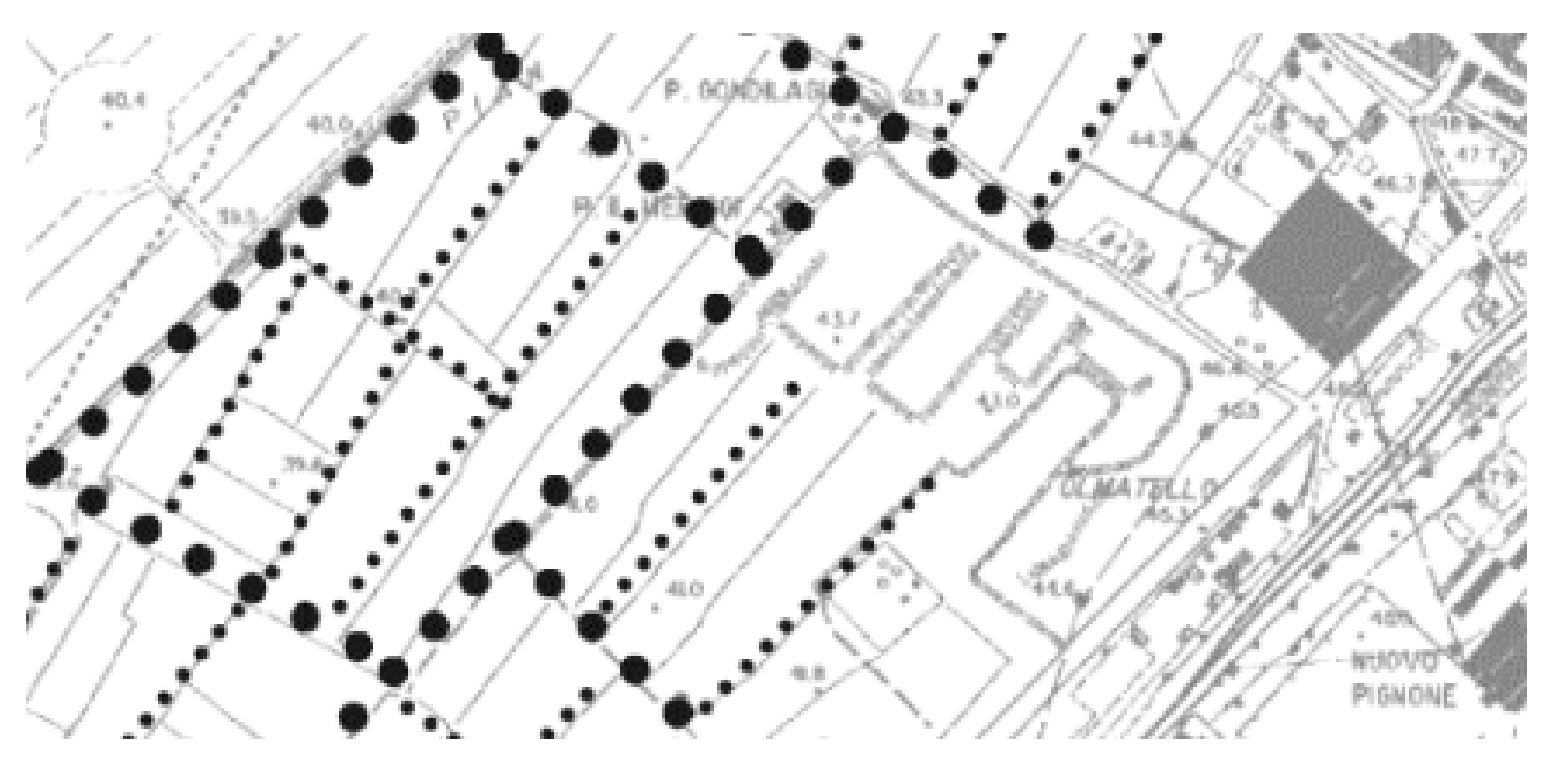

A(15) Protezione ambientale e regolazione microclimatica urbane - idoneità massima

Relativamente agli obiettivi di equipaggiamento vegetale del paesaggio urbano per la fissazione delle polveri e la depurazione dell'aria dalle emissioni inquinanti e per il miglioramento del microclima, le formazioni vegetali dei sistemi semplici possono avere una elevata capacità di adattamento ed inserimento in tessuti generalmente carenti di spazio: la loro efficacia è funzione del grado di diffusione complessivo delle piantagioni.

A(16) Protezione ambientale delle acque superficiali - idoneità massima

Conservate o realizzate con particolare attenzione alla loro densità presso i corsi d'acqua, le formazioni vegetali dei sistemi semplici, secondo una opportuna composizione fitosociologica, possono svolgere funzioni di filtro per la depurazione delle acque di scolo dei campi e delle strade extraurbane e delle acque di scolo non captate dai sistemi di smaltimento e depurazione a servizio degli spazi urbanizzati, prima della loro confluenza nel reticolo idrografico superficiale.

A(17) Protezione ambientale delle acque di falda - idoneità media

Le formazioni vegetali dei sistemi semplici, secondo una opportuna composizione fitosociologica, contribuendo al trattenimento delle acque meteoriche e garantendo nelle loro sedi suoli permeabili, possono costituire con le altre piantagioni, un presidio ambientale per gli acquiferi sotterranei: la loro efficacia è però strettamente dipendente dal grado di densità territoriale delle piantagioni, fino a divenire irrilevante nel caso in cui esse siano limitate a scansioni spaziali molto dilatate e/o siano caratterizzate da dimensioni trasversali esigue. 
$\mathrm{B}(1) \quad$ Costituzione di riserze di risorse - idoneità massima

La progressiva riduzione degli spazi non mineralizzati, in particolare nelle aree urbane e periurbane, richiede la protezione delle ultime risorse disponibili. La formazione di sistemi complessi costituiti da spazi aperti differenziati, evitandone la marginalizzazione e la banalizzazione, ne può elevare l'importanza, contribuendo a proteggerli dai processi di consumo. In tal senso è determinante che agli spazi aperti siano riferiti scenari di uso e gestione contenenti opzioni alternative, appartenenti a categorie compatibili con la funzione primaria complessiva del sistema. Al contrario l'assenza di un esplicito e riconosciuto destino, pone queste aree in condizioni di grave rischio di aggressione da parte dei processi di sviluppo urbano della infrastrutturazione e della edificazione, tradizionalmente prevaricanti.

$\mathrm{B}(2) \quad$ Incremento della biomassa vegetale - idoneità massima

La differenziazione degli spazi aperti nell'ambito dei sistemi complessi permette, anche nelle aree a forte presenza insediativa, di incrementare le opportunità di sistemazione a verde $\mathrm{e}$, conseguentemente, secondo opportune scelte progettuali, di incrementare la biomassa vegetale.

B(3) Costituzione di habitat zegetali - idoneità massima

Per il precedente criterio di idoneità $\mathrm{B}(2)$ e per le possibilità di diversificazione tipologica degli spazi aperti caratteristiche dei sistemi complessi, è possibile ottenere attraverso di essi un sostanziale incremento di habitat vegetali.

B(4) Costituzione di habitat animali - idoneità media In particolare nel caso del paesaggio rurale, ma anche nelle aree urbane di margine, l'articolazione raggiungibile attraverso la messa in rete di più spazi aperti secondo una strategia unitaria afferente ad uno o più sistemi complessi, può contribuire alla conservazione ed allo sviluppo di habitat animali che, nelle aree ad elevata pressione insediativa ed impoverimento biologico, possono divenire importanti stazioni di passo e/o permanenza.

$\mathrm{B}(5) \quad$ Connessione biotica di aree di interesse naturalistico e/ o ecologico - idoneità massima

La costituzione di sistemi complessi di spazi aperti, in molte situazioni tipiche delle realtà territoriali metropolitane, può risultare una via strategica per la continuità biotica del paesaggio, concepita in questo caso, anziché attraverso strutture omogenee, attraverso mosaici differenziati, caratterizzati da destinazioni d'uso compatibili con la funzione paesistica primaria.

B(6) Costituzione di habitat umano con spazi aperti di servizio - idoneità massima

Gli stati di deficit di spazio che caratterizzano le aree urbane e metropolitane fanno sì che $\mathrm{i}$ sistemi complessi di spazi aperti possano costituire strutture strategiche di incremento delle dimensioni unitarie dei complessi continui di spazi verdi e di collegamento di aree di rilevante interesse sociale, anche in termini di sviluppo di itinerari alternativi a quelli congestionati della viabilità carrabile.

B(7) Collegamento funzionale di parchi e spazi verdi - idoneità massima

Attraverso sistemi complessi di spazi aperti, è possibile mettere in continuità funzionale aree verdi isolate. Questa operazione determina, all'atto della connessione, sistemi di scala spaziale maggiore, a cui possono corrispondere elementi di rilievo dal punto di vista dei servizi sociali che gli spazi svolgono e/o ospitano.

B(8) Definizione morfologica degli spazi - idoneità massima

La considerazione a scala paesistica della valenza dell'effetto del limite diviene di fondamentale importanza in riferimento alle problematiche di identità morfologica dei margini urbani. I sistemi complessi di spazi aperti possono configurarsi anche come fasce o cinture verdi che, nel più ampio quadro generale del paesaggio urbano, possono acquisire rinnovati significati 
ed efficacie, rispetto ai modelli storici, rispondenti ai canoni strutturali della città monocentrica.

B(9) Separazione visuale di usi del suolo conflittuali - idoneità massima

La costituzione di sistemi complessi di spazi aperti, secondo opportune modalità di equipaggiamento vegetale, può permettere anche la totale eliminazione di fenomeni di intervisibilità negativa. Nello schema è confrontata una situazione di intervisibilità di tessuti insediativi industriali e residenziali nella frequente condizione con interclusione di seminativi o terreni nudi abbandonati (schema in alto), talvolta coincidenti con aree verdi di standard urbanistico, con la separazione visuale completa, ottenibile attraverso spazi con piantagioni di alto fusto, a cui possono corrispondere diverse destinazioni d'uso, sia di servizio, che produttive, in funzione della estensione (schema in basso).

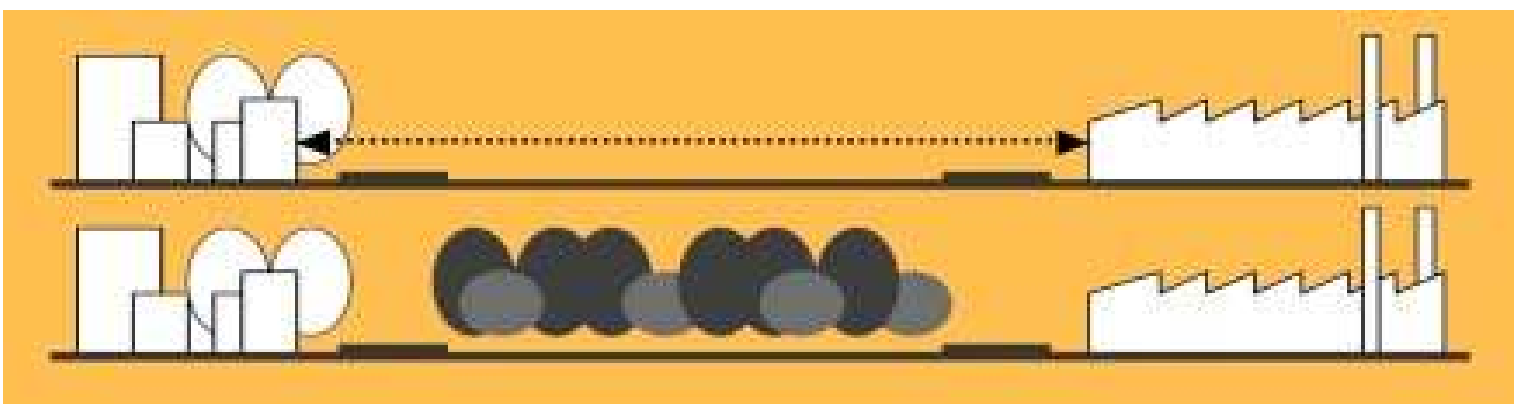

B(10) Separazione acustica e/ o olfattiza di usi del suolo conflittuali - idoneità media

La costituzione di sistemi complessi di spazi aperti, secondo opportune modalità di equipaggiamento vegetale, può permettere anche la totale eliminazione di fenomeni di disturbo acustico e/o olfattivo. Tale idoneità è limitata alla previsione di spazi che escludano l'utilizzo umano in quanto, in caso contrario, essi stessi diverrebbero aree critiche, ed è funzione delle dimensioni minime degli spazi di separazione.

Lo schema descrive la funzione di filtro che, con diversi gradi di efficacia, un sistema di spazi verdi con piantagioni arboree di alto fusto ed arbustive può svolgere rispetto agli impatti acustici ed olfattivi di un'area produttiva. A queste tipologie di sistemi di spazi verdi afferiscono anche importanti potenziali di fissazione delle polveri e di depurazione dell'aria dalle emissioni inquinanti. In ragione della estensione di tali sistemi l'idoneità può complessivamente elevarsi in maniera sostanziale.

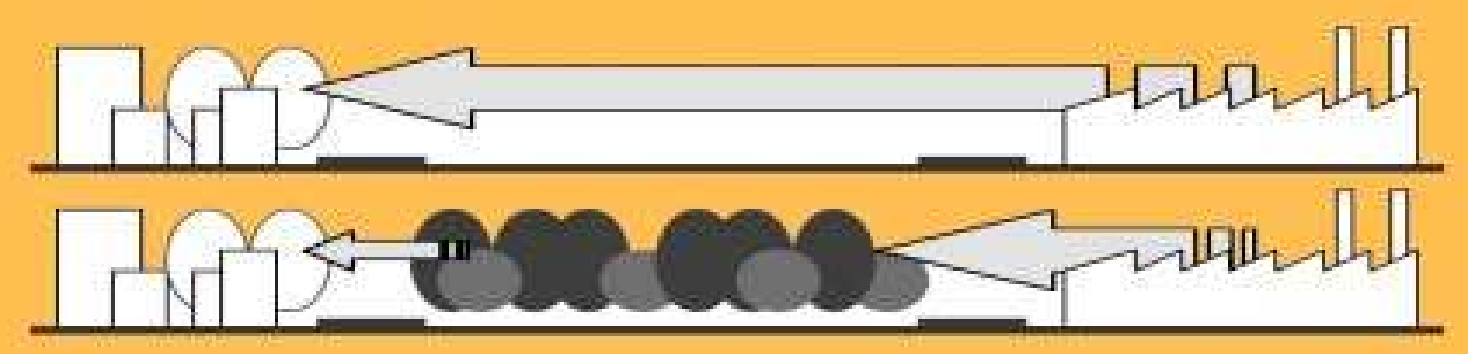

$\mathrm{B}(11)$ Equipagoiamento zegetale di infrastrutture viarie - idoneità massima

La considerazione di una infrastruttura viaria all'interno di uno o più sistemi complessi di spazi aperti può permettere di orientarne la pianificazione e la progettazione secondo obiettivi di protezione ambientale, contribuendo alla fissazione delle polveri ed alla depurazione dell'aria dalle emissioni inquinanti, come alla depurazione delle acque di scolo, prima della immissione nel reticolo idrografico superficiale e della penetrazione in falda, compatibilmente con obiettivi complementari di miglioramento microclimatico e percettivo degli spazi stradali 
e delle condizioni di percezione del paesaggio di pertinenza visuale immediata, come dello scenario complessivo.

$\mathrm{B}(12)$ Inserimento visuale di infrastrutture viarie - idoneità massima

La considerazione di una infrastruttura viaria all'interno di uno o più sistemi complessi di spazi aperti può permettere di orientarne la pianificazione e la progettazione secondo obiettivi di mitigazione degli effetti negativi che l'infrastruttura produce sul paesaggio, come di quelli dovuti a detrattori preesistenti, percepibili durante l'utilizzo dell'infrastruttura stessa.

$\mathrm{B}(13)$ Equipagoiamento zegetale del paesagoio rurale - idoneità massima

La costituzione, lungo i corsi d'acqua principali o attraverso spazi agricoli marginali, di sistemi complessi di spazi aperti con destinazioni diversificate e compatibili con le produzioni agricole, può contribuire ad elevare la qualità ambientale e morfolgica del paesaggio rurale di pianura, sviluppandone inoltre il potenziale ricreativo rispetto alla domanda sociale delle aree urbane e metropolitane.

$\mathrm{B}(14)$ Regolazione anemometrica e mitigazione degli impatti sul suolo - idoneità massima

Nel paesaggio rurale, la presenza di sistemi complessi di spazi aperti, ad esempio lungo i corsi d'acqua, permette di ottenere effetti di regolazione dei venti, che possono costituire integrazioni sostanziali delle funzioni del reticolo diffuso dell'equipaggiamento vegetale minuto degli spazi colturali, ove presente, e possono assumere il ruolo strategico di dotazione di base, in sua assenza. Si vedano anche i criteri di attenzione relativi agli elementi di idoneità $\mathrm{A}(14)$.

$\mathrm{B}(15)$ Protezione ambientale e regolazione microclimatica urbane - idoneità massima

La quantità di biomassa vegetale ottenibile attraverso la costituzione di sistemi complessi di spazi aperti e la loro capacità di permeazione dei tessuti insediativi urbani e periurbani, fanno sì che essi possano costituire importanti strategie per il miglioramento e la protezione ambientale dei territori ad elevata pressione insediativa, con efficacie specifiche per la fissazione delle polveri e la depurazione dell'aria dalle emissioni inquinanti e per il miglioramento del microclima.

B(16) Protezione ambientale delle acque superficiali - idoneità massima

L'incremento della continuità e della quantità di spazi verdi ottenibile attraverso sistemi complessi di spazi aperti fa sì che essi possano costituire una importante politica di miglioramento e protezione ambientale (complementare al riequilibrio idraulico di bacino ed alla depurazione delle acque reflue urbane civili ed industriali) dei corsi d'acqua nel territorio di pianura ed in particolare negli insediamenti. Gli spazi diversificati afferenti a tali sistemi possono contribuire sostanzialmente alla depurazione, prima della loro confluenza nel reticolo idrografico superficiale, sia delle acque di scolo dei campi e delle strade extraurbane, che delle acque di scolo non captate dai sistemi di smaltimento e depurazione a servizio degli spazi urbanizzati.

B(17) Protezione ambientale delle acque di falda - idoneità massima

Relativamente all'elevato grado di impermeabilizzazione del suolo caratteristico delle aree urbane e periurbane, la costituzione di sistemi complessi di spazi aperti con una opportuna composizione fitosociologica delle piantagioni, contribuendo al'incremento o almeno alla conservazione dei suoli non mineralizzati, può svolgere funzioni di presidio ambientale delle falde acquifere. La copertura vegetale degli spazi aperti può nello specifico contribuire efficacemente al trattenimento ed alla depurazione delle acque meteoriche e di dilavazione: tali funzioni sono caratterizzate da gradi di efficacia in relazione inversa con la strutturazione artificiale ed il carico di uso degli spazi ed in relazione diretta con la composizione vegetale. 
Figura 50 a

IDONEITÀ DEI SISTEMI LINEARI DI SPAZI APERTI PER IL CONTENIMENTO E LA RIDUZIONE DELLA FRAMMENT AZIONE PAESISTICA

OBIETTIVI

DI RIQUALIFICAZIONE PAESISTICA

\section{(A) - SISTEMI SEMPLICI DI SPAZI APERTI} (identità "morf ologica")
IDONEITÀ

(01) - COSTITUZIONE DI RISERVE DI RISORSE

massima

(02) - INCREMENTO DELLA BIOMASSA VEGETALE massima

(03) - COSTITUZIONE DI HABITAT VEGETALI media

media massima

(05) - CONNESSIONE BIOTICA

DI AREE DI INTERESSE NATURALISTICO E/O ECOLOGICO

(06) - COSTITUZIONE DI HABITAT UMANO CON SPAZI APERTI DI SERVIZIO

media

(07) - COLLEGAMENTO FUNZIONALE
DI PARCHI E SPAZI VERDI

(08) - DEFINIZIONE MORFOLOGICA
DEGLI SPAZI

massima

(09) - SEPARAZIONE VISUALE DI USI DEL SUOLO CONFLITTUAL

media

(10) - SEPARAZIONE ACUSTICA E/O OLFATTIVA DI USI DEL SUOLO CONFLITTUALI

minima

filari risultano singolarmente non idonei a garantire requisiti soddisfacenti.

L'idoneità complessiva può divenire comunque apprezzabile nel caso siano presenti rilevati in terra.

Possono migliorare il microclima e la percepibilità degli spazi stradali, differenziandoli in ragione dell'intorno e contribuendo alla piacevolezza ed

(11) - EQUIPAGGIAMENTO VEGETALE DI INFRASTRUTTURE VIARIE

massima

alla sicurezza del percorso, e svolgere funzioni di protezione ambientale, contribuendo alla fissazione delle polveri ed alla depurazione dell'aria dalle emissioni inquinanti. come alla depurazione delle acque di scolo.

Opportunamente individuati nel paesaggio circostante infrastrutture viarie d grande impatto, possono limitarne la percezione a vedute parziali, riducendo la compresenza di elementi conflittuali, dovuti all'infrastruttura stessa,comea detrattori preesistenti. Si veda anche l'elemento di idoneità $A(11)$.

(12) - INSERIMENTO VISUALE DI INFRASTRUTTURE VIARIE

massima

Possono migliorare la qualità ambientale e morfologica del paesaggio rurale di pianura, sviluppandone inoltre il potenziale ricreativo rispetto alladomanda sociale delle aree urbane e metropolitane. Si vedano anche gli elementi di idoneità $A(1), A(2), A(8)$

(13) - EQUIPAGGIAMENTO VEGETALE DEL PAESAGGIO RURALE

massima

L'effetto frangivento può ridurre i fenomeni di erosione eolica dei suoliequelli L'effetto frangivento può ridurre i fenomeni di erosione eolica dei suoliequelli
di inaridimento per evapotraspirazione e di esposizione al gelo. Particolare di inaridimento per evapotraspirazione e di esposizione al gelo. Particolare
attenzione deve essere riservata alla valutazione preventiva degli effetti sugli insediamenti limitrofi.

(14) - REGOLAZIONE ANEMOMETRICA E MITIGAZIONE DEGLI IMPATTI SUL SUOLO massima

Possono avere una elevata capacità di adattamento ed inserimento intessudi generalmente carenti di spazio, per la fissazione delle polveri e la depurazione dell'aria dalle emissioni inquinanti e per il miglioramento del microclima: Ia loro efficacia è funzione del grado di diffusione complessivo delle piantagioni.

Secondo una opportuna composizione fitosociologica, possono svolgere funzioni di filtro per la depurazione delle acque di scolo dei campi e delle

(16) - PROTEZIONE AMBIENTALE DELLE ACQUE SUPERFICIALI

massima strade extraurbane e delle acque di scolo non captate dai sistemi di smaltimento e depurazione a servizio degli spazi urbanizzati, prima dellaloro confluenza nel reticolo idrografico superficiale.

(17) - PROTEZIONE AMBIENTALE DELLE ACQUE DI FALDA
Secondo una opportuna composizione fitosociologica, possono costitureun presidio ambientale per gli acquiferi sotterranei: la loro efficacia è però strettamente dipendente dal grado di densità territoriale delle piantagioni. 
Figura 50 b

IDONEITÀ DEI SISTEMI LINEARI DI SPAZI APERTI PER IL CONTENIMENTO E LA RIDUZIONE DELLA FRAMMENTAZIONE PAESISTICA

OBIETTIVI

DI RIQUALIFICAZIONE PAESISTICA

\section{(B) - SISTEMI COMPLESSI DI SPAZI APERTI}

(identità "strategica")

\section{IDONEITÀ DESCRIZIONE SOMMARIA DEGLI ELEMENTI DI IDONEITÀ}

\begin{tabular}{|c|c|c|}
\hline (01) - COSTITUZIONE DI RISERVE DI RISORSE & massima & $\begin{array}{l}\text { Evitando la marginalizzazione e la banalizzazione degli spazi aperti, ne } \\
\text { possono elevare l'importanza, contribuendo a proteggerli dai processi di } \\
\text { consumo. L'assenza di un esplicito e riconosciuto destino, determina } \\
\text { condizioni di grave rischio di aggressione da parte dei processi disviluppo } \\
\text { urbano prevaricanti. }\end{array}$ \\
\hline (02) - INCREMENTO DELLA BIOMASSA VEGETALE & massima & $\begin{array}{l}\text { La differenziazione degli spazi aperti permette, anche nelle aree a forte } \\
\text { presenza insediativa, di incrementare le opportunità di sistemazione a } \\
\text { verde e, conseguentemente, la biomassa, anzitutto di tipo vegetale. }\end{array}$ \\
\hline (03) - COSTITUZIONE DI HABITAT VEGETALI & massima & $\begin{array}{l}\text { Per il precedente criterio di idoneità } B(2) \text { e per le possibilità di } \\
\text { diversificazione tipologica degli spazi aperti, è possibile ottenere un } \\
\text { sostanziale incremento di habitat vegetali. }\end{array}$ \\
\hline (04) - COSTITUZIONE DI HABITAT ANIMALI & media & $\begin{array}{l}\text { L'articolazione raggiungibile attraverso la messa in rete di più spazi apeti } \\
\text { secondo una strategia unitaria afferente ad uno o più sistemi, può } \\
\text { contribuire alla conservazione ed allo sviluppo di habitat animali che,nelle } \\
\text { aree ad elevata pressione insediativa ed impoverimento biologico, } \\
\text { possono divenire importanti stazioni di passo e/o permanenza. }\end{array}$ \\
\hline $\begin{array}{l}\text { (05) - CONNESSIONE BIOTICA } \\
\text { DI AREE DI INTERESSE NATURALISTICO } \\
\text { E/O ECOLOGICO }\end{array}$ & massima & $\begin{array}{l}\text { In molte situazioni tipiche delle realtà territoriali metropolitane, possono } \\
\text { risultare una via strategica per la continuità biotica del paesaggio, } \\
\text { concepita attraverso mosaici differenziati, caratterizzati da destinazioni } \\
\text { d'uso compatibili con la funzione paesistica primaria. }\end{array}$ \\
\hline $\begin{array}{l}\text { (06) - COSTITUZIONE DI HABITAT UMANO } \\
\text { CON SPAZI APERTI DI SERVIZIO }\end{array}$ & massima & $\begin{array}{l}\text { Per gli stati di deficit di spazio che caratterizzano le aree urbane e } \\
\text { metropolitane, possono costituire strutture strategiche di incrementodelle } \\
\text { dimensioni unitarie dei complessi continui di spazi verdi e di collegamento } \\
\text { di aree di rilevante interesse sociale, anche in termini di sviluppo di } \\
\text { itinerari alternativi a quelli congestionati della viabilità carrabile. }\end{array}$ \\
\hline $\begin{array}{l}\text { (07) - COLLEGAMENTO FUNZIONALE } \\
\text { DI PARCHI E SPAZI VERDI }\end{array}$ & massima & $\begin{array}{l}\text { Possono mettere in continuità funzionale aree verdi isolate. Questa } \\
\text { operazione determina, all'atto della connessione, sistemi di scalaspażale } \\
\text { maggiore, a cui possono corrispondere elementi di rilievo dal punto di } \\
\text { vista dei servizi sociali che gli spazi svolgono e/o ospitano. }\end{array}$ \\
\hline $\begin{array}{l}\text { (08) - DEFINIZIONE MORFOLOGICA } \\
\text { DEGLI SPAZI }\end{array}$ & massima & $\begin{array}{l}\text { Per la considerazione a scala paesistica della valenza dell' effetto del } \\
\text { limite, in riferimento alle problematiche di identità morfologica dei margin } \\
\text { urbani, possono configurarsi anche come fasce o cinture verdi ed } \\
\text { acquisire rinnovati significati ed efficacie, rispetto ai modelli storici, } \\
\text { rispondenti ai canoni strutturali della città monocentrica. }\end{array}$ \\
\hline $\begin{array}{l}\text { (09) - SEPARAZIONE VISUALE } \\
\text { DI USI DEL SUOLO CONFLITTUALI }\end{array}$ & massima & $\begin{array}{l}\text { Secondo opportune modalità di equipaggiamento vegetale, possono } \\
\text { permettere anche la totale eliminazione di fenomeni di intervisibilità } \\
\text { negativa. }\end{array}$ \\
\hline $\begin{array}{l}\text { (10) - SEPARAZIONE ACUSTICA E/O OLFATTIVA } \\
\text { DI USI DEL SUOLO CONFLITTUALI }\end{array}$ & media & $\begin{array}{l}\text { Secondo opportune modalità di equipaggiamento vegetale, possono } \\
\text { permettere anche la totale eliminazione di fenomeni di disturbo acustico } \\
\text { e/o olfattivo. Tale idoneità è limitata alla previsione di spazi cheescludano } \\
\text { l'utilizzo umano in quanto, in caso contrario, essi stessi diverrebberoaree } \\
\text { critiche. ed è funzione delle dimensioni minime deali spazi di separazione. }\end{array}$ \\
\hline $\begin{array}{l}\text { (11) - EQUIPAGGIAMENTO VEGETALE } \\
\text { DI INFRASTRUTTURE VIARIE }\end{array}$ & massima & $\begin{array}{l}\text { La considerazione di una infrastruttura viaria all'interno di uno o più } \\
\text { sistemi può permettere di orientarne la pianificazione e la progettazione } \\
\text { secondo obiettivi di protezione ambientale e di miglioramento } \\
\text { microclimatico e percettivo degli spazi stradali e delle condizioni di } \\
\text { percezione del paesaggio di pertinenza visuale immediata, come dello } \\
\text { scenario complessivo. }\end{array}$ \\
\hline $\begin{array}{l}\text { (12) - INSERIMENTO VISUALE } \\
\text { DI INFRASTRUTTURE VIARIE }\end{array}$ & massima & $\begin{array}{l}\text { La considerazione di una infrastruttura viaria all'interno di uno o più } \\
\text { sistemi può permettere di orientarne la pianificazione e la progettazione } \\
\text { secondo obiettivi di mitigazione degli effetti negativi che l'infrastruttura } \\
\text { produce sul paesaggio, come di quelli dovuti a detrattori preesistenti, } \\
\text { percepibili durante l'utilizzo dell'infrastruttura stessa. }\end{array}$ \\
\hline $\begin{array}{l}\text { (13) - EQUIPAGGIAMENTO VEGETALE } \\
\text { DEL PAESAGGIO RURALE }\end{array}$ & massima & $\begin{array}{l}\text { La loro costituzione, lungo i corsi d'acqua principali o attraverso spazi } \\
\text { agricoli marginali, può contribuire ad elevare la qualità ambientale e } \\
\text { morfologica del paesaggio rurale di pianura, sviluppandone inoltre il } \\
\text { potenziale ricreativo rispetto alla domanda sociale delle aree urbane e } \\
\text { metropolitane. }\end{array}$ \\
\hline $\begin{array}{l}\text { (14) - REGOLAZIONE ANEMOMETRICA } \\
\text { E MITIGAZIONE DEGLI IMPATTI SUL SUOLO }\end{array}$ & massima & $\begin{array}{l}\text { Nel paesaggio rurale, la loro presenza, ad esempio lungo i corsi d'acqua, } \\
\text { permette di ottenere effetti di regolazione dei venti, che possono costuire } \\
\text { integrazioni sostanziali delle funzioni del reticolo diffuso } \\
\text { dell'equipaggiamento vegetale minuto degli spazi colturali, ove presente, } \\
\text { e possono assumere il ruolo strategico di dotazione di base, in sua } \\
\text { assenza. Si vedano anche i criteri di attenzione relativi agli elementi di } \\
\text { idoneità } \mathrm{A}(14) \text {. }\end{array}$ \\
\hline $\begin{array}{l}\text { (15) - PROTEZIONE AMBIENTALE } \\
\text { E REGOLAZIONE MICROCLIMATICA URBANE }\end{array}$ & massima & $\begin{array}{l}\text { Possono costituire importanti strategie per il miglioramento e la protejione } \\
\text { ambientale dei territori ad elevata pressione insediativa con efficacie } \\
\text { specifiche per la fissazione delle polveri e la depurazione dell'aria dalle } \\
\text { emissioni inquinanti e per il miglioramento del microclima. }\end{array}$ \\
\hline $\begin{array}{l}\text { (16) - PROTEZIONE AMBIENTALE } \\
\text { DELLE ACQUE SUPERFICIALI }\end{array}$ & massima & $\begin{array}{l}\text { Possono costituire una importante politica di miglioramento e protezione } \\
\text { ambientale dei corsi d'acqua nel territorio di pianura ed in particolarenegli } \\
\text { insediamenti, contribuendo alla depurazione delle acque di scolo, dei } \\
\text { campi e delle strade extraurbane, e delle acque di scolo non captate dai } \\
\text { sistemi di smaltimento e depurazione a servizio degli spazi urbanizzati. }\end{array}$ \\
\hline $\begin{array}{l}\text { (17) - PROTEZIONE AMBIENTALE } \\
\text { DELLE ACQUE DI FALDA }\end{array}$ & massima & $\begin{array}{l}\text { Con una opportuna composizione fitosociologica delle piantagioni, } \\
\text { possono svolgere funzioni di presidio ambientale delle falde acquifere, } \\
\text { contribuendo efficacemente al trattenimento ed alla depurazione delle } \\
\text { acque meteoriche e di dilavazione. }\end{array}$ \\
\hline
\end{tabular}




\section{Scenario di riqualificazione diffusa del paesagoio periurbano della piana di Castello a Firenze}

L'ambito considerato, coincidente con la cosiddetta piana di Castello, ad ovest di Firenze, è compreso nelle aree a cui sono state riferite le considerazioni analitiche e diagnostiche e quelle relative agli scenari teorici, riportate nei precedenti paragrafi.

Il quadro sperimentale ipotizzato si riferisce alla considerazione congiunta delle osservazioni territoriali generali derivate dagli scenari teorici e di quelle tipologiche specifiche, relative ai criteri di idoneità dei sistemi lineari di spazi aperti per il contenimento e la riduzione della frammentazione paesistica.

La complessa vicenda della realizzazione del nuovo centro direzionale del capoluogo toscano, non è argomento di studio della ricerca, che ne considera esclusivamente gli esiti sotto il proprio specifico punto di vista, astraendo l'applicazione dall'ampio spettro delle situazioni politiche, amministrative, economiche e progettuali, al fine di ottenere una congrua indipendenza metodologica da aspetti contingenti o non strettamente attinenti. Preme, in tal senso, rimarcare come quanto proposto non costituisca una nuova progettazione od una riprogettazione del paesaggio urbano del nuovo insediamento di Castello, bensì una simulazione delle modalità e delle opportunità di applicazione del modello progettuale elaborato, in riferimento alle finalità primarie di contenimento e riduzione della frammentazione nelle aree periurbane.

Il caso studio risulta particolarmente significativo in relazione al tema della individuazione di possibili modalità di indirizzo degli interventi in ambiti soggetti ad operazioni di trasformazione complessiva, dove le analisi e le diagnosi di frammentazione e le conseguenti indicazioni progettuali, afferiscono sostanzialmente ai processi ed agli strumenti di pianificazione, oltre che agli attuali stati del paesaggio. Questo permette di sviluppare in ottica preventiva un'ampia gamma di misure di controllo e compensazione degli effetti potenziali di frammentazione, finalizzata a conferire ai piani congrui requisiti qualitativi e quantitativi di attenzione agli aspetti paesistico-ambientali. Tali condizioni possono essere raggiunte più facilmente intervenendo nelle fasi di elaborazione dei piani generali ed attuativi, ma vi sono margini di operatività anche per l'indirizzo e la revisione delle configurazioni da essi predefinite.

Nello specifico caso in questione si tratta prevalentemente di spazi interessati dal Piano urbanistico esecutivo di Castello (si vedano i paragrafi 2.2 e 3.4), rispetto al quale lo scenario indica criteri di indirizzo progettuale compatibili con la struttura spaziale e l'articolazione tipologica del tessuto urbano proposte. Pertanto, la formula con cui viene sviluppata la simulazione parte dall'acquisizione del piano come dato di fatto, da considerare nel complesso dei fattori di frammentazione che lo identificano e delle relative politiche territoriali, insieme alle caratteristiche di altri spazi di importanza strategica, appartenenti agli attuali margini urbani. La descrizione complessiva del soggetto di studioè riportata nei precedenti capitoli 1, 2, 3, in funzione dei rispettivi temi specifici.

Lo scenario, finalizzato alla verifica complessiva della capacità di diffusione territoriale delle azioni di riqualificazione, considera sia misure di prevenzione e indirizzo progettuale, relative sostanzialmente ai fattori potenziali, che misure di intervento, relative ad alcuni dei fattori in essere in questo ambito del paesaggio di pianura fiorentino, sviluppando una configurazione tendente ad un modello spaziale reticolare, che potrebbe assumere ruoli strutturali nella ipotesi dell'adozione di politiche di piano incentrate sulla differenziazione degli usi degli spazi aperti per l'equilibrio paesistico-ambientale.

Gli scenari teorici, illustrati nel precedente paragrafo 4.1, hanno reso evidente il potenziale di diffusione territoriale che caratterizzerebbe le eventuali politiche strategiche di strutturazione di sistemi paesistici lineari afferenti ad alcune tipologie spaziali e funzionali fondamentali, come le infrastrutture viarie, di diverso ordine, ed i corsi d'acqua, sia quelli artificiali, che quelli naturali regimati. 
Nell'ottica di una pianificazione sensibile alla valenza strategica della conservazione e dello sviluppo dei caratteri di continuità della struttura spaziale e funzionale del paesaggio, nelle diverse articolazioni biotiche ed abiotiche, è possibile pensare alla definizione di un quadro di governo territoriale caratterizzato da una struttura primaria costituita da una rete di sistemi differenziati di spazi aperti, con funzioni prioritarie di conservazione, miglioramento e compensazione paesistico-ambientali. Tali sistemi paesistici, sia nelle configurazioni semplici, che in quelle complesse, possono afferire a scale diverse, sia per le dimensioni spaziali che assumono, che per le relazioni territoriali che sostengono.

Nel caso indagato, non sono stati riscontrati margini di costituzione di sistemi lineari complessi idonei a supportare relazioni di continuità paesistica di scala vasta, essendo a questo livello decisamente profondi e strutturati gli stati di frammentazione che caratterizzano i rapporti della pianura metropolitana con il paesaggio pedecollinare e collinare, a nord ed a sud delle conurbazioni di Sesto Fiorentino e di Osmannoro (si vedano le considerazioni interpretative dei paragrafi 1.1 e 3.2). In relazione agli assetti esistenti e previsti ${ }^{142}$ nell'area risulta invece una articolata possibilità di intervento in termini di costituzione di sistemi paesistici lineari di livello locale, sia appartenenti alla tipologia complessa, che a quella semplice. La loro specifica descrizione ${ }^{143}$, riportata di seguito, fa riferimento alla cartografia generale della figura 51 .

I principali tipi di interventi di strutturazione paesistica che ricorrono nella definizione di indirizzo progettuale relativa ai diversi sistemi di spazi aperti considerati dallo scenario sono essenzialmente:

1. movimenti di terra per la mitigazione dei carichi ambientali da inquinamento acustico (non abbattibili attraverso l'esclusivo uso della vegetazione) e/o per la ridefinizione morfologica e funzionale di assetti spaziali esistenti caratterizzati da rilevati in terra;

2. piantagioni arboree, arbustive e di vegetazione tappezzante, di spazi verdi, di margini stradali e di parcheggi e piazze, per la mitigazione dei carichi ambientali dovuti a diverse fonti inquinanti (fissazione delle polveri, depurazione dell'aria e delle acque di scolo, integrazione delle funzionidi riduzione dei rumori), il miglioramento morfologico degli spazi (conferimento di effetto del limite e di identità morfologica e dimensionale) e la limitazione delle intervisibilità critiche (separazione visuale e frammentazione delle visibilità critiche);

3. percorsi ciclabili e pedonali, su sede propria o su sede promiscua protetta, privi di barriere architettoniche (piani di movimento continui; raccordi di livello dei piani carrabili) per la costituzione o l'integrazione del sistema della mobilità, secondo requisiti di separazione dei traffici, e l'ottimizzazione delle opportunità di utilizzo delle diverse modalità di spostamento private e pubbliche.

Lo scenario costituisce un quadro territoriale organico per la riqualificazione diffusa del paesaggio periurbano, identificato da una articolazione spaziale di tipo reticolare e da una articolazione tipologica dei sistemi di spazi aperti, a cui fanno riferimento specifici indirizzi generali con finalità primarie di contenimento e riduzione delle forme reali e potenziali di frammentazione paesistica.

Il confronto dello scenario (figura 51) con l'articolazione del sistema del verde previsto dal Piano urbanistico esecutivo di Castello (figura 13), rende evidente le potenzialità di intervento strutturalesul sistema degli spazi aperti proprie di una concezione di tipo sistemico e le potenzialità di adattamento ai tessuti urbani e periurbani proprie specificamente dei sistemi lineari di spazi aperti.

142 Ci si riferisce, come premesso, agli elementi di specificazione del Piano regolatore generale di Firenze contenuti nel Piano urbanistico esecutivo relativo all'area della piana di Castello, ovvero alle aree comprese tra la ferrovia Firenze-Prato, a nord, la ferrovia Firenze-E mpoli, ad est, l'autostrada Firenze-Mare, a sud, e l'aereoporto, ad ovest.

${ }^{143}$ Le sigle identificative delle diverse tipologie di intervento fanno riferimento, nella seconda e terza indicazione, alle seguenti abbreviazioni: "E" per "esistente"; "P" per "previsto"; "sc" per "sistemi complessi"; "ss" per "sistemi semplici". La prima indicazione è relativa alla semplice numerazione progressiva delle singole tipologie al fine di facilitarne l'immediata individuazione e denominazione. 


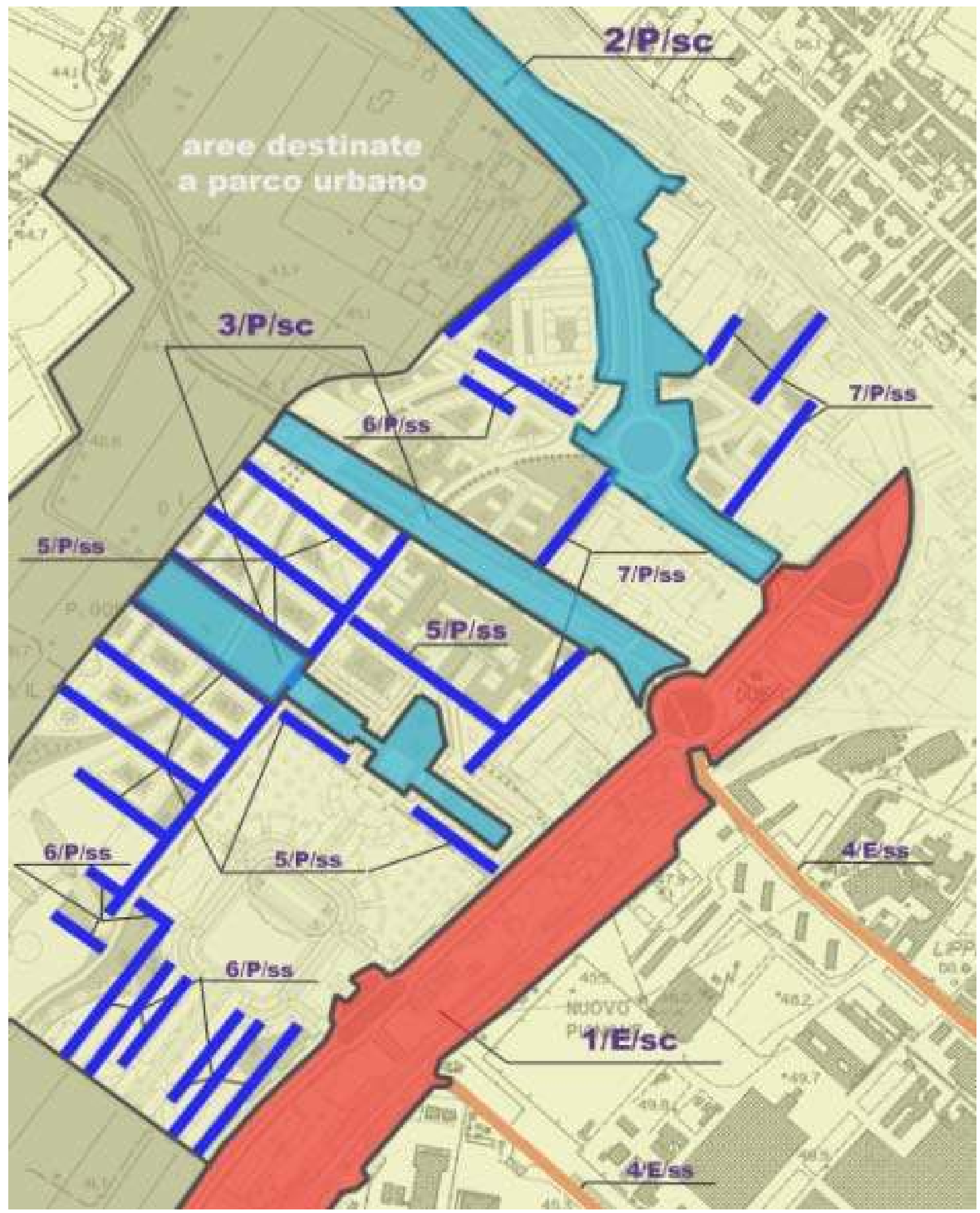

Figura 51 - Simulazione di scenario di riqualificazione diffusa del paesaggio periurbano della piana di Firenze, con obiettivi primari di contenimento e riduzione della frammentazione paesistica. La prima indicazione è relativa alla semplicenumerazione progressiva delle singole tipologie al fine di facilitarne l'immediata individuazione e denominazione. Le sigle identificativedelle diverse tipologie di intervento (seconda e terza indicazione) fanno riferimento alle seguenti abbreviazioni: " $\mathrm{E}$ " per "esistente"; "P" per "previsto"; "sc" per "sistemi complessi"; "ss" per "sistemi semplici". Le funzioni paesistiche primarie dei sistemi territoriali ed i relativi indirizzi per la progettazione del paesaggio sono specificati alle pagine seguenti (cartografia ridotta da originale 1:10000 - CTR Regione Toscana). 
1/E/sc - Sistema lineare complesso, costituito da spazi aperti propri di tessuti urbani esistenti, da sottoporrea misure prioritarie di riqualificazione paesistica, per il miglioramento ambientale, funzionale e morfologico degli insediamenti. Le funzioni primarie del sistema, legate alle sue caratteristiche di localizzazione e di destinazione d'uso degli spazi, sono individuabili nelle funzioni insediative di margine (attuale) e di separazione (futura, conseguente agli sviluppi insediativi previsti) e nella funzione di dorsale occidentale del trasporto pubblico urbano su rotaia (nella prospettiva di una apertura e conversione parziale in forma di ferrovie e tramvie metropolitane).

Nella progettazione degli spazi aperti appartenenti al sistema, occorre privilegiare le sistemazioni vegetali ed in particolare l'adozione di specie di alto fusto ed arbustive, laddove le esigenze di separazione visuale o di definizione morfologica lo richiedano e/o le condizioni d'uso e le dimensioni degli spazi permettano di assecondare la finalità generale di incremento della biomassa territoriale. Negli spazi compresi nei rilevati infrastrutturali, in ragione della collocazione pensile rispetto al piano di campagna, occorre che l'utilizzo di specie di alto fusto sia limitato ad eventuali esemplari o gruppi isolati. È invece opportuno ricorrere alle specie arboree di alto fusto nella sistemazione degli spazi ai piedi dei rilevati, per le caratteristiche dimensionali che esse sono in grado di sviluppare, idonee al riequilibrio morfologico di queste configurazioni. I rilevati in terra costituiscono una componente radicata in questi paesaggi, dominati dalle infrastrutture e dai canali di bonifica, è pertanto possibile considerare correttamente questo tipo di sistemazione degli spazi aperti come praticabile nell'ambito degli interventi di miglioramento morfologico e funzionale, anche in ragione alle eventuali esigenze di raccordo di piani posti a quote diverse. Per la posizione pensile delle infrastrutture ferroviarie, i movimenti di terra non risultano idonei alla mitigazione dell'inquinamento acustico. Essi possono invece assolvere tale funzione rispetto agli effetti della viabilità ad elevato carico di traffico con orientamento nord-sud, esistente sul piano di campagna, in margine alle aree dove sono previsti gli sviluppi insediativi.

2/ P/sc - Sistema lineare complesso, costituito da spazi aperti propri di tessuti urbani prezisti, da sottoporrea misure prioritarie di inserimento paesistico delle infrastrutture viarie urbane.

Il sistema è caratterizzato da funzioni primarie connesse alla mobilità e permette di integrare quelle relative al precedente sistema $E / s c / 1$ con collegamenti ciclabili e pedonali con la stazione ferroviaria di Castello.

Nella progettazione degli spazi aperti appartenenti al sistema, occorre privilegiare le sistemazioni vegetali ed in particolare l'adozione di specie di alto fusto ed arbustive, laddove le esigenze di separazione visuale o di definizione morfologica lo richiedano. In ragione di esigenze di congruenza con le caratteristiche geomorfologiche del paesaggio di pianura, i rilevati in terra sono da limitare ai casi di assoluta necessità tecnica, oppure alle localizzazioni ove possano essere trattati come eventi di land art, inseriti nel quadro complessivo delle misure di qualificazione del paesaggio periurbano e nello specifico degli spazi di pertinenza della viabilità primaria. L'efficacia delle misure di inserimento paesistico è complessivamente legata, oltre che all'adozione di criteri integrati di progettazione delle opere di urbanizzazione (funzionali, statici, economici, ecologici), all'impegno di spazi aperti a margine delle carreggiate in quantità e qualità congrue, in termini di estensione trasversale e di continuità longitudinale.

3/ P/sc - Sistemi lineari complessi, costituiti da spazi aperti propri di tessuti urbani previsti, da sottoporre a misure prioritarie di salzaguardia e definizione del ruolo strategico di connessione dei tessuti insediativi con il parco urban. La funzione prioritaria di questi sistemi, di relazione del tessuto insediativo con il parco urbano previsto ad ovest (figura 51), richiede prioritariamente la salvaguardia degli spazi aperti ed una loro definizione morfologica unitaria, rispondente alla scala paesistica a cui sono riferibili, pur nella considerazione delle diversità dei singoli spazi componenti il sistema. La funzione di connessione del parco con il tessuto urbano risulta transcalare, investendo l'ambito locale del nuovo insediamento di 
Castello, come l'ambito urbano fiorentino nel suo complesso, in ragione del ruolo del parco e delle opportunità di collegamento date dai sistemi infrastrutturali ad esso limitrofi.

Nella progettazione degli spazi aperti appartenenti al sistema, occorre privilegiare le sistemazioni vegetali ed in particolare l'adozione di specie di alto fusto ed alto-arbustive, laddove le esigenze di separazione visuale o di definizione morfologica lo richiedano e/o le condizioni d'uso e le dimensioni degli spazi permettano di assecondare la finalità generale di incremento della biomassa territoriale.

Occorre riferire particolari attenzioni, in termini di quantità e qualità degli spazi, ai sistemi funzionali riservati alla mobilità pedonale e ciclabile, in ragione della loro importanza a livello locale, ma anche come sistemi di raccordo tra la città ed il parco urbano previsto, integrati al sistema primario dei trasporti pubblici (sistema $E / s c / 1$ ).

4/ E/ss - Sistemi lineari semplici, costituiti da spazi aperti propri di strade esistenti, da sottoporre ad interzenti di equipagiamento paesistico, per il miglioramento dei requisiti di qualità ambientale, funzionale e morfologica degli insediamenti.

A questi sistemi afferisce la funzione primaria di relazione dell'ambito urbano con prevalente destinazioni a servizi ed attività produttive di cui fanno parte e, attraverso questo, della città, con il nuovo centro direzionale, previsto ad ovest nell'area di Castello, e con il relativo parco urbano. Nell'ottica complessiva del quadro territoriale definito dallo scenario, tale funzione è complementare a quella dei precedenti sistemi $3 / \mathrm{P} / \mathrm{sc}$, nel garantire la continuità di congrui livelli di qualità morfologica, funzionale ed ambientale degi spazi stradali, che hanno ruoli strategici nella struttura delle relazioni urbane.

Nella progettazione degli spazi aperti appartenenti al sistema, occorre privilegiare le sistemazioni vegetali ed in particolare l'adozione di filari di specie di alto fusto ed arbustive. L'efficacia delle misure di inserimento paesistico è complessivamente legata all'estensione trasversale ed alla continuità longitudinale degli spazi aperti effettivamente coinvolgibili. L'equipaggiamento vegetale degli spazi stradali dovrà, ove possibile, essere affiancato da interventi di integrazione e miglioramento dei sistemi di mobilità ciclabile e pedonale, in ragione delle funzioni di collegamento con il parco urbano ed in generale dei suddetti ruoli nell'ambito del sistema della mobilità.

5/ P/ss - Sistemi lineari semplici, costituiti da spazi aperti propri di strade previste, da sottoporre ad interzenti di equipaggiamento paesistico per la dotazione di congrui requisiti di qualità ambientale, funzionale e morfologica deģi insediamenti.

Questi sistemi trovano, per funzioni primarie e per tipologia degli spazi, elementi di analogia e complementarietà con i precedenti sistemi complessi di relazione con il parco $(3 / \mathrm{P} / \mathrm{sc})$ e semplici di connessione con i tessuti urbani consolidati $(4 / E / s s)$. Il loro orientamento gli conferisce infatti funzioni di relazione del tessuto insediativo con il parco urbano, in questo caso limitate però alla scala locale degli ambiti di sviluppo insediativo previsti dal Piano urbanistico esecutivo e la tipologia spaziale della strada comporta precisi criteri e tipi di intervento.

Nella progettazione degli spazi aperti appartenenti al sistema, occorre privilegiare le sistemazioni vegetali ed in particolare l'adozione di filari o fasce di specie di alto fusto ed arbustive. L'efficacia delle misure di inserimento paesistico è complessivamente legata, oltre che all'adozione di criteri integrati di progettazione delle opere di urbanizzazione (funzionali, statici, economici, ecologici), all'impegno di spazi aperti a margine delle carreggiate in quantità e qualità congrue in termini di estensione trasversale e di continuità longitudinale. L'equipaggiamento vegetale degli spazi stradali dovrà essere integrato ad interventi di equipaggiamento funzionale per la realizzazione di sistemi di mobilità ciclabile e pedonale in condizioni di continuità con il parco urbano. 
6/ P/ss - Sistemi lineari semplici, costituiti da spazi aperti propri di piazze e parcheogi previsti e da spazi apertidi diretta pertinenza di edifici e complessi di edifici previsti, da sottoporre ad interzenti di equipagoiamentopaesisticoper la dotazione di congrui requisiti di qualità ambientale, funzionale e morfologica degli insediamenti.

Questi sistemi fanno riferimento a funzioni primarie di dotazione interna dei tessuti insediativi, non hanno funzioni strategiche a livello urbano, ma possono assumere importanti funzioni di spazi di relazione e di centralità insediativa. Ad essi afferiscono sia le piazze pubbliche, che gli spazi di pertinenza di edifici e complessi di edifici.

Nella progettazione degli spazi aperti appartenenti al sistema, sono da privilegiare le percorrenze ciclabili e pedonali e le relative sistemazioni degli spazi aperti e, con particolare riferimento agli interventi di equipaggiamento vegetale, le loro funzioni di definizione morfologica e di dotazione ambientale per la regolazione del microclima e la riduzione degli effetti dell'inquinamento atmosferico delle aree insediative, attraverso la fissazione delle polveri e la depurazione dell'aria. Occorre che tali attenzioni siano in questo caso portate ai massimi livelli di efficacia ottenibili in ragione dell'uso prevalente di questi spazi aperti per la sosta e la ricreazione.

7/P/ss - Sistemi lineari semplici, costituiti da spazi aperti propri dei margini di aree di trasformazione paesistica previste, da sottoporre ad interzenti di separazione visuale, per la riduzione delle condizioni di criticità in essere e/ o potenziali.

Questi sistemi fanno riferimento a funzioni primarie di separazione visuale di fonti di disturbo rispetto a spazi sensibili. Nella progettazione delle sistemazioni idonee ad assolvere alle funzioni primarie del sistema, sono da privilegiare le piantagioni vegetali con specie di alto fusto ed arbustive in filare o a fascia, secondo modalità di composizione vegetale idonee a mantenere l'efficacia di schermatura durante tutto l'arco dell'anno. 


\section{5 - La frammentazione del paesaggio periurbano: esiti ed indirizzi di sviluppodella ricerca}

Dipende da una sorta di fisiologia del processo speculativo il fatto, pressoché generalizzato nelle sperimentazioni scientifiche, che le maglie della riflessione teorica e delle applicazioni modellistiche si allargano e si restringono ciclicamente secondo un ragionamento unitario, con obiettivi specifici ed esplicitamente circoscritti. Si è ritenuto utile perciò ricondurre la ricerca ad uno spazio conclusivo di sintesi, in cui i presupposti teorici, i passaggi peculiari e gli esiti di studio, vengono messi in relazione all'interno di un quadro unitario.

Lo studio si è articolato in tre fasi che hanno sviluppato argomentazioni concatenate, dalle ipotesi teoriche iniziali alla definizione conclusiva, con il costante supporto di applicazioni sperimentali riferite alle specificità del paesaggio periurbano di pianura di Firenze.

La prima fase ha riguardato l'estensione teorica del concetto di frammentazione dalla accezione ambientale, riguardante gli habitat e le popolazioni delle specie animali e vegetali, allo studio delle alterazioni strutturali del paesaggio ed ha sviluppato specifiche osservazioni inerenti i significati e le opportunità speculative ed applicative del concetto di frammentazione paesistica.

In secondo luogo, sono state condotte le osservazioni inerenti l'intensità e le forme di diffusione della frammentazione paesistica nei territori di pianura ad elevata pressione insediativa ed, in particolare, nelle aree periurbane, dove il tessuto degli spazi aperti è connotato da gravi deficienze e non risulta in grado di assolvere molte delle importanti funzioni paesistico-ambientali che gli sono proprie. Alla conoscenza della fenomenologia sono state finalizzate l'individuazione dei temi e delle modalità di analisi della frammentazione paesistica, attraverso la definizione di specifici fattori territoriali causali ed indicatori, e l'individuazione delle modalità interpretative, attraverso la definizione di un modello diagnostico preposto alla correlazione dei dati conoscitivi di tipo analitico. Infine, all'obiettivo dell'individuazione di criteri di progettazione paesistica congruenti con la fenomenologia della frammentazione e con i caratteri strutturali dei paesaggi periurbani di pianura, sono state riferite le osservazioni sulle idoneità dei sistemi paesistici a configurazione lineare per la riduzione ed il contenimento degi stati e delle dinamiche di frammentazione del paesaggio. Nell'ambito della definizione dei suddetti criteri di idoneità, sono stati distinti i sistemi paesistici a configurazione semplice (sistemi relativamente omogenei, afferenti ad una componente paesistica lineare dominante come un fiume o una strada, identificabili in termini morfologici) ed a configurazione complessa (sistemi fortemente differenziati, composti da più tipi di componenti paesistiche, non necessariamente caratterizzati da componenti dominanti, ma sostanzialmente afferenti ad una organizzazione unitaria, identificabili in termini strategici), individuando per entrambe le tipologie una serie di caratteristiche di risposta rispetto alle specifiche criticità proprie degli stati e delle dinamiche di frammentazione paesistica.

L'articolazione dei ruoli individuati per tali sistemi si basa su principi teorici di diversificazione delle modalità di uso e gestione degli spazi per l'equilibrio paesistico-ambientale. Piuttosto che ad una visione monocorde di fasce verdi omogenee, rispetto alle quali sarebbe improbabile immaginare l'assenza di conflitti con la gran parte delle attività umane, si fa riferimento a scenari differenziati, calibrati sulla congestione e sul deficit di risorse dei paesaggi periurbani ed urbani di pianura, in cui i 
sistemi lineari di spazi aperti fanno capo ad una politica, unitaria e gerachizzata, di controllo e compensazione degli elevati livelli di pressione insediativa, che da alcuni decenni riducono progressivamente le disponibilità di spazi e le loro qualità paesistiche ed ambientali.

Secondo questi requisiti, i sistemi lineari di continuità paesistica costituiscono tipologie di intervento il cui impiego strategico, dalla pianificazione di scala vasta a quella comunale generale, a quella attuativa, fino alla definizione progettuale dei singoli siti, può dare sostanziali esisti di contenimentoe riduzione della frammentazione del paesaggio periubano di pianura, contribuendo efficacemente alla sua riqualificazione diffusa e producendo equilibri paesistico-ambientali favorevoli alle esigenze di conservazione della biodiversità.

Ai fini della applicabilità di questi elementi di definizione metodologica occorre porre in evidenza alcuni aspetti che sono risultati determinanti nella connotazione della problematica indagata.

La frammentazione paesistica è una alterazione strutturale complessa, la cui interpretazione conoscitiva e progettuale non può essere esaurita nell'ambito settoriale degli aspetti ecologici afferenti le condizioni e le relazioni degli habitat e delle popolazioni animali e vegetali. Essa mostra una fenomenologia molto più vasta ed articolata, responsabile di alterazioni delle funzioni ambientali determinanti per l'ecologia umana, come delle condizioni di permanenza storica, e di articolazione morfologica del paesaggio.

Alle finalità di riduzione e contenimento della frammentazione paesistica corrisponde uno spettro molto ampio di interventi di miglioramento paesistico-ambientale con efficacia di riqualificazione diffusa, che non coincidono in modo diretto ed aprioristico con la conservazione e la neocostituzione delle reti ecologiche, sebbene siano in larga misura favorevoli e necessari rispetto al perseguimento di questi obiettivi. All'interno delle condizioni di equilibrio paesistico-ambientale attendibili dalla attuazione di politiche di contenimento e riduzione della frammentazione paesistica, le esigenze di conservazione della biodiversità troverebbero presumibilmente ampi spazi di compatibilità e di sviluppo, anche ove non direttamente supportate da apposite misure.

Lo studio conoscitivo, come la definizione progettuale, relativi alla frammentazione paesistica sono connotati da una decisa transcalarità. Questo carattere è evidentemente attestato dalla forte differenziazione e complementarietà degli esisti interpretativi prodotti dai diversi livelli diagnostici sperimentati. Se la transcalarità è un carattere tipico delle applicazioni di interpretazione conoscitivae progettuale del paesaggio, relativamente alla problematica in questione, essa assume il ruolo di criterio metodologico ineludibile e determinante nello sviluppo dei processi di articolazione delle strategie di intervento.

Diverse questioni aperte potrebbero essere indagate nell'ambito di altre esperienze di studio teorico ed applicato. In tal senso si vogliono evidenziare, a conclusione del lavoro, gli aspetti ritenuti importanti relativamente a specifici sviluppi, che possono costituire nuovi indirizzi di ricerca.

Una prima e fondamentale questione riguarda i fattori territoriali della frammentazione paesistica ed in particolare la verifica e l'articolazione della classificazione proposta, in funzione del suo impiego a più scale di analisi.

Ancora nell'ambito delle acquisizioni conoscitive, si distingue inoltre un secondo importante indirizzo di ricerca riguardante gli indicatori di frammentazione paesistica ed in particolare la valutazione della opportunità e/o delle necessità di utilizzo di altri indicatori primari o secondari e lo sviluppo delle tecniche di misurazione e sintesi, attraverso idonei indici e sistemi di calcolo informatizzato.

In entrambi questi casi, si ritiene fondamentale pensare alla applicazione dei sistemi informativi geografici che, ancorchè non impiegati in questo lavoro di impostazione teorica e metodologica, divengono strumenti tecnici estremamente efficaci nelle applicazioni che vedono la gestione interrelata di dati numerici e geografici su aree vaste. 
Si è detto inoltre dell'importanza dello studio dei processi socioeconomici sotto il punto di vista dei loro effetti e potenziali di frammentazione del paesaggio, sia relativamente alle dinamiche caratteristiche delle aree urbane e periurbane, che per quanto riguarda i paesaggi agrari industrializzati ed i paesaggi rurali marginali. In questo ambito risulta determinante lo studio degli stati e delle dinamiche che caratterizzano l'articolazione della proprietà fondiaria, sia in termini cartografici, relativi alla distribuzione territoriale, che in termini tipologici, relativi alla definizione dei diversiattori delle trasformazioni paesistiche.

Per quanto riguarda gli aspetti progettuali non si ritiene utile evidenziare un indirizzo di ricerca piuttosto che un altro, essendo per definizione questo un campo aperto alla creatività scientifica. La scelta specifica di questo studio di occuparsi delle configurazioni paesistiche lineari, suggerisce in ogni caso la curiosità di indagare che tipo di contributi possano essere portati allo studio delle alterazioni di frammentazione paesistica da approcci che prediligano le proprietà di configurazioni paesistiche puntuali o areali, oppure che neghino a priori tale atteggiamento selettivo, indagando altre opportunità di definizione di scenari paesistici idonei alla riduzione ed al contenimento di queste manifestazioni di alterazione del paesaggio.

Fra le possibilità di impiego degli esiti definitori e metodologici della ricerca vi sono diversi generi di applicazioni territoriali, dalle attività di formazione dei piani regolatori generali comunali di nuova generazione, a quelle relative ai piani territoriali provinciali di coordinamento, come, ad un livello superiore, ai piani regionali di indirizzo territoriale ed ai piani di parchi ed aree protette. Ma occorre considerare che importanti spazi di applicazione vi possono essere nella progettazione delle infrastrutture viarie e tecnologiche, come nelle sistemazioni di bonifica idraulica e di messa in sicurezza idrogeologica, di riordino agricolo ed infine nei progetti per interventi di sviluppo e completamento insediativo. Un utilizzo strettamente finalizzato dei modelli di conoscenza sperimentati riguarda le possibili applicazioni nell'ambito delle valutazioni strategiche interne ai processi di formazione dei piani, come in quelle ex post. Soprattutto nelle valutazioni ex ante, è possibile sviluppare applicazioni funzionali ad obiettivi di pianificazione di politiche di uso differenziato degli spazi per l'equilibrio paesistico-ambientale.

Considerando infine l'origine della problematica di studio, per come è stata descritta nei paragrafi iniziali ed approfondita in merito alle teorie ed alle applicazioni intorno ai sistemi paesistici lineari, l'utilizzo di modelli di questo tipo nelle attività di pianificazione e progettazione in corso di sviluppo intorno alle tematiche delle reti ecologiche, può allargare progressivamente l'attenzione, dal prevalere di un'accezione esclusivamente ambientale alla considerazione complessiva del paesaggio secondo i suoi caratteri strutturali, e mirare alla definizione di piani e progetti opportunamente dotati della necessaria transdisciplinarietà, governati nel loro complesso da un approccio generalista profondamente formato e sviluppato, che può trovare nella pianificazione e progettazione paesistica validi paradigmi disciplinari di riferimento. 


\section{Bibliografia}

\section{Riferimenti di interesse generale per lo studio del paesaggio e degi insediamenti}

Lo studio delle condizioni di frammentazione paesistica dei territori soggetti ad elevata pressione insediativa si avvale innanzitutto dei contributi interpretativi che la letteratura scientifica mette a disposizione intorno alle caratteristiche strutturali ed alle dinamiche dei processi di urbanizzazione ed infrastrutturazione. La forte articolazione della bibliografia e la complessità di questi studi richiedono, nel caso della loro applicazione ad una specifica problematica di ricerca, una selezione ragionata dei lavori maggiormente attinenti.

Un contributo di interesse generale è costituito dagli sviluppi della ricerca Itaten, che restituisce un panorama articolato delle forme insediative del territorio nazionale, fornendo un'ampia base di dati interpretabili anche sotto punti di vista specifici, non espressamente affrontati nella ricerca stessa. Una interessante sintesi di presentazione del lavoro, curata da A. Clementi (1996), si aggiunge alla pubblicazione dei primi esiti della ricerca, a cura dello stesso A. Clementi, con P.C. Palermo e G. Dematteis (1996).

Legata ai modelli territoriali contemporanei delle aree metropolitane, la questione del policentrismo insediativo risulta specificamente indagata in un recente lavoro di A. Moretti (1999), relativo al territorio lombardo, fra i più significativi per tali tematiche nella realtà italiana.

Sulle diversità di fondo tra le forme di diffusione insediativa si trovano contributi nell'ultimo lavoro di E. Piroddi (2000), nel quale viene affrontata anche la complessa tematica del degrado dei tessuti urbani periferici e dei significati contemporanei del loro recupero.

Alle problematiche delle aree di margine urbano ed alle relative strategie di recupero e miglioramento paesistico ambientale è espressamente rivolto il panorama fornito dalla pubblicazione degli esiti di alcune ricerche condotte presso il Politecnico di Milano, curata da A. Tosi (1999). Nello stesso lavoro viene illustrato un approccio conoscitivo in cui lo studio del mosaico degli spazi aperti assume piena centralità nell'interpretazione delle dinamiche delle aree periurbane (P. Properzi, 1999).

Su questo stesso tema sono significativi i lavori di F. Boscacci e R. Camagni (1994), di P.L. Paolillo (1994) ed ancora dello stesso A. Tosi con V . Borachia, R. Camagni, A. Moretti, P.L . Paolillo (1988), per quanto riguarda le problematiche del consumo di spazio.

Suggerimenti di sperimentazione teorica possono essere derivati anche dagli sviluppi delle applicazioni territoriali degli studi sui sistemi complessi. I contributi di C.S. Bertuglia e L. Staricco (2000), con specifico riferimento alla interpretazione della città come sistema complesso, non mostrano elementi di incompatibilità con l'estensione di tali concetti alla campagna, nelle aree di pianura caratterizzata dalla sempre più difficile individuazione di esatti limiti. Il soggetto più propriamente adatto ad applicazioni sperimentali di tali modelli interpretativi potrebbe essere pertanto proprio il paesaggio, inteso come configurazione di ordine superiore che assume, a secondo della realtà territoriale, i caratteri di urbano storico, urbano periferico, urbano di margine, agrourbano o con urbanizzazione diffusa, oppure, in realtà a minor pressione insediativa, rurale o forestale.

Il confronto dei caratteri del modello storico città-campagna e del modello contemporaneo "a corridoi e cunei" nell'ambito delle esperienze di studio del New Urbanism (Duany e Plater-Zyberk, 1997) 
mette in risalto il ruolo significativo della scala regionale rispetto alla interpretazione conoscitiva e progettuale del paesaggio ed i relativi forti connotati ecologici delle problematiche territoriali che caratterizzano i modelli insediativi.

Di particolare interesse per la specifica attenzione ai rapporti tra il paesaggio ed i processi insediativi, risulta l'ultimo lavoro di E. Turri (2000), riguardante la lettura del territorio della Val Padana secondo i connotati identificativi e le dinamiche della megalopoli.

Si riportano di seguito riferimenti bibliografici di interesse generale utili alla interpretazione dei modelli e dei processi insediativi.

Astengo A., NuCCI C., IT. URB. 80 Rapporto sullo stato dell'urbanizzazione in Italia, volume primo, Quaderni di Urbanistica Informazioni, n. 8, 1990.

BALBO M., La città di frammenti, Urbanistica, 100, 1990.

Bertuglia C. S., STARICCO L., Complessità, autoorganizzazione, città, Franco Angeli, Milano, 2000.

BOATTI A., L'urbanistica tra piano e progetto, Franco Angeli, Milano, 2001.

Borachia V., MOREtTi A., PAOlillo P.L., TOSi A. (a cura), Il parametro suolo: dalla misura del consumo alle politiche di utilizo, Grafo, Brescia, 1988.

Bortolotti L., De LuCA G., Come nasce un'area metropolitana - Firenze Prato Pistoia: 1848-2000, Alinea, Firenze, 2000.

BOSCACCI F., CAMAGNI R. (a cura), Tra città e campagna - Periurbanizzazione e politiche territoriali, Il Mulino, Bologna, 1994.

CLEMENTI A., La ricerca Itaten: forme del territorio italiano, Urbanistica, 106, 1996.

Clementi A., Dematteis G., PALERMo P.C., Le forme del territorio italiano, 2 voll., Laterza, Roma-Bari, 1996.

DE RitA G., Proliferazioni e addensamenti del territorio: 1985-1995, in: A. Clementi, P.C. Palermo, G. Dematteis, (a cura), Le forme del territorio italiano, I volume, Laterza, Bari, 1996.

Dematteis G., Indovina F., Magnaghi A., Piroddi E., ScANDURRA E., SECCHI B., I futuri della città, tesi a confronto, Angeli, Milano, 1999.

DuANY A., Plater-ZYBerk, Il lessico del New Urbanism, Urbanistica, 108, 1997.

GAMBINO R., Reti urbane e spazi naturali, in: Salzano E., La città sostenibile, Ed. delle Autonomie, Roma, 1992.

LYNCH K., Progettare la città: la qualità della forma urbana, ETAS libri (The MIT Press), Milano (Cambridge, Massachusetts), 1990 (1981).

Moretti A. (a cura), Il paradigma del policentrismo. Conoscenza e trasformazione del territorio, Quaderni del Dipartimento di Scienze del Territorio del Politecnico di Milano, Angeli, Milano, 1999.

PaOlillo P.L., Contenimento degli sprechi e qualità morfologica territoriale, una correlazione inseparabile, in: Boscacci F., CamagniR (a cura), Tra città e campagna - Periurbanizzazione e politiche territoriali, Il Mulino, Bologna, 1994.

PIRODDI E., Le regole della ricomposizione urbana, Angeli, Milano, 1999.

Piroddi E., SCANDURRA E., De BONIS L. (a cura), I futuri della città. Mutamenti, nuozi sogeetti e progetti, Angeli, Milano, 1999.

PROPERZI P., Classificazione dei suoli e pianificazione operatiza nel restauro del territorio: il caso del periurbano dell'A quila, in Tosi A, (a cura), Degrado ambientale periurbano e restauro naturalistico, Quaderni del Dipartimento di Scienze del Territorio del Politecnico di Milano, Angeli, Milano, 1999.

Tosi A. (a cura), Degrado ambientale periurbano e restauro naturalistico, Quaderni del Dipartimento di Scienze del Territorio del Politecnico di Milano, Angeli, Milano, 1999.

TURRI. E., La megalopoli padana, Marsilio, Venezia, 2000.

Lo studio del paesaggio ha sviluppato una gamma assai articolata di metodologie ed esperienze applicative, sia per quanto riguarda gli aspetti conoscitivi, delle attività di analisi e valutazione diagnostica, che per quelli decisionali, dei piani e progetti per l'uso e la gestione delle risorse. Ogni lavoro, di ricerca scientifica, come di applicazione tecnica, ha a disposizione una letteratura specialistica vasta, alla quale occorre consapevolmente riferisi in maniera mirata. Nell'ambito di questo studio, il tema generale della riqualificazione del paesaggio è inquadrato, anche dal punto di vista bibliografico, sotto l'obiettivo specifico, pur assai vasto, della prevenzione e del recupero della frammentazione paesistica. Questo fa sì che i testi utilizzati siano in netta prevalenza afferenti agli autori che propongono un approccio scientifico comprensivo ${ }^{144}$.

Gli aspetti generali di concezione strutturale, di conoscenza e di progettazione paesistica fanno riferimento ai molti contributi derivanti dalle esperienze statunitensi e nord europee, fra cui gli studi

144 Il termine comprensivo si riferisce alla accezione letterale in uso negi Stati Uniti (comprehensize planning). Si veda la definizione riportata in Steiner F., Costruire il paesaggio, McGraw Hill Italia, Milano 1994. 
di W.E. Dramstad, R.T.T. Forman, L. Finke, J.D. Olson, F. Steiner, H.N. V an Lier, per i recenti sviluppi dell'ecologia del paesaggio e della pianificazione e progettazione paesistica, nonché ad importanti contributi prodotti in Italia negli ultimi venti anni.

Rimandando per la disamina dei molti contributi significativi alla consultazione dei singoli testi, si riportano di seguito alcuni riferimenti bibliografici di interesse generale utilizzati nella definzione dell'approccio conoscitivo e progettuale della ricerca ed alcuni importanti documenti istituzionali.

AHERN J., Planning and design for an extensize open space system: linking landscape structure to function, Landscape and Urban Planning, 33, 1991.

AIAPP-FEDAP, Carta di Napoli. Il parere degli specialisti sulla riforma degli ordinamenti di tutela del paesagoio in Italia, Raccomandazioni per la redazione di una carta del paesaggio avanzate al Convegno Nazionale FEDAP-AIAPP, La trasformazione sostenibile del paesagoio, con il patrocinio del Ministero per i Beni e le Attività Culturali e del Ministero dell'Ambiente, Napoli, 8 ottobre 1999.

ASSUNTO R., Il paesagoio e l'estetica, vol. I e II, Giannini, Napoli, 1973.

BALDI M. E. (a cura), La riqualificazione del paesaggio, La Zisa, Palermo, 1999.

BETTINI V., Elementi di ecologia urbana, E inaudi, Torino, 1996.

BOATTI A., Verde e metropoli. Milano e l'E uropa, Città Studi, Milano, 1991.

BOATti A., PAPA D., Parchi e protezione del territorio. Realtà e progetti europei, nazionali e regionali, Franco Angeli, Milano, 1995.

BRUSCHI S., FALINI P., Il paesagoio, in BRUSCHI S., GisOtTI G., V alutare l'ambiente, La Nuova Italia Scientifica, Roma, 1990.

CARAVELlo G., E cologia del paesaggio ed ecologia umana: gerarchie di rapporti, Genio Rurale n. 4, 1992.

CAlzolari V., Concetto di paesaggio e paesistica, in: AA.VV., A rchitettura del paesaggio, La Nuova Italia, Firenze, 1975.

Colombo A.G., MALCEvSCHI S. (a cura), Manuale A A A degli Indicatori per la V alutazione di Impatto A mbientale - Indicatoride paesaggio (coordinatore: Delsante S.), Vol. 5, Centro VIA Italia, Associazione Analisti Ambientali, Federazione delle Associazioni Scientifiche e Tecniche, Milano, 1999.

Comitato per la BellezZa e Centro StUdi TCI (a cura), Un Paese spaesato. Rapporto sullo stato del paesagoio italiano, I Libri Bianchi del Touring Club Italiano, n. 12, Milano, 2001.

COOK E.A., V AN LIER H.N., Landscape planning and ecological networks, E lsevier, Amsterdam, 1994.

D'APONTE P. (et al.), Il paesagoio italiano - patrimonio identità gestione, Bollettino della Società Geografica Italiana (numero monografico), 2, 1999.

Di FIDIO M., A rchitettura del paesagoio, Pirola, Milano, 1993.

DramstaD W.E., OlsON J.D., FORMAN R.T.T., Landscape E cology Principles in Landscape A rchitecture and Land-Use Planning Harvard University Graduate School of Design - Island Press - American Society of Landscape Architects, Washington, 1996.

FABBRI P., Natura e cultura del paesagoio agrario, CittàStudi, Milano, 1997.

FERRARA G. (a cura), Risorse del territorio e politica di piano, Marsilio, Venezia, 1976.

FINKE L., Introduzione all'E cologia del paesaggio, (Landschaftokologie), Franco Angeli, Milano, 1993 (Verlags-GmbH Holler und Zwick, Braunscweig, 1986).

FINKE L., E cologia del paesaggio e pianificazione degli spazi aperti, Urbanistica 107, 1996.

Flink C.A., SEARns R.M., SchwarZ L. LAB. (a cura), Greenumy. A guide to Planning Design, and Dezelopment, The Conservation Fund, Island Press, Washington, 1993.

Forman R.T.T., Hersperger A. M., E cologia del paesaggio e pianificazione: una potente combinazione, Urbanistica 108, 1997.

FORMAN R.T.T., Land mosaics, the ecology of landscapes and regions, Cambridge, 1995.

G AMBINO R., I parchi naturali, La Nuova I talia Scientifica, Roma, 1991.

GHERSi A., SESSAREgO A. (a cura), Sistema del zerde E cosistema urbano, Alinea, Firenze, 1996.

Giacomini V., I parchi nazionali, in Per la salzezza dei beni culturali in Italia, Atti e documenti della Commissione di indagine per la tutela e la valorizzazione del patrimonio storico, archeologico, artistico e del paesaggio, vol. II, Colombo, Roma, 1967.

GREGORETTI V. (et al.), Il disegno del paesaggio italiano, Casabella (numero monografico), 575/576, 1991.

INGEGNOLI V., Fondamenti di ecologia del paesagogio, CittàStudi, Milano, 1993.

LEWIS P.H. JR., L'eroluzione della progettazione regionale, Urbanistica, 108, 1997.

MALCEVSCHI S., Qualità ed impatto ambientale, E taslibri, Milano, 1990.

Maniglio Calcagno A., A rchitettura del paesagoio. E woluzione storica, Calderini, Bologna, 1983.

MCHARG I., Progettare con la natura (Design with nature), Muzzio, Padova, 1989 (Natural History Press, Garden City, New York, 1969).

MCHARG I., Human ecological planning at Pennsyzania, Landscape and Urban Planning, 8, 1986.

MinNINNI M.V., L'analisi del paesagoio attrazerso i principi dell'E cologia del paesagoio e i metodi della pianificazione paesistica, Genio Rurale n. 4, 1992.

PIRANi A. (a cura), Progetti della natura e dell'uomo, Franco Angeli, Milano, 1998.

RitTer J., Paesagoio - Uomo e natura nell'età moderna, Guerini, Milano, 1994. 
Rizzo G.G. (a cura), Tuscia Romana - Paesaggio e territorio - Metodi e modelli di zalutazione, Gangemi E ditore, Roma, 1990.

ROMANI V., Il paesagoio dell'A lto Garda Bresciano: studio per un piano paesistico, Grafo, Brescia, 1988.

ROMANI V., Ecologia del paesaggio e pianificazione, Genio Rurale n. 4, 1992.

ROMANI V., Il paesagoio: teoria e pianificazione, Franco Angeli, Milano, 1994.

SCAZZOSI L. (a cura), Politiche e culture del paesaggio - esperienze internazionali a confronto, Gangemi, Roma, 1999.

SERENI E., Storia del paesaggio agrario italiano, Laterza, Bari, 1961-1999.

STEINER F., L'immaginazione ecologica. Dall'interzento nel nostro intorno all'integrazione con il paesagoio zizente, Urbanistica 108, 1997.

STEINER F., Costruire il paesaggio (The lizing landscape - an ecological approach to landscape planning), McG raw Hill Italia, Milano, 1994 (McGraw Hill, New York, 1991).

TOURING Club It AliANO, Il paesaggio italiano. Idee Contributi Immagini, Touring Club Italiano, Milano 2000.

TURRI E., Il paesagoio come teatro, Marsilio, Venezia, 1998.

VAN DER LubBe M., VAN MANSVELT J.D., Lista di controllo per la gestione sostenibile del paesagoio. Rapporto finale dell'azione concertata dell'Unione E uropea A IR3-CT93-1210 (Checklist for Sustainable Landscape Management), traduzione ed edizione italiana a cura di Rossi R., Regione Toscana, Dipartimento delle politiche territoriali ed ambientali, Firenze 1999 (Elsevier Science B.V., Amsterdam, 1999).

VAN LANGVELDE F., Conceptual integration of landscape planning and landscape ecology, with a focus on the Netherland, in: Cook E.A., Van Lier H.N., Landscape planning and ecological networks, E lsevier E d., Amsterdam, 1994.

\section{Riferimenti di interesse specifico per lo studio della frammentazione paesistica}

Alcune importanti tematiche di studio del paesaggio forniscono contributi di specifico interesse per l'interpretazione della fenomenologia della frammentazione paesistica. Fra questi un campo fondamentale di indagine riguarda la riflessione, in corso da tempo anche nel nostro paese, sul tema della naturalità diffusa, sviluppato con specifici riferimenti ai paesaggi culturali.

Gli aspetti specificamente riguardanti la frammentazione paesistica fanno riferimento ad esperienze prevalentemente estere, i cui sviluppi più evoluti sono dovuti a studi statunitensi e nordeuropei. Importanti lavori di inquadramento della problematica e di sviluppo di punti di vista diversi sono dovuti fra gli altri a J. A hern, E.A. Cook, J.G. Fabos, C.A. Flink, R.T.T. Forman, R.H.G. Jongman, C.E. Little, L. LaB Schuarz, R.M. Searns, H.N. $V$ an L ier. In Italia questa tematica, di sviluppo estremamente recente, ha assunto rilievo con autori come B. Romano, nel campo della pianificazione, $C$. Blasi, $L$. Boitani, S. Malcerschi, nel campo della ricerca ecologica applicata. Nell'ambito di questi lavori unavasta serie di contributi tecnicoscientifici riguarda gli aspetti di conoscenza e progetto dei corridoi paesistici. Per questo tema, come anche per le reti ecologiche in genere, la bibliografia qui riportata esclude la ricca produzione di contributi teorici ed empirici sviluppata nell'ambito dell'ecologia del paesaggio e della biologia conservazionale, selezionando quanto ha diretta attinenza con gli aspetti di conoscenza e progetto del paesaggio, con particolare riferimento alle finalità della pianificazione paesistica espressamente considerate in questo lavoro. Si segnala comunque il database bibliografico denominato LYNX, elaborato dall'European Centre for Nature Conservation (ECNC), con una vasta gamma di pubblicazioni inerenti gli aspetti di ecologia e biologia conservazionale.

Se molti elementi di approfondimento sono stati elaborati, nell'ambito delle discipline della pianificazione paesistica e ambientale, in relazione agli sviluppi degli studi di biologia conservazionale e delle applicazioni di pianificazione delle reti ecologiche, gli studi angloamericani riguardanti le greenuays hanno dato nel tempo sempre più importanza agli obiettivi di conservazione e miglioramento ecologico e ambientale insieme a quelli, per così dire fondativi, afferenti le attività ricreative all'aperto. La diversità di scala tra i paesaggi americani e quelli europei, ed italiani in particolare, impone in ogni caso molta accortezza nel considerare queste esperienze.

Si riportano di seguito alcuni riferimenti bibliografici di interesse specifico suddivisi per temi chiave: paesagoi culturali e naturalità diffusa, frammentazione paesistico-ambientale e reti ecologiche, greenuays, corridoi paesistici e corridoi ambientali. 


\section{Paesaggi culturali e naturalità diffusa}

Bagnaresi U., MinOtTa G., Problemi e orientamenti operatizi per la rinaturalizzazione delle aree rurali, Genio Rurale n. 4, 1994.

CAMPIONI G., FERRARA G., Tutela della naturalità diffusa, pianificazione degli spazi aperti e crescita metropolitana, Il Verde E ditoriale, Milano, 97.

Caravello G., Giacomini F., E terogeneità ed ewoluzione di un paesaggio rurale: V egoiano, Padova, Genio Rurale n. 11, 1996.

COLANTONIO R., CONTI G., SANTOLINI R., La pianificazione ecosistemica del paesagogio e la naturalità diffusa, Genio Rurale n.11, 1996.

COlANTONio Venturelli R., A proposito della naturalità nei paesagoi antropizzati, Genio Rurale n. 11, 1997.

FABBRI P., Natura e cultura del paesaggio agrario, CittàStudi, Milano, 1997.

FARINA A., Ruolo storico-ecologico della siepe nel paesaggio agrario mediterraneo, Genio Rurale n. 1, 1995.

FEDOROWICK J.M., A landscape restoration framework for wildlife and agriculture in the rural landcsape, Landscape and Urban Planning, 27, 1993.

FORMAN R.T.T., BAUDRY J., Hedgerous and hedgerow networks in landscape ecology, Environmental Management, 8, 1984.

Gibelli M. G., Paesaggi culturali e naturalità diffusa, Genio Rurale n. 10, 1995.

Guccione B., Kroegmeier L., Martelli S., Paolinelli G., Valentini A., Venturi P., L'interpretazione diagnostica e progettuale del mosaico degli spazi aperti nel piano generale comunale, in: Guccione B., Paolinelli G. (a cura), Piani del Verde $\mathcal{E}$ Pani del Paesaggio - Elementi di ewoluzione metodologica nell'ambito del dibattito sui nuori piani comunali, Alinea, Firenze, 2001.

MiNISTERO DELL'AMBIENTE, Comunicazione nazionale per la lotta alla siccità ed alla desertificazione, Comitato nazionale per la lotta alla desertificazione, Ministero dell'ambiente, Roma, 1999.

PIGNATTI S., Paesaggio zegetazionale e paesaggio agricolo, in Il disegno del paesaggio italiano, Casabella n. 575-576, 1991.

UfFICIO Di PIANO PER LA REDAZIONE DELLO SCHEMA DIRETTORE - SDTM, Primo rapporto sulle condizioni di sostenibilità delle prezisioni urbanistiche dell'area zasta bolognese, Bologna, 1997.

\section{Frammentazione paesistico-ambientale e reti ecologiche}

Asero A., Cacciatore L, De Santis L, Siracusa G, I Monti Rossi di Nicolosi, una "buffer zone" per i corridoi ecologici comunali, atti del workshop nazionale Reti e corridoi ecologici per gi interzenti di conserzazione e salzaguardia della natura in ambiente urbano e suburbano, Agenzia Nazionale per la Protezione Ambientale (ANPA), Catania, 1-3 ottobre 1999.

BASCHAK L.A., BROWN R.D., A n ecological framework for the planning design and management of urban rizer greenumy, Landscape and Urban Planning, 33, 1995.

BASCHAK L.A., BROWN R.D., Rizer systems and landscape networks, in: Cook E.A., Van Lier H.N., (a cura), Landscape planning and ecological networks, Elsevier, Amsterdam, 1994.

BATTISTI C., Le connessioni tra aree naturali attorno alla città di Roma, in: Dimaggio C., Ghiringhelli R., Reti ecologiche in aree urbanizzate, atti del seminario di Milano, 5.2.99, Agenzia Nazionale per la Protezione Ambientale (ANPA), Provincia di Milano, Angeli, Milano, 1999.

BATTISTI C., FIGLIUOLI F., ROMANO B., La continuità ambientale a scala nazionale: spunti da studi di pianificazione e da analisi faunistiche, atti del workshop nazionale Paesagoi rurali di domani: la gestione degli ecosistemi agrosilwopastorali e la tutela della connettizità ecologica del territorio extraurbano, Agenzia Nazionale per la Protezione Ambientale (ANPA), Torino, 10 settembre 1999.

Bisogni L.G., GaRibOldi A., MalCEvsCHI S., Reti ecologiche ed interzenti di miglioramento ambientale, Il Verde Editoriale, Milano, 1996.

BLASI C., L'analisi della connettizità e della frammentazione nella definizione dei corridoi ecologici in ambiente urbano e suburbano, Attidel workshop nazionale Reti e corridoi ecologici per gli interzenti di conserazione e salzaguardia della natura in ambiente urbano $e$ suburbano, Agenzia Nazionale per la Protezione Ambientale (ANPA), Catania, 1-3 ottobre 1999.

BOITANI L., La tutela e la zalorizzazione della biodizersità terrestre in Italia: appunti per la Rete Ecologica Nazionale, atti del seminario nazionale Conserazione della natura e suiluppo locale: il sistema delle aree protette e la Rete Ecologica Nazionale, ECOLAVORO99, Legambiente, Ministero dell'Ambiente, Federazione Italiana Parchi e Riserve Naturali, Firenze, 14 dicembre 1999.

Brucculeri R., Di ludovico D., Perrotti A., Properzi P., Romano B., TAmburini G., La continuità ambientale in A bruzzo. Riferimenti e criteri metodologici per la pianificazione della rete ecologica regionale, ANPA, Agenzia Nazionale per la Protezione dell'Ambiente, Piano Stralcio per lo sviluppo del sistema nazionale conoscitivo e dei controlli in campo ambientale, Progetto Monitoraggio delle Retri Ecologiche, Regione Abruzzo, Università dell'Aquila, sintesi del rapporto tecnico finale consegnato il 10 novembre 1999, 2000.

BUUREN M. VAN, The hydrological landscape structure as a basis for netuork formulation; a case study for the Rege catchment (NL), in: Cook E.A., Van Lier H.N. (a cura), Landscape planning and ecological networks, E lsevier, Amsterdam, 1994.

COOK E.A., VAN LIER H.N. (a cura), Landscape planning and ecological networks, E lsevier, Amsterdam, 1994.

CRAIG S., Beyond park boundaries, in: Cook E.A., Van Lier H.N., (a cura), Landscape planning and ecological networks, E lsevier, Amsterdam, 1994. 
DietVORST A., BOLHUIS P. VAN, Design principles for a combination of recreation and nature, in: Cook E.A., Van Lier H.N., (a cura), Landscape planning and ecological networks, E lsevier, Amsterdam, 1994.

Di FIDIO M., Teoria e prassi delle reti ecologiche. Dall'isolamento all'integrazione della difesa della natura, Seminario di studio del Dottorato di ricerca in Progettazione paesistica, Università degli Studi di Firenze, Dipartimento di Urbanistica e Pianificazione del territorio, Firenze, 26 maggio 1999.

Dramstad W.E., Olson J.D., Forman R.T.T., Landscape E cology Principles in Landscape A rchitecture and Land-Use Planning Harvard University Graduate School of Design - Island Press - American Society of Landscape Architects, Washington, 1996.

Dimaggio C., GHIRINGHelli R. (a cura), Reti ecologiche in aree urbanizzate, Provincia di Milano - Agenzia Nazionale per la Protezione dell'Ambiente, (atti del seminario di Milano del 5 febbraio 1999), Franco Angeli, Milano, 1999.

FORMAN R.T.T., BAUDRY J., Hedgerous and hedgerow networks in landscape ecology, Environmental Management, 8, 1984.

FORMAN R.T.T., Land mosaics, the ecology of landscapes and regions, Cambridge, 1995.

GIMONA A., Theoretical framework and practical tools for conserzation of biodizersity at the landscape scale, in PLANECO Project. Planning in ecological network, ricerca nazionale MURST, pubblicazione WE B, sito Planeco, Newsletter 2, 1999.

HANSEN-MOLleR J., Recreation, reproduction and ecological restoration in the Greater Copenhagen region, in: Cook E.A., Van Lier H.N., (a cura), Landscape planning and ecological networks, E lsevier, Amsterdam, 1994.

INFRA ECO NETWORK EUROPE (a), Input from the 3rd meeting "Roads and wildlife", 30 Sept. to 2 Oct. 1998 - Council of E urope - Strasbourg - France, in: Summary of the IENE and COST 341 activities in different countries, IENE WE B site, 1999.

INFRA ECO NETWORK EUROPE (b), Suitzerland's uddlife corridors, in: Summary of the IENE and COST 341 activities in different countries, IENE WEB site, 1999.

INFRA ECO NETWORK EUROPE (c), Habitat fragmentation due to transportation infrastructure in A ustria, in: Summary of the IENE and COST 341 activities in different countries, IENE WEB site, 1999.

IUCN, Corridors, transition zones and buffers: tools for enhancing the effectizeness of protected areas, Parks for life, Workshop III.9, IUCN, Gland, Switzerland, 1993.

JONGMAN R.H.G., An ecological main structure for the European Union, in: Cook E.A., Van Lier H.N., (a cura), Landscape planning and ecological networks, Elsevier, Amsterdam, 1994.

JONGMAN R.H.G., Nature conserzation planning in E urope: dezeloping ecological networks, Landscape and Urban Planning 32, 1995.

JONGMAN R.H.G., Buffer zones and ecological corridors, in: ECNC, Dezelopment of habitat specific recommendations for bufferzonesand corridors, ECNC Newsletter vol. 3, nr. 1, ECNC WE B site, 1996.

JONGMAN R.H.G., TROUMBIS A.Y, The wider landscape for nature conseration: ecological corridors and buffer zones, EQNC,MN2.7 project report, 1995.

KLEYER M., Habitat network schemes in Stuttgart, in: Cook E.A., Van Lier H.N., (a cura), Landscape planning and ecological networks, E lsevier, Amsterdam, 1994.

KNAAPEN J.P., SCHEFFER M., HARMS B., Estimating habitat isolation in landscape planning Landscape and Urban Planning, 23, 1992.

LAMMERS W., A new strategy in nature policy. touards a national ecological network in the Netherlands, in: Cook E.A., Van Lier H.N., (a cura), Landscape planning and ecological networks, Elsevier, Amsterdam, 1994.

LANGEVELDE F. VAN, Landscape ecological planning: conceptual integration of landscape planning and landscape ecology, with a fous on ecological networks in the Netherlands, in: Cook E.A., van Lier H.N., (a cura), Landscape planning and ecological networks, ISOMUL, Elsevier, Amsterdam, 1994.

LINEHAN J., GROSS M., FINN J., Greenuay planning: dezeloping a landscape ecological network approach, Landscape and Urban Planning 33, 1995.

Minissale P., Ronsisvalle G.A., SPAmpanAto S.G., Sistema di ambienti umidi circumetnei; ipotesi per una rete ecologica della prozincia di Catania, Atti del workshop nazionale Reti e corridoi ecologici per gli interzenti di conserazione e saluaguardia della natura in ambiente urbano e suburbano, Agenzia Nazionale per la Protezione Ambientale (ANPA), Catania, 1-3 ottobre 1999.

Ministero Dell'Ambiente, Servizio CONSERVAZIOne Della NATURA, La zalorizzazione delle risorse ambientali nelle politiche di sziluppo "La rete ecologica nazionale", Note informative, Ministero dell'Ambiente, Roma, 1999.

Provincia di Bologna, Assessorato Ambiente, Servizio PianificaZione Paesistica, Piano programmatico per la conserzazione e il mighioramento degli spazi naturali nella prozincia di Bologna, Bologna, 2000.

ROMANO B. (a), Oltre i parchi. La rete zerde regionale. Una ricerca sulle idoneità territoriali per $i$ corridoi ecologici dell'A ppennino Centrale, Università degli Studi dell'Aquila, Dipartimento di Architettura e Urbanistica, Andromeda E ditrice, Teramo, 1996.

ROMANO B. (b), Planning of enzironmental continuity in Italy, in: PLANE CO Project. Planning in ecological netuork, ricerca nazionale MURST, pubblicazione WE B, sito Planeco, Newsletter 1, 1998.

ROMANO B. (c), Continuità ambientale ed interferenza antropica tra le grandi aree protette e le città dell'A ppennino, Attidelworkshop nazionale Reti e corridoi ecologici per gli interzenti di conserzazione e saluguardia della natura in ambiente urbano e suburbano, Agenzia Nazionale per la Protezione Ambientale (ANPA), Catania, 1-3 ottobre 1999. 
ROMANO B. (d), L'ecologia nella pianificazione. Il progetto PLANECO, in: PLANECO Project. Planning in ecological network, ricerca nazionale MURST, pubblicazione WE B, sito Planeco, Newsletter 3, 1999.

ROMANO B. (e), La continuità ambientale nella pianificazione, Urbanistica, n. 112, 1999.

Romano B. (f), Continuità ambientale. Pianificare per il riassetto ecologico del territorio, Andromeda E ditrice, Teramo, 2000.

Romano B. (g), Politiche ambientali nell'A bruzzo dei parchi, Urbanistica Informazioni, n. 169, 2000.

ROMANO B. (h), Frammentazione ambientale e politiche di gozerno territoriale - sionificati e potenzialità delle reti ecologiche nella pianificazione dei paesagoi culturali, Seminario di studio del Dottorato di ricerca in Progettazione paesistica, Università degli Studi di Firenze, Dipartimento di Urbanistica e Pianificazione del territorio, Firenze, 5 aprile 2000.

ROSE D., STEINER F., JACKSON J., A $n$ applied human ecological planning approach to regional planning Landscape Planning, 5 , 1978-79.

SANTAROSSA L. (a), E cological networks from an "anthropological" point of riew. ideas for further thought, in: PLANECO Project. Planning in ecological network, ricerca nazionale MURST, pubblicazione WE B, sito Planeco, Newsletter 2, 1999.

SANT AROSSA L. (b), Le implicazioni socioeconomiche delle reti ecologiche, in: PLANECO Project. Planning in ecological network, ricerca nazionale MURST, pubblicazione WE B, sito Planeco, Newsletter 3, 1999.

SCHILLECI F., La rete ecologica, uno strumento per la riqualificazione del territorio, in: Baldi M.E., La riqualificazione del paesagoio, La Zisa Ed., Palermo, 1999.

SLUIS VAN DER T., PEDROLI B., Ecological networks in the Netherlands: implementation, research and results after 10 years, in: PLANECO Project. Planning in ecological network, ricerca nazionale MURST, pubblicazione WEB, sito Planeco, Newsletter 3, 1999.

TOSI A. (a cura), Degrado ambientale periurbano e restauro naturalistico, Franco Angeli, Quaderni del Dipartimento di scienze del territorio, Milano, 1999.

VRIJLANDT P., KERKSTRAK., A strategy for ecological and urban dezelopment, in: Cook E.A., Van Lier H.N., (a cura), Landkape planning and ecological networks, Elsevier, Amsterdam, 1994.

\section{Greenuays}

AHERN J., Greenumys as ecological networks in rural areas, in: Cook E.A., van Lier H.N., (a cura), Landscape Planning and Ecological Network, ISOMUL, Elsevier, Amsterdam, 1994.

AHERN J., Greenumys as a planning strategy, Landscape and Urban Planning, 33, 1995.

AHERN J., FABOS J.G., Greenways The beginning of an international mozement, Special Issue of Landscape and Urban Planning 33, Elsevier, Amsterdam, 1995.

ANGRILLI L., Greenuays, Urbanistica, 113, 1999.

BASCHAK L.A., BROWN R.D., A n ecological frameuork for the planning design and management of urban rizer greenumy, Landscape and Urban Planning, 33, 1995.

BisCHOF F A., Greenumys as wehicles for expression, Landscape and Urban Planning, 33, 1995.

BUREL F., BAUDRY J., Social, aesthetic and ecological aspects of hedgerous in rural landscapes as a frameurk for greenumis, Landscape and Urban Planning, 33, 1995.

D AWSON K.J., A comprehensize conserzation strategy for Georgia's greenumy, Landscape and Urban Planning, 33, 1995.

GOBSTER P.H., Perception and use of a metropolitan greenway system for recreation, Landscape and Urban Planning, 33, 1995.

KENT R.L., E LLIOT C.L., Scenic routes linking and protecting natural and cultural landscape features: a greenumy skeleton, Landscape and Urban Planning, 33, 1995.

LINEHAN J., GROSS M., FINN J., Greenuay planning: dezeloping a landscape ecological network approach, Landscape and Urban Planning 33, 1995.

LUYMES D.T., TAMMINGA K., Integrating public safety and use into planning urban greenumys, Landscape and Urban Planning33, 1995.

MAternini G., PeZZAgno M., Le caratteristiche funzionali delle greenumis: un primo approccio metodologico, Atti del $2^{\circ}$ convegno europeo sulle greenways, Milano 1999.

Maternini G., PeZZAGno M., La progettazione delle greenumy, “Le strade”, n.1/2, 2000.

MACHADO J.R., RIBEIRO L.P., LANGSTON A.T.R., Greenumys in a metropolitan context: the metropolitan area of Lisbon, Attide $2^{\circ}$ convegno europeo sulle greenways, Milano 1999.

NDUBisi F., DEMEO T., DiTTO N.D., E nzironmentally sensitize areas: a template for dezeloping greenzuay corridors, Landscape and Urban Planning 33, 1995.

QUAYLE M., Urban greenumys and public umys: realizing public ideas in a fragmented world, Landscape and Urban Planning 33, 1995.

RYAN K.L., "Trails for the first tuenty-century. Planning design and management. Manual for multiuse trails", Island Press, Washington DC, 1993.

RYDER B.A., Greenuay palnning and grouth management: partners in conserzation?, Landscape and Urban Planning 33, 1995.

SCHRADER C.C., Rural greenway planning: the role of streamland perception in landowner acceptance of landmanagement strategies, Landscape and Urban Planning 33, 1995.

SEARNS R.M., The ewolution of greenuays as an adaptize urban landscape form, Landscape and Urban Planning 33, 1995. 
SHANNON S., SMARDON R., KNUDSON M., Using zisual assessment as a fundation for greenumy planning in the St. Laurence Rizer V alley, Landscape and Urban Planning 33, 1995.

SPELlERBERG I. G AYWOOD M., Linear landscape features, Landscape Design, n. 223, 1993.

TAYLOR J., PAINE C., FitzGibBOn J., Form greenbelt to greenumys: four Canadian cases studies, Landscape and Urban Planning 33, 1995.

TURNER T., Greenuays, blueways, skyuays and other ways to a better London, Landscape and Urban Planning 33, 1995.

Tzolova G.V., A n experiment in greenumy analyois and assessment: the Danube Rizer, Landscape and Urban Planning, 33,1995.

ZUBE E.H, Greenumys and the US National Park System, Landscape and Urban Planning, 33, 1995.

WALMSLEY A., Greenuays and the making of urban form, Landscape and Urban Planning 33, 1995.

\section{Corridoi paesistici e corridoi ambientali}

Asero A., Cacciatore L., De Santis L., Siracusa G., I Monti Rossi di Nicolosi, una "buffer zone" per i corridoi ecologici comunali, Atti del workshop nazionale Reti e corridoi ecologici per gi interzenti di conserzazione e salnguardia della natura in ambiente urbano e suburbano, Agenzia Nazionale per la Protezione Ambientale (ANPA), Catania, 1-3 ottobre 1999.

BLASI C., L'analisi della connettizità e della frammentazione nella definizione dei corridoi ecologici in ambiente urbano e suburbano, attidel workshop nazionale Reti e corridoi ecologici per gli interzenti di conserazione e salaguardia della natura in ambiente urbano $e$ suburbano, Agenzia Nazionale per la Protezione Ambientale (ANPA), Catania, 1-3 ottobre 1999.

BonTEMPo C.G., Dai Macconi di Gela a Torre Manfria. Tutela della costa e corridoi ecologici, Atti del workshop nazionale Reti e corridoi ecologici per gli interzenti di conserzazione e salzaguardia della natura in ambiente urbano e suburbano, Agenzia Nazionale per la Protezione Ambientale (ANPA), Catania, 1-3 ottobre 1999.

CLIGNEZ M., Un corridoio ripristinato dall'uomo: i dintorni delle zie di comunicazione, Naturopa, n. 87, 1998.

FARJON J.M.J., BAKKER J.B., E cological corridors and buffer zones: role, practical experience and planning. Preliminary Report, Project MN2.7, ECNC, E TC Nature, 1995.

Foppen R., GEILEN N., VAN DER SlUIS T., Rizers: green corridors for E urope, in: PLANE CO Project. Planning in ecological network, ricerca nazionale MURST, pubblicazione WE B, sito Planeco. Newsletter 3, 1999.

FURLANETTO D., Un corridoio zerde per eliminare le barriere, Acer n. 4, 2000.

GACKA-GRZESIKIEWIEZ E., Il corridoio ecologico della alle della V istola, Naturopa, n. 87, 1998.

GHIRINGHelli R., GuSSONI S., Corridoi ecologici di connessione tra i boschi del Ticino e l'ambito dei boschi e dei fontanilidel sudorest di Milano, Atti del workshop nazionale Paesagoi rurali di domani: la gestione degli ecosistemi agrosilwopastorali e la tutela della connettizità ecologica del territorio extraurbano, Agenzia Nazionale per la Protezione Ambientale (ANPA), Torino, 10 settembre 1999.

GROOME D., Green corridors: a discussion of a planning concept, Landscape and Urban Planning, 19, 1990.

IUCN, Corridors, transition zones and buffers: tools for enhancing the effectizeness of protected areas, Parks for life, Workshop III.9, IUCN, Gland, Switzerland, 1993.

LEWIS P.H. JR., Quality corridors in Wisconsin, Landscape Architecture, 54, 1964.

JONGMAN R.H.G., Buffer zones and ecological corridors, in: ECNC, Dezelopment of habitat specific recommendations for buffer zonesand corridors, ECNC Newsletter vol. 3, nr. 1, ECNC WE B site, 1996.

Jongman R.H.G., Corridoi ecologici: dall'esperienza nei Paesi Bassi alle prospettize di sziluppo delle politiche di tutela in E uropa, attidel workshop nazionale Gozerno sostenibile del territorio e conserazione della natura in relazione agli strumenti di pianificazione in E uropa, Agenzia Nazionale per la Protezione Ambientale (ANPA), Roma, 13 novembre 1997.

JONGMAN R.H.G., Ecological corridors in Europe, in: PLANECO Project. Planning in ecological network, ricerca nazionale MURST, pubblicazione WE B, sito Planeco, Newsletter 1, 1998.

Martinelli N., MininNi M., I zalloni erosizi del carsismo in Puglia come corridoi di connessione ecologica e itinerari naturalistia ollinamare, Atti $2^{\circ}$ Convegno Internazionale di Studio Sentieri, le reti di interconnessione delle risorse naturali e culturali, Cagliari, 1997.

Miklós L., Koren M., Steffek J., E cological corridors and buffer zones. Preliminary Report, Project MN2.7, ECNC, E TC Nature, 1995.

NDUBisi F., DEMEO T., DiTTO N.D., Enzironmentally sensitize areas: a template for dezelopping greenuay corridors, Landscapeand Urban Planning 33, 1995.

Отт J., Collegamenti fra biotopi e reti ecologiche nelle aree urbane della Germania. Che senso hanno i corridoi zerdi nelle città?, in Ghiringhelli R., Dimaggio C. (a cura), Reti ecologiche in aree urbanizzate, Provincia di Milano - Agenzia Nazionale per la Protezione dell'Ambiente (atti del seminario di Milano del 5 febbraio 1999), Franco Angeli, Milano, 1999.

ROMANO B., La continuità ambientale in Italia, corridoi ecologici per i parchi e le aree protette, Atti XVIII Conferenza Italiana di Scienze Regionali, E uropa e Mediterraneo, Siracusa ottobre 1997, Volume 3.

ROMANO B., Politiche ambientali nell'A bruzzo dei parchi, Urbanistica Informazioni, n. 169, 2000.

Ronsisvalle G.A., Siracusa G., La caza del Prainito (Sicilia sudorientale), modello di corridoi ecologico degli Iblei, Atti del workshop nazionale Reti e corridoi ecologici per gli interventi di conservazione e salvaguardia della natura in ambiente urbano e suburbano, Agenzia Nazionale per la Protezione Ambientale (ANPA), Catania, 1-3 ottobre 1999. 
SiraCUSA G., La Timpa di A cireale, modello di corridoio ecologico costiero, Atti del workshop nazionale Reti e corridoi ecologici per gii interventi di conservazione e salvaguardia della natura in ambiente urbano e suburbano, Agenzia Nazionale per la Protezione Ambientale (ANPA), Catania, 1-3 ottobre 1999.

SLUIS VAN DER T., FOPPEN R., GEILEN N., Rizers: Green corridors for Europe, in: PLA NE CO Project. Planning in ecological network, ricerca nazionale MURST, pubblicazione WE B, sito Planeco, Newsletter 3, 1999.

TROUMBiS A.Y., KARDAKARI N., Ecological corridors and buffer zones in Greece, Italy and France. Preliminary Report, Project MN2.7, ECNC, E TC Nature, 1995.

\section{Riferimenti relativi al territorio di indagine}

BARBIERI G., CANIGIANI F., FONNESU J., ROMBAI L., I zalori geografico-storici del paesaggio fiorentino - proposte di uso e tutel, Atti dell'Istituto di Geografia dell'Università di Firenze, quaderno n. 11, 1982.

Bellia P. (a cura), Le carte delle prozincie di Firenze e Prato. Repertorio cartografico, Università degii Studi di Firenze, Dipartimento di Urbanistica e Pianificazione del territorio, Centro di documentazione cartografica, Tipografia ABC, Sesto Fiorentino FI, 1997.

BiAgioli G., L'agricoltura e la popolazione in Toscana all'inizio dell'Ottocento. Un'indagine sul catasto particellare, Pacini, Pisa, 1975.

BIANCHI F.R., FAVI E., Le caratteristiche agricole della piana, Quaderni di Urbanistica Informazioni, n. 7, 1990

Boggiano A., Zoppi M., Toscana, in: Astengo A., Nucci C., IT URB. 80 Rapporto sullo stato dell'urbanizzazione in Italia, volume primo, Quaderni di Urbanistica Informazioni, n. 8, 1990.

Bortolotti L., De LuCA G., Come nasce un'area metropolitana Firenze Prato Pistoia: 1848-2000, Alinea, Firenze, 2000.

CAROCCI G., I dintorni di Firenze, 2 voll., Multigrafica, Roma, 1968.

Clemente C., Città e periferia nell'area metropolitana fiorentina, Bollettino del Dipartimento di Urbanistica e Pianificazione del territorio dell'Università degli Studi di Firenze, n. 1, 1996.

Clemente C., InNOCENTI R. (a cura), La formazione del nuozo piano di Firenze, Franco Angeli, Milano, 1994.

Clemente C., Innocenti R., L'idea di città nei piani di Firenze da Detti a Vittorini, Bollettino del Dipartimento di Urbanistica e Pianificazione del territorio dell'Università degli Studi di Firenze, n. 1-2, 1999.

CONFERENZA PER IL COORDINAMENTO DEGLI INTERVENTI DI PIANIFICAZIONE TERRITORIALE, Processo di urbanizzazione nell'area Firenze-Prato-Pistoia, La Casa Usher, Firenze, 1984.

CONFERENZA PER IL COORDINAMENTO DEGLI INTERVENTI DI PIANIFICAZIONE TERRITORIALE, Le aree a rischio idraulico nella piana Firenze-Prato-Pistoia, Regione Toscana, Firenze, 1986.

CONFERENZA PER IL COORDINAMENTO DEGLI INTERVENTI DI PIANIFICAZIONE TERRITORIALE, Terza fase: L'assetto agricolo della piana Firenze-Prato-Pistoia, Regione Toscana, Giunta Regionale, Firenze, 27-29 aprile 1988.

COMUnE Di FIRENZE (a cura), Castello. Campagna medicea, periferia urbana, Studio GE A, Firenze, 1984.

COMUNE Di FIRENZE (a cura), Firenzecologia Conoscere e capire l'ambiente del comune di Firenze, Il Ventagiio, Roma, 1987.

COMUne di Firenze (a cura), Sette idee per il parco urbano di Castello, Servizio Pianificazione Grandi Progetti, Comune di Firenze, Firenze, 2001.

CONTI E. (a), La formazione della struttura agraria moderna nel contado fiorentino. Monografie e tazole statistiche (secoli XV-XIX), Istituto Storico Italiano per il Medioevo, Roma, 1965.

CONTI E. (b), I catasti agrari della Repubblica fiorentina e il catasto particellare toscano (secoli XIV-XIX), Istituto Storico I taliano per il Medioevo, Roma, 1966.

Conti M., Sestini S., Del Perugia G., Firenze a Ozest. Cent'anni di confronti fotografici, Tipografia Il Fiorino, Firenze, 1990.

CONTi R., Ulivieri L., Piano Territoriale di Coordinamento Prozinciale, Provincia di Firenze, Settore Pianificazione territoriale, Franco Angeli, Milano, 2000.

De LuCA G., Costa M. (a cura), Schema strutturale per l'area metropolitana Firenze-Prato-Pistoia, Quaderni di Urbanistica Informazioni, 7, 1990.

DE' RICCI L., Della coltizazione dei contorni di Firenze, Giornale Agrario Toscano, n. 4, 1830.

Di Pietro G., Un progetto per Firenze - la nuoza città nella piana di Castello, Ponte alle Grazie, Firenze, 1990.

Di Pietro G., Greppi C., Pettini P., Sica P., Dall'ERba G. (collaboratore), Piano Intercomunale del Comprensorio Fiorentino, Ufficio Tecnico del Piano Intercomunale Fiorentino, Studi Ricerche Documenti, Firenze, 1965.

FERrARA G., Il progetto Grandi Cascine a Firenze, in MANIGLIO CALCAGNO A. (et al.), Nuozi parchi per la città storica, Maria Pacini Fazzi, Lucca, 1995.

GELLINI R., GROSSONI P., V alori culturali e ambientali del zerde urbano e periurbano, in: Ulivieri L. (a cura), PTCP conoscere per progettare: woci, frammenti, esperienze nel "cantiere" del Piano, ad Arnum, Quaderni dell'Assessorato all'Urbanistica e Assetto del Territorio della Provincia di Firenze, 2, 1994.

Gorelli G., Le tracce deboli delle strutture profonde, Bollettino del Dipartimento di Urbanistica e Pianificazione del territorio dell'Università degii Studi di Firenze, n. 1-2, 1998.

Greggio A., Hofmann A., Rossi R., Trevisani M., Vincia A., Una nuoza base dati di uso del suolo: la sperimentazione fatta nel bacino pilota del fiume Serchio, Documenti del territorio, 39, 1998.

OMODEI ORINI L., L'agricoltura nelle aree perturbane: il caso della piana Firenze-Prato-Pistoia, Firenze, 1989. 
PaZZAGLi C., Per la storia dell'agricoltura toscana nei secoli XIX e XX. Dal catasto particellare lorenese al catasto agrario del 1929, Fondazione Luigi E inaudi, Torino, 1979.

PAZZAGLi C., La terra delle città. Le campagne toscane dell'Ottocento, Ponte alle Grazie, Firenze, 1992.

PicCARDi M., Tra A mo e Bisenzio. Cartografia storica, fonti documentarie e trasformazioni del territorio, Tipografia Nova, Signa, Firenze, 2001.

PiNTO G., La campagna toscana nel tardo Medioewo, Sansoni, Firenze, 1986.

PINTo G., Toscana medioezale. Paesagoi e realtà sociali, Sansoni, Firenze, 1986.

PizZiolo G., La Toscana wolando, Sansoni, Firenze, 1986.

POLI D., La piana fiorentina. Una biografia territoriale narrata dalle colline di Castello, Alinea, Firenze, 1999.

Repetti E., Dizionario geografico fisico e storico della Toscana, Federazione delle Casse di Risparmio della Toscana, Firenze, 1972 (edizione originale 1845).

Repetti E., Compendio storico della città di Firenze. Sua Comunità-Diocesi e Compartimento, Forni, Bologna, 1972 (edizione originale 1849).

RePeTti E., Dizionario corografico della Toscana, Cassa di Risparmio di Firenze, Firenze, 1977 (edizione originale 1855).

Richard Rogers Partnership, Piana di Castello. Piano Guida, Comune di Firenze, Direzione Urbanistica, Servizio Pianificazione urbanistica, Firenze, 1998.

RoDOLICO F., Scritti di aria cultura urbana, Saffe, Firenze, 1976.

ROMBAI D., Le strade prozinciali di Firenze, Firenze, 1992.

ROMBAI L., Insediamenti e paesagoio agrario dall'età comunale al XIX sec., in: Barbieri G., Canigiani F., Fonnesu J., Rombai L., I zalori geografico-storici del paesagoio fiorentino - proposte di uso e tutela, Atti dell'Istituto di Geografia dell'Università di Firenze, quaderno n. 11, 1982.

STOPANI R., Il paesaggio agrario della Toscana. Tradizione e mutamento, FMG Stud. Imm., Firenze, 1989.

SuMmer M. (a cura), Il parco metropolitano dell'area fiorentina, Quaderni di Urbanistica Informazioni, 12, supplemento ad Urbanistica Informazioni n. 125/126, settembre-dicembre 1992.

Targioni TozZetti G., Notizie sulla storia delle scienze fisiche in Toscana, Biblioteca Palatina, Firenze, 1852.

UliVIERI L., PTCP Conoscere per progettare: woi, frammenti, esperienze nel "cantiere" del Piano, Ad Arnum, Quaderni dell'Assessorato all'Urbanistica e all'Assetto del territorio della Provincia di Firenze, Firenze, 1994.

V ALENTINI A., Il sistema del zerde fluziale fiorentino: riflessioni sul tema, Bollettino di Studi Fiorentini, n. 1, 1997.

ZuCCAGNi ORLANDini A., A tlante geografico, fisico e storico del Granducato di Toscana, Cassa di Risparmio di Firenze, Firenze, 1974 (edizione originale 1832). 
Appendice 


\section{A - Schede di identificazione morfologica dei fattori territoriali di frammentazione paesistic ${ }^{145}$}

La classificazione fa riferimento a due criteri principali di identificazione morfologica dei fattori territoriali di frammentazione paesistica: in base all'articolazione spaziale, i fattori sono distinti in lineari, areali e puntuali, in base all'omogeneità di composizione, i fattori sono distinti in semplici e complessi. Dal censimento territoriale condotto nell'ambito di studio sulla scorta di un'ipotesi teorica di impostazione preliminare, sono risultati cinque generi e diciassette tipi di fattori, elencati nella lista di controllo riportata nelle pagine successive. I generi si distinguono, in base ai suddetti criteri morfologici, in:

1. fattori areali complessi: caratterizzati dalla compresenza di più componenti in configurazioni areali sviluppate su significative superfici;

2. fattori areali semplici: caratterizzati dalla dominanza di una componente con configurazione areale sviluppata su significative superfici;

3. fattori lineari complessi: caratterizzati dalla compresenza di più componenti in configurazioni lineari sviluppate su significative lunghezze;

4. fattori lineari semplici: caratterizzati dalla dominanza di una componente con configurazione lineare sviluppata su significative lunghezze;

5. fattori puntuali: caratterizzati dalla dominanza di una sola componente con configurazione a sviluppo circoscritto.

Pare utile evidenziare alcuni criteri e termini, adottati nella classificazione tipologica per la denominazione delle classi dei fattori e la descrizione dei loro caratteri identificativi.

Le definizioni di configurazione sviluppata su significatize superfici o lunghezze e di configurazione a sviluppo decisamente circoscritto, si riferiscono a caratteri quantitativi di tipo relativo. Il significato dei termini citati è infatti strettamente dipendente dalle dimensioni caratteristiche del mosaico paesistico del soggetto di studio.

I fattori responsabili di processi e stati di frammentazione nei quali il paesaggio mantiene una composizione strutturale ed un funzionamento caratterizzati sostanzialmente anche da componenti biotiche devono essere distinti da quelli in cui queste vengono rimosse in modo dominante e praticamente non reversibile, portando alla progressiva mineralizzazione degli spazi a seguito della urbanizzazione e della infrastrutturazione. A tal fine è utile classificare i fattori individuati in ragione del livello di presenza di spazi mineralizzati. Teoricamente è possibile fare riferimento a quattro livelli principali: prevalenza di spazi mineralizzati, presenza rilevante di spazi mineralizzati, presenza mediamente rilevante di spazi mineralizzati, presenza irrilevante di spazi mineralizzati.

Relativamente agli insediamenti, l'attributo agoregato viene utilizzato in contrapposizione a diffuso nella descrizione complessiva della configurazione dell'insediamento di cui la porzione indagata è parte. Gli attributi continuo e discontinuo costituiscono invece i termini opposti di descrizione delle caratteristiche spaziali del tessuto dell'insediamento nei casi delle configurazioni aggregate. Tali

${ }^{145}$ Indagine condotta nel 2001. Fotografie di Gabriele Paolinelli. 
caratteristiche non fanno tanto riferimento alle diverse articolazioni tipologiche dei tessuti urbani consolidati che la storia ci consegna, quanto alla differenza netta tra il raggruppamento di tutti questi, classificati come tessuti continui, rispetto al tessuto di lottizzazione, con articolazione spaziale dilatata, caratteristico prevalentemente degli sviluppi insediativi degli ultimi quattro decenni.

Relativamente alle infrastrutture viarie e tecnologiche, diviene essenziale, nelle fasi di approfondimento conoscitivo preprogettuale finalizzato alla pianificazione e progettazione di interventi, la considerazione delle tipologie di sezione trasversale (a raso, su terrapieno, su struttura verticale puntiforme) rispetto allo sviluppo longitudinale, per l'individuazione dei relativi effetti di alterazione paesistica.

Relativamente ai corsi d'acqua, la presenza irrilezante di spazi mineralizzati, attributita nella classificazione, è ovviamente relativa alle aree extraurbane. Nelle aree urbane infatti i corsi d'acqua presentano un elevato grado di artificializzazione, fino alla completa mineralizzazione dell'alveo in alcuni tratti, ove non se ne riscontri la completa tombatura.

Nella esecuzione delle analisi i fattori lineari semplici vengono rilevati solo nel caso in cui si trovino a contatto con spazi caratterizzati da minori livelli di criticità e, a maggior ragione in tutti i contesti di pregio paesistico-ambientale. Tale tipo di fattore non assume invece rilievo quando risulta parte integrante di paesaggi caratterizzati da potenziali di frammentazione pari o maggiori a quelli che lo caratterizzano. Il caso limite è costituito evidentemente dalla strada interna al tessuto urbano denso, che viene considerata come parte del fattore complesso di tipo areale o lineare rappresentato dall'insediamento.

Il concetto di fattore puntuale non fa tanto riferimento ad un astratto tetto di dimensione superficiale, che risulterebbe poco idoneo a rispondere alla variazione di articolazione spaziale del paesaggio nelle diverse aree geografiche, quanto alla localizzazione isolata nel contesto per assenza di reiterazione della forma di uso del suolo considerata o per una sua presenza estremamente limitata relativamente all'articolazione paesistica complessiva.

I fattori sono stati identificati anche secondo il livello di presenza di spazi mineralizzati (per l'accezione del termine mineralizzazione adottata si veda la nota 22 a pag. 19). 


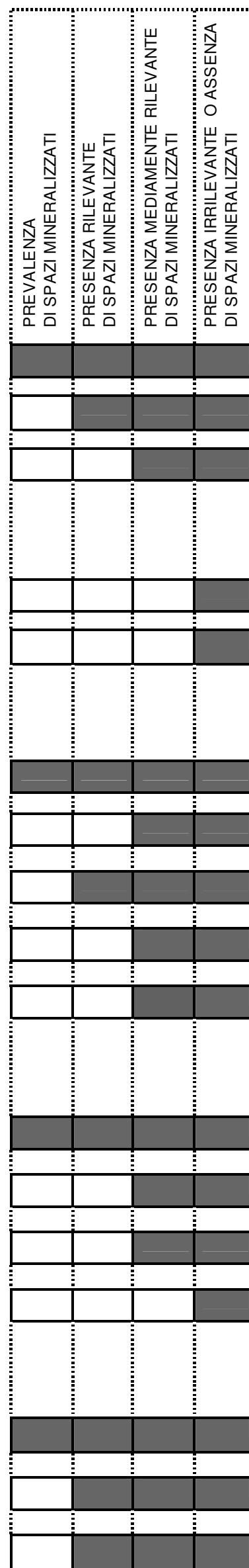

\section{FATTORI TERRITORIALI DI FRAMMENTAZIONE PAESISTICA}

\section{FATTORI AREALI COMPLESSI \\ 1.1 Insediamenti urbani con tessuto continuo \\ 1.2 Insediamenti urbani con tessuto discontinuo \\ 1.3 Insediamenti urbani diffusi \\ 2 FATTORI AREALI SEMPLICI \\ 2.1 Spazi aperti per attiv ità produttiv e agricole intensive \\ 2.2 Spazi aperti soggetti a vincoli di inutilizzazione}

3 FATTORI LINEARI COMPLESSI

3.1 Insediamenti urbani con tessuto continuo

3.2 Insediamenti urbani con tessuto discontinuo

3.3 Fasci di inf rastrutture viarie

3.4 Fasci di inf rastrutture tecnologiche

3.5 Fasci di corsi d'acqua

4 FATTORI LINEARI SEMPLICI

4.1 Inf rastrutture viarie

4.2 Inf rastrutture tecnologiche

4.3 Canali artificiali di bonif ica idraulica

4.4 Corsi d'acqua naturali regimati

$5 \quad$ FATTORI PUNTUALI

5.1 Edifici e complessi di edifici isolati

5.2 Spazi aperti per attiv ità produttive non agricole

5.3 Spazi aperti per servizi di igiene urbana e ambientale 


\section{FATTORI AREALI COMPLESSI}

\subsection{Insediamenti urbani con tessuto continuo}

\section{ESEMPLIFICAZIONI: VEDUTE}

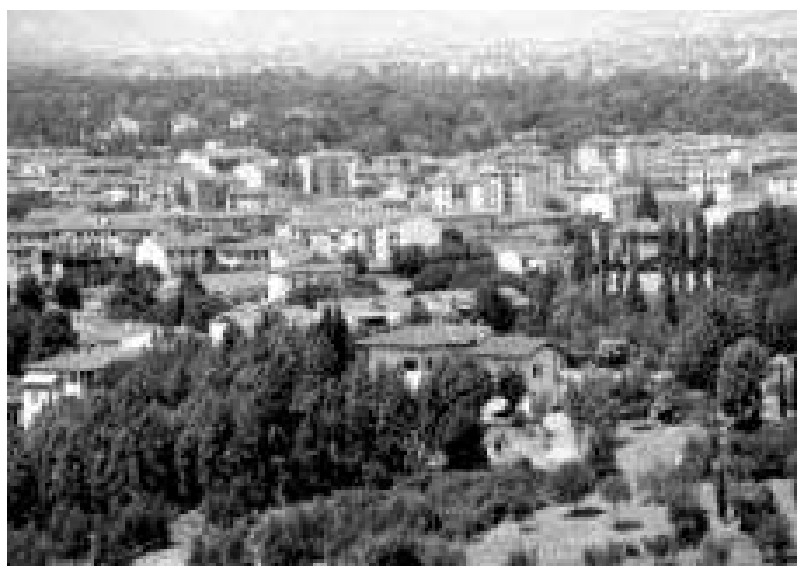

Dal colle di Monteoliveto il tessuto urbano di Firenze sembra immerso nel verde per la presenza sullo sfondo del parco delle Cascine, che in realtà, per quanto di dimensioni apprezzabili, è l'unico episodio di rilievo del paesaggio urbano di pianura

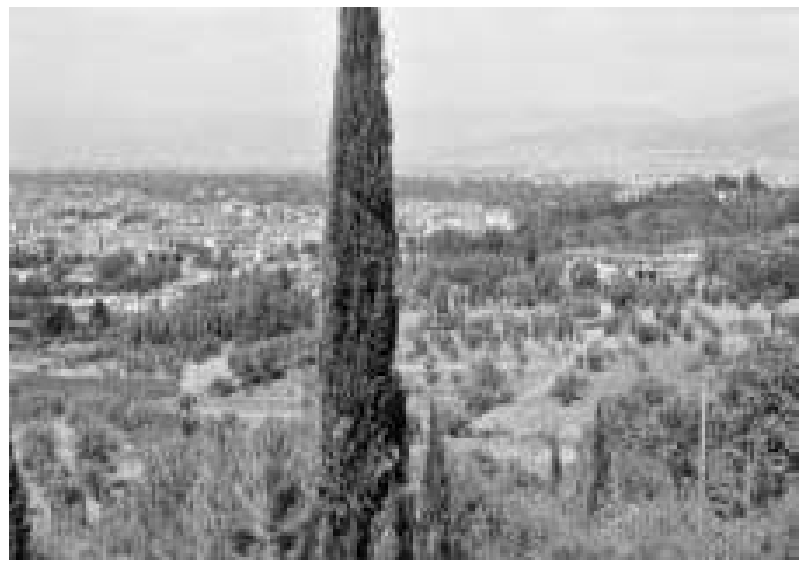

La vista sulla piana occidentale di Firenze spazia fino ai rilievi collinari e submontani a nord

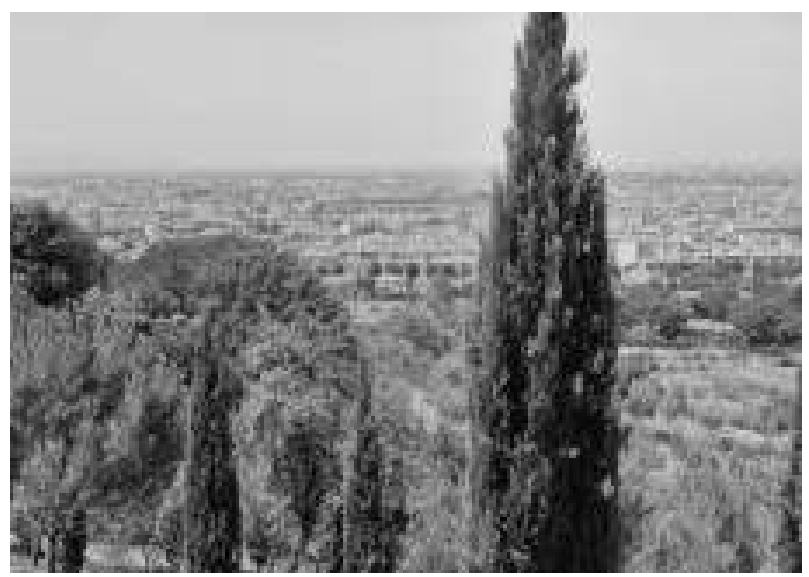

C.s.

\section{DESCRIZIONE TIPOLOGICA DEL FATTORE}

Insediamenti o parti di insediamenti urbani consolidati, con impianto aggregato. Continuità del tessuto degli spazi edificati, costituito da isolati o comunque da complessi di edifici aggregati. Prevalenza di spazi mineralizzati a seguito della urbanizzazione ed edificazione.

Le diversità fisionomico-strutturali del tessuto insediativo derivano dalla stratificazione storica di queste configurazioni ed in particolare dal relativo awicendarsi di tipologie diverse legate alle specificità socioeconomiche e tecnologiche dei diversi periodi.

Sebbene a tali diversità strutturali corrispondano necessariamente diverse situazioni paesistico-ambientali degli insediamenti, è comunque riscontrabile una omogeneità macrostrutturale caratteristica dei tessuti urbani aggregati relativamente ai loro effetti di frammentazione paesistica. Tale omogeneità può essere identificata in due caratteri identificativi comuni:

- la rilevante estensione superficiale degli spazi progressivamente interessati, rispetto all'estensione che caratterizza le matrici paesistiche che con essi si interfacciano e sono correlate;

- l'assenza di sistemi di spazi verdi con dimensioni e requisiti prestazionali idonei a compensare gli effetti negativ che la continuità indifferenziata dei tessuti insediativi genera, sugli stessi insediamenti e sul paesaggio di cui sono parte.

\section{CRITERI INTERPRETATIVI DEGLI EFFETTI}

Occorre fare riferimento a due generi principali di effetti di frammentazione paesistica di cui questa tipologia di fattore risulta responsabile.

II primo effetto di modificazione profonda della struttura del paesaggio è relativo alla sostituzione completa e praticamente irreversibile di parti della matrice paesistica preesistente, che viene ad essere così frammentata. Questo non costituisce necessariamente un fattore di alterazione negativa (si pensi ai caratteri spaziali e dimensionali della città storica), ma si configura progressivamente come tale a seguito della crescita a macchia, connessa normalmente ad una caduta di qualità degli spazi e delle articolazioni strutturali (si pensi ai caratteri spaziali e dimensionali della città contemporanea ed all'incidenza quantitativa e qualitativa degli sviluppi degli ultimi cinque decenni). Alla sostituzione della matrice paesistica preesistente è generalmente connessa la perdita di permanenza storica.

II secondo genere di alterazione riguarda le articolazioni funzionali del paesaggio, i cui apparati si trovano spesso fortemente condizionati dalla progressiva invadenza dell'insediamento urbano, normalmente ad essi indifferente anche quando rivestono ruoli strutturali primari (corsi d'acqua, rete viaria rurale, spazi aperti di regolazione microclimatica e di protezione ambientale). 
ESEMPLIFICAZIONI: FOTOGRAFIA ZENITALE E CARTOGRAFIA

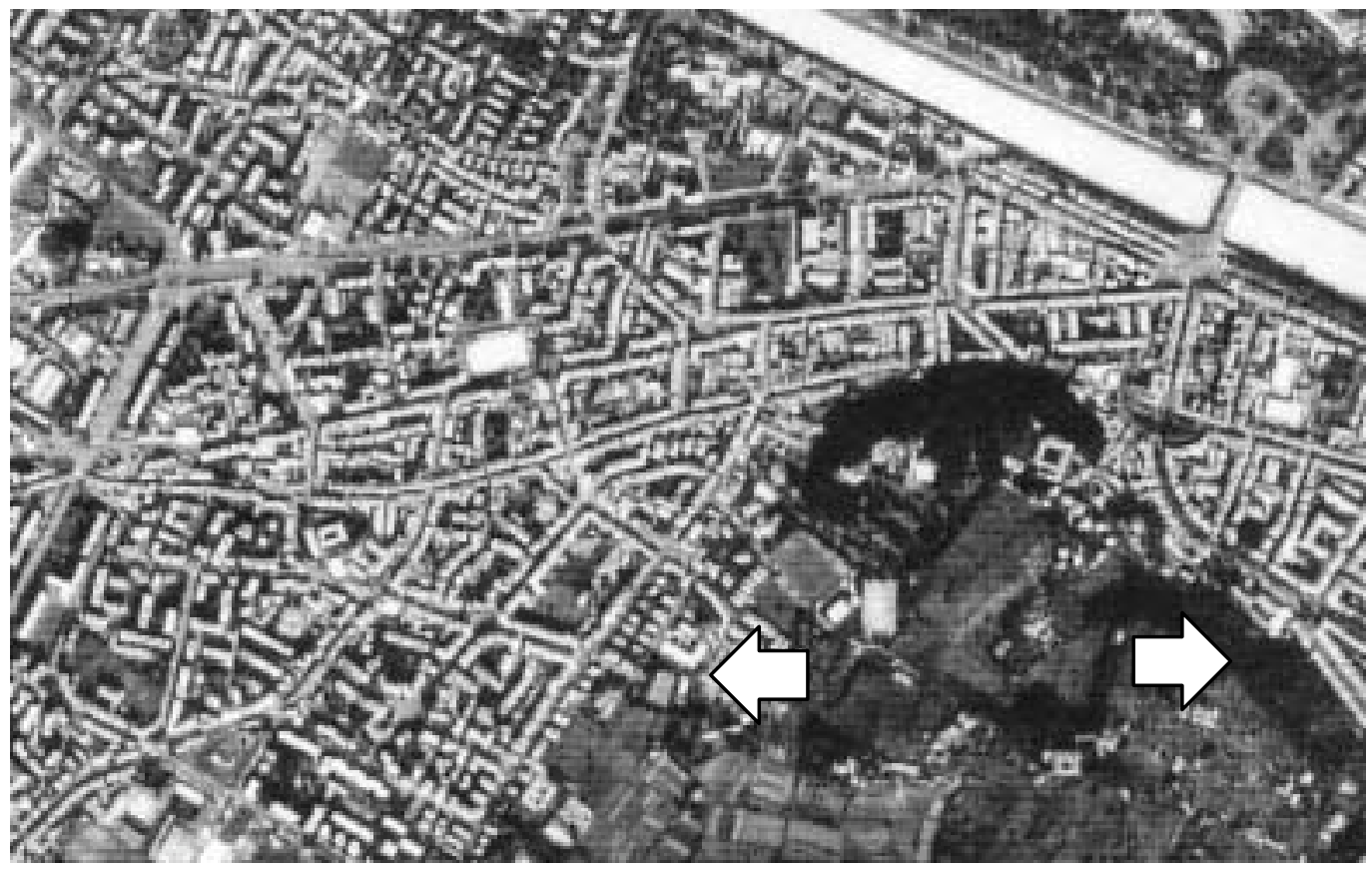

L'area di Monte Uliveto, Legnaia e Isolotto a Firenze.

Alla netta distinzione delle matrici paesistiche urbana e rurale determinata dalla presenza dei rilievi collinari a sud della città, corrisponde anche una decisa continutà del tessuto degli spazi urbanizzati, privo di sistemi paesistici di mediazione e penetrazione.

Fra gli effetti macroscopici si noti come il vicino Parco delle Cascine, nella foto, in alto a destra, risulti completamente privo di connession funzionali e biotiche con il Parco di Monte Uliveto ed il paesaggio collinare di cui questo costituisce la testata più settentrionale a ridosso de

(da formato digitale 1:30000 - Regione Toscana).

Rappresentazione planimetrica 1:5000 (da CTR 1:10000 - Regione Toscana).
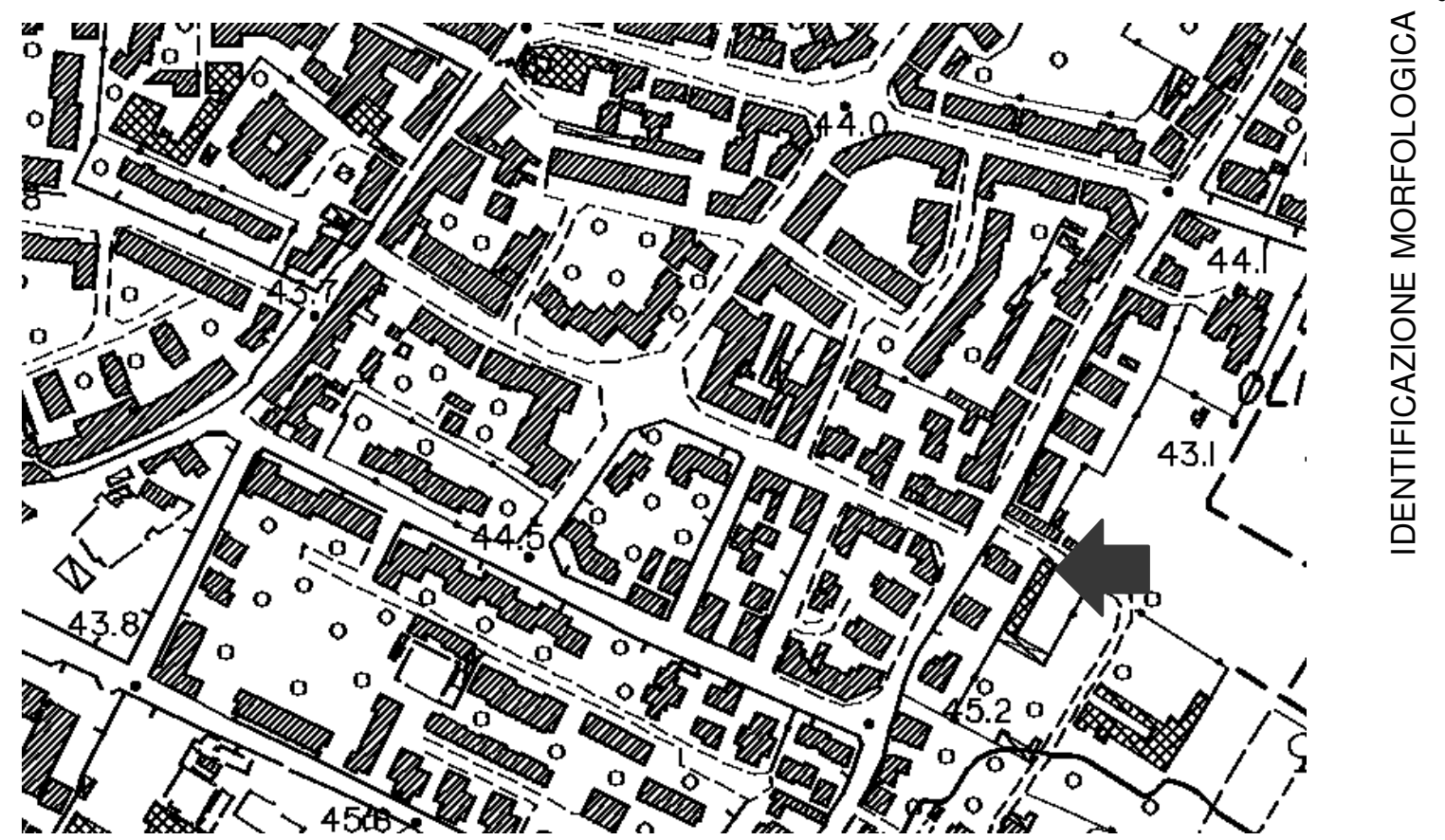


\section{FATTORI AREALI COMPLESSI}

\subsection{Insediamenti urbani con tessuto discontinuo}

\section{ESEMPLIFICAZIONI: VEDUTE}

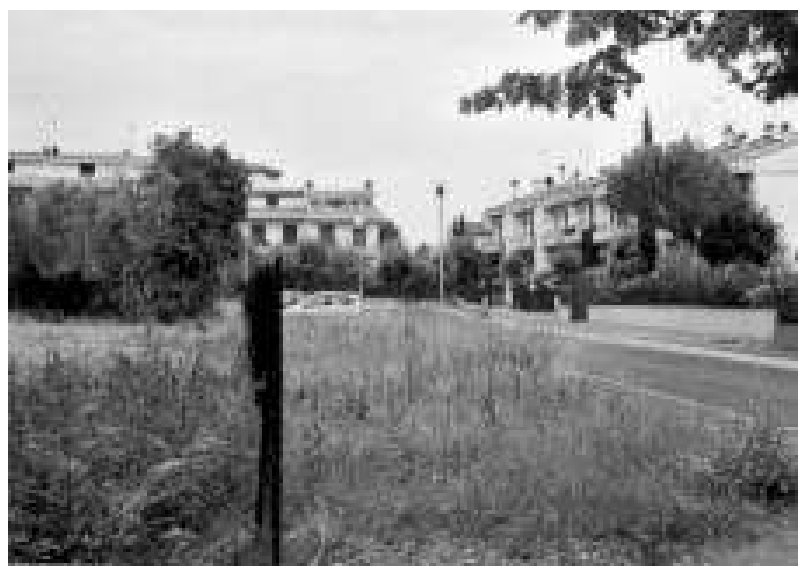

Fra gli elementi di maggior fragilità dei tessuti urbani prodotti dal modello della lottizzazione vi è senz'altro l'articolazione degli spazi aperti ed in particolare la qualità degli spazi verdi, che spesso oltre che frammentati ed omologati, risultano in condizioni di degrado ed inutilizzo, ove non privi di idonee sistemazioni.

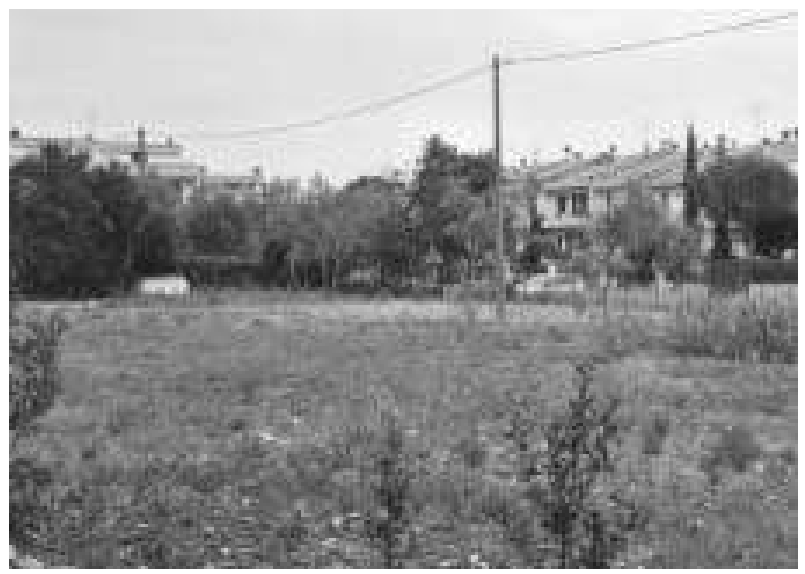

C.s.

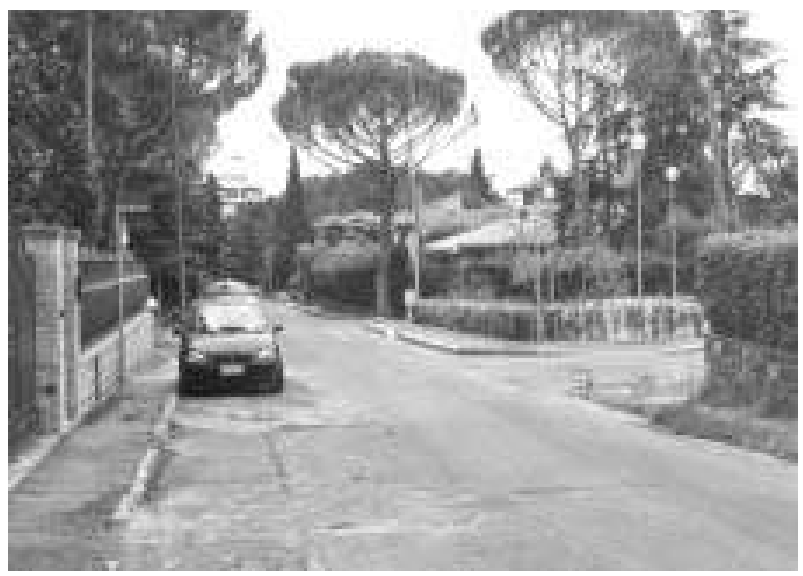

Quantità apprezzabili di spazi verdi sono costituite dalle pertinenze private, al di fuori di idonee regolamentazioni ed ancor meno politiche paesistiche.

\section{DESCRIZIONE TIPOLOGICA DEL FATTORE}

Insediamenti o parti di insediamenti urbani di sviluppo recente con impianto aggregato. Discontinuità del tessuto degli spazi edificati, costituito da edifici isolati in lotti. Presenza rilevante di spazi mineralizzati a seguito della urbanizzazione ed edificazione.

Si tratta per lo più di sviluppi insediativi databili fra gli anni '60 e la fine del secolo scorso, caratterizzati dall'impianto articolato in lotti con edifici isolati.

Queste configurazioni, salvo diversità specifiche non influenti sugli stati di frammentazione paesistica presentano una decisa omogeneità ed i seguenti caratteri identificativi comuni:

- scarsa definizione morfologica e gerarchizzazione funzionale degli spazi aperti, che risultano generalmente costituire un tessuto omologato, le cui componenti sono reiterate, senza essere comprese in un impianto sistemico; ne consegue normalmente una limitata qualità del paesaggio urbano, pur in presenza talvolta di quantità rilevanti di spazi aperti privati e pubblici;

- presenza di equipaggiamento vegetale degli spazi aperti pubblici e privati apprezzabile sul piano quantitativo, normalmente non caratterizzata da livelli qualitativi positivi, sia per aspetti di composizione spaziale, che di composizione veg etazionale;

- scarsa efficacia nella costituzione di configurazioni in scala con il paesaggio sul piano semiologico, con particolare criticità delle aree di margine.

\section{CRITERI INTERPRETATIVI DEGLI EFFETTI}

Come per la precedente tipologia 1.1 (insediamenti urbani con tessuto continuo), il primo effetto di modificazione profonda della struttura del paesaggio è relativo alla sostituzione completa e praticamente irreversibile di parti della matrice paesistica preesistente, che viene ad essere così frammentata.

A differenza della tipologia 1.1 , in questo caso si riscontrano generalmente condizioni critiche dal punto di vista della qualità del paesaggio, identificate in sintesi nella descrizione tipologica riportata sopra.

Alla sostituzione della matrice paesistica preesistente è generalmente connessa la perdita di permanenza storica che nei casi dei tessuti di lottizzazione awiene per lo più al di fuori delle più elementari attenzioni progettuali alla struttura paesistica.

Le alterazioni incidenti sulle articolazioni funzionali del paesaggio fanno sì che anche in questi casi si riscontrino decise manifestazioni di incidenza sui relativi apparati. 


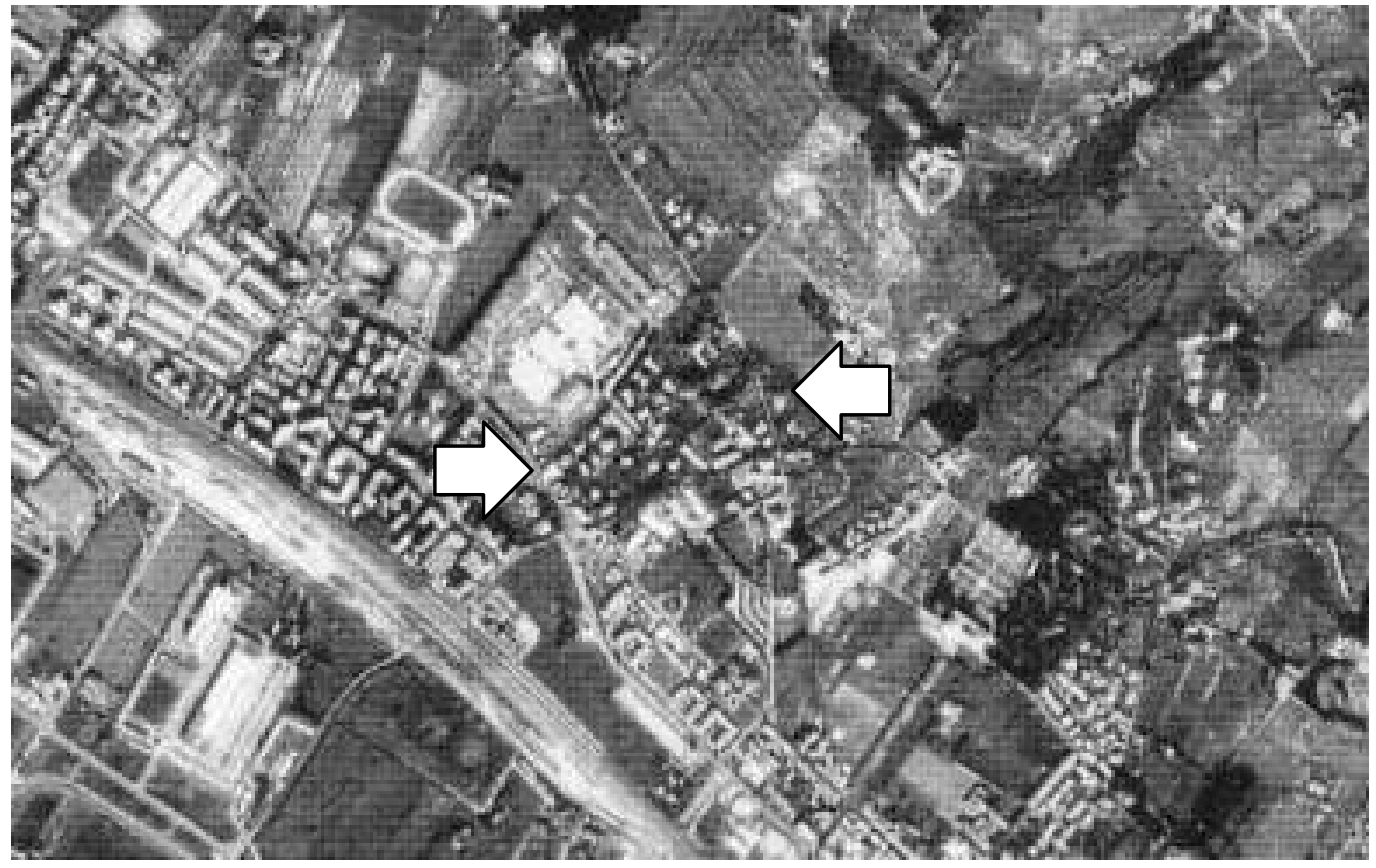

L'area pedecollinare limitrofa alla Villa Reale di Castello, nella foto, a destra.

I caratteri che identificano queste configurazioni producono generalmente situazioni problematiche sul piano della qualità del paesaggio. Se nelle aree di pianura la loro criticità è riconducibile, oltre che agli aspetti funzionali ecosistemici e urbanistici, agli aspetti fisionomici di inconsistenza come espressioni mature di paesaggi urbani contemporanei, nelle aree collinari e pedecollinari esse danno luogo ai fenomeni di "svillettamento" di cui è noto limpatto paesistico, particolarmente severo nei casi in cui si sia in presenza di beni e complessi di architettura del paesaggio di

Rappresentazione fotografica zenitale 1:15000 (da formato digitale 1:30000 - Regione Toscana).

Rappresentazione planimetrica 1:5000 (da CTR 1:10000 - Regione Toscana).

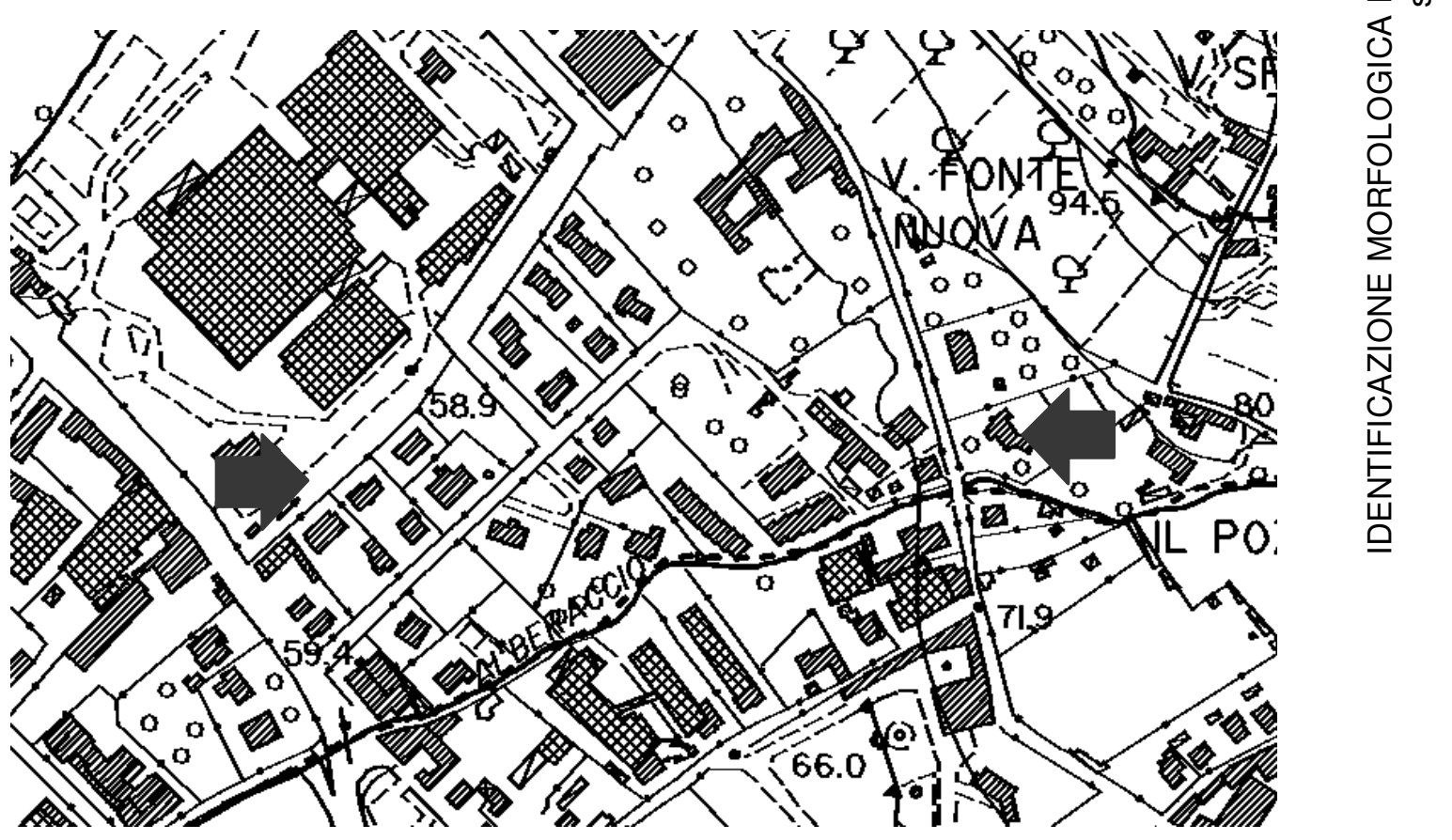




\section{FATTORI AREALI COMPLESSI}

\subsection{Insediamenti urbani diffusi}

NOTA

La tipologia viene inserita, pur non essendo riscontrabile nell'area di studio, per la nota importanza in termini di alterazioni paesistiche dei paesaggi di pianura e per il conseguente interesse ai fini teorici della presente classificazione.

Oltre che i fenomeni macroscopici della pianura Padana, nella Toscana settentrionale si trovano manifestazioni di questa tipologia insediativa in Valdinievole, fra i margini meridionali della conurbazione che corre a ridosso dei riliev collinari da Monsummano Terme, a Montecatini Terme, fino a Pescia e la corona delle fattorie storiche del margine settentrionale del Padule di Fucecchio.

Ancora questa tipologia identifica in modo deciso, sulla costa tirrenica, il paesaggio compreso tra il nucleo storico di Massa ed il litorale (questa realtà viene riportata, come esemplificazione nell'immagine a destra).

\section{DESCRIZIONE TIPOLOGICA DEL FATTORE}

Insediamenti, con caratteri funzionali prettamente urbani, con impianto diffuso. Presenza mediamente rilevante di spazi mineralizzati.

Forma insediativa sviluppatasi in epoca contemporanea, in particolare negli ultimi decenni, tipicamente nei paesaggi di pianura. Dà luogo a fenomeni di diffusione edilizia ed infrastrutturale per i quali al paesaggio della campagna e della città si aggiunge una ulteriore tipologia che, proprio per l'assenza dei caratteri distintivi tipici di questi, non risulta classificabile, se non come una nuova forma di paesaggio culturale contemporaneo. Pur assumendo configurazioni diverse a secondo della aree geografiche, ed in particolare in funzione dei fattori socioeconomici territoriali trainanti, il processo insediativo, risponde ad alcuni caratteri identificativi comuni:

- si sviluppa in stretta relazione ad insediamenti consolidati di centri urbani 0 aree metropolitane, costituendone la principale forma contemporanea;

- costituisce un fenomeno complesso a cui afferiscono più funzioni, complementari o connesse: dalla residenza, alle attività artigianali ed industriali, agli insediamenti specializzati commerciali e/o ricreativi, ai servizi alle imprese ed ai servizi pubblici urbani;

- per definizione, non interessa parti concentrate di territorio, ma occupa vaste aree con assetti insediativi omologati, pur variando, da un'area geografica all'altra, la specializzazione funzionale dal punto di vista socioeconomico.

\section{CRITERI INTERPRETATIVI DEGLI EFFETTI}

Costituiscono un fattore di frammentazione assolutamente distinto dagli altri, in quanto non provocano tanto un tipo di situazione critica circoscrivibile ad una determinata area di influenza 0 ad un determinato tipo di articolazione funzionale del paesaggio, bensì producono effetti di alterazione della struttura e dei processi funzionali del paesaggio nel complesso. Pur non trattandosi di una sostituzione integrale di una matrice paesistica ad un'altra come nel caso delle tipologie 1.1 (insediamenti urbani con tessuto continuo) e 1.2 (insediamenti urbani con tessuto discontinuo), non è possibile non rilevare l'entità del fenomeno, considerandolo erroneamente una evoluzione contemporanea della matrice del paesaggio rurale di pianura. Di fatto si assiste alla nascita di un tipo di matrice a mosaico misto in cui la presenza di spazi urbanizzati ed edificati, pur essendo subordinata dal punto di vista quantitativo a quella delle colture agricole, risulta decisamente articolata ed interconnessa da fitte reti infrastrutturali. La frequente concomitanza di questi fattori con quelli connessi alle pratiche agrarie industriali e con l'incremento degli sviluppi infrastrutturali, è responsabile dei processi di severa alterazione morfologica, storica ed ecosistemica del paesaggio contemporaneo di pianura. $\mathrm{Si}$ tratta di processi in costante progressione, generalmente al di fuori di politiche paesistiche idonee a costituirne una guida consapevole ed efficace. 
ESEMPLIFICAZIONI: FOTOGRAFIA ZENITALE E CARTOGRAFIA

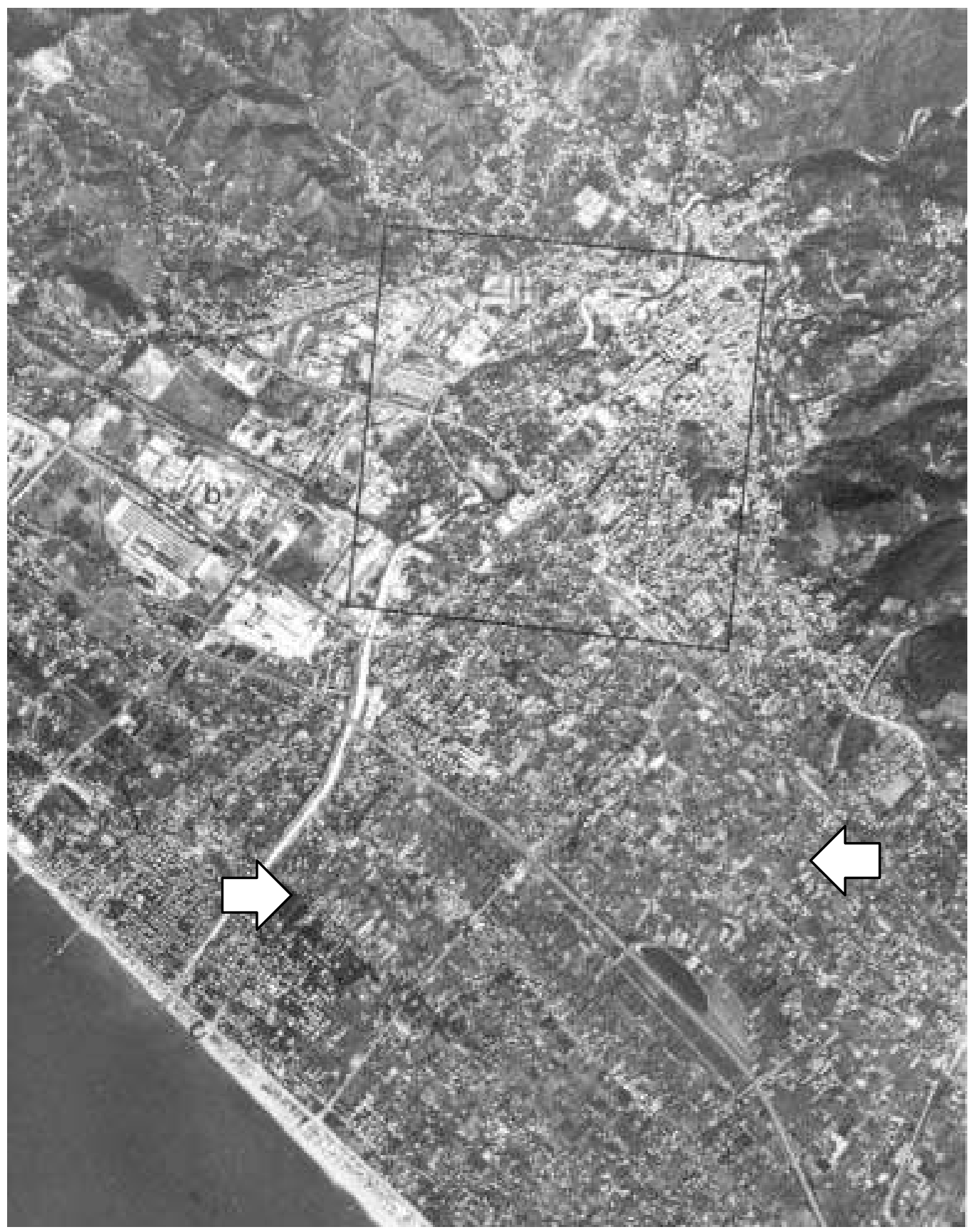

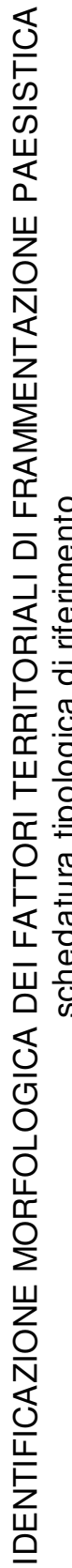

La costa tirrenica a Massa.

Sono ben evidenti $\mathrm{i}$ caratteri identificativi dellinsediamento diffuso (sotto il quadrato che evidenzia un fotogramma relativo al nucleo urbano di Massa, e che non interessa questa esemplificazione), sia sul piano della articolazione spaziale del paesaggio che su quello dellimpegno quantitativo di territorio.

(Foto tratta da: Pizziolo G., La Toscana, volando, Sansoni, Giunta Regionale della Toscana, Firenze, 1986). 


\section{FATTORI AREALI SEMPLICI}

\subsection{Spazi aperti per attività produttive agricole intensive}

\section{ESEMPLIFICAZIONI: VEDUTE}

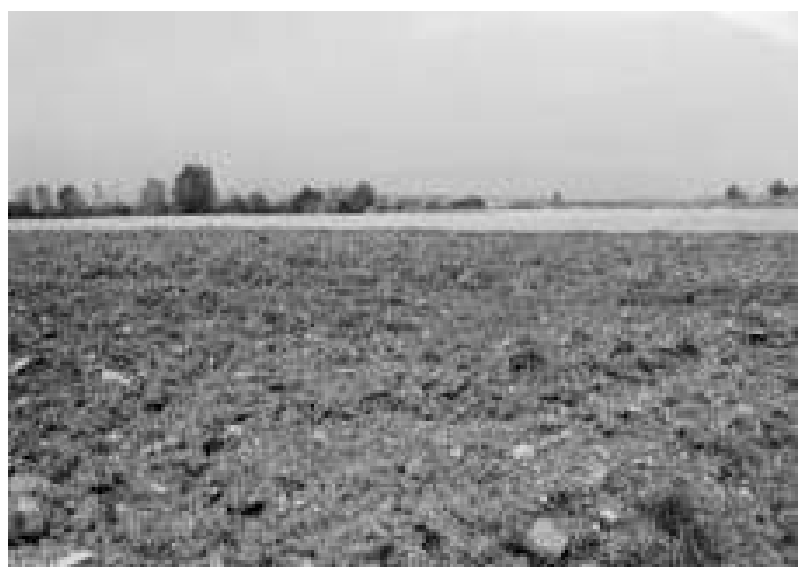

Effetti della meccanizzazione agraria: alle monocolture è stata associata anche la progressiva rimozione delle formazioni agroforestali per cui la vegetazione arborea ed arbustiva oggi presente nel paesaggio di pianura si limita ad episodiche ed arbustiva oggi presente nel paesaggio di pianu
formazioni a sviluppo spontaneo lungo i corsi d'acqua.

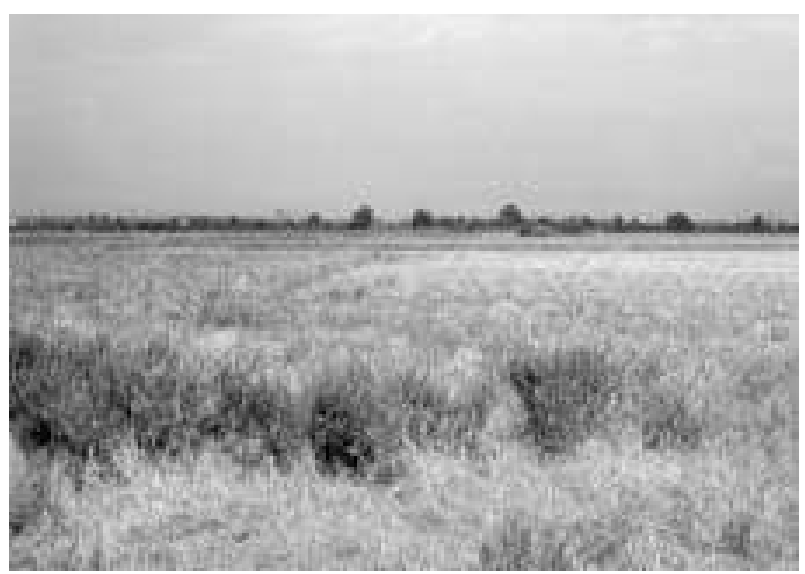

Nelle aree ad elevata pressione insediativa è esteso il fenomeno dell'abbandono dei campi per le pressioni economiche e la connessa marginalità dell'agricoltura.

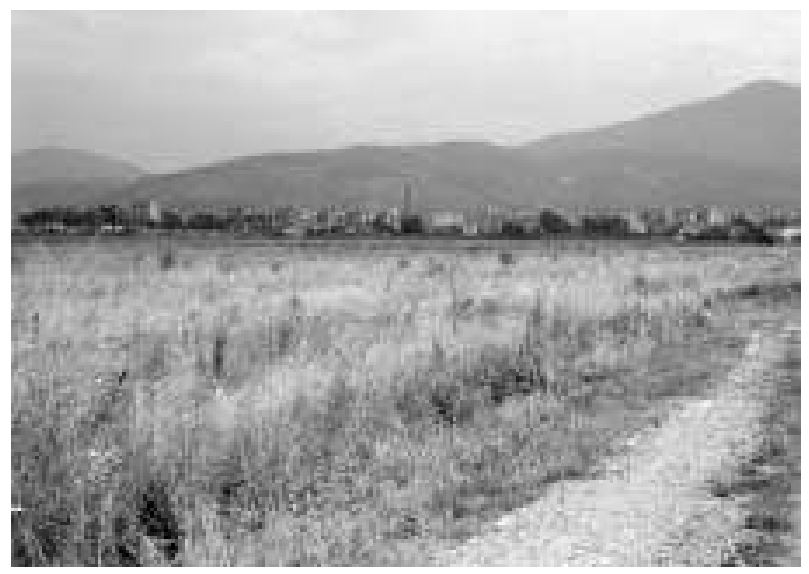

C.s.

\section{DESCRIZIONE TIPOLOGICA DEL FATTORE}

Colture agricole di tipo intensivo. Presenza irrilevante di spazi mineralizzati.

$\mathrm{Si}$ tratta di monocolture agricole di tipo industrializzato. Sebbene, a secondo delle aree geografiche, questi fattori assumano livelli di strutturazione e di estensione diversi, presentano alcuni caratteri identificativi comuni:

- progressiva eliminazione dell'equipaggiamento vegetale del paesaggio;

- abbandono di parte della rete viaria rurale ed adeguamento dei tratti utilizzati alle esigenze della meccanizzazione agraria;

abbandono dell'insediamento rurale sparso e costruzione di edifici per il rimessaggio dei mezzi ed il deposito dei prodotti;

- intenso utilizzo di risorse idriche per l'irrigazione delle colture;

- intenso utilizzo di sostanze chimiche per il trattamento fitosanitario delle colture.

\section{CRITERI INTERPRETATIVI DEGLI EFFETTI}

Dalla progressiva eliminazione dell'equipaggiamento vegetale del paesaggio, awenuta a seguito di politiche agrarie e conoscenze tecniche ormai datate e diffusamente contestate, dipendono sostanzialmente molti degli effetti di alterazione paesistica di cui sono responsabili questi fattori:

- perdita di identità spaziale del paesaggio e dei conseguenti caratteri complessivi di qualità paesistica e di idoneità funzionale come habitat umano, sia per la residenza stabile, che per le attività turistiche e ricreative in genere;

- perdita di efficienza ecosistemica del paesaggio, dal punto di vista delle capacità di protezione ambientale delle risorse primarie (suoli fertili e falde acquifere), come anche della protezione delle stesse colture agrarie dagli agenti di aggressione esogena ed endogena ed, infine, dal punto di vista della biodiversità, per le funzioni di habitat animale e veg etale.

La severa perdita di identità storica del paesaggio e dei conseguenti valori culturali, non è invece riconducibile alla sola eliminazione delle strutture vegetali. Awiene a seguito della complessa e profonda erosione del contesto e degli stessi elementi strutturali di tipo antropico dell'insediamento rurale, dalla viabilità, ai sistemi di regolazione e protezione idraulica, agli edifici privati minori ed agli edifici e manufatti di interesse storicoarchitettonico. 


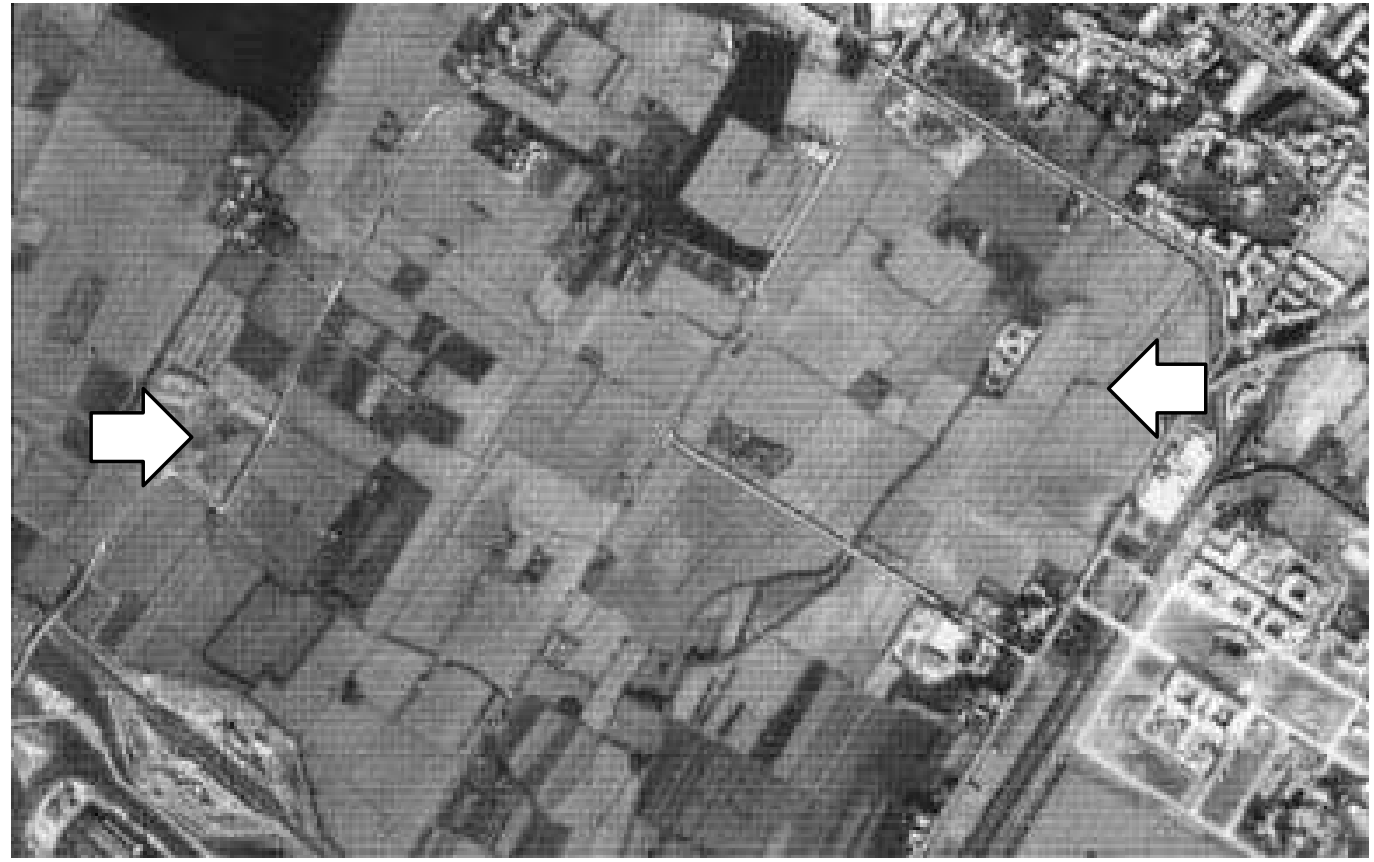

L'area ad ovest del nuovo polo scientifico universitario di Firenze a Sesto Fiorentino.

È evidente la pressoché totale disarticolazione spaziale dovuta all'assenza di sistemi con effetto del limite, ormai prodotto quasi esclusivamente dai rilevati stradali e dagli argini dei canali di bonifica e dei corsi d'acqua naturali regimati. come habitat animale. Le spiccate idoneità di questi spazi aperti e dei suoli fertili di pianura come habitat vegetale risultano infine direttamente condizionate dallevoluzione delle forme di uso del suolo.

Rappresentazione fotografica zenitale 1:15000 (da formato digitale 1:30000 - Regione Toscana).

Rappresentazione planimetrica 1:5000 (da CTR 1:10000 - Regione Toscana).

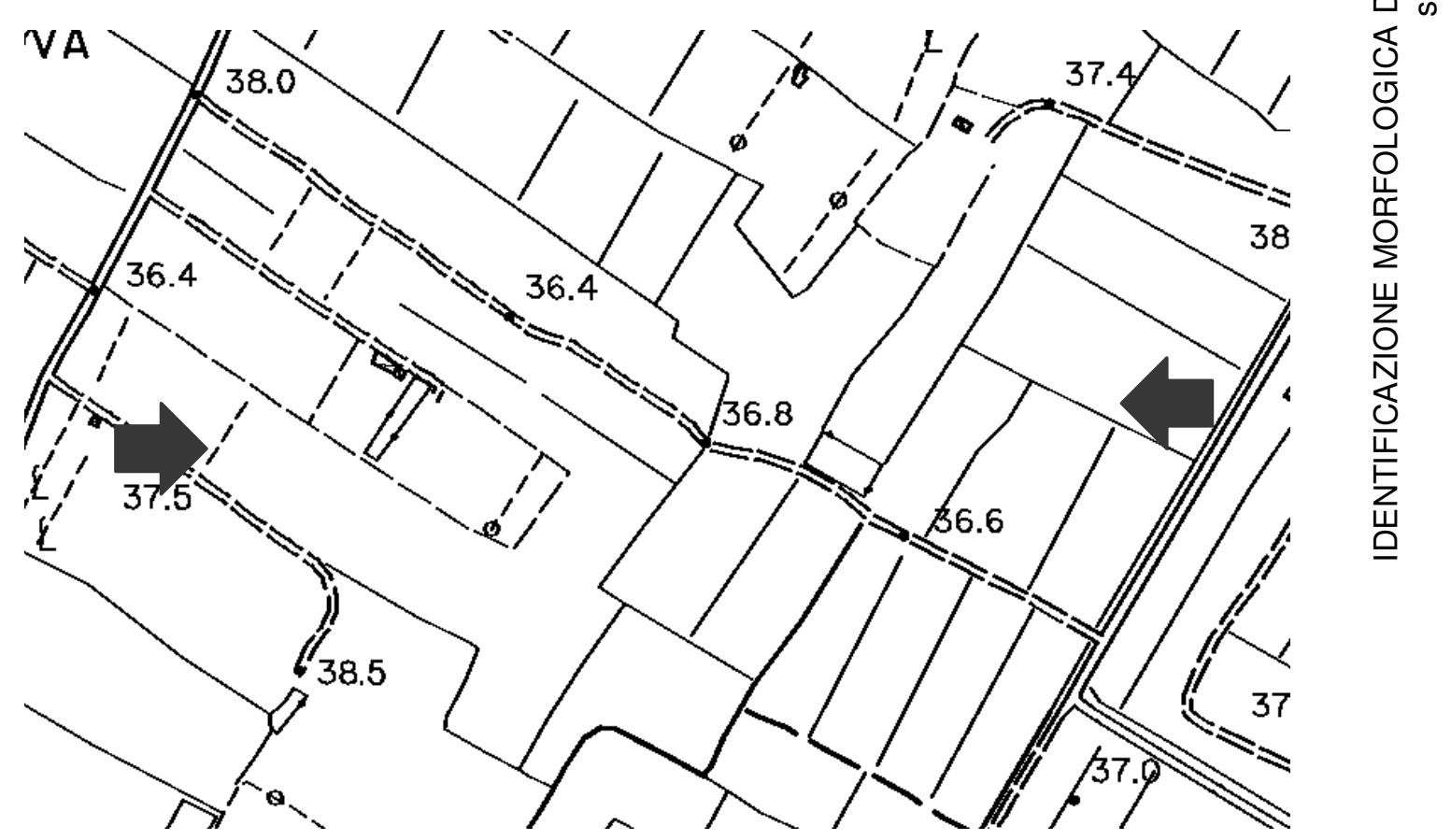




\section{FATTORI AREALI SEMPLICI}

\subsection{Spazi aperti soggetti a vincoli di inutilizzazione}

\section{ESEMPLIFICAZIONI: VEDUTE}

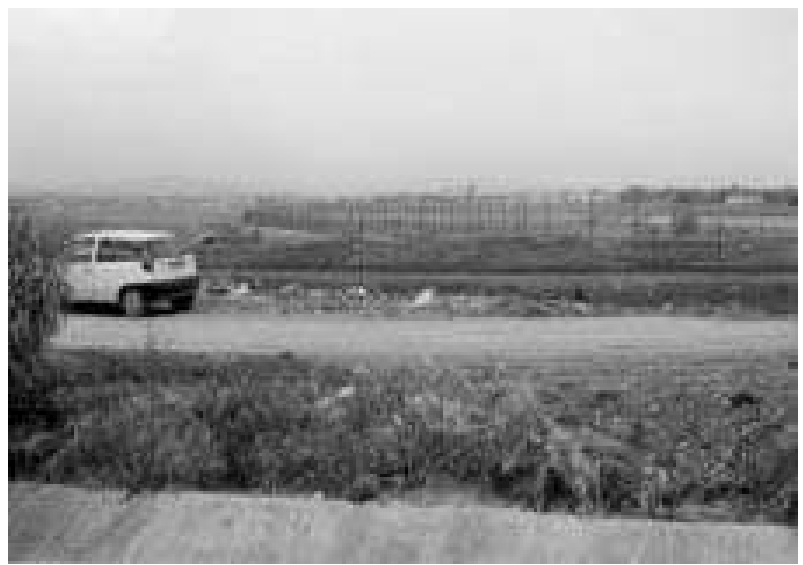

In presenza di vincoli di inutilizzazione degli spazi per motivi di sicurezza infrastrutturale, il paesaggio di pianura risulta protetto dalla dispersione insediativa, quanto privo di sistemazioni atte a conferire un congruo grado di qualità.

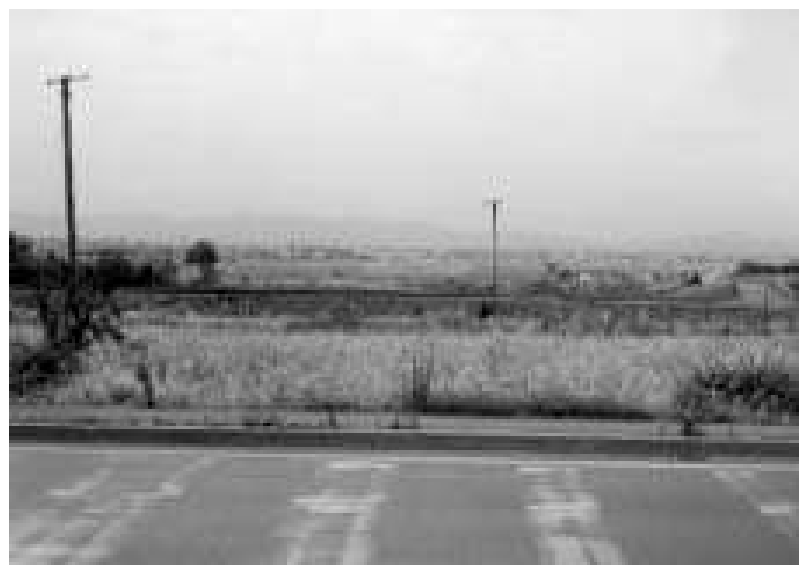

Gli unici elementi presenti sono costituiti dagli apparati funzionali necessari agli utilizzi, fra cui le recinzioni per la protezione delle aree: in assenza di misure di sistemazione, questi elementi contribuiscono ulterioremente al degrado del paesaggio.

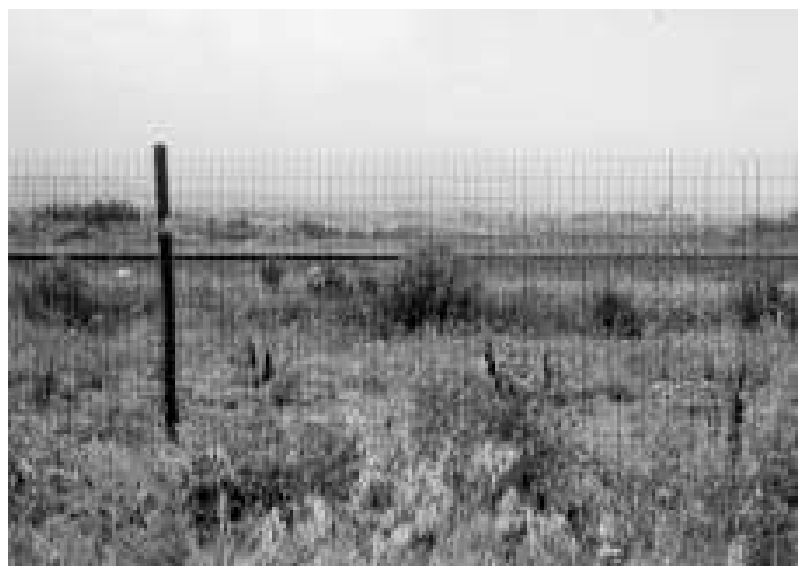

C.s.

\section{DESCRIZIONE TIPOLOGICA DEL FATTORE}

Spazi aperti limitrofi a grandi infrastrutture o strutture, sottoposti a limitazioni totali o parziali di utilizzo per motivazioni di sicurezza e/o igiene ambientale. Presenza irrilevante di spazi aperti mineralizzati.

$\mathrm{Si}$ tratta generalmente di spazi non mineralizzati da urbanizzazioni o da utilizzi pregressi.

Possono essere pertinenze specifiche delle infrastrutture, come le fasce di margine alle autostrade o le aree intorno alle piste aereoportuali, ed in tal caso risultano inaccessibili e recintati. Oppure possono essere spazi che, per le ragioni istitutive dei vincoli su essi gravanti, risultano soggetti a limitazioni d'uso, oltre che per quanto riguarda le possibilità di edificazione, anche, ad esempio, per la piantagione di alberi di alto fusto: si considerino i margini stradali dei tratti viari extraurbani sottoposti alle norme del Codice della strada.

Nonostante i diversi tipi e livelli di vincolo, si riscontra una caratterizzazione di base di questi spazi nella realtà paesistica contemporanea: sono generalmente in condizioni di marginalità, con soprassuoli prevalentemente erbacei, privi di connotazione spaziale e funzioni paesistico-ambientali attribuite e sviluppate sulla base di politiche sistemiche che, nel rispetto delle esigenze di prevenzione, garantiscano un idoneo inserimento nel contesto paesistico.

\section{CRITERI INTERPRETATIVI DEGLI EFFETTI}

Gli effetti di alterazione paesistica, sostanzialmente coincidenti con quelli della precedente tipologia 2.1 (spazi aperti per attività produttive agricole intensive), possono avere in questo caso risposte di segno decisamente diverso in funzione delle limitazioni di utilizzo del suolo. Da una parte la loro marginalità diviene un elemento di interesse nell'eventualità della progettazione ed attuazione di politiche organiche di miglioramento paesisticoambientale. Dall'altra però, in assenza di strategie di questo genere, la stessa marginalità, precludendo 0 limitando sostanzialmente l'intervento umano di tipo produttivo, destina questi spazi al degrado, a cui va soggetta ogni forma di paesaggio culturale in assenza delle attività di manutenzione e modificazione, ove queste non siano connesse alle gestione delle strutture ed infrastrutture di cui costituiscono spazi di rispetto. Si consideri ad esempio la differenza che può esservi tra gli ambiti di rispetto cimiteriale, ove il vincolo grava solo come limitazione dell'uso degli spazi, che restano completamente sotto la gestione dei proprietari, ed il caso invece delle aree di prima pertinenza di piste aereoportuali, dove gli spazi vengono acquisiti e mantenuti a carico delle società di gestione delle strutture. 
ESEMPLIFICAZIONI: FOTOGRAFIA ZENITALE E CARTOGRAFIA

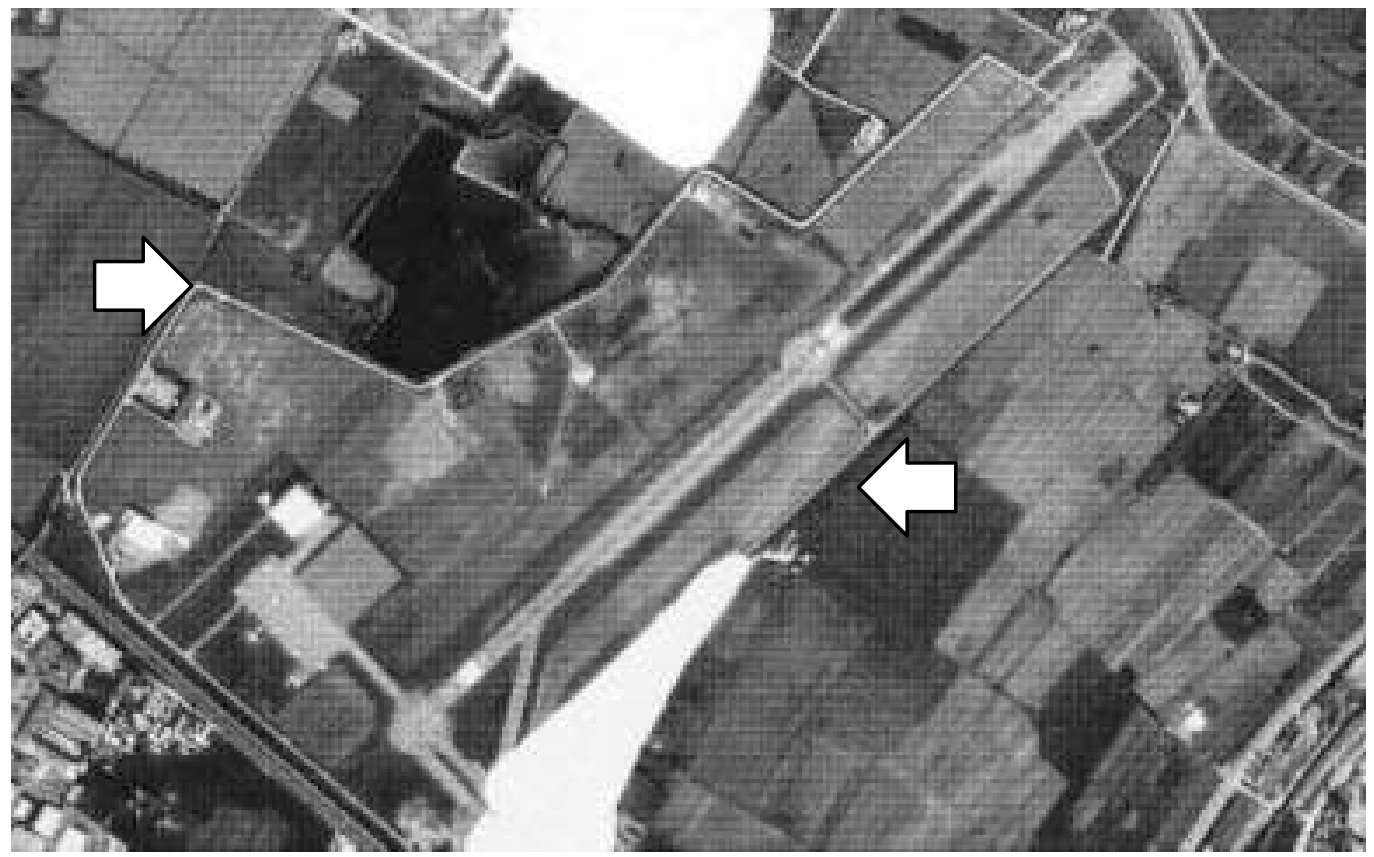

L'area dell'aereoporto Amerigo Vespucci a Firenze.

L'area di pertinenza dell'aereoporto è chiaramente individuabile per la presenza di una strada lungo il perimetro. In questo caso, appare evidente anche la coincidenza delle tessiture di questi spazi con quelle delle colture intensive della precedente tipologia 2.1 limitrofe all'area aereoportuale. Le differenze sostanziali sono date essenzialmente dalla presenza di recinzioni che impediscono l'accesso e dalla preclusione ci qualunque forma di utilizzo del suolo.

Rappresentazione fotografica zenitale 1:15000 (da formato digitale 1:30000 - Regione Toscana).

Rappresentazione planimetrica 1:5000 (da CTR 1:10000 - Regione Toscana).

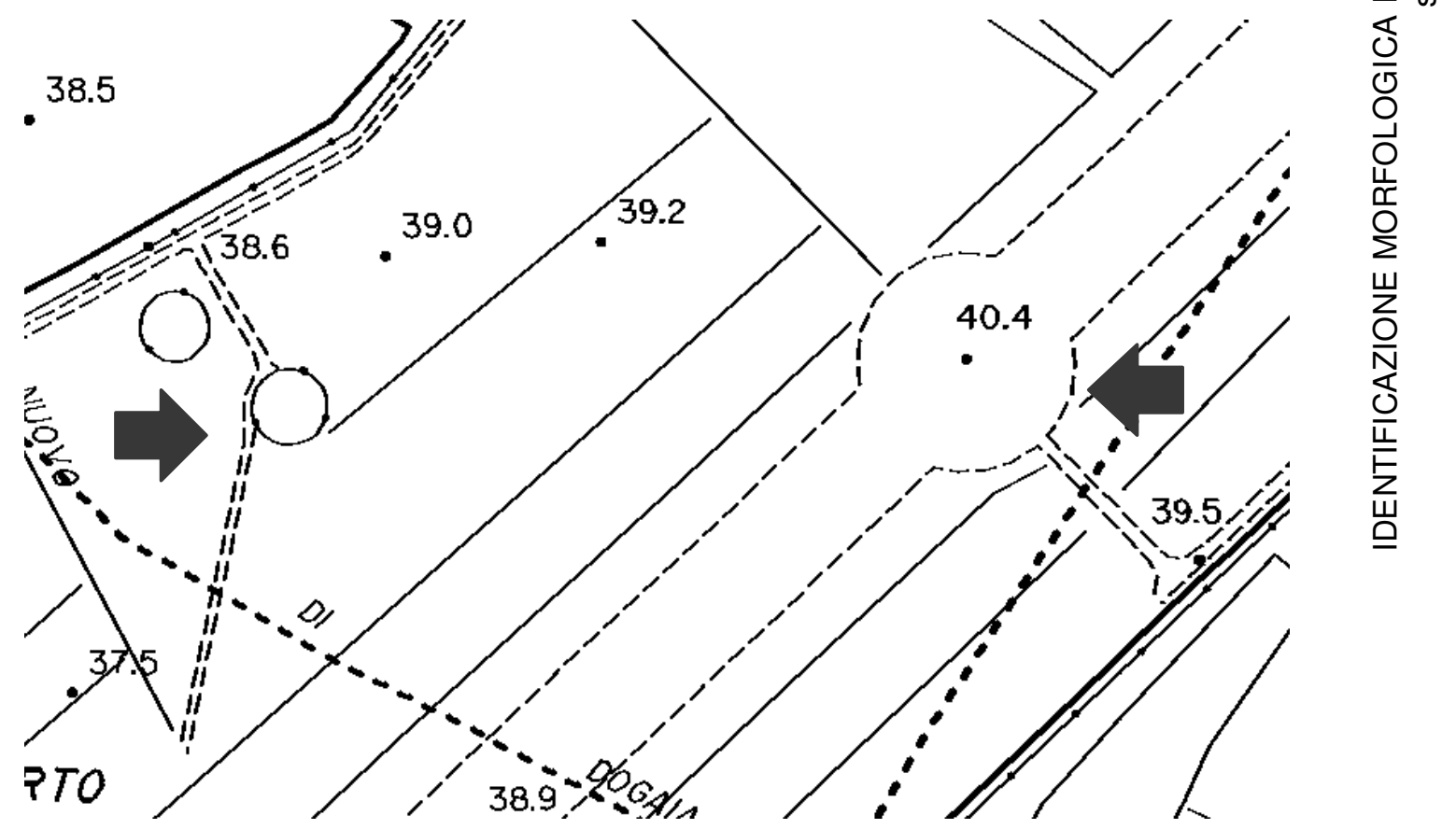




\section{FATTORI LINEARI COMPLESSI}

\subsection{Insediamenti urbani con tessuto continuo}

\section{ESEMPLIFICAZIONI: VEDUTE}

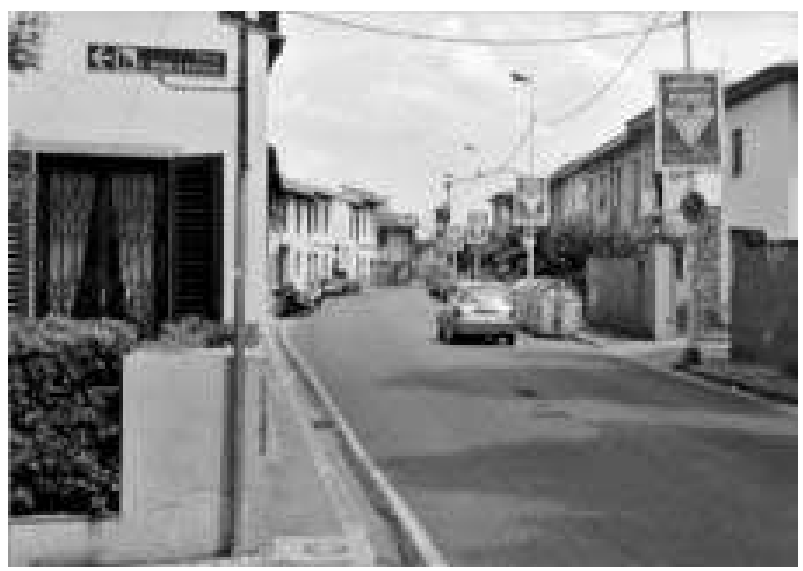

L'insediamento lineare di origine storica è caratterizzato da una specifica identità spaziale che oggi si scontra con un carico infrastrutturale sovralocale incompatibile con la sua configurazione.

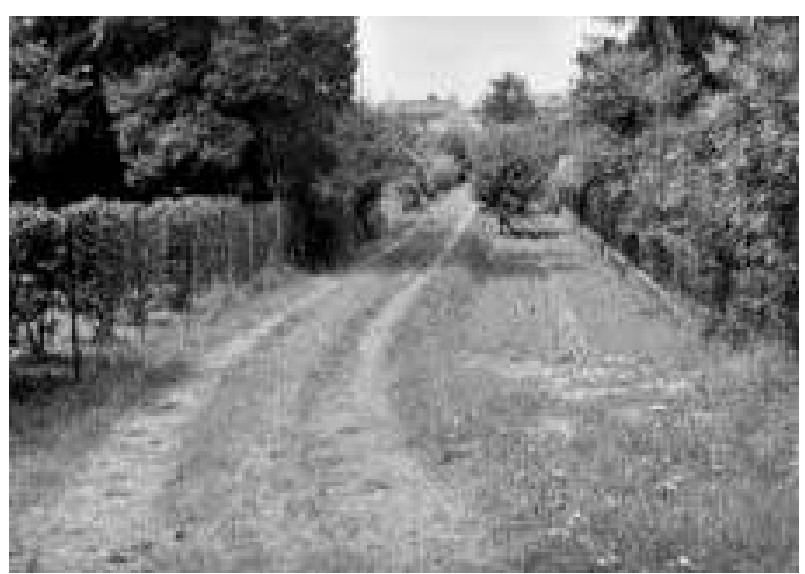

La continuità delle pertinenze, che costituiscono fasce di rilevanti dimensioni, limita identità paesistica.

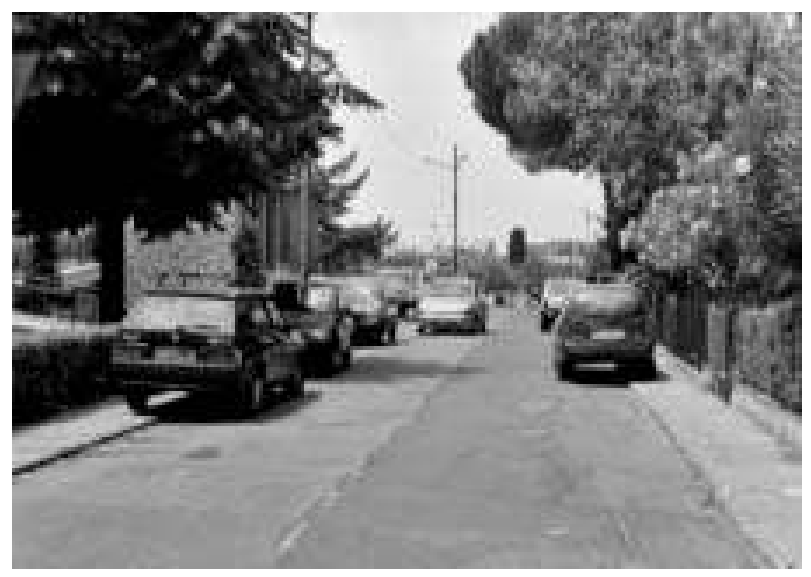

Modificazioni recenti hanno prodotto aberrazioni dei rapporti trasversali con il paesaggio rurale circostante: monconi stradali terminano in campi aperti con ambigui risultati di "città interrotta".

\section{DESCRIZIONE TIPOLOGICA DEL FATTORE}

Insediamenti o parti di insediamenti urbani consolidati, con impianto aggregato. Continuità del tessuto degli spazi edificati, costituito da isolati o comunque da complessi di edifici aggregati. Prevalenza di spazi mineralizzati a seguito della urbanizzazione ed edificazione.

Come per la precedente tipologia 1.1 di fattori areali (insediamenti urbani con tessuto continuo), le diversità fisionomico-strutturali del tessuto insediativo derivano dalla stratificazione storica di queste configurazioni ed, in particolare, dal relativo awicendarsi di tipologie insediative ed edilizie diverse, legate alle specificità socioeconomiche e tecnologiche dei diversi periodi. Nel caso dell'impianto lineare è però presumibile che tali differenze risultino di fatto sensibilmente ridotte per la minor variabilità di soluzioni insediative che su questo modello si sono sviluppate.

È comunque riscontrabile una omogeneità strutturale che fa capo a due caratteri identificativi comuni:

- la rilevante estensione longitudinale degli spazi interessati dagli sviluppi insediativi, relativamente all'estensione trasversale degli stessi;

- la dipendenza di queste configurazioni dalla dotazione funzionale di altre parti del territorio, per le carenze strutturali che le caratterizzano.

\section{CRITERI INTERPRETATIVI DEGLI EFFETTI}

Occorre fare riferimento a due generi principali di effetti di alterazione paesistica di cui questa tipologia di fattore risulta responsabile.

II primo, di modificazione profonda della struttura del paesaggio, è relativo alla sottrazione di spazi aperti e delle relative funzioni paesistico-ambientali, impegnati per l'edificazione e l'urbanizzazione. Ad essa è generalmente connessa la perdita di permanenza storica.

II secondo genere di alterazione riguarda le articolazioni funzionali del paesaggio, i cui apparati ed habitat risultano condizionati dall'incremento dell'effetto di barriera di discontinuità provocato dall'urbanizzazione e dalla edificazione rispetto ai livelli caratteristici della sola presenza dell'infrastruttura lungo la quale gli insediamenti si sviluppano. 
ESEMPLIFICAZIONI: FOTOGRAFIA ZENITALE E CARTOGRAFIA

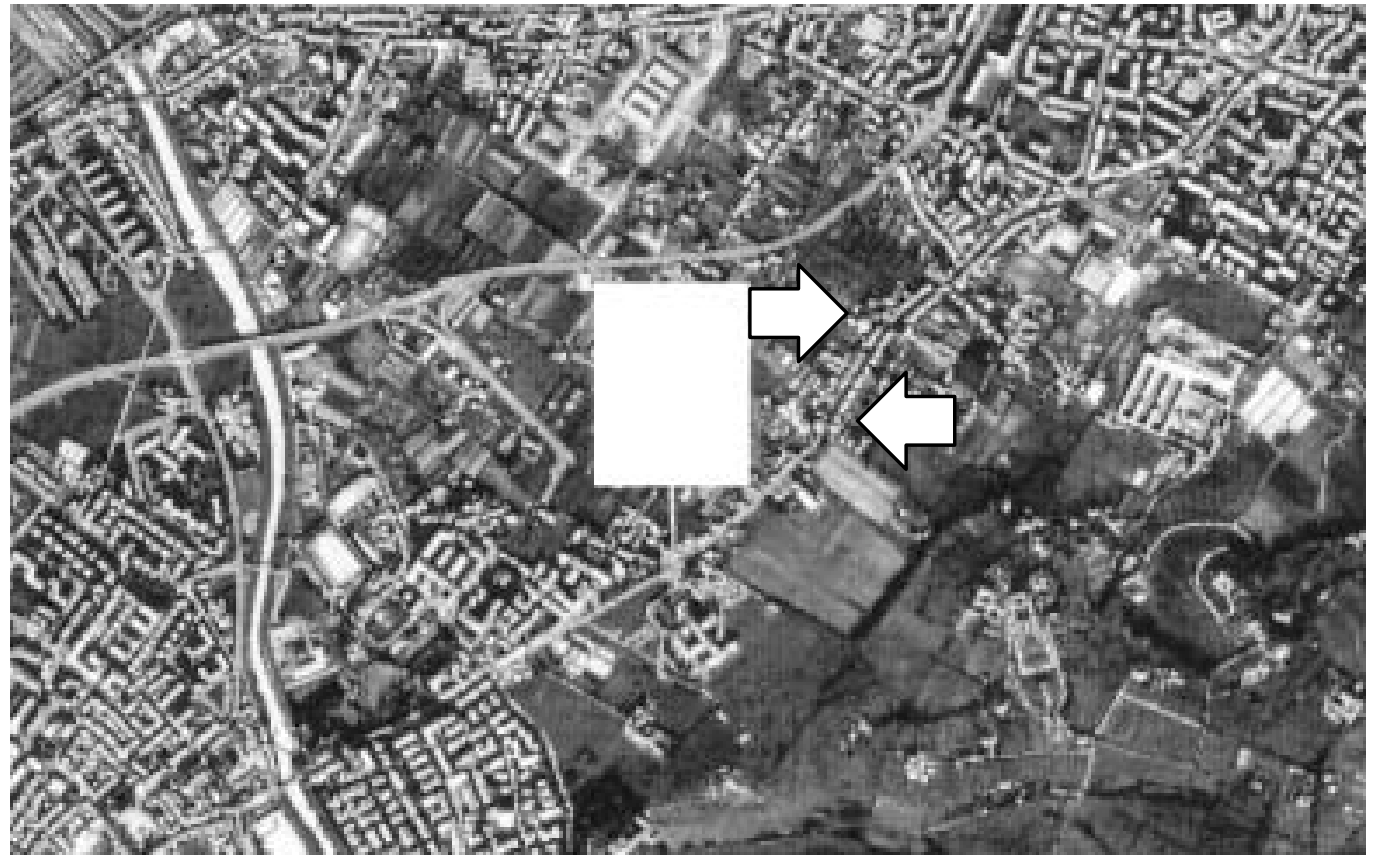

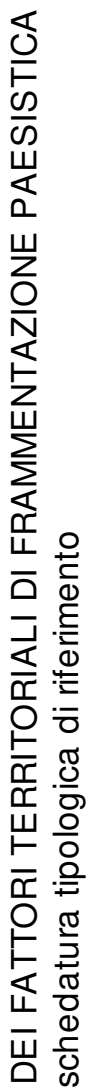

L'area di Torre Galli, tra Firenze e Scandicci.

La stretta dipendenza dellimpianto insediativo dallinfrastruttura ne determina la geometria e le stesse dimensioni trasversali al suo sviluppo .

(da formato digitale 1:30000 - Regione Toscana).

Rappresentazione planimetrica 1:5000 (da CTR 1:10000 - Regione Toscana).

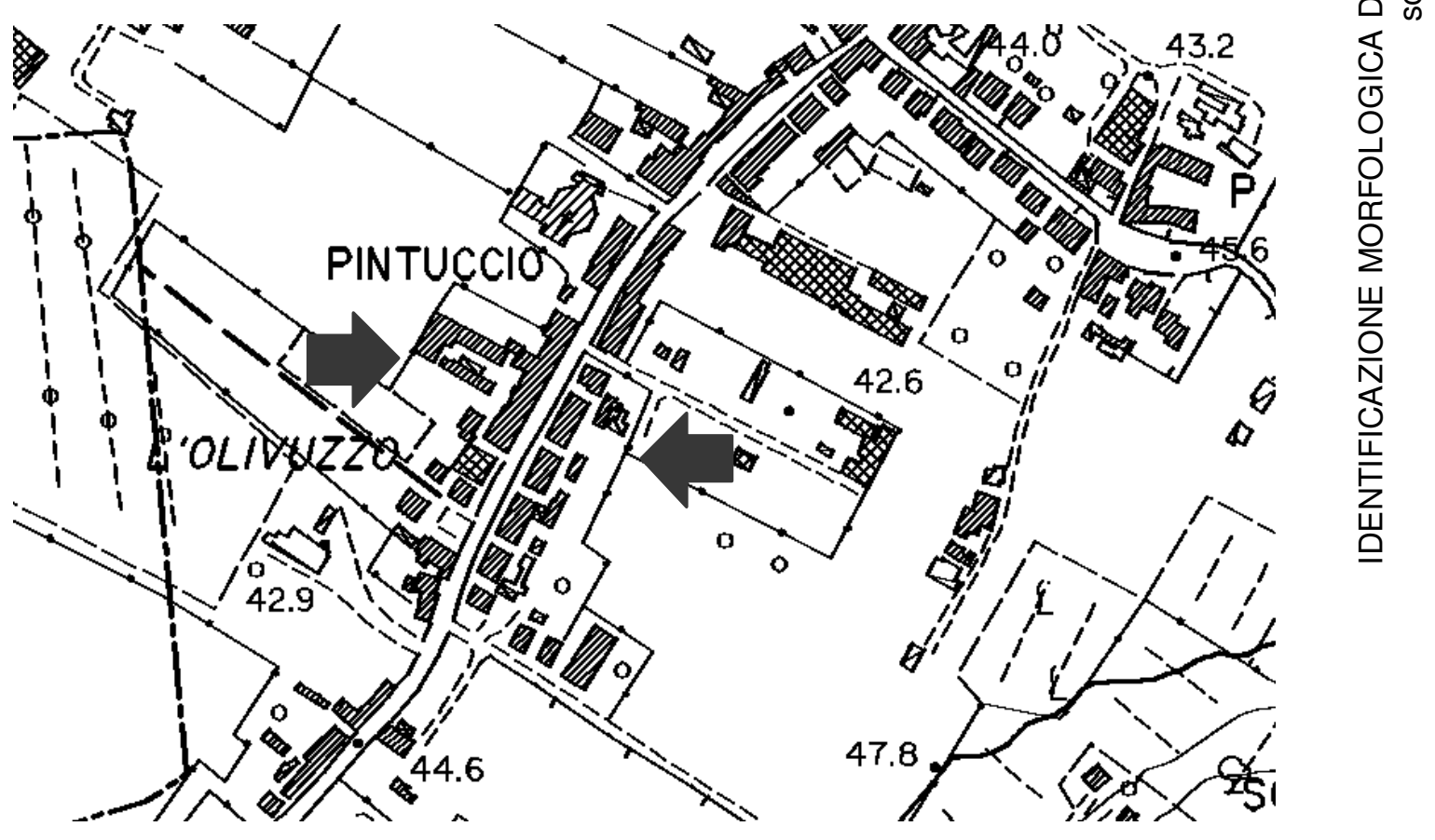




\section{FATTORI LINEARI COMPLESSI}

\subsection{Insediamenti urbani con tessuto discontinuo}

ESEMPLIFICAZIONI: VEDUTE

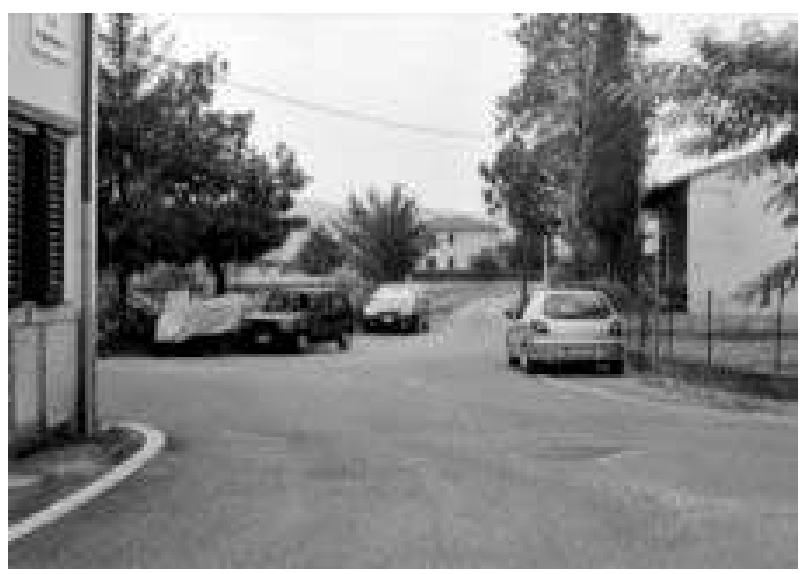

L'evoluzione degli insediamenti lungo le strade ha prodotto spesso la saldatura di nuclei preesistenti, determinando configurazioni lineari che, per l'origine recente di nuclei presistenti, determinando configurazioni lineari che, per lorigine recente di
molti edifici e la natura rurale di quelli storici, sono caratterizzate da un tessuto spaziale discontinuo.

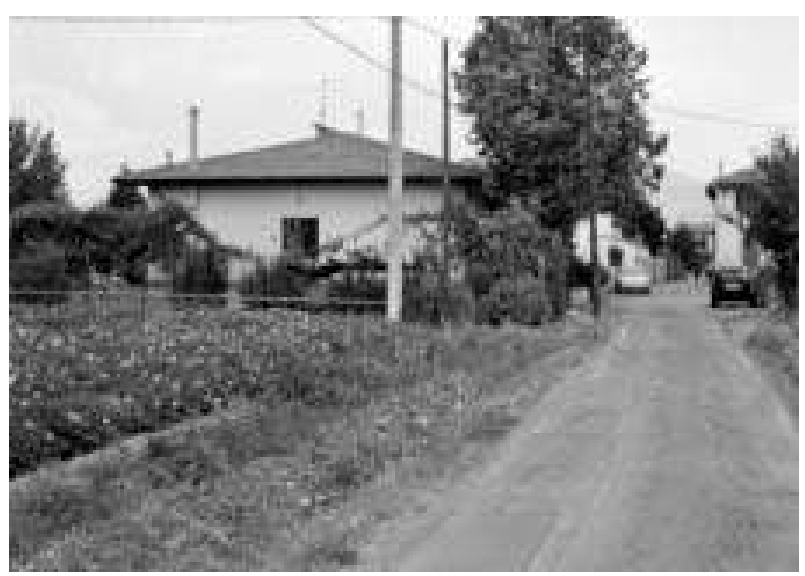

Insediamento e infrastrutture viarie e tecnologiche hanno prodotto lurbanizzazione della campagna che presenta oggi frequenti elementi di ambiguità della propria identità paesistica.

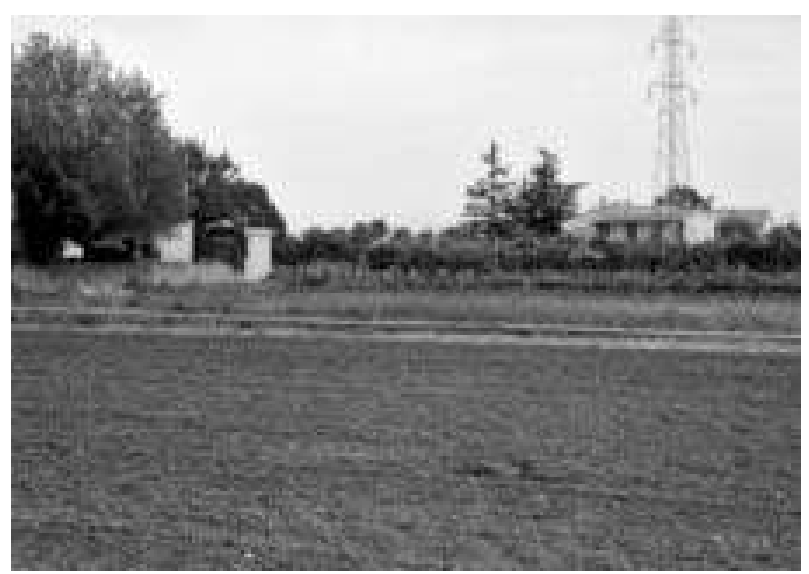

C.s.

\section{DESCRIZIONE TIPOLOGICA DEL FATTORE}

Insediamenti o parti di insediamenti urbani di sviluppo recente con impianto aggregato. Discontinuità del tessuto degli spazi edificati, costituito da edifici isolati in lotti. Presenza mediamente rilevante di spazi mineralizzati a seguito della urbanizzazione ed edificazione.

Queste configurazioni possono avere origine storica di tipo rurale, oppure possono essersi sviluppate lungo la viabilità in epoca contemporanea per ragioni legate all'insediamento di attività commerciali, artigianali 0 industriali, oppure possono derivare dalla edificazione episodica dei fronti stradali compresi tra piccoli borghi preesistenti.

Ė comunque riscontrabile una omogeneità strutturale che fa capo a tre caratteri identificativi comuni:

- la rilevante estensione longitudinale degli spazi interessati dagli sviluppi insediativi, relativamente all'estensione trasversale degli stessi;

- la dipendenza di queste configurazioni dalla dotazione funzionale di altre parti del territorio, per le carenze strutturali che le caratterizzano;

- le carenze di articolazione morfologica degli spazi secondo configurazioni paesistiche con valenze semiologiche positive.

\section{CRITERI INTERPRETATIVI DEGLI EFFETTI}

$\mathrm{Si}$ veda anche la tipologia 3.1 (insediamenti urbani con tessuto continuo).

L'intensità degli effetti di frammentazione paesistica, che tipologicamente risultano in sostanza coincidenti con quelli della precedente tipologia 3.1 , è in questo caso minore per la discontinuità del tessuto insediativo e la connessa presenza di spazi mineralizzati in quantità minori. 
ESEMPLIFICAZIONI: FOTOGRAFIA ZENITALE E CARTOGRAFIA

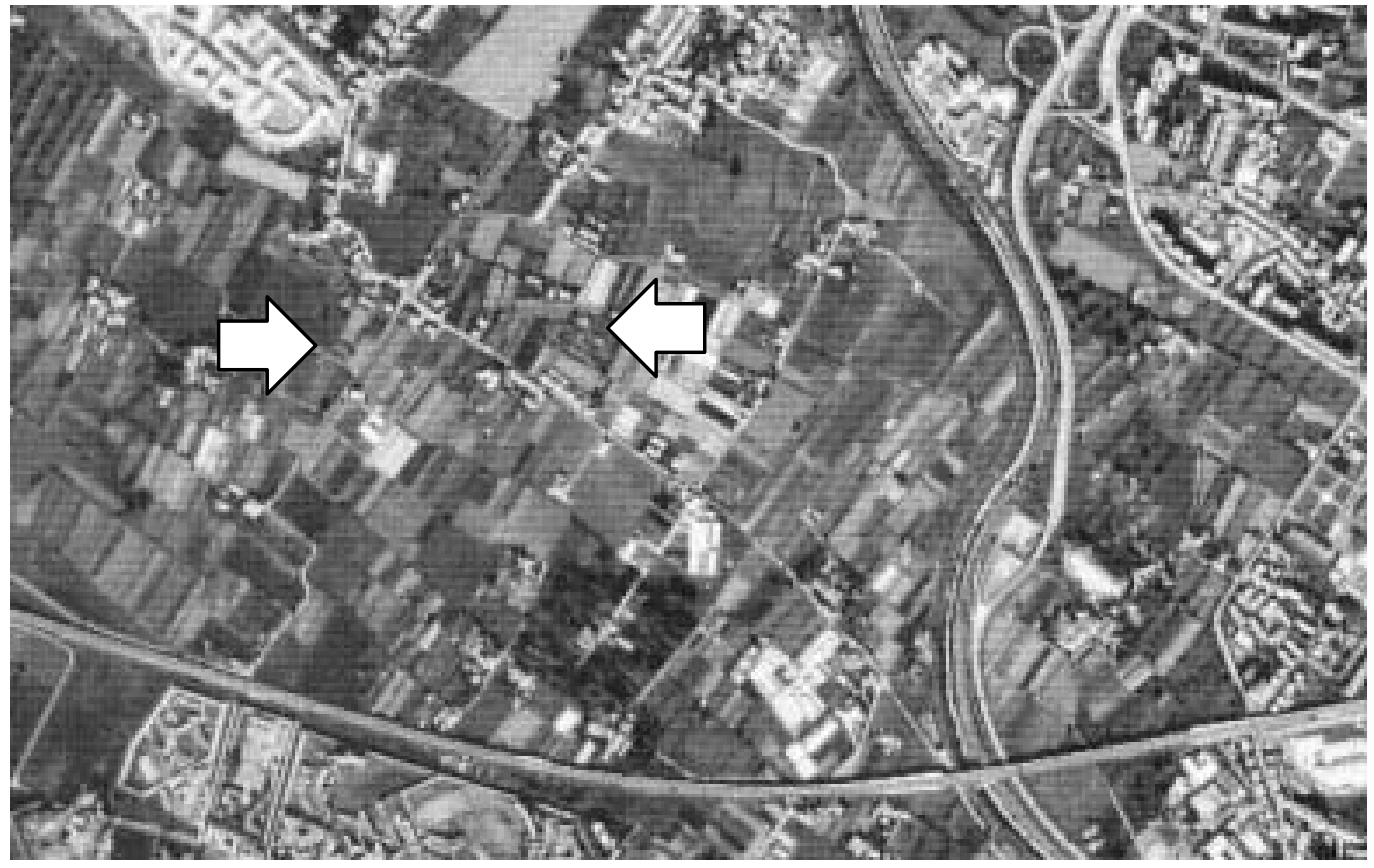

L'area di Borgo Galli, Castelnuovo e II Chiuso, in sx d'Arno, nel comune di Firenze, a valle della città

In questo caso la configurazione lineare a tessuto discontinuo è data dalla presenza ravvicinata lungo una strada di più borghi di origine rurale $e$

Rappresentazione fotografica zenitale 1:15000 (da formato digitale 1:30000 - Regione Toscana).

Rappresentazione planimetrica 1:5000 (da CTR 1:10000 - Regione Toscana).

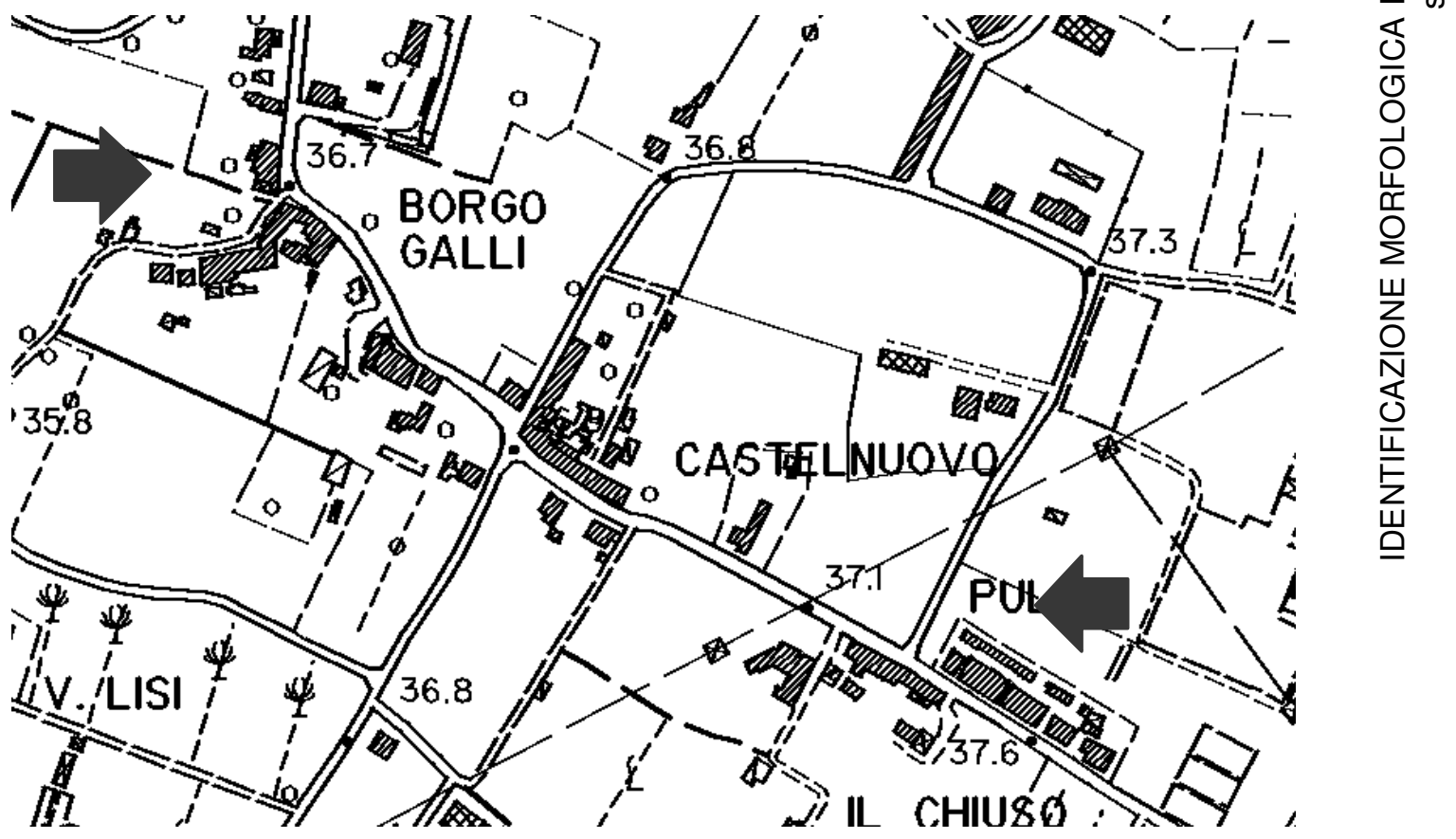




\section{FATTORI LINEARI COMPLESSI}

\subsection{Fasci di infrastrutture viarie}

ESEMPLIFICAZIONI: VEDUTE

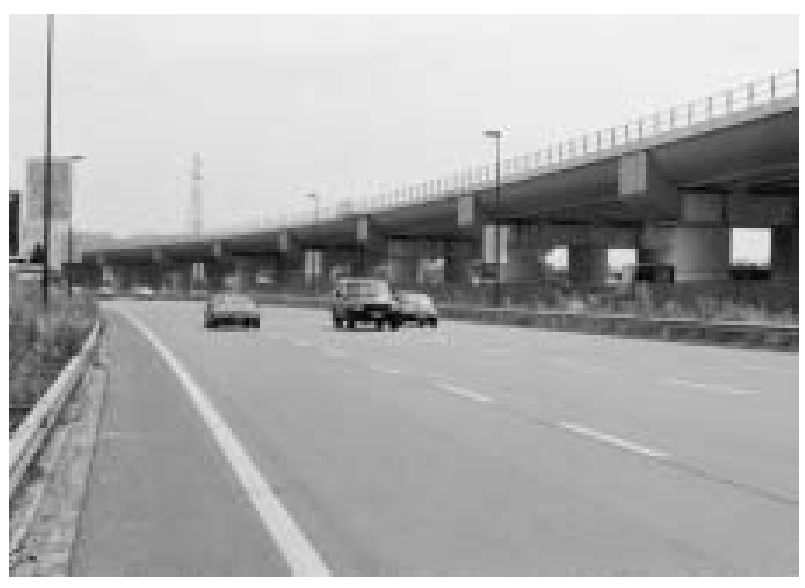

L'assenza di idonee misure di riserva di spazi e le esigenze di adeguamento del sistema della mobilità determinano facilmente situazioni in cui più infrastrutture $s i$ sovrappongono e/o si affiancano.

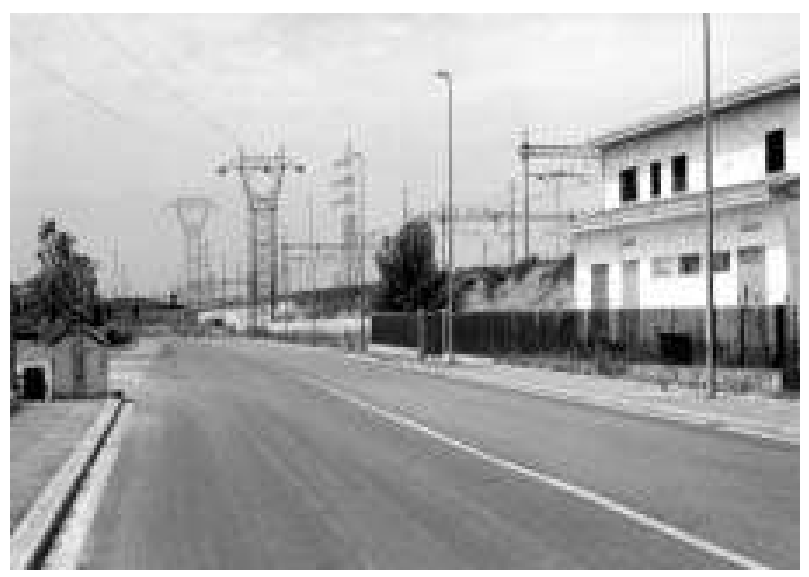

Infrastrutture tecnologiche possono affiancarsi a fasci di infratrutture viarie, incrementando la congestione degli spazi.

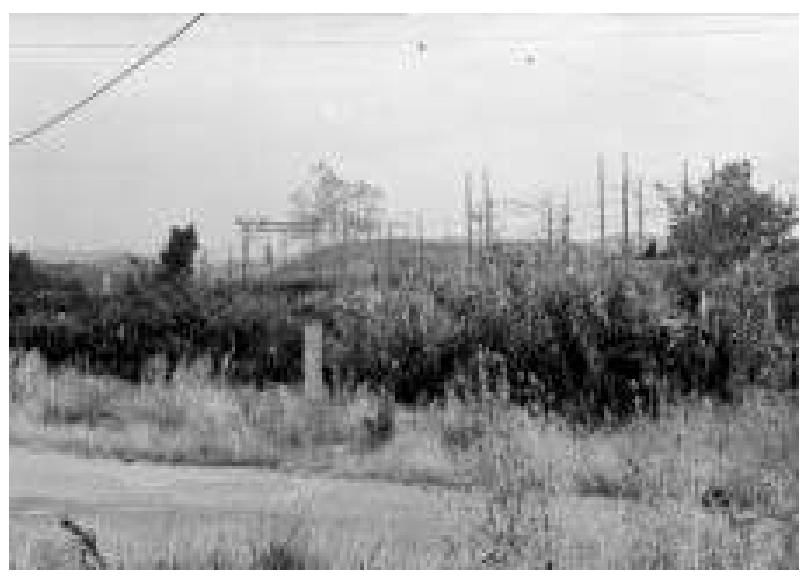

Gli spazi aperti marginali o interstiziali in genere, anziché essere prioritariamente destinati a sistemazioni di inserimento e compensazione paesistici, versano in gravi condizioni di degrado.

\section{DESCRIZIONE TIPOLOGICA DEL FATTORE}

Fasci di infrastrutture viarie. Presenza rilevante di spazi mineralizzati.

Le infrastrutture viarie costituiscono una delle tipologie di componenti antropiche di maggior rilievo nel paesaggio di pianura, anche in ragione dell'intenso sviluppo che a queste reti è stato dato nel $X X$ secolo. La densità e la connessione della rete viaria e le configurazioni di sezione trasversale ne fanno uno dei più importanti fattori di frammentazione paesistica.

Le configurazioni a cui danno luogo possono essere omogenee, ad esempio più strade su terrapieno affiancate, ma si riscontrano frequentemente anche miste, per tipo di trasporto o di sezione o per entrambi i caratteri.

Rispondono essenzialmente a quattro caratteri identificativi comuni:

- la configurazione planimetrica, tendenzialmente rettilinea o comunque geometrica;

- la configurazione altimetrica, caratterizzata spesso in pianura da sezioni in rilevato;

- l'assenza pressoché totale di equipaggiamento vegetale, sia arboreo che arbustivo;

- la presenza di spazi interstiziali, generalmente privi di sistemazioni atte a conferire ruoli paesistico-ambientali di compensazione e protezione.

\section{CRITERI INTERPRETATIVI DEGLI EFFETTI}

Occorre considerare i differenti effetti di queste configurazioni sulla articolazione del paesaggio. Esse costituiscono, per motiv diversi, fattori di crisi strutturale, contribuendo sostanzialmente ai seguenti stati:

- dal punto di vista ecosistemico, frammentazione degli spazi e conseguente riduzione delle vie di connessione di habitat animali;

dal punto di vista fisionomico-percettivo, frammentazione degli spazi e conseguente congestione visuale, che spesso sfocia in forme di conflitto, per l'intervisibilità di configurazioni non compatibili;

- dal punto di vista storico, frammentazione della struttura insediativa storica, alla quale in particolare le infrastrutture si sovrappongono in genere senza alcun riferimento, generando una severa perdita di permanenze.

Esse sono anche però fra le poche configurazioni con effetto del limite o comunque di rilievo semiologico, contribuendo, pur parzialmente e spesso in termini più potenziali che reali, ad una articolazione spaziale del paesaggio di pianura, desertificato dalla perdita dell'equipag giamento veg etale ag roforestale. 


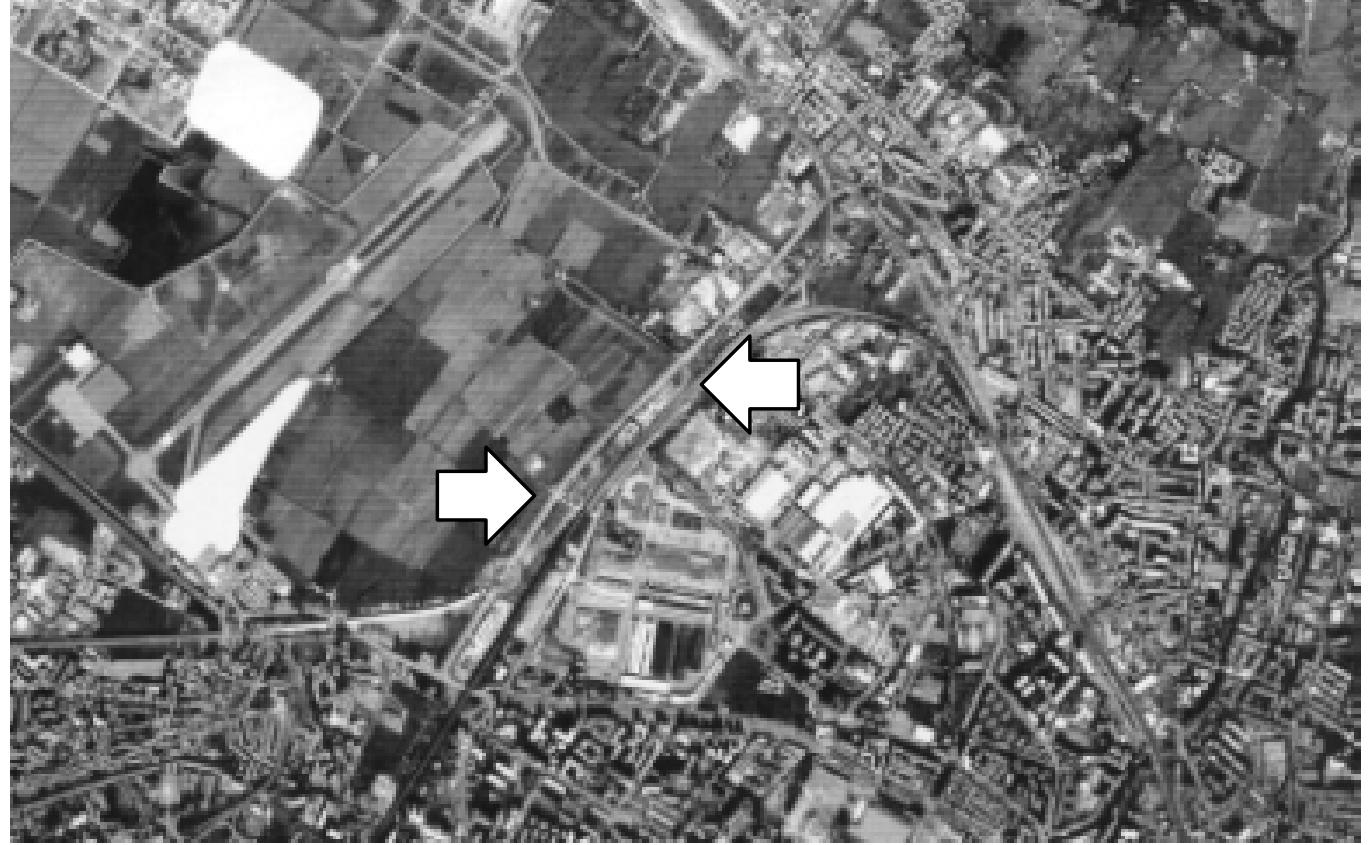

Larea periurbana della piana di Castello ad ovest di Firenze.

Da est verso ovest, la sezione di questo fascio di infrastrutture comprende la strada di distribuzione dell'area industriale e commerciale, la ferrovia a piú linee, di collegamento con Pisa, la strada tangenziale, a più corsie, che da nord, dove incontra la via Sestese, collega le zone di Sesto Fiorentino, Careggi e Rifredi, con l'autostrada e con l'aereoporto, e prosegue, attraverso il viadotto dell'Indiano, fino allinnesto con la superstrada Firenze-Pisa ed a Scandicci. A queste si aggiunge il nuovo tracciato ferroviario su viadotto e terrapieno.

Rappresentazione fotografica zenitale 1:15000 (da formato digitale 1:30000 - Regione Toscana).

Rappresentazione planimetrica 1:5000 (da CTR 1:10000 - Regione Toscana).

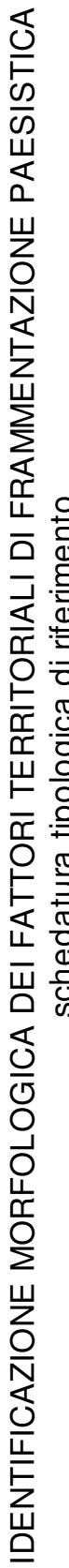

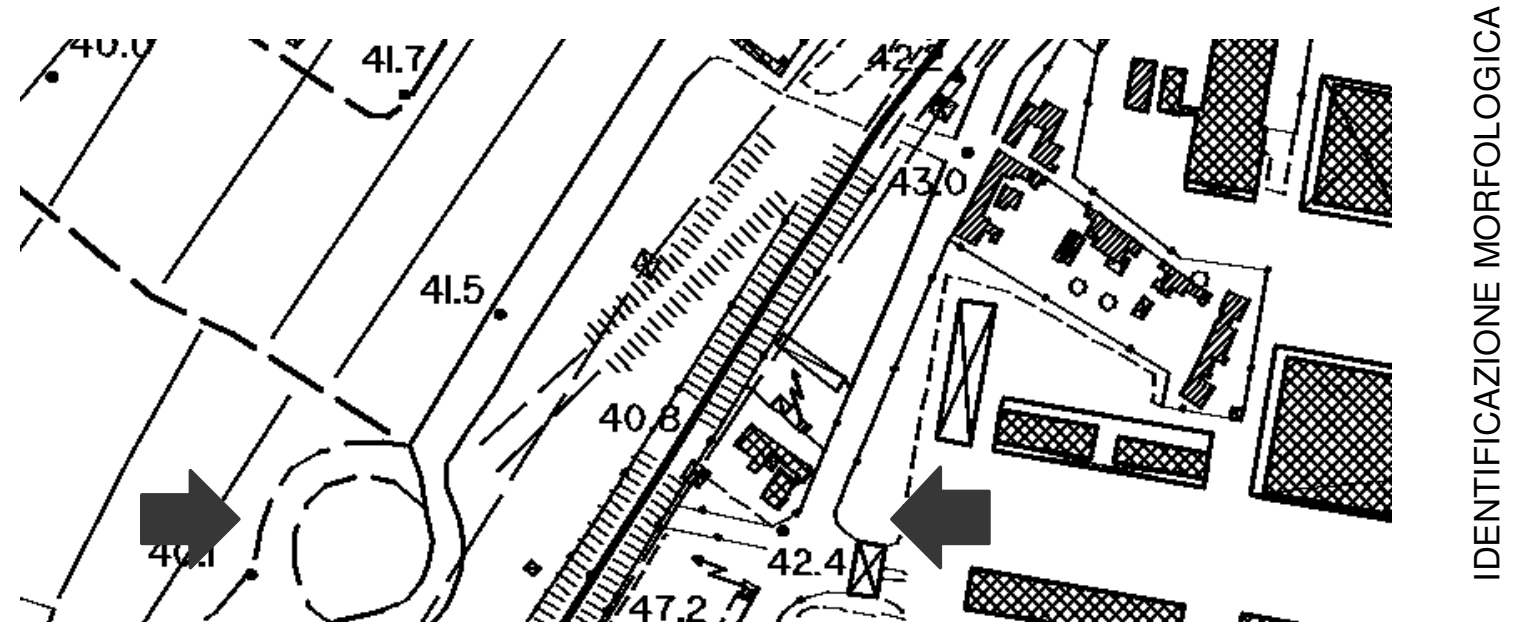




\section{FATTORI LINEARI COMPLESSI}

\subsection{Fasci di infrastrutture tecnologiche}

ESEMPLIFICAZIONI: VEDUTE

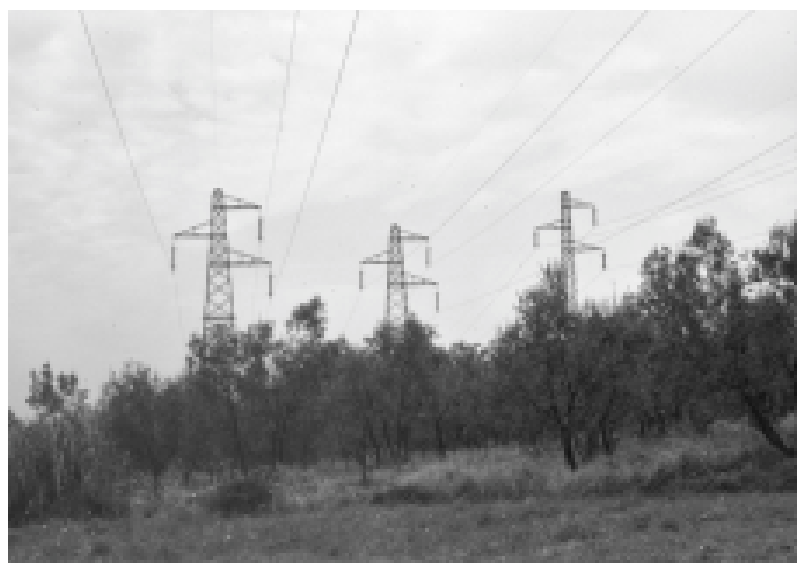

Dal punto di vista paesistico, gli elettrodotti aerei costituiscono la tipologia di infrastruttura tecnologica responsabile dei maggiori effetti di alterazione.

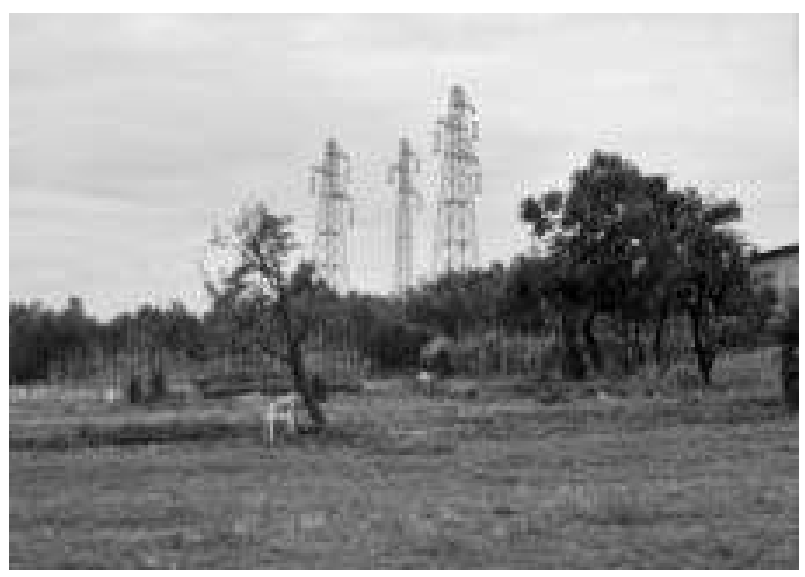

Queste infrastrutture attraversano spesso paesaggi rurali con effetti di grave criticità visuale.

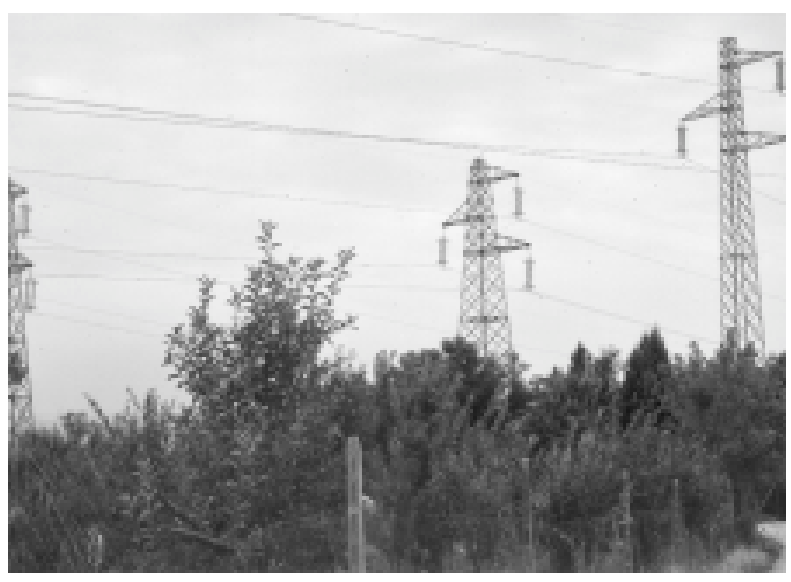

La formazione di più elettrodotti in fascio determina, in particolare per la rilevante moltiplicazione dei tralicci, una decisa amplificazione degli effetti sul paesaggio.
DESCRIZIONE TIPOLOGICA DEL FATTORE

Fasci di infrastrutture tecnologiche. Presenza mediamente rilevante di spazi mineralizzati a cui, nel caso delle tipologie aeree su strutture portanti puntiformi, si sostituisce una presenza rilevante di apparati impiantistici. Le infrastrutture tecnologiche costituiscono, con le infrastrutture viarie, una delle tipologie di componenti antropiche di maggior rilievo nel paesaggio di pianura, anche in ragione dell'intenso sviluppo che a queste reti è stato dato nel XX secolo. Si tratta essenzialmente di reti per il trasporto di energia (elettrodotti), di informazioni (telefonia e cablaggio telematico), di combustibili (metanodotti), di acqua (acquedotti civili e industriali) o infine di reflui (fognature). Tolti gli effetti eminentemente ambientali (inquinamento elettromagnetico, idrico), la rete di trasferimento e di distribuzione locale dell'energia elettrica resta quella caratterizzata dai più importanti effetti di alterazione paesistica, doviti all'inserimento spaziale degli impianti, in relazione anche alla commistione tipica delle aree di pianura.

Tali infrastrutture possono dare luogo a configurazioni complesse in cui più linee aeree si concentrano in un fascio di elettrodotti. Rispondono essenzialmente a due caratteri identificativi comuni:

- localizzazione talvolta impropria rispetto alla articolazione spaziale del paesaggio (ad esempio nel mezzo di un'area boscata o coltivata anziché nell'area di margine tra il bosco ed i coltivi);

- prevalenza della tipologia aerea nelle aree extraurbane o con insediamento diffuso, talvolta riscontrabile anche all'interno del tessuto insediativo urbano di formazione recente.

\section{CRITERI INTERPRETATIVI DEGLI EFFETTI}

Gli effetti principali di alterazione paesistica imputabili a questa tipologia di fattore investono l'articolazione spaziale del paesaggio ed i relativi caratteri visuali.

La fragilità del paesaggio rispetto a tali fattori non è inversamente proporzionale al grado di antropizzazione dello stesso. Infatti se un fascio di elettrodotti in un area montana di paesaggio forestale $\mathrm{o}$ in un'area collinare di paesaggio rurale di pregio, incide con effetti di detrattore sull'elevato grado di qualità paesistica caretteristico dei luoghi, lo stesso fattore, in un'area fortemente urbanizzata di pianura, determina un pesante picco della già elevata congestione degli spazi, che non hanno capacità illimitate di resistenza solo perché è ormai venuta meno la loro configurazione naturale o seminaturale. Se pertanto nei paesaggi rurali o forestali è possibile parlare di inserimento paesistico di queste grandi opere infrastrutturali, ciò diviene del tutto aleatorio proprio nei paesaggi di pianura ad elevata pressione insediativa, dove la carenza di spazi e l'assenza di articolazione geomorfologica in bacini visuali separati, riducono sensibilmente l'efficacia di ogni misura di inserimento e mitig azione che non sia l'interramento deg li elettrodotti. 


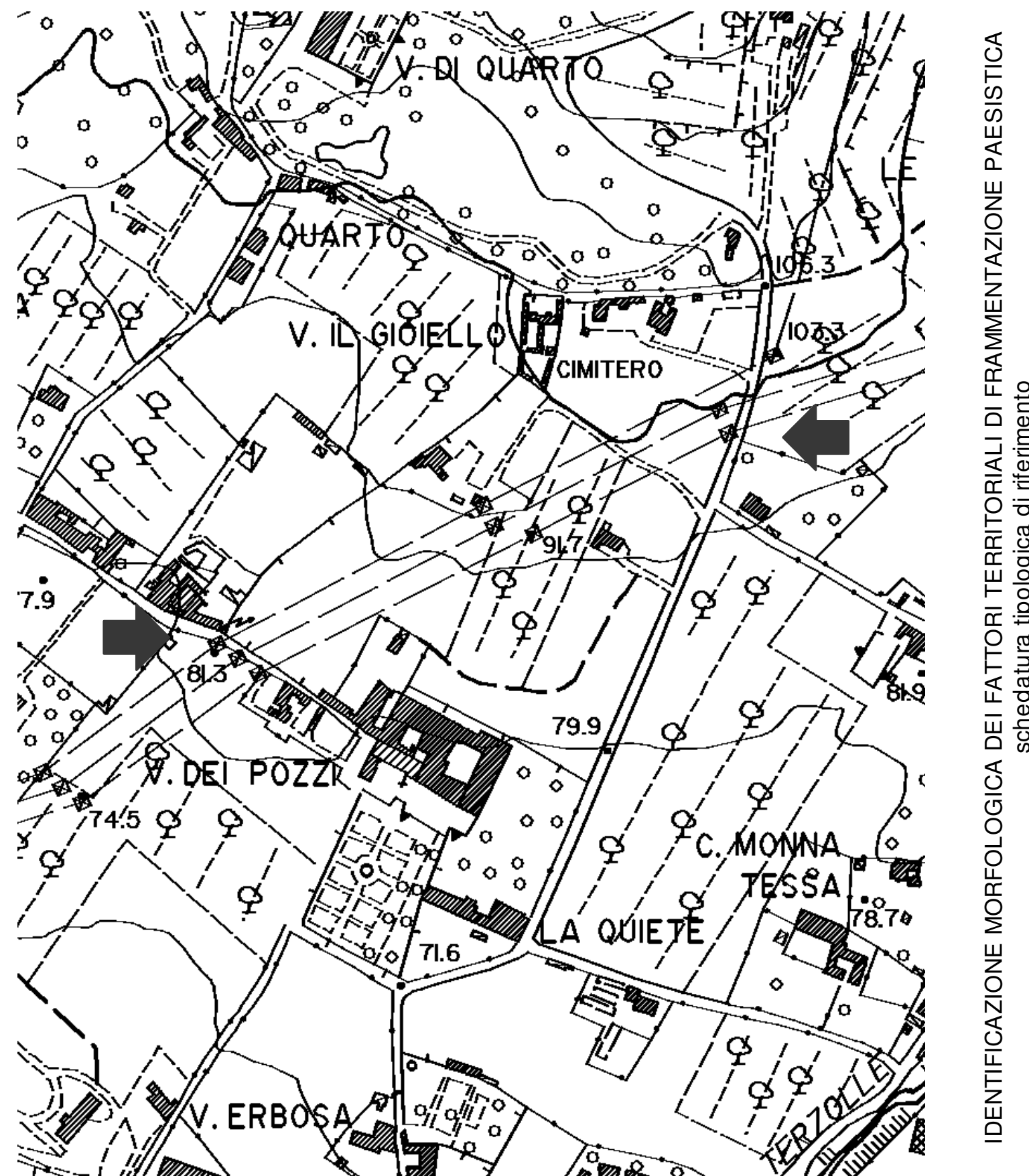

L'area pedecollinare di Quarto a Firenze, ad ovest di Careggi.

A seguito dell'esigenza di elettrodotti di adduzione o trasferimento, impianti di grandi dimensioni si trovano frequentemente nel paesaggio collinare e montano, anche in prossimità di siti di rilevante interesse storico o naturalistico. Localizzazioni assolutamente improprie o misure di inserimento paesistico assenti, determinano spesso forti livelli di conflittualità visuale. Nel paesaggio di pianura le condizioni di criticità visuale vengono ad essere aggravate da questi fattori che, con andamenti decisamente trasversali all'articolazione degli spazi, ne incrementano la congestione.

Rappresentazione planimetrica 1:5000 (da CTR 1:10000 - Regione Toscana). 


\section{FATTORI LINEARI COMPLESSI}

\subsection{Fasci di corsi d'acqua}

ESEMPLIFICAZIONI: VEDUTE

La tipologia viene inserita, pur non essendo riscontrabile nell'area di studio, per il rilievo paesistico che le configurazioni ad essa riferibili assumono nel paesaggio di pianura.

$\grave{E}$ direttamente connessa alla presenza di canali di bonifica ed opere di regimazione idraulica, non essendo proprio della struttura idrografica di origine naturale il parallelismo di linee di compluvio.

Riscontrabile nelle aree di bonifica della Toscana come di altre regioni, si trova anche nell'area metropolitana Firenze-Prato-Pistoia, a pochi chilometri ad ovest del soggetto di studio, ad esempio nell'area in destra d'Arno a nord dell'insediamento di Signa, dove confluiscono il fiume Bisenzio ed il Fosso Reale, canale collettore principale del sistema di bonifica della piana di Sesto Fiorentino (si riporta nella fotografia una veduta di quest'area, come esemplificazione).

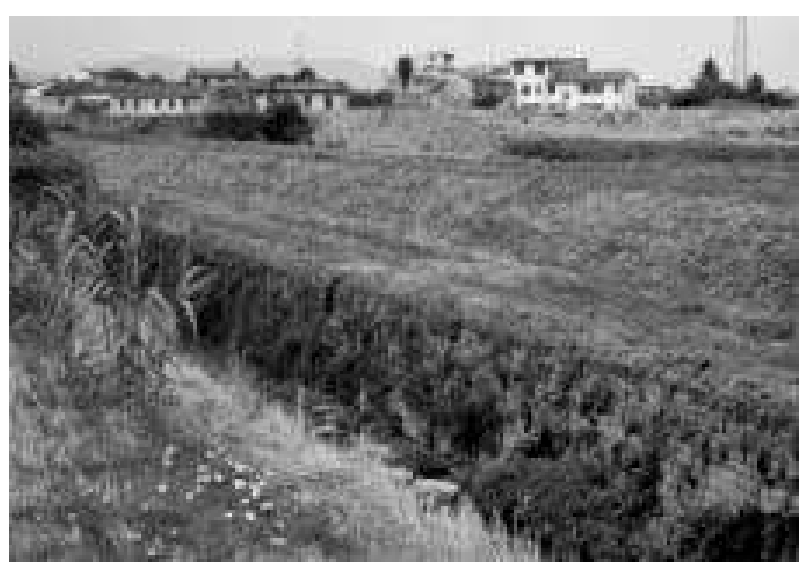

\section{DESCRIZIONE TIPOLOGICA DEL FATTORE}

Fasci di corsi d'acqua di origine artificiale o misti, naturale e artificiale. Presenza mediamente rilevante di spazi mineralizzati, dovita al forte peso relativo alla sensibilità caratteristica dei corsi d'acqua (situazioni generalmente localizzate, coincidenti con opere idrauliche).

L'intensità delle opere di bonifica e la loro stratificazione storica fanno sì che esse costituiscano sistemi di decisa caratterizzazione del paesaggio di pianura sia urbano che extraurbano.

I caratteri identificativi comuni di questi fattori, douti sostanzialmente ad esigenze dettate dagli equilibri idraulici delle pianure e dalle modalità di gestione adottate, sono essenzialmente quattro:

- la configurazione planimetrica, tendenzialmente rettilinea o comunque geometrica;

- la configurazione altimetrica, caratterizzata da argini e golene in terra, a sezione trapezoidale fortemente geometrizzata e con forti pendenze;

- la presenza di spazi interstiziali, generalmente privi di sistemazioni atte a conferire ruoli paesistico-ambientali di protezione e miglioramento, indipendenti dalla interdizione all'uso umano;

- l'assenza pressochè totale di equipaggiamento vegetale, sia arboreo che arbustivo.

\section{CRITERI INTERPRETATIVI DEGLI EFFETTI}

Occorre considerare i differenti effetti di queste configurazioni sulla articolazione del paesaggio. Esse costituiscono, per motivi diversi, fattori di alterazione strutturale, contribuendo sostanzialmente ai seguenti stati:

- dal punto di vista ecosistemico, frammentazione degli spazi e conseguente riduzione delle vie di connessione di habitat animali e riduzione del potenziale di connessione intrinseco, per l'assenza di vegetazione ripariale e di diversificazione ecologica dell'alveo;

- dal punto di vista fisionomico-percettivo, esclusione dell'acqua e delle formazioni fluviali nel loro complesso dallo scenario paesistico.

Esse sono anche però fra le poche configurazioni con effetto del limite o comunque di rilievo semiologico, contribuendo, pur parzialmente, ad una articolazione spaziale del paesaggio di pianura, desertificato dalla perdita dell'equipaggiamento veg etale ag roforestale. 
ESEMPLIFICAZIONI: FOTOGRAFIA ZENITALE E CARTOGRAFIA

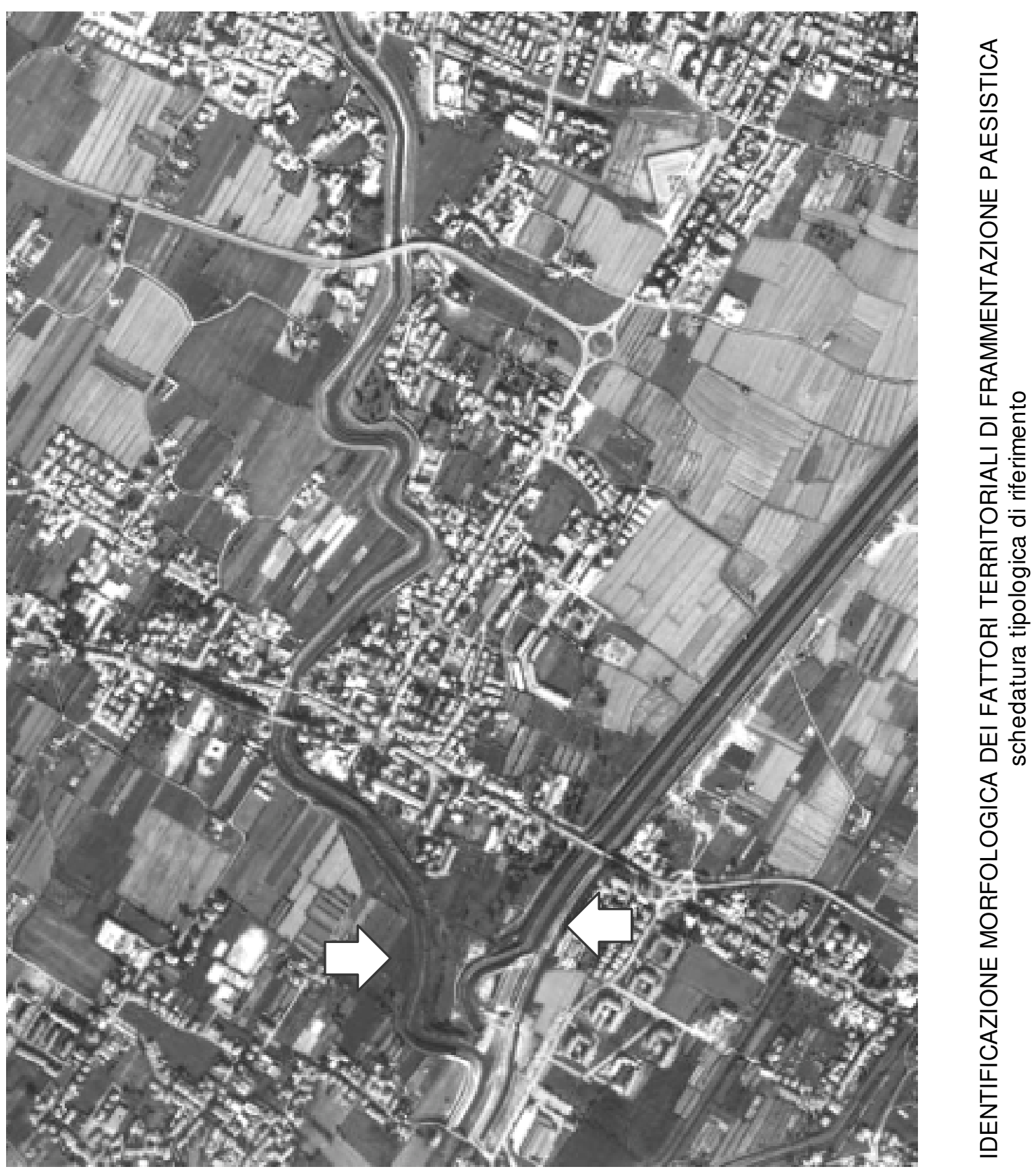

L'area in destra d'Arno a nord dellinsediamento di Signa.

In questo caso di confluenza di corsi d'acqua si trova una sezione con corpo triplo per un totale di sei argini in rilevato di terra tra il fiume Bisenzio ad ovest ed il fosso Reale ad est.

Rappresentazione fotografica zenitale 1:15000 (da formato digitale 1:30000 - Regione Toscana). 


\section{$4 \quad$ FATTORI LINEARI SEMPLICI}

\subsection{Infrastrutture viarie}

\section{ESEMPLIFICAZIONI: VEDUTE}

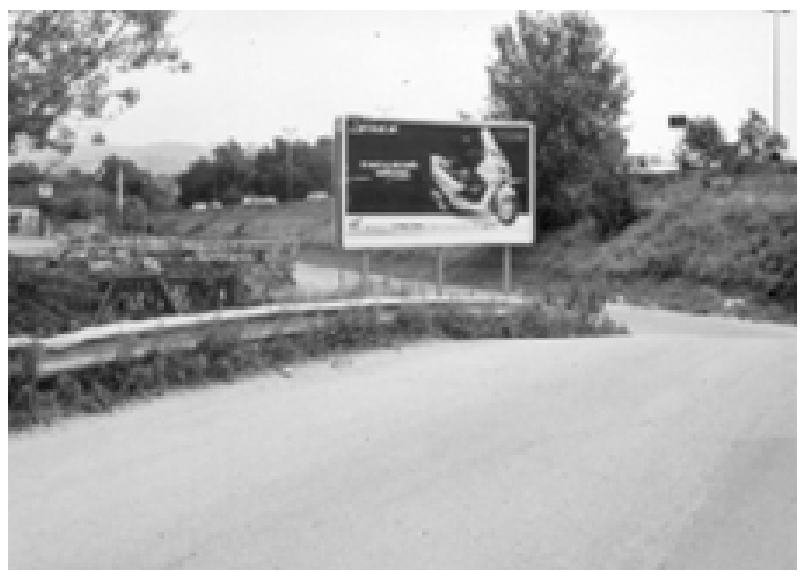

Nel paesaggio di pianura le maggiori infrastrutture stradali sono in genere caratterizzate da sezioni in rilevato, più frequentemente su terrapieno, ma talvolta anche su viadotto.

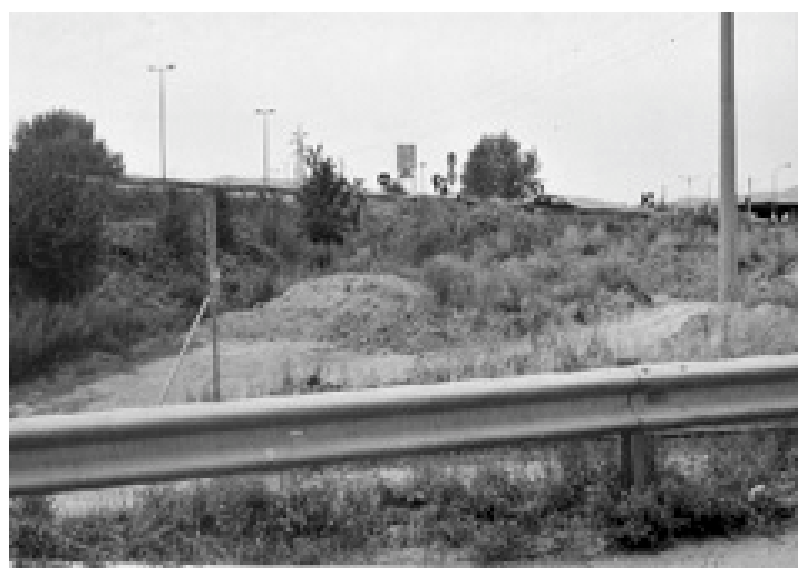

I sistemi di svincolo e raccordo con la viabilità locale danno luogo a spazi pertinenziali inutilizzati che generalmente risultano in condizioni di degrado.

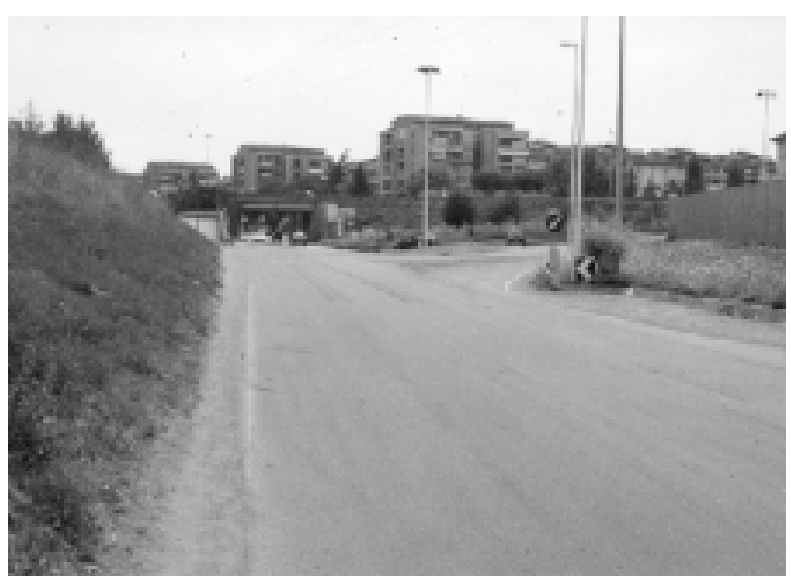

I sistemi di svincolo e raccordo con la viabilità locale sono apparati dellinfrastruttura che ne incrementano gli effetti di impegno e mineralizzazione di spazi.

\section{DESCRIZIONE TIPOLOGICA DEL FATTORE}

Infrastrutture viarie. Prevalenza di spazi mineralizzati. Si veda anche la tipologia 3.3 (fasci di infrastrutture viarie). La configurazione come fattore semplice, pur di minor impatto rispetto a quella della tipologia complessa 3.3 , è caratterizzata da un elevato grado di diffusione territoriale a cui è direttamente connessa l'importanza relativamente agli effetti paesistici cumulativi. La densità del reticolo viario, particolarmente elevata nelle aree di pianura, ne fa uno dei più importanti fattori di frammentazione paesistica.

I caratteri identificativi comuni sono essenzialmente tre:

- la configurazione planimetrica che, in particolare a seguito dei forti sviluppi della seconda metà del $X X$ sec., non risente dell'articolazione semiologica della matrice paesistica in cui le infrastrutture sono inserite e si sviluppano;

- la configurazione altimetrica in rilevato, generalmente su terrapieno, ma anche su viadotto, nei casi di infrastrutture veloci $\mathrm{e} / \mathrm{o}$ di aree particolarmente congestionate dal punto di vista spaziale;

- l'assenza pressoché totale di equipaggiamento vegetale sia arboreo che arbustivo.

\section{CRITERI INTERPRETATIVI DEGLI EFFETTI}

Si veda anche la tipologia 3.3 (fasci di infrastrutture viarie). L'intensità degli effetti di frammentazione paesistica, che tipologicamente risultano in sostanza coincidenti con quelli della precedente tipologia 3.3, è in questo caso minore per la "semplicità" della configurazione per la quale non si verifica la reiterazione di componenti trasversalmente allo sviluppo longitudinale. La barriera di discontinuità assume pertanto una sezione trasversale omogenea, impegnando spazi sensilbilmente minori. 


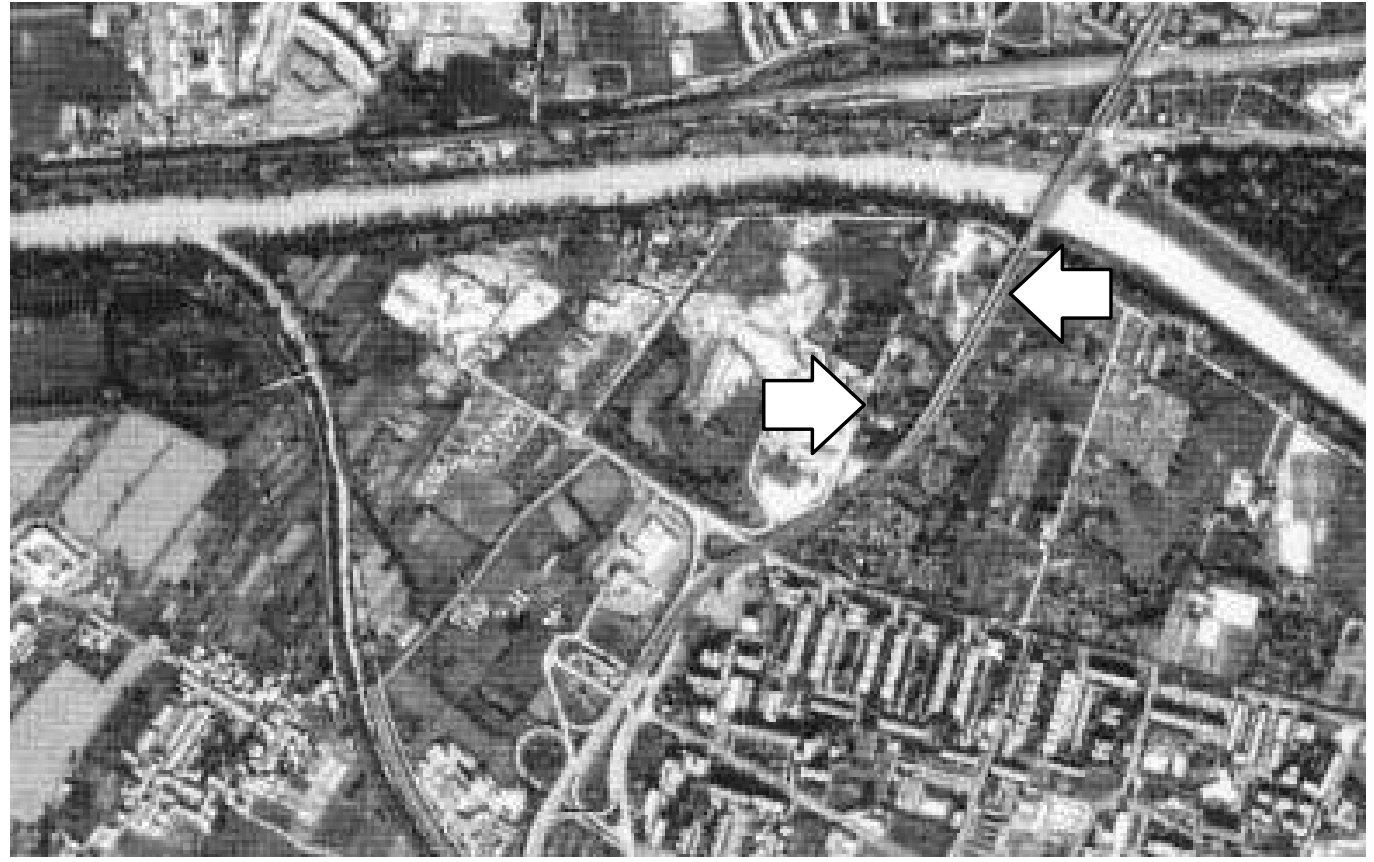

L'area di Argingrosso a Firenze.

II collegamento stradale veloce dell'area di Peretola in destra d'Arno con quella di Torri a Cintoia in sinistra, costituisce la maggiore infrastruttura stradale che interessa l'area di Argingrosso. Essa risulta indubbiamente anche uno dei principali sistemi di caratterizzazione semiologica di questa parte del paesaggio urbano di Firenze.

Rappresentazione fotografica zenitale 1:15000 (da formato digitale 1:30000 - Regione Toscana).

Rappresentazione planimetrica 1:5000 (da CTR 1:10000 - Regione Toscana).

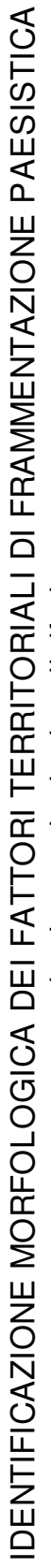

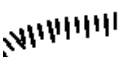

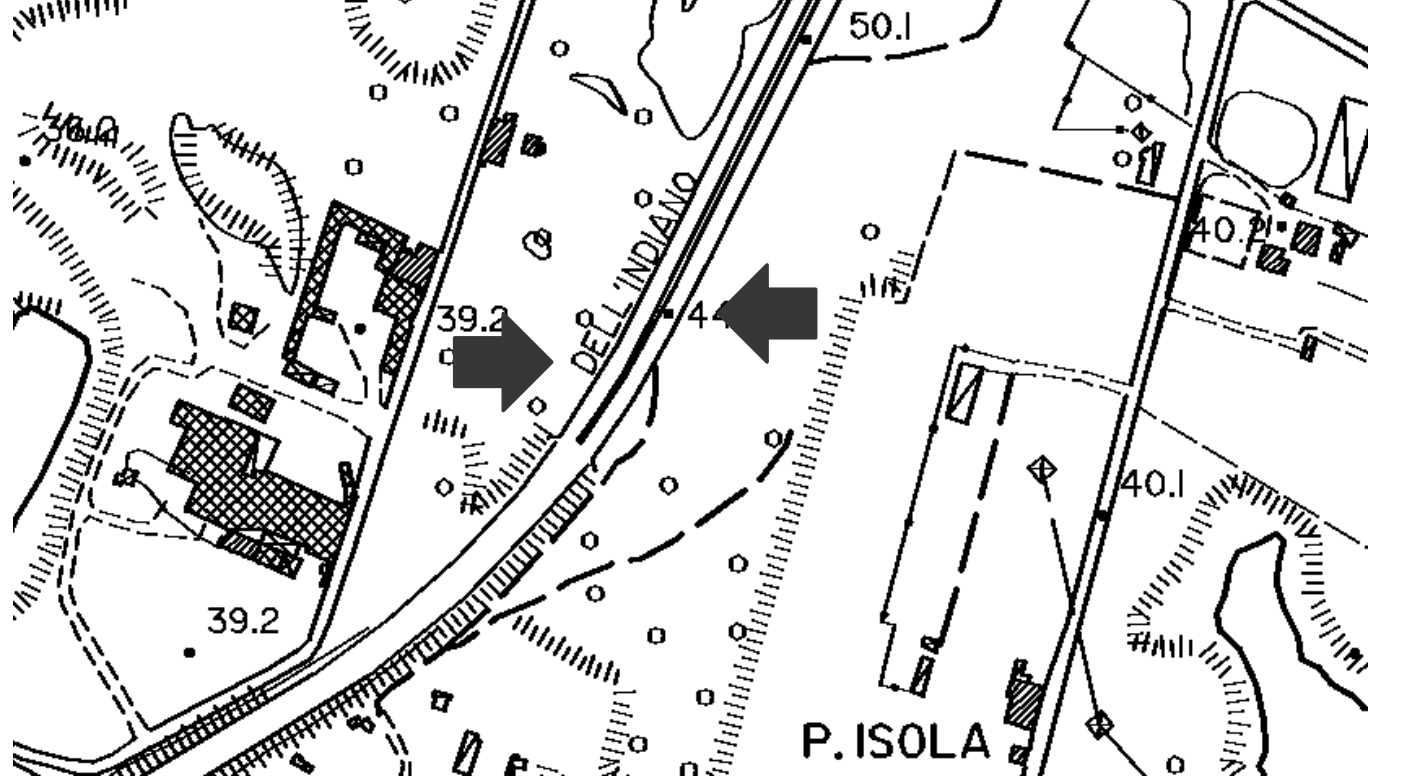




\section{$4 \quad$ FATTORI LINEARI SEMPLICI}

\subsection{Infrastrutture tecnologiche}

ESEMPLIFICAZIONI: VEDUTE

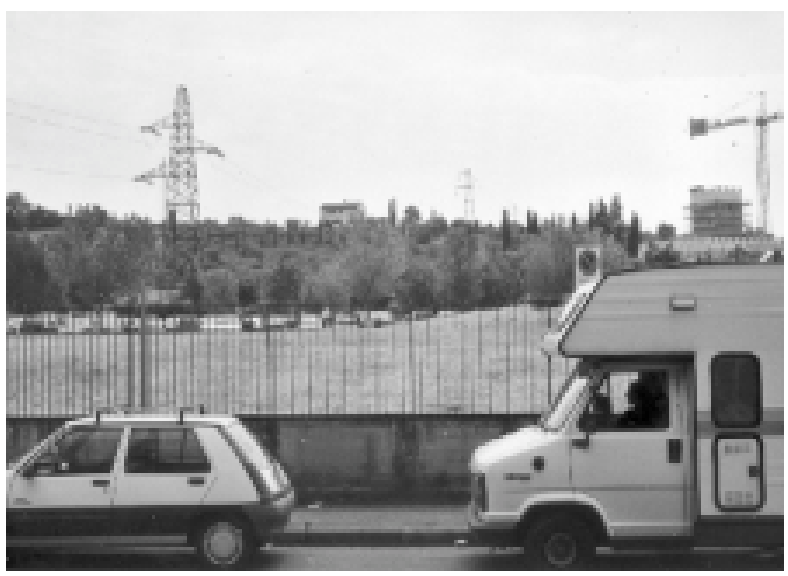

A seguito degli sviluppi urbani e della mancanza di misure di pianificazione integrata dei tessuti insediativi e degli apparati tecnologici, il paesaggio periurbano mostra frequentemente situazioni di congestione e di promiscuità nelle quali non è raro trovare elettrodotti sovralocali fra condomini residenziali (sotto) o allinterno questione, un importante ospedale (sopra e sotto).

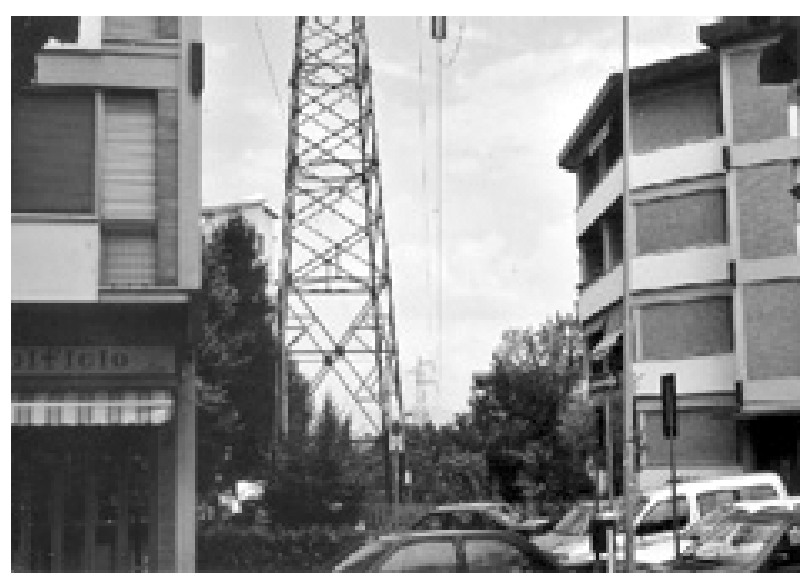

C.s.

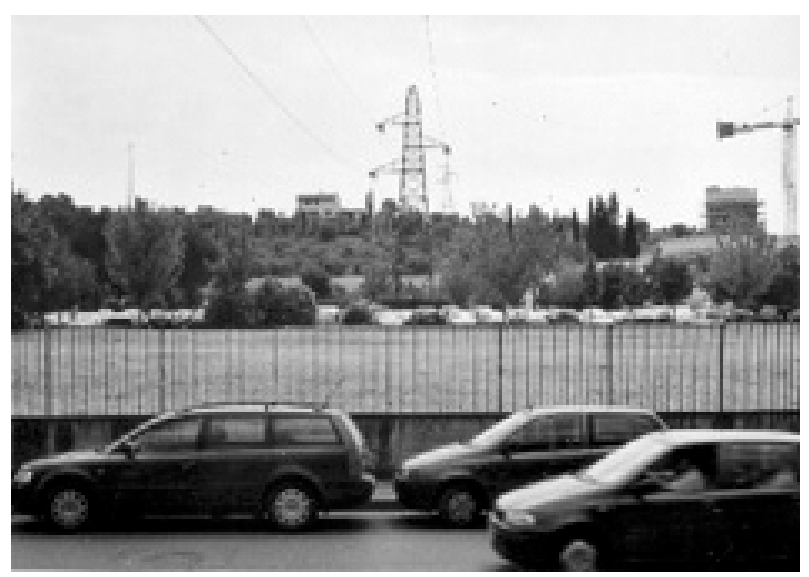

C.s.
DESCRIZIONE TIPOLOGICA DEL FATTORE

Infrastrutture tecnologiche. Presenza mediamente rilevante di spazi mineralizzati a cui, nel caso delle tipologie aeree su strutture portanti puntiformi, si sostituisce una presenza rilevante di apparati impiantistici. $\mathrm{Si}$ veda anche la tipologia 3.4 (fasci di infrastrutture tecnologiche).

La configurazione come fattore semplice, pur di minor impatto rispetto a quella della tipologia complessa 3.4, è caratterizzata da un elevato grado di diffusione territoriale a cui è direttamente connessa l'importanza relativamente agli effetti paesistici cumulativi.

I caratteri identificativi comuni coincidono con quelli della tipologia 3.4.

\section{CRITERI INTERPRETATIVI DEGLI EFFETTI}

Si veda anche la tipologia 3.4 (fasci di infrastrutture tecnologiche).

L'intensità degli effetti di frammentazione paesistica, che tipologicamente risultano in sostanza coincidenti con quelli della precedente tipologia 3.4, è in questo caso minore per la "semplicità" della configurazione per la quale non si verifica la reiterazione di componenti trasversalmente allo sviluppo longitudinale. Queste condizioni determinano una diminuzione di peso nello scenario paesistico a parità di localizzazione, di dimensione e di configurazione dell'infrastruttura. 
ESEMPLIFICAZIONI: FOTOGRAFIA ZENITALE E CARTOGRAFIA

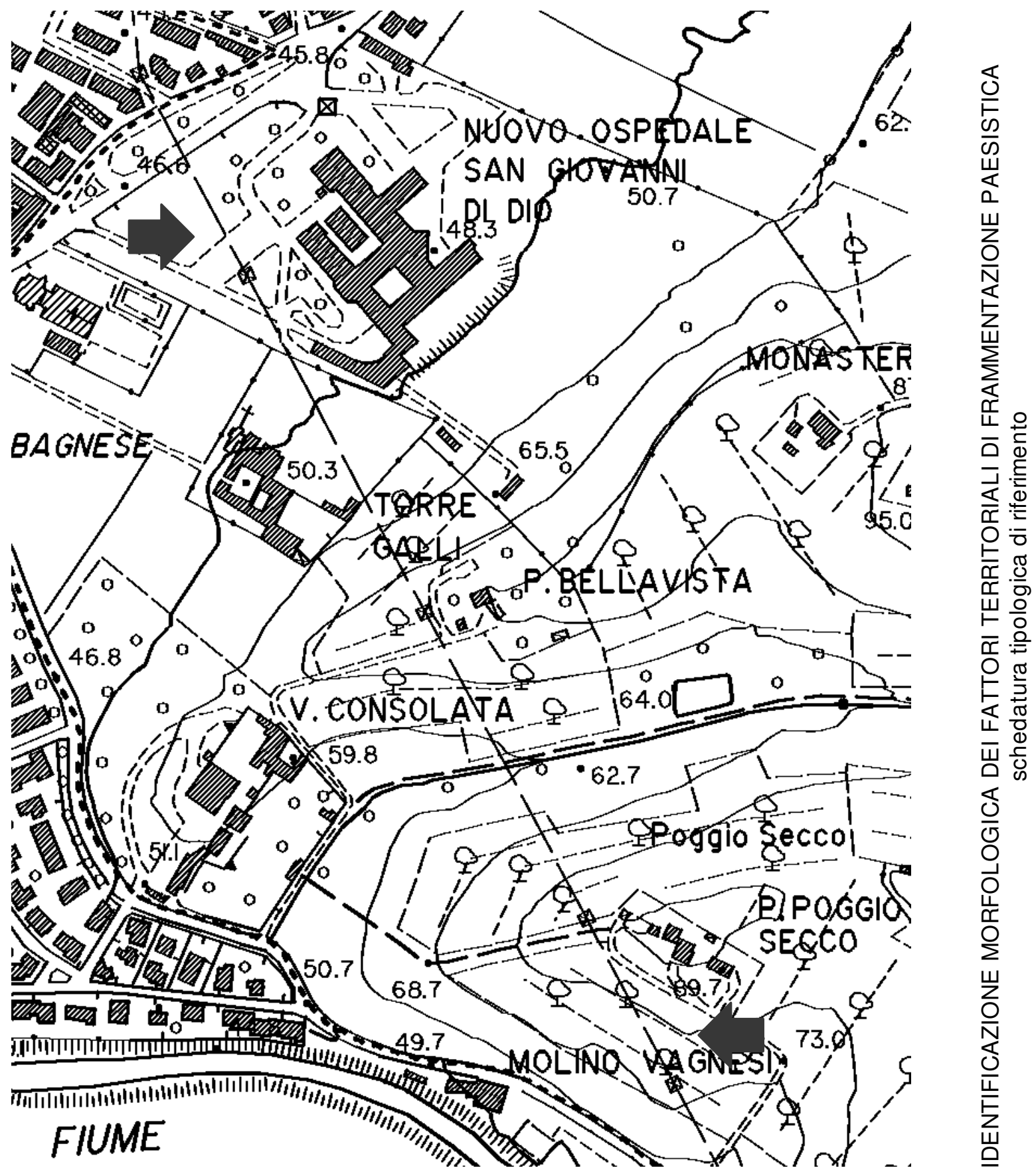

L'area di Torre Galli a Firenze, ad est di Scandicci.

Nel paesaggio pedecollinare e collinare un ulteriore motivo di conflitto indotto da queste infrastrutture è dovuto alle caratteristiche geomorfologiche, per le quali gli elettrodotti assumo in genere una visibilità elevata.
Rappresentazione planimetrica 1:5000 (da CTR 1:10000 - Regione Toscana). 


\section{$4 \quad$ FATTORI LINEARI SEMPLICI}

\subsection{Canali artificiali di bonifica idraulica}

\section{ESEMPLIFICAZIONI: VEDUTE}

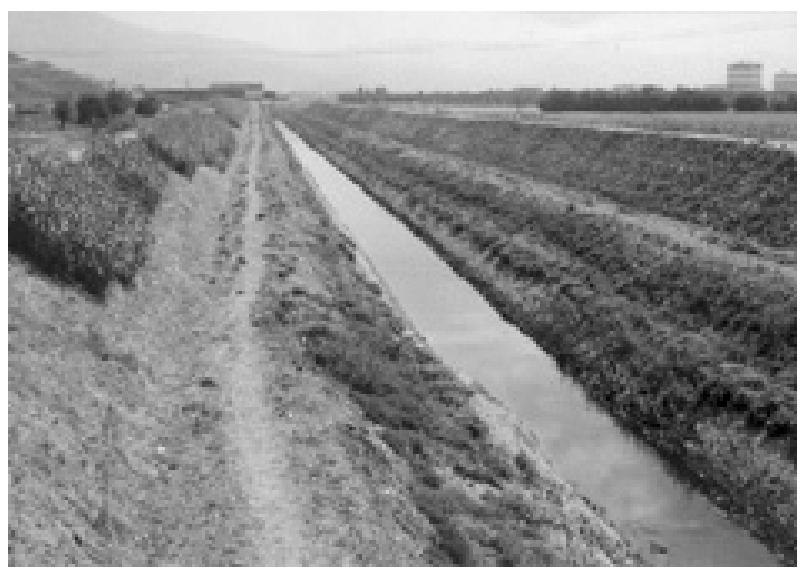

L'origine artificiale dei sistemi di bonifica idraulica è denunciata dalla geometria delle sezioni e dalla loro costituzione, che talvolta arriva alla completa mineralizzazione. L'assenza di vegetazione arborea ed arbustiva fa somigliare questi corsi d'acqua ad infrastrutture tecnologiche.

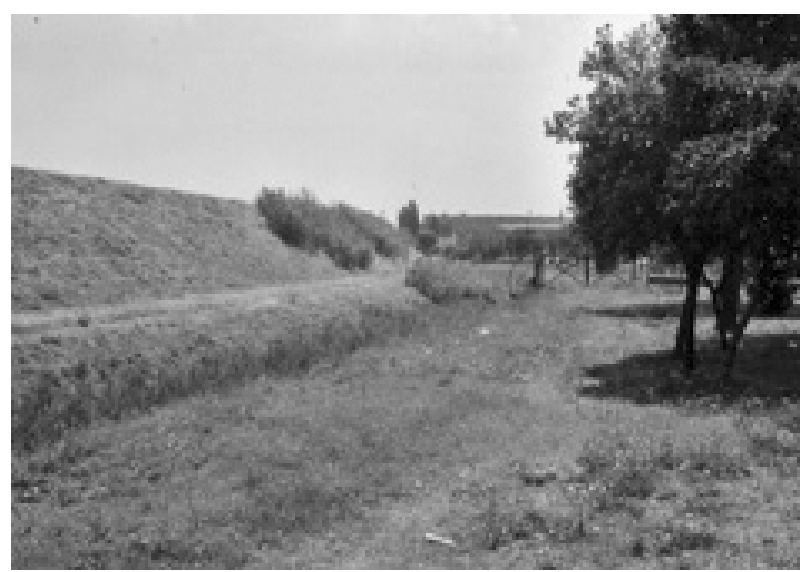

Gli argini in rilevato dei canali costituiscono formazioni caratterizzanti il paesaggio di pianura, ma escludono decisamente la presenza dell'acqua come risorsa naturale primaria.

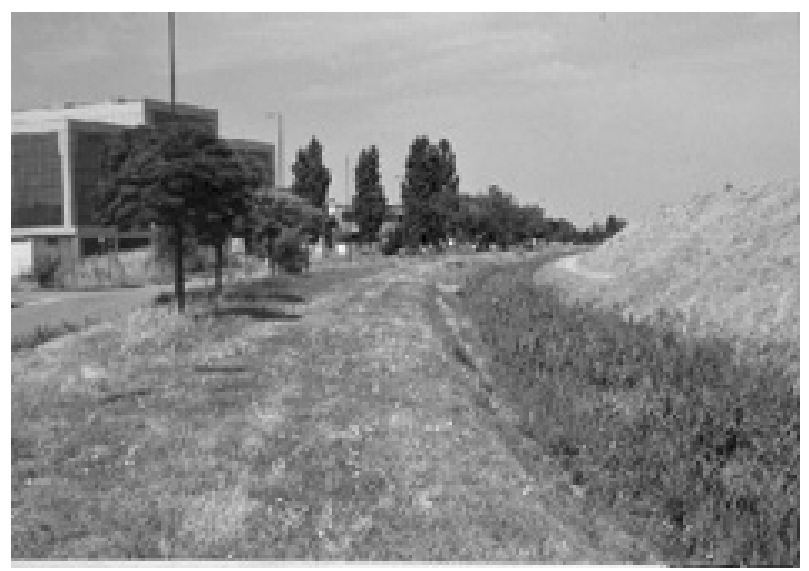

C.s.

\section{DESCRIZIONE TIPOLOGICA DEL FATTORE}

Corsi d'acqua di origine artificiale. Presenza mediamente rilevante di spazi mineralizzati, donta al forte peso relativo alla sensibilità caratteristica dei corsi d'acqua (situazioni generalmente localizzate, coincidenti con opere idrauliche). Si veda anche la tipologia 3.5 (fasci di corsi d'acqua).

L'intensità delle opere di bonifica e la loro stratificazione storica fanno sì che esse costituiscano sistemi di decisa caratterizzazione del paesaggio di pianura, sia urbano, che extraurbano.

Nei tratti di maggior criticità idraulica e/o limitrofi ad aree edificate o urbanizzate, questi corsi d'acqua risultano spesso fortemente mineralizzati da opere artificiali di canalizzazione e messa in sicurezza idraulica o di regolazione delle portate.

I caratteri identificativi comuni di questi fattori, douti sostanzialmente ad esigenze dettate dagli equilibri idraulici delle pianure e dalle modalità di gestione adottate, sono essenzialmente tre:

- Ia configurazione planimetrica, tendenzialmente rettilinea o comunque geometrica;

- la configurazione altimetrica, caratterizzata da argini in rilevati di terra a sezione trapezoidale, fortemente geometrizzata e con forti pendenze;

- l'assenza pressochè totale di equipaggiamento veg etale, sia arboreo che arbustivo.

\section{CRITERI INTERPRETATIVI DEGLI EFFETTI}

Si veda anche la tipologia 3.5 (fasci di corsi d'acqua).

L'intensità degli effetti di frammentazione paesistica, che tipologicamente risultano in sostanza coincidenti con quelli della precedente tipologia 3.4 , è in questo caso minore per l'assenza della reiterazione di componenti trasversalmente allo sviluppo longitudinale. La barriera di discontinuità assume pertanto una sezione trasversale omogenea impegnando spazi sensilbilmente minori. 
ESEMPLIFICAZIONI: FOTOGRAFIA ZENITALE E CARTOGRAFIA

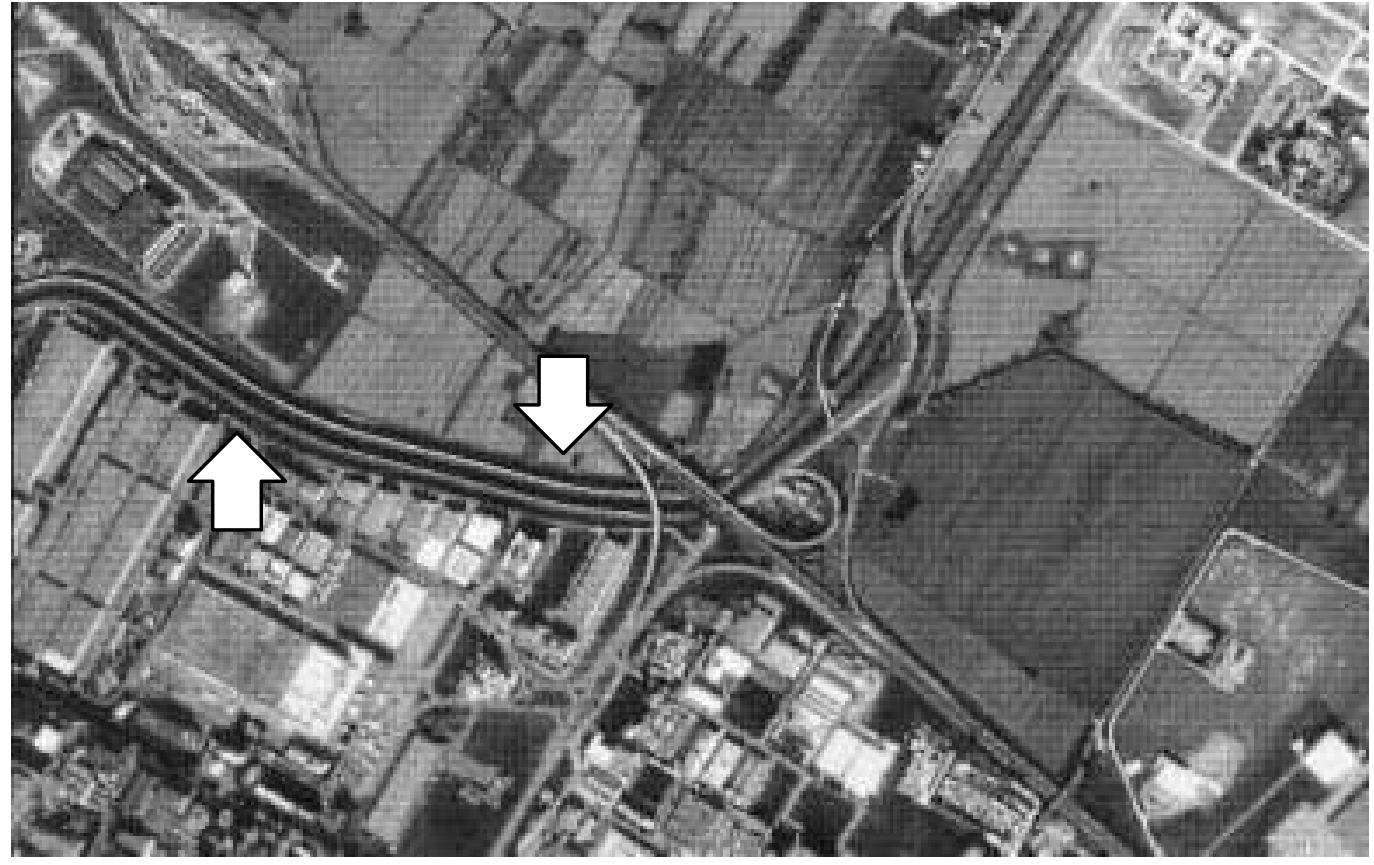

L'area di Osmannoro ad ovest di Firenze.

(a)

Rappresentazione fotografica zenitale 1:15000 (da formato digitale 1:30000 - Regione Toscana).

Rappresentazione planimetrica 1:5000 (da CTR 1:10000 - Regione Toscana).
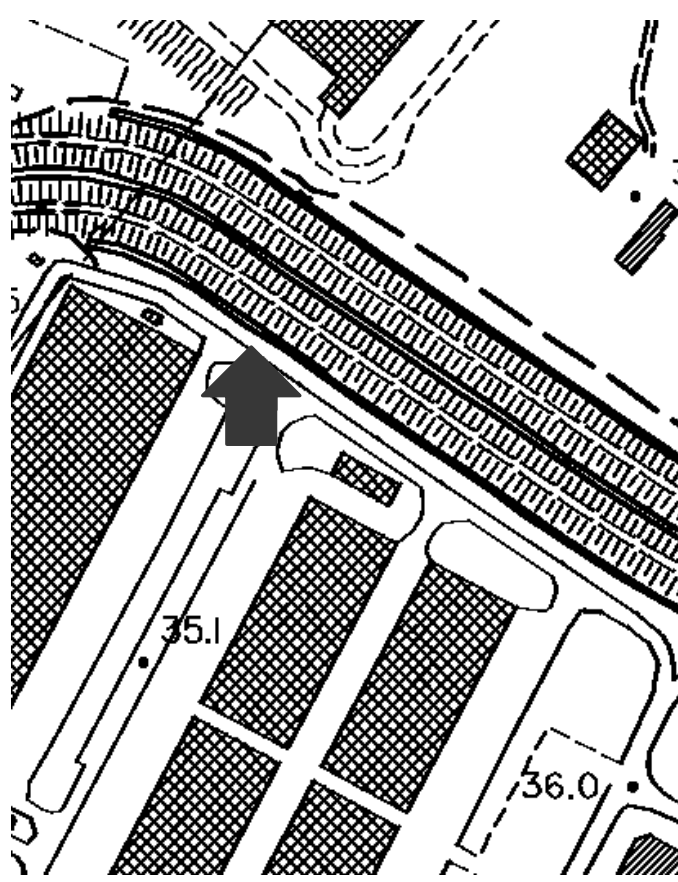

36.1
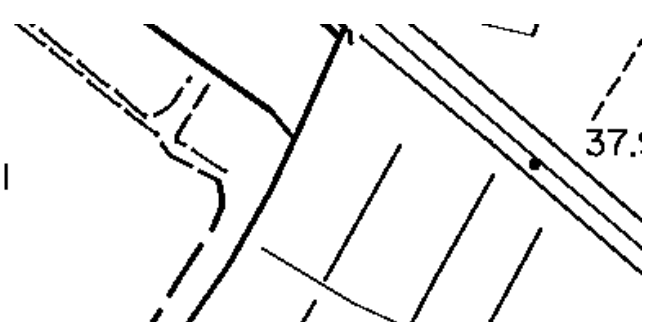

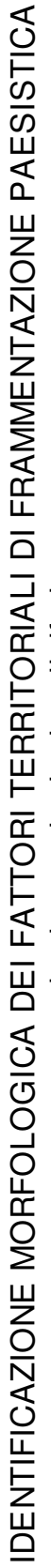




\section{$4 \quad$ FATTORI LINEARI SEMPLICI}

\subsection{Corsi d'acqua naturali regimati}

\section{ESEMPLIFICAZIONI: VEDUTE}

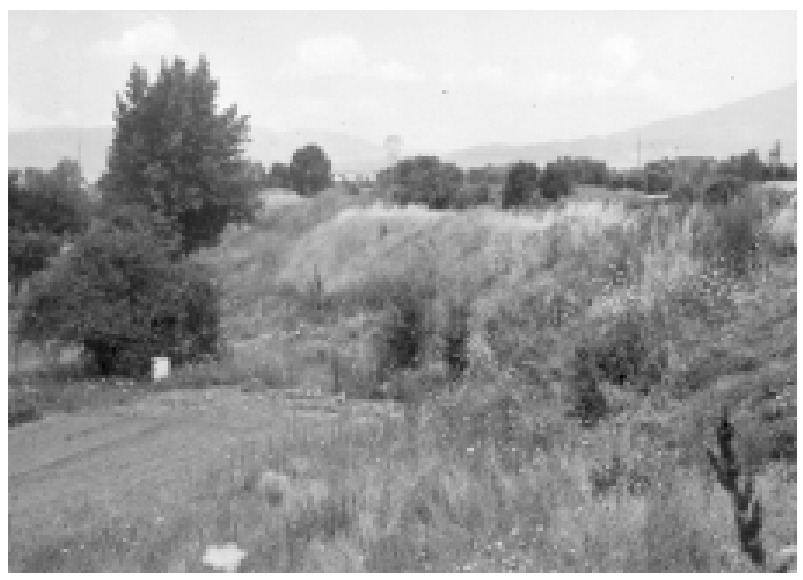

Come per i canali di bonifica, gli argini in rilevato costituiscono formazioni caratterizzanti il paesaggio di pianura, ma escludono decisamente la presenza dell'acqua come risorsa naturale primaria.

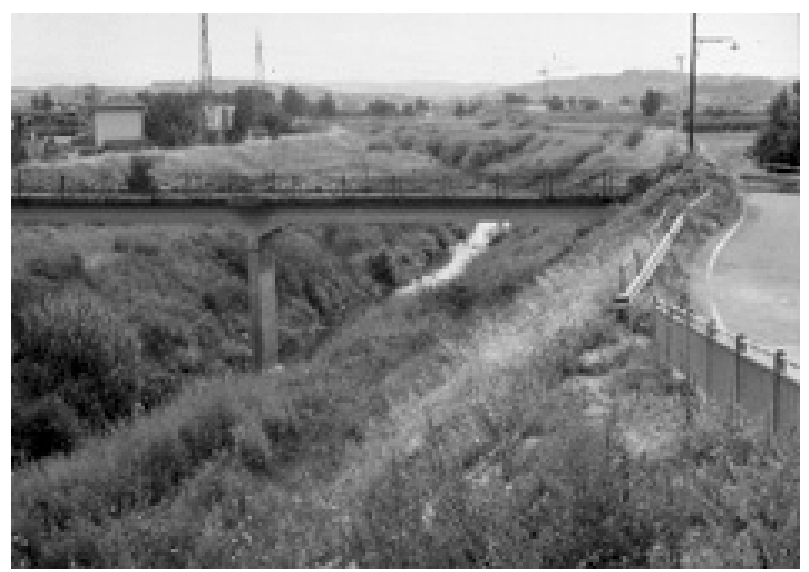

La canalizzazione dei corsi d'acqua naturali ha semplificato l'andamento meandrico, secondo la finalità idraulica di aumentare la velocità di deflusso delle

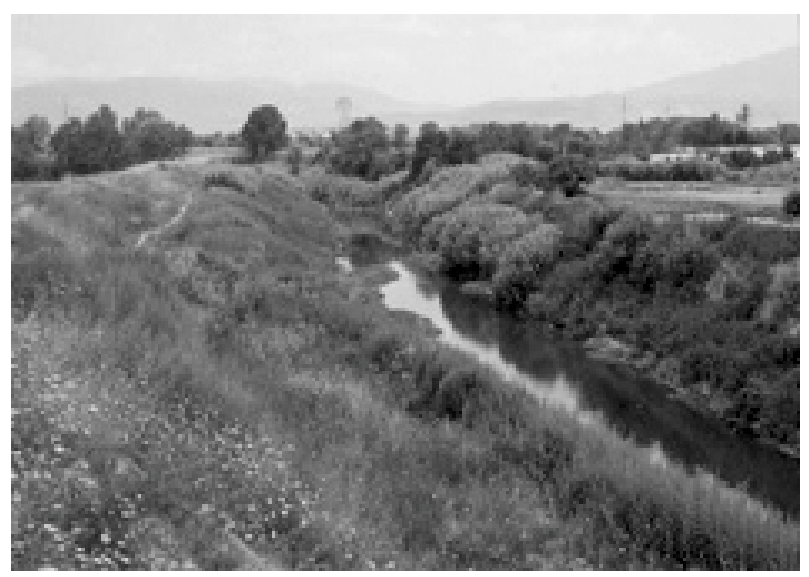

In alcuni tratti, rispetto ai canali di bonifica, la presenza di vegetazione risulta relativamente apprezzabile, nonostante sia soggetta a periodici tagli di manutenzione idraulica.

\section{DESCRIZIONE TIPOLOGICA DEL FATTORE}

Corsi d'acqua di origine naturale. Presenza irrilevante di spazi mineralizzati nelle aree extraurbane.

Nelle aree urbane i corsi d'acqua presentano un elevato grado di artificializzazione, fino alla completa mineralizzazione dell'alveo in alcuni tratti, ove non se ne riscontri la completa tombatura.

Si vedano anche le tipologie 3.5 (fasci di corsi d'acqua) e 4.3 (canali artificiali di bonifica idraulica).

Occorre considerare la diversità sostanziale rispetto ai canali artificiali, dovita all'origine naturale di questi corsi d'acqua, relativamente alla struttura ed ai processi di funzionamento e cambiamento del paesaggio.

Nonostante questo, i corsi d'acqua naturali nelle aree di pianura sono andati soggetti agli interventi di regimazione e di bonifica idraulica, fino a raggiungere talvolta la canalizzazione, con esiti di artificializzazione più o meno pesante.

Pertanto, anche i caratteri identificativi comuni di questi fattori sono doviti sostanzialmente ad esigenze dettate dagli equilibri idraulici delle pianure e dalle modalità di gestione adottate:

- la configurazione planimetrica, tendenzialmente geometrica anche se con minor rigidità di quella dei canali artificiali;

- la configurazione altimetrica, caratterizzata da argini in rilevati di terra a sezione trapezoidale fortemente geometrizzata e con forti pendenze;

- la carenza di equipaggiamento vegetale.

\section{CRITERI INTERPRETATIVI DEGLI EFFETTI}

Si vedano anche le tipologie 3.5 (fasci di corsi d'acqua) e 4.3 (canali artificiali di bonifica idraulica).

La presenza irrilevante di spazi mineralizzati nei tratti extraurbani fa sì che il peso di alterazione di questi fattori sia minore, a parità di condizioni dimensionali e di localizzazione, rispetto a tutte le tipologie precedenti di fattori che interessano i corsi d'acqua. 


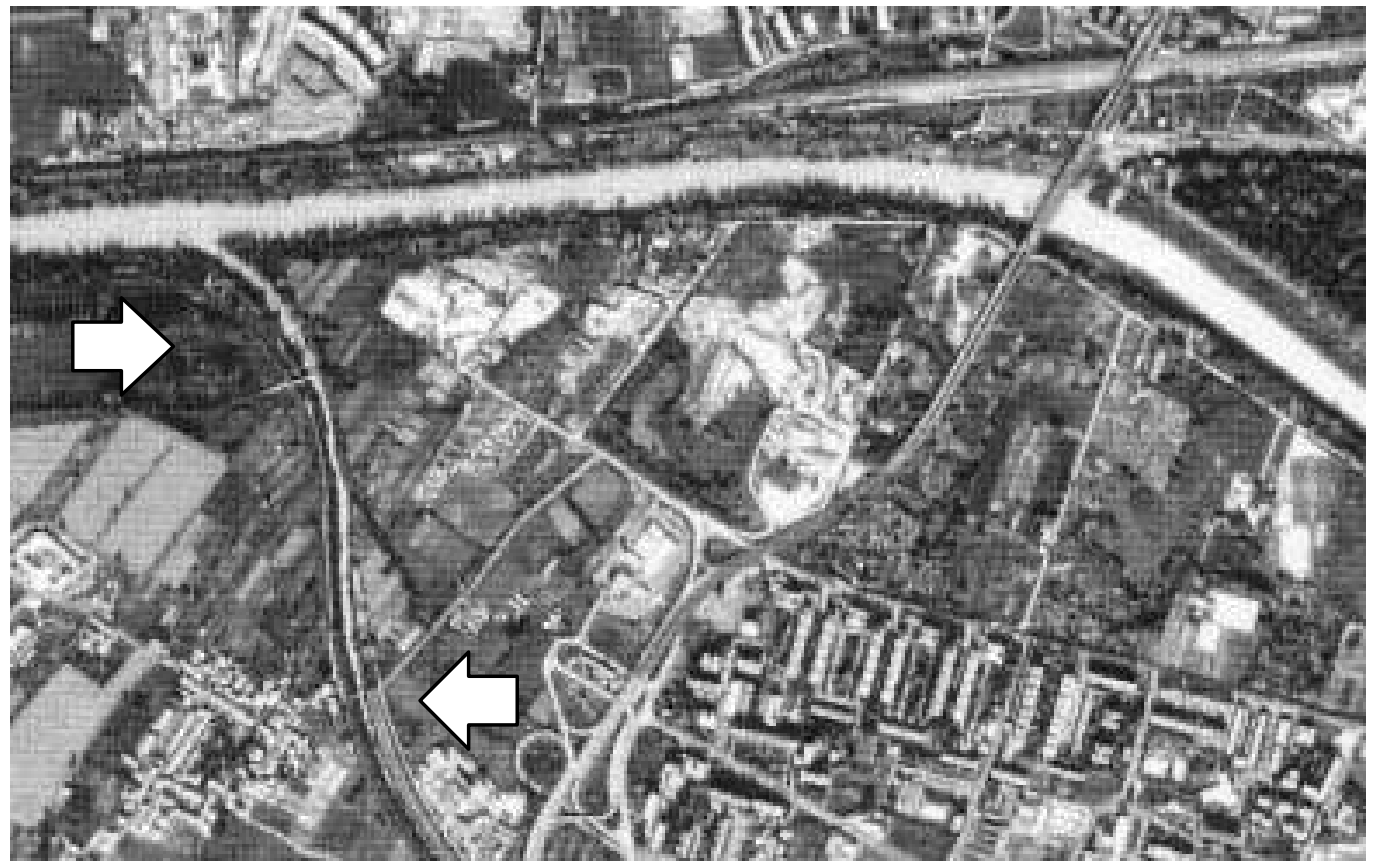

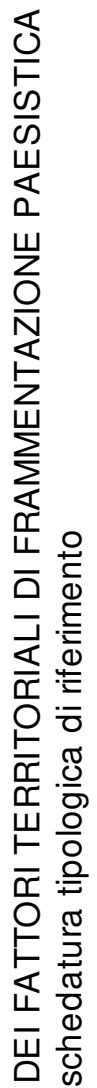

Ev evidente lan di regimazione idraulica Rappresentaziona fotografica zenital 1:15000 (da formato digitale 1:30000 - Regione Toscana).

Rappresentazione planimetrica 1:5000 (da CTR 1:10000 - Regione Toscana).

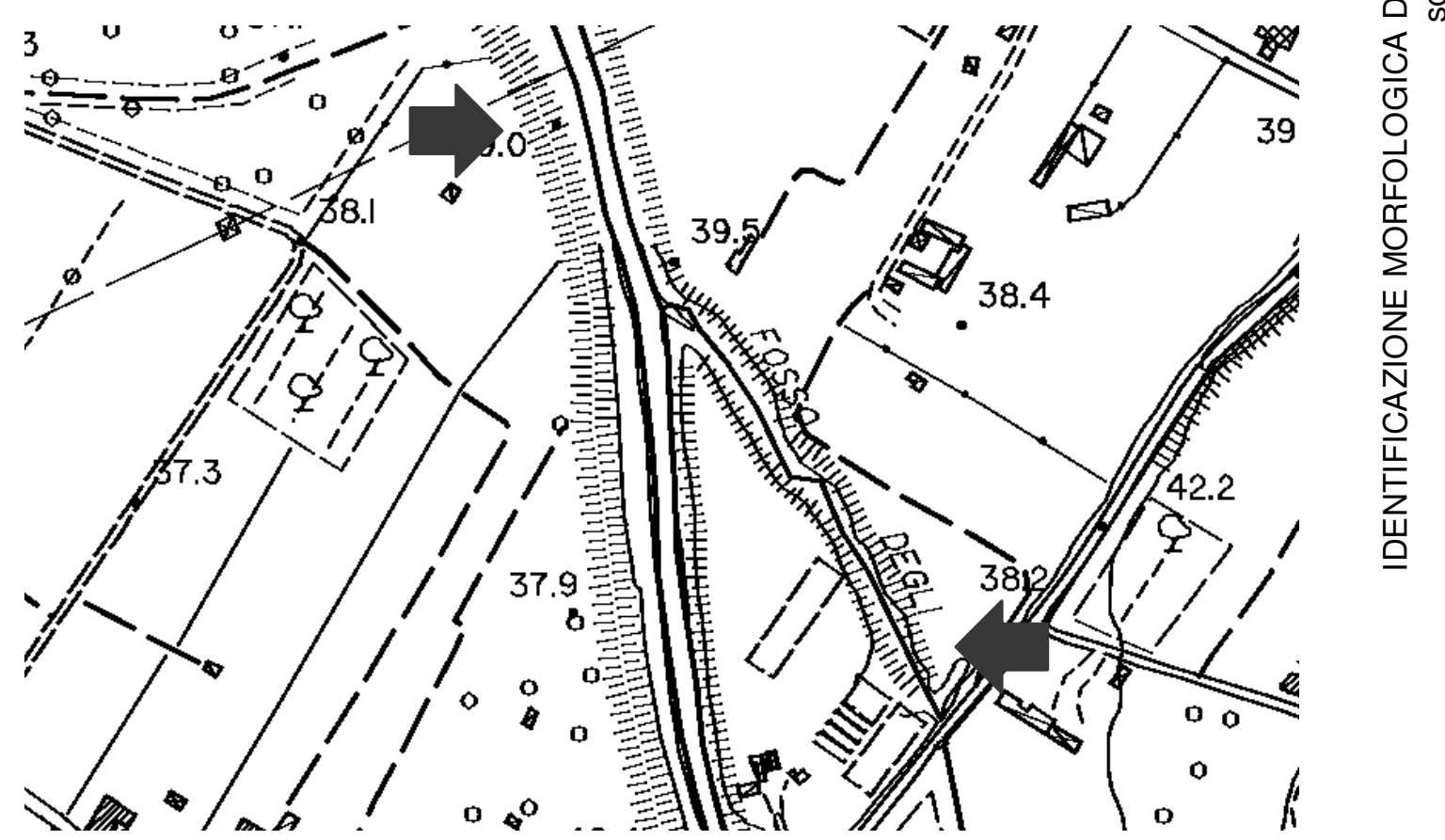




\section{$5 \quad$ FATTORI PUNTUALI}

\subsection{Edifici e complessi di edifici isolati}

\section{ESEMPLIFICAZIONI: VEDUTE}

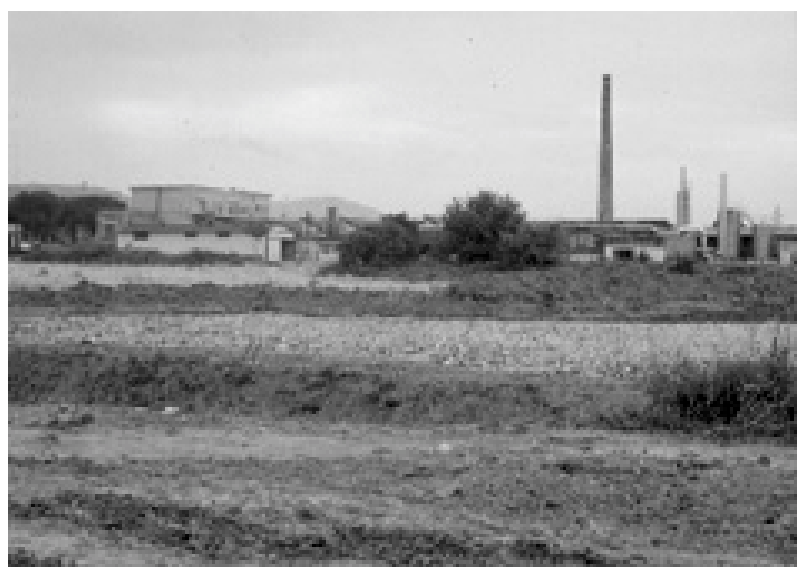

Fra le più invasive patologie del paesaggio periurbano di pianura contemporaneo vi è certamente la dispersione di edifici e spazi aperti di generi diversi e talvolta incompatibili ed inoltre privi di efficaci misure di inserimento paesistico.

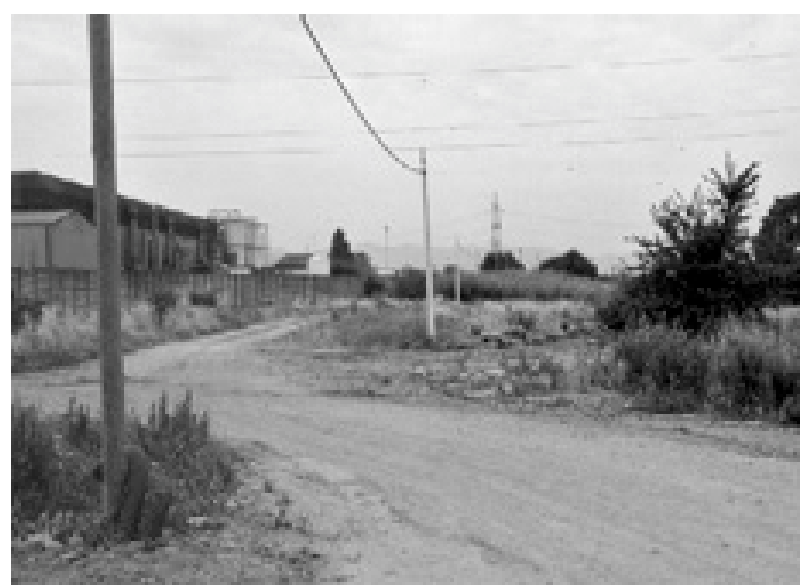

II livello di degrado che il paesaggio raggiunge in presenza di queste configurazioni deve essere considerato nel peso di questi fattori localizzati in ragione degli effetti indotti sullintorno, ben oltre il limite delle proprie aree di pertinenza.

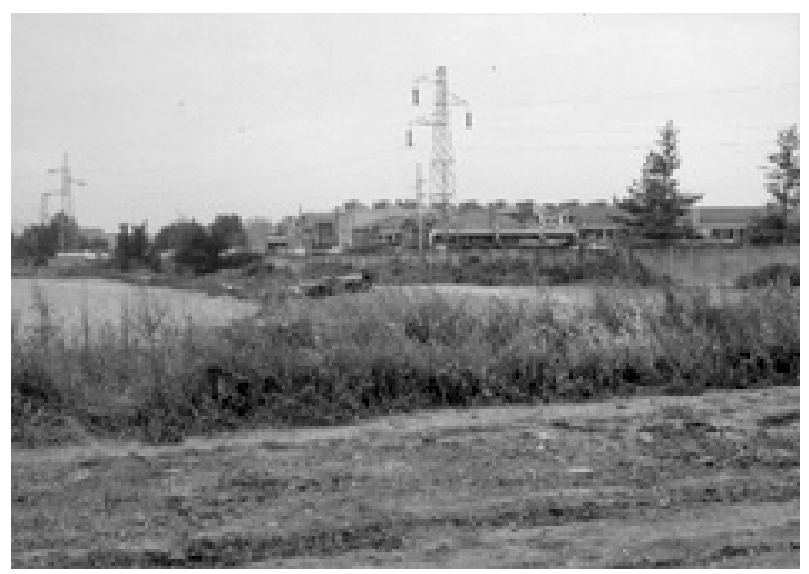

C.s.

\section{DESCRIZIONE TIPOLOGICA DEL FATTORE}

Edifici e complessi di edifici isolati. Prevalenza di spazi mineralizzati. Rappresentano la tipologia di fattori puntuali con minor grado di reversibilità delle trasformazioni. Anche le opportunità di conversione in caso di dismissione delle attività sono fortemente condizionate dal valore dei suoli $\mathrm{e}$ degli immobili, nonché dai costi di demolizione e bonifica. In queste condizioni rientra praticamente anche tutta la casistica delle aree produttive urbane dismesse, nelle quali le reali possibilità di recupero paesistico-ambientale di siti urbani strategici sono fortemente condizionate da fattori socioeconomici e culturali.

I caratteri identificativi comuni sono essenzialmente:

- la completa subalternità degli spazi aperti rispetto agli edifici, dei quali costituiscono pertinenze esclusivamente funzionali;

- la forte parcellizzazione degli spazi aperti di pertinenza degli edifici, per la quale non si costituisce un tessuto urbano, ma un mosaico disorganico di episodi limitrofi;

- la carenza quantitativa e qualitativa di equipaggiamento vegetale degli spazi aperti.

\section{CRITERI INTERPRETATIVI DEGLI EFFETTI}

Fanno parte delle configurazioni di minor impatto unitario dal punto di vista degli effetti di frammentazione paesistica, ma occorre che siano censiti e considerati a livello diagnostico relativamente agli effetti cumulativi doviti alla loro reiterazione.

Gli effetti di frammentazione sono sostanzialmente riconducibili alle seguenti categ orie:

- sottrazione di spazi non mineralizzati e delle relative configurazioni paesistiche e capacità intrinseche di rig enerazione paesistico-ambientale;

- congestione funzionale degli spazi e conseguente carico infrastrutturale, con relativi rischi di necessità di potenziamento della rete e di effetti indotti anche sulle aree circostanti;

disarticolazione dello scenario paesistico, con effetti di criticità di inserimento. 
ESEMPLIFICAZIONI: FOTOGRAFIA ZENITALE E CARTOGRAFIA

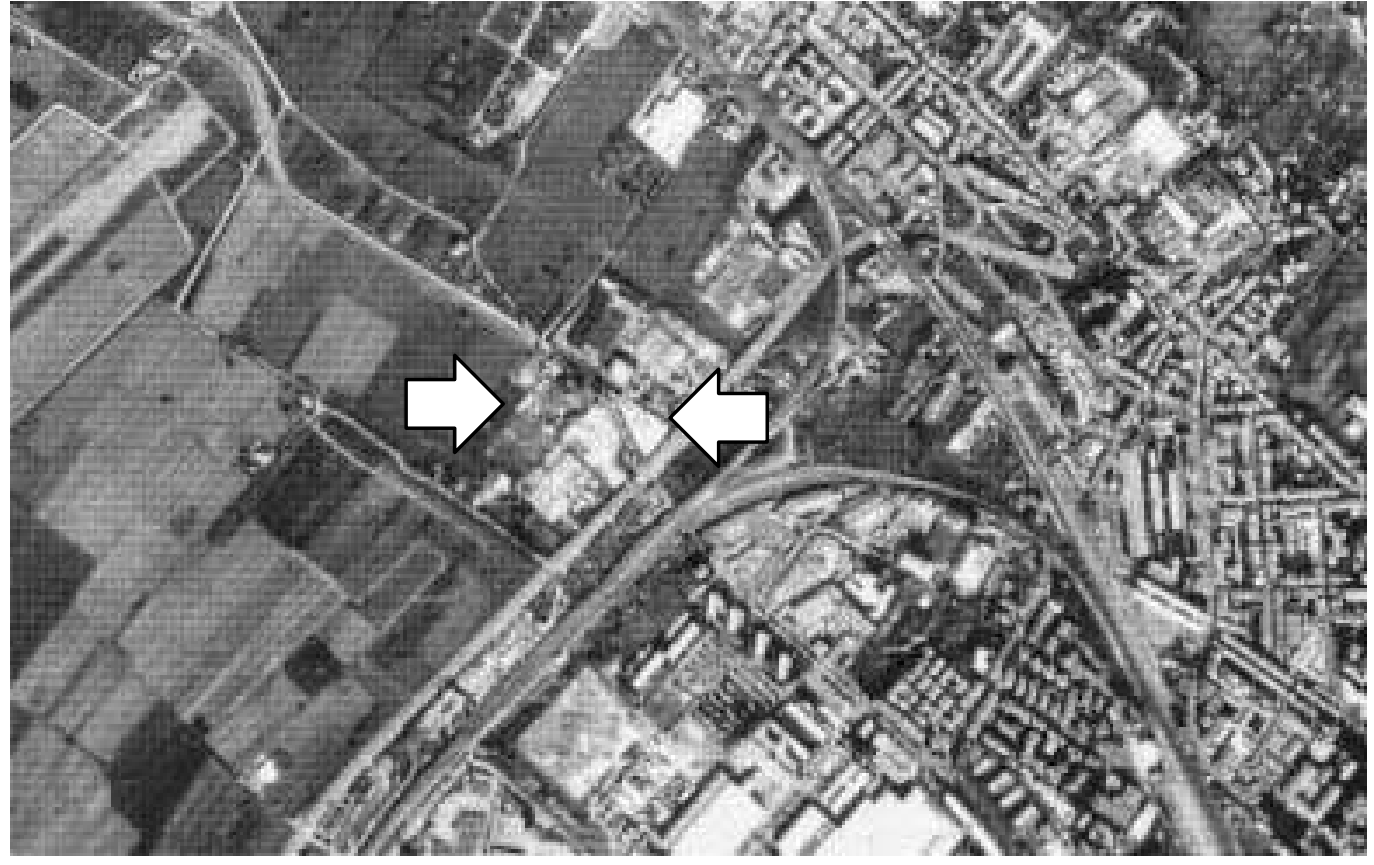

La piana di Castello ad ovest di Firenze.

La crescita disordinata di edifici e gruppi di edifici nelle aree periurbane trova generalmente nella viabilità stradale, apparato funzionale strettamente necessario, l'unico sistema del contesto con cui tesse relazioni non casuali. Gli effetti di sottrazione di paesaggio determinati da questo tin

Rappresentazione fotografica zenitale 1:15000 (da formato digitale 1:30000 - Regione Toscana).

Rappresentazione planimetrica 1:5000 (da CTR 1:10000 - Regione Toscana).

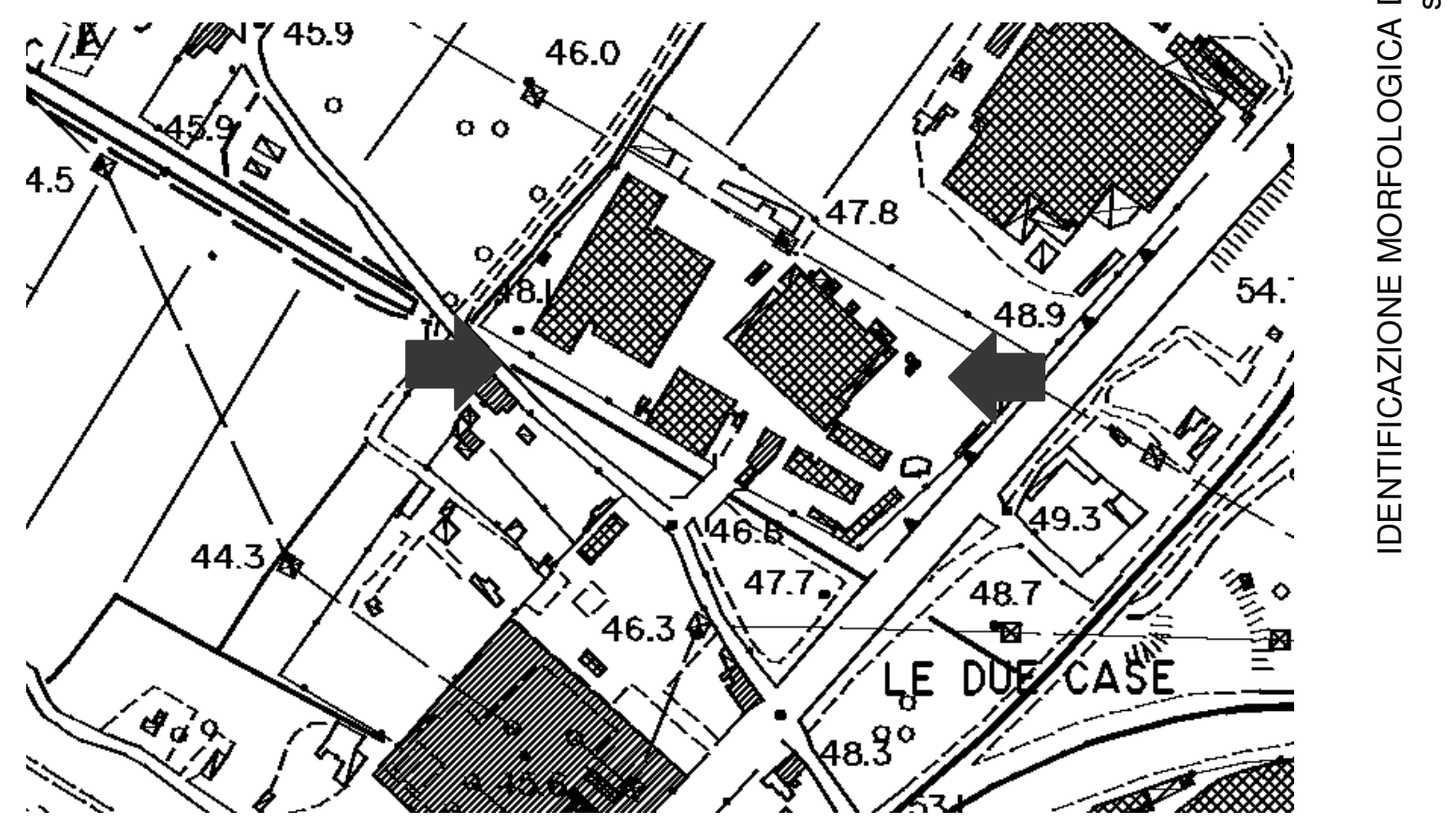




\section{$5 \quad$ FATTORI PUNTUALI}

\subsection{Spazi aperti per attività produttive non agricole}

\section{ESEMPLIFICAZIONI: VEDUTE}

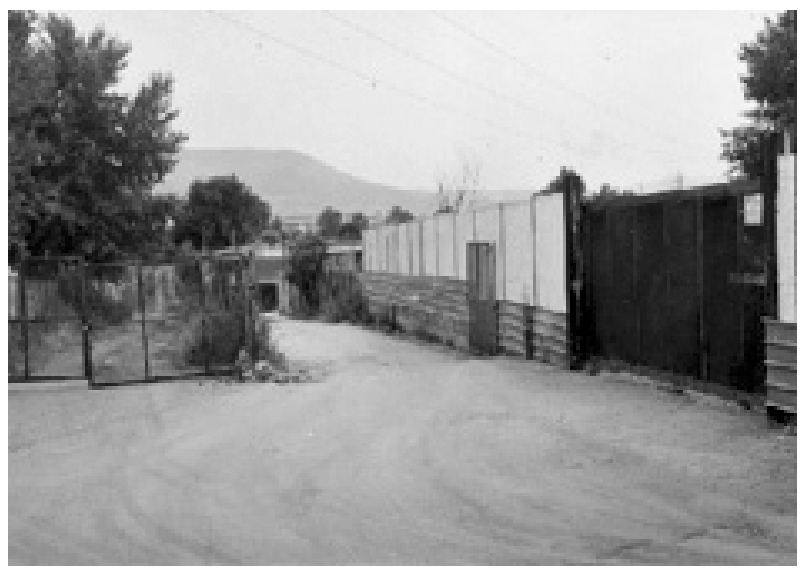

Gli spazi aperti utilizzati per attività produttive non agricole determinano effetti di rilevante alterazione paesistica e possono essere fonti di gravi fenomeni di inquinamento ambientale per gestione impropria.

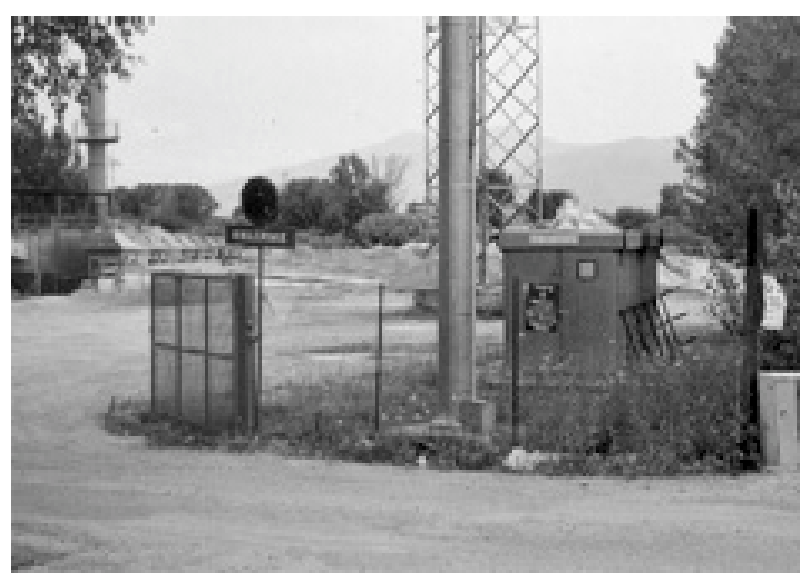

II livello di degrado che il paesaggio raggiunge in presenza di queste configurazioni deve essere considerato nel peso di questi fattori in ragione degli effetti indotti sullintorno, ben oltre il limite delle proprie aree di pertinenza.

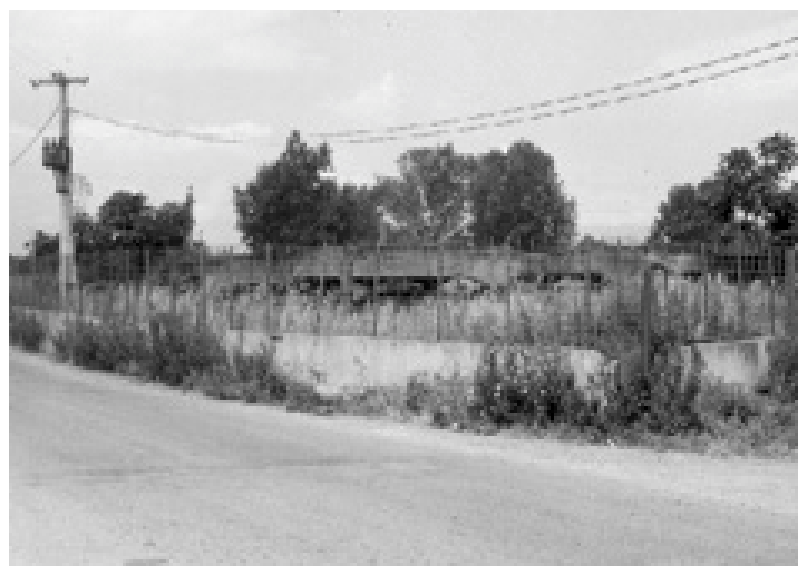

C.s.

\section{DESCRIZIONE TIPOLOGICA DEL FATTORE}

Attività produttive non agricole che si awalgono prevalentemente di spazi aperti.

Possono avere un minore grado di mineralizzazione della precedente tipologia 5.1 (edifici o complessi di edifici isolati), ma risultano comunque tendenzialmente caratterizzati da una presenza rilevante di spazi mineralizzati o a rischio di mineralizzazione.

Resta basso il grado di reversibilità delle trasformazioni, pur essendovi in questi casi maggiori opportunità di conversione e recupero paesistico-ambientale dei siti, una volta che risultino dismessi.

L'inserimento paesistico-ambientale durante il periodo di esercizio delle attività risulta invece assai più problematico che nel caso della precedente tipologia 5.1.

Queste configurazioni sono generalmente caratterizzate dalla chiusura, attraverso recinzioni, di spazi che possono assumere anche estensioni di rilievo, pur rimanendo ascrivibili alla tipologia puntuale, relativamente all'estensione sensibilmente maggiore, delle tipologie areali.

I caratteri identificativi comuni sono essenzialmente:

- criticità della articolazione degli spazi;

- criticità dei caratteri geomorfologici ed ecologici in genere;

- assenza di equipaggiamento veg etale.

\section{CRITERI INTERPRETATIVI DEGLI EFFETTI}

Fanno parte delle configurazioni di minor impatto unitario dal punto di vista degli effetti di frammentazione paesistica, ma occorre che siano censiti e considerati a livello diagnostico complessivo, relativamente agli effetti cumulativi donuti alla loro reiterazione.

Gli effetti di frammentazione sono sostanzialmente riconducibili alle seguenti categ orie:

- possibile sottrazione di spazi non mineralizzati e delle relative configurazioni paesistiche e capacità intrinseche di rigenerazione paesistico-ambientale;

alterazione geomorfologica, idrogeologica ed ecologica di componenti strutturali del paesaggio fra cui le biomasse vegetali, i suoli fertili, le falde acquifere, con i connessi rischi ambientali;

- congestione funzionale degli spazi e conseguente carico infrastrutturale con relativi rischi di necessità di potenziamento della rete e di effetti indotti anche sulle aree circostanti;

- disarticolazione dello scenario paesistico con effetti di criticità di inserimento. 


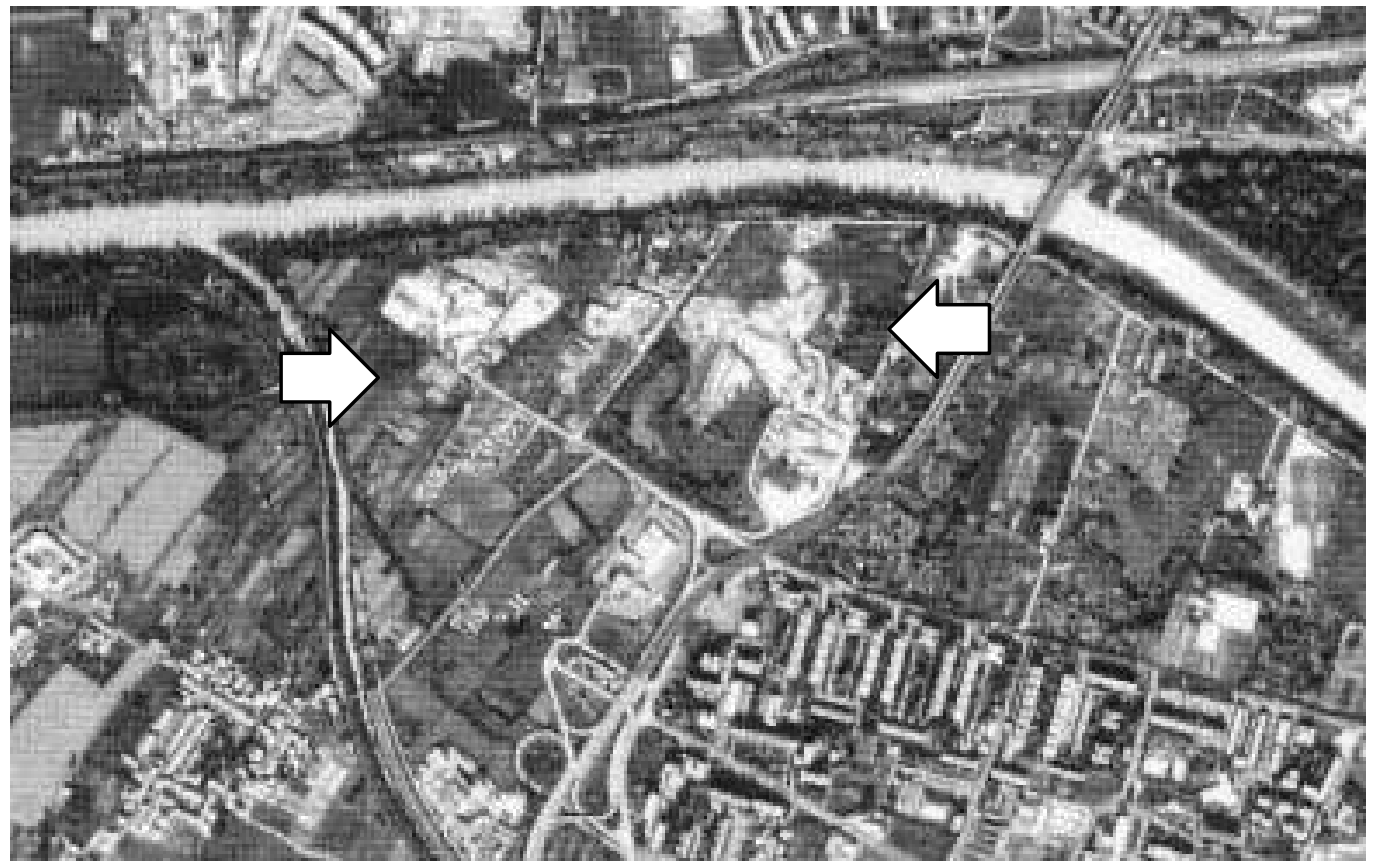

'area di Argingrosso a Firenze.

Sono evidenti le condizioni critiche del paesaggio in questa area dove operano impianti di lavorazione e stoccaggio di inerti. Gli effetti di tali fattori risultano come per le altre tipologie di maggior gravità nei casi di localizzazioni limitrofe 0 in relazione a componenti paesistiche ad elevata sensibilità storica e/o ecosistemica.

Rappresentazione fotografica zenitale 1:15000 (da formato digitale 1:30000 - Regione Toscana).

Rappresentazione planimetrica 1:5000 (da CTR 1:10000 - Regione Toscana).

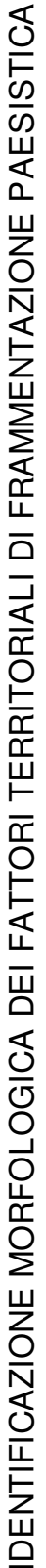

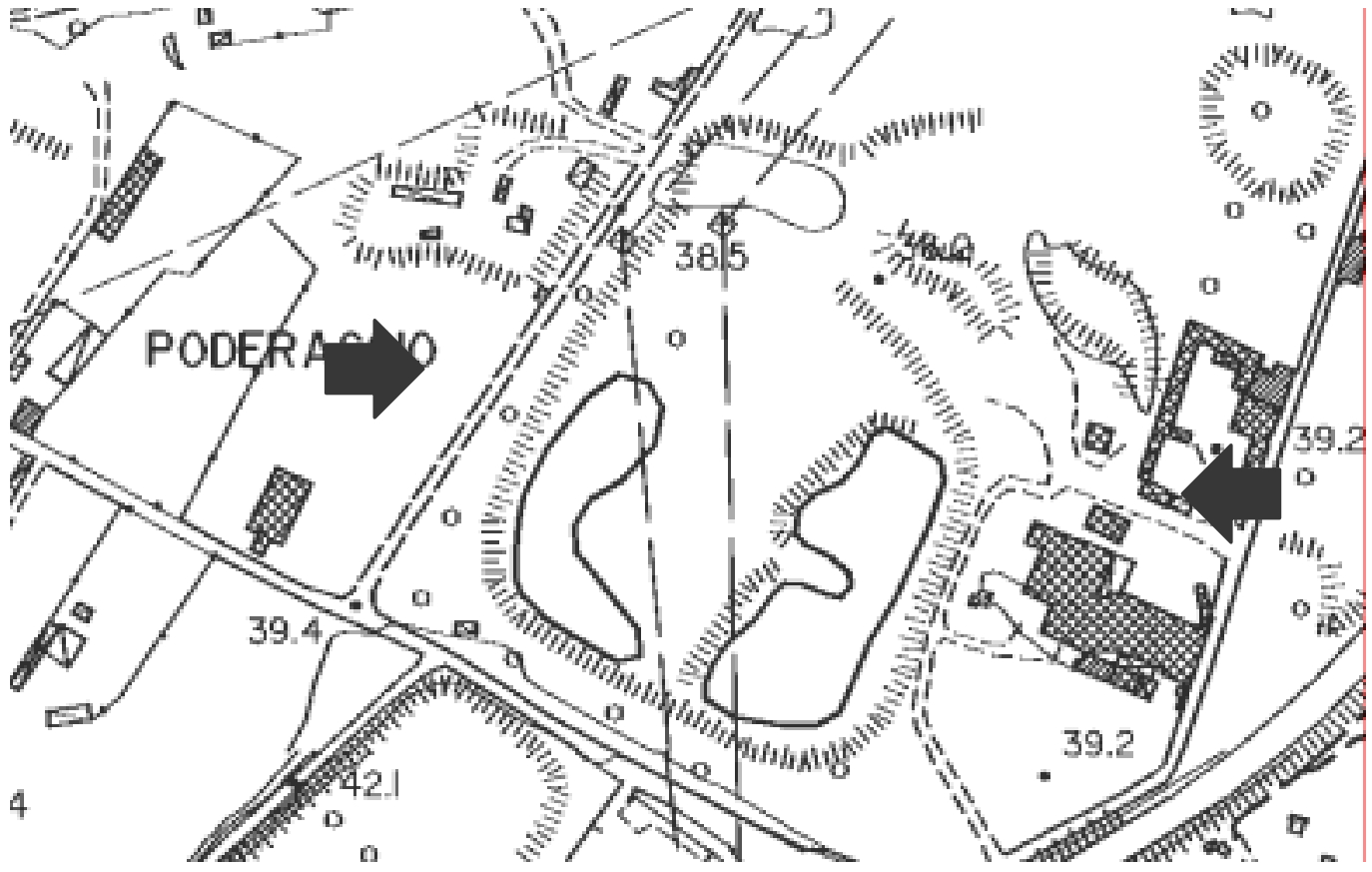




\section{$5 \quad$ FATTORI PUNTUALI}

\subsection{Spazi aperti per servizi di igiene urbana e ambientale}

\section{ESEMPLIFICAZIONI: VEDUTE}

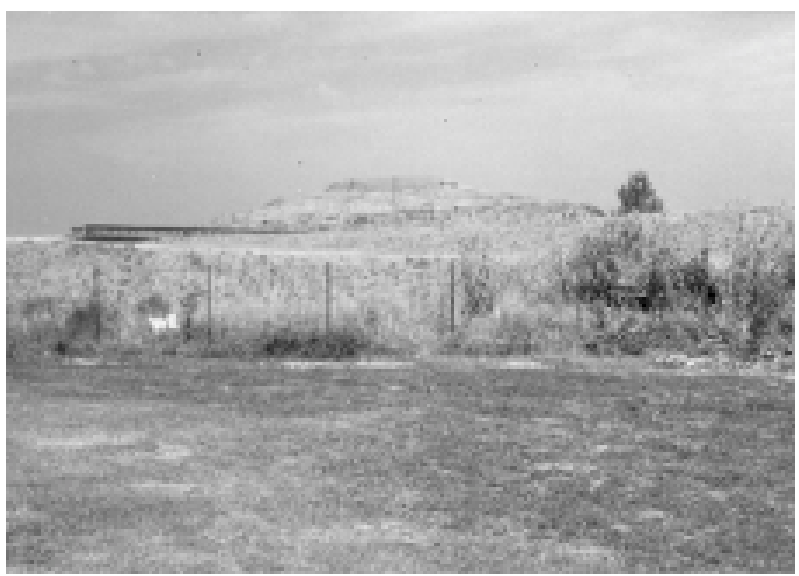

Gli spazi aperti utilizzati per servizi di igiene urbana ed ambientale determinano effetti di rilevante alterazione paesistica e possono essere fonti di gravi fenomeni di inquinamento ambientale per gestione impropria.

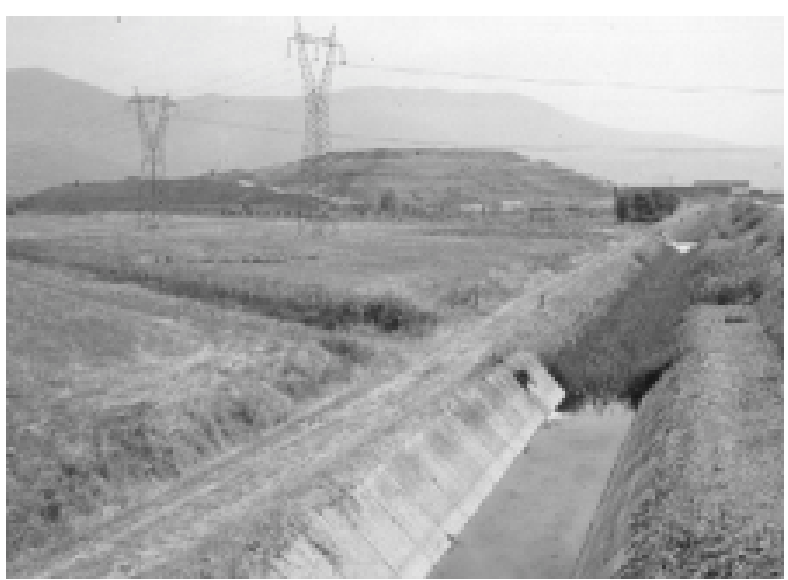

La collocazione e la sistemazione di questi spazi devono essere oggetto di pianificazione e progettazione paesistica al fine di mitigarne gli effetti negativi ed ove possibile tradurli in occasioni di progettazione di nuovi paesaggi.

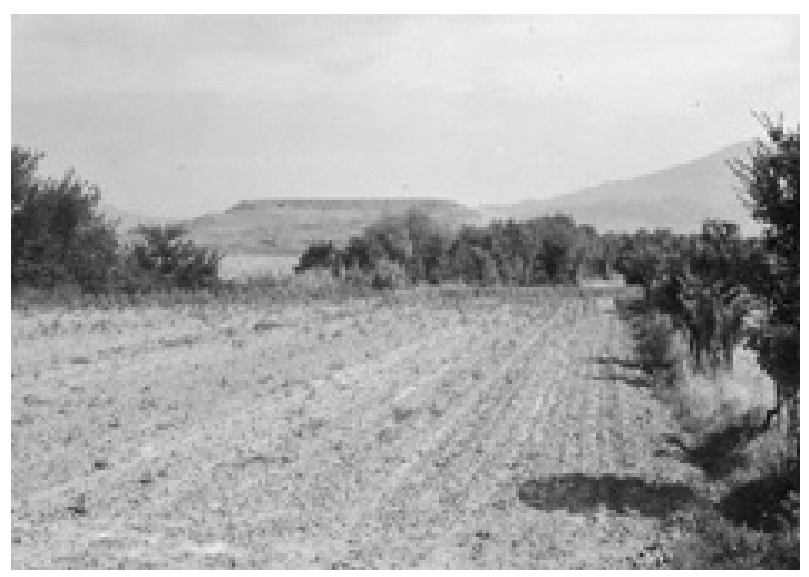

C.s.

\section{DESCRIZIONE TIPOLOGICA DEL FATTORE}

Servizi di igiene urbana e ambientale che si awalgono prevalentemente di spazi aperti. Presenza rilevante di spazi mineralizzati o comunque profondamente alterati dal punto di vista biotico.

Possono avere un minore grado di mineralizzazione delle precedenti tipologie 5.1 (edifici o complessi di edifici isolati) e 5.2 (spazi aperti per attività produttive non agricole), fino ad arrivare ad una presenza mediamente o scarsamente rilevante di spazi mineralizzati nel caso della attuazione di idonee misure di gestione, nelle fasi di esercizio, e di recupero, nelle fasi di dismissione ed a dismissione awenuta.

Resta basso il grado di reversibilità delle trasformazioni, pur essendovi in questi casi maggiori opportunità di conversione e recupero paesistico-ambientale dei siti dismessi.

L'inserimento paesistico-ambientale di queste configurazioni durante il periodo di esercizio delle attività risulta invece assai più problematico che nel caso della precedente tipologia 5.1.

Queste configurazioni sono generalmente caratterizzate dalla chiusura attraverso recinzioni di spazi che possono assumere anche estensioni di rilievo, pur rimanendo ascrivibili alla tipologia puntuale relativamente all'estensione sensibilmente maggiore delle tipologie areali.

II maggior controllo presumibile in ragione della gestione pubblica di queste attività non costituisce di fatto una garanzia sufficiente nei confronti della congruità paesistico-ambientale delle opere, che peraltro sono notoriamente oggetto di battaglie tra gli enti locali per la Ioro localizzazione e le modalità di realizzazione.

I caratteri identificativi comuni sono essenzialmente:

- criticità della articolazione degli spazi;

- criticità degli equilibri ecologici.

\section{CRITERI INTERPRETATIVI DEGLI EFFETTI}

Si veda anche la tipologia 5.2 (spazi aperti per attività produttive non agricole).

L'intensità delle alterazioni paesistiche dovite a questi fattori può essere presunta minore di quella realisticamente ad oggi tipica della precedente tipologia 5.2, a condizione che siano realmente messe in atto le misure di prevenzione e compensazione dei danni paesistico-ambientali previste dalla legislazione, dalle normative e dai regolamenti afferenti ai vari livelli di governo del territorio. 
ESEMPLIFICAZIONI: FOTOGRAFIA ZENITALE E CARTOGRAFIA

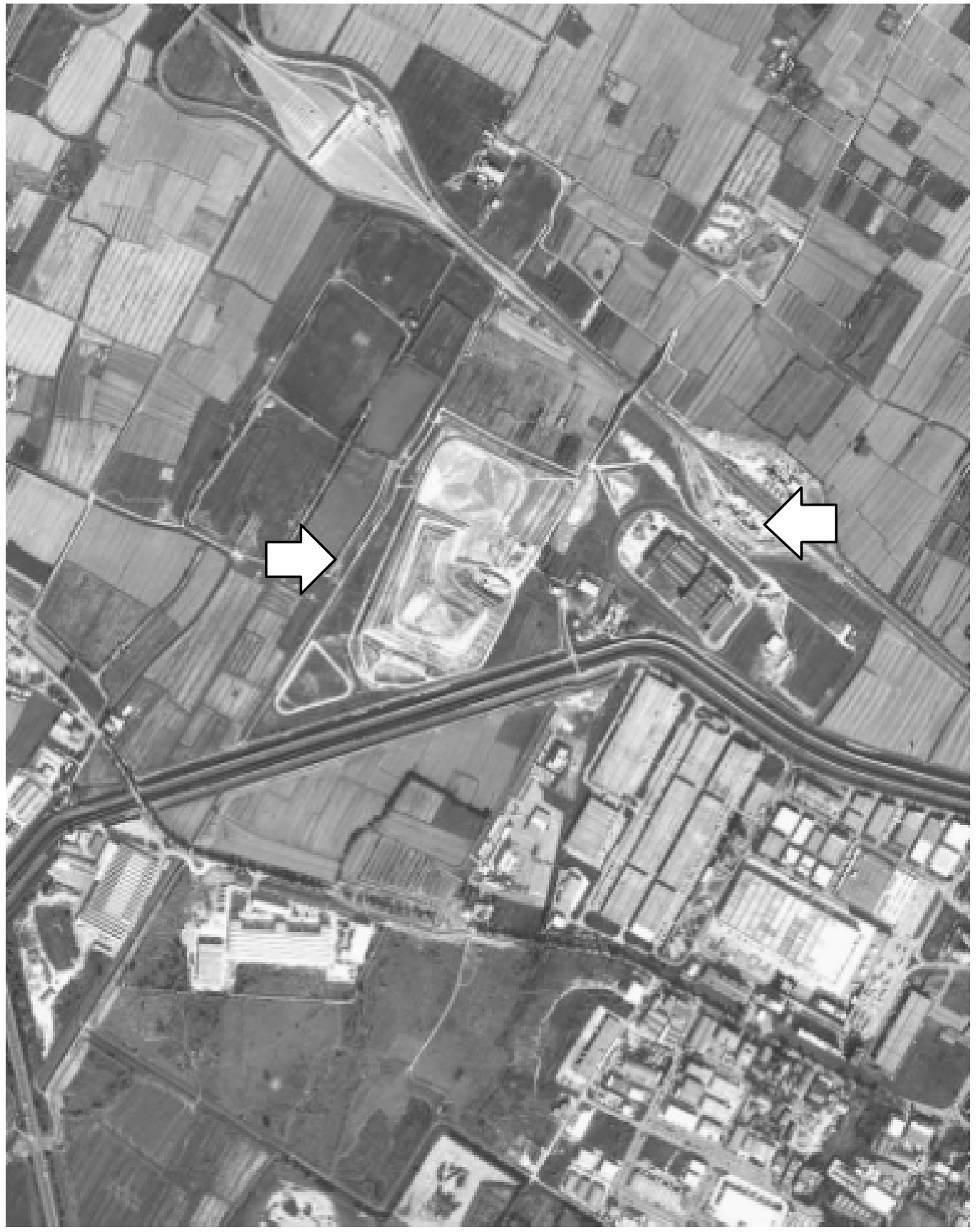

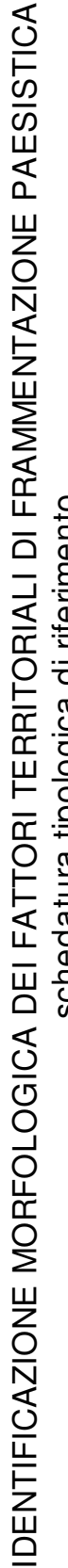

L'area di Case Passerini dove ha sede il maggiore impianto di stoccaggio e trattamento di rifiuti solidi urbani dell'area fiorentina.

L'immagine mostra l'area della discarica, fra il Fosso Reale a sud e l'autostrada A11 a nord.

II vicino impianto di trattamento di rifiuti solidi urbani e relativi spazi aperti di pertinenza, ha molti elementi in comune con i fattori della precedente tipologia 5.1 relativa alle attività produttive.

Rappresentazione fotografica zenitale 1:15000 (da formato digitale 1:30000 - Regione Toscana). 


\section{B - I corridoi paesistici secondo alcuni casi studio di piani e progetti per la conservazione della natura e del paesaggio in Italia ${ }^{146}$}

I casi studio presentati nel quadro di sintesi e nella schedatura riportati di seguito costituiscono una selezione ragionata di contributi tecnicoscientifici afferenti al panorama delle esperienze italiane riferibili alle problematiche di conoscenza, pianificazione e progettazione connesse alla frammentazione paesistica e ambientale, nell'ambito dei quali sono stati riscontrati elementi significativi rispetto allo specifico tema dei ruoli e della costituzione dei sistemi di continuità, ovvero dei corridoi paesistici.

La schedatura è stata concepita come strumento di approfondimento conoscitivo mirato e non costituisce pertanto un contributo esaustivo di descrizione dei singoli lavori, non essendo tanto questi soggetto di studio, quanto pre-testo e base di discussione ed ordinamento metodologico.

La scelta di limitare i casi studio alla realtà italiana, ha ridotto inevitabilmente l'ampiezza della gamma di esperienze disponibili, ma è stata ritenuta un criterio sostanziale di selezione, in ragione del peso determinante che hanno i contesti socio-culturale e territoriale nella discussione degli argomenti trattati.

La schedatura non costituisce un pacchetto chiuso e completo, bensì un riferimento ampliabile, al quale la scelta delle esperienze riportate ha voluto conferire una rappresentatività delle diverse scale alle quali può essere riconosciuta e gestita la problematica della frammentazione paesistica ed ambientale. Pertanto, l'omogeneità dei casi studio è data esclusivamente dal tema generale della frammentazione e dai contenuti di considerazione dei sistemi lineari di continuità, mentre differiscono tutti reciprocamente di scala, passando da quella locale sub-comunale, alla quale si esplicano le decisioni finali di progetto e quelle di attuazione, fino a quella regionale che, comprendendo ancora valutazioni e definizioni progettuali riferite agli strumenti della pianificazione del territorio, permette di gestire le relazioni con la scala nazionale e transnazionale, inevitabilmente connessa alle problematiche considerate, ma allo stesso tempo idonea solamente a scenari di regolamentazione e di programmazione, non direttamente riferibili alle realtà territoriali per la connaturata parzialità del grado di definizione topologica e strategica che li caratterizza.

Due casi (caso studio 1 e caso studio 5) costituiscono esperienze edite uniche in Italia nel loro genere di scala, così, nel caso dell'Abruzzo, l'interesse prescinde per questa ragione dalla coincidenza tipologica dei soggetti territoriali trattati con quello a cui si riferiscono le applicazioni di questa ricerca. Il fatto che il territorio abruzzese, in assenza di bacini di pianura con pressione insediativa corrispondente a quella del soggetto qui considerato, non toglie infatti validità alle argomentazioni derivabili dallo studio di tali materiali, pur richiedendone la relativizzazione alle differenze territoriali.

${ }^{146}$ Indagine condotta nel 1999 e 2000. 


\begin{tabular}{|c|c|c|c|}
\hline N & zona (scala) & IDENTIFICABILITÀ MORFOLOGICA & IDENTIFICABILITÀ STRATEGICA \\
\hline 1 & $\begin{array}{c}\text { LOMBARDIA } \\
\text { TORNAVENTO } \\
\text { (locale sub comunale) }\end{array}$ & $\begin{array}{l}\text { NON RISCONTRATA } \\
\text { La funzione di continuità biotica della struttura del } \\
\text { paesaggio riconosciuta alle formazioni } \\
\text { paesistiche lineari è subordinata alla } \\
\text { considerazione del corridoio come strategia } \\
\text { complessa. }\end{array}$ & $\begin{array}{l}\text { RISCONTRATA } \\
\text { ॥l corridoio è costituito da un complesso di } \\
\text { spazi con destinazione d'uso e funzioni } \\
\text { paesistico-ambientali distinte, per i quali il } \\
\text { progetto prevede una serie di interventi } \\
\text { diversificati. }\end{array}$ \\
\hline 2 & $\begin{array}{c}\text { EMILIA ROMAGNA } \\
\text { BOLOGNA } \\
\text { (comunale/provinciale) }\end{array}$ & $\begin{array}{l}\text { RISCONTRATA } \\
\text { I corridoi sono costituiti da formazioni paesistiche } \\
\text { lineari, con funzioni prioritarie di miglioramento } \\
\text { della continuità biotica del paesaggio e di } \\
\text { miglioramento ambientale degli ecosistemi agrari } \\
\text { di pianura. }\end{array}$ & $\begin{array}{l}\text { NON RISCONTRATA } \\
\text { La previsione di un sistema binario di } \\
\text { corridoi primari, lungo i corsi d'acqua, e } \\
\text { secondari, terrestri, trasversali ai primi, } \\
\text { costituisce una politica strategica parte } \\
\text { integrante del progetto di rete ecologica, } \\
\text { ma essa non si riferisce ai corridoi come } \\
\text { entità bensì al complesso di effetti } \\
\text { paesistici attendibile dalla progressiva } \\
\text { realizzazione dell'insieme delle previsioni. }\end{array}$ \\
\hline 3 & $\begin{array}{l}\text { LOMBARDIA } \\
\text { PAVIA } \\
\text { (provinciale) }\end{array}$ & $\begin{array}{l}\text { RISCONTRATA } \\
\text { idem n. } 2\end{array}$ & $\begin{array}{l}\text { NON RISCONTRATA } \\
\text { idem n. } 2\end{array}$ \\
\hline 4 & $\begin{array}{c}\text { LOMBARDIA } \\
\text { MILANO } \\
\text { (provinciale metropolitana) }\end{array}$ & $\begin{array}{l}\text { NON RISCONTRATA } \\
\text { idem n. } 1\end{array}$ & $\begin{array}{l}\text { RISCONTRATA } \\
\text { I corridoi sono previsti nel progetto direttore } \\
\text { a scala provinciale e definiti nei progetti di } \\
\text { approfondimento come complessi di spazi } \\
\text { con destinazione d'uso e funzioni } \\
\text { paesistico-ambientali distinte, per i quali il } \\
\text { progetto prevede una serie di interventi } \\
\text { diversificati. }\end{array}$ \\
\hline 5 & $\begin{array}{l}A B R \cup Z Z O \\
\text { (regionale) }\end{array}$ & $\begin{array}{l}\text { NON RISCONTRATA } \\
\text { La scala di lettura e progettazione non considera } \\
\text { la funzione di continuità biotica della struttura del } \\
\text { paesaggio specificamente afferente alle } \\
\text { formazioni paesistiche lineari. }\end{array}$ & $\begin{array}{l}\text { RISCONTRATA } \\
\text { La previsione di un sistema di corridoi } \\
\text { primari lungo le principali direttrici territoriali } \\
\text { di continuità paesistica ed ambientale } \\
\text { (biocanali) costituisce parte integrante del } \\
\text { disegno strategico della rete ecologica } \\
\text { regionale. }\end{array}$ \\
\hline
\end{tabular}


Caso studio 1 - Lombardia (scala locale sub comunale)

Il corridoio ecologico di Tornavento: un progetto di compensazione ambientale delle infrastrutture lineari dell'aereoporto di Malpensa $2000^{147}$

Tipologia e finalità dello studio

Studio di progettazione paesistica finalizzato alla definizione delle misure di mitigazione e compensazione degli interventi di realizzazione del tratto di strada a scorrimento veloce "Nuova S.S. 336" di collegamento dell'aereoporto Malpensa 2000 con la S.S. 527 Lonate-Oleggio. II progetto è finalizzato alla salvaguardia ed al miglioramento della continuità biotica e spaziale del paesaggio tra le brughiere ed i boschi di Somma Lombardo e la valle del Ticino attraverso la realizzazione di un corridoio ecologico in un territorio ad elevato incremento della pressione antropica donita agli sviluppi infrastrutturali.

\section{Elaborazione}

Parco Regionale Lombardo della Valle del Ticino Dott. arch. L. Giamporcaro

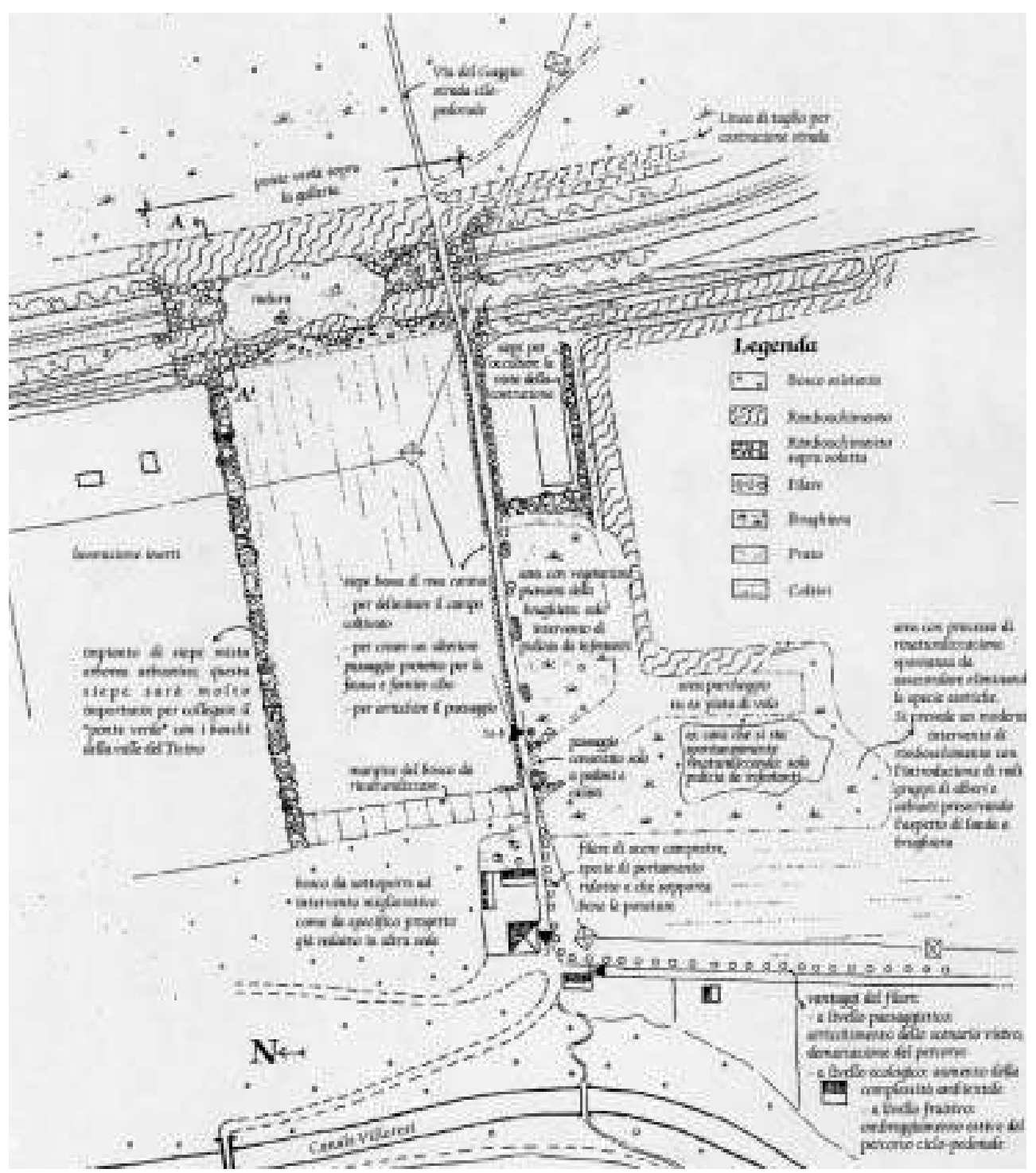

Figura B1 -

Progetto preliminare (riduzione della planimetria generale, originale in scala 1:2000).

${ }^{147}$ Le citazioni riportate fra virgolette e le immagini sono tratte da D. Furlanetto, in C. Dimaggio, R. Ghiringhelli (a cura), Reti ecologiche in aree urbanizzate, atti del Seminario promosso dalla Provincia di Milano e da ANPA, Milano, 5 febbraio 1999, Franco Angeli, Milano, 1999 e dalla relazione di D. Furlanetto per il Convegno Internazionale Reti ecologiche - Azioni locali di gestione territoriale per la conservazione dell'ambiente, Gargnano, 12-13 ottobre 2000. 


\section{Area di studio}

Lo studio riguarda la località Tornavento nel comune di Lonate Bozzolo in provincia di Varese e considera un'area di intervento che varia da un minimo di $200 \mathrm{ml}$ circa nella direzione dell'asse stradale in corrispondenza delle strade stesse, dove è previsto I'interramento della viabilità di progetto e di quella preesistente, a $1 \mathrm{~km}$ circa nella direzione di connessione della brughiera alla Valle del Ticino, trasversalmente alle strade.

Impostazione metodologica ed elementi di interesse relativi alla considerazione dei caratteri paesistici

Accanto al significato ecologico, l'intervento si prospetta anche come un vero e proprio "restauro del paesaggio, dove ci si è preoccupati di realizzare spazi, forme, materiali, in questo caso viventi, come lo sono i vegetali, per costruire una scenografia dalle molte valenze (paesaggistica, contemplativa, sociale, ecosistemica). II corridoio ecologico di Tornavento si presenta come un'occasione per rinaturalizzare un'intera porzione di territorio: da un progetto a fini prevalentemente faunistici-ecologici è scaturito un miglioramento ambientale complessivo (...)".

II progetto prevede misure di recupero quantitativo e qualitativo dell'equipaggiamento vegetale del paesaggio che, attraverso le tipologie principali del bosco, del filare e della siepe, mirano alla acquisizione di caratteri di interesse spaziale e visuale, oltre che di funzionalità ecosistemica per il miglioramento della connessione degli habitat. Tali misure sono improntate alla valorizzazione ed al rispetto delle pratiche colturali agrarie in essere ed al miglioramento delle condizioni complessive del paesaggio, comprese le esigenze di mitigazione dell'impatto visuale di attività problematiche, quali la lavorazione di inerti. In tal senso la simulazione riportata nella figura B2 costituisce una descrizione del rapporto positivo che esiste tra la semplicità degli interventi e la loro efficacia morfologica.
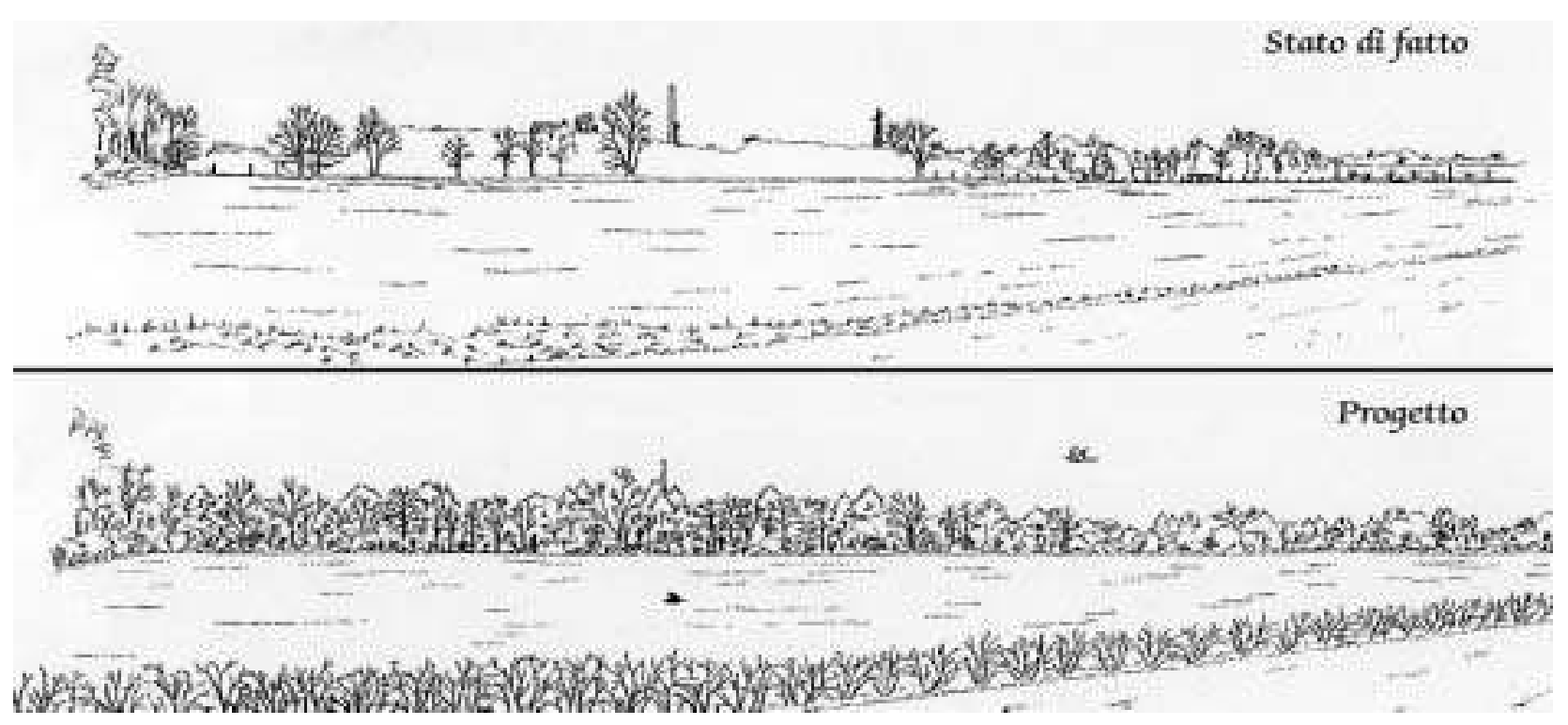

Figura B2 - Le siepi arboree e/o arbustive, oltre a svolgere funzioni di continuità biotica, permettono la mitigazione degli effetti visuali critici, in questo caso donuti ad un impianto di lavorazione di inerti di cui il grafico delle stato di fatto indica lo skyline dei rilevati di accumulo. Progetto preliminare (riduzione di simulazione prospettica degli effetti delle sistemazioni vegetali sullo scenario paesistico del paesaggio di pianura).

Elementi di interesse per il tema specifico della ricerca con riferimento alla tipologia del corridoio nell'ambito delle problematiche della frammentazione paesistica

In ragione dell'impostazione metodologica che lo caratterizza, lo studio individua una articolazione del corridoio rispondente a più finalità ed usi. "Gli elementi che fanno parte del corridoio ecologico sono:

- il ponte verde, owero il tratto di galleria artificiale rimboschito soprasoletta, che permette di superare la SS 336 e la SP 52;

- le siepi che consentono al "ponte verde" di connettere i boschi della brughiera di Lonate Bozzolo con i boschi della Valle del Ticino;

- la strada sterrata che congiunge Via del Gaggio con Cascina Parravicino;

- il parcheggio dei visitatori del Parco".

II corridoio si configura pertanto come una strategia unitaria finalizzata a costituire e gestire un sistema differenziato di spazi aperti, individuati e progettati per essere idonei, nelle loro caratteristiche intrinseche e relazionali, alle funzioni complessive primarie di connessione paesistica della brug hiera alla valle del Ticino.

"Se il "ponte verde" costituisce il nucleo centrale dell'intervento, per integrare la brughiera lonatese nel resto del Parco del Ticino risultano altrettanto importanti le siepi di connessione fra il rinverdimento soprasoletta e l'ambiente circostante. In effetti il corridoio ecologico resterebbe strutturalmente carente se non si pensasse anche a connettere il rimboschimento sopra la galleria con i boschi che si affacciano sulla valle fluviale. (...) In questo modo si creerà un collegamento privo di grossolane interferenze antropiche tra la brughiera di Lonate Bozzolo ed il resto del Parco e si manterrà l'antico tracciato ed una porzione di paesaggio circostante via del Gaggio, strada storica di collegamento tra Lonate e la Cascina Parravicino - ex dogana austroungarica - e il fiume. Oggi la via del Gaggio è stata resa pista ciclopedonale dal Comune di Lonate Bozzolo ed è particolarmente cara agli abitanti del luogo, oltre che per la memoria storica, anche per gli aspetti ricreativi ad essa connessi". 


\section{Caso studio 2 - Emilia Romagna (scala provinciale/comunale) Piano programmatico per la conservazione e il miglioramento degli spazi naturali in provincia di Bologna ${ }^{148}$}

\section{Tipologia e finalità dello studio}

Studio di pianificazione paesistica, preliminare alla elaborazione del Piano Territoriale di Coordinamento Provinciale, finalizzato alla definizione di uno scenario di rete ecologica con particolare riferimento ai sistemi locali di connessione degli habitat nei territori di pianura ad elevata pressione antropica.

\section{Elaborazione}

Provincia di Bologna, Assessorato Ambiente, Servizio Pianificazione Pesistica.

Dott. arch. Paola Altobelli, dirigente del Serv. Pianificazione Paesistica: direzione; dott. arch. Giuseppe De Togni: coordinamento tecnico-scientifico; dott. Rossella Ghedini: coordinamento tecnico-organizzativo; Andrea Morrisi - Centro Agricoltura Ambiente; Roberto Tinarelli: consulenza naturalistica; dott. Claudio Zoppellari e Francesco Marucci, coop Alveo: progettazione e realizzazione GIS; dott. Paolo Roli, geom. Antonio Landini: collaborazione.

Periodo: 1998-2000

\section{Area di studio}

Lo studio riguarda l'intero territorio di competenza amministrativa della Provincia di Bologna. L'area comprende pertanto i tre tipi principali di paesaggio di questo ambito geografico: (a) il paesaggio a matrice forestale e agrosilvopastorale dei rilievi montani $e$ submontani del versante settentrionale della dorsale appenninica; (b) il paesaggio a matrice mista agro-urbana generato dallo sviluppo insediativo lungo la via Emilia e la parallela autostrada e ferrovia; (c) il paesaggio a matrice agricola della pianura Padana a nord del precedente.

Impostazione metodologica ed elementi di interesse relativi alla considerazione dei caratteri paesistici

Alla rete ecologica sono attribute funzioni paesistiche ed ambientali diversificate ${ }^{149}$ in considerazione del fatto che "soprattutto per le aree altamente antropizzate e importanti dal punto di vista produttivo (...) appaiono improponibili soluzioni alternative per la conservazione diffusa del patrimonio ambientale e paesaggistico (...) dal momento che la creazione di grandi aree protette non troverebbe né la concreta possibilità spaziale, né l'effettiva presenza di vaste estensioni da tutelare".

La rete è proposta pertanto come politica articolata di conservazione della natura e del paesaggio improntata alla concreta praticabilità relativamente alle condizioni dei territori di pianura, nella misura in cui distingue il valore delle risorse ed attribuisce ruoli prioritari, secondo obiettivi di equilibrio tra le istanze di conservazione e quelle di produzione. Pur riguardando un territorio ad elevata antropizzazione e caratterizzato da presenze insediative di rilievo metropolitano, lo studio non ha sviluppato un sistema di analisi e diagnosi idoneo alla conoscenza profonda dei caratteri culturali del paesaggio, sia relativamente alle condizioni ambientali della campagna e degli insediamenti, che alle condizioni di equipaggiamento paesistico e fruibilità ricreativa della rete degli spazi rurali, che alle condizioni di conservazione dei gradi di permanenza storica. L'argomento compare nella progettazione delle modalità di uso e gestione delle risorse alla scala locale, nell'ipotesi elaborata sul territorio del comune di Bentivoglio, dove "i segni del paesaggio contemporaneo" diveng ono elementi strutturali di appog gio e confronto per la definizione della rete.

Gli interventi di modificazione e gestione del paesaggio sono distinti secondo le due tipologie primarie della "rinaturazione" e della "rinaturalizzazione" ${ }^{\prime 150}$ e descritti dal punto di vista tecnico prog ettuale e gestionale.

Rientrano nelle misure di "rinaturazione" (finalizzate all'incremento della naturalità di un ecosistema preesistente): le modalità di intervento sugli agroecosistemi delle colture agrarie, le modalità di conservazione dei boschi, le modalità di conservazione $e$ piantagione di alberi, le modalità di conservazione dei maceri abbandonati o in abbandono.

Rientrano nelle misure di "rinaturalizzazione" (finalizzate alla realizzazione di neoecosistemi): le modalità di piantagione e/o gestione di siepi, boschi e macchie, filari e alberate stradali, unità erbacee (prati, pascoli, terreni saldi incolti); le modalità di realizzazione e gestione di zone umide.

Elementi di interesse per il tema specifico della ricerca con riferimento alla tipologia del corridoio nell'ambito delle problematiche della frammentazione paesistica

Lo studio individua fra i criteri significativi di valutazione delle condizioni di frammentazione del paesag gio:

- i fattori limitanti intrinseci alla frammentazione degli habitat: relativamente alle dirette conseguenze di riduzione complessiva della superficie territoriale riferibile a spazi seminaturali relitti;

- la geometria degli spazi seminaturali relitti: relativamente ai diversi effetti di cui essa risulta responsabile nella insularizzazione biog eog rafica;

- le caratteristiche delle specie presenti negli spazi seminaturali relitti: relativamente alle specifiche modalità di dispersione $e$ conseguenti capacità di colonizzazione;

- la natura delle barriere intermedie: relativamente agli effetti delle diverse tipologie di barriera in funzione della loro collocazione in paesag gi con matrici diverse e delle sing ole categ orie di specie naturali;

- l'esistenza di "corridoi eco-biolog ici": relativamente alle funzioni di connessione di habitat ad essi attribuite.

Le funzioni di corridoio ecologico considerate nel piano fanno riferimento alle seguenti configurazioni paesistiche lineari:

- $\quad$ "sistemi di siepi e fasce arboreo-arbustive tra terreni coltivati;

- sistemi ripari a veg etazione arboreo-arbustiva lung o corsi d'acqua che scorrono in matrici territoriali artificializzate;

${ }^{148}$ Le citazioni riportate fra virg olette e le immagini sono tratte dai documenti originali dello studio.

${ }^{149}$ Si veda Malcevschi S., Reti ecologiche, lo stato dell'arte, ACER, 4, 1998.

150 Per le definizioni oltre a quanto riportato nei documenti originali dello studio nella loro versione integrale si veda: Bisogni L.G., Gariboldi A., Malcevschi S., Reti ecologiche ed interventi di miglioramento ambientale, II Verde Editoriale, Milano, 1996. 
- $\quad$ fasce arboree ed arbustive connesse ad infrastrutture lineari che attraversano una matrice antropizzata;

- $\quad$ sistemi di spazi aperti lineari con veg etazione erbacea entro matrici boscate".

II corridoio è pertanto considerato nella accezione morfologica di formazione paesistica con soprassuolo prevalentemente vegetale arboreo e/o arbustivo.

Lo studio evidenzia la necessità di articolare la rete differenziando i corridoi:

- $\quad$ "è in genere opportuno individuare un primo sistema di corridoi ecologici imperniato sulla rete idrografica dal momento che quest'ultima, oltre a costituire un sistema comprendente habitat solitamente di un certo interesse, è spesso affiancata ad aree di proprietà pubblica (consorzi di bonifica, demanio statale), elementi e soggetti che dovrebbero garantire una semplificazione delle possibilità di intervento;

- conseguentemente è generalmente opportuno individuare un secondo sistema integrativo di corridoi "terrestri", tendenzialmente trasversale al precedente, che ne attenua la struttura "a pettine", evitando in questo modo sia di trascurare le situazioni ecologiche mesofile e xerofile ad esclusivo vantaggio di quelle igrofile ed idrofile, sia di lasciare i diversi corridoi idrog rafici relativamente segregati l'uno rispetto all'altro"

Questo ultimo punto può costituire, se sviluppato sulla base di idonee conoscenze paesistiche, un ampio spazio di incontro delle istanze di miglioramento delle condizioni ecologiche degli habitat animali e vegetali con quelle di riqualificazione del paesaggio di pianura e conseguentemente di conservazione e miglioramento ambientale degli ecosistemi agrari e urbani.
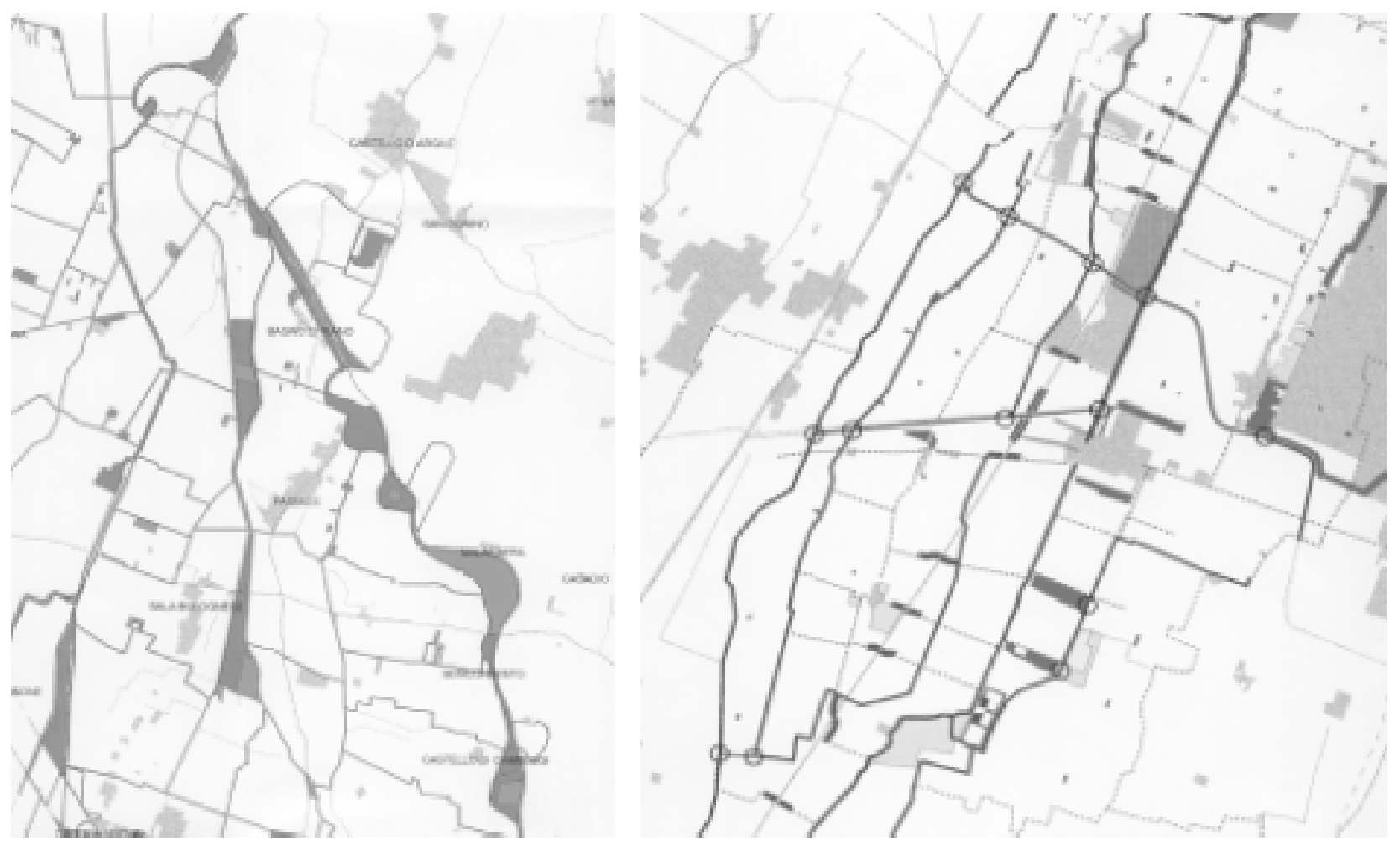

Figura B3 - Progetto alla scala sovracomunale dei sistemi locali della rete ecologica della provincia di Bologna (a sinistra); progetto alla scala comunale dei sistemi locali della rete ecologica della provincia di Bologna nel comune di Bentivoglio (stralci ridotti della cartografia originale dell'Ipotesi di Progetto di Rete Ecologica - da: Provincia di Bologna, Assessorato Ambiente, Servizio Pianificazione Paesistica, Piano programmatico per la conservazione e il miglioramento degli spazi naturali in provincia di Bologna, 2000). 


\section{Caso studio 3 - Lombardia (scala provinciale) Il piano della rete ecologica della provincia di Pavia ${ }^{151}$}

Tipologia e finalità dello studio

Studio tematico di riqualificazione e miglioramento ambientale compreso nel Piano faunistico-venatorio e di miglioramento ambientale previsto dalla Legge della Regione Lombardia del 16 agosto 1993 n. 26 "Norme per la protezione della fauna selvatica e per la tutela dell'equilibrio ambientale e disciplina dell'attività venatoria".

\section{Elaborazione}

Provincia di Pavia

Prof. S. Malcevschi, Università di Pavia

Periodo: 1995

\section{Area di studio}

Lo studio riguarda l'intero territorio della provincia, costituente il lembo meridionale della Lombardia, compreso fra Piemonte e Veneto. "La superficie complessiva è di $2.964,70 \mathrm{kmq}$ di cui $2 / 3$ sono costituiti da una vasta area di pianura che si estende da est ad ovest a cavallo di un asse principale costituito dal fiume Po. (...) La collina pavese occupa circa il $16 \%$ del territorio provinciale. (...) La demarcazione tra collina e montagna è di difficile individuazione, per consuetudine questa viene posta lungo la direttrice VarziPietragravina-Zavattarello. La porzione montana della provincia posta a sud di questa direttrice occupa circa il $10 \%$ della superficie provinciale complessiva".

Impostazione metodologica ed elementi di interesse relativi alla considerazione dei caratteri paesistici

L'analisi del paesaggio, condotta in termini esclusivamente ecologici, ha riguardato innanzitutto i caratteri generali dell'intero territorio provinciale, distinguendo le condizioni del paesaggio di pianura, collina e montagna, secondo criteri idrogeomorfologici, vegetazionali, floristici e faunistici.

II paesaggio è stato ulteriormente indagato in termini di censimento delle componenti ed analisi della struttura ecosistemica con "un'analisi mirata degli ecomosaici esistenti attraverso sopralluoghi, letture di aereofoto e consultazione di elaborati ed informazioni già esistenti".

I dati raccolti sono stati sintetizzati in una cartografia in scala 1:100.000 secondo le seguenti "tipologie ambientali: pioppeti; vigneti; risaie; formazioni vegetali resinose; formazioni vegetali a latifoglie di alto fusto; formazioni vegetali governate a ceduo; prati $e$ pascoli; incolti rocciosi; altri incolti; aree sabbiose; rocce e calanchi; zone umide; riserve naturali; altre aree naturali; corsi d'acqua; cave e discariche.

La lettura della carta delle unità ecosistemiche attuali ha consentito il riconoscimento alla scala definita e per territorio provinciale di ambiti ecologici caratterizzati da propri ecomosaici".

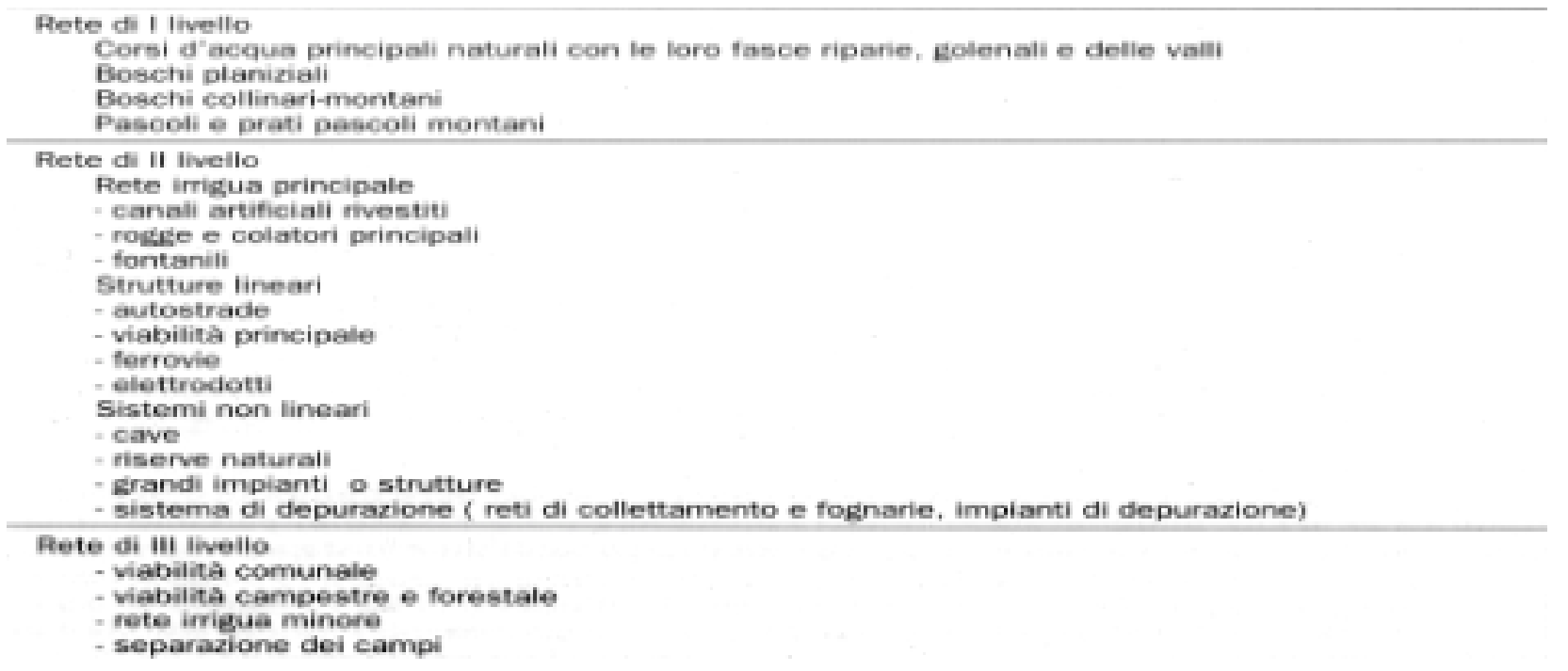

Figura B4 - Elementi di appoggio della rete ecologica della provincia di Pavia suddivisi per livelli di importanza. Gli interventi per l'equipaggiamento vegetale, la realizzazione di neoecosistemi, il miglioramento e la conservazione di ecosistemi di pregio, costituiscono una ricca serie di opportunità di riqualificazione diffusa del paesaggio, a patto che trovino una definizione progettuale completa in quanto derivante da un approccio di prog ettazione paesistica.

Il prog etto prevede due diversi sistemi di corridoi ecologici in relazione gerarchica e di complementarietà:

- "sistema dei corridoi ecologici diversificati imperniato sui principali corsi d'acqua" - dall'asse del Po la struttura della rete ecologica assume "una struttura a pettine attraverso la pianura, costituendo una serie di corridoi fondamentali connessi con quello padano, delimitando subsistemi di intervento" nei quali mirare ad ottenere la massima continuità biotica, possibile attraverso opere di "rinaturazione delle fasce laterali degli alvei".

151 Le citazioni riportate fra virgolette e le immagini sono tratte da Bisogni L.G., Gariboldi A., Malcevschi S., Reti ecologiche ed interventi di miglioramento ambientale, II Verde Editoriale, Milano, 1996. 
- "sistema integrativo di corridoi ecologici diversificati trasversali in grado di aggiungere valenze di tipo "terrestre", per una maggiore interconnessione di quelli portanti in modo da attenuarne la struttura a pettine" - questo sistema si colloca con andamento approssimativamente parallelo al corso del Po a circa $10 \mathrm{~km}$ da questo.

Il progetto direttore della rete ecologica è integrato da una serie di approfondimenti progettuali condotti per tipologia di intervento elaborando:

- "moduli tipo" relativi alle modalità di piantagione di alberi ed arbusti;

- "progetti tipo" relativi alle modalità di intervento attraverso l'applicazione dei necessari moduli.

Relativamente alle formazioni lineari sono stati definiti tre moduli tipo, utilizzati nel Progetto Tipo 1 - Impianto di siepi e filari: "A siepe arboreo-arbustiva lineare; B - siepe arboreo-arbustiva a struttura mediamente complessa; C - fascia arboreo-arbustiva".

Nell'ambito del disegno complessivo questo progetto interessa una superficie di 10 ha, tutti di paesaggio planiziale. Le componenti paesistiche direttamente interessate sono corsi d'acqua, viabilità rurale, divisioni di campi. La distribuzione prevista per il secondo e terzo modulo tipo fa riferimento ad una distanza maggiore di $100 \mathrm{ml}$.

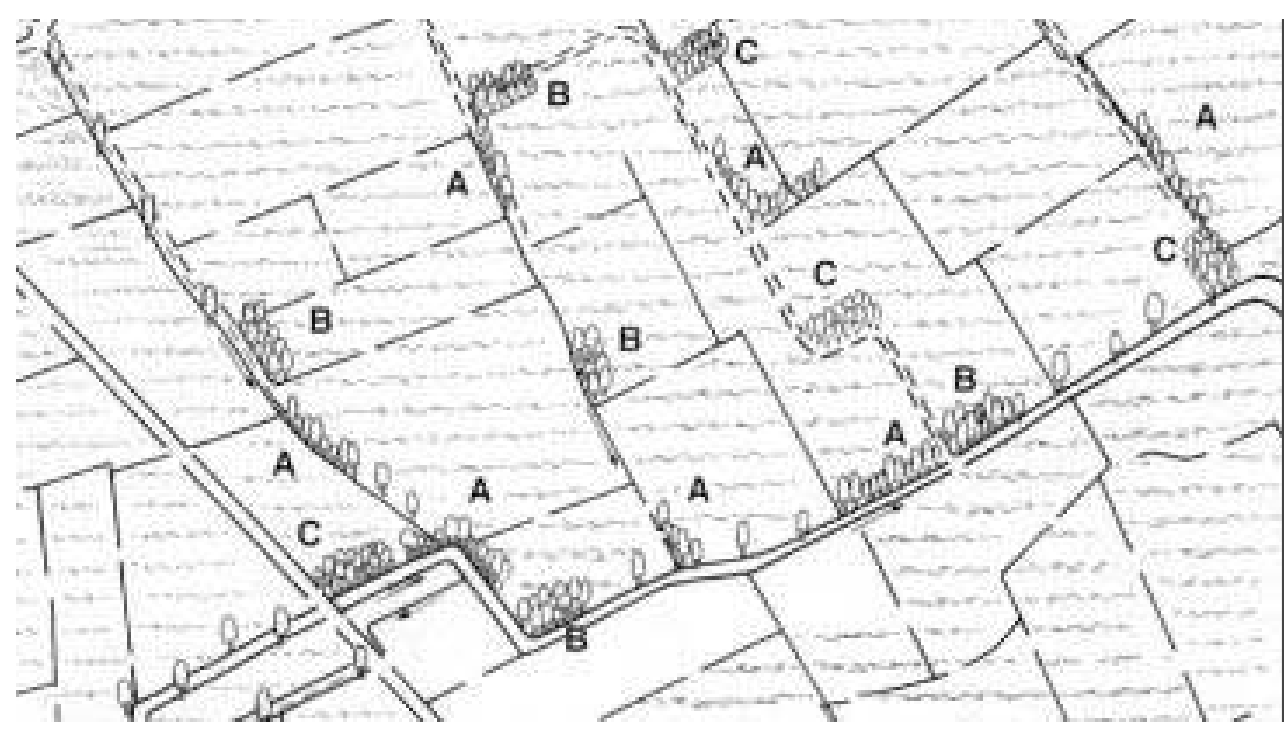

Figura B5 - Progetto di piantagioni lineari ottenuto con l'impiego dei moduli A, B, C esemplificativo della situazione in un'area di pianura ad intensa coltivazione con matrice paesistica caratterizzata dalle risaie. 
Caso studio 4 - Lombardia (scala provinciale metropolitana)

Studi di approfondimento del piano della rete ecologica della provincia di Milano: i corridoi ecologici di connessione tra i boschi del Ticino e l'ambito dei boschi e dei fontanili del sud-ovest di Milano e tra il Parco delle Groane ed il Parco della Valle del Lambro ${ }^{152}$

\section{Tipologia e finalità dello studio}

Studi di ricerca metodologica applicata per la definizione di linee guida per la pianificazione e la progettazione di corridoi ecologici nell'ambito delle fasce di reperimento indicate nel piano provinciale della rete ecologica. Gli studi hanno anto come base di riferimento la carta delle unità ecosistemiche e la carta della rete ecologica della Provincia di Milano.

\section{Elaborazione}

Agenzia Nazionale per la Protezione dell'Ambiente ANPA - Gruppo di lavoro per le Reti Ecologiche

Provincia di Milano - Ufficio di Pianificazione Paesistica

Periodo: 1998-2000

\section{Aree di studio}

Una prima area di studio (qui denominata A) comprende il territorio della pianura padana occidentale e più esattamente "il settore sud-ovest della provincia di Milano, delimitato dal fiume Ticino ad ovest, dalla SS 11 Milano-Novara a nord, dalla tangenziale di Milano e dal Naviglio Pavese a est, dai confini provinciali a sud. Le zone oggetto di studio ricadono nei territori di competenza del Parco Lombardo della Valle del Ticino e del Parco Agricolo Sud Milano. Interne a detta area di studio sono anche da segnalare, per la loro particolare valenza naturalistica, il Parco dei fontanili, che è un parco naturale di interesse sovracomunale nella zona di Bareggio e Cornaredo, e tre Siti di Interesse Comunitario (SIC): il Fontanile Nuovo, il Bosco di Cusago e i boschi de "La Fagiana".

L'altra area di studio considerata (qui denominata B) riguarda il settore nord della provincia, "delimitato dal Parco delle Groane a ovest, il Parco della Valle del Lambro a est, dai confini provinciali a nord e dal tratto di autostrada della MI-VE (A4) che va da Novate Milanese a Monza, a sud. Si tratta di un territorio di alta pianura, piuttosto povero in corsi d'acqua, che ha presentato negli ultimi decenni una fortissima espansione urbanistica ed industriale che rischia di produrre una completa sutura tra i vari centri abitati”.

Impostazione metodologica ed elementi di interesse relativi alla considerazione dei caratteri paesistici

$\mathrm{Nel}$ caso (A), ad una prima fase di ricerca bibliografica di approfondimento delle conoscenze edite sui caratteri vegetazionali, faunistici ed ecologici della zona, è seguita una fase di approfondimento conoscitivo e di interazione con gli operatori presenti sul territorio, sia pubbliche amministrazioni che aziende agricole, utilizzando questionari opportunamente costruiti. La verifica cartografica di dettaglio in scala 1:5000 dei progetti, vincoli ed opportunità ricadenti nell'area di studio ha portato alla individuazione di maggior dettaglio delle direttrici in cui sono stati effettuati ulteriori rilievi ecologici di approfondimento conoscitivo, con valutazioni di idoneità faunistica attraverso specie guida.

Le tipologie di intervento proposte costituiscono un programma complesso di misure di miglioramento paesistico-ambientale:

_ "ampliamento delle unità ambientali boschive con presenza di specie guida o già in parte potenzialmente idonee per esse;

- incremento delle superfici con copertura legnosa delle aree attualmente insufficienti rispetto alle esigenze ecologiche delle specie guida (stepping stones);

- miglioramenti ambientali diffusi in aree ag ricole per il miglioramento della connettività all'interno delle fasce dei corridoi;

- ricostituzione e/o miglioramento delle fasce ripariali dei corsi d'acqua;

- costituzione di fasce buffer ripariali lungo i principali corsi d'acqua delle direttrici con l'obiettivo di:

a. ottenere strutture efficaci per la formazione degli elementi costitutivi del corridoio;

b. migliorare la compatibilità ambientale di opere esistenti;

c. contribuire al miglioramento della capacità di autodepurazione del territorio nei confronti dell'inquinamento diffuso;

- miglioramento dell'inserimento ambientale delle aree di cava;

- riduzione delle interferenze reciproche e miglioramento delle fasce di contatto tra aree urbanizzate ed aree agricole;

- previsione di ecosistemi filtro per la riduzione degli impatti negativi dei residui derivanti dai sistemi di trattamento delle acque;

- miglioramento della permeabilità delle barriere più significative presenti”.

$\mathrm{Nel}$ caso (B), "l'elevata frammentazione non è un problema solo faunistico, ma riguarda direttamente la vivibilità dei luoghi da parte dell'uomo e la qualità urbana in generale. In questo contesto la realizzazione di una rete ecologica (...) può diventare un reale strumento per la riorganizzazione di alcune parti di territorio, a patto di incidere sugli assetti urbanistici che troppo spesso hanno seguito, invece che pianificato, lo sviluppo urbano e non hanno mai considerato le funzioni di servizio degli ecosistemi anche nei confronti dei sistemi antropici." La complessità dell'area ha richiesto una prima articolazione in ambiti cartografati in scala 1:25000 e schedati. Questa prima analisi ha permesso attraverso l'interpretazione della cartografia e delle foto aeree la definizione preliminare degli ambiti in cui approfondire l'analisi che è stata successivamente condotta con rilievi di campagna ed elaborazione di cartografia in scala 1:5000. Anche per l'analisi faunistica "la scelta delle specie guida è stata funzionale alla necessità di scomporre il sistema ambientale secondo diversi livelli di complessità (scale) sia in senso strutturale che in senso spaziale".

Elementi di interesse per il tema specifico della ricerca con riferimento alla tipologia del corridoio nell'ambito delle problematiche della frammentazione paesistica

Nel primo caso (A), sono stati previsti diversi tipi di interventi: piantagioni vegetali (formazioni boschive ripariali, fasce boschive igrofile, fasce boschive mesofile, fasce arbustive termoeliofile di mantello, cotico erbaceo, ed "un modello di successione dinamicoevolutiva direttamente influenzata dal gradiente acqua"), manufatti infrastrutturali e sistemi di superamento di corsi d'acqua per la fauna, interventi di miglioramento ambientale legati alle pratiche agricole, ecosistemi filtro costituiti da ambienti palustri, "oasi familiari" periurbane. "Nelle parcelle individuate dovranno essere messi in atto interventi di infrastrutturazione (sentieri) e di arredo

152 Le citazioni riportate fra virgolette sono tratte dalla relazione della ricerca ANPA Progetto Monitoraggio Reti Ecologiche (programma triennale 1998-2001); le immagini relative al progetto della rete ecologica provinciale sono tratte da Romano B., 2000, Continuità ambientale. Pianificare per il riassetto ecologico del territorio, Andromeda Editrice, Teramo. 
ecologico (siepi, filari) che definiranno l'unità di fruizione. Tutti questi moduli progettuali sono stati poi calati all'interno dei corridoi individuati precedentemente rappresentando sempre in scala 1:5000, le aree dove si prevedono i vari interventi (...) L'esecuzione delle proposte progettuali non è stata configurata con un sistema di vincoli aggiuntivi e presenta un certo grado di flessibilità attuativa, in modo da essere vista come un orientamento ed un obiettivo da rag giung ere più che come un obbligo di azione".

Nel secondo caso (B), la forte pressione insediativa ha suggerito la definizione di un sistema di corridoi gerarchizzato con componenti primarie, con funzioni prioritarie per la funzionalità faunistica, e componenti secondarie, a cui sono affidate sia funzioni di complemento dei corridoi primari, in termini di zone buffer e/o tampone per la mitigazione dei disturbi antropici, sia funzioni assimilabili a quelle delle aree sink, sia funzioni, strettamente legate al paesaggio urbano e dell'urbanizzazione diffusa, di servizi ricreativi ed attività agricole compatibili, nonché di generici spazi verdi a servizio del tessuto urbano denso.

In entrambi questi casi, nel primo in maniera del tutto esplicita, il corridoio si configura come strategia unitaria di riqualificazione e mig lioramento paesistico, articolata in una serie differenziata di interventi complementari, multifunzionali o specializzati.

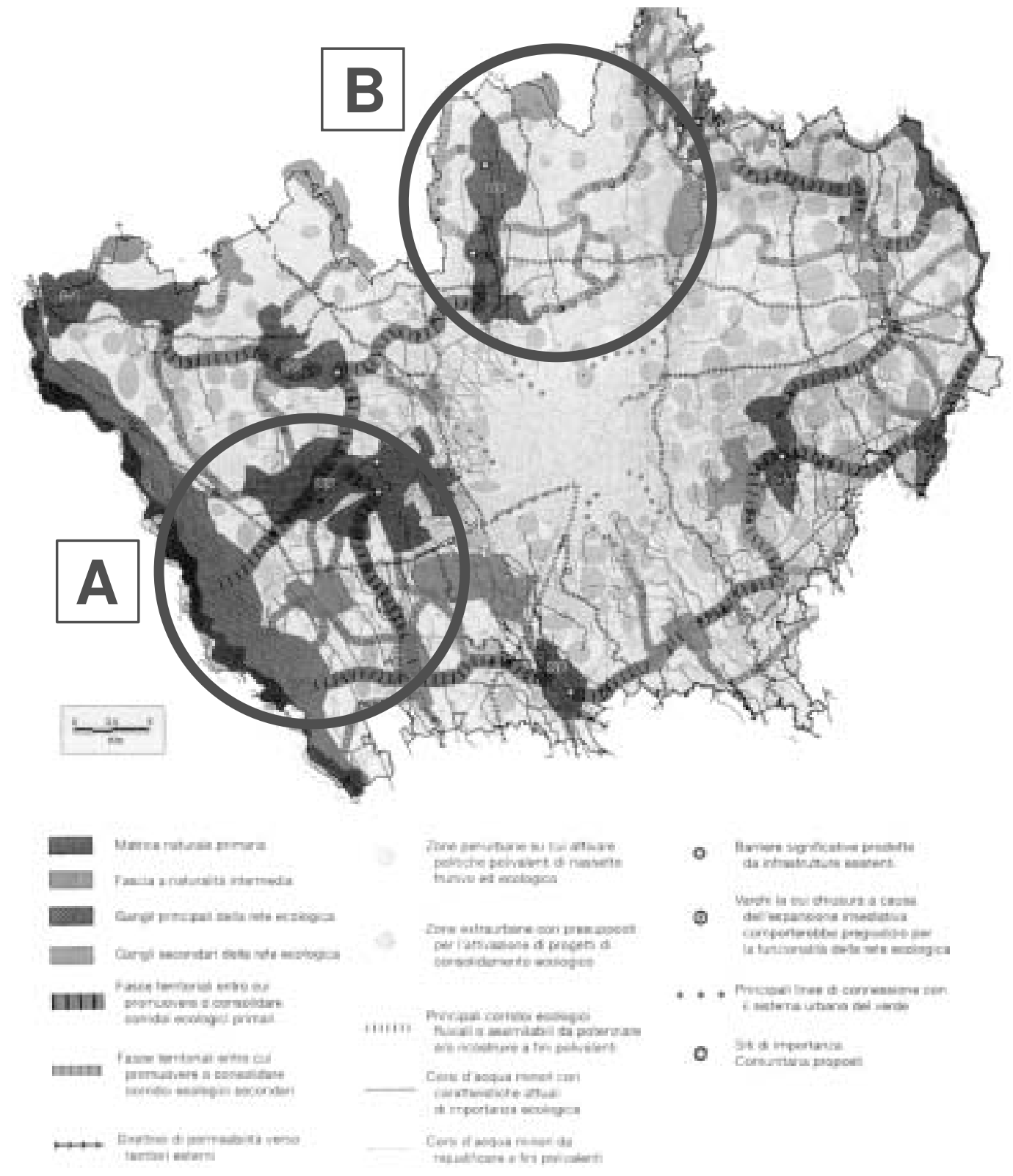

Figura B6 - Progetto generale della Rete Ecologica della Provincia di Milano costituente parte del relativo Piano Territoriale di Coordinamento Provinciale (i cerchi e gli indici A e B si riferiscono al territorio dei casi studio qui considerati). 


\section{Caso studio 5 - Abruzzo (scala regionale) Idoneità territoriali per i corridoi ecologici dell'Appennino Centrale $e^{153}$}

Tipologia e finalità dello studio

Studio di ricerca metodologica applicata per la definizione dei sistemi di continuità ambientale dell'Appennino Centrale per la connessione delle aree protette e la costituzione della rete ecologica regionale.

\section{Elaborazione}

Ministero dell'Università e della Ricerca Scientifica (ricerca di interesse nazionale)

Coordinatore nazionale: Prof. R. Gambino

Coordinatore unità locale dell'Università dell'Aquila: Prof. G. Tamburini

Componente del Gruppo di lavoro: Prof. B. Romano

Periodo: 1996

\section{Area di studio}

Lo studio riguarda il territorio regionale abruzzese, notoriamente caratterizzato da elevato grado di naturalità, ma allo stesso tempo interessato da fenomeni di frammentazione ambientale reale e potenziale non trascurabili, proprio in ragione dei valori presenti e dei requisiti di efficacia dei relativi sistemi di protezione (parchi e riserve naturali).

Impostazione metodologica ed elementi di interesse relativi alla considerazione dei caratteri paesistici

La ricerca riguarda innanzitutto la "valutazione preliminare di quegli ambiti per i quali è ancora ipotizzabile una funzione biologica significativa, quanto meno in termini di collegamento. Gli elementi che sono stati interpretati al fine di determinare un livello qualitativo di biopermeabilità degli spazi territoriali sono sostanzialmente le modalità di uso del suolo e le barriere infrastrutturali ed insediative".

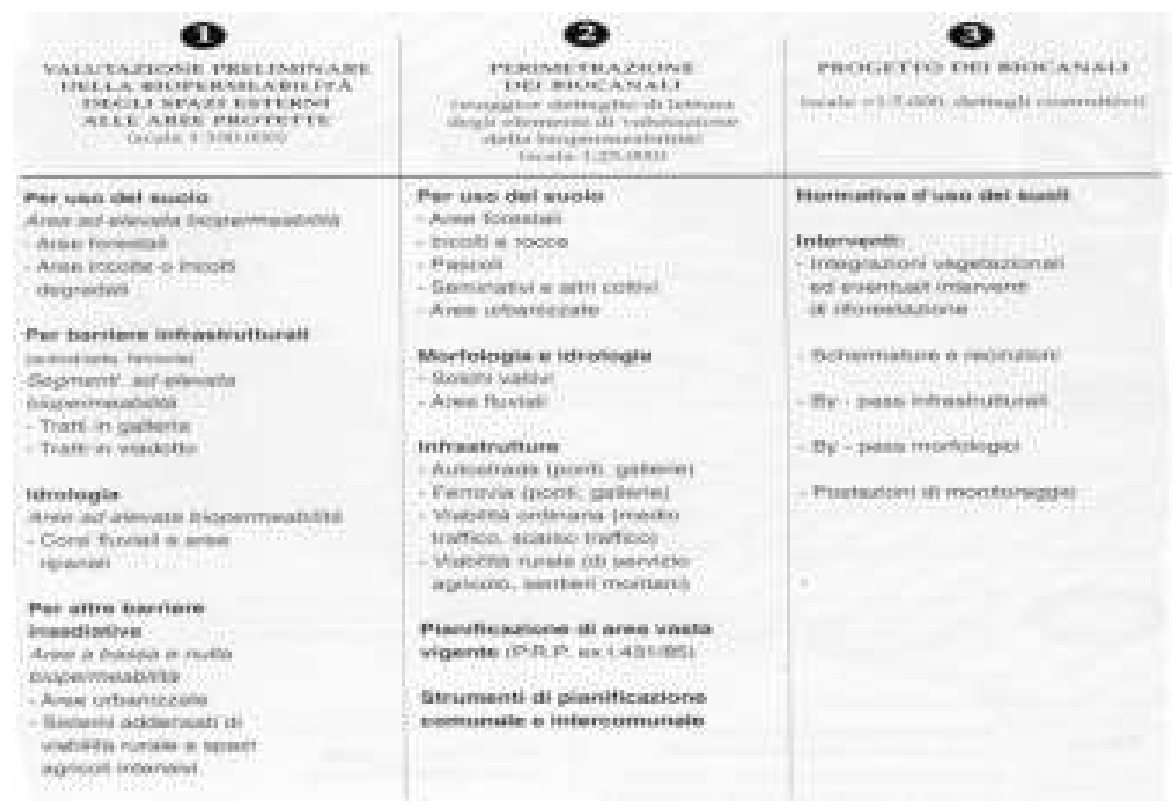

Figura B7 - Schema generale della ricerca.

"Poste in evidenza le grandi aree protette della regione, l'attenzione è stata centrata sull'insieme degli ambiti interstiziali, correlando la biopermeabilità ag li aspetti di uso del suolo, alle barriere infrastrutturali e alle barriere insediative".

Lo studio affronta le problematiche della frammentazione ambientale dal punto di vista tematico faunistico delle limitazioni ed opportunità di spostamento e stazionamento delle popolazioni animali, ma "(...) le condizioni che sono state rilevate non hanno considerato particolari habitat legati a specifiche zoocenosi (...) - questo approfondimento potrà (...) awenire con l'apporto sul tema di competenze disciplinari specifiche".

II modello interpretativo adottato ha una chiara matrice di pianificazione ambientale, per la quale la diagnosi di biopermeabilità awiene attraverso la lettura delle condizioni del paesaggio e la conseguente associazione di un grado più o meno elevato di permeabilità biotica, ancor prima che attraverso rilievi ecolog ici specialistici.

${ }^{153}$ Le citazioni riportate fra virgolette e le immagini sono tratte da Romano B., Oltre i parchi. La rete verde regionale. Una ricerca sulle idoneità territoriali per $i$ corridoi ecologici dell'Appennino Centrale, Università degli Studi dell'Aquila, Dipartimento di Architettura e Urbanistica, Andromeda Editrice, Teramo, 1996. Si trovano inoltre notizie su questi studi in Romano B., Continuità ambientale. Pianificare per il riassetto ecologico del territorio, Andromeda Editrice, Teramo, 2000. 
Questo fa sì che la valenza delle considerazioni analitiche e progettuali sia ben più ampia della specifica motivazione primaria, costituendo un insieme organico di considerazioni sulla continuità paesistico-ambientale della regione e sulle relative necessità/opportunità di salvaguardia e sviluppo.

Questo approccio è stato ulteriormente sviluppato nell'ambito della ricerca nazionale PLANECO Project, attraverso l'impostazione su tecnologie informatiche GIS, applicate in Abruzzo nell'ambito della ricerca "La continuità ambientale in Abruzzo. Riferimenti e criteri metodologici per la pianificazione della rete ecologica regionale". ${ }^{154}$

Elementi di interesse per il tema specifico della ricerca con riferimento alla tipologia del corridoio nell'ambito delle problematiche della frammentazione paesistica

"L'obiettivo della ricerca condotta è stato quello di definire una fisionomia territoriale attendibile per i biocanali regionali attraverso lo studio dei principali fattori che condizionano la biopermeabilità, almeno in termini generali".

"Relativamente alle tipologie di uso del suolo sono state evidenziate quelle alle quali corrisponde teoricamente il minor grado di disturbo o nelle quali sia più facile per le specie animali dissimulare la propria presenza e trovare rifugio. Per questo motivo sono state poste in rilievo le aree forestali, quale ambiente indubbiamente elettivo per la maggior parte delle specie faunistiche, e le aree incolte degradate. Queste ultime, oltre ad essere soggette probabilisticamente a limitati effetti di disturbo, in quanto non più asservite ad utilizzazione antropica, spesso presentano anche la caratteristica di contenere numerose specie vegetali, in particolare arboree, tipiche degli ex coltivi, spesso fonte di cibo per alcuni animali. (...) Sulla base di questa considerazione, tendente a classificare gli ambiti biopermeabili sulla scorta del livello di disturbo antropico probabilisticamente riscontrabile in essi, è evidente che le aree intensamente coltivate o urbanizzate corrispondono ad una limitatissima biopermeabilità. II secondo aspetto considerato riguarda le barriere infrastrutturali. Da questo punto di vista è stata considerata, quale elemento decisamente condizionante la biopermeabilità l'autostrada che, a differenza di ogni altro tipo di infrastruttura, è fisicamente insuperabile a meno dei tratti in galleria o in viadotto. (...) Altre barriere di rilevante peso sono poi quelle più genericamente insediative: le aree urbanizzate e $\mathrm{i}$ sistemi addensati di infrastrutture per uso agricolo e rurale associate a spazi agricoli intensivi. (...) Un esame della regione condotto sulla base di questi presupposti ha posto in luce la presenza di alcuni e generalmente ben definiti spazi territoriali che assolvono già spontaneamente la funzione di biocanali, e di alcuni altri per i quali invece la funzione è solo potenziale e va pertanto attivata mediante interventi progettuali finalizzati”.

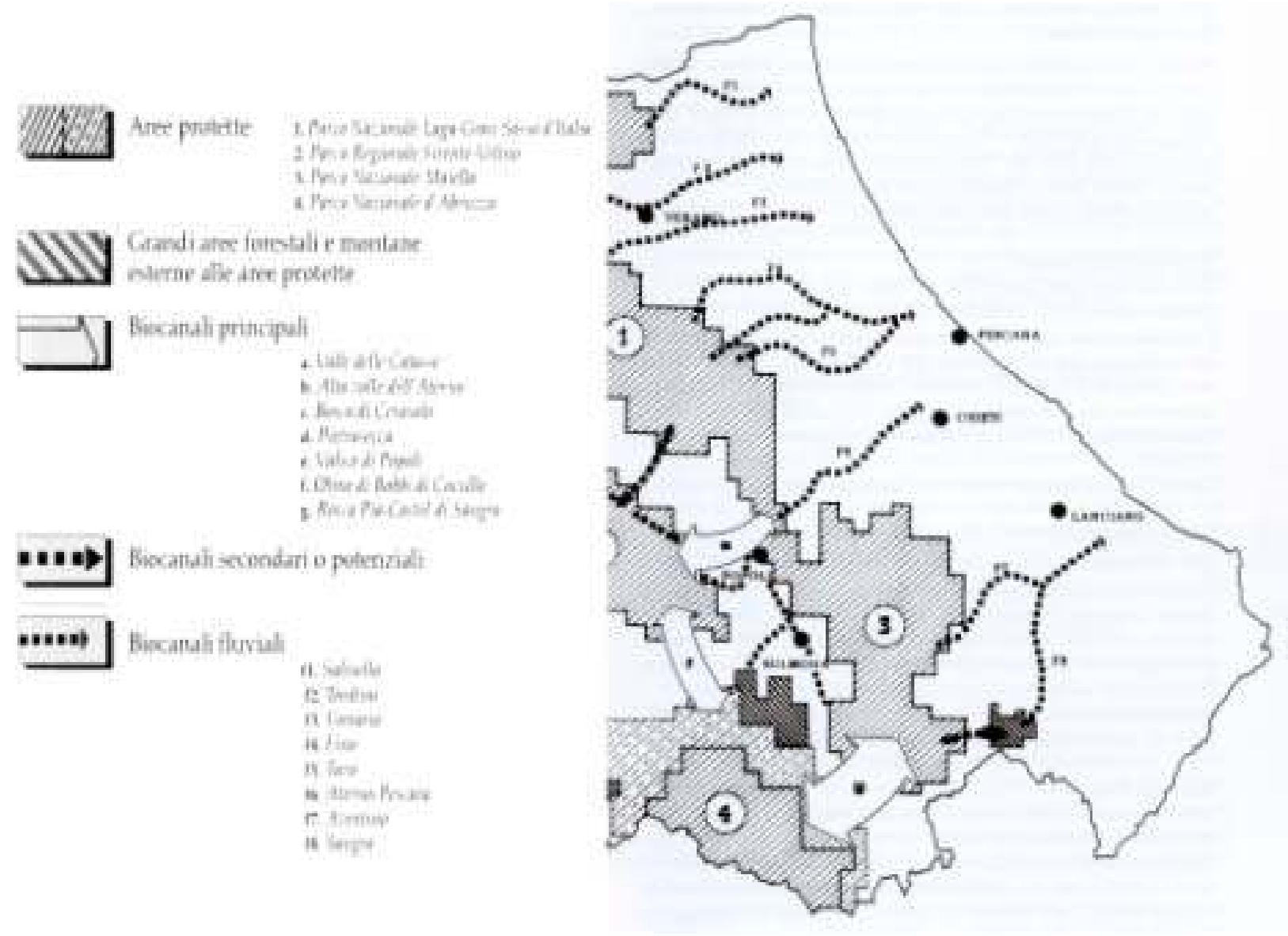

Figura B8 - Schema regionale dei biocanali: costituisce uno scenario di base per la definizione del quadro strategico dei sistemi di continuità ambientale tra le aree protette e tra queste ed il territorio soggetto al regime ordinario dei Piani Regolatori Generali Comunali e dei vari piani di settore - stralcio.

154 La ricerca è stata promossa dall'ANPA nell'ambito del Progetto Monitoraggio Reti Ecologiche (programma triennale 1998-2001) di concerto con la Regione Abruzzo ed elaborata dal Dipartimento di Architettura e Urbanistica dell'Università dell'Aquila. 
